\title{
GEOTECHNICAL BOREHOLE AND WELL-LOG DATABASE FOR ANCHORAGE, ALASKA
}

\author{
by \\ R.A. Combellick, J.L. Mayer, K.D. Sink-Blair, and S.M. Weems
}

\begin{abstract}
THIS REPORT HAS NOT BEEN REVIEWED FOR TECHNICAL CONTENT OR FOR CONFORMITY TO THE EDITORIAL STANDARDS OF DGGS
\end{abstract}

Alaska Department of Natural Resources Division of Geological \& Geophysical Surveys 794 University Ave. \#200

Fairbanks, Alaska 99709

February 2001

Research supported by the U.S. Geological Survey (USGS), Department of the Interior, under USGS award number 98HQGR1047. The views and conclusions contained in this document are those of the author and should not be interpreted as necessarily representing the official policies, either expressed or implied, of the U.S. Government. 



\title{
GEOTECHNICAL BOREHOLE AND WELL-LOG DATABASE FOR ANCHORAGE, ALASKA
}

\author{
R.A. Combellick, J.L. Mayer, K.D. Sink-Blair, and S.M. Weems \\ Alaska Division of Geological \& Geophysical Surveys \\ 2001
}

\begin{abstract}
The Alaska Division of Geological \& Geophysical Surveys has finished the compilation of subsurface geotechnical-borehole and well-log data for Anchorage, Alaska, in a Geographic Information System (GIS) database. Many private companies and government agencies contributed to this database. It contains downhole geologic or geotechnical data for more 2,500 boreholes and water wells, plus locations and reference information for an additional 1,700. We believe that the database is complete for geotechnical boreholes $50 \mathrm{ft}$ or deeper and for available digital data from water wells $100 \mathrm{ft}$ or deeper; data for many shallower holes are also included. When remaining issues of distribution formats and permissions are resolved with contributing organizations, nonproprietary data will be made available to the public in digital and hard-copy formats.
\end{abstract}

\section{INTRODUCTION}

The objectives of this 2-phase project are to (1) complete the compilation of geotechnical borehole data for Anchorage, Alaska, and make the nonproprietary data accessible to the public, (2) produce a detailed (1:25,000-scale) liquefaction-susceptibility map of Anchorage, and (3) produce a map of NEHRP $^{1}$-recommended seismic soil-types for Anchorage (also at 1:25,000 scale). The GIS database and resulting maps will be useful to engineers, developers, planners, and emergency managers in Anchorage for construction planning, site selection, structural design, and emergency-response planning. Our previous NEHRP-supported project (Engineering-geology maps and cross sections of Anchorage, Alaska; award 1434-HQ-96-GR-02713) resulted in compilation of more than 1,500 geotechnical borehole logs, 1,500 digital water-well logs, mostly for central and east Anchorage, and publication of a geologic map and seven cross sections for that area (Combellick, 1999). This is the final report for the first phase of this project, item (1) above. We have completed entry of the geotechnical data for west Anchorage, and have added many shallow boreholes and recently-drilled boreholes for the entire area. This completes the subsurface database for the entire Anchorage study area, including entry of downhole data for all known geotechnical boreholes deeper than $50 \mathrm{ft}$ and all available digital well-log data for water wells deeper than $100 \mathrm{ft}$. Additionally, we have manually entered more than 200 shallow $(<50 \mathrm{ft})$ boreholes and nondigital well-log data to supplement the database in areas of sparse coverage. Hundreds of shallow boreholes for which downhole data have not been entered are nonetheless located and indexed in GIS for rapid retrieval. The current status of data compilation is shown in Table 1. These data will be used to complete a simplified geologic map and cross sections for the entire city.

A complementary project is being conducted by the University of Alaska Geophysical Institute (UAGI) to collect and analyze seismic data for the purpose of generating seismic siteamplification maps. The project, Seismic Microzonation of Metropolitan Anchorage (Biswas, 1997), which is funded by the Alaska Council on Science and Technology, is making extensive

\footnotetext{
${ }^{1}$ National Earthquake Hazard Reduction Program of the Federal Emergency Management Agency and U.S. Geological Survey
} 
Table 1 - Status of subsurface data compilation for Anchorage, Alaska, as of December 2000.

$\begin{array}{lccc} & \begin{array}{c}\text { Geotechnical } \\ \text { Holes* }\end{array} & \text { Water } & \text { Wells } \\ \text { Located \& indexed } & 2,816 & 1,426 & 4,242 \\ \text { Lithologic (stratigraphic) log } & 1,147 & 1,357 & 2,504 \\ \text { Penetration test data } & 906 & -- & 906 \\ \text { Laboratory analysis data } & 602 & -- & 602 \\ \text { Seismic velocity } & 13 & -- & 13 \\ \text { Static water level } & 799 & 1,271 & 2,070 \\ \text { *includes test pits and natural geologic exposures } & & & \end{array}$

use of subsurface data from this NEHRP project and, in turn, will provide important shear-wave velocity data that will be used along with standard penetration test data from the borehole database for the delineation of NEHRP seismic soil types. In phase 2 of the project, for which funding was recently awarded (USGS award 01HQGR0006), we will use the database to complete items (2) and (3) above (liquefaction-susceptibility map and seismic soil-type map). The seismic soil-type map is being prepared in cooperation with the UAGI to make optimum use of available shear-wave velocity data.

\section{METHODS OF DATA COMPILATION}

We obtained borehole and well-log data from the files of private geotechnical consulting firms and from federal, state, and local agencies (see Acknowledgments). Many of the reports obtained from private companies contain proprietary data because the drilling was performed under contract to private individuals or companies that have not released the data for public use. Under Alaska state law, we are authorized to retain as proprietary any data sets that the providing companies request to remain proprietary; these companies give the Alaska Division of Geological $\&$ Geophysical Surveys (DGGS) permission to use the data internally for interpretive purposes in preparing maps and reports but not permission to distribute the original data in any form.

Our goals were to compile not only sufficient data to prepare geologic cross sections, a liquefaction-susceptibility map, and seismic soil-type map, but to make provide nonproprietary subsurface data on request to researchers, planners, designers, and developers. We strived for a level of completeness that would include all known geotechnical boreholes of $50 \mathrm{ft}$ and greater depth. Although not complete, we have also entered data for several hundred shallower boreholes to provide near-surface information where few deeper boreholes have been drilled.

Similarly, for water wells, manual entry of thousands of well logs was beyond the scope of this project. Therefore, our goal for water wells was to ensure completeness for all available digital well-log data from wells $100 \mathrm{ft}$ and deeper. Although some wells were manually entered where needed and available, the bulk of the water-well database comes from the U.S. Geological Survey's digital Ground Water Site Index (GWSI). Unfortunately, because the GWSI database has not been maintained in recent years, these data are mostly pre-1988. Several more recent deep water wells, particularly in the downtown area and near Anchorage International Airport, have been manually entered. 
We entered borehole data into a Microsoft Access database, which was linked to MapInfo GIS software through an open database connection (ODBC). The relational database in Access consists of the individual data tables listed in table 2. With the exception of median grain diameter, which is given in millimeters, all data are reported in English units because this is the system which, until very recently, has been used almost universally by geotechnical engineering organizations in the U.S.

Table 2 - Content of data tables in relational database. Not all data are available for all boreholes (see table 1).

\begin{tabular}{|c|c|c|c|}
\hline Table & Description & $\underline{\text { Key field(s) }}$ & $\underline{\text { Records }}$ \\
\hline Reports & $\begin{array}{l}\text { Project information (organization, } \\
\text { project number, report year, maximum } \\
\text { borehole depth, where filed); one record } \\
\text { per report or project }\end{array}$ & $\begin{array}{l}\text { Project number (linked } \\
\text { to Borehole) }\end{array}$ & 671 \\
\hline Borehole & $\begin{array}{l}\text { Index data (DGGS ID, project number, } \\
\text { hole name, hole type, UTM location, } \\
\text { location accuracy, reported elevation, } \\
\text { date drilled, total depth, types of data } \\
\text { entered); one record per borehole }\end{array}$ & $\begin{array}{l}\text { Project number (linked } \\
\text { to Reports), DGGS ID } \\
\text { (linked to all other } \\
\text { tables) }\end{array}$ & $4,479 *$ \\
\hline Lithology & $\begin{array}{l}\text { Lithologic logs (DGGS ID, depth to top, } \\
\text { depth to bottom, lithologic description, } \\
\text { unified soil class); one record per layer }\end{array}$ & $\begin{array}{l}\text { DGGS ID (linked to } \\
\text { Borehole) }\end{array}$ & 15,392 \\
\hline SPT data & $\begin{array}{l}\text { Standard penetration test data (DGGS } \\
\text { ID, depth to top, depth to bottom, } \\
\text { material type, blows/ft, sample diameter, } \\
\text { hammer weight, drop distance); one } \\
\text { record per test }\end{array}$ & $\begin{array}{l}\text { DGGS ID (linked to } \\
\text { Borehole) }\end{array}$ & 8,982 \\
\hline Sample Test & $\begin{array}{l}\text { Laboratory analytical data (DGGS ID, } \\
\text { depth to top, depth to bottom, unit } \\
\text { weight, dry density, moisture content, } \\
\text { liquid limit, plastic limit, plasticity } \\
\text { index, median grain diameter, percent } \\
\text { gravel, percent fines, percent clay); one } \\
\text { record per sample }\end{array}$ & $\begin{array}{l}\text { DGGS ID (linked to } \\
\text { Borehole) }\end{array}$ & 5,680 \\
\hline Water level & $\begin{array}{l}\text { Measured water level (DGGS ID, date } \\
\text { measured, depth to water, comments); } \\
\text { one record per measurement) }\end{array}$ & $\begin{array}{l}\text { DGGS ID (linked to } \\
\text { Borehole) }\end{array}$ & 2,152 \\
\hline Velocity & $\begin{array}{l}\text { Seismic velocity (DGGS ID, depth to top } \\
\text { of interval, depth to bottom of interval, } \\
\text { shear-wave velocity); one record per } \\
\text { interval }\end{array}$ & $\begin{array}{l}\text { DGGS ID (linked to } \\
\text { Borehole) }\end{array}$ & 164 \\
\hline
\end{tabular}




\section{Borehole Records}

With the exception of digital well logs, for which geographic positions were provided, all borehole locations were digitized in GIS from project site maps provided with the drilling reports. The scale and detail of these site maps are highly variable, resulting in some variation in accuracy of the digitized locations. The borehole location, in Universal Transverse Mercator (UTM) coordinates, was recorded in the Borehole header record in the database (see table 2), along with a code indicating relative accuracy. In addition to the reported elevation, if any, a corrected elevation was derived from the digital elevation model (DEM), based on the borehole's location in GIS. This corrected elevation was derived for every borehole except those in areas where artificial fill had been emplaced after the drilling date and before the issue date of the topographic map on which the DEM was based. The purpose of including the DEM elevations was to enable automated placement of the borehole logs on a cross-section view in their correct positions relative to the surface topography.

The DEM elevations helped identify erroneous locations or elevations recorded in the digital water-well data as obtained from USGS Ground Water Site Inventory. By obtaining original well logs, we determined that where a reported elevation differed significantly from the DEM elevation, it was usually because the location was in error, most often by one township (6 mi).

After digitizing the hole location in GIS and automatically recording the UTM coordinates in the Borehole table, other general information about the borehole or well was also recorded in the Borehole record (see table 2). The original hard-copy borehole data are retrievable through a link to the Reports records provided by the project number. Original reports are organized by Municipality of Anchorage grid square number.

\section{Lithology records}

The most basic geotechnical information entered for each borehole is the stratigraphic description, or lithologic log. For more than half of the geotechnical boreholes, the lithologic log is the only type of data available (see table 1). However, for every borehole for which any other data are recorded (sample analyses, penetration data, etc.), there is also a lithologic log in the database. Lithologic logs were entered verbatim, one data record per layer, except that technical details of the drilling process were excluded, as were codes regarding the frozen versus unfrozen state and frost susceptibility of the material. In a few cases, lithologic logs consisted only of individual sample descriptions. In these cases, we subdivided the logs into logical stratigraphic layers, estimated the depths of stratigraphic contacts, and wrote composite descriptions from the sample descriptions.

\section{$\underline{\text { SPT data records }}$}

Although procedures for the standard penetration test are prescribed by the American Society for Testing and Materials (ASTM, 2001), methods of reporting the results vary widely among different organizations. Because this database provides a capability for providing penetration data from multiple sources for analytical purposes, such as evaluating the susceptibility of an area to earthquake-induced liquefaction, we devoted considerable effort to ensuring consistency of data reporting and excluding tests that did not appear to conform to the ASTM standard.

The standard penetration test consists of driving a 2.0-in outside diameter (O.D.) split-spoon sampler with a 140-lb weight that is repeatedly dropped from a height of $30 \mathrm{in}$. Two purposes are achieved during the test: collecting a sediment sample and determining the penetration resistance of the material. The driller counts the number of blows required to drive the sample each 6-in. interval for a total of $18 \mathrm{in}$. The reported penetration resistance, or blow count, is the sum of blows for the second and third 6-in. intervals. Because some penetration tests are made with 
larger samplers (typically 3-in. O.D.) and heavier hammers (typically $340 \mathrm{lb}$ ), we recorded the sampler size, hammer weight, and drop distance for each test.

In most reports, the test result was given in blows per foot. In others, the blow count was reported as the driller recorded it for each 6-in. or, in a few cases, 3-in. interval. In these latter cases, we calculated the blow counts from the given data so that all values we recorded in the database represented the sum of blows in the second and third 6-in. intervals as required by the ASTM standard. From the lithologic or textural data, we also recorded material characteristics for each test that are significant for interpreting the penetration value: predominantly sand with minor gravel, high gravel content, predominantly silt or clay, or high organic content. For many, but not all tests, grain-size data are also available in the sample test records.

For many split-spoon sampling intervals, the penetration test was incomplete. The driller stopped the count because of very hard material or refusal, or the material was so soft that the sampler penetrated it with a single blow or under the weight of the drill stem with no blows required; an 'easy push' condition. In the case of very hard material, the driller may have recorded a high blow count but not for the standard 12-in. interval of an 18-in. test. The recorded number may have been for only for the first few inches of penetration. Although the reported value is not a standard value according to the ASTM standard, we did not wish to disregard the test because knowing the relative hardness of the material is important information for interpreting its engineering characteristics. Similarly, we did not wish to discard 'easy push' information or very low blow counts because they give important information about the softness of the material. To accommodate both types of data, we developed a decision tree (fig. 1) to allow

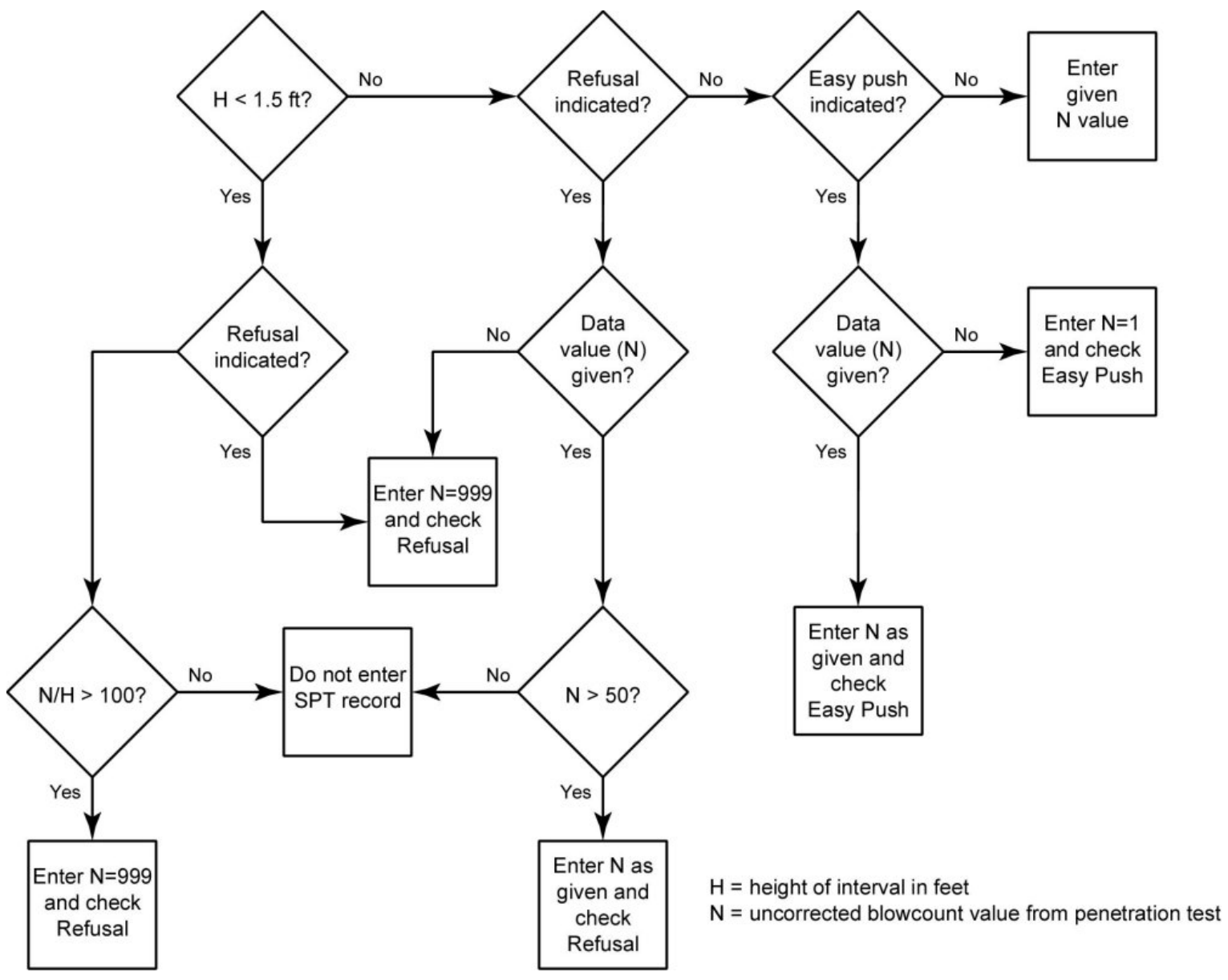

Figure 1 - Decision tree for entering standard penetration test data. 
for consistent reporting of data for both very hard and very soft materials. Among other uses, this allows the data to be used to develop graphical displays of penetration resistance with depth that convey information about the relative hardness of the material.

Although use of the decision tree resulted in some data for soft or hard materials being discarded because of ambiguous reporting, the remaining data that could not be reported in terms of standard blow counts according to ASTM were given either a value of 1, for easy push condition, or 999 , for very hard or refusal condition.

\section{Sample test records}

We recorded laboratory analytical data for individual samples where available. Data most commonly available included grain-size distribution, moisture content, Atterberg limits, and either wet unit weight or dry density. Each of these analyses is associated with a sample for which depth to top and depth to bottom were recorded in the database.

Grain-size data were typically obtained from sieve analyses and presented either in tabular or graphic form showing cumulative weight percent passing. Rather than attempting to record the entire analysis, we recorded percent gravel (retained on no. 4 sieve), percent fines (passing no. 200 sieve), and percent clay (portion of fines smaller than $0.005 \mathrm{~mm}$ ) where available. The user can calculate percent sand by subtracting from 100 the total percent gravel and fines. If the data were presented graphically, we also determined and recorded the median grain diameter in millimeters (diameter at which the curve crosses the 50-percent line; half of the sample by weight is coarser or finer than this diameter).

We recorded moisture content only if Atterberg limits or density data were also provided. Atterberg limits (liquid limit and plastic limit) were recorded as given. Plasticity index was calculated automatically as the difference between liquid and plastic limits. For some samples, more than one Atterberg limit test was performed and recorded.

Wet unit weight or bulk density values are not commonly provided but were recorded where available. From all available data sources, density values are given in pounds per cubic foot. If wet unit weight was not given but dry density and moisture content were provided, we used a macro to automatically calculate and enter the wet unit weight, $\gamma_{w}$, using the formula $\gamma_{w}=$ $\gamma_{d}(1+w)$, where $\gamma_{d}$ is the dry density and $w$ is the fractional moisture content (Lambe and Whitman, 1969).

For a small fraction of samples, other data are given such as shear strength and sensitivity. Because of the wide variety of methods used to determine these values, we did not feel confident in including them in this database. These analyses may be added at a later date.

\section{Water level records}

We recorded depth to water wherever reported in a borehole or well log. In a few cases, more than one depth was recorded because the measurement was made at different times, such as one while drilling and one after drilling. Recorded data include date of measurement, depth to water, and, where provided, any comments regarding conditions or timing of the measurement.

\section{Velocity records}

Continuous downhole shear-wave velocity measurements were available for 13 boreholes in the Anchorage area. Each data record contains depth to top of interval, depth to bottom of interval, and reported shear-wave velocity in feet per second. 


\section{INDEX MAP AND TABLE}

Sheet 1 shows locations, hole types, and total depths of all boreholes and well logs in the database. Also shown on this map is the Municipality of Anchorage grid system, which divides the city into numbered quarter-sections that are $0.5 \mathrm{mi}$ by $0.5 \mathrm{mi}$ square. We used GIS to automatically determine the grid number for each borehole and to record these numbers in the borehole records. All original hard-copy data reports are filed by this number to facilitate retrieval. In areas where there are no assigned grid squares, we determined the grid numbers by extrapolating the municipality's numbering system.

Table 3 (in .pdf and .xls formats on 3.5-in. diskette) provides DGGS number, hole type, date completed, location, total depth, and types of data entered for all holes in the database, sorted by Municipality of Anchorage grid number. Locations are given in UTM coordinates, for which U.S. Geological Survey standard topographic maps show reference marks along the margins. Date completed is provided where available. Where only the month and year are given in the original data, the first of the month is used. If only the year is known, we entered the date as January 1.

\section{DATABASE MAINTENANCE AND AVAILABILITY}

DGGS maintains the Anchorage geotechnical database on a desktop computer and generates backups each time it is modified. We will continue to update the database as more data become available, and will enter additional data from existing hard-copy records as needed. As of this writing, we are negotiating with the original providers regarding what data can be released to the public, what data should be released only in original hard-copy form, and what data must remain proprietary. Data requests should be directed to the senior author. We invite organizations to submit any subsurface geotechnical data for the Anchorage area, regardless whether new or old, for inclusion in the database.

\section{ACKNOWLEDGMENTS}

We gratefully acknowledge the contributions of geotechnical and well-log data from the following organizations:

Alaska Department of Transportation \& Public Facilities

Dames \& Moore

DOWL Engineers \& Alaska Testlab

Golder Associates

Harding Lawson Associates

Hart Crowser

M-W Drilling

Municipality of Anchorage

R\&M Consultants

Shannon \& Wilson, Inc.

U.S. Army Corps of Engineers

U.S. Geological Survey

Woodward-Clyde Consultants

We especially thank David Cole, Lorie Dilley, Bob Dugan, Maria Kampsen, Mike Krueger, Dan Pavey, Charles Riddle, Jim Rooney, Robert Scher, David Stanley, Randall Updike, Wayne Westberg, and Cara Wright for their personal interest and contributions in building this database.

This project was partially supported by the U.S. Geological Survey (USGS), Department of the Interior, under USGS award number 98HQGR1047. 


\section{REFERENCES CITED}

ASTM, 2001, D1586-99 Standard Test Method for Penetration Test and Split-Barrel Sampling of Soils: American Society for Testing and Materials, West Conshohocken, PA, 5 pages.

Biswas, N.N., 1997, Seismic Microzonation: Metropolitan Anchorage, Alaska: Proposal to the Alaska Science and Technology Foundation, 95 p.

Combellick, R.A., 1999, Simplified geologic map and cross sections of central and east Anchorage, Alaska: Alaska Division of Geological \& Geophysical Surveys Preliminary Interpretive Report 1999-1, 12 p., 2 sheets, scale 1:25,000.

Lambe, T.W., and Whitman,R.V., 1969, Soil Mechanics: New York, John Wiley \& Sons, p. 30. 
Table 3 - Index listing of geotechnical boreholes and water wells shown on Sheet 1. See text for explanation.

\begin{tabular}{|c|c|c|c|c|c|c|c|c|c|c|c|}
\hline \multirow[b]{2}{*}{$\begin{array}{c}\text { MOA } \\
\text { grid }\end{array}$} & \multirow[b]{2}{*}{$\begin{array}{c}\text { DGGS } \\
\text { number }\end{array}$} & \multirow[b]{2}{*}{ Hole Type } & \multirow[b]{2}{*}{$\begin{array}{c}\text { Date } \\
\text { Completed }\end{array}$} & \multirow[b]{2}{*}{$\begin{array}{c}\text { Total } \\
\text { depth (ft) }\end{array}$} & \multicolumn{2}{|c|}{ UTM coordinates (m) } & \multicolumn{5}{|c|}{ Data entered } \\
\hline & & & & & East & North & Lithology & SPT & $\begin{array}{l}\text { Sample } \\
\text { test }\end{array}$ & $\begin{array}{l}\text { Water } \\
\text { level }\end{array}$ & Velocity \\
\hline 831 & 1658 & Hollow stem borehole w/SPT & $4 / 25 / 1988$ & 77.0 & 345471 & 6793411 & $\checkmark$ & $\checkmark$ & $\nabla$ & $\square$ & $\square$ \\
\hline 831 & 1659 & Hollow stem borehole w/SPT & $4 / 24 / 1988$ & 38.0 & 345560 & 6793634 & $\square$ & $\square$ & $\square$ & $\square$ & $\square$ \\
\hline 831 & 1660 & Hollow stem borehole w/SPT & $4 / 22 / 1988$ & 36.5 & 345641 & 6793859 & $\square$ & $\square$ & $\square$ & $\square$ & $\square$ \\
\hline 831 & 2040 & Hollow stem borehole w/SPT & $11 / 5 / 1996$ & 81.5 & 345559 & 6793457 & $\nabla$ & $\nabla$ & $\nabla$ & $\nabla$ & $\square$ \\
\hline 831 & 2041 & Hollow stem borehole w/SPT & 11/6/1996 & 81.5 & 345586 & 6793512 & $\nabla$ & $\nabla$ & $\square$ & $\nabla$ & $\square$ \\
\hline 831 & 2043 & Hollow stem borehole w/SPT & $11 / 13 / 1996$ & 81.5 & 345591 & 6793430 & $\nabla$ & $\nabla$ & $\nabla$ & $\nabla$ & $\square$ \\
\hline 831 & 2045 & Hollow stem borehole w/SPT & $11 / 14 / 1996$ & 41.5 & 345614 & 6793596 & $\nabla$ & $\nabla$ & $\nabla$ & $\square$ & $\square$ \\
\hline 930 & 001 & Hollow stem borehole w/SPT & $10 / 1 / 1965$ & 23.0 & 345233 & 6793099 & $\nabla$ & $\nabla$ & $\square$ & $\square$ & $\square$ \\
\hline 930 & 002 & Hollow stem borehole w/SPT & $10 / 1 / 1965$ & 88.3 & 345198 & 6792998 & $\nabla$ & $\nabla$ & $\square$ & $\square$ & $\square$ \\
\hline 930 & 003 & Hollow stem borehole w/SPT & $10 / 1 / 1965$ & 55.4 & 345234 & 6792969 & $\nabla$ & $\nabla$ & $\square$ & $\square$ & $\square$ \\
\hline 930 & 004 & Hollow stem borehole w/SPT & $10 / 1 / 1965$ & 55.9 & 345210 & 6792921 & $\nabla$ & $\nabla$ & $\square$ & $\square$ & $\square$ \\
\hline 930 & 005 & Hollow stem borehole w/SPT & $10 / 1 / 1965$ & 57.5 & 345179 & 6792953 & $\nabla$ & $\nabla$ & $\square$ & $\square$ & $\square$ \\
\hline 930 & 006 & Hollow stem borehole w/SPT & $10 / 1 / 1965$ & 59.1 & 345167 & 6792957 & $\nabla$ & $\nabla$ & $\square$ & $\square$ & $\square$ \\
\hline 930 & 007 & Hollow stem borehole w/SPT & $10 / 1 / 1965$ & 97.5 & 345159 & 6792919 & $\nabla$ & $\nabla$ & $\square$ & $\square$ & $\square$ \\
\hline 930 & 011 & Hollow stem borehole, no SPT & $10 / 1 / 1965$ & 35.7 & 345155 & 6792887 & $\square$ & $\square$ & $\square$ & $\square$ & $\square$ \\
\hline 930 & 012 & Hollow stem borehole w/SPT & 10/1/1965 & 119.7 & 345141 & 6792872 & $\nabla$ & $\nabla$ & $\square$ & $\square$ & $\square$ \\
\hline 930 & 013 & Hollow stem borehole w/SPT & $10 / 1 / 1965$ & 50.0 & 345127 & 6792871 & $\nabla$ & $\nabla$ & $\square$ & $\square$ & $\square$ \\
\hline 930 & 014 & Hollow stem borehole, no SPT & $10 / 1 / 1965$ & 54.5 & 345139 & 6792840 & $\nabla$ & $\square$ & $\square$ & $\square$ & $\square$ \\
\hline 930 & 015 & Hollow stem borehole, no SPT & 10/1/1965 & 100.0 & 345122 & 6792841 & $\nabla$ & $\square$ & $\square$ & $\square$ & $\square$ \\
\hline 930 & 016 & Hollow stem borehole, no SPT & $10 / 1 / 1965$ & 34.0 & 345117 & 6792845 & $\square$ & $\square$ & $\square$ & $\square$ & $\square$ \\
\hline 930 & 017 & Hollow stem borehole w/SPT & $1 / 1 / 1965$ & 114.8 & 345112 & 6792820 & $\nabla$ & $\nabla$ & $\square$ & $\square$ & $\square$ \\
\hline 930 & 018 & Hollow stem borehole, no SPT & $1 / 1 / 1955$ & 51.8 & 345071 & 6792831 & $\nabla$ & $\square$ & $\square$ & $\square$ & $\square$ \\
\hline 930 & 019 & Hollow stem borehole, no SPT & $1 / 1 / 1957$ & 111.4 & 345056 & 6792757 & $\nabla$ & $\square$ & $\square$ & $\square$ & $\square$ \\
\hline 930 & 020 & Hollow stem borehole, no SPT & $1 / 1 / 1957$ & 62.0 & 345108 & 6792734 & $\nabla$ & $\square$ & $\square$ & $\square$ & $\square$ \\
\hline 930 & 021 & Hollow stem borehole, no SPT & $1 / 1 / 1957$ & 128.0 & 345143 & 6792780 & $\nabla$ & $\square$ & $\square$ & $\square$ & $\square$ \\
\hline 930 & 022 & Hollow stem borehole, no SPT & $8 / 28 / 1964$ & 160.0 & 345161 & 6792748 & $\nabla$ & $\square$ & $\nabla$ & $\square$ & $\square$ \\
\hline 930 & 023 & Hollow stem borehole, no SPT & $1 / 1 / 1955$ & 100.3 & 345279 & 6792754 & $\nabla$ & $\square$ & $\square$ & $\square$ & $\square$ \\
\hline 930 & 025 & Hollow stem borehole w/SPT & 8/28/1964 & 156.5 & 345121 & 6792692 & $\nabla$ & $\nabla$ & $\nabla$ & $\square$ & $\square$ \\
\hline 930 & 026 & Hollow stem borehole, no SPT & $1 / 1 / 1957$ & 66.0 & 345112 & 6792645 & $\nabla$ & $\square$ & $\square$ & $\square$ & $\square$ \\
\hline 930 & 028 & Hollow stem borehole, no SPT & $1 / 1 / 1957$ & 152.6 & 345074 & 6792650 & $\nabla$ & $\square$ & $\square$ & $\square$ & $\square$ \\
\hline 930 & 030 & Hollow stem borehole, no SPT & $1 / 1 / 1964$ & 102.6 & 345036 & 6792659 & $\nabla$ & $\square$ & $\square$ & $\square$ & $\square$ \\
\hline 930 & 031 & Hollow stem borehole, no SPT & $1 / 1 / 1957$ & 114.0 & 345012 & 6792659 & $\nabla$ & $\square$ & $\square$ & $\square$ & $\square$ \\
\hline 931 & 024 & Hollow stem borehole, no SPT & $1 / 1 / 1955$ & 86.1 & 345397 & 6792741 & $\nabla$ & $\square$ & $\square$ & $\square$ & $\square$ \\
\hline 931 & 2042 & Hollow stem borehole w/SPT & $11 / 7 / 1996$ & 81.5 & 345541 & 6793413 & $\nabla$ & $\nabla$ & $\nabla$ & $\nabla$ & $\square$ \\
\hline
\end{tabular}




\begin{tabular}{|c|c|c|c|c|c|c|c|c|c|c|c|}
\hline \multirow[b]{2}{*}{$\begin{array}{c}\text { MOA } \\
\text { grid }\end{array}$} & \multirow[b]{2}{*}{$\begin{array}{c}\text { DGGS } \\
\text { number }\end{array}$} & \multirow[b]{2}{*}{ Hole Type } & \multirow[b]{2}{*}{$\begin{array}{c}\text { Date } \\
\text { Completed }\end{array}$} & \multirow[b]{2}{*}{$\begin{array}{c}\text { Total } \\
\text { depth (ft) }\end{array}$} & \multicolumn{2}{|c|}{ UTM coordinates (m) } & \multicolumn{5}{|c|}{ Data entered } \\
\hline & & & & & East & North & Lithology & SPT & $\begin{array}{c}\text { Sample } \\
\text { test }\end{array}$ & $\begin{array}{c}\text { Water } \\
\text { level }\end{array}$ & Velocity \\
\hline 931 & 2044 & Hollow stem borehole w/SPT & $11 / 14 / 1996$ & 81.5 & 345567 & 6793360 & $\boldsymbol{V}$ & $\nabla$ & $\nabla$ & $\checkmark$ & $\square$ \\
\hline 931 & 237 & Hollow stem borehole, no SPT & $5 / 1 / 1996$ & 163.0 & 345880 & 6793357 & $\boldsymbol{V}$ & $\square$ & $\nabla$ & $\square$ & $\square$ \\
\hline 931 & 238 & Hollow stem borehole, no SPT & $5 / 1 / 1965$ & 159.8 & 345741 & 6792747 & $\boldsymbol{V}$ & $\square$ & $\nabla$ & $\nabla$ & $\square$ \\
\hline 938 & 1827 & Hollow stem borehole, no SPT & $4 / 1 / 1985$ & 31.5 & 351241 & 6792722 & $\square$ & $\square$ & $\square$ & $\square$ & $\square$ \\
\hline 938 & 1828 & Hollow stem borehole, no SPT & $4 / 2 / 1985$ & 30.0 & 351438 & 6792843 & $\square$ & $\square$ & $\square$ & $\square$ & $\square$ \\
\hline 938 & 1829 & Hollow stem borehole w/SPT & $9 / 21 / 1986$ & 45.0 & 351132 & 6792852 & $\square$ & $\square$ & $\square$ & $\square$ & $\square$ \\
\hline 938 & 1830 & Hollow stem borehole w/SPT & $9 / 22 / 1986$ & 50.0 & 351218 & 6793075 & $\checkmark$ & $\checkmark$ & $\square$ & $\checkmark$ & $\square$ \\
\hline 938 & 1831 & Hollow stem borehole w/SPT & 9/19/1986 & 41.5 & 351061 & 6792681 & $\square$ & $\square$ & $\square$ & $\square$ & $\square$ \\
\hline 940 & 3004 & Water well & 8/6/1982 & 119.0 & 353181 & 6792607 & $\nabla$ & $\square$ & $\square$ & $\square$ & $\square$ \\
\hline 940 & 3005 & Water well & $8 / 4 / 1982$ & 162.0 & 352952 & 6792493 & $\boldsymbol{V}$ & $\square$ & $\square$ & $\square$ & $\square$ \\
\hline 941 & 3003 & Water well & $1 / 14 / 1982$ & 264.4 & 354062 & 6792632 & $\boldsymbol{V}$ & $\square$ & $\square$ & $\nabla$ & $\square$ \\
\hline 942 & 3001 & Water well & $8 / 24 / 1983$ & 218.0 & 354675 & 6792979 & $\boldsymbol{V}$ & $\square$ & $\square$ & $\square$ & $\square$ \\
\hline 942 & 3006 & Water well & $8 / 21 / 1984$ & 182.0 & 354379 & 6792340 & $\boldsymbol{V}$ & $\square$ & $\square$ & $\square$ & $\square$ \\
\hline 1030 & 027 & Hollow stem borehole, no SPT & $8 / 28 / 1964$ & 160.0 & 345112 & 6792633 & $\checkmark$ & $\square$ & $\checkmark$ & $\square$ & $\square$ \\
\hline 1030 & 032 & Hollow stem borehole, no SPT & $8 / 28 / 1964$ & 108.8 & 344998 & 6792580 & $\boldsymbol{V}$ & $\square$ & $\square$ & $\square$ & $\square$ \\
\hline 1030 & 033 & Hollow stem borehole, no SPT & $1 / 1 / 1955$ & 102.9 & 344996 & 6792488 & $\checkmark$ & $\square$ & $\square$ & $\square$ & $\square$ \\
\hline 1030 & 036 & Hollow stem borehole w/SPT & $6 / 1 / 1964$ & 142.0 & 345359 & 6792259 & $\boldsymbol{V}$ & $\checkmark$ & $\boldsymbol{V}$ & $\nabla$ & $\square$ \\
\hline 1030 & 037 & Hollow stem borehole w/SPT & $6 / 1 / 1964$ & 141.5 & 345285 & 6792248 & $\boldsymbol{V}$ & $\checkmark$ & $\nabla$ & $\nabla$ & $\square$ \\
\hline 1030 & 1001 & Hollow stem borehole w/SPT & 11/1/1994 & 51.1 & 345234 & 6792287 & $\boldsymbol{V}$ & $\checkmark$ & $\nabla$ & $\nabla$ & $\square$ \\
\hline 1030 & 1002 & Hollow stem borehole w/SPT & 11/1/1994 & 50.9 & 345234 & 6792261 & $\boldsymbol{V}$ & $\nabla$ & $\nabla$ & $\nabla$ & $\square$ \\
\hline 1030 & 1081 & Hollow stem borehole w/SPT & $10 / 1 / 1969$ & 75.0 & 344894 & 6791932 & $\boldsymbol{V}$ & $\checkmark$ & $\nabla$ & $\nabla$ & $\square$ \\
\hline 1030 & 124 & Solid stem borehole (no cores or SP & $2 / 28 / 1973$ & 21.0 & 345330 & 6792005 & $\square$ & $\square$ & $\square$ & $\square$ & $\square$ \\
\hline 1030 & 125 & Solid stem borehole (no cores or SP & $2 / 28 / 1973$ & 19.0 & 345287 & 6792007 & $\square$ & $\square$ & $\square$ & $\square$ & $\square$ \\
\hline 1030 & 126 & Hollow stem borehole w/SPT & $12 / 1 / 1966$ & 40.0 & 345196 & 6791963 & $\nabla$ & $\checkmark$ & $\square$ & $\nabla$ & $\square$ \\
\hline 1030 & 127 & Hollow stem borehole w/SPT & $12 / 1 / 1966$ & 57.5 & 345257 & 6791865 & $\checkmark$ & $\checkmark$ & $\checkmark$ & $\square$ & $\square$ \\
\hline 1030 & 140 & Hollow stem borehole, no SPT & $3 / 3 / 1977$ & 15.0 & 344934 & 6791892 & $\square$ & $\square$ & $\square$ & $\square$ & $\square$ \\
\hline 1030 & 141 & Hollow stem borehole, no SPT & $3 / 3 / 1977$ & 15.5 & 344919 & 6791876 & $\square$ & $\square$ & $\square$ & $\square$ & $\square$ \\
\hline 1030 & 1623 & Hollow stem borehole w/SPT & $7 / 2 / 1996$ & 26.5 & 344843 & 6791913 & $\square$ & $\square$ & $\square$ & $\square$ & $\square$ \\
\hline 1030 & 1624 & Hollow stem borehole w/SPT & $7 / 2 / 1996$ & 26.5 & 344814 & 6791908 & $\square$ & $\square$ & $\square$ & $\square$ & $\square$ \\
\hline 1030 & 1625 & Hollow stem borehole w/SPT & $7 / 2 / 1996$ & 21.5 & 344935 & 6792007 & $\square$ & $\square$ & $\square$ & $\square$ & $\square$ \\
\hline 1030 & 1683 & Hollow stem borehole w/SPT & $9 / 10 / 1980$ & 31.5 & 345215 & 6792514 & $\checkmark$ & $\checkmark$ & $\square$ & $\square$ & $\square$ \\
\hline 1030 & 217 & Hollow stem borehole w/SPT & $5 / 20 / 1964$ & 52.0 & 345019 & 6791880 & 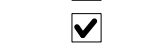 & $\checkmark$ & $\checkmark$ & $\square$ & $\square$ \\
\hline 1030 & 219 & Solid stem borehole (no cores or SP & $3 / 8 / 1966$ & 19.0 & 345212 & 6791873 & $\square$ & $\square$ & $\square$ & $\square$ & $\square$ \\
\hline 1030 & 220 & Solid stem borehole (no cores or SP & 3/8/1966 & 18.0 & 345280 & 6791931 & $\square$ & $\square$ & $\square$ & $\square$ & $\square$ \\
\hline 1030 & 221 & Solid stem borehole (no cores or SP & $3 / 8 / 1966$ & 19.0 & 345213 & 6791900 & $\square$ & $\square$ & $\square$ & $\square$ & $\square$ \\
\hline 1030 & 222 & Solid stem borehole (no cores or SP & $3 / 8 / 1966$ & 19.0 & 345172 & 6791891 & $\square$ & $\square$ & $\square$ & $\square$ & $\square$ \\
\hline
\end{tabular}




\begin{tabular}{|c|c|c|c|c|c|c|c|c|c|c|c|}
\hline \multirow[b]{2}{*}{$\begin{array}{l}\text { MOA } \\
\text { grid }\end{array}$} & \multirow[b]{2}{*}{$\begin{array}{l}\text { DGGS } \\
\text { number }\end{array}$} & \multirow[b]{2}{*}{ Hole Type } & \multirow[b]{2}{*}{$\begin{array}{c}\text { Date } \\
\text { Completed }\end{array}$} & \multirow[b]{2}{*}{$\begin{array}{c}\text { Total } \\
\text { depth }(\mathrm{ft})\end{array}$} & \multicolumn{2}{|c|}{ UTM coordinates (m) } & \multicolumn{5}{|c|}{ Data entered } \\
\hline & & & & & East & North & Lithology & SPT & $\begin{array}{c}\text { Sample } \\
\text { test }\end{array}$ & $\begin{array}{l}\text { Water } \\
\text { level }\end{array}$ & Velocity \\
\hline 1030 & 223 & Solid stem borehole (no cores or SP & 3/8/1966 & 19.0 & 345291 & 6791955 & $\square$ & & $\square$ & $\square$ & \\
\hline 1030 & 224 & Solid stem borehole (no cores or SP & 3/8/1966 & 19.0 & 345187 & 6791908 & $\square$ & $\square$ & $\square$ & $\square$ & $\square$ \\
\hline 1030 & 225 & Solid stem borehole (no cores or SP & $3 / 8 / 1966$ & 19.0 & 345148 & 6791908 & $\square$ & $\square$ & $\square$ & $\square$ & \\
\hline 1030 & 227 & Hollow stem borehole w/SPT & $10 / 21 / 1972$ & 76.5 & 345155 & 6791841 & $\checkmark$ & $\checkmark$ & $\square$ & $\square$ & $\square$ \\
\hline 1030 & 228 & Hollow stem borehole w/SPT & $10 / 23 / 1972$ & 47.0 & 345117 & 6791865 & $\square$ & $\square$ & $\square$ & $\square$ & $\square$ \\
\hline 1030 & 229 & Hollow stem borehole w/SPT & $10 / 23 / 1972$ & 26.5 & 345139 & 6791868 & $\square$ & $\square$ & $\square$ & $\square$ & $\square$ \\
\hline 1031 & 034 & Hollow stem borehole, no SPT & $10 / 1 / 1974$ & 16.5 & 345405 & 6792357 & $\square$ & $\square$ & $\square$ & $\square$ & $\square$ \\
\hline 1031 & 035 & Hollow stem borehole, no SPT & $10 / 1 / 1974$ & 16.5 & 345394 & 6792358 & $\square$ & $\square$ & $\square$ & $\square$ & \\
\hline 1031 & 038 & Hollow stem borehole w/SPT & $8 / 28 / 1964$ & 131.0 & 345789 & 6792196 & $\nabla$ & $\checkmark$ & $\checkmark$ & $\square$ & $\square$ \\
\hline 1031 & 039 & Solid stem borehole (no cores or SP & $10 / 3 / 1973$ & 25.0 & 345765 & 6792118 & $\checkmark$ & $\square$ & $\square$ & $\square$ & $\square$ \\
\hline 1031 & 040 & Hollow stem borehole w/SPT & $1 / 11 / 1977$ & 25.5 & 345763 & 6792073 & $\checkmark$ & 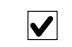 & $\bar{\nabla}$ & $\square$ & $\square$ \\
\hline 1031 & 041 & Solid stem borehole (no cores or SP & $7 / 3 / 1973$ & 24.0 & 345739 & 6792100 & $\square$ & & $\square$ & $\square$ & \\
\hline 1031 & 042 & Hollow stem borehole w/SPT & $1 / 13 / 1977$ & 31.5 & 345748 & 6792072 & $\square$ & & $\square$ & $\square$ & $\square$ \\
\hline 1031 & 043 & Hollow stem borehole w/SPT & $1 / 14 / 1977$ & 26.5 & 345746 & 6792051 & $\square$ & 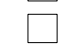 & $\square$ & $\square$ & $\square$ \\
\hline 1031 & 044 & Hollow stem borehole w/SPT & $1 / 11 / 1977$ & 26.5 & 345762 & 6792022 & $\square$ & & $\square$ & $\square$ & $\square$ \\
\hline 1031 & 045 & Hollow stem borehole w/SPT & $3 / 11 / 1978$ & 30.3 & 345749 & 6792010 & $\square$ & & $\square$ & $\square$ & $\square$ \\
\hline 1031 & 046 & Hollow stem borehole, no SPT & $3 / 14 / 1978$ & 8.2 & 345754 & 6791992 & \begin{tabular}{|r|r} 
\\
\end{tabular} & & 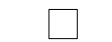 & $\square$ & \\
\hline 1031 & 047 & Hollow stem borehole w/SPT & $3 / 11 / 1978$ & 29.5 & 345738 & 6791993 & $\square$ & & $\square$ & $\square$ & $\square$ \\
\hline 1031 & 048 & Hollow stem borehole w/SPT & $3 / 10 / 1978$ & 30.1 & 345738 & 6791983 & $\square$ & $\square$ & $\square$ & $\square$ & $\square$ \\
\hline 1031 & 049 & Hollow stem borehole, no SPT & $3 / 14 / 1978$ & 7.5 & 345737 & 6791965 & $\square$ & $\checkmark$ & $\square$ & $\square$ & $\square$ \\
\hline 1031 & 050 & Hollow stem borehole w/SPT & $3 / 14 / 1978$ & 31.0 & 345724 & 6791979 & $\square$ & & $\square$ & $\square$ & \\
\hline 1031 & 051 & Hollow stem borehole w/SPT & $3 / 10 / 1978$ & 29.8 & 345721 & 6791967 & $\square$ & & $\square$ & $\square$ & $\square$ \\
\hline 1031 & 052 & Hollow stem borehole w/SPT & 3/9/1978 & 30.0 & 345712 & 6791963 & $\square$ & & $\square$ & $\square$ & $\square$ \\
\hline 1031 & 053 & Hollow stem borehole, no SPT & $3 / 14 / 1978$ & 4.0 & 345710 & 6791948 & $\square$ & $\checkmark$ & $\square$ & $\square$ & $\square$ \\
\hline 1031 & 054 & Hollow stem borehole w/SPT & 3/8/1978 & 29.5 & 345695 & 6791952 & $\square$ & & $\square$ & $\square$ & $\square$ \\
\hline 1031 & 055 & Hollow stem borehole w/SPT & $6 / 15 / 1977$ & 28.5 & 345677 & 6792074 & $\square$ & & $\square$ & $\square$ & 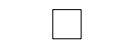 \\
\hline 1031 & 056 & Solid stem borehole (no cores or SP & $7 / 3 / 1973$ & 7.0 & 345691 & 6792100 & $\square$ & 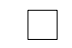 & $\square$ & $\square$ & $\square$ \\
\hline 1031 & 057 & Solid stem borehole (no cores or SP & $7 / 3 / 1973$ & 13.0 & 345703 & 6792117 & $\square$ & & $\square$ & $\square$ & $\square$ \\
\hline 1031 & 058 & Hollow stem borehole w/SPT & $6 / 13 / 1977$ & 36.5 & 345673 & 6792108 & $\square$ & & $\square$ & $\square$ & $\square$ \\
\hline 1031 & 059 & Hollow stem borehole w/SPT & $6 / 14 / 1977$ & 36.5 & 345655 & 6792124 & $\square$ & & $\square$ & $\square$ & $\square$ \\
\hline 1031 & 060 & Solid stem borehole (no cores or SP & $7 / 3 / 1973$ & 8.0 & 345661 & 6792106 & $\square$ & $\square$ & $\square$ & $\square$ & $\square$ \\
\hline 1031 & 061 & Hollow stem borehole w/SPT & $6 / 10 / 1977$ & 36.5 & 345658 & 6792090 & $\square$ & $\square$ & $\square$ & $\square$ & $\square$ \\
\hline 1031 & 062 & Hollow stem borehole w/SPT & $6 / 10 / 1977$ & 26.5 & 345653 & 6792088 & $\square$ & $\square$ & $\square$ & $\square$ & $\square$ \\
\hline 1031 & 063 & Hollow stem borehole w/SPT & $6 / 14 / 1977$ & 36.5 & 345632 & 6792116 & $\square$ & $\square$ & $\square$ & $\square$ & $\square$ \\
\hline 1031 & 064 & Hollow stem borehole w/SPT & $6 / 10 / 1977$ & 26.5 & 345638 & 6792104 & $\square$ & $\square$ & $\square$ & $\square$ & $\square$ \\
\hline 1031 & 065 & Solid stem borehole (no cores or SP & $7 / 5 / 1973$ & 8.0 & 345636 & 6792094 & $\square$ & $\square$ & $\square$ & $\square$ & $\square$ \\
\hline
\end{tabular}




\begin{tabular}{|c|c|c|c|c|c|c|c|c|c|c|c|}
\hline \multirow[b]{2}{*}{$\begin{array}{l}\text { MOA } \\
\text { grid }\end{array}$} & \multirow[b]{2}{*}{$\begin{array}{c}\text { DGGS } \\
\text { number }\end{array}$} & \multirow[b]{2}{*}{ Hole Type } & \multirow[b]{2}{*}{$\begin{array}{c}\text { Date } \\
\text { Completed }\end{array}$} & \multirow[b]{2}{*}{$\begin{array}{c}\text { Total } \\
\text { depth (ft) }\end{array}$} & \multicolumn{2}{|c|}{ UTM coordinates (m) } & \multicolumn{5}{|c|}{ Data entered } \\
\hline & & & & & East & North & Lithology & SPT & $\begin{array}{c}\text { Sample } \\
\text { test }\end{array}$ & $\begin{array}{l}\text { Water } \\
\text { level }\end{array}$ & Velocity \\
\hline 1031 & 066 & Solid stem borehole (no cores or SP & $7 / 5 / 1973$ & 15.0 & 345635 & 6792061 & $\square$ & & $\square$ & $\square$ & \\
\hline 1031 & 067 & Hollow stem borehole w/SPT & 6/7/1977 & 36.5 & 345627 & 6792062 & $\square$ & $\square$ & $\square$ & $\square$ & $\square$ \\
\hline 1031 & 068 & Hollow stem borehole w/SPT & 6/7/1977 & 36.5 & 345625 & 6792030 & $\square$ & & $\square$ & $\square$ & $\square$ \\
\hline 1031 & 069 & Solid stem borehole (no cores or SP & $7 / 5 / 1973$ & 6.0 & 345637 & 6792006 & $\square$ & & $\square$ & $\square$ & $\square$ \\
\hline 1031 & 070 & Hollow stem borehole w/SPT & $6 / 8 / 1977$ & 45.5 & 345610 & 6792057 & $\square$ & & $\square$ & $\square$ & $\square$ \\
\hline 1031 & 071 & Solid stem borehole (no cores or SP & $7 / 5 / 1973$ & 15.0 & 345602 & 6792057 & $\square$ & $\square$ & $\square$ & $\square$ & $\square$ \\
\hline 1031 & 072 & Hollow stem borehole w/SPT & 6/8/1977 & 36.5 & 345590 & 6792056 & $\square$ & $\square$ & $\square$ & $\square$ & $\square$ \\
\hline 1031 & 073 & Hollow stem borehole w/SPT & 6/9/1977 & 36.5 & 345581 & 6792087 & $\square$ & & $\square$ & $\square$ & $\square$ \\
\hline 1031 & 074 & Solid stem borehole (no cores or SP & $7 / 5 / 1973$ & 4.0 & 345600 & 6792101 & $\square$ & $\square$ & $\square$ & $\square$ & $\square$ \\
\hline 1031 & 075 & Hollow stem borehole w/SPT & 6/9/1977 & 36.5 & 345611 & 6792097 & $\square$ & $\square$ & $\square$ & $\square$ & $\square$ \\
\hline 1031 & 076 & Solid stem borehole (no cores or SP & $6 / 1 / 1956$ & 5.0 & 345570 & 6792012 & $\square$ & $\square$ & $\square$ & $\square$ & $\square$ \\
\hline 1031 & 077 & Solid stem borehole (no cores or SP & $6 / 1 / 1956$ & 9.0 & 345569 & 6791988 & $\square$ & $\square$ & $\square$ & $\square$ & $\square$ \\
\hline 1031 & 078 & Solid stem borehole (no cores or SP & $6 / 1 / 1956$ & 20.0 & 345567 & 6791974 & $\square$ & & $\square$ & $\square$ & $\square$ \\
\hline 1031 & 079 & Solid stem borehole (no cores or SP & $6 / 1 / 1956$ & 20.0 & 345567 & 6791954 & $\square$ & & $\square$ & $\square$ & $\square$ \\
\hline 1031 & 080 & Hollow stem borehole, no SPT & $6 / 1 / 1956$ & 24.0 & 345561 & 6791955 & $\square$ & & $\square$ & $\square$ & $\square$ \\
\hline 1031 & 081 & Solid stem borehole (no cores or SP & $6 / 1 / 1956$ & 13.0 & 345549 & 6791956 & $\square$ & & $\square$ & $\square$ & $\square$ \\
\hline 1031 & 082 & Solid stem borehole (no cores or SP & $6 / 1 / 1956$ & 12.0 & 345532 & 6791956 & $\square$ & & $\square$ & $\square$ & $\square$ \\
\hline 1031 & 083 & Hollow stem borehole, no SPT & $6 / 1 / 1956$ & 84.0 & 345543 & 6791979 & $\checkmark$ & & $\square$ & $\square$ & $\square$ \\
\hline 1031 & 084 & Hollow stem borehole, no SPT & $6 / 1 / 1956$ & 93.0 & 345546 & 6792010 & $\checkmark$ & & $\square$ & $\boldsymbol{V}$ & \\
\hline 1031 & 085 & Solid stem borehole (no cores or SP & $6 / 1 / 1956$ & 4.0 & 345520 & 6791980 & $\square$ & $\checkmark$ & $\square$ & $\square$ & $\square$ \\
\hline 1031 & 086 & Solid stem borehole (no cores or SP & $7 / 5 / 1973$ & 17.0 & 345549 & 6792061 & $\square$ & 5 & $\square$ & $\square$ & $\square$ \\
\hline 1031 & 087 & Solid stem borehole (no cores or SP & $7 / 5 / 1973$ & 25.0 & 345534 & 6792102 & $\square$ & & $\square$ & $\square$ & $\square$ \\
\hline 1031 & 088 & Solid stem borehole (no cores or SP & $7 / 5 / 1973$ & 14.0 & 345521 & 6792085 & $\square$ & & $\square$ & $\square$ & \\
\hline 1031 & 089 & Water well & $12 / 7 / 1973$ & 295.0 & 345511 & 6792126 & $\checkmark$ & $\square$ & $\square$ & $\bar{\nabla}$ & $\square$ \\
\hline 1031 & 090 & Solid stem borehole (no cores or SP & $7 / 5 / 1973$ & 17.0 & 345507 & 6792099 & $\square$ & & $\square$ & $\square$ & $\square$ \\
\hline 1031 & 091 & Solid stem borehole (no cores or SP & $7 / 5 / 1973$ & 25.0 & 345507 & 6792092 & $\square$ & & $\square$ & $\square$ & $\square$ \\
\hline 1031 & 092 & Solid stem borehole (no cores or SP & $7 / 5 / 1973$ & 25.0 & 345483 & 6792084 & $\square$ & & $\square$ & $\square$ & $\square$ \\
\hline 1031 & 093 & Solid stem borehole (no cores or SP & $7 / 5 / 1973$ & 23.0 & 345458 & 6792088 & $\square$ & & $\square$ & $\square$ & $\square$ \\
\hline 1031 & 094 & Solid stem borehole (no cores or SP & $7 / 5 / 1973$ & 21.0 & 345435 & 6792046 & $\square$ & $\square$ & $\square$ & $\square$ & $\square$ \\
\hline 1031 & 095 & Solid stem borehole (no cores or SP & $7 / 5 / 1973$ & 6.0 & 345469 & 6792057 & $\square$ & & $\square$ & $\square$ & $\square$ \\
\hline 1031 & 096 & Hollow stem borehole, no SPT & $6 / 1 / 1956$ & 190.0 & 345487 & 6792041 & $\checkmark$ & & $\square$ & $\checkmark$ & $\square$ \\
\hline 1031 & 097 & Hollow stem borehole, no SPT & $6 / 1 / 1956$ & 37.0 & 345502 & 6792030 & $\square$ & & $\square$ & $\square$ & $\square$ \\
\hline 1031 & 098 & Hollow stem borehole, no SPT & $6 / 1 / 1956$ & 48.0 & 345503 & 6792018 & $\square$ & $\square$ & $\square$ & $\square$ & $\square$ \\
\hline 1031 & 099 & Hollow stem borehole, no SPT & $6 / 1 / 1956$ & 59.0 & 345505 & 6791997 & $\checkmark$ & $\square$ & $\square$ & $\square$ & $\square$ \\
\hline 1031 & 100 & Hollow stem borehole, no SPT & $6 / 1 / 1956$ & 50.0 & 345496 & 6791997 & $\checkmark$ & $\square$ & $\square$ & $\square$ & $\square$ \\
\hline 1031 & 1003 & Hollow stem borehole w/SPT & $9 / 14 / 1982$ & 52.0 & 345821 & 6791992 & $\checkmark$ & $\checkmark$ & $\checkmark$ & $\checkmark$ & $\square$ \\
\hline
\end{tabular}




\begin{tabular}{|c|c|c|c|c|c|c|c|c|c|c|c|}
\hline \multirow[b]{2}{*}{$\begin{array}{l}\text { MOA } \\
\text { grid }\end{array}$} & \multirow[b]{2}{*}{$\begin{array}{l}\text { DGGS } \\
\text { number }\end{array}$} & \multirow[b]{2}{*}{ Hole Type } & \multirow[b]{2}{*}{$\begin{array}{c}\text { Date } \\
\text { Completed }\end{array}$} & \multirow[b]{2}{*}{$\begin{array}{c}\text { Total } \\
\text { depth }(\mathrm{ft})\end{array}$} & \multicolumn{2}{|c|}{ UTM coordinates (m) } & \multicolumn{5}{|c|}{ Data entered } \\
\hline & & & & & East & North & Lithology & SPT & $\begin{array}{c}\text { Sample } \\
\text { test }\end{array}$ & $\begin{array}{l}\text { Water } \\
\text { level }\end{array}$ & Velocity \\
\hline 1031 & 1004 & Hollow stem borehole w/SPT & $9 / 14 / 1982$ & 51.5 & 345910 & 6791929 & $\checkmark$ & $\checkmark$ & $\checkmark$ & $\checkmark$ & 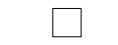 \\
\hline 1031 & 1005 & Hollow stem borehole w/SPT & $9 / 13 / 1982$ & 51.5 & 345880 & 6791964 & $\checkmark$ & $\nabla$ & $\checkmark$ & $\checkmark$ & \\
\hline 1031 & 101 & Hollow stem borehole, no SPT & $6 / 1 / 1956$ & 50.0 & 345492 & 6791996 & $\checkmark$ & & $\square$ & $\bar{\nabla}$ & \\
\hline 1031 & 102 & Solid stem borehole (no cores or SP & $6 / 1 / 1956$ & 8.0 & 345500 & 6791976 & $\square$ & $\square$ & $\square$ & $\square$ & $\square$ \\
\hline 1031 & 103 & Solid stem borehole (no cores or SP & $6 / 1 / 1956$ & 11.0 & 345501 & 6791957 & $\square$ & $\square$ & $\square$ & $\square$ & $\square$ \\
\hline 1031 & 104 & Hollow stem borehole w/SPT & $7 / 8 / 1977$ & 100.5 & 345485 & 6791955 & $\checkmark$ & $\checkmark$ & $\checkmark$ & $\square$ & $\square$ \\
\hline 1031 & 105 & Solid stem borehole (no cores or SP & $6 / 1 / 1956$ & 8.0 & 345485 & 6791975 & $\square$ & $\square$ & $\square$ & $\square$ & $\square$ \\
\hline 1031 & 106 & Hollow stem borehole, no SPT & $6 / 1 / 1956$ & 50.0 & 345477 & 6791990 & $\checkmark$ & & $\square$ & $\nabla$ & \\
\hline 1031 & 107 & Hollow stem borehole, no SPT & $6 / 1 / 1956$ & 65.0 & 345482 & 6792007 & $\checkmark$ & $\square$ & $\square$ & $\checkmark$ & $\square$ \\
\hline 1031 & 109 & Hollow stem borehole, no SPT & $6 / 1 / 1956$ & 45.0 & 345456 & 6792014 & $\square$ & $\square$ & $\square$ & $\square$ & \\
\hline 1031 & 110 & Hollow stem borehole, no SPT & $6 / 1 / 1956$ & 50.0 & 345461 & 6792005 & $\checkmark$ & $\square$ & $\square$ & $\square$ & $\square$ \\
\hline 1031 & 111 & Hollow stem borehole, no SPT & $6 / 1 / 1956$ & 40.0 & 345458 & 6791996 & $\square$ & $\square$ & $\square$ & $\square$ & $\square$ \\
\hline 1031 & 112 & Hollow stem borehole, no SPT & $6 / 1 / 1956$ & 50.0 & 345458 & 6791986 & $\checkmark$ & $\square$ & $\square$ & $\checkmark$ & $\square$ \\
\hline 1031 & 113 & Solid stem borehole (no cores or SP & $6 / 1 / 1956$ & 8.0 & 345470 & 6791973 & $\square$ & $\square$ & $\square$ & $\square$ & $\square$ \\
\hline 1031 & 114 & Solid stem borehole (no cores or SP & $6 / 1 / 1956$ & 11.0 & 345472 & 6791954 & $\square$ & $\square$ & $\square$ & $\square$ & $\checkmark$ \\
\hline 1031 & 115 & Solid stem borehole (no cores or SP & $6 / 1 / 1956$ & 20.0 & 345456 & 6791953 & $\square$ & $\square$ & $\square$ & $\square$ & $\square$ \\
\hline 1031 & 116 & Hollow stem borehole, no SPT & $6 / 1 / 1956$ & 76.0 & 345421 & 6791967 & $\checkmark$ & $\square$ & $\square$ & $\square$ & $\square$ \\
\hline 1031 & 117 & Hollow stem borehole w/SPT & $7 / 5 / 1977$ & 100.5 & 345503 & 6791897 & $\checkmark$ & $\nabla$ & $\checkmark$ & $\nabla$ & \\
\hline 1031 & 118 & Hollow stem borehole w/SPT & $2 / 28 / 1978$ & 60.7 & 345444 & 6791888 & $\checkmark$ & $\nabla$ & $\checkmark$ & $\square$ & \\
\hline 1031 & 119 & Hollow stem borehole w/SPT & $1 / 11 / 1978$ & 61.2 & 345411 & 6791875 & $\checkmark$ & $\checkmark$ & $\checkmark$ & $\checkmark$ & $\checkmark$ \\
\hline 1031 & 120 & Hollow stem borehole w/SPT & 3/1/1978 & 59.0 & 345395 & 6791865 & $\checkmark$ & $\checkmark$ & $\bar{\nabla}$ & $\square$ & $\square$ \\
\hline 1031 & 121 & Hollow stem borehole w/SPT & $2 / 10 / 1978$ & 30.5 & 345368 & 6791894 & $\square$ & $\square$ & $\square$ & $\square$ & $\square$ \\
\hline 1031 & 122 & Hollow stem borehole w/SPT & $3 / 3 / 1978$ & 30.0 & 345358 & 6791869 & $\square$ & & $\square$ & $\square$ & $\square$ \\
\hline 1031 & 123 & Hollow stem borehole w/SPT & $2 / 10 / 1978$ & 30.5 & 345349 & 6791885 & $\square$ & 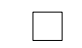 & $\square$ & $\square$ & $\square$ \\
\hline 1031 & 2028 & Solid stem borehole (no cores or SP & $8 / 13 / 1964$ & 18.5 & 346038 & 6792051 & $\checkmark$ & ᄂ & $\square$ & 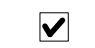 & $\square$ \\
\hline 1031 & 2029 & Solid stem borehole (no cores or SP & $8 / 13 / 1964$ & 16.0 & 346031 & 6792081 & $\checkmark$ & & $\square$ & $\square$ & $\square$ \\
\hline 1031 & 2030 & Solid stem borehole (no cores or SP & $8 / 13 / 1964$ & 18.0 & 346066 & 6792011 & $\checkmark$ & & $\square$ & $\checkmark$ & $\square$ \\
\hline 1031 & 207 & Hollow stem borehole w/SPT & $5 / 1 / 1973$ & 20.0 & 345881 & 6791825 & $\square$ & & $\square$ & $\square$ & \\
\hline 1031 & 208 & Hollow stem borehole w/SPT & $5 / 1 / 1973$ & 20.0 & 345889 & 6791853 & $\square$ & $\square$ & $\square$ & $\square$ & $\square$ \\
\hline 1031 & 209 & Hollow stem borehole w/SPT & $5 / 2 / 1973$ & 19.0 & 345839 & 6791843 & $\square$ & $\square$ & $\square$ & $\square$ & $\square$ \\
\hline 1031 & 210 & Hollow stem borehole w/SPT & $5 / 2 / 1973$ & 19.5 & 345844 & 6791819 & $\square$ & $\square$ & $\square$ & $\square$ & $\square$ \\
\hline 1031 & 211 & Hollow stem borehole w/SPT & $5 / 2 / 1973$ & 30.0 & 345858 & 6791834 & $\checkmark$ & $\checkmark$ & $\checkmark$ & $\checkmark$ & $\square$ \\
\hline 1031 & 212 & Hollow stem borehole w/SPT & $5 / 2 / 1973$ & 20.0 & 345781 & 6791851 & $\square$ & $\square$ & $\square$ & $\square$ & $\square$ \\
\hline 1031 & 239 & Hollow stem borehole, no SPT & $5 / 1 / 1965$ & 165.5 & 345778 & 6792425 & $\checkmark$ & $\square$ & $\checkmark$ & $\bar{\nabla}$ & $\square$ \\
\hline 1034 & JL004 & Hollow stem borehole w/SPT & $8 / 28 / 1964$ & 200.0 & 347920 & 6791772 & $\checkmark$ & $\checkmark$ & $\checkmark$ & $\square$ & $\square$ \\
\hline 1035 & 3007 & Water well & $6 / 16 / 1970$ & 166.0 & 348809 & 6791956 & $\checkmark$ & $\square$ & $\square$ & $\checkmark$ & $\square$ \\
\hline
\end{tabular}




\begin{tabular}{|c|c|c|c|c|c|c|c|c|c|c|c|}
\hline \multirow[b]{2}{*}{$\begin{array}{c}\text { MOA } \\
\text { grid }\end{array}$} & \multirow[b]{2}{*}{$\begin{array}{l}\text { DGGS } \\
\text { number }\end{array}$} & \multirow[b]{2}{*}{ Hole Type } & \multirow[b]{2}{*}{$\begin{array}{c}\text { Date } \\
\text { Completed }\end{array}$} & \multirow[b]{2}{*}{$\begin{array}{c}\text { Total } \\
\text { depth }(\mathrm{ft})\end{array}$} & \multicolumn{2}{|c|}{ UTM coordinates (m) } & \multicolumn{5}{|c|}{ Data entered } \\
\hline & & & & & East & North & Lithology & SPT & $\begin{array}{c}\text { Sample } \\
\text { test }\end{array}$ & $\begin{array}{l}\text { Water } \\
\text { level }\end{array}$ & Velocity \\
\hline 1040 & 1021 & Hollow stem borehole w/SPT & $11 / 5 / 1970$ & 61.5 & 353285 & 6791551 & $\checkmark$ & $\checkmark$ & $\square$ & $\square$ & $\square$ \\
\hline 1040 & 1022 & Hollow stem borehole w/SPT & $10 / 26 / 1970$ & 60.0 & 353352 & 6791731 & $\checkmark$ & $\checkmark$ & $\square$ & $\square$ & $\square$ \\
\hline 1040 & 1023 & Hollow stem borehole w/SPT & $10 / 29 / 1970$ & 60.5 & 353353 & 6791690 & $\checkmark$ & $\nabla$ & $\square$ & $\square$ & \\
\hline 1040 & 1024 & Hollow stem borehole w/SPT & $11 / 7 / 1970$ & 61.5 & 353352 & 6791607 & $\checkmark$ & 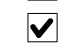 & $\square$ & $\square$ & $\square$ \\
\hline 1040 & 1025 & Hollow stem borehole w/SPT & $11 / 6 / 1970$ & 61.5 & 353352 & 6791550 & $\checkmark$ & $\checkmark$ & $\square$ & $\square$ & $\square$ \\
\hline 1040 & 1029 & Hollow stem borehole w/SPT & $11 / 15 / 1982$ & 50.5 & 353374 & 6791786 & $\checkmark$ & $\checkmark$ & $\checkmark$ & $\checkmark$ & $\square$ \\
\hline 1040 & 1030 & Hollow stem borehole w/SPT & $11 / 9 / 1982$ & 50.5 & 353374 & 6791746 & $\checkmark$ & $\checkmark$ & $\checkmark$ & $\checkmark$ & $\square$ \\
\hline 1040 & 306 & Hollow stem borehole w/SPT & $4 / 12 / 1994$ & 266.0 & 352650 & 6791560 & $\checkmark$ & $\checkmark$ & $\checkmark$ & $\nabla$ & \\
\hline 1040 & 307 & Water well & $2 / 24 / 1997$ & 278.0 & 353000 & 6792100 & $\nabla$ & $\square$ & $\square$ & $\square$ & $\square$ \\
\hline 1040 & 309 & Hollow stem borehole w/SPT & $2 / 29 / 1992$ & 151.3 & 352800 & 6791960 & $\checkmark$ & $\checkmark$ & $\square$ & $\checkmark$ & $\checkmark$ \\
\hline 1041 & 1026 & Hollow stem borehole w/SPT & $10 / 30 / 1970$ & 61.0 & 353391 & 6791499 & $\checkmark$ & 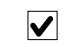 & $\square$ & $\square$ & $\square$ \\
\hline 1041 & 1027 & Hollow stem borehole w/SPT & $10 / 22 / 1970$ & 61.0 & 353430 & 6791690 & $\checkmark$ & 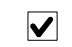 & $\square$ & $\square$ & $\square$ \\
\hline 1041 & 1028 & Hollow stem borehole w/SPT & $11 / 2 / 1970$ & 60.5 & 353493 & 6791565 & $\nabla$ & $\checkmark$ & $\checkmark$ & $\nabla$ & $\square$ \\
\hline 1129 & 1656 & Hollow stem borehole w/SPT & $4 / 18 / 1989$ & 55.5 & 344146 & 6791163 & $\checkmark$ & $\checkmark$ & $\checkmark$ & $\nabla$ & $\square$ \\
\hline 1129 & 1657 & Hollow stem borehole w/SPT & $4 / 18 / 1989$ & 63.5 & 344110 & 6791183 & $\checkmark$ & $\bar{\square}$ & $\bar{\nabla}$ & $\square$ & $\square$ \\
\hline 1130 & 1006 & Hollow stem borehole w/SPT & $6 / 18 / 1973$ & 61.5 & 344873 & 6791484 & $\checkmark$ & $\checkmark$ & $\square$ & 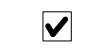 & $\square$ \\
\hline 1130 & 1009 & Hollow stem borehole w/SPT & $1 / 28 / 1969$ & 178.0 & 345206 & 6791146 & $\nabla$ & $\checkmark$ & $\checkmark$ & $\nabla$ & 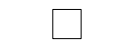 \\
\hline 1130 & 1010 & Hollow stem borehole w/SPT & $1 / 10 / 1969$ & 157.0 & 345154 & 6791291 & $\nabla$ & $\checkmark$ & $\checkmark$ & $\checkmark$ & \\
\hline 1130 & 1011 & Hollow stem borehole w/SPT & $1 / 23 / 1969$ & 102.0 & 345150 & 6791405 & $\nabla$ & 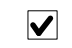 & $\checkmark$ & $\checkmark$ & \\
\hline 1130 & 1012 & Hollow stem borehole w/SPT & $1 / 20 / 1969$ & 102.0 & 345038 & 6791404 & $\checkmark$ & $\checkmark$ & $\checkmark$ & $\checkmark$ & $\checkmark$ \\
\hline 1130 & 1017 & Hollow stem borehole w/SPT & $12 / 2 / 1991$ & 52.0 & 344863 & 6791812 & $\checkmark$ & $\bar{\nabla}$ & $\square$ & $\bar{\nabla}$ & $\square$ \\
\hline 1130 & 128 & Hollow stem borehole w/SPT & $11 / 1 / 1966$ & 102.5 & 345289 & 6791818 & $\checkmark$ & $\checkmark$ & $\checkmark$ & $\square$ & $\square$ \\
\hline 1130 & 129 & Hollow stem borehole w/SPT & $12 / 1 / 1966$ & 137.5 & 345325 & 6791761 & $\nabla$ & $\bar{\nabla}$ & $\nabla$ & $\square$ & $\square$ \\
\hline 1130 & 130 & Solid stem borehole (no cores or SP & $6 / 1 / 1966$ & 11.0 & 345176 & 6791725 & $\square$ & $\square$ & $\square$ & $\square$ & $\square$ \\
\hline 1130 & 131 & Solid stem borehole (no cores or SP & $6 / 1 / 1966$ & 11.0 & 345158 & 6791720 & $\square$ & $\square$ & $\square$ & $\square$ & $\square$ \\
\hline 1130 & 132 & Solid stem borehole (no cores or SP & $6 / 1 / 1966$ & 11.0 & 345140 & 6791719 & $\square$ & $\square$ & $\square$ & $\square$ & $\square$ \\
\hline 1130 & 134 & Hollow stem borehole, no SPT & $8 / 28 / 1964$ & 110.0 & 345161 & 6791626 & $\checkmark$ & $\square$ & $\checkmark$ & $\square$ & $\square$ \\
\hline 1130 & 135 & Solid stem borehole (no cores or SP & $6 / 10 / 1966$ & 7.0 & 345141 & 6791611 & $\square$ & $\square$ & $\square$ & $\square$ & \\
\hline 1130 & 136 & Solid stem borehole (no cores or SP & $6 / 10 / 1966$ & 13.0 & 345009 & 6791715 & $\square$ & $\square$ & $\square$ & $\square$ & $\square$ \\
\hline 1130 & 137 & Solid stem borehole (no cores or SP & $6 / 10 / 1966$ & 11.0 & 345002 & 6791715 & $\square$ & $\square$ & $\square$ & $\square$ & $\square$ \\
\hline 1130 & 138 & Solid stem borehole (no cores or SP & $6 / 10 / 1966$ & 11.0 & 344993 & 6791715 & $\square$ & $\square$ & $\square$ & $\square$ & $\square$ \\
\hline 1130 & 139 & Solid stem borehole (no cores or SP & $6 / 10 / 1966$ & 11.0 & 344981 & 6791715 & $\square$ & $\square$ & $\square$ & $\square$ & $\square$ \\
\hline 1130 & 142 & Hollow stem borehole w/SPT & $11 / 1 / 1966$ & 52.5 & 344961 & 6791569 & $\checkmark$ & $\checkmark$ & $\checkmark$ & $\nabla$ & $\square$ \\
\hline 1130 & 143 & Hollow stem borehole w/SPT & $11 / 1 / 1966$ & 82.5 & 345004 & 6791549 & $\nabla$ & $\checkmark$ & $\checkmark$ & $\checkmark$ & $\square$ \\
\hline 1130 & 144 & Hollow stem borehole w/SPT & $12 / 1 / 1966$ & 102.5 & 345029 & 6791536 & $\checkmark$ & $\checkmark$ & $\checkmark$ & $\square$ & $\square$ \\
\hline 1130 & 145 & Hollow stem borehole w/SPT & $12 / 1 / 1966$ & 152.0 & 345063 & 6791522 & $\checkmark$ & $\checkmark$ & $\checkmark$ & $\checkmark$ & $\square$ \\
\hline
\end{tabular}




\begin{tabular}{|c|c|c|c|c|c|c|c|c|c|c|c|}
\hline \multirow[b]{2}{*}{$\begin{array}{l}\text { MOA } \\
\text { grid }\end{array}$} & \multirow[b]{2}{*}{$\begin{array}{l}\text { DGGS } \\
\text { number }\end{array}$} & \multirow[b]{2}{*}{ Hole Type } & \multirow[b]{2}{*}{$\begin{array}{c}\text { Date } \\
\text { Completed }\end{array}$} & \multirow[b]{2}{*}{$\begin{array}{c}\text { Total } \\
\text { depth }(\mathrm{ft})\end{array}$} & \multicolumn{2}{|c|}{ UTM coordinates (m) } & \multicolumn{5}{|c|}{ Data entered } \\
\hline & & & & & East & North & Lithology & SPT & $\begin{array}{c}\text { Sample } \\
\text { test }\end{array}$ & $\begin{array}{l}\text { Water } \\
\text { level }\end{array}$ & Velocity \\
\hline 1130 & 147 & Solid stem borehole (no cores or SP & $6 / 10 / 1966$ & 5.0 & 345157 & 6791502 & $\square$ & $\square$ & $\square$ & $\square$ & 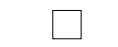 \\
\hline 1130 & 148 & Hollow stem borehole w/SPT & $12 / 20 / 1967$ & 105.0 & 345156 & 6791394 & $\checkmark$ & $\nabla$ & $\bar{\nabla}$ & $\checkmark$ & \\
\hline 1130 & 149 & Hollow stem borehole w/SPT & $12 / 4 / 1967$ & 124.0 & 345174 & 6791253 & $\checkmark$ & $\checkmark$ & $\nabla$ & $\square$ & \\
\hline 1130 & 150 & Hollow stem borehole w/SPT & $10 / 16 / 1967$ & 172.0 & 345212 & 6791087 & $\nabla$ & $\nabla$ & $\checkmark$ & $\checkmark$ & $\square$ \\
\hline 1130 & 158 & Hollow stem borehole w/SPT & $11 / 24 / 1967$ & 150.0 & 345248 & 6791290 & $\checkmark$ & $\checkmark$ & $\checkmark$ & $\checkmark$ & $\square$ \\
\hline 1130 & 159 & Solid stem borehole (no cores or SP & $6 / 10 / 1966$ & 5.0 & 345261 & 6791518 & $\square$ & & $\square$ & $\square$ & 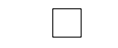 \\
\hline 1130 & 164 & Solid stem borehole (no cores or SP & $6 / 10 / 1966$ & 9.0 & 345312 & 6791621 & $\square$ & $\square$ & $\square$ & $\square$ & $\square$ \\
\hline 1130 & 165 & Solid stem borehole (no cores or SP & $6 / 10 / 1966$ & 8.0 & 345294 & 6791647 & $\square$ & & $\square$ & $\square$ & \\
\hline 1130 & 166 & Solid stem borehole (no cores or SP & $6 / 10 / 1966$ & 5.0 & 345243 & 6791629 & $\square$ & $\square$ & $\square$ & $\square$ & $\square$ \\
\hline 1130 & 167 & Solid stem borehole (no cores or SP & $6 / 10 / 1966$ & 5.0 & 345283 & 6791662 & $\square$ & $\square$ & $\square$ & $\square$ & \\
\hline 1130 & 168 & Solid stem borehole (no cores or SP & $6 / 10 / 1966$ & 11.0 & 345289 & 6791666 & $\square$ & $\square$ & $\square$ & $\square$ & $\square$ \\
\hline 1130 & 169 & Solid stem borehole (no cores or SP & $6 / 10 / 1966$ & 11.0 & 345308 & 6791676 & $\square$ & $\square$ & $\square$ & $\square$ & $\square$ \\
\hline 1130 & 170 & Solid stem borehole (no cores or SP & $6 / 10 / 1966$ & 11.0 & 345316 & 6791680 & $\square$ & $\square$ & $\square$ & $\square$ & $\square$ \\
\hline 1130 & 171 & Solid stem borehole (no cores or SP & $6 / 10 / 1966$ & 11.0 & 345323 & 6791684 & $\square$ & $\square$ & $\square$ & $\square$ & $\square$ \\
\hline 1130 & 206 & Solid stem borehole (no cores or SP & $6 / 1 / 1966$ & 6.0 & 345086 & 6791513 & $\square$ & $\square$ & $\square$ & $\square$ & $\square$ \\
\hline 1130 & 213 & Hollow stem borehole w/SPT & $5 / 20 / 1964$ & 31.5 & 345158 & 6791798 & $\square$ & $\square$ & $\square$ & $\square$ & $\square$ \\
\hline 1130 & 214 & Hollow stem borehole w/SPT & $5 / 20 / 1964$ & 31.5 & 345083 & 6791822 & $\square$ & $\square$ & $\square$ & $\square$ & $\square$ \\
\hline 1130 & 215 & Hollow stem borehole w/SPT & $5 / 20 / 1964$ & 61.5 & 345226 & 6791813 & $\checkmark$ & $\nabla$ & $\checkmark$ & $\square$ & $\square$ \\
\hline 1130 & 216 & Hollow stem borehole w/SPT & $5 / 20 / 1964$ & 96.0 & 345187 & 6791790 & $\checkmark$ & $\nabla$ & $\checkmark$ & $\square$ & \\
\hline 1130 & 218 & Hollow stem borehole w/SPT & $5 / 20 / 1964$ & 61.5 & 345114 & 6791779 & $\checkmark$ & $\checkmark$ & $\square$ & $\square$ & $\square$ \\
\hline 1130 & 226 & Hollow stem borehole w/SPT & $6 / 21 / 1969$ & 52.0 & 345193 & 6791762 & $\checkmark$ & $\checkmark$ & $\bar{\nabla}$ & $\square$ & $\square$ \\
\hline 1130 & 230 & Hollow stem borehole, no SPT & 2/9/1977 & 10.0 & 345068 & 6791801 & $\square$ & $\square$ & $\square$ & $\square$ & $\square$ \\
\hline 1130 & 231 & Hollow stem borehole, no SPT & $2 / 10 / 1977$ & 10.0 & 345043 & 6791828 & $\square$ & $\square$ & $\square$ & $\square$ & $\square$ \\
\hline 1130 & 232 & Hollow stem borehole, no SPT & $4 / 15 / 1977$ & 21.0 & 345170 & 6791807 & $\square$ & & $\square$ & $\square$ & $\square$ \\
\hline 1130 & 233 & Hollow stem borehole, no SPT & $4 / 18 / 1977$ & 15.0 & 345157 & 6791754 & $\square$ & 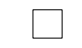 & $\square$ & $\square$ & $\square$ \\
\hline 1130 & 234 & Hollow stem borehole, no SPT & $4 / 18 / 1977$ & 21.0 & 345152 & 6791756 & $\square$ & & $\square$ & $\square$ & $\square$ \\
\hline 1130 & 235 & Hollow stem borehole, no SPT & 2/3/1977 & 10.0 & 345145 & 6791738 & $\square$ & & $\square$ & $\square$ & $\square$ \\
\hline 1130 & 236 & Hollow stem borehole, no SPT & 2/3/1977 & 10.0 & 345162 & 6791742 & $\square$ & & $\square$ & $\square$ & \\
\hline 1130 & 240 & Hollow stem borehole, no SPT & $7 / 26 / 1978$ & 27.0 & 345177 & 6791667 & $\square$ & $\square$ & $\square$ & $\square$ & $\square$ \\
\hline 1130 & 241 & Hollow stem borehole, no SPT & $7 / 26 / 1978$ & 21.0 & 345177 & 6791675 & $\square$ & $\square$ & $\square$ & $\square$ & $\square$ \\
\hline 1130 & 242 & Hollow stem borehole, no SPT & $7 / 26 / 1978$ & 30.0 & 345176 & 6791689 & $\square$ & $\square$ & $\square$ & $\square$ & $\square$ \\
\hline 1130 & 243 & Hollow stem borehole, no SPT & $7 / 26 / 1978$ & 30.0 & 345112 & 6791663 & $\square$ & $\square$ & $\square$ & $\square$ & $\square$ \\
\hline 1130 & 244 & Hollow stem borehole, no SPT & $7 / 26 / 1978$ & 16.5 & 345111 & 6791685 & $\square$ & $\square$ & $\square$ & $\square$ & $\square$ \\
\hline 1130 & 245 & Hollow stem borehole w/SPT & $7 / 26 / 1978$ & 15.0 & 345129 & 6791686 & $\square$ & $\square$ & $\square$ & $\square$ & $\square$ \\
\hline 1130 & 246 & Hollow stem borehole, no SPT & $7 / 26 / 1978$ & 15.0 & 345153 & 6791687 & $\square$ & $\square$ & $\square$ & $\square$ & $\square$ \\
\hline 1130 & JN009 & Hollow stem borehole, no SPT & $8 / 28 / 1964$ & 142.0 & 345104 & 6791502 & $\checkmark$ & $\square$ & $\checkmark$ & $\square$ & $\square$ \\
\hline
\end{tabular}




\begin{tabular}{|c|c|c|c|c|c|c|c|c|c|c|c|}
\hline \multirow[b]{2}{*}{$\begin{array}{l}\text { MOA } \\
\text { grid }\end{array}$} & \multirow[b]{2}{*}{$\begin{array}{l}\text { DGGS } \\
\text { number }\end{array}$} & \multirow[b]{2}{*}{ Hole Type } & \multirow[b]{2}{*}{$\begin{array}{c}\text { Date } \\
\text { Completed }\end{array}$} & \multirow[b]{2}{*}{$\begin{array}{c}\text { Total } \\
\text { depth }(\mathrm{ft})\end{array}$} & \multicolumn{2}{|c|}{ UTM coordinates (m) } & \multicolumn{5}{|c|}{ Data entered } \\
\hline & & & & & East & North & Lithology & SPT & $\begin{array}{c}\text { Sample } \\
\text { test }\end{array}$ & $\begin{array}{l}\text { Water } \\
\text { level }\end{array}$ & Velocity \\
\hline 1131 & 160 & Solid stem borehole (no cores or SP & $6 / 10 / 1966$ & 4.0 & 345404 & 6791484 & $\square$ & & $\square$ & $\square$ & $\square$ \\
\hline 1131 & 161 & Solid stem borehole (no cores or SP & $6 / 10 / 1966$ & 4.5 & 345350 & 6791567 & $\square$ & $\square$ & $\square$ & $\square$ & $\square$ \\
\hline 1131 & 162 & Solid stem borehole (no cores or SP & $6 / 10 / 1966$ & 10.0 & 345344 & 6791571 & $\square$ & & $\square$ & $\square$ & \\
\hline 1131 & 163 & Solid stem borehole (no cores or SP & $6 / 10 / 1966$ & 8.5 & 345339 & 6791581 & $\square$ & $\square$ & $\square$ & $\square$ & $\square$ \\
\hline 1131 & 172 & Solid stem borehole (no cores or SP & $6 / 10 / 1966$ & 11.0 & 345358 & 6791711 & $\square$ & & $\square$ & $\square$ & $\square$ \\
\hline 1131 & 173 & Solid stem borehole (no cores or SP & $6 / 10 / 1966$ & 11.0 & 345369 & 6791716 & $\square$ & & $\square$ & $\square$ & $\square$ \\
\hline 1131 & 174 & Solid stem borehole (no cores or SP & $6 / 10 / 1966$ & 6.0 & 345381 & 6791723 & $\square$ & & $\square$ & $\square$ & $\square$ \\
\hline 1131 & 175 & Solid stem borehole (no cores or SP & $6 / 10 / 1966$ & 11.0 & 345391 & 6791731 & $\square$ & & $\square$ & $\square$ & $\square$ \\
\hline 1131 & 176 & Solid stem borehole (no cores or SP & $6 / 10 / 1966$ & 11.0 & 345413 & 6791744 & $\square$ & 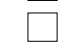 & $\square$ & $\square$ & $\square$ \\
\hline 1131 & 177 & Solid stem borehole (no cores or SP & $6 / 10 / 1966$ & 11.0 & 345428 & 6791752 & $\square$ & & $\square$ & $\square$ & \\
\hline 1131 & 178 & Solid stem borehole (no cores or SP & $6 / 10 / 1966$ & 11.0 & 345453 & 6791769 & $\square$ & $\square$ & $\square$ & $\square$ & $\square$ \\
\hline 1131 & 179 & Solid stem borehole (no cores or SP & $6 / 10 / 1966$ & 6.0 & 345469 & 6791781 & $\square$ & & $\square$ & $\square$ & $\square$ \\
\hline 1131 & 180 & Solid stem borehole (no cores or SP & $6 / 10 / 1966$ & 11.0 & 345479 & 6791787 & $\square$ & & $\square$ & $\square$ & $\square$ \\
\hline 1131 & 181 & Solid stem borehole (no cores or SP & $6 / 10 / 1966$ & 11.0 & 345505 & 6791804 & $\square$ & $\square$ & $\square$ & $\square$ & $\square$ \\
\hline 1131 & 182 & Solid stem borehole (no cores or SP & $6 / 10 / 1966$ & 8.0 & 345400 & 6791608 & $\square$ & & $\square$ & $\square$ & $\square$ \\
\hline 1131 & 183 & Solid stem borehole (no cores or SP & $6 / 10 / 1966$ & 7.0 & 345422 & 6791621 & $\square$ & & $\square$ & $\square$ & $\square$ \\
\hline 1131 & 184 & Solid stem borehole (no cores or SP & $6 / 10 / 1966$ & 5.0 & 345436 & 6791632 & $\square$ & & $\square$ & $\square$ & $\square$ \\
\hline 1131 & 185 & Solid stem borehole (no cores or SP & $6 / 10 / 1966$ & 4.0 & 345498 & 6791541 & $\square$ & & $\square$ & $\square$ & $\square$ \\
\hline 1131 & 186 & Solid stem borehole (no cores or SP & $6 / 10 / 1966$ & 6.5 & 345461 & 6791649 & $\square$ & & $\square$ & $\square$ & $\square$ \\
\hline 1131 & 187 & Solid stem borehole (no cores or SP & $6 / 10 / 1966$ & 6.5 & 345474 & 6791655 & $\square$ & $\checkmark$ & $\square$ & $\square$ & $\square$ \\
\hline 1131 & 188 & Solid stem borehole (no cores or SP & $6 / 10 / 1966$ & 6.5 & 345512 & 6791681 & $\square$ & & $\square$ & $\square$ & \\
\hline 1131 & 189 & Solid stem borehole (no cores or SP & $6 / 10 / 1966$ & 5.0 & 345528 & 6791692 & $\square$ & & $\square$ & $\square$ & $\square$ \\
\hline 1131 & 190 & Solid stem borehole (no cores or SP & $6 / 10 / 1966$ & 6.0 & 345591 & 6791600 & $\square$ & & $\square$ & $\square$ & $\square$ \\
\hline 1131 & 191 & Solid stem borehole (no cores or SP & $6 / 10 / 1966$ & 5.0 & 345645 & 6791634 & $\square$ & $\checkmark$ & $\square$ & $\square$ & $\square$ \\
\hline 1131 & 192 & Solid stem borehole (no cores or SP & $6 / 10 / 1966$ & 7.0 & 345676 & 6791654 & $\square$ & & $\square$ & $\square$ & $\square$ \\
\hline 1131 & 193 & Solid stem borehole (no cores or SP & $6 / 10 / 1966$ & 10.0 & 345684 & 6791659 & $\square$ & & $\square$ & $\square$ & $\square$ \\
\hline 1131 & 194 & Solid stem borehole (no cores or SP & $6 / 10 / 1966$ & 5.0 & 345694 & 6791666 & $\square$ & $\square$ & $\square$ & $\square$ & $\square$ \\
\hline 1131 & 195 & Solid stem borehole (no cores or SP & $6 / 10 / 1966$ & 6.0 & 345621 & 6791748 & $\square$ & & $\square$ & $\square$ & $\square$ \\
\hline 1131 & 196 & Solid stem borehole (no cores or SP & $6 / 10 / 1966$ & 11.0 & 345714 & 6791804 & $\square$ & & $\square$ & $\square$ & $\square$ \\
\hline 1131 & 197 & Hollow stem borehole, no SPT & $8 / 28 / 1964$ & 45.0 & 345785 & 6791384 & $\square$ & & $\square$ & $\square$ & $\square$ \\
\hline 1131 & 198 & Hollow stem borehole, no SPT & $8 / 28 / 1964$ & 236.0 & 345802 & 6791453 & $\nabla$ & $\square$ & $\checkmark$ & $\square$ & $\square$ \\
\hline 1131 & 199 & Hollow stem borehole, no SPT & $8 / 28 / 1964$ & 142.0 & 345825 & 6791479 & $\checkmark$ & $\square$ & $\square$ & $\square$ & $\square$ \\
\hline 1131 & 200 & Hollow stem borehole, no SPT & $8 / 28 / 1964$ & 177.0 & 345837 & 6791532 & $\checkmark$ & $\square$ & $\bar{\nabla}$ & $\square$ & $\square$ \\
\hline 1131 & 201 & Hollow stem borehole, no SPT & $8 / 28 / 1964$ & 180.0 & 345859 & 6791560 & $\checkmark$ & $\square$ & $\square$ & $\square$ & $\square$ \\
\hline 1131 & 202 & Hollow stem borehole w/SPT & $8 / 28 / 1964$ & 200.0 & 345940 & 6791531 & $\checkmark$ & $\boldsymbol{V}$ & $\checkmark$ & $\nabla$ & $\square$ \\
\hline 1131 & 203 & Hollow stem borehole, no SPT & $8 / 28 / 1964$ & 180.0 & 345936 & 6791510 & $\checkmark$ & $\square$ & $\square$ & $\square$ & $\square$ \\
\hline
\end{tabular}




\begin{tabular}{|c|c|c|c|c|c|c|c|c|c|c|c|}
\hline \multirow[b]{2}{*}{$\begin{array}{l}\text { MOA } \\
\text { grid }\end{array}$} & \multirow[b]{2}{*}{$\begin{array}{l}\text { DGGS } \\
\text { number }\end{array}$} & \multirow[b]{2}{*}{ Hole Type } & \multirow[b]{2}{*}{$\begin{array}{c}\text { Date } \\
\text { Completed }\end{array}$} & \multirow[b]{2}{*}{$\begin{array}{c}\text { Total } \\
\text { depth }(\mathrm{ft})\end{array}$} & \multicolumn{2}{|c|}{ UTM coordinates (m) } & \multicolumn{5}{|c|}{ Data entered } \\
\hline & & & & & East & North & Lithology & SPT & $\begin{array}{c}\text { Sample } \\
\text { test }\end{array}$ & $\begin{array}{l}\text { Water } \\
\text { level }\end{array}$ & Velocity \\
\hline 1131 & 204 & Hollow stem borehole, no SPT & $8 / 28 / 1964$ & 135.0 & 345914 & 6791435 & $\checkmark$ & $\square$ & $\checkmark$ & $\checkmark$ & \\
\hline 1131 & 205 & Water well & $6 / 11 / 1958$ & 280.0 & 345786 & 6791106 & $\checkmark$ & $\square$ & $\square$ & $\checkmark$ & $\square$ \\
\hline 1132 & 2147 & Hollow stem borehole w/SPT & $2 / 16 / 1997$ & 111.5 & 346871 & 6791380 & $\checkmark$ & $\nabla$ & $\nabla$ & $\bar{\nabla}$ & \\
\hline 1132 & 2148 & Hollow stem borehole w/SPT & 2/12/1997 & 111.5 & 346352 & 6791354 & $\nabla$ & $\checkmark$ & $\checkmark$ & $\nabla$ & $\square$ \\
\hline 1132 & 2149 & Hollow stem borehole w/SPT & $2 / 10 / 1997$ & 111.5 & 346351 & 6791324 & $\checkmark$ & $\checkmark$ & $\checkmark$ & $\nabla$ & $\square$ \\
\hline 1132 & 2150 & Hollow stem borehole w/SPT & 2/17/1997 & 81.5 & 346864 & 6791335 & $\checkmark$ & $\checkmark$ & $\checkmark$ & $\checkmark$ & $\square$ \\
\hline 1132 & 2152 & Hollow stem borehole w/SPT & $7 / 18 / 1996$ & 51.5 & 346924 & 6791294 & $\checkmark$ & $\checkmark$ & $\checkmark$ & $\checkmark$ & $\square$ \\
\hline 1132 & 2153 & Hollow stem borehole w/SPT & $7 / 19 / 1996$ & 51.5 & 346604 & 6791273 & $\checkmark$ & 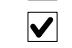 & $\nabla$ & $\bar{\nabla}$ & \\
\hline 1132 & 2154 & Hollow stem borehole w/SPT & $7 / 20 / 1996$ & 51.5 & 346347 & 6791262 & $\checkmark$ & $\checkmark$ & $\checkmark$ & $\checkmark$ & $\square$ \\
\hline 1133 & 2145 & Hollow stem borehole w/SPT & 2/13/1997 & 111.5 & 347167 & 6791386 & $\checkmark$ & $\checkmark$ & $\checkmark$ & $\checkmark$ & \\
\hline 1133 & 2146 & Hollow stem borehole w/SPT & $2 / 14 / 1997$ & 111.5 & 347181 & 6791344 & $\checkmark$ & $\checkmark$ & $\checkmark$ & $\checkmark$ & $\square$ \\
\hline 1133 & 2151 & Hollow stem borehole w/SPT & $7 / 18 / 1996$ & 51.5 & 347192 & 6791310 & $\checkmark$ & 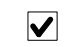 & $\bar{\nabla}$ & $\bar{\nabla}$ & $\square$ \\
\hline 1133 & IV002 & Hollow stem borehole w/SPT & 2/1/1968 & 121.5 & 347143 & 6790958 & $\checkmark$ & $\checkmark$ & $\checkmark$ & $\square$ & $\square$ \\
\hline 1133 & IV009 & Cone Penetration Test (CPT) & $3 / 28 / 1986$ & 144.0 & 347208 & 6790954 & $\square$ & $\square$ & $\square$ & $\square$ & $\square$ \\
\hline 1133 & JL001 & Hollow stem borehole w/SPT & $1 / 26 / 1968$ & 122.0 & 347154 & 6791157 & $\checkmark$ & $\bar{\square}$ & $\bar{\nabla}$ & $\checkmark$ & $\square$ \\
\hline 1133 & JL002 & Hollow stem borehole w/SPT & $1 / 22 / 1968$ & 122.0 & 347162 & 6791284 & $\checkmark$ & $\checkmark$ & $\checkmark$ & $\nabla$ & $\square$ \\
\hline 1133 & JL003 & Hollow stem borehole w/SPT & 2/7/1968 & 119.5 & 347162 & 6791321 & $\checkmark$ & $\checkmark$ & $\checkmark$ & $\nabla$ & $\square$ \\
\hline 1133 & JL005 & Water well & $9 / 17 / 1953$ & 850.0 & 347720 & 6791632 & $\checkmark$ & $\square$ & $\square$ & $\checkmark$ & \\
\hline 1134 & IW002 & Hollow stem borehole, no SPT & $5 / 6 / 1976$ & 20.0 & 348016 & 6790935 & $\square$ & $\square$ & $\square$ & $\square$ & \\
\hline 1134 & JK001 & Hollow stem borehole w/SPT & $5 / 31 / 1977$ & 32.0 & 348135 & 6791307 & $\square$ & $\square$ & $\square$ & $\square$ & $\square$ \\
\hline 1134 & JK002 & Hollow stem borehole w/SPT & $5 / 26 / 1977$ & 117.0 & 348130 & 6791332 & $\checkmark$ & $\bar{\nabla}$ & $\checkmark$ & $\bar{\nabla}$ & $\square$ \\
\hline 1134 & JK003 & Hollow stem borehole w/SPT & $5 / 24 / 1977$ & 147.0 & 348132 & 6791354 & $\checkmark$ & $\checkmark$ & $\checkmark$ & $\nabla$ & $\square$ \\
\hline 1134 & JK004 & Hollow stem borehole w/SPT & $5 / 18 / 1977$ & 102.0 & 348134 & 6791387 & $\nabla$ & $\bar{\nabla}$ & $\bar{\nabla}$ & $\nabla$ & $\square$ \\
\hline 1134 & JK005 & Hollow stem borehole w/SPT & 6/6/1977 & 142.0 & 348138 & 6791408 & $\checkmark$ & $\bar{\square}$ & $\bar{\nabla}$ & $\checkmark$ & $\square$ \\
\hline 1134 & JK006 & Solid stem borehole (no cores or SP & $5 / 7 / 1976$ & 15.5 & 348109 & 6791153 & $\square$ & $\square$ & $\square$ & $\square$ & $\square$ \\
\hline 1134 & JL006 & Hollow stem borehole w/SPT & $10 / 14 / 1980$ & 31.5 & 347660 & 6791260 & $\checkmark$ & $\checkmark$ & $\checkmark$ & $\nabla$ & $\square$ \\
\hline 1135 & 2035 & Hollow stem borehole w/SPT & $5 / 18 / 1983$ & 21.5 & 348608 & 6791153 & $\checkmark$ & $\checkmark$ & $\square$ & $\square$ & $\square$ \\
\hline 1136 & 1076 & Pit or trench & $9 / 16 / 1992$ & 10.0 & 349800 & 6790946 & $\square$ & $\square$ & $\square$ & $\square$ & \\
\hline 1136 & 1077 & Pit or trench & $9 / 16 / 1992$ & 10.0 & 349810 & 6790960 & $\square$ & $\square$ & $\square$ & $\square$ & $\square$ \\
\hline 1136 & 1078 & Pit or trench & $9 / 16 / 1992$ & 9.0 & 349799 & 6790972 & $\square$ & $\square$ & $\square$ & $\square$ & $\square$ \\
\hline 1136 & 1669 & Hollow stem borehole, no SPT & 4/7/1982 & 10.0 & 349780 & 6790920 & $\square$ & $\square$ & $\square$ & $\square$ & $\square$ \\
\hline 1136 & 1670 & Hollow stem borehole, no SPT & 4/7/1982 & 10.0 & 349782 & 6790965 & $\square$ & $\square$ & $\square$ & $\square$ & $\square$ \\
\hline 1136 & 1671 & Hollow stem borehole, no SPT & 4/7/1982 & 10.0 & 349785 & 6791015 & $\square$ & $\square$ & $\square$ & $\square$ & $\square$ \\
\hline 1137 & 1020 & Hollow stem borehole w/SPT & $1 / 17 / 1985$ & 62.0 & 350213 & 6790865 & $\checkmark$ & $\checkmark$ & $\checkmark$ & $\nabla$ & $\square$ \\
\hline 1137 & 1079 & Hollow stem borehole w/SPT & $12 / 12 / 1984$ & 61.0 & 350936 & 6790869 & $\checkmark$ & $\checkmark$ & $\checkmark$ & $\nabla$ & $\square$ \\
\hline 1137 & 1080 & Hollow stem borehole w/SPT & $12 / 13 / 1984$ & 61.0 & 350936 & 6790837 & $\checkmark$ & $\checkmark$ & $\checkmark$ & $\checkmark$ & $\square$ \\
\hline
\end{tabular}




\begin{tabular}{|c|c|c|c|c|c|c|c|c|c|c|c|}
\hline \multirow[b]{2}{*}{$\begin{array}{c}\text { MOA } \\
\text { grid }\end{array}$} & \multirow[b]{2}{*}{$\begin{array}{c}\text { DGGS } \\
\text { number }\end{array}$} & \multirow[b]{2}{*}{ Hole Type } & \multirow[b]{2}{*}{$\begin{array}{c}\text { Date } \\
\text { Completed }\end{array}$} & \multirow[b]{2}{*}{$\begin{array}{c}\text { Total } \\
\text { depth }(\mathrm{ft})\end{array}$} & \multicolumn{2}{|c|}{ UTM coordinates (m) } & \multicolumn{5}{|c|}{ Data entered } \\
\hline & & & & & East & North & Lithology & SPT & $\begin{array}{l}\text { Sample } \\
\text { test }\end{array}$ & $\begin{array}{l}\text { Water } \\
\text { level }\end{array}$ & Velocity \\
\hline 1137 & 4443 & Water well & $5 / 1 / 1967$ & 172.0 & 350644 & 6791227 & $\checkmark$ & $\square$ & $\square$ & $\checkmark$ & $\square$ \\
\hline 1137 & 4444 & Water well & $6 / 14 / 1967$ & 180.0 & 350585 & 6791230 & $\nabla$ & $\square$ & $\square$ & $\square$ & $\square$ \\
\hline 1138 & 1018 & Hollow stem borehole w/SPT & $12 / 21 / 1984$ & 62.5 & 350237 & 6790832 & $\checkmark$ & $\checkmark$ & $\checkmark$ & $\nabla$ & $\square$ \\
\hline 1140 & 1031 & Hollow stem borehole w/SPT & $11 / 21 / 1973$ & 51.5 & 353346 & 6791168 & $\nabla$ & $\checkmark$ & $\checkmark$ & $\nabla$ & $\square$ \\
\hline 1140 & 1869 & Hollow stem borehole w/SPT & 2/1/1974 & 25.0 & 353259 & 6791055 & $\square$ & $\square$ & $\square$ & $\square$ & $\square$ \\
\hline 1140 & 1870 & Hollow stem borehole w/SPT & 2/1/1974 & 23.5 & 353023 & 6790922 & $\checkmark$ & $\checkmark$ & $\square$ & $\checkmark$ & $\square$ \\
\hline 1141 & 1083 & Hollow stem borehole w/SPT & $11 / 18 / 1982$ & 200.2 & 354116 & 6791228 & $\checkmark$ & $\checkmark$ & $\checkmark$ & $\checkmark$ & $\checkmark$ \\
\hline 1141 & 1867 & Hollow stem borehole, no SPT & 2/1/1974 & 25.0 & 353401 & 6790968 & $\nabla$ & $\square$ & $\square$ & $\nabla$ & $\square$ \\
\hline 1141 & 1868 & Hollow stem borehole w/SPT & 2/1/1974 & 25.0 & 353660 & 6791156 & $\square$ & $\square$ & $\square$ & $\square$ & $\square$ \\
\hline 1141 & 3013 & Water well & $12 / 19 / 1967$ & 143.0 & 354136 & 6791173 & $\checkmark$ & $\square$ & $\square$ & $\checkmark$ & $\square$ \\
\hline 1141 & 3014 & Water well & $6 / 2 / 1967$ & 298.0 & 354132 & 6791080 & $\checkmark$ & $\square$ & $\square$ & $\checkmark$ & $\square$ \\
\hline 1142 & 1084 & Hollow stem borehole w/SPT & $12 / 5 / 1982$ & 51.5 & 354292 & 6791069 & $\checkmark$ & $\checkmark$ & $\checkmark$ & $\checkmark$ & $\square$ \\
\hline 1142 & 1085 & Hollow stem borehole w/SPT & $2 / 7 / 1983$ & 65.5 & 354319 & 6791057 & $\checkmark$ & $\nabla$ & $\nabla$ & $\nabla$ & $\square$ \\
\hline 1142 & 1086 & Hollow stem borehole w/SPT & $2 / 3 / 1983$ & 51.5 & 354281 & 6791041 & $\checkmark$ & $\checkmark$ & $\checkmark$ & $\checkmark$ & $\square$ \\
\hline 1142 & 1299 & Hollow stem borehole w/SPT & 2/3/1994 & 41.4 & 354559 & 6791059 & $\checkmark$ & $\checkmark$ & $\checkmark$ & $\square$ & $\square$ \\
\hline 1143 & 3011 & Water well & $5 / 1 / 1969$ & 129.0 & 355014 & 6791106 & $\checkmark$ & $\square$ & $\square$ & $\checkmark$ & $\square$ \\
\hline 1143 & 4434 & Water well & $9 / 1 / 1969$ & 105.0 & 355117 & 6790699 & $\checkmark$ & $\square$ & $\square$ & $\checkmark$ & $\square$ \\
\hline 1143 & 4438 & Water well & $9 / 1 / 1969$ & 105.0 & 355702 & 6791108 & $\nabla$ & $\square$ & $\square$ & $\nabla$ & $\square$ \\
\hline 1143 & 4442 & Water well & $9 / 1 / 1969$ & 105.0 & 355147 & 6791069 & $\checkmark$ & $\square$ & $\square$ & $\checkmark$ & $\square$ \\
\hline 1144 & 1864 & Solid stem borehole (no cores or SP & 2/5/1974 & 100.0 & 356137 & 6791137 & $\checkmark$ & $\square$ & $\checkmark$ & $\square$ & $\square$ \\
\hline 1144 & 1865 & Solid stem borehole (no cores or SP & 2/13/1974 & 110.0 & 355954 & 6791276 & $\checkmark$ & $\square$ & $\checkmark$ & $\checkmark$ & $\square$ \\
\hline 1144 & 1866 & Solid stem borehole (no cores or SP & $2 / 1 / 1974$ & 85.0 & 356017 & 6791344 & $\checkmark$ & $\square$ & $\nabla$ & $\nabla$ & $\square$ \\
\hline 1144 & 3009 & Water well & $3 / 31 / 1973$ & 126.0 & 356357 & 6791051 & 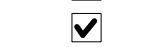 & $\square$ & $\square$ & 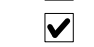 & $\square$ \\
\hline 1144 & 3010 & Water well & $3 / 18 / 1969$ & 205.0 & 356206 & 6791026 & $\checkmark$ & $\square$ & $\square$ & $V$ & $\square$ \\
\hline 1144 & 4437 & Water well & 2/13/1974 & 110.0 & 356081 & 6791248 & $\checkmark$ & $\square$ & $\square$ & $V$ & $\square$ \\
\hline 1144 & 4440 & Water well & 4/5/1974 & 112.0 & 356400 & 6791018 & $\checkmark$ & $\square$ & $\square$ & $V$ & $\square$ \\
\hline 1144 & 4441 & Water well & $3 / 18 / 1969$ & 205.0 & 356206 & 6791026 & $\nabla$ & $\square$ & $\square$ & $\nabla$ & $\square$ \\
\hline 1145 & 3008 & Water well & $4 / 17 / 1973$ & 190.0 & 356597 & 6791103 & $\checkmark$ & $\square$ & $\square$ & $\checkmark$ & $\square$ \\
\hline 1146 & 2006 & Natural exposure & 6/1/1997 & 16.0 & 357627 & 6790970 & $\boldsymbol{V}$ & $\square$ & $\square$ & $\square$ & $\square$ \\
\hline 1229 & 1032 & Hollow stem borehole w/SPT & $10 / 2 / 1985$ & 100.0 & 344240 & 6790561 & $\checkmark$ & $\checkmark$ & $\square$ & $\checkmark$ & $\square$ \\
\hline 1229 & 1033 & Hollow stem borehole w/SPT & $5 / 9 / 1996$ & 51.5 & 344137 & 6790357 & $\boldsymbol{V}$ & $\checkmark$ & $\square$ & $\checkmark$ & $\square$ \\
\hline 1229 & 1034 & Hollow stem borehole w/SPT & $5 / 19 / 1984$ & 126.5 & 344389 & 6790530 & 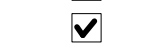 & $\checkmark$ & $\checkmark$ & $\sqrt{v}$ & $\square$ \\
\hline 1229 & 1035 & Hollow stem borehole w/SPT & $5 / 20 / 1984$ & 101.5 & 344388 & 6790456 & $\nabla$ & $\nabla$ & $\nabla$ & $\nabla$ & $\square$ \\
\hline 1229 & 1036 & Hollow stem borehole w/SPT & $5 / 22 / 1984$ & 101.5 & 344432 & 6790480 & $\checkmark$ & $\checkmark$ & $\checkmark$ & $\checkmark$ & $\square$ \\
\hline 1229 & 1037 & Hollow stem borehole w/SPT & $5 / 24 / 1984$ & 101.5 & 344496 & 6790490 & $\nabla$ & $\nabla$ & $\nabla$ & $\checkmark$ & $\square$ \\
\hline 1229 & 1038 & Hollow stem borehole w/SPT & $5 / 29 / 1984$ & 125.0 & 344410 & 6790566 & $\checkmark$ & $\checkmark$ & $\checkmark$ & $\checkmark$ & $\square$ \\
\hline
\end{tabular}




\begin{tabular}{|c|c|c|c|c|c|c|c|c|c|c|c|}
\hline \multirow[b]{2}{*}{$\begin{array}{c}\text { MOA } \\
\text { grid }\end{array}$} & \multirow[b]{2}{*}{$\begin{array}{l}\text { DGGS } \\
\text { number }\end{array}$} & \multirow[b]{2}{*}{ Hole Type } & \multirow[b]{2}{*}{$\begin{array}{c}\text { Date } \\
\text { Completed }\end{array}$} & \multirow[b]{2}{*}{$\begin{array}{c}\text { Total } \\
\text { depth }(\mathrm{ft})\end{array}$} & \multicolumn{2}{|c|}{ UTM coordinates (m) } & \multicolumn{5}{|c|}{ Data entered } \\
\hline & & & & & East & North & Lithology & SPT & $\begin{array}{c}\text { Sample } \\
\text { test }\end{array}$ & $\begin{array}{l}\text { Water } \\
\text { level }\end{array}$ & Velocity \\
\hline 1229 & 1039 & Hollow stem borehole w/SPT & $5 / 23 / 1975$ & 62.0 & 344161 & 6790309 & $\checkmark$ & $\checkmark$ & $\checkmark$ & $\checkmark$ & \\
\hline 1229 & 1040 & Hollow stem borehole w/SPT & $5 / 24 / 1975$ & 102.0 & 344162 & 6790284 & $\checkmark$ & $\checkmark$ & $\checkmark$ & $\checkmark$ & $\square$ \\
\hline 1229 & 1041 & Hollow stem borehole w/SPT & $5 / 21 / 1975$ & 56.5 & 344147 & 6790286 & $\checkmark$ & $\nabla$ & $\nabla$ & $\bar{\nabla}$ & \\
\hline 1229 & 1042 & Hollow stem borehole w/SPT & $5 / 21 / 1975$ & 96.5 & 344129 & 6790288 & $\nabla$ & $\checkmark$ & $\checkmark$ & $\nabla$ & $\square$ \\
\hline 1229 & 1043 & Hollow stem borehole w/SPT & $5 / 23 / 1975$ & 82.0 & 344161 & 6790259 & $\checkmark$ & $\checkmark$ & $\checkmark$ & $\nabla$ & $\square$ \\
\hline 1229 & 1044 & Hollow stem borehole w/SPT & $5 / 22 / 1975$ & 82.0 & 344146 & 6790259 & $\checkmark$ & $\checkmark$ & $\checkmark$ & $\checkmark$ & \\
\hline 1229 & 1135 & Hollow stem borehole w/SPT & $3 / 28 / 1977$ & 82.0 & 344387 & 6790383 & $\checkmark$ & $\checkmark$ & $\square$ & $\checkmark$ & $\square$ \\
\hline 1229 & 1136 & Hollow stem borehole w/SPT & $3 / 27 / 1977$ & 81.5 & 344391 & 6790380 & $\checkmark$ & 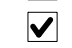 & $\square$ & $\nabla$ & \\
\hline 1229 & 1661 & Hollow stem borehole w/SPT & $3 / 24 / 1986$ & 84.0 & 344339 & 6790542 & $\checkmark$ & $\checkmark$ & $\checkmark$ & $\checkmark$ & $\square$ \\
\hline 1229 & 1662 & Hollow stem borehole w/SPT & $3 / 23 / 1986$ & 91.0 & 344376 & 6790498 & $\checkmark$ & $\checkmark$ & $\checkmark$ & $\checkmark$ & \\
\hline 1229 & 1663 & Hollow stem borehole w/SPT & $3 / 27 / 1986$ & 84.0 & 344312 & 6790450 & $\checkmark$ & $\checkmark$ & $\checkmark$ & $\checkmark$ & $\square$ \\
\hline 1229 & 1665 & Hollow stem borehole w/SPT & $3 / 22 / 1986$ & 88.0 & 344397 & 6790475 & $\checkmark$ & 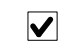 & $\square$ & $\bar{\nabla}$ & $\square$ \\
\hline 1229 & 2049 & Hollow stem borehole w/SPT & $12 / 8 / 1997$ & 99.0 & 344196 & 6790238 & $\checkmark$ & $\checkmark$ & $\checkmark$ & $\nabla$ & $\square$ \\
\hline 1229 & 2086 & Hollow stem borehole w/SPT & & 62.0 & 344095 & 6790476 & $\checkmark$ & $\checkmark$ & $\checkmark$ & $\nabla$ & $\square$ \\
\hline 1229 & 4433 & Water well & $5 / 17 / 1982$ & 291.0 & 344496 & 6790999 & $\checkmark$ & $\square$ & $\square$ & $\square$ & $\square$ \\
\hline 1229 & IS010 & Hollow stem borehole, no SPT & $8 / 28 / 1964$ & 217.0 & 344393 & 6790560 & $\checkmark$ & $\square$ & $\checkmark$ & $\square$ & $\square$ \\
\hline 1229 & IS011 & Hollow stem borehole, no SPT & $8 / 28 / 1964$ & 155.0 & 344271 & 6790478 & $\checkmark$ & $\square$ & $\square$ & $\square$ & $\square$ \\
\hline 1229 & IS012 & Hollow stem borehole, no SPT & $8 / 28 / 1964$ & 133.0 & 344398 & 6790518 & $\checkmark$ & $\square$ & $\checkmark$ & $\square$ & $\square$ \\
\hline 1229 & IS013 & Hollow stem borehole, no SPT & $8 / 28 / 1964$ & 131.0 & 344052 & 6790326 & $\checkmark$ & $\square$ & $\square$ & $\square$ & \\
\hline 1229 & IS014 & Hollow stem borehole, no SPT & $8 / 28 / 1964$ & 148.0 & 344132 & 6790295 & $\checkmark$ & $\square$ & $\checkmark$ & $\square$ & $\square$ \\
\hline 1229 & IS016 & Hollow stem borehole w/SPT & $5 / 20 / 1975$ & 31.5 & 344190 & 6790550 & $\checkmark$ & $\bar{\nabla}$ & $\square$ & $\bar{\nabla}$ & $\square$ \\
\hline 1229 & IS017 & Hollow stem borehole w/SPT & $5 / 20 / 1975$ & 29.5 & 344157 & 6790566 & $\checkmark$ & $\checkmark$ & $\square$ & $\nabla$ & $\square$ \\
\hline 1229 & IS018 & Hollow stem borehole w/SPT & $3 / 31 / 1978$ & 41.0 & 344362 & 6790306 & $\square$ & $\square$ & $\square$ & $\square$ & $\square$ \\
\hline 1229 & IS019 & Hollow stem borehole w/SPT & $3 / 31 / 1978$ & 41.5 & 344383 & 6790303 & $\square$ & $\square$ & $\square$ & $\square$ & $\square$ \\
\hline 1229 & IS020 & Hollow stem borehole w/SPT & $3 / 30 / 1978$ & 87.0 & 344371 & 6790292 & $\checkmark$ & $\checkmark$ & $\square$ & $\checkmark$ & $\square$ \\
\hline 1229 & IS021 & Hollow stem borehole w/SPT & $3 / 29 / 1978$ & 42.0 & 344354 & 6790284 & $\square$ & $\square$ & $\square$ & $\square$ & $\square$ \\
\hline 1229 & IS022 & Hollow stem borehole w/SPT & $3 / 29 / 1978$ & 42.0 & 344379 & 6790271 & $\square$ & $\square$ & $\square$ & $\square$ & $\square$ \\
\hline 1229 & IS023 & Hollow stem borehole w/SPT & $6 / 10 / 1976$ & 52.0 & 344147 & 6790395 & $\checkmark$ & $\bar{\nabla}$ & $\square$ & $\nabla$ & \\
\hline 1229 & ISO24 & Hollow stem borehole w/SPT & $6 / 10 / 1976$ & 52.0 & 344177 & 6790395 & $\checkmark$ & $\checkmark$ & $\square$ & $\checkmark$ & $\square$ \\
\hline 1229 & ISO25 & Hollow stem borehole w/SPT & $6 / 10 / 1976$ & 111.5 & 344151 & 6790382 & $\nabla$ & $\bar{\nabla}$ & $\square$ & $\checkmark$ & $\square$ \\
\hline 1229 & IS026 & Hollow stem borehole w/SPT & $6 / 10 / 1976$ & 52.0 & 344147 & 6790370 & $\nabla$ & $\checkmark$ & $\square$ & $\nabla$ & $\square$ \\
\hline 1229 & IS027 & Hollow stem borehole w/SPT & $6 / 10 / 1976$ & 52.0 & 344176 & 6790369 & $\nabla$ & $\checkmark$ & $\square$ & $\checkmark$ & $\square$ \\
\hline 1229 & ISO28 & Hollow stem borehole w/SPT & $12 / 12 / 1977$ & 27.0 & 344160 & 6790432 & $\square$ & $\square$ & $\square$ & $\square$ & $\square$ \\
\hline 1229 & IS029 & Hollow stem borehole w/SPT & $12 / 12 / 1977$ & 62.0 & 344161 & 6790414 & $\checkmark$ & $\checkmark$ & $\checkmark$ & $\nabla$ & $\square$ \\
\hline 1229 & IS048 & Hollow stem borehole w/SPT & $8 / 28 / 1964$ & 81.0 & 344494 & 6790704 & $\checkmark$ & $\checkmark$ & $\checkmark$ & $\square$ & $\square$ \\
\hline 1229 & IS064 & Hollow stem borehole w/SPT & $4 / 28 / 1984$ & 98.0 & 344293 & 6790610 & $\checkmark$ & $\checkmark$ & $\checkmark$ & $\checkmark$ & $\square$ \\
\hline
\end{tabular}




\begin{tabular}{|c|c|c|c|c|c|c|c|c|c|c|c|}
\hline \multirow[b]{2}{*}{$\begin{array}{c}\text { MOA } \\
\text { grid }\end{array}$} & \multirow[b]{2}{*}{$\begin{array}{c}\text { DGGS } \\
\text { number }\end{array}$} & \multirow[b]{2}{*}{ Hole Type } & \multirow[b]{2}{*}{$\begin{array}{c}\text { Date } \\
\text { Completed }\end{array}$} & \multirow[b]{2}{*}{$\begin{array}{c}\text { Total } \\
\text { depth }(\mathrm{ft})\end{array}$} & \multicolumn{2}{|c|}{ UTM coordinates (m) } & \multicolumn{5}{|c|}{ Data entered } \\
\hline & & & & & East & North & Lithology & SPT & $\begin{array}{c}\text { Sample } \\
\text { test }\end{array}$ & $\begin{array}{c}\text { Water } \\
\text { level }\end{array}$ & Velocity \\
\hline 1229 & IS065 & Hollow stem borehole w/SPT & $4 / 25 / 1984$ & 102.0 & 344212 & 6790612 & $\checkmark$ & $\checkmark$ & $\checkmark$ & $\square$ & $\square$ \\
\hline 1229 & IS066 & Hollow stem borehole w/SPT & $4 / 28 / 1984$ & 98.0 & 344213 & 6790562 & $\nabla$ & $\checkmark$ & $\square$ & $\checkmark$ & $\square$ \\
\hline 1229 & IS074 & Cone Penetration Test (CPT) & $11 / 2 / 1984$ & 100.0 & 344045 & 6790248 & $\square$ & $\square$ & $\square$ & $\square$ & $\square$ \\
\hline 1229 & IS075 & Cone Penetration Test (CPT) & $10 / 5 / 1984$ & 190.0 & 344201 & 6790363 & $\square$ & $\square$ & $\square$ & $\square$ & $\square$ \\
\hline 1230 & 1007 & Hollow stem borehole w/SPT & 2/7/1969 & 151.0 & 345194 & 6790663 & $\checkmark$ & $\checkmark$ & $\checkmark$ & $\checkmark$ & $\square$ \\
\hline 1230 & 1008 & Hollow stem borehole w/SPT & $2 / 17 / 1969$ & 148.5 & 345193 & 6790746 & $\nabla$ & $\checkmark$ & $\checkmark$ & $\nabla$ & $\square$ \\
\hline 1230 & 1045 & Hollow stem borehole w/SPT & 6/6/1974 & 60.0 & 344927 & 6790401 & $\checkmark$ & $\checkmark$ & $\checkmark$ & $\checkmark$ & $\square$ \\
\hline 1230 & 1046 & Hollow stem borehole w/SPT & $6 / 7 / 1974$ & 101.0 & 344932 & 6790382 & $\nabla$ & $\nabla$ & $\nabla$ & $\square$ & $\square$ \\
\hline 1230 & 1047 & Hollow stem borehole w/SPT & $12 / 19 / 1969$ & 107.0 & 344852 & 6790513 & $\nabla$ & $\checkmark$ & $\checkmark$ & $\checkmark$ & $\square$ \\
\hline 1230 & 1048 & Hollow stem borehole w/SPT & $2 / 1 / 1984$ & 111.5 & 344743 & 6790519 & $\checkmark$ & $\checkmark$ & $\checkmark$ & $\square$ & $\square$ \\
\hline 1230 & 1049 & Hollow stem borehole w/SPT & $8 / 28 / 1964$ & 60.5 & 344977 & 6790518 & $\checkmark$ & $\checkmark$ & $\square$ & $\square$ & $\square$ \\
\hline 1230 & 1050 & Hollow stem borehole w/SPT & $8 / 28 / 1964$ & 75.0 & 345042 & 6790464 & $\checkmark$ & $\checkmark$ & $\square$ & $\square$ & $\square$ \\
\hline 1230 & 1051 & Hollow stem borehole w/SPT & $8 / 28 / 1964$ & 83.0 & 345080 & 6790706 & $\checkmark$ & $\nabla$ & $\square$ & $\square$ & $\square$ \\
\hline 1230 & 1052 & Hollow stem borehole w/SPT & $8 / 28 / 1964$ & 85.5 & 344805 & 6790706 & $\checkmark$ & $\checkmark$ & $\checkmark$ & $\square$ & $\square$ \\
\hline 1230 & 1053 & Hollow stem borehole w/SPT & $4 / 30 / 1990$ & 102.5 & 345044 & 6791015 & $\checkmark$ & $\checkmark$ & $\checkmark$ & $\checkmark$ & $\square$ \\
\hline 1230 & 1054 & Hollow stem borehole w/SPT & $5 / 2 / 1990$ & 101.5 & 345052 & 6790975 & $\checkmark$ & $\checkmark$ & $\checkmark$ & $\checkmark$ & $\square$ \\
\hline 1230 & 1055 & Hollow stem borehole w/SPT & 9/4/1981 & 62.0 & 344618 & 6790262 & $\checkmark$ & $\checkmark$ & $\checkmark$ & $\checkmark$ & $\square$ \\
\hline 1230 & 1073 & Hollow stem borehole w/SPT & $11 / 1 / 1969$ & 112.0 & 344581 & 6790287 & $\nabla$ & $\checkmark$ & $\square$ & $\nabla$ & $\square$ \\
\hline 1230 & 1137 & Hollow stem borehole w/SPT & $3 / 3 / 1981$ & 51.0 & 344740 & 6790331 & $\checkmark$ & $\checkmark$ & $\checkmark$ & $\checkmark$ & $\square$ \\
\hline 1230 & 1138 & Hollow stem borehole w/SPT & $3 / 6 / 1981$ & 50.0 & 344740 & 6790361 & $\checkmark$ & $\checkmark$ & $\checkmark$ & $\checkmark$ & $\square$ \\
\hline 1230 & 1139 & Hollow stem borehole w/SPT & $3 / 6 / 1981$ & 52.0 & 344782 & 6790361 & $\checkmark$ & $\checkmark$ & $\checkmark$ & $\square$ & $\square$ \\
\hline 1230 & 1140 & Hollow stem borehole w/SPT & $3 / 9 / 1981$ & 51.1 & 344782 & 6790332 & $\checkmark$ & $\nabla$ & $\nabla$ & $\nabla$ & $\square$ \\
\hline 1230 & 1141 & Hollow stem borehole w/SPT & $3 / 10 / 1981$ & 50.0 & 344824 & 6790369 & 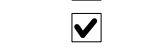 & $\nabla$ & $\nabla$ & $\square$ & $\square$ \\
\hline 1230 & 1142 & Hollow stem borehole w/SPT & $3 / 5 / 1981$ & 51.5 & 344824 & 6790334 & $\checkmark$ & $\checkmark$ & $\checkmark$ & $\checkmark$ & $\square$ \\
\hline 1230 & 1169 & Hollow stem borehole w/SPT & $4 / 22 / 1964$ & 154.0 & 344956 & 6790359 & $\checkmark$ & $\checkmark$ & $\checkmark$ & $\square$ & $\square$ \\
\hline 1230 & 1170 & Hollow stem borehole w/SPT & $4 / 16 / 1964$ & 105.0 & 344958 & 6790466 & $\checkmark$ & $\checkmark$ & $\checkmark$ & $\square$ & $\square$ \\
\hline 1230 & 1171 & Hollow stem borehole w/SPT & $4 / 15 / 1964$ & 150.0 & 344958 & 6790514 & $\nabla$ & $\checkmark$ & $\checkmark$ & $\square$ & $\square$ \\
\hline 1230 & 1172 & Hollow stem borehole w/SPT & $4 / 23 / 1964$ & 170.0 & 344959 & 6790577 & $\checkmark$ & $\checkmark$ & $\checkmark$ & $\square$ & $\square$ \\
\hline 1230 & 153 & Hollow stem borehole w/SPT & $10 / 1 / 1967$ & 166.0 & 345190 & 6790943 & $\boldsymbol{V}$ & $\nabla$ & $\nabla$ & $\square$ & $\square$ \\
\hline 1230 & 155 & Hollow stem borehole w/SPT & $10 / 13 / 1967$ & 160.0 & 345202 & 6790817 & $\checkmark$ & $\checkmark$ & $\checkmark$ & $\square$ & $\square$ \\
\hline 1230 & 156 & Hollow stem borehole w/SPT & $8 / 28 / 1964$ & 83.0 & 345197 & 6790696 & $\boldsymbol{V}$ & $\checkmark$ & $\nabla$ & $\square$ & $\square$ \\
\hline 1230 & 157 & Hollow stem borehole, no SPT & $8 / 28 / 1964$ & 210.0 & 345193 & 6790693 & 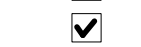 & $\square$ & $\square$ & $\square$ & $\square$ \\
\hline 1230 & 1664 & Hollow stem borehole w/SPT & $3 / 29 / 1986$ & 88.0 & 344533 & 6790420 & $\nabla$ & $\nabla$ & $\nabla$ & $\nabla$ & $\square$ \\
\hline 1230 & 305 & Water well & $4 / 1 / 1993$ & 902.0 & 344520 & 6790420 & $\checkmark$ & $\square$ & $\square$ & $\checkmark$ & $\square$ \\
\hline 1230 & 4446 & Water well & $2 / 24 / 1965$ & 190.0 & 344943 & 6790298 & $\checkmark$ & $\square$ & $\square$ & $\checkmark$ & $\square$ \\
\hline 1230 & IS030 & Hollow stem borehole w/SPT & 6/9/1982 & 71.5 & 344979 & 6790600 & $\nabla$ & $\checkmark$ & $\square$ & $\nabla$ & $\square$ \\
\hline
\end{tabular}




\begin{tabular}{|c|c|c|c|c|c|c|c|c|c|c|c|}
\hline \multirow[b]{2}{*}{$\begin{array}{c}\text { MOA } \\
\text { grid }\end{array}$} & \multirow[b]{2}{*}{$\begin{array}{l}\text { DGGS } \\
\text { number }\end{array}$} & \multirow[b]{2}{*}{ Hole Type } & \multirow[b]{2}{*}{$\begin{array}{c}\text { Date } \\
\text { Completed }\end{array}$} & \multirow[b]{2}{*}{$\begin{array}{c}\text { Total } \\
\text { depth (ft) }\end{array}$} & \multicolumn{2}{|c|}{ UTM coordinates (m) } & \multicolumn{5}{|c|}{ Data entered } \\
\hline & & & & & East & North & Lithology & SPT & $\begin{array}{c}\text { Sample } \\
\text { test }\end{array}$ & $\begin{array}{c}\text { Water } \\
\text { level }\end{array}$ & Velocity \\
\hline 1230 & IS031 & Hollow stem borehole w/SPT & $6 / 5 / 1982$ & 81.5 & 344981 & 6790542 & $\boldsymbol{V}$ & $\nabla$ & $\square$ & $\square$ & $\square$ \\
\hline 1230 & IS032 & Hollow stem borehole w/SPT & $6 / 8 / 1982$ & 81.5 & 344968 & 6790424 & $\nabla$ & $\nabla$ & $\square$ & $\checkmark$ & $\square$ \\
\hline 1230 & IS032C & Cone Penetration Test (CPT) & $5 / 22 / 1982$ & 177.9 & 344968 & 6790424 & $\square$ & $\square$ & $\square$ & $\square$ & $\square$ \\
\hline 1230 & IS033 & Hollow stem borehole w/SPT & $5 / 27 / 1982$ & 80.5 & 344946 & 6790275 & $\boldsymbol{V}$ & $\nabla$ & $\square$ & $\nabla$ & $\square$ \\
\hline 1230 & IS033C & Cone Penetration Test (CPT) & $5 / 22 / 1982$ & 171.4 & 344946 & 6790275 & $\square$ & $\square$ & $\square$ & $\square$ & $\square$ \\
\hline 1230 & IS035 & Hollow stem borehole, no SPT & $8 / 28 / 1964$ & 225.0 & 344748 & 6790360 & $\boldsymbol{V}$ & $\square$ & $\nabla$ & $\square$ & $\square$ \\
\hline 1230 & IS036 & Hollow stem borehole, no SPT & $8 / 28 / 1964$ & 170.0 & 344877 & 6790552 & $\boldsymbol{V}$ & $\square$ & $\nabla$ & $\square$ & $\square$ \\
\hline 1230 & IS037 & Hollow stem borehole, no SPT & $8 / 28 / 1964$ & 164.0 & 344927 & 6790634 & $\boldsymbol{V}$ & $\square$ & $\nabla$ & $\square$ & $\square$ \\
\hline 1230 & IS038 & Hollow stem borehole w/SPT & $8 / 28 / 1964$ & 85.0 & 344874 & 6790696 & $\boldsymbol{v}$ & $\nabla$ & $\nabla$ & $\square$ & $\square$ \\
\hline 1230 & IS039 & Hollow stem borehole, no SPT & 8/28/1964 & 240.0 & 344961 & 6790636 & $\boldsymbol{V}$ & $\square$ & $\square$ & $\square$ & $\square$ \\
\hline 1230 & IS040 & Hollow stem borehole, no SPT & $8 / 28 / 1964$ & 200.0 & 344800 & 6790458 & $\boldsymbol{V}$ & $\square$ & $\square$ & $\square$ & $\square$ \\
\hline 1230 & IS041 & Hollow stem borehole, no SPT & 8/28/1964 & 165.0 & 344972 & 6790728 & $\boldsymbol{V}$ & $\square$ & $\square$ & $\square$ & $\square$ \\
\hline 1230 & IS042 & Hollow stem borehole w/SPT & $8 / 28 / 1964$ & 68.0 & 344979 & 6790462 & $\boldsymbol{V}$ & $\nabla$ & $\square$ & $\square$ & $\square$ \\
\hline 1230 & IS043 & Hollow stem borehole w/SPT & $8 / 28 / 1964$ & 126.5 & 344748 & 6790712 & $\checkmark$ & $\checkmark$ & $\square$ & $\square$ & $\square$ \\
\hline 1230 & IS044 & Hollow stem borehole, no SPT & $8 / 28 / 1964$ & 145.0 & 344754 & 6790582 & $\boldsymbol{V}$ & $\square$ & $\nabla$ & $\square$ & $\square$ \\
\hline 1230 & IS045 & Hollow stem borehole, no SPT & $8 / 28 / 1964$ & 190.0 & 344534 & 6790594 & $\boldsymbol{V}$ & $\square$ & $\square$ & $\square$ & $\square$ \\
\hline 1230 & IS046 & Hollow stem borehole, no SPT & $8 / 28 / 1964$ & 144.0 & 344535 & 6790481 & $\boldsymbol{V}$ & $\square$ & $\square$ & $\square$ & $\square$ \\
\hline 1230 & IS047 & Hollow stem borehole w/SPT & $8 / 28 / 1964$ & 83.0 & 344621 & 6790736 & $\boldsymbol{V}$ & $\checkmark$ & $\boldsymbol{V}$ & $\square$ & $\square$ \\
\hline 1230 & IS049 & Hollow stem borehole w/SPT & $8 / 28 / 1964$ & 145.0 & 344968 & 6790420 & $\boldsymbol{V}$ & $\checkmark$ & $\boldsymbol{V}$ & $\nabla$ & $\square$ \\
\hline 1230 & IS052 & Hollow stem borehole w/SPT & $5 / 1 / 1964$ & 150.0 & 344950 & 6790196 & $\boldsymbol{V}$ & $\checkmark$ & $\nabla$ & $\square$ & $\square$ \\
\hline 1230 & IS054 & Hollow stem borehole w/SPT & $3 / 13 / 1981$ & 121.5 & 344788 & 6790330 & $\boldsymbol{V}$ & $\checkmark$ & $\nabla$ & $\checkmark$ & $\square$ \\
\hline 1230 & IS059 & Hollow stem borehole w/SPT & $9 / 8 / 1981$ & 38.0 & 344630 & 6790300 & $\square$ & $\square$ & $\square$ & $\square$ & $\square$ \\
\hline 1230 & IS060 & Hollow stem borehole w/SPT & 9/9/1981 & 41.5 & 344708 & 6790299 & $\square$ & $\square$ & $\square$ & $\square$ & $\square$ \\
\hline 1230 & IS061 & Hollow stem borehole w/SPT & $9 / 11 / 1981$ & 77.0 & 344669 & 6790258 & $\nabla$ & $\checkmark$ & $\nabla$ & $\nabla$ & $\square$ \\
\hline 1230 & IS062 & Hollow stem borehole w/SPT & 9/3/1981 & 53.5 & 344621 & 6790219 & $\boldsymbol{V}$ & $\checkmark$ & $\boldsymbol{V}$ & $\checkmark$ & $\square$ \\
\hline 1230 & IS063 & Hollow stem borehole w/SPT & 9/13/1981 & 68.0 & 344679 & 6790212 & $\boldsymbol{V}$ & $\checkmark$ & $\nabla$ & $\nabla$ & $\square$ \\
\hline 1230 & IT009 & Hollow stem borehole w/SPT & 6/6/1982 & 92.0 & 345187 & 6790564 & $\boldsymbol{V}$ & $\checkmark$ & $\square$ & $\square$ & $\square$ \\
\hline 1230 & IT009C & Cone Penetration Test (CPT) & $5 / 22 / 1982$ & 157.0 & 345187 & 6790564 & $\square$ & $\square$ & $\square$ & $\square$ & $\square$ \\
\hline 1230 & IT010C & Cone Penetration Test (CPT) & $5 / 25 / 1982$ & 150.0 & 345282 & 6790563 & $\square$ & $\square$ & $\square$ & $\square$ & $\square$ \\
\hline 1230 & IT015 & Hollow stem borehole w/SPT & $6 / 5 / 1982$ & 81.2 & 345084 & 6790469 & $\boldsymbol{V}$ & $\checkmark$ & $\square$ & $\square$ & $\square$ \\
\hline 1230 & IT015C & Cone Penetration Test (CPT) & $6 / 6 / 1982$ & 144.4 & 345084 & 6790469 & $\square$ & $\square$ & $\square$ & $\square$ & $\square$ \\
\hline 1230 & IT016 & Hollow stem borehole w/SPT & 6/6/1982 & 80.3 & 345280 & 6790446 & $\boldsymbol{V}$ & $\checkmark$ & $\square$ & $\square$ & $\square$ \\
\hline 1230 & IT016C & Cone Penetration Test (CPT) & $5 / 22 / 1982$ & 150.0 & 345280 & 6790446 & $\square$ & $\square$ & $\square$ & $\square$ & $\square$ \\
\hline 1230 & IT020 & Hollow stem borehole w/SPT & $6 / 4 / 1982$ & 80.5 & 345079 & 6790328 & $\boldsymbol{V}$ & $\nabla$ & $\square$ & $\nabla$ & $\square$ \\
\hline 1230 & IT021 & Hollow stem borehole w/SPT & $6 / 10 / 1982$ & 80.5 & 345170 & 6790305 & $\boldsymbol{v}$ & $\checkmark$ & $\square$ & $\nabla$ & $\square$ \\
\hline 1230 & IT027C & Cone Penetration Test (CPT) & $6 / 5 / 1982$ & 100.0 & 345170 & 6790188 & $\square$ & $\square$ & $\square$ & $\square$ & $\square$ \\
\hline
\end{tabular}




\begin{tabular}{|c|c|c|c|c|c|c|c|c|c|c|c|}
\hline \multirow[b]{2}{*}{$\begin{array}{l}\text { MOA } \\
\text { grid }\end{array}$} & \multirow[b]{2}{*}{$\begin{array}{l}\text { DGGS } \\
\text { number }\end{array}$} & \multirow[b]{2}{*}{ Hole Type } & \multirow[b]{2}{*}{$\begin{array}{c}\text { Date } \\
\text { Completed }\end{array}$} & \multirow[b]{2}{*}{$\begin{array}{c}\text { Total } \\
\text { depth }(\mathrm{ft})\end{array}$} & \multicolumn{2}{|c|}{ UTM coordinates (m) } & \multicolumn{5}{|c|}{ Data entered } \\
\hline & & & & & East & North & Lithology & SPT & $\begin{array}{c}\text { Sample } \\
\text { test }\end{array}$ & $\begin{array}{l}\text { Water } \\
\text { level }\end{array}$ & Velocity \\
\hline 1230 & IT037 & Hollow stem borehole, no SPT & $8 / 28 / 1964$ & 143.0 & 345192 & 6790521 & $\nabla$ & & $\checkmark$ & $\square$ & \\
\hline 1230 & IT038 & Hollow stem borehole, no SPT & $8 / 28 / 1964$ & 185.0 & 345204 & 6790576 & $\checkmark$ & L & $\square$ & $\square$ & $\square$ \\
\hline 1230 & IT042 & Hollow stem borehole, no SPT & $8 / 28 / 1964$ & 207.0 & 345186 & 6790454 & $\checkmark$ & & $\nabla$ & $\square$ & \\
\hline 1230 & IT043 & Hollow stem borehole, no SPT & $8 / 28 / 1964$ & 225.0 & 345181 & 6790341 & $\checkmark$ & $\square$ & $\nabla$ & $\square$ & $\square$ \\
\hline 1230 & IT046 & Hollow stem borehole w/SPT & $8 / 28 / 1964$ & 61.0 & 345014 & 6790496 & $\checkmark$ & $\checkmark$ & $\square$ & $\nabla$ & $\square$ \\
\hline 1230 & IT049 & Hollow stem borehole, no SPT & $8 / 28 / 1964$ & 155.0 & 345225 & 6790401 & $\checkmark$ & $\square$ & $\checkmark$ & $\square$ & $\square$ \\
\hline 1230 & IT050 & Hollow stem borehole w/SPT & $8 / 28 / 1964$ & 135.0 & 345020 & 6790565 & $\checkmark$ & $\checkmark$ & $\checkmark$ & $\checkmark$ & \\
\hline 1230 & IT051 & Hollow stem borehole, no SPT & $8 / 28 / 1964$ & 160.0 & 345207 & 6790233 & $\checkmark$ & & $\square$ & $\square$ & $\square$ \\
\hline 1230 & IT053 & Hollow stem borehole, no SPT & $8 / 28 / 1964$ & 231.0 & 345019 & 6790699 & $\nabla$ & 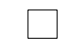 & $\square$ & $\checkmark$ & $\square$ \\
\hline 1231 & 1056 & Hollow stem borehole w/SPT & $4 / 13 / 1972$ & 18.0 & 345857 & 6790847 & $\square$ & $\square$ & $\square$ & $\square$ & $\square$ \\
\hline 1231 & 1057 & Hollow stem borehole w/SPT & $7 / 6 / 1976$ & 36.5 & 345413 & 6790548 & $\square$ & $\square$ & $\square$ & $\square$ & $\square$ \\
\hline 1231 & 1058 & Hollow stem borehole w/SPT & 6/7/1976 & 51.5 & 345442 & 6790549 & $\checkmark$ & $\checkmark$ & $\square$ & $\bar{\nabla}$ & \\
\hline 1231 & 1059 & Hollow stem borehole w/SPT & $7 / 6 / 1976$ & 41.5 & 345450 & 6790532 & $\square$ & $\square$ & $\square$ & $\square$ & $\square$ \\
\hline 1231 & 1060 & Hollow stem borehole w/SPT & $7 / 6 / 1976$ & 50.0 & 345413 & 6790520 & $\checkmark$ & $\checkmark$ & $\square$ & $\square$ & $\square$ \\
\hline 1231 & 1061 & Hollow stem borehole w/SPT & $7 / 12 / 1994$ & 21.5 & 345581 & 6790772 & $\square$ & 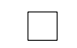 & $\square$ & $\square$ & $\square$ \\
\hline 1231 & 1062 & Hollow stem borehole w/SPT & 7/13/1994 & 31.5 & 345718 & 6790770 & $\square$ & & $\square$ & $\square$ & $\square$ \\
\hline 1231 & 1063 & Hollow stem borehole w/SPT & $7 / 12 / 1994$ & 32.0 & 345828 & 6790754 & $\square$ & 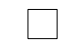 & $\square$ & $\square$ & 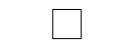 \\
\hline 1231 & 1064 & Hollow stem borehole w/SPT & $7 / 12 / 1994$ & 25.0 & 345748 & 6790697 & $\square$ & $\square$ & $\square$ & $\square$ & $\square$ \\
\hline 1231 & 1065 & Hollow stem borehole w/SPT & $7 / 11 / 1994$ & 37.0 & 345821 & 6790697 & $\square$ & $\square$ & $\square$ & $\square$ & $\square$ \\
\hline 1231 & 1066 & Hollow stem borehole w/SPT & $7 / 12 / 1994$ & 82.0 & 345814 & 6790647 & $\checkmark$ & $\nabla$ & $\bar{\nabla}$ & $\checkmark$ & $\square$ \\
\hline 1231 & 1068 & Hollow stem borehole w/SPT & $7 / 15 / 1994$ & 21.5 & 346149 & 6790688 & $\square$ & & $\square$ & $\square$ & \\
\hline 1231 & 1190 & Hollow stem borehole w/SPT & $9 / 4 / 1990$ & 40.0 & 346020 & 6790290 & $\square$ & $\square$ & $\square$ & $\square$ & $\square$ \\
\hline 1231 & 1832 & Hollow stem borehole w/SPT & $1 / 1 / 1976$ & 151.5 & 345645 & 6790204 & $\checkmark$ & $\checkmark$ & $\sqrt{V}$ & $\checkmark$ & $\square$ \\
\hline 1231 & 1833 & Hollow stem borehole w/SPT & $1 / 1 / 1976$ & 101.5 & 345611 & 6790177 & $\checkmark$ & $\nabla$ & $\bar{\nabla}$ & $\checkmark$ & $\square$ \\
\hline 1231 & 1834 & Hollow stem borehole w/SPT & $1 / 1 / 1976$ & 101.5 & 345661 & 6790175 & $\checkmark$ & $\checkmark$ & $\square$ & $\checkmark$ & $\square$ \\
\hline 1231 & 1835 & Hollow stem borehole w/SPT & $1 / 1 / 1976$ & 102.0 & 345613 & 6790235 & $\checkmark$ & $\checkmark$ & $\square$ & $\checkmark$ & $\square$ \\
\hline 1231 & 1836 & Hollow stem borehole w/SPT & $1 / 1 / 1976$ & 102.0 & 345696 & 6790256 & $\checkmark$ & $\checkmark$ & $\checkmark$ & $\checkmark$ & $\square$ \\
\hline 1231 & IT004 & Solid stem borehole (no cores or SP & $11 / 8 / 1974$ & 6.0 & 345494 & 6790817 & $\square$ & $\sqrt{2}$ & $\square$ & $\square$ & \\
\hline 1231 & IT005 & Solid stem borehole (no cores or SP & 9/23/1976 & 10.0 & 345564 & 6790818 & $\square$ & $\square$ & $\square$ & $\square$ & $\square$ \\
\hline 1231 & IT006 & Solid stem borehole (no cores or SP & 9/23/1976 & 10.0 & 345607 & 6790821 & $\square$ & $\square$ & $\square$ & $\square$ & $\square$ \\
\hline 1231 & IT007 & Solid stem borehole (no cores or SP & 9/20/1976 & 6.0 & 345691 & 6790826 & $\square$ & $\square$ & $\square$ & $\square$ & $\square$ \\
\hline 1231 & IT008 & Solid stem borehole (no cores or SP & 9/20/1976 & 8.0 & 345808 & 6790831 & $\square$ & $\square$ & $\square$ & $\square$ & $\square$ \\
\hline 1231 & IT011 & Hollow stem borehole w/SPT & $6 / 2 / 1982$ & 81.5 & 345392 & 6790538 & $\checkmark$ & $\nabla$ & $\square$ & $\square$ & $\square$ \\
\hline 1231 & IT011C & Cone Penetration Test (CPT) & $8 / 5 / 1982$ & 150.0 & 345392 & 6790538 & $\square$ & $\square$ & $\square$ & $\square$ & $\square$ \\
\hline 1231 & IT012C & Cone Penetration Test (CPT) & $6 / 5 / 1982$ & 100.0 & 345512 & 6790618 & $\square$ & $\square$ & $\square$ & $\square$ & $\square$ \\
\hline 1231 & IT013C & Cone Penetration Test (CPT) & $5 / 28 / 1982$ & 150.0 & 345503 & 6790552 & $\square$ & $\square$ & $\square$ & $\square$ & $\square$ \\
\hline
\end{tabular}




\begin{tabular}{|c|c|c|c|c|c|c|c|c|c|c|c|}
\hline \multirow[b]{2}{*}{$\begin{array}{c}\text { MOA } \\
\text { grid }\end{array}$} & \multirow[b]{2}{*}{$\begin{array}{l}\text { DGGS } \\
\text { number }\end{array}$} & \multirow[b]{2}{*}{ Hole Type } & \multirow[b]{2}{*}{$\begin{array}{c}\text { Date } \\
\text { Completed }\end{array}$} & \multirow[b]{2}{*}{$\begin{array}{c}\text { Total } \\
\text { depth (ft) }\end{array}$} & \multicolumn{2}{|c|}{ UTM coordinates (m) } & \multicolumn{5}{|c|}{ Data entered } \\
\hline & & & & & East & North & Lithology & SPT & $\begin{array}{l}\text { Sample } \\
\text { test }\end{array}$ & $\begin{array}{l}\text { Water } \\
\text { level }\end{array}$ & Velocity \\
\hline 1231 & IT014C & Cone Penetration Test (CPT) & $5 / 24 / 1982$ & 150.0 & 345693 & 6790580 & $\square$ & $\square$ & $\square$ & $\square$ & $\square$ \\
\hline 1231 & IT017 & Hollow stem borehole w/SPT & $6 / 2 / 1982$ & 102.0 & 345384 & 6790444 & $\boldsymbol{V}$ & $\nabla$ & $\square$ & $\nabla$ & $\square$ \\
\hline 1231 & IT018 & Hollow stem borehole w/SPT & 6/8/1982 & 81.2 & 345510 & 6790452 & $\boldsymbol{V}$ & $\checkmark$ & $\square$ & $\square$ & $\square$ \\
\hline 1231 & IT018C & Cone Penetration Test (CPT) & $5 / 27 / 1982$ & 150.0 & 345510 & 6790452 & $\square$ & $\square$ & $\square$ & $\square$ & $\square$ \\
\hline 1231 & IT019 & Hollow stem borehole w/SPT & 6/7/1982 & 81.5 & 345618 & 6790449 & $\checkmark$ & $\checkmark$ & $\square$ & $\checkmark$ & $\square$ \\
\hline 1231 & IT022 & Hollow stem borehole w/SPT & $6 / 1 / 1982$ & 81.5 & 345374 & 6790347 & $\nabla$ & $\checkmark$ & $\square$ & $\checkmark$ & $\square$ \\
\hline 1231 & IT022C & Cone Penetration Test (CPT) & $5 / 4 / 1982$ & 150.0 & 345374 & 6790347 & $\square$ & $\square$ & $\square$ & $\square$ & $\square$ \\
\hline 1231 & IT023C & Cone Penetration Test (CPT) & $5 / 25 / 1982$ & 150.0 & 345595 & 6790298 & $\square$ & $\square$ & $\square$ & $\square$ & $\square$ \\
\hline 1231 & IT024 & Hollow stem borehole w/SPT & $5 / 31 / 1982$ & 102.0 & 345286 & 6790258 & $\boldsymbol{v}$ & $\nabla$ & $\square$ & $\nabla$ & $\square$ \\
\hline 1231 & IT024C & Cone Penetration Test (CPT) & $6 / 5 / 1982$ & 150.0 & 345286 & 6790258 & $\square$ & $\square$ & $\square$ & $\square$ & $\square$ \\
\hline 1231 & IT025C & Cone Penetration Test (CPT) & $6 / 5 / 1982$ & 100.2 & 345380 & 6790266 & $\square$ & $\square$ & $\square$ & $\square$ & $\square$ \\
\hline 1231 & IT026 & Hollow stem borehole w/SPT & $5 / 29 / 1982$ & 73.5 & 345492 & 6790252 & $\boldsymbol{V}$ & $\nabla$ & $\square$ & $\checkmark$ & $\square$ \\
\hline 1231 & IT026C & Cone Penetration Test (CPT) & $6 / 6 / 1982$ & 150.0 & 345492 & 6790252 & $\square$ & $\square$ & $\square$ & $\square$ & $\square$ \\
\hline 1231 & IT028C & Cone Penetration Test (CPT) & $6 / 5 / 1982$ & 100.0 & 345316 & 6790211 & $\square$ & $\square$ & $\square$ & $\square$ & $\square$ \\
\hline 1231 & IT029 & Hollow stem borehole w/SPT & $6 / 1 / 1982$ & 153.0 & 345374 & 6790222 & $\boldsymbol{V}$ & $\checkmark$ & $\square$ & $\square$ & $\square$ \\
\hline 1231 & IT029C & Cone Penetration Test (CPT) & $5 / 25 / 1982$ & 150.0 & 345374 & 6790222 & $\square$ & $\square$ & $\square$ & $\square$ & $\square$ \\
\hline 1231 & IT030C & Cone Penetration Test (CPT) & $6 / 5 / 1982$ & 100.0 & 345460 & 6790205 & $\square$ & $\square$ & $\square$ & $\square$ & $\square$ \\
\hline 1231 & IT034 & Hollow stem borehole w/SPT & $6 / 7 / 1982$ & 81.3 & 345588 & 6790171 & $\boldsymbol{V}$ & $\nabla$ & $\square$ & $\nabla$ & $\square$ \\
\hline 1231 & IT034C & Cone Penetration Test (CPT) & $5 / 24 / 1982$ & 150.0 & 345588 & 6790171 & $\square$ & $\square$ & $\square$ & $\square$ & $\square$ \\
\hline 1231 & IT044 & Hollow stem borehole w/SPT & $8 / 28 / 1964$ & 109.0 & 345489 & 6790328 & $\boldsymbol{V}$ & $\checkmark$ & $\nabla$ & $\square$ & $\square$ \\
\hline 1231 & IT045 & Hollow stem borehole, no SPT & 8/28/1964 & 208.0 & 345381 & 6790586 & $\boldsymbol{V}$ & $\square$ & $\nabla$ & $\square$ & $\square$ \\
\hline 1231 & IT047 & Hollow stem borehole, no SPT & $8 / 28 / 1964$ & 194.0 & 345369 & 6790324 & $\boldsymbol{V}$ & $\square$ & $\nabla$ & $\square$ & $\square$ \\
\hline 1231 & IT048 & Hollow stem borehole, no SPT & $8 / 28 / 1964$ & 194.0 & 345696 & 6790577 & $\boldsymbol{V}$ & $\square$ & $\square$ & $\square$ & $\square$ \\
\hline 1231 & IT052 & Hollow stem borehole w/SPT & $8 / 28 / 1964$ & 85.0 & 345375 & 6790675 & $\nabla$ & $\checkmark$ & $\nabla$ & $\square$ & $\square$ \\
\hline 1231 & IT054 & Hollow stem borehole, no SPT & $8 / 28 / 1964$ & 150.0 & 345555 & 6790440 & $\boldsymbol{V}$ & $\square$ & $\square$ & $\square$ & $\square$ \\
\hline 1231 & IT055 & Hollow stem borehole w/SPT & $9 / 1 / 1978$ & 52.0 & 345556 & 6790547 & $\boldsymbol{V}$ & $\checkmark$ & $\square$ & $\nabla$ & $\square$ \\
\hline 1231 & IU001 & Solid stem borehole (no cores or SP & $5 / 24 / 1977$ & 10.0 & 346021 & 6790843 & $\square$ & $\square$ & $\square$ & $\square$ & $\square$ \\
\hline 1231 & IU002 & Hollow stem borehole w/SPT & $8 / 28 / 1964$ & 170.0 & 346102 & 6790584 & $\boldsymbol{V}$ & $\checkmark$ & $\nabla$ & $\square$ & $\square$ \\
\hline 1231 & IU003 & Hollow stem borehole, no SPT & $8 / 28 / 1964$ & 166.0 & 346086 & 6790633 & $\boldsymbol{V}$ & $\square$ & $\checkmark$ & $\square$ & $\square$ \\
\hline 1231 & IU004 & Hollow stem borehole, no SPT & 8/28/1964 & 144.0 & 346123 & 6790646 & 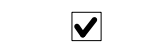 & $\square$ & $\nabla$ & $\square$ & $\square$ \\
\hline 1231 & IU005 & Hollow stem borehole w/SPT & $8 / 28 / 1964$ & 142.0 & 346119 & 6790686 & $\checkmark$ & $\checkmark$ & $\nabla$ & $\square$ & $\square$ \\
\hline 1231 & IU010 & Hollow stem borehole, no SPT & $8 / 28 / 1964$ & 169.5 & 346069 & 6790580 & $\boldsymbol{v}$ & $\square$ & $\nabla$ & $\nabla$ & $\square$ \\
\hline 1231 & IU011 & Hollow stem borehole, no SPT & $8 / 28 / 1964$ & 49.0 & 346135 & 6790668 & $\nabla$ & $\square$ & $\nabla$ & $\square$ & $\square$ \\
\hline 1232 & 1067 & Hollow stem borehole w/SPT & 7/14/1994 & 72.0 & 346128 & 6790741 & $\boldsymbol{V}$ & $\nabla$ & $\nabla$ & $\nabla$ & $\square$ \\
\hline 1232 & 1069 & Hollow stem borehole w/SPT & $6 / 1 / 1980$ & 51.5 & 346641 & 6790362 & $\boldsymbol{v}$ & $\checkmark$ & $\nabla$ & $\nabla$ & $\square$ \\
\hline 1232 & 1070 & Hollow stem borehole w/SPT & $6 / 1 / 1980$ & 51.5 & 346729 & 6790361 & $\boldsymbol{V}$ & $\boldsymbol{V}$ & $\nabla$ & $\checkmark$ & $\square$ \\
\hline
\end{tabular}




\begin{tabular}{|c|c|c|c|c|c|c|c|c|c|c|c|}
\hline \multirow[b]{2}{*}{$\begin{array}{l}\text { MOA } \\
\text { grid }\end{array}$} & \multirow[b]{2}{*}{$\begin{array}{l}\text { DGGS } \\
\text { number }\end{array}$} & \multirow[b]{2}{*}{ Hole Type } & \multirow[b]{2}{*}{$\begin{array}{c}\text { Date } \\
\text { Completed }\end{array}$} & \multirow[b]{2}{*}{$\begin{array}{c}\text { Total } \\
\text { depth }(\mathrm{ft})\end{array}$} & \multicolumn{2}{|c|}{ UTM coordinates (m) } & \multicolumn{5}{|c|}{ Data entered } \\
\hline & & & & & East & North & Lithology & SPT & $\begin{array}{c}\text { Sample } \\
\text { test }\end{array}$ & $\begin{array}{l}\text { Water } \\
\text { level }\end{array}$ & Velocity \\
\hline 1232 & 1071 & Hollow stem borehole w/SPT & $6 / 1 / 1980$ & 51.5 & 346642 & 6790293 & $\checkmark$ & $\nabla$ & $\square$ & $\checkmark$ & $\square$ \\
\hline 1232 & 1072 & Hollow stem borehole w/SPT & $6 / 1 / 1980$ & 51.5 & 346717 & 6790307 & $\checkmark$ & $\nabla$ & $\square$ & $\checkmark$ & $\square$ \\
\hline 1232 & 1655 & Hollow stem borehole w/SPT & $11 / 3 / 1986$ & 117.5 & 346285 & 6790522 & $\checkmark$ & $\checkmark$ & $\nabla$ & $\checkmark$ & $\square$ \\
\hline 1232 & IU006 & Hollow stem borehole w/SPT & $7 / 1 / 1980$ & 190.0 & 346723 & 6790250 & $\checkmark$ & $\checkmark$ & $\nabla$ & $\boldsymbol{V}$ & $\checkmark$ \\
\hline 1232 & IU007 & Hollow stem borehole w/SPT & $7 / 1 / 1980$ & 150.0 & 346725 & 6790345 & $\checkmark$ & $\checkmark$ & $\checkmark$ & $\checkmark$ & $\checkmark$ \\
\hline 1233 & 4429 & Water well & $4 / 24 / 1965$ & 140.0 & 347056 & 6790793 & $\checkmark$ & $\square$ & $\square$ & $\square$ & $\square$ \\
\hline 1233 & 4432 & Water well & $1 / 1 / 1965$ & 172.0 & 347164 & 6790882 & $\checkmark$ & $\square$ & $\square$ & $\square$ & $\square$ \\
\hline 1233 & IV001 & Hollow stem borehole w/SPT & $1 / 11 / 1968$ & 120.6 & 347121 & 6790459 & $\checkmark$ & $\checkmark$ & $\checkmark$ & $\checkmark$ & $\square$ \\
\hline 1233 & IV005 & Cone Penetration Test (CPT) & $10 / 31 / 1984$ & 126.0 & 347384 & 6790484 & $\square$ & $\square$ & $\square$ & $\square$ & $\square$ \\
\hline 1233 & IV006 & Hollow stem borehole w/SPT & $5 / 20 / 1982$ & 21.5 & 347623 & 6790834 & $\square$ & $\square$ & $\square$ & $\square$ & \\
\hline 1233 & IV007 & Hollow stem borehole w/SPT & $5 / 20 / 1982$ & 32.0 & 347604 & 6790866 & $\checkmark$ & $\nabla$ & $\square$ & $\nabla$ & $\square$ \\
\hline 1233 & IV008 & Hollow stem borehole w/SPT & $5 / 21 / 1982$ & 21.5 & 347598 & 6790910 & $\square$ & $\square$ & $\square$ & $\square$ & $\square$ \\
\hline 1234 & 2031 & Hollow stem borehole w/SPT & $3 / 22 / 1972$ & 21.0 & 347993 & 6790201 & $\checkmark$ & $\nabla$ & $\square$ & $\square$ & $\square$ \\
\hline 1234 & 2032 & Hollow stem borehole w/SPT & $3 / 23 / 1977$ & 20.7 & 348011 & 6790270 & $\checkmark$ & $\checkmark$ & $\square$ & $\square$ & $\square$ \\
\hline 1234 & 2033 & Hollow stem borehole w/SPT & 8/9/1993 & 21.5 & 347852 & 6790209 & $\checkmark$ & $\checkmark$ & $\square$ & $\square$ & $\square$ \\
\hline 1234 & 2034 & Hollow stem borehole w/SPT & $3 / 22 / 1977$ & 21.5 & 348029 & 6790229 & $\checkmark$ & $\square$ & $\square$ & $\square$ & $\square$ \\
\hline 1234 & IV003 & Solid stem borehole (no cores or SP & $5 / 5 / 1976$ & 15.0 & 347767 & 6790426 & $\square$ & $\square$ & $\square$ & $\square$ & $\square$ \\
\hline 1234 & IV004 & Solid stem borehole (no cores or SP & $5 / 5 / 1976$ & 20.0 & 347892 & 6790712 & $\checkmark$ & $\square$ & $\nabla$ & $\vec{\nabla}$ & $\square$ \\
\hline 1235 & 1013 & Hollow stem borehole w/SPT & 3/8/1989 & 50.0 & 348858 & 6790849 & $\checkmark$ & $\nabla$ & $\square$ & $\nabla$ & $\square$ \\
\hline 1235 & 1014 & Hollow stem borehole w/SPT & 3/8/1989 & 46.5 & 348882 & 6790849 & $\square$ & $\square$ & $\square$ & $\square$ & $\square$ \\
\hline 1235 & 1015 & Hollow stem borehole w/SPT & 3/9/1989 & 50.0 & 348897 & 6790848 & $\checkmark$ & $\checkmark$ & $\square$ & $\bar{\nabla}$ & \\
\hline 1235 & 1016 & Hollow stem borehole w/SPT & 3/9/1989 & 50.0 & 348880 & 6790804 & $\checkmark$ & $\nabla$ & $\square$ & $\nabla$ & $\square$ \\
\hline 1235 & 1074 & Hollow stem borehole w/SPT & 8/3/1992 & 31.5 & 348982 & 6790145 & $\square$ & $\square$ & $\square$ & $\square$ & $\square$ \\
\hline 1235 & 1075 & Hollow stem borehole w/SPT & $7 / 31 / 1992$ & 31.5 & 349071 & 6790144 & $\checkmark$ & $\nabla$ & $\bar{\nabla}$ & $\checkmark$ & $\square$ \\
\hline 1235 & 1202 & Hollow stem borehole w/SPT & $8 / 17 / 1978$ & 16.5 & 348602 & 6790076 & $\square$ & $\square$ & $\square$ & $\square$ & $\square$ \\
\hline 1235 & 1203 & Hollow stem borehole w/SPT & $1 / 10 / 1979$ & 40.0 & 348694 & 6790122 & $\nabla$ & $\checkmark$ & $\square$ & $\nabla$ & $\square$ \\
\hline 1235 & 1631 & Hollow stem borehole w/SPT & $7 / 18 / 1996$ & 26.5 & 349183 & 6790312 & $\checkmark$ & $\checkmark$ & $\checkmark$ & $\checkmark$ & $\square$ \\
\hline 1235 & 1632 & Hollow stem borehole w/SPT & $7 / 18 / 1996$ & 26.5 & 349242 & 6790251 & $\square$ & $\square$ & $\square$ & $\square$ & $\square$ \\
\hline 1236 & 1143 & Hollow stem borehole w/SPT & $4 / 28 / 1976$ & 21.5 & 349784 & 6790745 & $\square$ & $\square$ & $\square$ & $\square$ & $\square$ \\
\hline 1236 & 1144 & Hollow stem borehole w/SPT & $4 / 28 / 1976$ & 21.5 & 349791 & 6790727 & $\square$ & $\square$ & $\square$ & $\square$ & $\square$ \\
\hline 1236 & 1145 & Hollow stem borehole w/SPT & $4 / 28 / 1976$ & 21.5 & 349839 & 6790765 & $\square$ & $\square$ & $\square$ & $\square$ & $\square$ \\
\hline 1236 & 1146 & Hollow stem borehole w/SPT & $4 / 28 / 1976$ & 21.5 & 349848 & 6790745 & $\checkmark$ & $\checkmark$ & $\square$ & $\nabla$ & $\square$ \\
\hline 1237 & 4431 & Water well & $2 / 16 / 1966$ & 132.0 & 350519 & 6790737 & $\checkmark$ & $\square$ & $\square$ & $\bar{\square}$ & $\square$ \\
\hline 1238 & 1019 & Hollow stem borehole w/SPT & $1 / 16 / 1985$ & 62.0 & 350226 & 6790805 & $\checkmark$ & $\checkmark$ & $\checkmark$ & $\bar{\nabla}$ & $\square$ \\
\hline 1239 & 1147 & Hollow stem borehole w/SPT & $3 / 4 / 1982$ & 31.5 & 351997 & 6790288 & $\square$ & $\square$ & $\square$ & $\square$ & $\square$ \\
\hline 1239 & 1148 & Hollow stem borehole w/SPT & $3 / 4 / 1982$ & 31.5 & 352020 & 6790309 & $\square$ & $\square$ & $\square$ & $\square$ & $\square$ \\
\hline
\end{tabular}




\begin{tabular}{|c|c|c|c|c|c|c|c|c|c|c|c|}
\hline \multirow[b]{2}{*}{$\begin{array}{c}\text { MOA } \\
\text { grid }\end{array}$} & \multirow[b]{2}{*}{$\begin{array}{c}\text { DGGS } \\
\text { number }\end{array}$} & \multirow[b]{2}{*}{ Hole Type } & \multirow[b]{2}{*}{$\begin{array}{c}\text { Date } \\
\text { Completed }\end{array}$} & \multirow[b]{2}{*}{$\begin{array}{c}\text { Total } \\
\text { depth }(\mathrm{ft})\end{array}$} & \multicolumn{2}{|c|}{ UTM coordinates (m) } & \multicolumn{5}{|c|}{ Data entered } \\
\hline & & & & & East & North & Lithology & SPT & $\begin{array}{l}\text { Sample } \\
\text { test }\end{array}$ & $\begin{array}{l}\text { Water } \\
\text { level }\end{array}$ & Velocity \\
\hline 1239 & 1149 & Hollow stem borehole w/SPT & $3 / 5 / 1982$ & 31.5 & 352036 & 6790285 & $\square$ & $\square$ & $\square$ & $\square$ & $\square$ \\
\hline 1239 & 1150 & Hollow stem borehole w/SPT & $3 / 5 / 1982$ & 31.5 & 351981 & 6790309 & $\square$ & $\square$ & $\square$ & $\square$ & $\square$ \\
\hline 1239 & 1151 & Hollow stem borehole w/SPT & $3 / 8 / 1982$ & 31.5 & 351953 & 6790280 & $\square$ & $\square$ & $\square$ & $\square$ & $\square$ \\
\hline 1239 & 1152 & Hollow stem borehole w/SPT & 3/9/1982 & 29.0 & 351963 & 6790261 & $\square$ & $\square$ & $\square$ & $\square$ & $\square$ \\
\hline 1239 & 1153 & Hollow stem borehole w/SPT & 3/9/1982 & 30.0 & 351940 & 6790245 & $\square$ & $\square$ & $\square$ & $\square$ & $\square$ \\
\hline 1239 & 1154 & Hollow stem borehole w/SPT & $3 / 10 / 1982$ & 30.0 & 351951 & 6790225 & $\square$ & $\square$ & $\square$ & $\square$ & $\square$ \\
\hline 1240 & 1155 & Hollow stem borehole w/SPT & $3 / 25 / 1983$ & 36.0 & 352557 & 6790684 & $\square$ & $\square$ & $\square$ & $\square$ & $\square$ \\
\hline 1240 & 1156 & Hollow stem borehole w/SPT & $3 / 24 / 1983$ & 36.0 & 352622 & 6790684 & $\square$ & $\square$ & $\square$ & $\square$ & $\square$ \\
\hline 1240 & 1157 & Hollow stem borehole w/SPT & $3 / 23 / 1983$ & 36.0 & 352566 & 6790646 & $\square$ & $\square$ & $\square$ & $\square$ & $\square$ \\
\hline 1240 & 1158 & Hollow stem borehole w/SPT & $3 / 24 / 1983$ & 41.5 & 352612 & 6790646 & $\checkmark$ & $\checkmark$ & $\square$ & $\checkmark$ & $\square$ \\
\hline 1240 & 1161 & Hollow stem borehole w/SPT & $5 / 25 / 1995$ & 51.5 & 353290 & 6790176 & $\checkmark$ & $\checkmark$ & $\checkmark$ & $\checkmark$ & $\square$ \\
\hline 1240 & 4427 & Water well & $3 / 1 / 1965$ & 122.0 & 352787 & 6789960 & $\checkmark$ & $\square$ & $\square$ & $\checkmark$ & $\square$ \\
\hline 1240 & 4430 & Water well & $8 / 12 / 1964$ & 121.0 & 352993 & 6790601 & $\boldsymbol{V}$ & $\square$ & $\square$ & 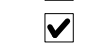 & $\square$ \\
\hline 1241 & 1159 & Hollow stem borehole w/SPT & $10 / 17 / 1975$ & 26.5 & 353394 & 6790322 & $\checkmark$ & $\checkmark$ & $\square$ & $\checkmark$ & $\square$ \\
\hline 1241 & 1160 & Hollow stem borehole w/SPT & 2/5/1982 & 26.5 & 353372 & 6790459 & $\square$ & $\square$ & $\square$ & $\square$ & $\square$ \\
\hline 1241 & 4428 & Water well & $12 / 3 / 1974$ & 134.0 & 353597 & 6790421 & $\checkmark$ & $\square$ & $\square$ & $\checkmark$ & $\square$ \\
\hline 1328 & 1162 & Hollow stem borehole w/SPT & $12 / 4 / 1995$ & 66.5 & 343120 & 6789571 & $\boldsymbol{V}$ & $\nabla$ & $\nabla$ & 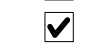 & $\square$ \\
\hline 1328 & 1163 & Hollow stem borehole w/SPT & $12 / 5 / 1995$ & 35.5 & 343124 & 6789593 & $\nabla$ & $\checkmark$ & $\checkmark$ & $\nabla$ & $\square$ \\
\hline 1328 & 1164 & Hollow stem borehole w/SPT & $1 / 9 / 1996$ & 61.0 & 343101 & 6789573 & $\checkmark$ & $\checkmark$ & $\checkmark$ & $\checkmark$ & $\square$ \\
\hline 1328 & 1165 & Hollow stem borehole w/SPT & $12 / 5 / 1995$ & 59.4 & 343103 & 6789582 & $\checkmark$ & $\checkmark$ & $\checkmark$ & $\checkmark$ & $\square$ \\
\hline 1328 & 1166 & Hollow stem borehole w/SPT & $4 / 6 / 1971$ & 61.5 & 343137 & 6789564 & $\checkmark$ & $\checkmark$ & $\square$ & $\checkmark$ & $\square$ \\
\hline 1328 & 1167 & Hollow stem borehole w/SPT & $3 / 2 / 1971$ & 57.5 & 343141 & 6789557 & $\sqrt{v}$ & $\sqrt{v}$ & $\square$ & $\checkmark$ & $\square$ \\
\hline 1328 & IN008 & Hollow stem borehole, no SPT & $8 / 28 / 1964$ & 162.0 & 343632 & 6789772 & 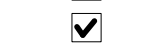 & $\square$ & $\square$ & $\square$ & $\square$ \\
\hline 1328 & IN010 & Hollow stem borehole w/SPT & $8 / 28 / 1964$ & 124.0 & 343552 & 6789640 & $\checkmark$ & $\checkmark$ & $\checkmark$ & $\square$ & $\square$ \\
\hline 1328 & IR005 & Hollow stem borehole, no SPT & $8 / 28 / 1964$ & 37.5 & 343636 & 6790116 & $\checkmark$ & $\square$ & $\checkmark$ & $\square$ & $\square$ \\
\hline 1329 & 1168 & Hollow stem borehole w/SPT & $8 / 27 / 1985$ & 72.5 & 344315 & 6790192 & $\checkmark$ & $\checkmark$ & $\checkmark$ & $\checkmark$ & $\square$ \\
\hline 1329 & 1611 & Hollow stem borehole w/SPT & 2/28/1997 & 21.5 & 343855 & 6790211 & $\square$ & $\square$ & $\square$ & $\square$ & $\square$ \\
\hline 1329 & 1612 & Hollow stem borehole w/SPT & 2/28/1997 & 21.5 & 343887 & 6790209 & $\square$ & $\square$ & $\square$ & $\square$ & $\square$ \\
\hline 1329 & 1666 & Hollow stem borehole w/SPT & $11 / 13 / 1984$ & 103.5 & 344072 & 6790104 & $\boldsymbol{V}$ & $\nabla$ & $\nabla$ & $\nabla$ & $\square$ \\
\hline 1329 & 1667 & Hollow stem borehole w/SPT & $11 / 27 / 1973$ & 60.0 & 344341 & 6789730 & $\checkmark$ & $\checkmark$ & $\checkmark$ & $\checkmark$ & $\square$ \\
\hline 1329 & 1668 & Hollow stem borehole w/SPT & $11 / 21 / 1973$ & 51.0 & 344283 & 6789689 & $\checkmark$ & $\nabla$ & $\nabla$ & $\nabla$ & $\square$ \\
\hline 1329 & 2046 & Hollow stem borehole w/SPT & $12 / 11 / 1997$ & 107.0 & 344403 & 6790082 & 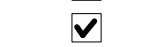 & $\checkmark$ & $\checkmark$ & $\sqrt{v}$ & $\square$ \\
\hline 1329 & 2047 & Hollow stem borehole w/SPT & $12 / 10 / 1997$ & 107.0 & 344347 & 6790122 & $\nabla$ & $\nabla$ & $\nabla$ & $\checkmark$ & $\square$ \\
\hline 1329 & 2048 & Hollow stem borehole w/SPT & $12 / 9 / 1997$ & 112.0 & 344271 & 6790192 & $\checkmark$ & $\checkmark$ & $\checkmark$ & $\checkmark$ & $\square$ \\
\hline 1329 & 2050 & Hollow stem borehole w/SPT & $12 / 12 / 1997$ & 66.5 & 344431 & 6790064 & $\boldsymbol{V}$ & $\nabla$ & $\square$ & $\checkmark$ & $\square$ \\
\hline 1329 & 2051 & Hollow stem borehole w/SPT & $12 / 12 / 1997$ & 66.5 & 344452 & 6790087 & $\checkmark$ & $\nabla$ & $\square$ & $\nabla$ & $\square$ \\
\hline
\end{tabular}




\begin{tabular}{|c|c|c|c|c|c|c|c|c|c|c|c|}
\hline \multirow[b]{2}{*}{$\begin{array}{c}\text { MOA } \\
\text { grid }\end{array}$} & \multirow[b]{2}{*}{$\begin{array}{c}\text { DGGS } \\
\text { number }\end{array}$} & \multirow[b]{2}{*}{ Hole Type } & \multirow[b]{2}{*}{$\begin{array}{c}\text { Date } \\
\text { Completed }\end{array}$} & \multirow[b]{2}{*}{$\begin{array}{c}\text { Total } \\
\text { depth }(\mathrm{ft})\end{array}$} & \multicolumn{2}{|c|}{ UTM coordinates (m) } & \multicolumn{5}{|c|}{ Data entered } \\
\hline & & & & & East & North & Lithology & SPT & $\begin{array}{c}\text { Sample } \\
\text { test }\end{array}$ & $\begin{array}{c}\text { Water } \\
\text { level }\end{array}$ & Velocity \\
\hline 1329 & 3016 & Water well & $7 / 1 / 1964$ & 200.0 & 343968 & 6789876 & $\checkmark$ & $\square$ & $\square$ & $\checkmark$ & $\square$ \\
\hline 1329 & 4424 & Water well & $6 / 21 / 1970$ & 233.0 & 344297 & 6789893 & $\nabla$ & $\square$ & $\square$ & $\checkmark$ & $\square$ \\
\hline 1329 & IM007 & Hollow stem borehole, no SPT & $8 / 28 / 1964$ & 175.0 & 344121 & 6789610 & $\checkmark$ & $\square$ & $\square$ & $\nabla$ & $\square$ \\
\hline 1329 & IM008 & Hollow stem borehole, no SPT & 8/28/1964 & 144.0 & 344288 & 6789857 & $\boldsymbol{V}$ & $\square$ & $\square$ & $\square$ & $\square$ \\
\hline 1329 & IM009 & Hollow stem borehole, no SPT & 8/28/1964 & 163.0 & 344148 & 6789978 & $\checkmark$ & $\square$ & $\square$ & $\square$ & $\square$ \\
\hline 1329 & IM010 & Hollow stem borehole, no SPT & $8 / 28 / 1964$ & 150.0 & 344183 & 6789463 & $\nabla$ & $\square$ & $\checkmark$ & $\square$ & $\square$ \\
\hline 1329 & IM011 & Hollow stem borehole w/SPT & $12 / 18 / 1979$ & 180.0 & 344343 & 6789758 & $\checkmark$ & $\checkmark$ & $\checkmark$ & $\checkmark$ & $\checkmark$ \\
\hline 1329 & IM012 & Hollow stem borehole w/SPT & $5 / 1 / 1964$ & 151.4 & 344203 & 6789990 & $\boldsymbol{V}$ & $\nabla$ & 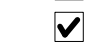 & $\square$ & $\square$ \\
\hline 1329 & IM013 & Cone Penetration Test (CPT) & 4/1/1982 & 105.0 & 344114 & 6789842 & $\square$ & $\square$ & $\square$ & $\square$ & $\square$ \\
\hline 1329 & IM014 & Cone Penetration Test (CPT) & $4 / 1 / 1982$ & 150.0 & 344287 & 6789828 & $\square$ & $\square$ & $\square$ & $\square$ & $\square$ \\
\hline 1329 & IM015 & Cone Penetration Test (CPT) & 4/1/1982 & 158.0 & 344482 & 6789818 & $\square$ & $\square$ & $\square$ & $\square$ & $\square$ \\
\hline 1329 & IN007 & Hollow stem borehole w/SPT & $8 / 28 / 1964$ & 157.5 & 343881 & 6789876 & $\checkmark$ & $\checkmark$ & $\checkmark$ & $\square$ & $\square$ \\
\hline 1329 & IN009 & Hollow stem borehole, no SPT & 8/28/1964 & 143.0 & 343947 & 6789818 & $\boldsymbol{V}$ & $\square$ & $\square$ & $\square$ & $\square$ \\
\hline 1329 & IN011 & Cone Penetration Test (CPT) & 4/1/1982 & 150.0 & 343740 & 6789874 & $\square$ & $\square$ & $\square$ & $\square$ & $\square$ \\
\hline 1329 & IN012 & Cone Penetration Test (CPT) & $4 / 1 / 1982$ & 150.0 & 343739 & 6789834 & $\square$ & $\square$ & $\square$ & $\square$ & $\square$ \\
\hline 1329 & IN013 & Hollow stem borehole, no SPT & $6 / 19 / 1964$ & 99.2 & 343781 & 6789881 & $\checkmark$ & $\square$ & $\checkmark$ & $\square$ & $\square$ \\
\hline 1329 & IN014 & Hollow stem borehole, no SPT & 6/17/1964 & 78.5 & 343783 & 6789856 & $\boldsymbol{V}$ & $\square$ & $\boldsymbol{V}$ & $\square$ & $\square$ \\
\hline 1329 & IN015 & Cone Penetration Test (CPT) & $4 / 1 / 1982$ & 150.0 & 343780 & 6789838 & $\square$ & $\square$ & $\square$ & $\square$ & $\square$ \\
\hline 1329 & IN016 & Cone Penetration Test (CPT) & $4 / 1 / 1982$ & 100.0 & 343923 & 6789852 & $\square$ & $\square$ & $\square$ & $\square$ & $\square$ \\
\hline 1329 & IR001 & Hollow stem borehole, no SPT & $8 / 28 / 1964$ & 290.0 & 343767 & 6790046 & $\boldsymbol{V}$ & $\square$ & $\square$ & $\square$ & $\square$ \\
\hline 1329 & IR002 & Hollow stem borehole, no SPT & $8 / 28 / 1964$ & 106.0 & 343841 & 6790010 & $\checkmark$ & $\square$ & $\checkmark$ & $\square$ & $\square$ \\
\hline 1329 & IR003 & Hollow stem borehole, no SPT & 8/28/1964 & 125.0 & 343764 & 6790123 & $\sqrt{v}$ & $\square$ & $\square$ & $\checkmark$ & $\square$ \\
\hline 1329 & IR004 & Hollow stem borehole, no SPT & $8 / 28 / 1964$ & 70.0 & 343739 & 6790214 & 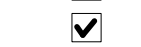 & $\square$ & $\nabla$ & $\square$ & $\square$ \\
\hline 1329 & IR006 & Hollow stem borehole w/SPT & $5 / 1 / 1964$ & 110.5 & 343912 & 6790235 & $\nabla$ & $\nabla$ & $\nabla$ & $\square$ & $\square$ \\
\hline 1329 & IS015 & Hollow stem borehole, no SPT & $8 / 28 / 1964$ & 146.0 & 344371 & 6790147 & $\checkmark$ & $\square$ & $\checkmark$ & $\square$ & $\square$ \\
\hline 1329 & IS050 & Hollow stem borehole w/SPT & $5 / 1 / 1964$ & 152.0 & 344071 & 6790102 & $\boldsymbol{V}$ & 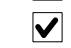 & $\boldsymbol{V}$ & $\square$ & $\square$ \\
\hline 1329 & IS051 & Hollow stem borehole w/SPT & $5 / 1 / 1964$ & 55.0 & 344009 & 6790170 & $\boldsymbol{V}$ & $\checkmark$ & $\square$ & 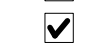 & $\square$ \\
\hline 1329 & IS072 & Cone Penetration Test (CPT) & $10 / 4 / 1984$ & 125.0 & 344158 & 6790098 & $\square$ & $\square$ & $\square$ & $\square$ & $\square$ \\
\hline 1329 & IS073 & Cone Penetration Test (CPT) & $10 / 4 / 1984$ & 160.0 & 344299 & 6790010 & $\square$ & $\square$ & $\square$ & $\square$ & $\square$ \\
\hline 1330 & 1173 & Hollow stem borehole w/SPT & $1 / 28 / 1981$ & 102.0 & 344591 & 6790090 & 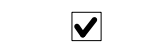 & $\nabla$ & $\nabla$ & $\nabla$ & $\square$ \\
\hline 1330 & 1174 & Hollow stem borehole w/SPT & $2 / 11 / 1981$ & 101.5 & 344596 & 6790058 & $\boldsymbol{V}$ & $\checkmark$ & $\nabla$ & $\checkmark$ & $\square$ \\
\hline 1330 & 1175 & Hollow stem borehole w/SPT & 2/4/1981 & 102.0 & 344553 & 6790091 & 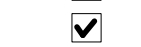 & $\checkmark$ & $\square$ & $\sqrt{v}$ & $\square$ \\
\hline 1330 & 1176 & Hollow stem borehole w/SPT & $1 / 30 / 1981$ & 102.0 & 344553 & 6790056 & 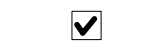 & $\nabla$ & $\square$ & $\checkmark$ & $\square$ \\
\hline 1330 & 1177 & Hollow stem borehole w/SPT & $1 / 1 / 1974$ & 65.0 & 344600 & 6790200 & $\checkmark$ & $\checkmark$ & $\square$ & $\checkmark$ & $\square$ \\
\hline 1330 & 1178 & Hollow stem borehole w/SPT & $1 / 1 / 1977$ & 102.0 & 344812 & 6790045 & $\nabla$ & $\checkmark$ & $\square$ & $\checkmark$ & $\square$ \\
\hline 1330 & 1179 & Hollow stem borehole w/SPT & $1 / 1 / 1977$ & 101.5 & 344785 & 6790046 & $\boldsymbol{V}$ & $\boldsymbol{V}$ & $\square$ & $\checkmark$ & $\square$ \\
\hline
\end{tabular}




\begin{tabular}{|c|c|c|c|c|c|c|c|c|c|c|c|}
\hline \multirow[b]{2}{*}{$\begin{array}{c}\text { MOA } \\
\text { grid }\end{array}$} & \multirow[b]{2}{*}{$\begin{array}{c}\text { DGGS } \\
\text { number }\end{array}$} & \multirow[b]{2}{*}{ Hole Type } & \multirow[b]{2}{*}{$\begin{array}{c}\text { Date } \\
\text { Completed }\end{array}$} & \multirow[b]{2}{*}{$\begin{array}{c}\text { Total } \\
\text { depth }(\mathrm{ft})\end{array}$} & \multicolumn{2}{|c|}{ UTM coordinates (m) } & \multicolumn{5}{|c|}{ Data entered } \\
\hline & & & & & East & North & Lithology & SPT & $\begin{array}{c}\text { Sample } \\
\text { test }\end{array}$ & $\begin{array}{c}\text { Water } \\
\text { level }\end{array}$ & Velocity \\
\hline 1330 & 1180 & Hollow stem borehole w/SPT & $7 / 28 / 1991$ & 52.0 & 344785 & 6790077 & $\checkmark$ & $\checkmark$ & $\checkmark$ & $\checkmark$ & $\square$ \\
\hline 1330 & 1181 & Hollow stem borehole w/SPT & $2 / 19 / 1982$ & 51.5 & 345164 & 6790014 & $\nabla$ & $\checkmark$ & $\checkmark$ & $\checkmark$ & $\square$ \\
\hline 1330 & 1182 & Hollow stem borehole w/SPT & $2 / 24 / 1982$ & 52.3 & 345241 & 6790003 & $\checkmark$ & $\checkmark$ & $\checkmark$ & $\checkmark$ & $\square$ \\
\hline 1330 & 1183 & Hollow stem borehole w/SPT & $2 / 18 / 1982$ & 52.0 & 345241 & 6789974 & $\nabla$ & $\checkmark$ & $\checkmark$ & $\nabla$ & $\square$ \\
\hline 1330 & 1184 & Hollow stem borehole w/SPT & 2/19/1982 & 52.0 & 345168 & 6789976 & $\checkmark$ & $\checkmark$ & $\checkmark$ & $\square$ & $\square$ \\
\hline 1330 & 1185 & Hollow stem borehole w/SPT & $10 / 2 / 1984$ & 92.0 & 344875 & 6789949 & $\checkmark$ & $\checkmark$ & $\checkmark$ & $\checkmark$ & $\square$ \\
\hline 1330 & IL001 & Cone Penetration Test (CPT) & $5 / 12 / 1982$ & 80.8 & 345020 & 6789969 & $\square$ & $\square$ & $\square$ & $\square$ & $\square$ \\
\hline 1330 & IM001 & Cone Penetration Test (CPT) & $5 / 12 / 1982$ & 101.3 & 344845 & 6789975 & $\square$ & $\square$ & $\square$ & $\square$ & $\square$ \\
\hline 1330 & IM002 & Hollow stem borehole w/SPT & $6 / 15 / 1982$ & 81.0 & 344897 & 6789992 & $\nabla$ & $\checkmark$ & $\square$ & $\checkmark$ & $\square$ \\
\hline 1330 & IM003 & Cone Penetration Test (CPT) & $5 / 13 / 1982$ & 150.0 & 344888 & 6789973 & $\square$ & $\square$ & $\square$ & $\square$ & $\square$ \\
\hline 1330 & IM004 & Cone Penetration Test (CPT) & $5 / 13 / 1982$ & 115.0 & 344948 & 6789972 & $\square$ & $\square$ & $\square$ & $\square$ & $\square$ \\
\hline 1330 & IM005 & Cone Penetration Test (CPT) & $5 / 12 / 1982$ & 101.3 & 344987 & 6789970 & $\square$ & $\square$ & $\square$ & $\square$ & $\square$ \\
\hline 1330 & IM016 & Cone Penetration Test (CPT) & $4 / 1 / 1982$ & 155.0 & 344715 & 6789814 & $\square$ & $\square$ & $\square$ & $\square$ & $\square$ \\
\hline 1330 & IM020 & Hollow stem borehole w/SPT & $4 / 28 / 1983$ & 52.0 & 344985 & 6789893 & $\checkmark$ & $\checkmark$ & $\checkmark$ & $\checkmark$ & $\square$ \\
\hline 1330 & IS001 & Hollow stem borehole w/SPT & $8 / 24 / 1982$ & 77.0 & 344833 & 6790054 & $\checkmark$ & $\checkmark$ & $\square$ & $\checkmark$ & $\square$ \\
\hline 1330 & ISO02 & Cone Penetration Test (CPT) & $5 / 13 / 1982$ & 163.0 & 344848 & 6790084 & $\square$ & $\square$ & $\square$ & $\square$ & $\square$ \\
\hline 1330 & IS003 & Cone Penetration Test (CPT) & $5 / 13 / 1982$ & 88.0 & 344900 & 6790078 & $\square$ & $\square$ & $\square$ & $\square$ & $\square$ \\
\hline 1330 & ISO04 & Cone Penetration Test (CPT) & $5 / 13 / 1982$ & 113.0 & 344924 & 6790080 & $\square$ & $\square$ & $\square$ & $\square$ & $\square$ \\
\hline 1330 & IS005 & Hollow stem borehole w/SPT & $8 / 5 / 1982$ & 77.1 & 344935 & 6790056 & $\checkmark$ & $\checkmark$ & $\square$ & $\checkmark$ & $\square$ \\
\hline 1330 & IS006 & Cone Penetration Test (CPT) & $8 / 5 / 1982$ & 115.0 & 344981 & 6790071 & $\square$ & $\square$ & $\square$ & $\square$ & $\square$ \\
\hline 1330 & IS007 & Cone Penetration Test (CPT) & $8 / 5 / 1982$ & 88.0 & 344893 & 6790028 & $\square$ & $\square$ & $\square$ & $\square$ & $\square$ \\
\hline 1330 & IS008 & Cone Penetration Test (CPT) & $8 / 5 / 1982$ & 90.0 & 344948 & 6790024 & $\square$ & $\square$ & $\square$ & $\square$ & $\square$ \\
\hline 1330 & ISO09 & Hollow stem borehole w/SPT & $6 / 3 / 1982$ & 87.0 & 344984 & 6790034 & 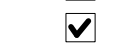 & $\checkmark$ & $\square$ & 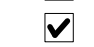 & $\square$ \\
\hline 1330 & IS034C & Cone Penetration Test (CPT) & $5 / 22 / 1982$ & 165.4 & 344929 & 6790070 & $\square$ & $\square$ & $\square$ & $\square$ & $\square$ \\
\hline 1330 & IS053 & Hollow stem borehole w/SPT & $2 / 26 / 1981$ & 198.0 & 344576 & 6790060 & $\checkmark$ & $\checkmark$ & $\checkmark$ & $\checkmark$ & $\checkmark$ \\
\hline 1330 & IS055 & Hollow stem borehole w/SPT & $2 / 28 / 1984$ & 87.0 & 344556 & 6790155 & $\boldsymbol{V}$ & 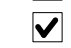 & $\nabla$ & $\checkmark$ & $\square$ \\
\hline 1330 & IS056 & Hollow stem borehole w/SPT & $2 / 29 / 1984$ & 80.0 & 344529 & 6790183 & $\checkmark$ & $\checkmark$ & $\checkmark$ & $\checkmark$ & $\square$ \\
\hline 1330 & IS057 & Hollow stem borehole w/SPT & 3/1/1984 & 70.0 & 344597 & 6790165 & $\checkmark$ & $\checkmark$ & $\checkmark$ & $\checkmark$ & $\square$ \\
\hline 1330 & IS058 & Hollow stem borehole w/SPT & $3 / 2 / 1984$ & 87.0 & 344529 & 6790153 & $\boldsymbol{V}$ & $\nabla$ & $\nabla$ & $\nabla$ & $\square$ \\
\hline 1330 & IS067 & Hollow stem borehole w/SPT & 8/4/1981 & 199.0 & 344748 & 6790047 & $\checkmark$ & $\checkmark$ & $\checkmark$ & $\checkmark$ & $\square$ \\
\hline 1330 & IS068 & Hollow stem borehole w/SPT & 9/29/1977 & 102.0 & 344778 & 6790050 & $\boldsymbol{V}$ & $\checkmark$ & $\square$ & $\checkmark$ & $\square$ \\
\hline 1330 & IS069 & Hollow stem borehole w/SPT & 9/29/1977 & 102.0 & 344810 & 6790062 & 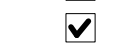 & $\checkmark$ & $\square$ & $\sqrt{v}$ & $\square$ \\
\hline 1330 & IT001 & Hollow stem borehole w/SPT & $6 / 10 / 1982$ & 75.5 & 345010 & 6790070 & $\checkmark$ & $\checkmark$ & $\square$ & $\checkmark$ & $\square$ \\
\hline 1330 & IT002 & Cone Penetration Test (CPT) & $6 / 1 / 1982$ & 77.0 & 345018 & 6790070 & $\square$ & $\square$ & $\square$ & $\square$ & $\square$ \\
\hline 1330 & IT003 & Cone Penetration Test (CPT) & 6/1/1982 & 147.0 & 345002 & 6790023 & $\square$ & $\square$ & $\square$ & $\square$ & $\square$ \\
\hline 1330 & IT031 & Hollow stem borehole w/SPT & $6 / 9 / 1982$ & 81.5 & 345256 & 6790152 & $\boldsymbol{V}$ & $\checkmark$ & $\square$ & $\checkmark$ & $\square$ \\
\hline
\end{tabular}




\begin{tabular}{|c|c|c|c|c|c|c|c|c|c|c|c|}
\hline \multirow[b]{2}{*}{$\begin{array}{c}\text { MOA } \\
\text { grid }\end{array}$} & \multirow[b]{2}{*}{$\begin{array}{l}\text { DGGS } \\
\text { number }\end{array}$} & \multirow[b]{2}{*}{ Hole Type } & \multirow[b]{2}{*}{$\begin{array}{c}\text { Date } \\
\text { Completed }\end{array}$} & \multirow[b]{2}{*}{$\begin{array}{c}\text { Total } \\
\text { depth (ft) }\end{array}$} & \multicolumn{2}{|c|}{ UTM coordinates (m) } & \multicolumn{5}{|c|}{ Data entered } \\
\hline & & & & & East & North & Lithology & SPT & $\begin{array}{c}\text { Sample } \\
\text { test }\end{array}$ & $\begin{array}{c}\text { Water } \\
\text { level }\end{array}$ & Velocity \\
\hline 1330 & IT031C & Cone Penetration Test (CPT) & $5 / 25 / 1982$ & 150.0 & 345256 & 6790152 & $\square$ & $\square$ & $\square$ & $\square$ & $\square$ \\
\hline 1330 & IT035C & Cone Penetration Test (CPT) & $5 / 27 / 1982$ & 148.2 & 345179 & 6790078 & $\square$ & $\square$ & $\square$ & $\square$ & $\square$ \\
\hline 1330 & IT056 & Hollow stem borehole w/SPT & $2 / 23 / 1982$ & 92.5 & 345215 & 6790114 & $\boldsymbol{V}$ & $\checkmark$ & $\nabla$ & $\checkmark$ & $\square$ \\
\hline 1331 & 1186 & Hollow stem borehole w/SPT & $10 / 15 / 1982$ & 27.2 & 345253 & 6789814 & $\boldsymbol{V}$ & $\checkmark$ & $\square$ & 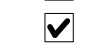 & $\square$ \\
\hline 1331 & 1491 & Hollow stem borehole w/SPT & $1 / 24 / 1984$ & 21.5 & 346063 & 6789886 & $\square$ & $\square$ & $\square$ & $\square$ & $\square$ \\
\hline 1331 & 1492 & Hollow stem borehole w/SPT & $1 / 24 / 1984$ & 21.5 & 346104 & 6789874 & $\square$ & $\square$ & $\square$ & $\square$ & $\square$ \\
\hline 1331 & 1493 & Hollow stem borehole w/SPT & $1 / 24 / 1984$ & 21.5 & 346063 & 6789865 & $\square$ & $\square$ & $\square$ & $\square$ & $\square$ \\
\hline 1331 & 1494 & Hollow stem borehole w/SPT & $1 / 24 / 1984$ & 31.5 & 346089 & 6789869 & $\boldsymbol{V}$ & $\nabla$ & $\square$ & $\nabla$ & $\square$ \\
\hline 1331 & 1495 & Hollow stem borehole w/SPT & $1 / 27 / 1984$ & 21.5 & 346109 & 6789861 & $\square$ & $\square$ & $\square$ & $\square$ & $\square$ \\
\hline 1331 & 1496 & Hollow stem borehole w/SPT & $1 / 27 / 1984$ & 21.5 & 346061 & 6789836 & $\square$ & $\square$ & $\square$ & $\square$ & $\square$ \\
\hline 1331 & 1497 & Hollow stem borehole w/SPT & $1 / 30 / 1984$ & 21.5 & 346108 & 6789827 & $\square$ & $\square$ & $\square$ & $\square$ & $\square$ \\
\hline 1331 & IL006 & Cone Penetration Test (CPT) & $10 / 31 / 1984$ & 110.0 & 345773 & 6789549 & $\square$ & $\square$ & $\square$ & $\square$ & $\square$ \\
\hline 1331 & IT032C & Cone Penetration Test (CPT) & $6 / 5 / 1982$ & 100.0 & 345383 & 6790171 & $\square$ & $\square$ & $\square$ & $\square$ & $\square$ \\
\hline 1331 & IT033 & Hollow stem borehole w/SPT & $6 / 29 / 1982$ & 102.0 & 345498 & 6790158 & $\checkmark$ & $\checkmark$ & $\square$ & $\checkmark$ & $\square$ \\
\hline 1331 & IT033C & Cone Penetration Test (CPT) & $5 / 25 / 1982$ & 150.0 & 345498 & 6790158 & $\square$ & $\square$ & $\square$ & $\square$ & $\square$ \\
\hline 1331 & IT036 & Hollow stem borehole w/SPT & $6 / 3 / 1982$ & 80.5 & 345379 & 6790079 & $\boldsymbol{V}$ & $\nabla$ & $\square$ & $\nabla$ & $\square$ \\
\hline 1331 & IT036C & Cone Penetration Test (CPT) & $5 / 25 / 1982$ & 150.0 & 345379 & 6790079 & $\square$ & $\square$ & $\square$ & $\square$ & $\square$ \\
\hline 1333 & 3015 & Water well & $5 / 6 / 1969$ & 305.0 & 347627 & 6789839 & $\boldsymbol{V}$ & $\square$ & $\square$ & $\nabla$ & $\square$ \\
\hline 1334 & 1187 & Hollow stem borehole w/SPT & $5 / 27 / 1983$ & 76.5 & 348287 & 6789448 & $\boldsymbol{V}$ & $\checkmark$ & $\nabla$ & $\nabla$ & $\square$ \\
\hline 1334 & 1188 & Hollow stem borehole w/SPT & $6 / 3 / 1983$ & 79.2 & 348307 & 6789447 & $\boldsymbol{V}$ & $\checkmark$ & $\checkmark$ & $\checkmark$ & $\square$ \\
\hline 1334 & 1189 & Hollow stem borehole w/SPT & $6 / 1 / 1983$ & 71.5 & 348310 & 6789434 & $\boldsymbol{V}$ & $\checkmark$ & $\nabla$ & $\checkmark$ & $\square$ \\
\hline 1334 & 1191 & Hollow stem borehole w/SPT & $9 / 18 / 1990$ & 71.0 & 348265 & 6789442 & $\boldsymbol{V}$ & $\nabla$ & $\square$ & $\nabla$ & $\square$ \\
\hline 1334 & 1192 & Hollow stem borehole w/SPT & $9 / 14 / 1990$ & 65.5 & 348228 & 6789445 & $\boldsymbol{V}$ & $\nabla$ & $\nabla$ & $\nabla$ & $\square$ \\
\hline 1334 & 1193 & Hollow stem borehole w/SPT & $9 / 17 / 1990$ & 70.7 & 348266 & 6789468 & $\nabla$ & $\checkmark$ & $\square$ & $\nabla$ & $\square$ \\
\hline 1334 & 1194 & Hollow stem borehole w/SPT & $4 / 23 / 1990$ & 66.0 & 348226 & 6789467 & $\boldsymbol{V}$ & $\checkmark$ & $\nabla$ & $\nabla$ & $\square$ \\
\hline 1334 & 1195 & Hollow stem borehole w/SPT & $9 / 27 / 1990$ & 70.5 & 348240 & 6789455 & $\boldsymbol{V}$ & $\checkmark$ & $\nabla$ & $\nabla$ & $\square$ \\
\hline 1334 & 1196 & Hollow stem borehole w/SPT & $9 / 27 / 1990$ & 70.5 & 348253 & 6789455 & $\boldsymbol{V}$ & $\checkmark$ & $\nabla$ & $\nabla$ & $\square$ \\
\hline 1334 & 1199 & Hollow stem borehole w/SPT & $3 / 21 / 1995$ & 31.5 & 348436 & 6790041 & $\square$ & $\square$ & $\square$ & $\square$ & $\square$ \\
\hline 1334 & 1200 & Hollow stem borehole, no SPT & $1 / 10 / 1979$ & 40.0 & 348454 & 6790036 & $\square$ & $\square$ & $\square$ & $\square$ & $\square$ \\
\hline 1334 & II001 & Hollow stem borehole w/SPT & $5 / 26 / 1983$ & 100.3 & 348302 & 6789461 & 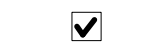 & $\nabla$ & $\nabla$ & $\nabla$ & $\boldsymbol{V}$ \\
\hline 1334 & II002 & Hollow stem borehole w/SPT & $6 / 2 / 1983$ & 65.5 & 348300 & 6789424 & $\checkmark$ & $\checkmark$ & $\square$ & $\checkmark$ & $\square$ \\
\hline 1334 & II003 & Hollow stem borehole w/SPT & $6 / 7 / 1983$ & 95.0 & 348325 & 6789440 & $\boldsymbol{v}$ & $\checkmark$ & $\nabla$ & $\checkmark$ & $\square$ \\
\hline 1334 & II004 & Hollow stem borehole w/SPT & $7 / 28 / 1983$ & 19.0 & 348387 & 6789466 & $\square$ & $\square$ & $\square$ & $\square$ & $\square$ \\
\hline 1334 & II006 & Hollow stem borehole w/SPT & $4 / 8 / 1980$ & 76.5 & 348107 & 6789854 & $\nabla$ & $\checkmark$ & $\nabla$ & $\nabla$ & $\square$ \\
\hline 1334 & II007 & Hollow stem borehole w/SPT & $4 / 2 / 1980$ & 61.5 & 348185 & 6789852 & $\boldsymbol{v}$ & $\checkmark$ & $\nabla$ & $\nabla$ & $\square$ \\
\hline 1334 & II008 & Hollow stem borehole w/SPT & $3 / 31 / 1980$ & 67.0 & 348143 & 6789825 & $\boldsymbol{V}$ & $\boldsymbol{V}$ & $\square$ & $\checkmark$ & $\square$ \\
\hline
\end{tabular}




\begin{tabular}{|c|c|c|c|c|c|c|c|c|c|c|c|}
\hline \multirow[b]{2}{*}{$\begin{array}{l}\text { MOA } \\
\text { grid }\end{array}$} & \multirow[b]{2}{*}{$\begin{array}{l}\text { DGGS } \\
\text { number }\end{array}$} & \multirow[b]{2}{*}{ Hole Type } & \multirow[b]{2}{*}{$\begin{array}{c}\text { Date } \\
\text { Completed }\end{array}$} & \multirow[b]{2}{*}{$\begin{array}{c}\text { Total } \\
\text { depth }(\mathrm{ft})\end{array}$} & \multicolumn{2}{|c|}{ UTM coordinates (m) } & \multicolumn{5}{|c|}{ Data entered } \\
\hline & & & & & East & North & Lithology & SPT & $\begin{array}{c}\text { Sample } \\
\text { test }\end{array}$ & $\begin{array}{l}\text { Water } \\
\text { level }\end{array}$ & Velocity \\
\hline 1334 & ІІ009 & Hollow stem borehole w/SPT & $4 / 7 / 1980$ & 66.5 & 348178 & 6789814 & $\nabla$ & $\checkmark$ & $\square$ & $\checkmark$ & \\
\hline 1334 & II010 & Hollow stem borehole w/SPT & $4 / 4 / 1980$ & 72.0 & 348108 & 6789802 & $\checkmark$ & $\checkmark$ & $\checkmark$ & $\checkmark$ & $\square$ \\
\hline 1334 & II011 & Solid stem borehole (no cores or SP & $4 / 1 / 1980$ & 24.0 & 348139 & 6789928 & $\square$ & & $\square$ & $\square$ & \\
\hline 1334 & II012 & Solid stem borehole (no cores or SP & $4 / 1 / 1980$ & 10.0 & 348294 & 6789874 & $\square$ & $\checkmark$ & $\square$ & $\square$ & $\square$ \\
\hline 1335 & 1197 & Hollow stem borehole w/SPT & $8 / 10 / 1983$ & 31.5 & 349240 & 6789587 & $\square$ & $\square$ & $\square$ & $\square$ & $\square$ \\
\hline 1335 & 1198 & Hollow stem borehole w/SPT & $8 / 10 / 1983$ & 31.5 & 349242 & 6789777 & $\square$ & $\square$ & $\square$ & $\square$ & $\square$ \\
\hline 1335 & 1201 & Hollow stem borehole w/SPT & $1 / 15 / 1979$ & 41.5 & 348528 & 6790015 & $\square$ & $\square$ & $\square$ & $\square$ & $\square$ \\
\hline 1335 & 1204 & Hollow stem borehole w/SPT & $1 / 11 / 1979$ & 40.0 & 348772 & 6790005 & $\square$ & & $\square$ & $\square$ & $\square$ \\
\hline 1335 & 1215 & Hollow stem borehole w/SPT & $3 / 16 / 1971$ & 13.0 & 349120 & 6789251 & $\square$ & $\square$ & $\square$ & $\square$ & $\square$ \\
\hline 1335 & 2016 & Hollow stem borehole, no SPT & $1 / 1 / 1979$ & 460.0 & 348650 & 6790070 & $\checkmark$ & & $\square$ & $\checkmark$ & $\checkmark$ \\
\hline 1335 & 4447 & Water well & $5 / 1 / 1977$ & 164.0 & 348580 & 6789767 & $\checkmark$ & $\square$ & $\square$ & $\checkmark$ & $\square$ \\
\hline 1335 & II016 & Solid stem borehole (no cores or SP & $3 / 16 / 1971$ & 13.0 & 348476 & 6789280 & $\square$ & & $\square$ & $\square$ & \\
\hline 1335 & ІІ017 & Solid stem borehole (no cores or SP & $3 / 16 / 1971$ & 13.0 & 348600 & 6789270 & $\square$ & & $\square$ & $\square$ & $\square$ \\
\hline 1335 & II018 & Solid stem borehole (no cores or SP & $3 / 16 / 1971$ & 13.0 & 348702 & 6789266 & $\square$ & $\square$ & $\square$ & $\square$ & $\square$ \\
\hline 1335 & II019 & Solid stem borehole (no cores or SP & $3 / 16 / 1971$ & 13.0 & 348820 & 6789268 & $\square$ & & $\square$ & $\square$ & $\square$ \\
\hline 1335 & IW001 & Hollow stem borehole w/SPT & $1 / 11 / 1979$ & 40.0 & 348678 & 6790028 & $\square$ & & $\square$ & $\square$ & $\square$ \\
\hline 1336 & 1205 & Hollow stem borehole w/SPT & $7 / 3 / 1990$ & 26.0 & 349810 & 6789295 & $\square$ & & $\square$ & $\square$ & $\square$ \\
\hline 1336 & 1206 & Hollow stem borehole w/SPT & $7 / 5 / 1994$ & 28.5 & 349857 & 6789422 & $\square$ & & $\square$ & $\square$ & $\square$ \\
\hline 1336 & 1207 & Hollow stem borehole w/SPT & $4 / 11 / 1991$ & 31.5 & 349524 & 6789452 & $\square$ & $\square$ & $\square$ & $\square$ & $\square$ \\
\hline 1336 & 1208 & Hollow stem borehole w/SPT & $4 / 10 / 1991$ & 31.5 & 349623 & 6789449 & $\square$ & $\checkmark$ & $\square$ & $\square$ & $\square$ \\
\hline 1336 & 1209 & Hollow stem borehole w/SPT & $4 / 10 / 1991$ & 32.0 & 349619 & 6789367 & $\square$ & & $\square$ & $\square$ & \\
\hline 1336 & 1218 & Hollow stem borehole w/SPT & $3 / 17 / 1971$ & 13.0 & 349642 & 6789232 & $\square$ & & $\square$ & $\square$ & $\square$ \\
\hline 1337 & 1210 & Hollow stem borehole w/SPT & 9/4/1969 & 17.5 & 350826 & 6789212 & $\square$ & & $\square$ & $\square$ & $\square$ \\
\hline 1337 & 1211 & Hollow stem borehole w/SPT & $4 / 29 / 1970$ & 25.0 & 350819 & 6789241 & $\square$ & $\square$ & $\square$ & $\square$ & $\square$ \\
\hline 1337 & 1212 & Hollow stem borehole w/SPT & $9 / 8 / 1969$ & 13.5 & 350843 & 6789441 & $\square$ & & $\square$ & $\square$ & $\square$ \\
\hline 1337 & 1213 & Hollow stem borehole w/SPT & 9/8/1969 & 10.0 & 350851 & 6789610 & $\square$ & & $\square$ & $\square$ & $\square$ \\
\hline 1337 & 1214 & Hollow stem borehole w/SPT & $9 / 8 / 1969$ & 18.0 & 350866 & 6789829 & $\square$ & $\square$ & $\square$ & $\square$ & $\square$ \\
\hline 1337 & 1220 & Hollow stem borehole w/SPT & $3 / 22 / 1971$ & 25.0 & 350491 & 6789208 & $\square$ & & $\square$ & $\square$ & \\
\hline 1338 & 1221 & Pit or trench & $4 / 24 / 1970$ & 16.0 & 351606 & 6789641 & $\square$ & & $\square$ & $\square$ & $\square$ \\
\hline 1338 & 1222 & Hollow stem borehole w/SPT & $3 / 3 / 1983$ & 21.5 & 351367 & 6789198 & $\square$ & & $\square$ & $\square$ & $\square$ \\
\hline 1339 & 1223 & Hollow stem borehole w/SPT & $8 / 15 / 1973$ & 15.5 & 352029 & 6789925 & $\square$ & $\square$ & $\square$ & $\square$ & $\square$ \\
\hline 1339 & 1224 & Hollow stem borehole w/SPT & $8 / 15 / 1973$ & 15.5 & 352024 & 6789838 & $\square$ & $\square$ & $\square$ & $\square$ & $\square$ \\
\hline 1339 & 1225 & Hollow stem borehole w/SPT & $8 / 15 / 1973$ & 15.5 & 351754 & 6789932 & $\square$ & $\square$ & $\square$ & $\square$ & $\square$ \\
\hline 1340 & 1226 & Hollow stem borehole w/SPT & $2 / 11 / 1992$ & 25.0 & 353246 & 6789106 & $\square$ & $\square$ & $\square$ & $\square$ & $\square$ \\
\hline 1340 & 1227 & Hollow stem borehole w/SPT & $2 / 10 / 1992$ & 29.5 & 353093 & 6789259 & $\square$ & $\square$ & $\square$ & $\square$ & $\square$ \\
\hline 1340 & 1228 & Hollow stem borehole w/SPT & $2 / 10 / 1992$ & 30.5 & 353131 & 6789291 & $\square$ & $\square$ & $\square$ & $\square$ & $\square$ \\
\hline
\end{tabular}




\begin{tabular}{|c|c|c|c|c|c|c|c|c|c|c|c|}
\hline \multirow[b]{2}{*}{$\begin{array}{l}\text { MOA } \\
\text { grid }\end{array}$} & \multirow[b]{2}{*}{$\begin{array}{l}\text { DGGS } \\
\text { number }\end{array}$} & \multirow[b]{2}{*}{ Hole Type } & \multirow[b]{2}{*}{$\begin{array}{c}\text { Date } \\
\text { Completed }\end{array}$} & \multirow[b]{2}{*}{$\begin{array}{c}\text { Total } \\
\text { depth }(\mathrm{ft})\end{array}$} & \multicolumn{2}{|c|}{ UTM coordinates (m) } & \multicolumn{5}{|c|}{ Data entered } \\
\hline & & & & & East & North & Lithology & SPT & $\begin{array}{c}\text { Sample } \\
\text { test }\end{array}$ & $\begin{array}{l}\text { Water } \\
\text { level }\end{array}$ & Velocity \\
\hline 1340 & 1229 & Hollow stem borehole w/SPT & 2/10/1992 & 29.0 & 353081 & 6789315 & $\square$ & & $\square$ & $\square$ & \\
\hline 1340 & 1230 & Hollow stem borehole w/SPT & $2 / 10 / 1992$ & 30.0 & 353174 & 6789317 & $\square$ & $\square$ & $\square$ & $\square$ & $\square$ \\
\hline 1341 & 4423 & Water well & $6 / 9 / 1982$ & 108.0 & 353343 & 6789317 & $\checkmark$ & 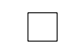 & $\square$ & $\square$ & \\
\hline 1341 & 4425 & Water well & $6 / 1 / 1967$ & 104.0 & 353654 & 6789614 & $\checkmark$ & $\square$ & $\square$ & $\boldsymbol{V}$ & $\square$ \\
\hline 1341 & 4426 & Water well & $9 / 30 / 1977$ & 142.0 & 353340 & 6789627 & $\checkmark$ & $\square$ & $\square$ & $\boldsymbol{V}$ & $\square$ \\
\hline 1421 & HK005 & Solid stem borehole (no cores or SP & $3 / 14 / 1969$ & 20.0 & 337930 & 6788970 & $\square$ & $\square$ & $\square$ & $\square$ & $\square$ \\
\hline 1422 & 2014 & Natural exposure & 6/5/1997 & 119.0 & 338105 & 6789056 & $\checkmark$ & $\square$ & $\square$ & $\square$ & $\square$ \\
\hline 1422 & HL010 & Solid stem borehole (no cores or SP & $7 / 3 / 1968$ & 17.0 & 338187 & 6788986 & $\square$ & $\square$ & $\square$ & $\square$ & \\
\hline 1422 & HL024 & Solid stem borehole (no cores or SP & $11 / 9 / 1973$ & 25.0 & 338321 & 6788967 & $\square$ & $\square$ & $\square$ & $\square$ & $\square$ \\
\hline 1422 & IP001 & Solid stem borehole (no cores or SP & $11 / 9 / 1973$ & 25.0 & 338032 & 6789026 & $\square$ & $\square$ & $\square$ & $\square$ & $\square$ \\
\hline 1426 & 2158 & Hollow stem borehole w/SPT & $5 / 15 / 1997$ & 51.5 & 341975 & 6788916 & $\checkmark$ & $\bar{\nabla}$ & $\square$ & $\square$ & $\square$ \\
\hline 1426 & 2159 & Hollow stem borehole w/SPT & $5 / 15 / 1997$ & 51.5 & 341986 & 6788911 & $\nabla$ & 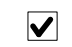 & $\checkmark$ & $\square$ & $\square$ \\
\hline 1426 & 2160 & Hollow stem borehole w/SPT & $5 / 15 / 1997$ & 51.5 & 341972 & 6788903 & $\nabla$ & $\checkmark$ & $\checkmark$ & $\checkmark$ & $\square$ \\
\hline 1426 & 2287 & Hollow stem borehole w/SPT & $12 / 16 / 1988$ & 66.8 & 341961 & 6788782 & $\checkmark$ & $\checkmark$ & $\checkmark$ & $\checkmark$ & $\square$ \\
\hline 1426 & 2288 & Hollow stem borehole w/SPT & $12 / 15 / 1988$ & 51.5 & 341944 & 6788822 & $\nabla$ & $\bar{\square}$ & $\bar{\nabla}$ & $\checkmark$ & $\square$ \\
\hline 1426 & 2289 & Hollow stem borehole w/SPT & $12 / 13 / 1988$ & 51.5 & 341925 & 6788861 & $\checkmark$ & $\checkmark$ & $\checkmark$ & $\checkmark$ & 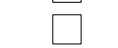 \\
\hline 1427 & 2161 & Hollow stem borehole w/SPT & 2/5/1998 & 51.5 & 342323 & 6788981 & $\checkmark$ & $\checkmark$ & $\checkmark$ & $\square$ & $\square$ \\
\hline 1427 & 2290 & Hollow stem borehole w/SPT & $1 / 17 / 1989$ & 61.7 & 342198 & 6788788 & $\checkmark$ & 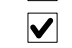 & $\nabla$ & $\checkmark$ & $\square$ \\
\hline 1427 & 2291 & Hollow stem borehole w/SPT & $12 / 19 / 1988$ & 52.2 & 342146 & 6788893 & $\checkmark$ & 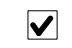 & $\checkmark$ & $\nabla$ & $\square$ \\
\hline 1427 & 2292 & Hollow stem borehole w/SPT & $12 / 8 / 1988$ & 64.6 & 342283 & 6788935 & $\checkmark$ & $\checkmark$ & $\checkmark$ & $\checkmark$ & $\square$ \\
\hline 1427 & 2293 & Hollow stem borehole w/SPT & $12 / 12 / 1988$ & 51.1 & 342261 & 6788980 & $\checkmark$ & $\checkmark$ & $\checkmark$ & $\checkmark$ & $\square$ \\
\hline 1427 & HP001 & Hollow stem borehole, no SPT & $3 / 2 / 1978$ & 19.0 & 342814 & 6788989 & $\square$ & & $\square$ & $\square$ & $\square$ \\
\hline 1427 & HP003 & Hollow stem borehole, no SPT & $3 / 2 / 1978$ & 19.0 & 342799 & 6788745 & $\square$ & & $\square$ & $\square$ & $\square$ \\
\hline 1428 & 3018 & Water well & 9/3/1974 & 222.0 & 342974 & 6789022 & $\checkmark$ & $\square$ & $\square$ & $\checkmark$ & $\square$ \\
\hline 1428 & HP002 & Hollow stem borehole, no SPT & $3 / 2 / 1978$ & 19.0 & 342860 & 6788898 & $\square$ & & $\square$ & $\square$ & $\square$ \\
\hline 1428 & IN001 & Solid stem borehole (no cores or SP & $5 / 1 / 1975$ & 7.5 & 343270 & 6789372 & $\square$ & & $\square$ & $\square$ & $\square$ \\
\hline 1428 & IN002 & Solid stem borehole (no cores or SP & $5 / 1 / 1975$ & 13.5 & 343265 & 6789335 & $\square$ & $\square$ & $\square$ & $\square$ & $\square$ \\
\hline 1428 & IN003 & Solid stem borehole (no cores or SP & $5 / 1 / 1975$ & 12.5 & 343273 & 6789264 & $\square$ & & $\square$ & $\square$ & $\square$ \\
\hline 1428 & IN004 & Solid stem borehole (no cores or SP & $5 / 1 / 1975$ & 13.8 & 343345 & 6789266 & $\square$ & - & $\square$ & $\square$ & $\square$ \\
\hline 1428 & IN005 & Solid stem borehole (no cores or SP & $5 / 1 / 1975$ & 29.0 & 343432 & 6789285 & $\square$ & $\square$ & $\square$ & $\square$ & $\square$ \\
\hline 1429 & 1837 & Hollow stem borehole w/SPT & $2 / 23 / 1995$ & 31.0 & 344027 & 6788863 & $\square$ & $\square$ & $\square$ & $\square$ & $\square$ \\
\hline 1429 & 1838 & Hollow stem borehole w/SPT & $2 / 24 / 1995$ & 27.0 & 344034 & 6788848 & $\square$ & $\square$ & $\square$ & $\square$ & $\square$ \\
\hline 1429 & HQ006 & Hollow stem borehole w/SPT & $6 / 8 / 1965$ & 28.0 & 343622 & 6788718 & $\square$ & $\square$ & $\square$ & $\square$ & $\square$ \\
\hline 1429 & HQ025 & Hollow stem borehole w/SPT & $6 / 23 / 1965$ & 20.0 & 343697 & 6788686 & $\square$ & $\square$ & $\square$ & $\square$ & $\square$ \\
\hline 1429 & HQ029 & Hollow stem borehole w/SPT & $7 / 3 / 1965$ & 23.0 & 343694 & 6788873 & $\square$ & $\square$ & $\square$ & $\square$ & $\square$ \\
\hline 1429 & HQ030 & Hollow stem borehole w/SPT & $7 / 2 / 1965$ & 21.0 & 343685 & 6788854 & $\square$ & $\square$ & $\square$ & $\square$ & $\square$ \\
\hline
\end{tabular}




\begin{tabular}{|c|c|c|c|c|c|c|c|c|c|c|c|}
\hline \multirow[b]{2}{*}{$\begin{array}{l}\text { MOA } \\
\text { grid }\end{array}$} & \multirow[b]{2}{*}{$\begin{array}{l}\text { DGGS } \\
\text { number }\end{array}$} & \multirow[b]{2}{*}{ Hole Type } & \multirow[b]{2}{*}{$\begin{array}{c}\text { Date } \\
\text { Completed }\end{array}$} & \multirow[b]{2}{*}{$\begin{array}{c}\text { Total } \\
\text { depth }(\mathrm{ft})\end{array}$} & \multicolumn{2}{|c|}{ UTM coordinates (m) } & \multicolumn{5}{|c|}{ Data entered } \\
\hline & & & & & East & North & Lithology & SPT & $\begin{array}{c}\text { Sample } \\
\text { test }\end{array}$ & $\begin{array}{l}\text { Water } \\
\text { level }\end{array}$ & Velocity \\
\hline 1429 & HQ031 & Hollow stem borehole w/SPT & $7 / 2 / 1965$ & 21.5 & 343673 & 6788826 & $\square$ & $\square$ & $\square$ & $\square$ & \\
\hline 1429 & HQ032 & Hollow stem borehole w/SPT & $7 / 2 / 1965$ & 23.0 & 343673 & 6788770 & $\square$ & $\square$ & $\square$ & $\square$ & $\square$ \\
\hline 1429 & HQ033 & Solid stem borehole (no cores or SP & $7 / 1 / 1965$ & 24.5 & 343639 & 6788774 & $\square$ & $\square$ & $\square$ & $\square$ & \\
\hline 1429 & HR011 & Solid stem borehole (no cores or SP & $7 / 1 / 1965$ & 23.0 & 344160 & 6788978 & $\square$ & $\square$ & $\square$ & $\square$ & $\square$ \\
\hline 1429 & HR012 & Hollow stem borehole w/SPT & $7 / 6 / 1965$ & 28.0 & 344252 & 6788964 & $\square$ & $\square$ & $\square$ & $\square$ & $\square$ \\
\hline 1429 & HR013 & Hollow stem borehole w/SPT & $7 / 7 / 1965$ & 29.5 & 344302 & 6788962 & $\square$ & $\square$ & $\square$ & $\square$ & $\square$ \\
\hline 1429 & HR014 & Hollow stem borehole w/SPT & $7 / 8 / 1965$ & 40.0 & 344312 & 6788998 & $\square$ & $\square$ & $\square$ & $\square$ & $\square$ \\
\hline 1429 & IN006 & Solid stem borehole (no cores or SP & $7 / 1 / 1965$ & 23.0 & 343948 & 6789138 & $\square$ & $\square$ & $\square$ & $\square$ & \\
\hline 1429 & IN017 & Cone Penetration Test (CPT) & $3 / 28 / 1986$ & 92.0 & 343943 & 6789461 & $\square$ & $\square$ & $\square$ & $\square$ & $\square$ \\
\hline 1430 & 1231 & Hollow stem borehole w/SPT & $10 / 19 / 1984$ & 60.5 & 345173 & 6789007 & $\checkmark$ & $\checkmark$ & $\square$ & $\checkmark$ & $\square$ \\
\hline 1430 & 1235 & Hollow stem borehole w/SPT & $1 / 27 / 1983$ & 81.8 & 345174 & 6789209 & $\checkmark$ & 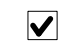 & $\nabla$ & $\nabla$ & $\square$ \\
\hline 1430 & 1238 & Hollow stem borehole w/SPT & $3 / 15 / 1983$ & 60.0 & 345171 & 6788730 & $\checkmark$ & $\checkmark$ & $\square$ & $\checkmark$ & $\square$ \\
\hline 1430 & 1239 & Hollow stem borehole w/SPT & $3 / 22 / 1983$ & 61.0 & 345148 & 6788689 & $\checkmark$ & $\nabla$ & $\square$ & $\vec{\nabla}$ & $\square$ \\
\hline 1430 & 1249 & Hollow stem borehole w/SPT & $3 / 12 / 1973$ & 46.5 & 345020 & 6789048 & $\square$ & $\square$ & $\square$ & $\square$ & $\square$ \\
\hline 1430 & 1302 & Hollow stem borehole w/SPT & $3 / 12 / 1974$ & 46.0 & 345020 & 6789079 & $\square$ & $\square$ & $\square$ & $\square$ & $\square$ \\
\hline 1430 & 2114 & Hollow stem borehole w/SPT & & 51.2 & 345023 & 6788925 & $\checkmark$ & $\checkmark$ & $\checkmark$ & $\checkmark$ & $\square$ \\
\hline 1430 & 2115 & Hollow stem borehole w/SPT & & 50.5 & 345071 & 6789003 & $\nabla$ & $\checkmark$ & $\checkmark$ & $\checkmark$ & $\square$ \\
\hline 1430 & 3017 & Water well & $8 / 1 / 1968$ & 110.0 & 345137 & 6788957 & $\nabla$ & $\square$ & $\square$ & $\square$ & $\square$ \\
\hline 1430 & 4450 & Water well & $12 / 1 / 1976$ & 118.0 & 345061 & 6788929 & $\checkmark$ & $\square$ & $\square$ & $\square$ & $\square$ \\
\hline 1430 & HR015 & Hollow stem borehole w/SPT & $1 / 1 / 1974$ & 31.1 & 344537 & 6788982 & $\checkmark$ & $\bar{\nabla}$ & 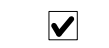 & $\checkmark$ & $\square$ \\
\hline 1430 & HR016 & Solid stem borehole (no cores or SP & $6 / 18 / 1968$ & 18.0 & 344981 & 6788927 & $\square$ & $\square$ & $\square$ & $\square$ & \\
\hline 1430 & HR017 & Solid stem borehole (no cores or SP & $6 / 18 / 1968$ & 18.0 & 344986 & 6788776 & $\square$ & $\square$ & $\square$ & $\square$ & $\square$ \\
\hline 1430 & HR018 & Solid stem borehole (no cores or SP & $3 / 11 / 1968$ & 16.5 & 344976 & 6788690 & $\square$ & $\square$ & $\square$ & $\square$ & $\square$ \\
\hline 1430 & IL005 & Hollow stem borehole w/SPT & 9/9/1982 & 26.0 & 345228 & 6789284 & $\square$ & $\square$ & $\square$ & $\square$ & $\square$ \\
\hline 1430 & IM017 & Hollow stem borehole w/SPT & $1 / 1 / 1974$ & 61.5 & 344542 & 6789000 & $\nabla$ & 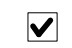 & $\checkmark$ & $\square$ & $\square$ \\
\hline 1430 & IM018 & Hollow stem borehole w/SPT & $1 / 1 / 1974$ & 66.5 & 344545 & 6789016 & $\nabla$ & $\checkmark$ & $\checkmark$ & $\nabla$ & $\square$ \\
\hline 1430 & IM019 & Hollow stem borehole w/SPT & $3 / 24 / 1983$ & 52.0 & 344955 & 6789140 & $\checkmark$ & $\checkmark$ & $\checkmark$ & $\checkmark$ & $\square$ \\
\hline 1431 & 1232 & Hollow stem borehole w/SPT & $8 / 25 / 1982$ & 52.0 & 345231 & 6789256 & $\checkmark$ & $\bar{\nabla}$ & $\nabla$ & $\nabla$ & $\square$ \\
\hline 1431 & 1233 & Hollow stem borehole w/SPT & $8 / 27 / 1982$ & 52.0 & 345248 & 6789239 & $\checkmark$ & $\checkmark$ & $\checkmark$ & $\checkmark$ & $\square$ \\
\hline 1431 & 1234 & Hollow stem borehole w/SPT & $9 / 10 / 1982$ & 52.0 & 345248 & 6789288 & $\checkmark$ & $\bar{\nabla}$ & $\checkmark$ & $\checkmark$ & $\square$ \\
\hline 1431 & 1273 & Hollow stem borehole w/SPT & $10 / 11 / 1974$ & 30.0 & 346003 & 6788801 & $\square$ & $\square$ & $\square$ & $\square$ & $\square$ \\
\hline 1431 & 1274 & Hollow stem borehole w/SPT & $10 / 16 / 1974$ & 42.0 & 345994 & 6788913 & $\square$ & $\square$ & $\square$ & $\square$ & $\square$ \\
\hline 1431 & 1275 & Hollow stem borehole w/SPT & $10 / 17 / 1974$ & 47.0 & 345968 & 6788977 & $\square$ & $\square$ & $\square$ & $\square$ & $\square$ \\
\hline 1431 & 2156 & Hollow stem borehole w/SPT & $6 / 19 / 1998$ & 36.5 & 345443 & 6788928 & $\checkmark$ & $\checkmark$ & $\checkmark$ & $\bar{\nabla}$ & $\square$ \\
\hline 1431 & HS013 & Hollow stem borehole w/SPT & $9 / 22 / 1977$ & 32.0 & 345858 & 6788612 & $\square$ & $\square$ & $\square$ & $\square$ & $\square$ \\
\hline 1431 & HS015 & Hollow stem borehole w/SPT & $9 / 22 / 1977$ & 31.5 & 345815 & 6788655 & $\square$ & $\square$ & $\square$ & $\square$ & $\square$ \\
\hline
\end{tabular}




\begin{tabular}{|c|c|c|c|c|c|c|c|c|c|c|c|}
\hline \multirow[b]{2}{*}{$\begin{array}{l}\text { MOA } \\
\text { grid }\end{array}$} & \multirow[b]{2}{*}{$\begin{array}{l}\text { DGGS } \\
\text { number }\end{array}$} & \multirow[b]{2}{*}{ Hole Type } & \multirow[b]{2}{*}{$\begin{array}{c}\text { Date } \\
\text { Completed }\end{array}$} & \multirow[b]{2}{*}{$\begin{array}{c}\text { Total } \\
\text { depth }(\mathrm{ft})\end{array}$} & \multicolumn{2}{|c|}{ UTM coordinates (m) } & \multicolumn{5}{|c|}{ Data entered } \\
\hline & & & & & East & North & Lithology & SPT & $\begin{array}{c}\text { Sample } \\
\text { test }\end{array}$ & $\begin{array}{l}\text { Water } \\
\text { level }\end{array}$ & Velocity \\
\hline 1431 & HS016 & Hollow stem borehole w/SPT & $9 / 22 / 1977$ & 30.5 & 345899 & 6788644 & $\square$ & $\square$ & $\square$ & $\square$ & \\
\hline 1431 & HS017 & Hollow stem borehole w/SPT & $1 / 2 / 1981$ & 118.0 & 345830 & 6788942 & $\checkmark$ & $\checkmark$ & $\checkmark$ & $\checkmark$ & $\square$ \\
\hline 1431 & HS018 & Hollow stem borehole w/SPT & $12 / 13 / 1980$ & 100.0 & 345752 & 6788946 & $\checkmark$ & $\checkmark$ & $\nabla$ & $\checkmark$ & \\
\hline 1431 & HS019 & Hollow stem borehole w/SPT & $1 / 15 / 1981$ & 97.0 & 345832 & 6788891 & $\nabla$ & $\nabla$ & $\checkmark$ & $\checkmark$ & $\square$ \\
\hline 1431 & HSO20 & Hollow stem borehole w/SPT & $1 / 7 / 1981$ & 85.0 & 345761 & 6788894 & $\checkmark$ & $\checkmark$ & $\checkmark$ & $\checkmark$ & $\square$ \\
\hline 1431 & IL003 & Hollow stem borehole w/SPT & $10 / 11 / 1982$ & 90.5 & 345239 & 6789176 & $\checkmark$ & $\checkmark$ & $\checkmark$ & $\checkmark$ & $\square$ \\
\hline 1431 & IL004 & Hollow stem borehole w/SPT & $10 / 8 / 1982$ & 94.0 & 345230 & 6789216 & $\checkmark$ & $\checkmark$ & $\checkmark$ & $\checkmark$ & $\square$ \\
\hline 1432 & 1240 & Hollow stem borehole w/SPT & $3 / 31 / 1983$ & 31.5 & 346574 & 6788986 & $\checkmark$ & $\checkmark$ & $\square$ & $\nabla$ & $\square$ \\
\hline 1432 & 1241 & Hollow stem borehole w/SPT & $3 / 31 / 1983$ & 31.5 & 346604 & 6788963 & $\square$ & $\square$ & $\square$ & $\square$ & $\square$ \\
\hline 1432 & 1242 & Hollow stem borehole w/SPT & $3 / 31 / 1983$ & 31.5 & 346669 & 6788963 & $\checkmark$ & $\nabla$ & $\square$ & $\checkmark$ & \\
\hline 1432 & 1243 & Hollow stem borehole, no SPT & $5 / 29 / 1983$ & 31.5 & 346596 & 6788931 & $\square$ & $\square$ & $\square$ & $\square$ & $\square$ \\
\hline 1432 & 1244 & Hollow stem borehole, no SPT & $3 / 31 / 1983$ & 30.5 & 346682 & 6788931 & $\square$ & $\square$ & $\square$ & $\square$ & $\square$ \\
\hline 1432 & 1246 & Hollow stem borehole w/SPT & $6 / 15 / 1982$ & 51.5 & 346032 & 6788974 & $\checkmark$ & $\checkmark$ & $\checkmark$ & $\square$ & $\square$ \\
\hline 1433 & 4449 & Water well & $4 / 1 / 1970$ & 102.0 & 347532 & 6788697 & $\checkmark$ & $\square$ & $\square$ & $\nabla$ & $\square$ \\
\hline 1434 & II014 & Solid stem borehole (no cores or SP & $3 / 16 / 1971$ & 13.0 & 348287 & 6789274 & $\square$ & $\square$ & $\square$ & $\square$ & $\square$ \\
\hline 1434 & II015 & Solid stem borehole (no cores or SP & $3 / 16 / 1971$ & 13.0 & 348372 & 6789276 & $\square$ & $\square$ & $\square$ & $\square$ & $\square$ \\
\hline 1434 & IJ002 & Hollow stem borehole w/SPT & $1 / 7 / 1983$ & 81.5 & 347903 & 6789102 & $\checkmark$ & $\checkmark$ & $\checkmark$ & $\checkmark$ & $\square$ \\
\hline 1436 & 1216 & Hollow stem borehole w/SPT & $3 / 17 / 1971$ & 13.0 & 349431 & 6789219 & $\square$ & $\square$ & $\square$ & $\square$ & $\square$ \\
\hline 1436 & 1217 & Hollow stem borehole w/SPT & $3 / 17 / 1971$ & 13.0 & 349465 & 6789217 & $\square$ & $\checkmark$ & $\square$ & $\square$ & $\square$ \\
\hline 1436 & 1219 & Hollow stem borehole w/SPT & $3 / 18 / 1971$ & 17.0 & 349969 & 6789191 & $\square$ & $\square$ & $\square$ & $\square$ & $\square$ \\
\hline 1436 & 1615 & Hollow stem borehole w/SPT & $5 / 22 / 1990$ & 21.5 & 349658 & 6788570 & $\square$ & $\square$ & $\square$ & $\square$ & $\square$ \\
\hline 1436 & 1616 & Hollow stem borehole w/SPT & $5 / 22 / 1990$ & 21.5 & 349572 & 6788470 & $\square$ & $\square$ & $\square$ & $\square$ & $\square$ \\
\hline 1436 & 1617 & Hollow stem borehole w/SPT & $5 / 22 / 1990$ & 21.5 & 349739 & 6788449 & $\square$ & $\square$ & $\square$ & $\square$ & $\square$ \\
\hline 1436 & 1618 & Hollow stem borehole w/SPT & $5 / 22 / 1990$ & 21.5 & 349829 & 6788568 & $\square$ & $\square$ & $\square$ & $\square$ & $\square$ \\
\hline 1436 & 1684 & Hollow stem borehole w/SPT & $3 / 26 / 1973$ & 56.0 & 349283 & 6788638 & $\checkmark$ & $\nabla$ & $\checkmark$ & 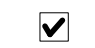 & $\square$ \\
\hline 1437 & 1250 & Hollow stem borehole w/SPT & $4 / 18 / 1988$ & 42.0 & 350826 & 6788404 & $\square$ & & $\square$ & $\square$ & $\square$ \\
\hline 1438 & 1251 & Hollow stem borehole w/SPT & $5 / 28 / 1985$ & 21.0 & 351003 & 6789007 & $\square$ & $\square$ & $\square$ & $\square$ & $\square$ \\
\hline 1438 & 1791 & Hollow stem borehole, no SPT & $9 / 25 / 1980$ & 25.0 & 350847 & 6788540 & $\square$ & & $\square$ & $\square$ & $\square$ \\
\hline 1438 & 4420 & Water well & $5 / 1 / 1969$ & 151.0 & 351273 & 6788443 & $\checkmark$ & $\square$ & $\square$ & $\checkmark$ & $\square$ \\
\hline 1439 & 1252 & Hollow stem borehole w/SPT & $8 / 12 / 1992$ & 21.5 & 351790 & 6789077 & $\square$ & $\square$ & $\square$ & $\square$ & $\square$ \\
\hline 1439 & 1253 & Hollow stem borehole w/SPT & $8 / 12 / 1992$ & 25.0 & 351850 & 6789064 & $\square$ & $\square$ & $\square$ & $\square$ & $\square$ \\
\hline 1440 & 4422 & Water well & $8 / 17 / 1971$ & 130.0 & 352712 & 6788909 & $\checkmark$ & $\square$ & $\square$ & $\nabla$ & $\square$ \\
\hline 1441 & 1254 & Hollow stem borehole w/SPT & $4 / 5 / 1988$ & 29.5 & 354060 & 6788585 & $\checkmark$ & $\nabla$ & $\square$ & $\bar{\square}$ & $\square$ \\
\hline 1441 & 1298 & Hollow stem borehole w/SPT & 8/19/1994 & 41.1 & 354055 & 6788378 & $\square$ & $\square$ & $\square$ & $\square$ & $\square$ \\
\hline 1441 & 1301 & Hollow stem borehole w/SPT & $4 / 30 / 1990$ & 21.5 & 353717 & 6788819 & $\checkmark$ & $\checkmark$ & $\square$ & $\nabla$ & $\square$ \\
\hline 1441 & 1394 & Hollow stem borehole, no SPT & $6 / 14 / 1974$ & 14.0 & 353264 & 6788572 & $\square$ & $\square$ & $\square$ & $\square$ & $\square$ \\
\hline
\end{tabular}




\begin{tabular}{|c|c|c|c|c|c|c|c|c|c|c|c|}
\hline \multirow[b]{2}{*}{$\begin{array}{l}\text { MOA } \\
\text { grid }\end{array}$} & \multirow[b]{2}{*}{$\begin{array}{l}\text { DGGS } \\
\text { number }\end{array}$} & \multirow[b]{2}{*}{ Hole Type } & \multirow[b]{2}{*}{$\begin{array}{c}\text { Date } \\
\text { Completed }\end{array}$} & \multirow[b]{2}{*}{$\begin{array}{c}\text { Total } \\
\text { depth }(\mathrm{ft})\end{array}$} & \multicolumn{2}{|c|}{ UTM coordinates (m) } & \multicolumn{5}{|c|}{ Data entered } \\
\hline & & & & & East & North & Lithology & SPT & $\begin{array}{c}\text { Sample } \\
\text { test }\end{array}$ & $\begin{array}{l}\text { Water } \\
\text { level }\end{array}$ & Velocity \\
\hline 1441 & 1608 & Hollow stem borehole w/SPT & $5 / 23 / 1996$ & 31.5 & 353806 & 6789007 & $\nabla$ & $\checkmark$ & $\checkmark$ & $\square$ & \\
\hline 1441 & 4421 & Water well & $4 / 15 / 1975$ & 280.0 & 353832 & 6788522 & $\checkmark$ & $\square$ & $\square$ & $\square$ & $\square$ \\
\hline 1521 & 2297 & Hollow stem borehole w/SPT & $3 / 5 / 1969$ & 56.5 & 337829 & 6788250 & $\checkmark$ & $\nabla$ & $\square$ & $\square$ & \\
\hline 1521 & 4452 & Water well & $11 / 2 / 1970$ & 356.0 & 337854 & 6788232 & $\nabla$ & $\checkmark$ & $\square$ & $\checkmark$ & $\square$ \\
\hline 1521 & 4453 & Water well & $12 / 18 / 1970$ & 102.0 & 337749 & 6788206 & $\checkmark$ & $\square$ & $\square$ & $\checkmark$ & $\square$ \\
\hline 1521 & HK003 & Solid stem borehole (no cores or SP & $9 / 10 / 1968$ & 23.5 & 337819 & 6788766 & $\square$ & $\square$ & $\square$ & $\square$ & $\square$ \\
\hline 1521 & HK004 & Solid stem borehole (no cores or SP & $3 / 13 / 1969$ & 24.0 & 337896 & 6788852 & $\square$ & $\square$ & $\square$ & $\square$ & $\square$ \\
\hline 1521 & HK006 & Solid stem borehole (no cores or SP & 9/9/1968 & 28.0 & 337931 & 6788420 & $\square$ & $\checkmark$ & $\square$ & $\square$ & $\square$ \\
\hline 1521 & HK007 & Solid stem borehole (no cores or SP & $9 / 10 / 1968$ & 18.0 & 337874 & 6788514 & $\square$ & $\square$ & $\square$ & $\square$ & $\square$ \\
\hline 1521 & HK008 & Solid stem borehole (no cores or SP & $11 / 7 / 1968$ & 48.0 & 337849 & 6788670 & $\square$ & $\square$ & $\square$ & $\square$ & $\square$ \\
\hline 1521 & HK009 & Solid stem borehole (no cores or SP & $11 / 9 / 1977$ & 15.0 & 337944 & 6788166 & $\square$ & $\square$ & $\square$ & $\square$ & $\square$ \\
\hline 1522 & 2070 & Hollow stem borehole w/SPT & $6 / 2 / 1983$ & 52.5 & 338521 & 6788149 & $\checkmark$ & $\bar{\nabla}$ & $\bar{\nabla}$ & $\square$ & \\
\hline 1522 & 2071 & Hollow stem borehole, no SPT & $7 / 1 / 1983$ & 40.0 & 338731 & 6788196 & $\nabla$ & & $\checkmark$ & $\square$ & $\square$ \\
\hline 1522 & 2072 & Hollow stem borehole, no SPT & $6 / 30 / 1983$ & 50.0 & 338679 & 6788431 & $\checkmark$ & $\square$ & $\checkmark$ & $\square$ & $\square$ \\
\hline 1522 & 2073 & Hollow stem borehole, no SPT & $6 / 30 / 1983$ & 50.0 & 338738 & 6788444 & $\checkmark$ & $\square$ & $\bar{\nabla}$ & $\square$ & $\square$ \\
\hline 1522 & 2074 & Hollow stem borehole w/SPT & $6 / 3 / 1983$ & 61.5 & 338761 & 6788450 & $\checkmark$ & $\square$ & $\checkmark$ & $\square$ & $\square$ \\
\hline 1522 & 2298 & Hollow stem borehole w/SPT & $1 / 28 / 1969$ & 116.5 & 338038 & 6788187 & $\checkmark$ & $\checkmark$ & $\checkmark$ & $\square$ & $\square$ \\
\hline 1522 & 2299 & Hollow stem borehole w/SPT & $2 / 13 / 1969$ & 111.5 & 338140 & 6788156 & $\checkmark$ & $\checkmark$ & $\nabla$ & $\square$ & $\square$ \\
\hline 1522 & 2300 & Hollow stem borehole w/SPT & $1 / 30 / 1969$ & 110.4 & 338241 & 6788125 & $\checkmark$ & 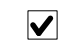 & $\checkmark$ & $\square$ & $\square$ \\
\hline 1522 & HL001 & Solid stem borehole (no cores or SP & $3 / 14 / 1969$ & 20.0 & 338558 & 6788534 & $\square$ & $\checkmark$ & $\square$ & $\square$ & $\square$ \\
\hline 1522 & HL004 & Solid stem borehole (no cores or SP & 8/1/1968 & 33.0 & 338271 & 6788152 & $\square$ & & $\square$ & $\square$ & \\
\hline 1522 & HL005 & Solid stem borehole (no cores or SP & $8 / 2 / 1968$ & 23.0 & 338246 & 6788298 & $\square$ & & $\square$ & $\square$ & $\square$ \\
\hline 1522 & HL006 & Solid stem borehole (no cores or SP & $8 / 2 / 1968$ & 33.0 & 338193 & 6788432 & $\square$ & & $\square$ & $\square$ & $\square$ \\
\hline 1522 & HL007 & Solid stem borehole (no cores or SP & $8 / 2 / 1968$ & 23.0 & 338149 & 6788592 & $\square$ & $\square$ & $\square$ & $\square$ & $\square$ \\
\hline 1522 & HL008 & Solid stem borehole (no cores or SP & $8 / 2 / 1968$ & 22.0 & 338101 & 6788760 & $\square$ & & $\square$ & $\square$ & $\square$ \\
\hline 1522 & HL009 & Solid stem borehole (no cores or SP & $8 / 5 / 1968$ & 33.0 & 338059 & 6788892 & $\square$ & & $\square$ & $\square$ & $\square$ \\
\hline 1522 & HL011 & Solid stem borehole (no cores or SP & $7 / 3 / 1968$ & 13.0 & 338238 & 6788824 & $\square$ & $\square$ & $\square$ & $\square$ & $\square$ \\
\hline 1522 & HL012 & Solid stem borehole (no cores or SP & $7 / 3 / 1968$ & 22.0 & 338277 & 6788694 & $\square$ & & $\square$ & $\square$ & \\
\hline 1522 & HL013 & Solid stem borehole (no cores or SP & $7 / 3 / 1968$ & 28.0 & 338325 & 6788546 & $\square$ & & $\square$ & $\square$ & $\square$ \\
\hline 1522 & HL014 & Solid stem borehole (no cores or SP & $7 / 3 / 1968$ & 32.0 & 338371 & 6788409 & $\square$ & & $\square$ & $\square$ & $\square$ \\
\hline 1522 & HL015 & Solid stem borehole (no cores or SP & $7 / 3 / 1968$ & 32.0 & 338428 & 6788248 & $\square$ & $\square$ & $\square$ & $\square$ & $\square$ \\
\hline 1522 & HL017 & Solid stem borehole (no cores or SP & $7 / 31 / 1968$ & 33.0 & 338610 & 6788128 & $\square$ & $\square$ & $\square$ & $\square$ & $\square$ \\
\hline 1522 & HL018 & Solid stem borehole (no cores or SP & 8/1/1968 & 23.0 & 338545 & 6788303 & $\square$ & $\square$ & $\square$ & $\square$ & $\square$ \\
\hline 1522 & HL019 & Solid stem borehole (no cores or SP & $8 / 2 / 1968$ & 23.0 & 338496 & 6788448 & $\square$ & $\square$ & $\square$ & $\square$ & $\square$ \\
\hline 1522 & HL020 & Solid stem borehole (no cores or SP & $8 / 2 / 1968$ & 33.0 & 338451 & 6788560 & $\square$ & $\square$ & $\square$ & $\square$ & $\square$ \\
\hline 1522 & HL021 & Solid stem borehole (no cores or SP & $8 / 2 / 1968$ & 33.0 & 338389 & 6788742 & $\square$ & $\square$ & $\square$ & $\square$ & $\square$ \\
\hline
\end{tabular}




\begin{tabular}{|c|c|c|c|c|c|c|c|c|c|c|c|}
\hline \multirow[b]{2}{*}{$\begin{array}{c}\text { MOA } \\
\text { grid }\end{array}$} & \multirow[b]{2}{*}{$\begin{array}{c}\text { DGGS } \\
\text { number }\end{array}$} & \multirow[b]{2}{*}{ Hole Type } & \multirow[b]{2}{*}{$\begin{array}{c}\text { Date } \\
\text { Completed }\end{array}$} & \multirow[b]{2}{*}{$\begin{array}{c}\text { Total } \\
\text { depth (ft) }\end{array}$} & \multicolumn{2}{|c|}{ UTM coordinates (m) } & \multicolumn{5}{|c|}{ Data entered } \\
\hline & & & & & East & North & Lithology & SPT & $\begin{array}{c}\text { Sample } \\
\text { test }\end{array}$ & $\begin{array}{c}\text { Water } \\
\text { level }\end{array}$ & Velocity \\
\hline 1522 & HL022 & Solid stem borehole (no cores or SP & $8 / 5 / 1968$ & 33.0 & 338306 & 6788890 & $\square$ & $\square$ & $\square$ & $\square$ & $\square$ \\
\hline 1522 & HL023 & Solid stem borehole (no cores or SP & $8 / 5 / 1968$ & 33.0 & 338421 & 6788874 & $\square$ & $\square$ & $\square$ & $\square$ & $\square$ \\
\hline 1522 & HL025 & Solid stem borehole (no cores or SP & 9/9/1968 & 33.0 & 338074 & 6788250 & $\square$ & $\square$ & $\square$ & $\square$ & $\square$ \\
\hline 1522 & HL026 & Solid stem borehole (no cores or SP & $1 / 24 / 1967$ & 42.0 & 338317 & 6788738 & $\square$ & $\square$ & $\square$ & $\square$ & $\square$ \\
\hline 1522 & HL029 & Hollow stem borehole w/SPT & $3 / 10 / 1973$ & 66.5 & 338162 & 6788250 & $\checkmark$ & $\checkmark$ & $\checkmark$ & $\nabla$ & $\square$ \\
\hline 1522 & HL030 & Solid stem borehole (no cores or SP & $2 / 5 / 1973$ & 30.0 & 338132 & 6788364 & $\square$ & $\square$ & $\square$ & $\square$ & $\square$ \\
\hline 1522 & HL031 & Solid stem borehole (no cores or SP & $2 / 5 / 1973$ & 30.0 & 338101 & 6788474 & $\square$ & $\square$ & $\square$ & $\square$ & $\square$ \\
\hline 1522 & HL032 & Solid stem borehole (no cores or SP & $2 / 5 / 1973$ & 25.0 & 338062 & 6788608 & $\square$ & $\square$ & $\square$ & $\square$ & $\square$ \\
\hline 1522 & HL033 & Solid stem borehole (no cores or SP & $2 / 5 / 1973$ & 25.0 & 338034 & 6788710 & $\square$ & $\square$ & $\square$ & $\square$ & $\square$ \\
\hline 1522 & HL035 & Solid stem borehole (no cores or SP & 2/8/1973 & 30.0 & 338029 & 6788172 & $\square$ & $\square$ & $\square$ & $\square$ & $\square$ \\
\hline 1522 & HL036 & Solid stem borehole (no cores or SP & 2/8/1973 & 25.0 & 337988 & 6788320 & $\square$ & $\square$ & $\square$ & $\square$ & $\square$ \\
\hline 1522 & HL037 & Hollow stem borehole w/SPT & $12 / 21 / 1966$ & 31.0 & 338399 & 6788116 & $\square$ & $\square$ & $\square$ & $\square$ & $\square$ \\
\hline 1522 & HL038 & Solid stem borehole (no cores or SP & $7 / 2 / 1968$ & 38.0 & 338430 & 6788130 & $\square$ & $\square$ & $\square$ & $\square$ & $\square$ \\
\hline 1523 & 2060 & Solid stem borehole (no cores or SP & $4 / 3 / 1984$ & 30.0 & 339334 & 6788292 & $\checkmark$ & $\square$ & $\square$ & $\checkmark$ & $\square$ \\
\hline 1523 & 2061 & Hollow stem borehole w/SPT & $6 / 26 / 1984$ & 62.0 & 339408 & 6788524 & $\boldsymbol{V}$ & $\checkmark$ & $\nabla$ & $\square$ & $\square$ \\
\hline 1523 & 2164 & Hollow stem borehole w/SPT & $2 / 22 / 1996$ & 42.0 & 339194 & 6788410 & $\checkmark$ & $\square$ & $\square$ & $\square$ & $\square$ \\
\hline 1523 & 2165 & Hollow stem borehole w/SPT & $2 / 22 / 1996$ & 42.0 & 339204 & 6788425 & $\boldsymbol{V}$ & $\square$ & $\boldsymbol{V}$ & $\square$ & $\square$ \\
\hline 1523 & 2166 & Hollow stem borehole w/SPT & $2 / 22 / 1996$ & 32.0 & 339212 & 6788382 & $\boldsymbol{V}$ & $\square$ & $\square$ & $\nabla$ & $\square$ \\
\hline 1523 & 2184 & Hollow stem borehole w/SPT & $7 / 19 / 1994$ & 41.5 & 339069 & 6788342 & $\boldsymbol{V}$ & $\checkmark$ & $\square$ & $\nabla$ & $\square$ \\
\hline 1523 & HM004 & Solid stem borehole (no cores or SP & $9 / 5 / 1968$ & 15.0 & 339360 & 6788198 & $\square$ & $\square$ & $\square$ & $\square$ & $\square$ \\
\hline 1523 & HM005 & Solid stem borehole (no cores or SP & $9 / 5 / 1968$ & 18.0 & 339368 & 6788362 & $\square$ & $\square$ & $\square$ & $\square$ & $\square$ \\
\hline 1524 & 2013 & Natural exposure & $6 / 5 / 1997$ & 46.0 & 339762 & 6788429 & $\boldsymbol{V}$ & $\square$ & $\square$ & $\square$ & $\square$ \\
\hline 1524 & HМ002 & Solid stem borehole (no cores or SP & $3 / 13 / 1969$ & 12.0 & 339852 & 6788330 & $\square$ & $\square$ & $\square$ & $\square$ & $\square$ \\
\hline 1524 & HМ006 & Solid stem borehole (no cores or SP & $9 / 6 / 1968$ & 20.0 & 339689 & 6788075 & $\square$ & $\square$ & $\square$ & $\square$ & $\square$ \\
\hline 1524 & HM007 & Solid stem borehole (no cores or SP & $9 / 6 / 1968$ & 20.0 & 339707 & 6788288 & $\square$ & $\square$ & $\square$ & $\square$ & $\square$ \\
\hline 1524 & HM008 & Hollow stem borehole, no SPT & $8 / 28 / 1964$ & 218.0 & 339810 & 6788137 & $\boldsymbol{V}$ & $\square$ & $\nabla$ & $\nabla$ & $\square$ \\
\hline 1524 & HM009 & Hollow stem borehole, no SPT & $8 / 28 / 1964$ & 125.0 & 339829 & 6788304 & $\boldsymbol{V}$ & $\square$ & $\square$ & $\nabla$ & $\square$ \\
\hline 1524 & HM010 & Hollow stem borehole, no SPT & $8 / 28 / 1964$ & 125.0 & 339831 & 6788368 & $\boldsymbol{V}$ & $\square$ & $\square$ & $\square$ & $\square$ \\
\hline 1524 & HN012 & Hollow stem borehole, no SPT & $8 / 28 / 1964$ & 140.5 & 340321 & 6788061 & $\boldsymbol{V}$ & $\square$ & $\square$ & $\square$ & $\square$ \\
\hline 1524 & HN013 & Hollow stem borehole, no SPT & $8 / 28 / 1964$ & 120.0 & 340313 & 6788120 & 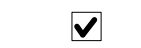 & $\square$ & $\square$ & $\nabla$ & $\square$ \\
\hline 1524 & HN014 & Hollow stem borehole, no SPT & $8 / 28 / 1964$ & 131.0 & 340312 & 6788250 & $\checkmark$ & $\square$ & $\square$ & $\square$ & $\square$ \\
\hline 1524 & HN015 & Hollow stem borehole, no SPT & $8 / 28 / 1964$ & 130.0 & 340305 & 6788386 & 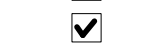 & $\square$ & $\square$ & $\square$ & $\square$ \\
\hline 1525 & HF003 & Hollow stem borehole, no SPT & $8 / 28 / 1964$ & 140.0 & 341159 & 6787990 & $\boldsymbol{V}$ & $\square$ & $\square$ & $\bar{\nabla}$ & $\square$ \\
\hline 1525 & HNO02 & Solid stem borehole (no cores or SP & $11 / 7 / 1968$ & 48.0 & 340703 & 6788290 & $\square$ & $\square$ & $\square$ & $\square$ & $\square$ \\
\hline 1525 & HN003 & Solid stem borehole (no cores or SP & $11 / 7 / 1968$ & 25.0 & 340752 & 6788478 & $\square$ & $\square$ & $\square$ & $\square$ & $\square$ \\
\hline 1525 & HN004 & Solid stem borehole (no cores or SP & $11 / 8 / 1968$ & 23.0 & 340369 & 6788085 & $\square$ & $\square$ & $\square$ & $\square$ & $\square$ \\
\hline
\end{tabular}




\begin{tabular}{|c|c|c|c|c|c|c|c|c|c|c|c|}
\hline \multirow[b]{2}{*}{$\begin{array}{c}\text { MOA } \\
\text { grid }\end{array}$} & \multirow[b]{2}{*}{$\begin{array}{l}\text { DGGS } \\
\text { number }\end{array}$} & \multirow[b]{2}{*}{ Hole Type } & \multirow[b]{2}{*}{$\begin{array}{c}\text { Date } \\
\text { Completed }\end{array}$} & \multirow[b]{2}{*}{$\begin{array}{c}\text { Total } \\
\text { depth (ft) }\end{array}$} & \multicolumn{2}{|c|}{ UTM coordinates (m) } & \multicolumn{5}{|c|}{ Data entered } \\
\hline & & & & & East & North & Lithology & SPT & $\begin{array}{c}\text { Sample } \\
\text { test }\end{array}$ & $\begin{array}{l}\text { Water } \\
\text { level }\end{array}$ & Velocity \\
\hline 1525 & HN005 & Solid stem borehole (no cores or SP & $11 / 8 / 1968$ & 20.0 & 340401 & 6788212 & $\square$ & $\square$ & $\square$ & $\square$ & $\square$ \\
\hline 1525 & HN006 & Hollow stem borehole, no SPT & $8 / 28 / 1964$ & 200.0 & 340948 & 6788248 & $\boldsymbol{V}$ & $\square$ & $\square$ & $\nabla$ & $\square$ \\
\hline 1525 & HN007 & Hollow stem borehole, no SPT & 8/28/1964 & 103.0 & 340655 & 6788078 & $\boldsymbol{V}$ & $\square$ & $\nabla$ & $\nabla$ & $\square$ \\
\hline 1525 & HN008 & Hollow stem borehole, no SPT & $8 / 28 / 1964$ & 120.0 & 340691 & 6788144 & $\boldsymbol{V}$ & $\square$ & $\square$ & $\square$ & $\square$ \\
\hline 1525 & HN009 & Hollow stem borehole, no SPT & 8/28/1964 & 128.0 & 340750 & 6788243 & $\checkmark$ & $\square$ & $\checkmark$ & $\nabla$ & $\square$ \\
\hline 1525 & HN010 & Hollow stem borehole, no SPT & $8 / 28 / 1964$ & 136.0 & 340758 & 6788393 & $\boldsymbol{V}$ & $\square$ & $\square$ & $\square$ & $\square$ \\
\hline 1525 & HN011 & Hollow stem borehole, no SPT & $8 / 28 / 1964$ & 104.0 & 340758 & 6788546 & $\checkmark$ & $\square$ & $\square$ & $\checkmark$ & $\square$ \\
\hline 1525 & HO015 & Hollow stem borehole, no SPT & 8/28/1964 & 115.5 & 341169 & 6788250 & $\boldsymbol{V}$ & $\square$ & $\square$ & $\square$ & $\square$ \\
\hline 1525 & HO016 & Hollow stem borehole, no SPT & $8 / 28 / 1964$ & 125.0 & 341178 & 6788506 & $\nabla$ & $\square$ & $\square$ & $\square$ & $\square$ \\
\hline 1525 & HO017 & Hollow stem borehole, no SPT & 8/28/1964 & 106.0 & 341185 & 6788582 & $\boldsymbol{V}$ & $\square$ & $\square$ & $\square$ & $\square$ \\
\hline 1526 & 2085 & Hollow stem borehole w/SPT & & 101.5 & 341907 & 6788619 & $\square$ & $\square$ & $\square$ & $\square$ & $\square$ \\
\hline 1526 & 2283 & Hollow stem borehole, no SPT & & 50.0 & 341526 & 6788583 & $\boldsymbol{V}$ & $\square$ & $\nabla$ & $\square$ & $\square$ \\
\hline 1526 & 2284 & Hollow stem borehole, no SPT & & 46.0 & 341564 & 6788550 & $\boldsymbol{V}$ & $\square$ & $\nabla$ & $\square$ & $\square$ \\
\hline 1526 & 2286 & Hollow stem borehole w/SPT & $12 / 20 / 1988$ & 66.5 & 341982 & 6788743 & $\checkmark$ & $\checkmark$ & $\checkmark$ & $\checkmark$ & $\square$ \\
\hline 1526 & HF001 & Hollow stem borehole, no SPT & $8 / 28 / 1964$ & 210.0 & 341963 & 6787989 & $\boldsymbol{V}$ & $\square$ & $\square$ & $\square$ & $\square$ \\
\hline 1526 & HF002 & Hollow stem borehole w/SPT & $8 / 28 / 1964$ & 125.0 & 341560 & 6787980 & $\checkmark$ & $\checkmark$ & $\square$ & $\checkmark$ & $\square$ \\
\hline 1526 & HO002 & Solid stem borehole (no cores or SP & $3 / 13 / 1969$ & 33.0 & 341601 & 6788570 & $\square$ & $\square$ & $\square$ & $\square$ & $\square$ \\
\hline 1526 & HO003 & Hollow stem borehole w/SPT & 8/28/1964 & 195.0 & 341567 & 6788262 & $\boldsymbol{V}$ & $\checkmark$ & $\square$ & $\checkmark$ & $\square$ \\
\hline 1526 & HO004 & Hollow stem borehole, no SPT & $8 / 28 / 1964$ & 175.0 & 341580 & 6788454 & $\boldsymbol{V}$ & $\square$ & $\square$ & $\nabla$ & $\square$ \\
\hline 1526 & HO005 & Hollow stem borehole w/SPT & $8 / 28 / 1964$ & 97.0 & 341551 & 6788559 & $\boldsymbol{V}$ & $\nabla$ & $\square$ & $\square$ & $\square$ \\
\hline 1526 & HO006 & Hollow stem borehole w/SPT & 8/28/1964 & 72.5 & 341504 & 6788594 & $\boldsymbol{V}$ & $\checkmark$ & $\square$ & $\square$ & $\square$ \\
\hline 1526 & HO007 & Hollow stem borehole, no SPT & $8 / 28 / 1964$ & 50.0 & 341463 & 6788642 & $\boldsymbol{V}$ & $\square$ & $\square$ & $\nabla$ & $\square$ \\
\hline 1526 & HO008 & Hollow stem borehole, no SPT & $8 / 28 / 1964$ & 137.0 & 341965 & 6788238 & 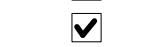 & $\square$ & $\square$ & $\nabla$ & $\square$ \\
\hline 1526 & HO009 & Hollow stem borehole w/SPT & $8 / 28 / 1964$ & 102.0 & 341964 & 6788478 & $\nabla$ & $\checkmark$ & $\square$ & $\square$ & $\square$ \\
\hline 1526 & HO010 & Hollow stem borehole, no SPT & 8/28/1964 & 155.3 & 341977 & 6788666 & $\boldsymbol{V}$ & $\square$ & $\square$ & $\nabla$ & $\square$ \\
\hline 1526 & HO011 & Hollow stem borehole w/SPT & $8 / 28 / 1964$ & 137.1 & 341946 & 6788704 & $\boldsymbol{V}$ & $\checkmark$ & $\nabla$ & $\nabla$ & $\square$ \\
\hline 1526 & HO013 & Hollow stem borehole, no SPT & $8 / 28 / 1964$ & 200.0 & 341387 & 6788414 & $\boldsymbol{V}$ & $\square$ & $\square$ & $\nabla$ & $\square$ \\
\hline 1526 & HO014 & Hollow stem borehole, no SPT & $8 / 28 / 1964$ & 200.0 & 341311 & 6788524 & $\boldsymbol{V}$ & $\square$ & $\square$ & $\nabla$ & $\square$ \\
\hline 1527 & 1300 & Hollow stem borehole w/SPT & $8 / 1 / 1984$ & 139.0 & 342729 & 6787935 & $\boldsymbol{V}$ & $\checkmark$ & $\checkmark$ & $\checkmark$ & $\square$ \\
\hline 1527 & 2285 & Hollow stem borehole w/SPT & $1 / 11 / 1989$ & 71.9 & 342003 & 6788698 & 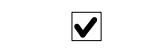 & $\nabla$ & $\nabla$ & $\nabla$ & $\square$ \\
\hline 1527 & HE011 & Hollow stem borehole w/SPT & $9 / 1 / 1984$ & 174.5 & 342550 & 6787948 & $\checkmark$ & $\checkmark$ & $\nabla$ & $\nabla$ & $\square$ \\
\hline 1527 & HP004 & Hollow stem borehole, no SPT & $3 / 2 / 1978$ & 18.0 & 342689 & 6788720 & $\square$ & $\square$ & $\square$ & $\square$ & $\square$ \\
\hline 1527 & HP005 & Hollow stem borehole, no SPT & $3 / 2 / 1978$ & 20.0 & 342689 & 6788603 & $\square$ & $\square$ & $\square$ & $\square$ & $\square$ \\
\hline 1527 & HP012 & Hollow stem borehole, no SPT & 8/28/1964 & 86.0 & 342303 & 6788530 & $\nabla$ & $\square$ & $\nabla$ & $\square$ & $\square$ \\
\hline 1527 & HP013 & Hollow stem borehole, no SPT & $8 / 28 / 1964$ & 87.0 & 342194 & 6788464 & $\boldsymbol{v}$ & $\square$ & $\nabla$ & $\square$ & $\square$ \\
\hline 1527 & HP014 & Hollow stem borehole, no SPT & $8 / 28 / 1964$ & 47.0 & 342440 & 6788616 & $\boldsymbol{V}$ & $\square$ & $\checkmark$ & $\square$ & $\square$ \\
\hline
\end{tabular}




\begin{tabular}{|c|c|c|c|c|c|c|c|c|c|c|c|}
\hline \multirow[b]{2}{*}{$\begin{array}{l}\text { MOA } \\
\text { grid }\end{array}$} & \multirow[b]{2}{*}{$\begin{array}{l}\text { DGGS } \\
\text { number }\end{array}$} & \multirow[b]{2}{*}{ Hole Type } & \multirow[b]{2}{*}{$\begin{array}{c}\text { Date } \\
\text { Completed }\end{array}$} & \multirow[b]{2}{*}{$\begin{array}{c}\text { Total } \\
\text { depth (ft) }\end{array}$} & \multicolumn{2}{|c|}{ UTM coordinates (m) } & \multicolumn{5}{|c|}{ Data entered } \\
\hline & & & & & East & North & Lithology & SPT & $\begin{array}{c}\text { Sample } \\
\text { test }\end{array}$ & $\begin{array}{l}\text { Water } \\
\text { level }\end{array}$ & Velocity \\
\hline 1527 & HP016 & Hollow stem borehole w/SPT & $3 / 7 / 1983$ & 32.5 & 342663 & 6788189 & & $\square$ & $\square$ & $\square$ & $\square$ \\
\hline 1528 & 1256 & Hollow stem borehole w/SPT & 2/8/1995 & 32.0 & 343280 & 6788494 & $\square$ & $\square$ & $\square$ & $\square$ & $\square$ \\
\hline 1528 & HD022 & Solid stem borehole (no cores or SP & $7 / 1 / 1965$ & 23.0 & 343559 & 6787930 & $\checkmark$ & $\square$ & $\square$ & $\checkmark$ & $\square$ \\
\hline 1528 & HE003 & Solid stem borehole (no cores or SP & $8 / 23 / 1971$ & 18.0 & 342867 & 6787915 & L & $\square$ & $\square$ & $\square$ & $\square$ \\
\hline 1528 & HQ001 & Hollow stem borehole, no SPT & $8 / 28 / 1964$ & 93.0 & 343500 & 6788432 & 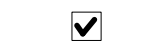 & $\square$ & $\checkmark$ & $\square$ & $\square$ \\
\hline 1528 & HQ002 & Hollow stem borehole, no SPT & 8/28/1964 & 103.0 & 343330 & 6788562 & $\boldsymbol{V}$ & $\square$ & $\square$ & $\square$ & $\square$ \\
\hline 1528 & HQ003 & Hollow stem borehole, no SPT & $8 / 28 / 1964$ & 112.0 & 343353 & 6788620 & $\nabla$ & $\square$ & $\checkmark$ & $\checkmark$ & $\square$ \\
\hline 1528 & HQ004 & Hollow stem borehole w/SPT & $8 / 28 / 1964$ & 103.0 & 343330 & 6788671 & $\boldsymbol{V}$ & $\nabla$ & $\square$ & $\square$ & $\square$ \\
\hline 1528 & HQ005 & Hollow stem borehole w/SPT & $8 / 28 / 1964$ & 113.0 & 343312 & 6788662 & $\boldsymbol{V}$ & $\checkmark$ & $\checkmark$ & $\square$ & $\square$ \\
\hline 1528 & HQ009 & Hollow stem borehole w/SPT & $6 / 7 / 1965$ & 75.0 & 343604 & 6788642 & $\boldsymbol{V}$ & $\checkmark$ & $\nabla$ & $\checkmark$ & $\square$ \\
\hline 1528 & HQ010 & Hollow stem borehole w/SPT & $6 / 22 / 1965$ & 46.5 & 343586 & 6788546 & $\square$ & $\square$ & $\square$ & $\square$ & $\square$ \\
\hline 1528 & HQ011 & Solid stem borehole (no cores or SP & $7 / 1 / 1965$ & 28.0 & 343575 & 6788494 & $\square$ & $\square$ & $\square$ & $\square$ & $\square$ \\
\hline 1528 & HQ012 & Solid stem borehole (no cores or SP & $7 / 1 / 1965$ & 28.0 & 343578 & 6788465 & & $\square$ & $\square$ & $\square$ & $\square$ \\
\hline 1528 & HQ014 & Solid stem borehole (no cores or SP & $7 / 1 / 1965$ & 28.0 & 343592 & 6788386 & & $\square$ & $\square$ & $\square$ & $\square$ \\
\hline 1528 & HQ015 & Solid stem borehole (no cores or SP & $7 / 1 / 1965$ & 23.0 & 343573 & 6788355 & $\square$ & $\square$ & $\square$ & $\square$ & $\square$ \\
\hline 1528 & HQ016 & Solid stem borehole (no cores or SP & $7 / 1 / 1965$ & 23.0 & 343568 & 6788262 & & $\square$ & $\square$ & $\square$ & $\square$ \\
\hline 1528 & HQ017 & Hollow stem borehole w/SPT & $6 / 15 / 1965$ & 26.0 & 343540 & 6788662 & $\square$ & $\square$ & $\square$ & $\square$ & $\square$ \\
\hline 1528 & HQ018 & Hollow stem borehole w/SPT & $6 / 11 / 1965$ & 20.0 & 343538 & 6788649 & & $\square$ & $\square$ & $\square$ & $\square$ \\
\hline 1528 & HQ019 & Hollow stem borehole w/SPT & $6 / 17 / 1965$ & 20.0 & 343514 & 6788638 & & $\square$ & $\square$ & $\square$ & $\square$ \\
\hline 1528 & HQ020 & Hollow stem borehole w/SPT & $6 / 10 / 1965$ & 30.0 & 343512 & 6788608 & $\square$ & $\square$ & $\square$ & $\square$ & $\square$ \\
\hline 1528 & HQ021 & Hollow stem borehole w/SPT & $6 / 16 / 1965$ & 30.0 & 343499 & 6788590 & $\square$ & $\square$ & $\square$ & $\square$ & $\square$ \\
\hline 1528 & HQ022 & Hollow stem borehole w/SPT & $6 / 17 / 1965$ & 35.0 & 343512 & 6788580 & & $\square$ & $\square$ & $\square$ & $\square$ \\
\hline 1528 & HQ023 & Hollow stem borehole w/SPT & $6 / 17 / 1965$ & 22.0 & 343515 & 6788572 & & $\square$ & $\square$ & $\square$ & $\square$ \\
\hline 1528 & HQ024 & Hollow stem borehole w/SPT & $7 / 1 / 1965$ & 28.0 & 343485 & 6788564 & $\square$ & $\square$ & $\square$ & $\square$ & $\square$ \\
\hline 1528 & HQ034 & Hollow stem borehole w/SPT & $10 / 28 / 1965$ & 107.0 & 343573 & 6788492 & $\nabla$ & $\checkmark$ & $\square$ & $\square$ & $\square$ \\
\hline 1528 & HQ035 & Hollow stem borehole w/SPT & $2 / 3 / 1983$ & 31.0 & 343284 & 6788092 & $\boldsymbol{V}$ & $\checkmark$ & $\square$ & $\nabla$ & $\square$ \\
\hline 1528 & HQ036 & Hollow stem borehole w/SPT & $2 / 3 / 1983$ & 30.5 & 343309 & 6788074 & & $\square$ & $\square$ & $\square$ & $\square$ \\
\hline 1528 & HQ037 & Hollow stem borehole w/SPT & $3 / 8 / 1983$ & 30.5 & 343324 & 6788100 & & $\square$ & $\square$ & $\square$ & $\square$ \\
\hline 1529 & 1257 & Hollow stem borehole w/SPT & $11 / 29 / 1990$ & 58.0 & 344365 & 6787904 & $\boldsymbol{V}$ & $\checkmark$ & $\square$ & $\checkmark$ & $\square$ \\
\hline 1529 & HQ007 & Hollow stem borehole w/SPT & $6 / 10 / 1965$ & 25.5 & 343612 & 6788677 & & $\square$ & $\square$ & $\square$ & $\square$ \\
\hline 1529 & HQ008 & Solid stem borehole (no cores or SP & $7 / 1 / 1965$ & 29.5 & 343608 & 6788659 & & $\square$ & $\square$ & $\square$ & $\square$ \\
\hline 1529 & HQ013 & Solid stem borehole (no cores or SP & $7 / 1 / 1965$ & 23.0 & 343602 & 6788446 & & $\square$ & $\square$ & $\square$ & $\square$ \\
\hline 1529 & HQ026 & Hollow stem borehole w/SPT & $6 / 25 / 1965$ & 20.0 & 343689 & 6788606 & $\square$ & $\square$ & $\square$ & $\square$ & $\square$ \\
\hline 1529 & HQ027 & Hollow stem borehole w/SPT & $6 / 30 / 1965$ & 32.0 & 343703 & 6788561 & $\square$ & $\square$ & $\square$ & $\square$ & $\square$ \\
\hline 1529 & HQ028 & Hollow stem borehole w/SPT & $7 / 1 / 1965$ & 28.0 & 343709 & 6788476 & $\square$ & $\square$ & $\square$ & $\square$ & $\square$ \\
\hline 1530 & 1258 & Hollow stem borehole w/SPT & $8 / 5 / 1985$ & 61.5 & 344815 & 6788125 & $\boldsymbol{V}$ & $\nabla$ & $\nabla$ & $\nabla$ & $\square$ \\
\hline
\end{tabular}




\begin{tabular}{|c|c|c|c|c|c|c|c|c|c|c|c|}
\hline \multirow[b]{2}{*}{$\begin{array}{l}\text { MOA } \\
\text { grid }\end{array}$} & \multirow[b]{2}{*}{$\begin{array}{l}\text { DGGS } \\
\text { number }\end{array}$} & \multirow[b]{2}{*}{ Hole Type } & \multirow[b]{2}{*}{$\begin{array}{c}\text { Date } \\
\text { Completed }\end{array}$} & \multirow[b]{2}{*}{$\begin{array}{c}\text { Total } \\
\text { depth }(\mathrm{ft})\end{array}$} & \multicolumn{2}{|c|}{ UTM coordinates (m) } & \multicolumn{5}{|c|}{ Data entered } \\
\hline & & & & & East & North & Lithology & SPT & $\begin{array}{c}\text { Sample } \\
\text { test }\end{array}$ & $\begin{array}{l}\text { Water } \\
\text { level }\end{array}$ & Velocity \\
\hline 1530 & 1259 & Hollow stem borehole w/SPT & $6 / 2 / 1976$ & 51.0 & 344749 & 6788373 & $\nabla$ & $\checkmark$ & $\square$ & $\square$ & \\
\hline 1530 & 1260 & Hollow stem borehole w/SPT & $8 / 18 / 1992$ & 51.0 & 344956 & 6788232 & $\checkmark$ & $\nabla$ & $\square$ & $\checkmark$ & $\square$ \\
\hline 1530 & 1261 & Hollow stem borehole w/SPT & $8 / 25 / 1992$ & 53.5 & 344958 & 6788221 & $\checkmark$ & $\nabla$ & $\square$ & $\checkmark$ & \\
\hline 1530 & 1262 & Hollow stem borehole w/SPT & $8 / 25 / 1992$ & 53.5 & 344946 & 6788212 & $\checkmark$ & 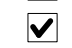 & $\square$ & $\boldsymbol{V}$ & $\square$ \\
\hline 1530 & 1263 & Hollow stem borehole w/SPT & $8 / 24 / 1992$ & 53.0 & 344939 & 6788192 & $\checkmark$ & $\checkmark$ & $\square$ & $\nabla$ & $\square$ \\
\hline 1530 & 1264 & Hollow stem borehole w/SPT & $8 / 17 / 1992$ & 53.5 & 344938 & 6788227 & $\checkmark$ & $\checkmark$ & $\square$ & $\checkmark$ & $\square$ \\
\hline 1530 & 1686 & Hollow stem borehole w/SPT & $6 / 30 / 1976$ & 41.5 & 345050 & 6788022 & $\square$ & $\square$ & $\square$ & $\square$ & $\square$ \\
\hline 1530 & 1687 & Hollow stem borehole w/SPT & $6 / 30 / 1976$ & 41.5 & 345019 & 6788008 & $\square$ & $\square$ & $\square$ & $\square$ & $\checkmark$ \\
\hline 1530 & HB026 & Solid stem borehole (no cores or SP & $7 / 9 / 1958$ & 5.5 & 345006 & 6787820 & $\square$ & $\square$ & $\square$ & $\square$ & $\square$ \\
\hline 1530 & HC053 & Hollow stem borehole w/SPT & $1 / 1 / 1976$ & 41.5 & 344872 & 6787976 & $\square$ & $\square$ & $\square$ & $\square$ & \\
\hline 1530 & $\mathrm{HC} 054$ & Hollow stem borehole w/SPT & $1 / 1 / 1976$ & 41.5 & 344924 & 6787949 & $\square$ & $\square$ & $\square$ & $\square$ & $\square$ \\
\hline 1530 & HC055 & Hollow stem borehole w/SPT & $1 / 1 / 1976$ & 41.5 & 344971 & 6787974 & $\square$ & $\square$ & $\square$ & $\square$ & \\
\hline 1530 & HC056 & Hollow stem borehole w/SPT & $1 / 1 / 1976$ & 41.5 & 344917 & 6787890 & $\square$ & $\square$ & $\square$ & $\square$ & $\square$ \\
\hline 1530 & $\mathrm{HC} 057$ & Hollow stem borehole w/SPT & $1 / 1 / 1976$ & 41.0 & 344909 & 6787842 & $\square$ & $\square$ & $\square$ & $\square$ & $\square$ \\
\hline 1530 & $\mathrm{HC} 058$ & Hollow stem borehole w/SPT & $1 / 1 / 1976$ & 30.0 & 344961 & 6787837 & $\square$ & $\square$ & $\square$ & $\square$ & $\square$ \\
\hline 1530 & HC059 & Hollow stem borehole w/SPT & $1 / 1 / 1976$ & 61.5 & 344962 & 6787888 & $\checkmark$ & $\checkmark$ & $\checkmark$ & $\checkmark$ & \\
\hline 1530 & HC060 & Hollow stem borehole w/SPT & $1 / 1 / 1976$ & 61.5 & 344928 & 6787833 & $\checkmark$ & $\checkmark$ & $\square$ & $\nabla$ & 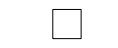 \\
\hline 1530 & HC061 & Hollow stem borehole w/SPT & $1 / 1 / 1976$ & 135.1 & 344938 & 6787862 & $\checkmark$ & 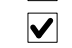 & $\nabla$ & $\vec{\nabla}$ & \\
\hline 1530 & HR008 & Solid stem borehole (no cores or SP & $7 / 2 / 1965$ & 15.0 & 344992 & 6788172 & $\square$ & $\square$ & $\square$ & $\square$ & $\square$ \\
\hline 1530 & HR009 & Solid stem borehole (no cores or SP & $6 / 18 / 1968$ & 28.0 & 344982 & 6788440 & $\square$ & $\square$ & $\square$ & $\square$ & $\square$ \\
\hline 1530 & HR010 & Solid stem borehole (no cores or SP & $3 / 11 / 1968$ & 22.0 & 344956 & 6788525 & $\square$ & $\square$ & $\square$ & $\square$ & \\
\hline 1530 & HS008 & Hollow stem borehole w/SPT & $11 / 30 / 1982$ & 52.0 & 345193 & 6788412 & $\checkmark$ & $\checkmark$ & $\checkmark$ & $\nabla$ & $\square$ \\
\hline 1530 & HSO09 & Hollow stem borehole w/SPT & $11 / 24 / 1982$ & 80.3 & 345192 & 6788352 & $\checkmark$ & $\nabla$ & $\nabla$ & $\nabla$ & $\square$ \\
\hline 1531 & 1236 & Hollow stem borehole w/SPT & 2/4/1983 & 50.0 & 345212 & 6788360 & $\nabla$ & $\bar{\square}$ & $\bar{\nabla}$ & $\checkmark$ & $\square$ \\
\hline 1531 & 1237 & Hollow stem borehole w/SPT & $8 / 19 / 1982$ & 51.5 & 345201 & 6788302 & $\checkmark$ & $\checkmark$ & $\checkmark$ & $\checkmark$ & $\square$ \\
\hline 1531 & 1265 & Hollow stem borehole w/SPT & $3 / 21 / 1978$ & 74.9 & 345491 & 6788012 & $\nabla$ & $\checkmark$ & $\square$ & $\nabla$ & $\square$ \\
\hline 1531 & 1266 & Hollow stem borehole w/SPT & $6 / 2 / 1978$ & 86.5 & 345473 & 6788028 & $\checkmark$ & $\checkmark$ & $\square$ & $\checkmark$ & $\square$ \\
\hline 1531 & 1790 & Solid stem borehole (no cores or SP & $7 / 21 / 1981$ & 26.0 & 345189 & 6787813 & $\square$ & $\square$ & $\square$ & $\square$ & $\square$ \\
\hline 1531 & 2116 & Hollow stem borehole w/SPT & & 66.5 & 345359 & 6787957 & $\checkmark$ & $\checkmark$ & $\square$ & $\square$ & $\square$ \\
\hline 1531 & HS010 & Hollow stem borehole w/SPT & $12 / 1 / 1982$ & 55.0 & 345191 & 6788246 & $\nabla$ & 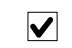 & $\checkmark$ & 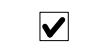 & $\square$ \\
\hline 1531 & HS011 & Hollow stem borehole w/SPT & $5 / 14 / 1981$ & 51.5 & 345891 & 6788026 & $\checkmark$ & $\checkmark$ & $\square$ & $\checkmark$ & $\square$ \\
\hline 1531 & HS012 & Hollow stem borehole w/SPT & $9 / 22 / 1977$ & 31.0 & 345816 & 6788568 & $\square$ & $\square$ & $\square$ & $\square$ & $\square$ \\
\hline 1531 & HS014 & Hollow stem borehole w/SPT & $9 / 27 / 1977$ & 30.0 & 345892 & 6788560 & $\square$ & $\square$ & $\square$ & $\square$ & $\square$ \\
\hline 1531 & HS021 & Hollow stem borehole w/SPT & $6 / 3 / 1978$ & 83.0 & 345474 & 6788008 & $\checkmark$ & $\checkmark$ & $\square$ & $\nabla$ & $\square$ \\
\hline 1532 & HA006 & Solid stem borehole (no cores or SP & $8 / 19 / 1971$ & 13.0 & 346530 & 6787766 & $\square$ & $\square$ & $\square$ & $\square$ & $\square$ \\
\hline 1532 & HA007 & Solid stem borehole (no cores or SP & $8 / 19 / 1971$ & 13.0 & 346677 & 6787744 & $\square$ & $\square$ & $\square$ & $\square$ & $\square$ \\
\hline
\end{tabular}




\begin{tabular}{|c|c|c|c|c|c|c|c|c|c|c|c|}
\hline \multirow[b]{2}{*}{$\begin{array}{c}\text { MOA } \\
\text { grid }\end{array}$} & \multirow[b]{2}{*}{$\begin{array}{c}\text { DGGS } \\
\text { number }\end{array}$} & \multirow[b]{2}{*}{ Hole Type } & \multirow[b]{2}{*}{$\begin{array}{c}\text { Date } \\
\text { Completed }\end{array}$} & \multirow[b]{2}{*}{$\begin{array}{c}\text { Total } \\
\text { depth (ft) }\end{array}$} & \multicolumn{2}{|c|}{ UTM coordinates (m) } & \multicolumn{5}{|c|}{ Data entered } \\
\hline & & & & & East & North & Lithology & SPT & $\begin{array}{c}\text { Sample } \\
\text { test }\end{array}$ & $\begin{array}{l}\text { Water } \\
\text { level }\end{array}$ & Velocity \\
\hline 1532 & HT002 & Cone Penetration Test (CPT) & $3 / 28 / 1986$ & 24.0 & 346358 & 6788450 & & $\square$ & $\square$ & $\square$ & $\square$ \\
\hline 1533 & GZ001 & Solid stem borehole (no cores or SP & $8 / 20 / 1971$ & 18.0 & 347024 & 6787762 & $\square$ & $\square$ & $\square$ & $\square$ & $\square$ \\
\hline 1533 & GZ002 & Solid stem borehole (no cores or SP & $8 / 20 / 1971$ & 13.0 & 347169 & 6787753 & & $\square$ & $\square$ & $\square$ & $\square$ \\
\hline 1533 & GZ003 & Solid stem borehole (no cores or SP & $8 / 20 / 1971$ & 8.0 & 347312 & 6787726 & 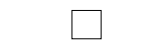 & $\square$ & $\square$ & $\square$ & $\square$ \\
\hline 1533 & GZ004 & Solid stem borehole (no cores or SP & 9/3/1971 & 4.0 & 347365 & 6787720 & $\square$ & $\square$ & $\square$ & $\square$ & $\square$ \\
\hline 1533 & GZ005 & Solid stem borehole (no cores or SP & $8 / 20 / 1971$ & 8.0 & 347416 & 6787735 & $\square$ & $\square$ & $\square$ & $\square$ & $\square$ \\
\hline 1533 & GZ006 & Solid stem borehole (no cores or SP & $8 / 20 / 1971$ & 8.0 & 347554 & 6787736 & $\square$ & $\square$ & $\square$ & $\square$ & $\square$ \\
\hline 1533 & HA008 & Solid stem borehole (no cores or SP & $8 / 19 / 1971$ & 13.0 & 346787 & 6787738 & & $\square$ & $\square$ & $\square$ & $\square$ \\
\hline 1533 & HA009 & Solid stem borehole (no cores or SP & $8 / 20 / 1971$ & 10.0 & 346902 & 6787743 & $\square$ & $\square$ & $\square$ & $\square$ & $\square$ \\
\hline 1533 & HA010 & Solid stem borehole (no cores or SP & $8 / 20 / 1971$ & 13.0 & 346957 & 6787738 & $\square$ & $\square$ & $\square$ & $\square$ & $\square$ \\
\hline 1533 & HA011 & Solid stem borehole (no cores or SP & $8 / 20 / 1971$ & 11.0 & 346978 & 6787740 & $\square$ & $\square$ & $\square$ & $\square$ & $\square$ \\
\hline 1534 & 1267 & Hollow stem borehole w/SPT & $3 / 30 / 1974$ & 20.0 & 347958 & 6787782 & $\boldsymbol{V}$ & $\checkmark$ & $\nabla$ & $\nabla$ & $\square$ \\
\hline 1534 & 1268 & Hollow stem borehole w/SPT & $3 / 30 / 1974$ & 20.0 & 347856 & 6787725 & $\boldsymbol{V}$ & $\nabla$ & $\square$ & $\nabla$ & $\square$ \\
\hline 1534 & 3021 & Water well & $4 / 18 / 1964$ & 400.0 & 348282 & 6787704 & $\checkmark$ & $\square$ & $\square$ & $\checkmark$ & $\square$ \\
\hline 1534 & HV001 & Solid stem borehole (no cores or SP & $8 / 1 / 1984$ & 760.0 & 348374 & 6788336 & $\boldsymbol{V}$ & $\square$ & $\square$ & $\square$ & $\square$ \\
\hline 1535 & 1276 & Hollow stem borehole w/SPT & $4 / 3 / 1981$ & 9.0 & 348533 & 6788064 & & $\square$ & $\square$ & $\square$ & $\square$ \\
\hline 1535 & 1277 & Hollow stem borehole w/SPT & $4 / 3 / 1981$ & 9.0 & 348780 & 6788067 & & $\square$ & $\square$ & $\square$ & $\square$ \\
\hline 1535 & 1278 & Hollow stem borehole w/SPT & $4 / 3 / 1981$ & 8.0 & 348904 & 6788063 & - & $\square$ & $\square$ & $\square$ & $\square$ \\
\hline 1535 & 1629 & Hollow stem borehole w/SPT & $7 / 24 / 1996$ & 26.5 & 348844 & 6788401 & $\square$ & $\square$ & $\square$ & $\square$ & $\square$ \\
\hline 1535 & 1630 & Hollow stem borehole w/SPT & 7/18/1996 & 26.5 & 348918 & 6788407 & $\square$ & $\square$ & $\square$ & $\square$ & $\square$ \\
\hline 1535 & 1839 & Hollow stem borehole w/SPT & $6 / 3 / 1981$ & 46.5 & 348468 & 6787991 & $\boldsymbol{V}$ & $\checkmark$ & $\square$ & $\nabla$ & $\square$ \\
\hline 1535 & 1840 & Hollow stem borehole w/SPT & $6 / 2 / 1981$ & 46.0 & 348440 & 6788036 & $\square$ & $\square$ & $\square$ & $\square$ & $\square$ \\
\hline 1536 & 1279 & Hollow stem borehole w/SPT & $4 / 3 / 1981$ & 8.0 & 349427 & 6788009 & $\square$ & $\square$ & $\square$ & $\square$ & $\square$ \\
\hline 1536 & 1280 & Hollow stem borehole w/SPT & $3 / 31 / 1981$ & 10.0 & 349750 & 6787958 & $\square$ & $\square$ & $\square$ & $\square$ & $\square$ \\
\hline 1536 & 1281 & Hollow stem borehole w/SPT & $3 / 31 / 1981$ & 10.0 & 349871 & 6787783 & $\square$ & $\square$ & $\square$ & $\square$ & $\square$ \\
\hline 1536 & 1282 & Hollow stem borehole w/SPT & $3 / 31 / 1981$ & 10.0 & 349871 & 6787734 & $\square$ & $\square$ & $\square$ & $\square$ & $\square$ \\
\hline 1536 & 2093 & Hollow stem borehole w/SPT & $6 / 15 / 1970$ & 63.9 & 349290 & 6788142 & $\boldsymbol{V}$ & $\checkmark$ & 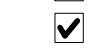 & $\square$ & $\square$ \\
\hline 1536 & 2094 & Hollow stem borehole w/SPT & 6/9/1970 & 61.5 & 349357 & 6788285 & $\boldsymbol{V}$ & $\checkmark$ & $\nabla$ & $\nabla$ & $\square$ \\
\hline 1536 & 2095 & Hollow stem borehole w/SPT & $6 / 11 / 1970$ & 68.0 & 349441 & 6788200 & $\boldsymbol{V}$ & $\checkmark$ & $\checkmark$ & $\checkmark$ & $\square$ \\
\hline 1537 & 1269 & Hollow stem borehole w/SPT & $12 / 11 / 1981$ & 20.0 & 350116 & 6787799 & $\boldsymbol{V}$ & $\nabla$ & $\nabla$ & $\nabla$ & $\square$ \\
\hline 1537 & 1270 & Hollow stem borehole w/SPT & $12 / 10 / 1981$ & 19.0 & 350288 & 6787793 & $\square$ & $\square$ & $\square$ & $\square$ & $\square$ \\
\hline 1537 & 1271 & Hollow stem borehole w/SPT & $12 / 10 / 1981$ & 20.0 & 350313 & 6787894 & $\square$ & $\square$ & $\square$ & $\square$ & $\square$ \\
\hline 1537 & 1272 & Hollow stem borehole w/SPT & $12 / 10 / 1981$ & 20.0 & 350364 & 6787927 & $\square$ & $\square$ & $\square$ & $\square$ & $\square$ \\
\hline 1537 & 1283 & Hollow stem borehole w/SPT & $3 / 20 / 1976$ & 12.5 & 350117 & 6788390 & $\square$ & $\square$ & $\square$ & $\square$ & $\square$ \\
\hline 1537 & 1284 & Hollow stem borehole w/SPT & $3 / 11 / 1976$ & 12.5 & 350148 & 6788389 & $\square$ & $\square$ & $\square$ & $\square$ & $\square$ \\
\hline 1537 & 1285 & Hollow stem borehole w/SPT & $3 / 11 / 1976$ & 13.0 & 350125 & 6788344 & $\square$ & $\square$ & $\square$ & $\square$ & $\square$ \\
\hline
\end{tabular}




\begin{tabular}{|c|c|c|c|c|c|c|c|c|c|c|c|}
\hline \multirow[b]{2}{*}{$\begin{array}{l}\text { MOA } \\
\text { grid }\end{array}$} & \multirow[b]{2}{*}{$\begin{array}{l}\text { DGGS } \\
\text { number }\end{array}$} & \multirow[b]{2}{*}{ Hole Type } & \multirow[b]{2}{*}{$\begin{array}{c}\text { Date } \\
\text { Completed }\end{array}$} & \multirow[b]{2}{*}{$\begin{array}{c}\text { Total } \\
\text { depth }(\mathrm{ft})\end{array}$} & \multicolumn{2}{|c|}{ UTM coordinates (m) } & \multicolumn{5}{|c|}{ Data entered } \\
\hline & & & & & East & North & Lithology & SPT & $\begin{array}{c}\text { Sample } \\
\text { test }\end{array}$ & $\begin{array}{l}\text { Water } \\
\text { level }\end{array}$ & Velocity \\
\hline 1537 & 1286 & Hollow stem borehole w/SPT & $3 / 22 / 1976$ & 12.0 & 350109 & 6788305 & $\square$ & & $\square$ & $\square$ & \\
\hline 1540 & 1287 & Hollow stem borehole w/SPT & $12 / 7 / 1987$ & 31.5 & 352855 & 6787597 & $\checkmark$ & $\bar{\nabla}$ & $\square$ & $\checkmark$ & $\square$ \\
\hline 1540 & 1288 & Hollow stem borehole w/SPT & 9/9/1981 & 30.0 & 353059 & 6787647 & $\square$ & & $\square$ & $\square$ & \\
\hline 1540 & 1289 & Hollow stem borehole w/SPT & 9/8/1981 & 30.0 & 353122 & 6787647 & $\square$ & $\square$ & $\square$ & $\square$ & $\checkmark$ \\
\hline 1540 & 1290 & Hollow stem borehole w/SPT & 9/4/1981 & 31.5 & 353138 & 6787593 & $\checkmark$ & $\checkmark$ & $\checkmark$ & $\boldsymbol{V}$ & $\square$ \\
\hline 1540 & 1291 & Hollow stem borehole w/SPT & $4 / 26 / 1982$ & 31.0 & 353181 & 6787524 & $\square$ & $\square$ & $\square$ & $\square$ & $\square$ \\
\hline 1540 & 1792 & Hollow stem borehole w/SPT & $3 / 14 / 1984$ & 31.5 & 353048 & 6787745 & $\square$ & $\square$ & $\square$ & $\square$ & $\square$ \\
\hline 1540 & 1793 & Hollow stem borehole w/SPT & $3 / 14 / 1984$ & 31.5 & 353047 & 6787706 & $\square$ & $\checkmark$ & $\square$ & $\square$ & $\checkmark$ \\
\hline 1540 & 1794 & Hollow stem borehole w/SPT & $3 / 16 / 1984$ & 31.5 & 353101 & 6787722 & $\square$ & $\checkmark$ & $\square$ & $\square$ & $\square$ \\
\hline 1540 & 1795 & Hollow stem borehole w/SPT & $10 / 28 / 1983$ & 31.0 & 353114 & 6787675 & $\square$ & $\square$ & $\square$ & $\square$ & $\square$ \\
\hline 1540 & 1796 & Hollow stem borehole w/SPT & $3 / 19 / 1984$ & 30.5 & 353131 & 6787747 & $\square$ & $\square$ & $\square$ & $\square$ & $\square$ \\
\hline 1540 & 1797 & Hollow stem borehole w/SPT & $10 / 31 / 1983$ & 31.5 & 353136 & 6787703 & $\square$ & & $\square$ & $\square$ & $\square$ \\
\hline 1540 & 1798 & Hollow stem borehole w/SPT & $10 / 28 / 1983$ & 31.5 & 353163 & 6787679 & $\square$ & $\square$ & $\square$ & $\square$ & $\square$ \\
\hline 1541 & 1255 & Hollow stem borehole w/SPT & $4 / 4 / 1988$ & 31.5 & 354049 & 6788226 & $\checkmark$ & $\checkmark$ & $\square$ & $\checkmark$ & $\square$ \\
\hline 1541 & 1295 & Hollow stem borehole w/SPT & 8/18/1994 & 41.5 & 354048 & 6788218 & $\nabla$ & $\bar{\square}$ & $\square$ & $\checkmark$ & $\square$ \\
\hline 1541 & 1296 & Hollow stem borehole w/SPT & 8/18/1994 & 41.5 & 354038 & 6788004 & $\checkmark$ & $\checkmark$ & $\square$ & $\square$ & $\square$ \\
\hline 1541 & 1297 & Hollow stem borehole w/SPT & $8 / 17 / 1994$ & 41.5 & 354030 & 6787810 & $\square$ & & $\square$ & $\square$ & \\
\hline 1541 & 1393 & Hollow stem borehole, no SPT & $5 / 23 / 1974$ & 15.0 & 353232 & 6787776 & $\square$ & $\square$ & $\square$ & $\square$ & $\square$ \\
\hline 1541 & 1609 & Hollow stem borehole w/SPT & $5 / 23 / 1996$ & 25.5 & 353679 & 6787559 & $\checkmark$ & 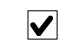 & $\square$ & $\nabla$ & $\square$ \\
\hline 1541 & 1697 & Hollow stem borehole, no SPT & $1 / 26 / 1976$ & 23.0 & 353440 & 6788253 & $\square$ & $\square$ & $\square$ & $\square$ & $\square$ \\
\hline 1541 & 1698 & Hollow stem borehole w/SPT & $1 / 26 / 1976$ & 23.0 & 353430 & 6788039 & $\square$ & & $\square$ & $\square$ & \\
\hline 1541 & 1699 & Hollow stem borehole w/SPT & $1 / 21 / 1976$ & 30.0 & 353315 & 6788168 & $\square$ & $\square$ & $\square$ & $\square$ & $\square$ \\
\hline 1541 & 4419 & Water well & $6 / 12 / 1964$ & 153.0 & 353311 & 6788203 & $\checkmark$ & $\square$ & $\square$ & $\checkmark$ & $\square$ \\
\hline 1541 & 4448 & Water well & $8 / 10 / 1971$ & 192.0 & 353486 & 6788103 & $\nabla$ & $\square$ & $\square$ & $\checkmark$ & $\square$ \\
\hline 1541 & 4451 & Water well & $6 / 10 / 1971$ & 219.0 & 353732 & 6787907 & $\checkmark$ & $\square$ & $\square$ & $\checkmark$ & $\square$ \\
\hline 1622 & 2301 & Hollow stem borehole w/SPT & $2 / 15 / 1969$ & 100.4 & 338358 & 6788090 & $\nabla$ & $\checkmark$ & $\square$ & $\square$ & $\square$ \\
\hline 1622 & 2302 & Hollow stem borehole w/SPT & $2 / 1 / 1969$ & 116.0 & 338475 & 6788054 & $\nabla$ & $\checkmark$ & $\checkmark$ & $\square$ & $\square$ \\
\hline 1622 & 2303 & Hollow stem borehole w/SPT & $2 / 11 / 1969$ & 86.5 & 338621 & 6788010 & $\checkmark$ & $\bar{\nabla}$ & $\nabla$ & $\square$ & $\square$ \\
\hline 1622 & HI001 & Solid stem borehole (no cores or SP & $7 / 12 / 1968$ & 33.0 & 338508 & 6787521 & $\square$ & & $\square$ & $\square$ & $\square$ \\
\hline 1622 & $\mathrm{HIOO2}$ & Solid stem borehole (no cores or SP & $7 / 12 / 1968$ & 13.0 & 338545 & 6787390 & $\square$ & & $\square$ & $\square$ & $\square$ \\
\hline 1622 & HI007 & Solid stem borehole (no cores or SP & $7 / 2 / 1968$ & 13.0 & 338710 & 6787369 & $\square$ & $\square$ & $\square$ & $\square$ & $\square$ \\
\hline 1622 & HI008 & Solid stem borehole (no cores or SP & $7 / 2 / 1968$ & 23.0 & 338656 & 6787512 & $\square$ & $\square$ & $\square$ & $\square$ & $\square$ \\
\hline 1622 & HI014 & Solid stem borehole (no cores or SP & $7 / 15 / 1968$ & 33.0 & 338460 & 6787650 & $\square$ & $\square$ & $\square$ & $\square$ & $\square$ \\
\hline 1622 & HI015 & Solid stem borehole (no cores or SP & $7 / 30 / 1968$ & 33.0 & 338420 & 6787756 & $\square$ & $\square$ & $\square$ & $\square$ & $\square$ \\
\hline 1622 & HI016 & Solid stem borehole (no cores or SP & $7 / 2 / 1968$ & 18.0 & 338613 & 6787655 & $\square$ & $\square$ & $\square$ & $\square$ & $\square$ \\
\hline 1622 & HI017 & Solid stem borehole (no cores or SP & $7 / 2 / 1968$ & 18.0 & 338568 & 6787794 & $\square$ & $\square$ & $\square$ & $\square$ & $\square$ \\
\hline
\end{tabular}




\begin{tabular}{|c|c|c|c|c|c|c|c|c|c|c|c|}
\hline \multirow[b]{2}{*}{$\begin{array}{l}\text { MOA } \\
\text { grid }\end{array}$} & \multirow[b]{2}{*}{$\begin{array}{l}\text { DGGS } \\
\text { number }\end{array}$} & \multirow[b]{2}{*}{ Hole Type } & \multirow[b]{2}{*}{$\begin{array}{c}\text { Date } \\
\text { Completed }\end{array}$} & \multirow[b]{2}{*}{$\begin{array}{c}\text { Total } \\
\text { depth }(\mathrm{ft})\end{array}$} & \multicolumn{2}{|c|}{ UTM coordinates (m) } & \multicolumn{5}{|c|}{ Data entered } \\
\hline & & & & & East & North & Lithology & SPT & $\begin{array}{c}\text { Sample } \\
\text { test }\end{array}$ & $\begin{array}{l}\text { Water } \\
\text { level }\end{array}$ & Velocity \\
\hline 1622 & HI018 & Solid stem borehole (no cores or SP & $7 / 2 / 1968$ & 28.0 & 338520 & 6787950 & $\square$ & & $\square$ & $\square$ & \\
\hline 1622 & HI019 & Solid stem borehole (no cores or SP & $7 / 12 / 1968$ & 23.0 & 338730 & 6787698 & $\square$ & $\square$ & $\square$ & $\square$ & $\square$ \\
\hline 1622 & $\mathrm{HIO} 20$ & Solid stem borehole (no cores or SP & $7 / 30 / 1968$ & 33.0 & 338687 & 6787852 & $\square$ & & $\square$ & $\square$ & \\
\hline 1622 & HI021 & Solid stem borehole (no cores or SP & $7 / 30 / 1968$ & 33.0 & 338648 & 6787988 & $\square$ & & $\square$ & $\square$ & $\checkmark$ \\
\hline 1622 & $\mathrm{HI} 026$ & Solid stem borehole (no cores or SP & 2/7/1973 & 25.0 & 338202 & 6787446 & $\square$ & & $\square$ & $\square$ & $\square$ \\
\hline 1622 & $\mathrm{HI} 027$ & Solid stem borehole (no cores or SP & 2/8/1973 & 30.0 & 338173 & 6787584 & $\square$ & & $\square$ & $\square$ & $\square$ \\
\hline 1622 & HIO28 & Solid stem borehole (no cores or SP & $2 / 5 / 1973$ & 35.0 & 338253 & 6787927 & $\square$ & & $\square$ & $\square$ & $\square$ \\
\hline 1622 & HIO29 & Solid stem borehole (no cores or SP & $2 / 5 / 1973$ & 35.0 & 338292 & 6787802 & $\square$ & & $\square$ & $\square$ & \\
\hline 1622 & HI030 & Solid stem borehole (no cores or SP & 2/8/1973 & 25.0 & 338144 & 6787715 & $\square$ & $\square$ & $\square$ & $\square$ & $\square$ \\
\hline 1622 & HI031 & Solid stem borehole (no cores or SP & 2/8/1973 & 35.0 & 338109 & 6787852 & $\square$ & & $\square$ & $\square$ & $\square$ \\
\hline 1622 & $\mathrm{HI} 032$ & Solid stem borehole (no cores or SP & $2 / 21 / 1973$ & 45.0 & 338430 & 6787854 & $\square$ & $\checkmark$ & $\square$ & $\square$ & $\square$ \\
\hline 1622 & $\mathrm{HI} 033$ & Solid stem borehole (no cores or SP & $11 / 9 / 1977$ & 15.0 & 337996 & 6787892 & $\square$ & & $\square$ & $\square$ & \\
\hline 1622 & HIO34 & Solid stem borehole (no cores or SP & $11 / 9 / 1977$ & 10.0 & 338028 & 6787714 & $\square$ & & $\square$ & $\square$ & $\square$ \\
\hline 1622 & HIO40 & Solid stem borehole (no cores or SP & $11 / 9 / 1977$ & 15.0 & 338085 & 6787419 & $\square$ & $\square$ & $\square$ & $\square$ & $\square$ \\
\hline 1622 & HI041 & Solid stem borehole (no cores or SP & $11 / 9 / 1977$ & 25.0 & 338052 & 6787574 & $\square$ & & $\square$ & $\square$ & $\square$ \\
\hline 1622 & HIO42 & Solid stem borehole (no cores or SP & $3 / 1 / 1973$ & 40.0 & 338262 & 6787404 & $\square$ & & $\square$ & $\square$ & $\square$ \\
\hline 1622 & HI043 & Solid stem borehole (no cores or SP & $7 / 12 / 1968$ & 28.0 & 338583 & 6787361 & $\square$ & & $\square$ & $\square$ & \\
\hline 1622 & HK010 & Solid stem borehole (no cores or SP & $11 / 9 / 1977$ & 15.0 & 337954 & 6788052 & $\square$ & & $\square$ & $\square$ & $\square$ \\
\hline 1622 & HL002 & Solid stem borehole (no cores or SP & $7 / 31 / 1968$ & 43.0 & 338392 & 6788040 & $\square$ & & $\square$ & $\square$ & $\square$ \\
\hline 1622 & HL003 & Solid stem borehole (no cores or SP & $8 / 5 / 1968$ & 33.0 & 338262 & 6788104 & $\square$ & $\square$ & $\square$ & $\square$ & $\square$ \\
\hline 1622 & HL016 & Solid stem borehole (no cores or SP & $7 / 2 / 1968$ & 38.0 & 338473 & 6788105 & $\square$ & & $\square$ & $\square$ & \\
\hline 1622 & HL027 & Solid stem borehole (no cores or SP & 9/9/1968 & 43.0 & 338061 & 6788058 & $\square$ & $\square$ & $\square$ & $\square$ & $\square$ \\
\hline 1622 & HL028 & Solid stem borehole (no cores or SP & $12 / 19 / 1972$ & 35.0 & 338203 & 6788084 & $\square$ & $\square$ & $\square$ & $\square$ & $\square$ \\
\hline 1622 & HL034 & Solid stem borehole (no cores or SP & 2/8/1973 & 25.0 & 338062 & 6788010 & $\square$ & $\square$ & $\square$ & $\square$ & $\square$ \\
\hline 1623 & 2058 & Hollow stem borehole w/SPT & $4 / 2 / 1984$ & 20.0 & 339377 & 6787501 & $\nabla$ & $\overline{5}$ & $\checkmark$ & $\square$ & $\square$ \\
\hline 1623 & 2059 & Solid stem borehole (no cores or SP & $4 / 3 / 1984$ & 25.0 & 339344 & 6788041 & $\nabla$ & & $\square$ & $\checkmark$ & \\
\hline 1623 & 2064 & Hollow stem borehole, no SPT & $8 / 12 / 1982$ & 20.0 & 339394 & 6787404 & $\checkmark$ & $\square$ & $\square$ & $\checkmark$ & $\square$ \\
\hline 1623 & 2065 & Hollow stem borehole, no SPT & $8 / 16 / 1982$ & 20.0 & 339352 & 6787755 & $\checkmark$ & $\square$ & $\square$ & $\nabla$ & \\
\hline 1623 & 2069 & Hollow stem borehole, no SPT & $6 / 13 / 1983$ & 40.0 & 338815 & 6788027 & $\checkmark$ & $\square$ & $\checkmark$ & $\square$ & $\square$ \\
\hline 1623 & 2304 & Hollow stem borehole w/SPT & $1 / 27 / 1969$ & 100.0 & 338768 & 6787965 & $\checkmark$ & $\bar{\nabla}$ & $\checkmark$ & $\square$ & $\square$ \\
\hline 1623 & 2305 & Hollow stem borehole w/SPT & $2 / 17 / 1969$ & 91.5 & 338855 & 6787937 & $\checkmark$ & $\checkmark$ & $\checkmark$ & $\square$ & $\square$ \\
\hline 1623 & 2306 & Hollow stem borehole w/SPT & $2 / 18 / 1969$ & 76.5 & 338929 & 6787916 & $\checkmark$ & $\checkmark$ & $\nabla$ & $\square$ & $\square$ \\
\hline 1623 & 2307 & Hollow stem borehole w/SPT & $2 / 14 / 1969$ & 51.5 & 339003 & 6787895 & $\checkmark$ & $\checkmark$ & $\checkmark$ & $\square$ & $\square$ \\
\hline 1623 & 2308 & Hollow stem borehole w/SPT & $2 / 15 / 1969$ & 51.5 & 339075 & 6787873 & $\checkmark$ & $\checkmark$ & $\checkmark$ & $\nabla$ & $\square$ \\
\hline 1623 & 2309 & Hollow stem borehole w/SPT & $1 / 29 / 1969$ & 50.0 & 339441 & 6787762 & $\checkmark$ & $\checkmark$ & $\square$ & $\square$ & $\square$ \\
\hline 1623 & 2313 & Hollow stem borehole w/SPT & $2 / 17 / 1969$ & 46.5 & 339148 & 6787851 & $\square$ & $\square$ & $\square$ & $\square$ & $\square$ \\
\hline
\end{tabular}




\begin{tabular}{|c|c|c|c|c|c|c|c|c|c|c|c|}
\hline \multirow[b]{2}{*}{$\begin{array}{l}\text { MOA } \\
\text { grid }\end{array}$} & \multirow[b]{2}{*}{$\begin{array}{l}\text { DGGS } \\
\text { number }\end{array}$} & \multirow[b]{2}{*}{ Hole Type } & \multirow[b]{2}{*}{$\begin{array}{c}\text { Date } \\
\text { Completed }\end{array}$} & \multirow[b]{2}{*}{$\begin{array}{c}\text { Total } \\
\text { depth }(\mathrm{ft})\end{array}$} & \multicolumn{2}{|c|}{ UTM coordinates (m) } & \multicolumn{5}{|c|}{ Data entered } \\
\hline & & & & & East & North & Lithology & SPT & $\begin{array}{c}\text { Sample } \\
\text { test }\end{array}$ & $\begin{array}{l}\text { Water } \\
\text { level }\end{array}$ & Velocity \\
\hline 1623 & 2321 & Hollow stem borehole w/SPT & $2 / 18 / 1969$ & 46.5 & 339236 & 6787824 & $\square$ & & $\square$ & $\square$ & \\
\hline 1623 & 2322 & Hollow stem borehole w/SPT & $2 / 17 / 1969$ & 41.5 & 339324 & 6787798 & - & & $\square$ & $\square$ & \\
\hline 1623 & HH004 & Solid stem borehole (no cores or SP & 9/4/1968 & 20.0 & 339434 & 6787340 & $\square$ & & $\square$ & $\square$ & \\
\hline 1623 & HH005 & Solid stem borehole (no cores or SP & $9 / 5 / 1968$ & 18.0 & 339380 & 6787500 & $\square$ & & $\square$ & $\square$ & $\square$ \\
\hline 1623 & HH006 & Solid stem borehole (no cores or SP & 9/5/1968 & 13.0 & 339334 & 6787570 & $\square$ & & $\square$ & $\square$ & $\square$ \\
\hline 1623 & HH007 & Solid stem borehole (no cores or SP & 9/5/1968 & 13.0 & 339320 & 6787700 & $\square$ & & $\square$ & $\square$ & \\
\hline 1623 & HH008 & Solid stem borehole (no cores or SP & 9/5/1968 & 18.0 & 339351 & 6787748 & $\square$ & 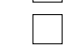 & $\square$ & $\square$ & $\square$ \\
\hline 1623 & HH009 & Solid stem borehole (no cores or SP & $9 / 5 / 1968$ & 13.0 & 339271 & 6787836 & $\square$ & & $\square$ & $\square$ & \\
\hline 1623 & HH010 & Solid stem borehole (no cores or SP & 9/5/1968 & 18.0 & 339354 & 6787880 & $\square$ & 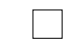 & $\square$ & $\square$ & $\square$ \\
\hline 1623 & HH011 & Solid stem borehole (no cores or SP & 9/5/1968 & 15.0 & 339235 & 6787946 & $\square$ & & $\square$ & $\square$ & \\
\hline 1623 & HH017 & Solid stem borehole (no cores or SP & $4 / 14 / 1972$ & 20.0 & 339510 & 6787522 & $\square$ & & $\square$ & $\square$ & $\square$ \\
\hline 1623 & HH018 & Solid stem borehole (no cores or SP & $4 / 14 / 1972$ & 20.0 & 339306 & 6787507 & $\square$ & $\square$ & $\square$ & $\square$ & $\square$ \\
\hline 1623 & HH019 & Solid stem borehole (no cores or SP & $4 / 14 / 1972$ & 20.0 & 339122 & 6787484 & $\square$ & & $\square$ & $\square$ & $\square$ \\
\hline 1623 & HH020 & Solid stem borehole (no cores or SP & $4 / 14 / 1972$ & 20.0 & 339145 & 6787365 & $\square$ & $\square$ & $\square$ & $\square$ & $\square$ \\
\hline 1623 & HH021 & Solid stem borehole (no cores or SP & $4 / 14 / 1972$ & 20.0 & 339360 & 6787382 & $\square$ & & $\square$ & $\square$ & $\square$ \\
\hline 1623 & HI009 & Solid stem borehole (no cores or SP & $7 / 12 / 1968$ & 18.0 & 338778 & 6787550 & $\square$ & 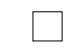 & $\square$ & $\square$ & $\square$ \\
\hline 1623 & HI010 & Solid stem borehole (no cores or SP & $7 / 10 / 1968$ & 8.0 & 338797 & 6787477 & $\square$ & & $\square$ & $\square$ & $\square$ \\
\hline 1623 & HI011 & Solid stem borehole (no cores or SP & $7 / 2 / 1968$ & 8.0 & 338845 & 6787342 & $\square$ & & $\square$ & $\square$ & \\
\hline 1623 & $\mathrm{HI} 044$ & Hollow stem borehole w/SPT & $12 / 21 / 1966$ & 21.5 & 338745 & 6787341 & $\square$ & & $\square$ & $\square$ & \\
\hline 1623 & HM003 & Solid stem borehole (no cores or SP & 9/5/1968 & 18.0 & 339359 & 6788017 & $\square$ & 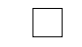 & $\square$ & $\square$ & $\square$ \\
\hline 1624 & 2310 & Hollow stem borehole w/SPT & $2 / 20 / 1969$ & 51.5 & 339910 & 6787619 & $\checkmark$ & $\checkmark$ & $\checkmark$ & $\bar{\nabla}$ & $\square$ \\
\hline 1624 & 2311 & Hollow stem borehole w/SPT & $1 / 20 / 1969$ & 51.5 & 340026 & 6787583 & $\checkmark$ & $\nabla$ & $\checkmark$ & $\nabla$ & $\square$ \\
\hline 1624 & 2323 & Hollow stem borehole w/SPT & $2 / 19 / 1969$ & 41.5 & 339558 & 6787727 & $\square$ & & $\square$ & $\square$ & $\square$ \\
\hline 1624 & 2324 & Hollow stem borehole w/SPT & $2 / 20 / 1969$ & 36.5 & 339675 & 6787691 & $\square$ & & $\square$ & $\square$ & $\square$ \\
\hline 1624 & 2325 & Hollow stem borehole w/SPT & $2 / 19 / 1969$ & 36.5 & 339793 & 6787655 & $\square$ & & $\square$ & $\square$ & $\square$ \\
\hline 1624 & 2326 & Hollow stem borehole w/SPT & $2 / 22 / 1969$ & 36.5 & 340142 & 6787550 & $\square$ & & $\square$ & $\square$ & \\
\hline 1624 & 2327 & Hollow stem borehole w/SPT & $2 / 22 / 1969$ & 31.5 & 340260 & 6787514 & $\square$ & & $\square$ & $\square$ & $\square$ \\
\hline 1624 & HG004 & Solid stem borehole (no cores or SP & $1 / 15 / 1970$ & 20.0 & 340266 & 6787918 & $\square$ & & $\square$ & $\square$ & \\
\hline 1624 & HG005 & Solid stem borehole (no cores or SP & $1 / 15 / 1970$ & 20.0 & 340265 & 6787855 & $\square$ & $\square$ & $\square$ & $\square$ & $\square$ \\
\hline 1624 & HG006 & Solid stem borehole (no cores or SP & $1 / 15 / 1970$ & 20.0 & 340265 & 6787782 & $\checkmark$ & $\square$ & $\square$ & $\checkmark$ & $\square$ \\
\hline 1624 & HG007 & Solid stem borehole (no cores or SP & $1 / 15 / 1970$ & 20.0 & 340198 & 6787921 & $\square$ & $\square$ & $\square$ & $\square$ & $\square$ \\
\hline 1624 & HG008 & Solid stem borehole (no cores or SP & $1 / 15 / 1970$ & 20.0 & 340194 & 6787850 & $\square$ & $\square$ & $\square$ & $\square$ & $\square$ \\
\hline 1624 & HG009 & Solid stem borehole (no cores or SP & $1 / 15 / 1970$ & 20.0 & 340196 & 6787781 & $\square$ & $\square$ & $\square$ & $\square$ & $\square$ \\
\hline 1624 & HG010 & Solid stem borehole (no cores or SP & $1 / 15 / 1970$ & 20.0 & 340132 & 6787919 & $\square$ & $\square$ & $\square$ & $\square$ & $\square$ \\
\hline 1624 & HG011 & Solid stem borehole (no cores or SP & $1 / 15 / 1970$ & 20.0 & 340131 & 6787848 & $\square$ & $\square$ & $\square$ & $\square$ & $\square$ \\
\hline 1624 & HG012 & Solid stem borehole (no cores or SP & $1 / 15 / 1970$ & 20.0 & 340125 & 6787782 & $\checkmark$ & $\square$ & $\square$ & $\checkmark$ & $\square$ \\
\hline
\end{tabular}




\begin{tabular}{|c|c|c|c|c|c|c|c|c|c|c|c|}
\hline \multirow[b]{2}{*}{$\begin{array}{l}\text { MOA } \\
\text { grid }\end{array}$} & \multirow[b]{2}{*}{$\begin{array}{l}\text { DGGS } \\
\text { number }\end{array}$} & \multirow[b]{2}{*}{ Hole Type } & \multirow[b]{2}{*}{$\begin{array}{c}\text { Date } \\
\text { Completed }\end{array}$} & \multirow[b]{2}{*}{$\begin{array}{c}\text { Total } \\
\text { depth }(\mathrm{ft})\end{array}$} & \multicolumn{2}{|c|}{ UTM coordinates (m) } & \multicolumn{5}{|c|}{ Data entered } \\
\hline & & & & & East & North & Lithology & SPT & $\begin{array}{c}\text { Sample } \\
\text { test }\end{array}$ & $\begin{array}{l}\text { Water } \\
\text { level }\end{array}$ & Velocity \\
\hline 1624 & HG015 & Hollow stem borehole, no SPT & $8 / 28 / 1964$ & 131.0 & 340331 & 6787981 & $\nabla$ & & $\square$ & $\square$ & \\
\hline 1624 & HG016 & Hollow stem borehole w/SPT & $2 / 22 / 1969$ & 31.5 & 340492 & 6787442 & $\square$ & $\square$ & $\square$ & $\square$ & $\square$ \\
\hline 1624 & HH012 & Solid stem borehole (no cores or SP & 9/6/1968 & 20.0 & 339692 & 6787306 & $\checkmark$ & & $\square$ & $\bar{\nabla}$ & \\
\hline 1624 & HH013 & Solid stem borehole (no cores or SP & 9/6/1968 & 20.0 & 339652 & 6787420 & $\square$ & $\square$ & $\square$ & $\square$ & $\square$ \\
\hline 1624 & HH014 & Solid stem borehole (no cores or SP & 9/6/1968 & 20.0 & 339670 & 6787572 & $\square$ & & $\square$ & $\square$ & $\square$ \\
\hline 1624 & HH015 & Solid stem borehole (no cores or SP & 9/6/1968 & 20.0 & 339690 & 6787726 & $\checkmark$ & $\square$ & $\square$ & $\checkmark$ & $\square$ \\
\hline 1624 & HH016 & Solid stem borehole (no cores or SP & $9 / 6 / 1968$ & 20.0 & 339687 & 6787871 & $\square$ & $\square$ & $\square$ & $\square$ & $\square$ \\
\hline 1624 & HH023 & Hollow stem borehole, no SPT & $8 / 28 / 1964$ & 117.5 & 339794 & 6787978 & $\checkmark$ & & $\square$ & $\checkmark$ & \\
\hline 1625 & 2328 & Hollow stem borehole w/SPT & $2 / 21 / 1969$ & 31.5 & 340375 & 6787478 & $\square$ & $\square$ & $\square$ & $\square$ & $\square$ \\
\hline 1625 & HF010 & Hollow stem borehole w/SPT & $2 / 6 / 1969$ & 51.5 & 341109 & 6787394 & $\checkmark$ & $\checkmark$ & $\square$ & $\checkmark$ & $\square$ \\
\hline 1625 & HG003 & Solid stem borehole (no cores or SP & $9 / 23 / 1968$ & 23.0 & 340392 & 6787710 & $\checkmark$ & $\square$ & $\square$ & $\nabla$ & $\square$ \\
\hline 1625 & HG013 & Hollow stem borehole, no SPT & $8 / 28 / 1964$ & 140.0 & 340609 & 6787993 & $\checkmark$ & & $\square$ & $\square$ & \\
\hline 1625 & HG014 & Hollow stem borehole, no SPT & $8 / 28 / 1964$ & 79.0 & 340553 & 6787880 & $\checkmark$ & $\square$ & $\square$ & $\checkmark$ & $\square$ \\
\hline 1625 & HG017 & Hollow stem borehole w/SPT & $1 / 30 / 1969$ & 51.5 & 340598 & 6787446 & $\checkmark$ & $\checkmark$ & $\checkmark$ & $\checkmark$ & $\square$ \\
\hline 1625 & HG018 & Hollow stem borehole w/SPT & $2 / 24 / 1969$ & 31.5 & 340720 & 6787409 & $\square$ & $\checkmark$ & $\square$ & $\square$ & $\square$ \\
\hline 1625 & HG019 & Hollow stem borehole w/SPT & $2 / 24 / 1969$ & 31.5 & 340837 & 6787401 & $\square$ & & $\square$ & $\square$ & $\square$ \\
\hline 1625 & HG020 & Hollow stem borehole w/SPT & $2 / 24 / 1969$ & 31.5 & 340972 & 6787398 & $\square$ & & $\square$ & $\square$ & $\square$ \\
\hline 1626 & 2329 & Hollow stem borehole w/SPT & $2 / 21 / 1969$ & 31.5 & 341926 & 6787255 & $\square$ & & $\square$ & $\square$ & $\square$ \\
\hline 1626 & HF004 & Hollow stem borehole w/SPT & $2 / 19 / 1969$ & 36.5 & 341263 & 6787384 & $\square$ & $\square$ & $\square$ & $\square$ & $\square$ \\
\hline 1626 & HF005 & Hollow stem borehole w/SPT & $2 / 19 / 1969$ & 36.5 & 341413 & 6787376 & $\square$ & $\square$ & $\square$ & $\square$ & $\square$ \\
\hline 1626 & HF006 & Hollow stem borehole w/SPT & $2 / 20 / 1969$ & 31.5 & 341544 & 6787370 & $\square$ & & $\square$ & $\square$ & \\
\hline 1626 & HF007 & Hollow stem borehole w/SPT & $2 / 20 / 1969$ & 31.5 & 341675 & 6787365 & $\square$ & $\square$ & $\square$ & $\square$ & $\square$ \\
\hline 1626 & HF008 & Hollow stem borehole w/SPT & $2 / 21 / 1969$ & 31.5 & 341797 & 6787358 & $\square$ & $\square$ & $\square$ & $\square$ & $\square$ \\
\hline 1626 & HF009 & Hollow stem borehole w/SPT & $2 / 5 / 1969$ & 51.5 & 341930 & 6787361 & $\checkmark$ & $\bar{\square}$ & $\bar{\nabla}$ & $\checkmark$ & $\square$ \\
\hline 1627 & HE001 & Solid stem borehole (no cores or SP & 8/9/1971 & 18.0 & 342715 & 6787882 & $\square$ & $\square$ & $\square$ & $\square$ & $\square$ \\
\hline 1627 & HE012 & Hollow stem borehole w/SPT & $8 / 17 / 1984$ & 166.0 & 342688 & 6787892 & $\checkmark$ & $\checkmark$ & $\checkmark$ & $\nabla$ & $\checkmark$ \\
\hline 1627 & HE015 & Cone Penetration Test (CPT) & $3 / 28 / 1986$ & 72.0 & 342666 & 6787142 & $\square$ & $\square$ & $\square$ & $\square$ & $\square$ \\
\hline 1628 & 2082 & Hollow stem borehole w/SPT & & 41.0 & 343332 & 6787612 & $\checkmark$ & $\bar{\nabla}$ & $\nabla$ & $\nabla$ & $\square$ \\
\hline 1628 & 2083 & Hollow stem borehole w/SPT & & 42.0 & 343289 & 6787599 & $\checkmark$ & $\checkmark$ & 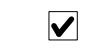 & $\checkmark$ & $\square$ \\
\hline 1628 & 4413 & Water well & 6/5/1964 & 151.0 & 343017 & 6787657 & $\checkmark$ & $\square$ & $\square$ & $\checkmark$ & $\square$ \\
\hline 1628 & 4414 & Water well & $6 / 7 / 1964$ & 150.0 & 343014 & 6787595 & $\checkmark$ & $\square$ & $\square$ & $\checkmark$ & $\square$ \\
\hline 1628 & HD001 & Solid stem borehole (no cores or SP & 8/9/1971 & 23.0 & 343054 & 6787873 & $\square$ & $\square$ & $\square$ & $\square$ & $\square$ \\
\hline 1628 & HD002 & Solid stem borehole (no cores or SP & $8 / 23 / 1971$ & 18.0 & 343121 & 6787866 & $\square$ & $\square$ & $\square$ & $\square$ & $\square$ \\
\hline 1628 & HD003 & Solid stem borehole (no cores or SP & 8/9/1971 & 13.0 & 343189 & 6787822 & $\square$ & $\square$ & $\square$ & $\square$ & $\square$ \\
\hline 1628 & HD004 & Solid stem borehole (no cores or SP & 8/9/1971 & 13.0 & 343277 & 6787752 & $\square$ & $\square$ & $\square$ & $\square$ & $\square$ \\
\hline 1628 & HD005 & Solid stem borehole (no cores or SP & 8/9/1971 & 13.0 & 343379 & 6787720 & $\square$ & $\square$ & $\square$ & $\square$ & $\square$ \\
\hline
\end{tabular}




\begin{tabular}{|c|c|c|c|c|c|c|c|c|c|c|c|}
\hline \multirow[b]{2}{*}{$\begin{array}{c}\text { MOA } \\
\text { grid }\end{array}$} & \multirow[b]{2}{*}{$\begin{array}{l}\text { DGGS } \\
\text { number }\end{array}$} & \multirow[b]{2}{*}{ Hole Type } & \multirow[b]{2}{*}{$\begin{array}{c}\text { Date } \\
\text { Completed }\end{array}$} & \multirow[b]{2}{*}{$\begin{array}{c}\text { Total } \\
\text { depth }(\mathrm{ft})\end{array}$} & \multicolumn{2}{|c|}{ UTM coordinates (m) } & \multicolumn{5}{|c|}{ Data entered } \\
\hline & & & & & East & North & Lithology & SPT & $\begin{array}{c}\text { Sample } \\
\text { test }\end{array}$ & $\begin{array}{l}\text { Water } \\
\text { level }\end{array}$ & Velocity \\
\hline 1628 & HD006 & Solid stem borehole (no cores or SP & 8/9/1971 & 13.0 & 343524 & 6787673 & $\square$ & 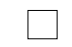 & $\square$ & $\square$ & \\
\hline 1628 & HD018 & Solid stem borehole (no cores or SP & $7 / 1 / 1965$ & 23.0 & 343543 & 6787747 & $\square$ & ᄂ & $\square$ & $\square$ & \\
\hline 1628 & HD023 & Hollow stem borehole w/SPT & $11 / 20 / 1984$ & 96.5 & 343025 & 6787138 & $\checkmark$ & $\nabla$ & 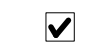 & $\square$ & \\
\hline 1628 & HE002 & Solid stem borehole (no cores or SP & 8/9/1971 & 13.0 & 342811 & 6787878 & $\square$ & $\square$ & $\square$ & $\square$ & $\square$ \\
\hline 1628 & HE004 & Solid stem borehole (no cores or SP & 8/9/1971 & 18.0 & 342983 & 6787875 & $\square$ & & $\square$ & $\square$ & $\square$ \\
\hline 1628 & HE013 & Hollow stem borehole w/SPT & $4 / 25 / 1983$ & 31.5 & 342909 & 6787510 & $\square$ & & $\square$ & $\square$ & \\
\hline 1628 & HE014 & Hollow stem borehole w/SPT & $4 / 26 / 1983$ & 16.5 & 342906 & 6787534 & $\square$ & $\square$ & $\square$ & $\square$ & $\square$ \\
\hline 1629 & 1303 & Hollow stem borehole w/SPT & $8 / 11 / 1985$ & 51.5 & 343735 & 6787664 & $\checkmark$ & & $\checkmark$ & $\nabla$ & \\
\hline 1629 & 1439 & Hollow stem borehole w/SPT & $8 / 10 / 1985$ & 50.6 & 343767 & 6787629 & $\checkmark$ & $\square$ & $\checkmark$ & $\checkmark$ & $\square$ \\
\hline 1629 & 4454 & Water well & $6 / 1 / 1977$ & 316.0 & 344324 & 6787475 & $\checkmark$ & & $\square$ & $\checkmark$ & \\
\hline 1629 & HC001 & Solid stem borehole (no cores or SP & $8 / 11 / 1971$ & 13.0 & 344134 & 6787701 & $\square$ & $\square$ & $\square$ & $\square$ & $\square$ \\
\hline 1629 & HC002 & Solid stem borehole (no cores or SP & $8 / 11 / 1971$ & 13.0 & 344311 & 6787730 & $\square$ & $\square$ & $\square$ & $\square$ & $\square$ \\
\hline 1629 & HC012 & Solid stem borehole (no cores or SP & $7 / 9 / 1958$ & 6.0 & 344026 & 6787848 & $\square$ & $\square$ & $\square$ & $\square$ & $\square$ \\
\hline 1629 & HC015 & Solid stem borehole (no cores or SP & $7 / 9 / 1958$ & 6.0 & 344119 & 6787843 & $\square$ & $\square$ & $\square$ & $\square$ & $\square$ \\
\hline 1629 & HC018 & Solid stem borehole (no cores or SP & $7 / 9 / 1958$ & 6.0 & 344180 & 6787830 & $\square$ & $\square$ & $\square$ & $\square$ & $\square$ \\
\hline 1629 & HC039 & Hollow stem borehole w/SPT & $7 / 15 / 1977$ & 26.0 & 344153 & 6787289 & $\square$ & $\square$ & $\square$ & $\square$ & $\square$ \\
\hline 1629 & $\mathrm{HC} 040$ & Hollow stem borehole w/SPT & $7 / 15 / 1977$ & 51.5 & 344153 & 6787262 & $\checkmark$ & $\checkmark$ & $\square$ & $\nabla$ & 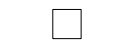 \\
\hline 1629 & $\mathrm{HC} 041$ & Hollow stem borehole w/SPT & $7 / 19 / 1977$ & 27.0 & 344171 & 6787230 & $\square$ & & $\square$ & $\square$ & \\
\hline 1629 & $\mathrm{HC} 042$ & Hollow stem borehole w/SPT & $7 / 19 / 1977$ & 41.5 & 344218 & 6787288 & $\square$ & & $\square$ & $\square$ & \\
\hline 1629 & $\mathrm{HC} 043$ & Hollow stem borehole w/SPT & $7 / 20 / 1977$ & 41.5 & 344216 & 6787264 & $\square$ & $\square$ & $\square$ & $\square$ & $\square$ \\
\hline 1629 & $\mathrm{HC} 044$ & Hollow stem borehole w/SPT & $7 / 21 / 1977$ & 41.5 & 344211 & 6787230 & $\square$ & $\square$ & $\square$ & $\square$ & $\square$ \\
\hline 1629 & $\mathrm{HC} 045$ & Hollow stem borehole w/SPT & $7 / 20 / 1977$ & 47.0 & 344249 & 6787289 & $\square$ & $\square$ & $\square$ & $\square$ & $\square$ \\
\hline 1629 & $\mathrm{HC} 046$ & Hollow stem borehole w/SPT & $7 / 18 / 1977$ & 61.5 & 344251 & 6787246 & $\checkmark$ & $\bar{\nabla}$ & $\bar{\nabla}$ & $\nabla$ & $\square$ \\
\hline 1629 & $\mathrm{HC} 047$ & Hollow stem borehole w/SPT & $7 / 21 / 1977$ & 51.5 & 344246 & 6787209 & $\checkmark$ & $\bar{\square}$ & $\bar{\nabla}$ & $\checkmark$ & $\square$ \\
\hline 1629 & $\mathrm{HC} 048$ & Hollow stem borehole w/SPT & $7 / 22 / 1977$ & 46.0 & 344288 & 6787271 & $\square$ & $\square$ & $\square$ & $\square$ & $\square$ \\
\hline 1629 & HC049 & Hollow stem borehole w/SPT & $7 / 25 / 1977$ & 51.0 & 344323 & 6787258 & $\checkmark$ & $\checkmark$ & $\checkmark$ & $\nabla$ & $\square$ \\
\hline 1629 & $\mathrm{HC} 050$ & Hollow stem borehole w/SPT & $7 / 27 / 1977$ & 37.0 & 344338 & 6787246 & $\square$ & & $\square$ & $\square$ & $\square$ \\
\hline 1629 & $\mathrm{HC} 051$ & Hollow stem borehole w/SPT & $7 / 25 / 1977$ & 49.0 & 344312 & 6787226 & $\square$ & & $\square$ & $\square$ & \\
\hline 1629 & $\mathrm{HC} 052$ & Hollow stem borehole w/SPT & $7 / 27 / 1977$ & 30.0 & 344298 & 6787190 & $\square$ & $\square$ & $\square$ & $\square$ & $\square$ \\
\hline 1629 & HD007 & Solid stem borehole (no cores or SP & $8 / 10 / 1971$ & 8.0 & 343779 & 6787710 & $\square$ & $\square$ & $\square$ & $\square$ & $\square$ \\
\hline 1629 & HD008 & Solid stem borehole (no cores or SP & $8 / 11 / 1971$ & 13.0 & 343906 & 6787716 & $\square$ & $\square$ & $\square$ & $\square$ & $\square$ \\
\hline 1629 & HD009 & Solid stem borehole (no cores or SP & $8 / 11 / 1971$ & 13.0 & 343991 & 6787697 & $\square$ & $\square$ & $\square$ & $\square$ & $\square$ \\
\hline 1629 & HD024 & Hollow stem borehole w/SPT & $7 / 17 / 1981$ & 81.5 & 343607 & 6787634 & $\checkmark$ & $\checkmark$ & $\square$ & $\nabla$ & $\square$ \\
\hline 1629 & HD025 & Hollow stem borehole w/SPT & $6 / 29 / 1982$ & 40.0 & 343590 & 6787556 & $\square$ & $\square$ & $\square$ & $\square$ & $\square$ \\
\hline 1630 & 1338 & Hollow stem borehole w/SPT & $8 / 19 / 1985$ & 61.5 & 344902 & 6787085 & $\checkmark$ & $\checkmark$ & $\checkmark$ & $\nabla$ & $\square$ \\
\hline 1630 & 1339 & Hollow stem borehole w/SPT & $10 / 14 / 1994$ & 51.5 & 345095 & 6787071 & $\checkmark$ & $\checkmark$ & $\checkmark$ & $\checkmark$ & $\square$ \\
\hline
\end{tabular}




\begin{tabular}{|c|c|c|c|c|c|c|c|c|c|c|c|}
\hline \multirow[b]{2}{*}{$\begin{array}{l}\text { MOA } \\
\text { grid }\end{array}$} & \multirow[b]{2}{*}{$\begin{array}{c}\text { DGGS } \\
\text { number }\end{array}$} & \multirow[b]{2}{*}{ Hole Type } & \multirow[b]{2}{*}{$\begin{array}{c}\text { Date } \\
\text { Completed }\end{array}$} & \multirow[b]{2}{*}{$\begin{array}{c}\text { Total } \\
\text { depth (ft) }\end{array}$} & \multicolumn{2}{|c|}{ UTM coordinates (m) } & \multicolumn{5}{|c|}{ Data entered } \\
\hline & & & & & East & North & Lithology & SPT & $\begin{array}{c}\text { Sample } \\
\text { test }\end{array}$ & $\begin{array}{c}\text { Water } \\
\text { level }\end{array}$ & Velocity \\
\hline 1630 & 1340 & Hollow stem borehole w/SPT & $10 / 12 / 1994$ & 51.5 & 345095 & 6787053 & $\boldsymbol{V}$ & $\nabla$ & $\boldsymbol{V}$ & $\checkmark$ & $\square$ \\
\hline 1630 & 4412 & Water well & $11 / 17 / 1964$ & 133.0 & 344623 & 6787462 & $\checkmark$ & $\square$ & $\square$ & $\sqrt{V}$ & $\square$ \\
\hline 1630 & 4415 & Water well & 9/30/1964 & 157.0 & 344917 & 6787696 & 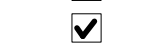 & $\square$ & $\square$ & $\square$ & $\square$ \\
\hline 1630 & 4416 & Water well & $4 / 18 / 1983$ & 154.0 & 344727 & 6787767 & $\checkmark$ & $\square$ & $\square$ & $\checkmark$ & $\square$ \\
\hline 1630 & HB001 & Solid stem borehole (no cores or SP & $5 / 30 / 1972$ & 20.0 & 345020 & 6787696 & $\square$ & $\square$ & $\square$ & $\square$ & $\square$ \\
\hline 1630 & HB002 & Solid stem borehole (no cores or SP & 8/17/1971 & 8.0 & 345033 & 6787717 & $\square$ & $\square$ & $\square$ & $\square$ & $\square$ \\
\hline 1630 & HB003 & Solid stem borehole (no cores or SP & $8 / 17 / 1971$ & 8.0 & 345071 & 6787692 & $\square$ & $\square$ & $\square$ & $\square$ & $\square$ \\
\hline 1630 & HB004 & Solid stem borehole (no cores or SP & $4 / 5 / 1972$ & 16.5 & 345101 & 6787685 & $\square$ & $\square$ & $\square$ & $\square$ & $\square$ \\
\hline 1630 & HB005 & Solid stem borehole (no cores or SP & 8/17/1971 & 23.0 & 345102 & 6787666 & $\square$ & $\square$ & $\square$ & $\square$ & $\square$ \\
\hline 1630 & HB006 & Solid stem borehole (no cores or SP & 8/17/1971 & 15.0 & 345140 & 6787700 & $\square$ & $\square$ & $\square$ & $\square$ & $\square$ \\
\hline 1630 & HB060 & Hollow stem borehole w/SPT & 4/7/1977 & 76.5 & 345113 & 6787746 & $\nabla$ & $\nabla$ & $\square$ & $\checkmark$ & $\square$ \\
\hline 1630 & HB061 & Hollow stem borehole w/SPT & $4 / 7 / 1977$ & 62.0 & 345075 & 6787776 & $\nabla$ & $\checkmark$ & $\square$ & $\checkmark$ & $\square$ \\
\hline 1630 & HB062 & Hollow stem borehole w/SPT & 4/7/1977 & 76.5 & 345071 & 6787726 & $\boldsymbol{V}$ & $\nabla$ & $\square$ & $\nabla$ & $\square$ \\
\hline 1630 & $\mathrm{HCOO3}$ & Solid stem borehole (no cores or SP & $8 / 11 / 1971$ & 13.0 & 344465 & 6787726 & $\square$ & $\square$ & $\square$ & $\square$ & $\square$ \\
\hline 1630 & HC004 & Solid stem borehole (no cores or SP & 8/11/1971 & 8.0 & 344504 & 6787744 & $\square$ & $\square$ & $\square$ & $\square$ & $\square$ \\
\hline 1630 & $\mathrm{HC} 005$ & Solid stem borehole (no cores or SP & $8 / 11 / 1971$ & 13.0 & 344542 & 6787716 & & $\square$ & $\square$ & $\square$ & $\square$ \\
\hline 1630 & HC006 & Solid stem borehole (no cores or SP & $8 / 11 / 1971$ & 13.0 & 344610 & 6787718 & & $\square$ & $\square$ & $\square$ & $\square$ \\
\hline 1630 & $\mathrm{HC} 007$ & Solid stem borehole (no cores or SP & $8 / 11 / 1971$ & 8.0 & 344655 & 6787719 & & $\square$ & $\square$ & $\square$ & $\square$ \\
\hline 1630 & HC008 & Solid stem borehole (no cores or SP & 8/16/1971 & 13.0 & 344709 & 6787740 & & $\square$ & $\square$ & $\square$ & $\square$ \\
\hline 1630 & HC009 & Solid stem borehole (no cores or SP & 8/16/1971 & 13.0 & 344752 & 6787742 & $\square$ & $\square$ & $\square$ & $\square$ & $\square$ \\
\hline 1630 & HC010 & Solid stem borehole (no cores or SP & $8 / 16 / 1971$ & 13.0 & 344864 & 6787702 & & $\square$ & $\square$ & $\square$ & $\square$ \\
\hline 1630 & HC011 & Solid stem borehole (no cores or SP & $8 / 16 / 1971$ & 13.0 & 344966 & 6787688 & & $\square$ & $\square$ & $\square$ & $\square$ \\
\hline 1630 & $\mathrm{HC} 024$ & Solid stem borehole (no cores or SP & $7 / 9 / 1958$ & 5.0 & 344428 & 6787836 & & $\square$ & $\square$ & $\square$ & $\square$ \\
\hline 1630 & $\mathrm{HC} 027$ & Solid stem borehole (no cores or SP & $7 / 9 / 1958$ & 6.0 & 344576 & 6787832 & $\square$ & $\square$ & $\square$ & $\square$ & $\square$ \\
\hline 1630 & $\mathrm{HC} 031$ & Solid stem borehole (no cores or SP & $7 / 9 / 1958$ & 6.0 & 344723 & 6787819 & $\square$ & $\square$ & $\square$ & $\square$ & $\square$ \\
\hline 1630 & $\mathrm{HC} 034$ & Solid stem borehole (no cores or SP & $7 / 9 / 1958$ & 5.2 & 344831 & 6787814 & $\square$ & $\square$ & $\square$ & $\square$ & $\square$ \\
\hline 1630 & HC036 & Solid stem borehole (no cores or SP & $7 / 9 / 1958$ & 5.0 & 344866 & 6787814 & $\square$ & $\square$ & $\square$ & $\square$ & $\square$ \\
\hline 1630 & HC062 & Hollow stem borehole w/SPT & $4 / 4 / 1984$ & 110.0 & 344924 & 6787756 & $\nabla$ & $\nabla$ & $\nabla$ & $\nabla$ & $\square$ \\
\hline 1630 & HC063 & Hollow stem borehole w/SPT & $3 / 6 / 1984$ & 31.5 & 344864 & 6787594 & $\square$ & $\square$ & $\square$ & $\square$ & $\square$ \\
\hline 1630 & HC064 & Hollow stem borehole w/SPT & $3 / 7 / 1984$ & 30.5 & 344901 & 6787596 & $\square$ & $\square$ & $\square$ & $\square$ & $\square$ \\
\hline 1630 & HC065 & Hollow stem borehole w/SPT & $2 / 28 / 1984$ & 31.5 & 344947 & 6787591 & $\square$ & $\square$ & $\square$ & $\square$ & $\square$ \\
\hline 1630 & HC066 & Hollow stem borehole w/SPT & $3 / 5 / 1984$ & 31.5 & 344883 & 6787568 & $\square$ & $\square$ & $\square$ & $\square$ & $\square$ \\
\hline 1630 & HC067 & Hollow stem borehole w/SPT & $2 / 29 / 1984$ & 61.5 & 344933 & 6787568 & $\nabla$ & $\bar{v}$ & $\nabla$ & 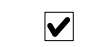 & $\square$ \\
\hline 1630 & HC068 & Hollow stem borehole w/SPT & $3 / 6 / 1984$ & 31.5 & 344862 & 6787552 & $\square$ & $\square$ & $\square$ & $\square$ & $\square$ \\
\hline 1630 & HC069 & Hollow stem borehole w/SPT & $4 / 10 / 1984$ & 31.5 & 344958 & 6787554 & $\square$ & $\square$ & $\square$ & $\square$ & $\square$ \\
\hline 1630 & HC070 & Hollow stem borehole w/SPT & $3 / 1 / 1984$ & 61.5 & 344937 & 6787534 & $\checkmark$ & $\checkmark$ & $\nabla$ & $\nabla$ & $\square$ \\
\hline
\end{tabular}




\begin{tabular}{|c|c|c|c|c|c|c|c|c|c|c|c|}
\hline \multirow[b]{2}{*}{$\begin{array}{c}\text { MOA } \\
\text { grid }\end{array}$} & \multirow[b]{2}{*}{$\begin{array}{l}\text { DGGS } \\
\text { number }\end{array}$} & \multirow[b]{2}{*}{ Hole Type } & \multirow[b]{2}{*}{$\begin{array}{c}\text { Date } \\
\text { Completed }\end{array}$} & \multirow[b]{2}{*}{$\begin{array}{c}\text { Total } \\
\text { depth (ft) }\end{array}$} & \multicolumn{2}{|c|}{ UTM coordinates (m) } & \multicolumn{5}{|c|}{ Data entered } \\
\hline & & & & & East & North & Lithology & SPT & $\begin{array}{c}\text { Sample } \\
\text { test }\end{array}$ & $\begin{array}{c}\text { Water } \\
\text { level }\end{array}$ & Velocity \\
\hline 1630 & HC071 & Hollow stem borehole w/SPT & $3 / 6 / 1984$ & 31.5 & 344858 & 6787526 & & $\square$ & $\square$ & $\square$ & $\square$ \\
\hline 1630 & HC072 & Hollow stem borehole w/SPT & $3 / 6 / 1984$ & 31.5 & 344899 & 6787524 & $\square$ & $\square$ & $\square$ & $\square$ & $\square$ \\
\hline 1630 & HC073 & Hollow stem borehole w/SPT & $3 / 1 / 1974$ & 51.5 & 344940 & 6787448 & $\boldsymbol{V}$ & $\checkmark$ & $\square$ & $\nabla$ & $\square$ \\
\hline 1631 & 1304 & Hollow stem borehole w/SPT & 8/20/1992 & 31.5 & 345215 & 6787526 & & $\square$ & $\square$ & $\square$ & $\square$ \\
\hline 1631 & 1305 & Hollow stem borehole w/SPT & 8/21/1992 & 31.5 & 345342 & 6787443 & $\square$ & $\square$ & $\square$ & $\square$ & $\square$ \\
\hline 1631 & 1306 & Hollow stem borehole w/SPT & $12 / 6 / 1994$ & 31.5 & 345753 & 6787007 & $\square$ & $\square$ & $\square$ & $\square$ & $\square$ \\
\hline 1631 & 1307 & Hollow stem borehole w/SPT & $12 / 7 / 1994$ & 29.8 & 345683 & 6787046 & $\square$ & $\square$ & $\square$ & $\square$ & $\square$ \\
\hline 1631 & 1308 & Hollow stem borehole w/SPT & $3 / 19 / 1985$ & 41.5 & 345628 & 6787755 & $\boldsymbol{V}$ & $\nabla$ & $\square$ & $\nabla$ & $\square$ \\
\hline 1631 & 1309 & Hollow stem borehole, no SPT & $3 / 19 / 1985$ & 41.5 & 345912 & 6787609 & 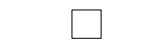 & $\square$ & $\square$ & $\square$ & $\square$ \\
\hline 1631 & 1342 & Hollow stem borehole w/SPT & $6 / 14 / 1975$ & 36.5 & 345314 & 6787696 & & $\square$ & $\square$ & $\square$ & $\square$ \\
\hline 1631 & 1343 & Hollow stem borehole w/SPT & $6 / 16 / 1975$ & 31.5 & 345237 & 6787764 & $\square$ & $\square$ & $\square$ & $\square$ & $\square$ \\
\hline 1631 & 1785 & Solid stem borehole (no cores or SP & $8 / 9 / 1982$ & 15.0 & 345142 & 6787098 & & $\square$ & $\square$ & $\square$ & $\square$ \\
\hline 1631 & 1787 & Solid stem borehole (no cores or SP & 8/9/1982 & 15.0 & 345155 & 6787382 & $\square$ & $\square$ & $\square$ & $\square$ & $\square$ \\
\hline 1631 & 1788 & Solid stem borehole (no cores or SP & $8 / 9 / 1982$ & 15.0 & 345163 & 6787533 & $\square$ & $\square$ & $\square$ & $\square$ & $\square$ \\
\hline 1631 & 1789 & Solid stem borehole (no cores or SP & $8 / 9 / 1982$ & 20.0 & 345169 & 6787723 & $\square$ & $\square$ & $\square$ & $\square$ & $\square$ \\
\hline 1631 & 4411 & Water well & $9 / 22 / 1966$ & 196.0 & 345548 & 6787421 & $\checkmark$ & $\square$ & $\square$ & $\square$ & $\square$ \\
\hline 1631 & HB007 & Solid stem borehole (no cores or SP & 8/17/1971 & 13.0 & 345199 & 6787667 & & L & L & $\square$ & $\square$ \\
\hline 1631 & HB008 & Solid stem borehole (no cores or SP & $4 / 3 / 1972$ & 16.5 & 345303 & 6787638 & & $\square$ & $\square$ & $\square$ & $\square$ \\
\hline 1631 & HB009 & Solid stem borehole (no cores or SP & $4 / 4 / 1972$ & 18.0 & 345346 & 6787646 & & $\square$ & $\square$ & $\square$ & $\square$ \\
\hline 1631 & HB010 & Solid stem borehole (no cores or SP & $4 / 4 / 1972$ & 14.0 & 345339 & 6787625 & & $\square$ & $\square$ & $\square$ & $\square$ \\
\hline 1631 & HB011 & Solid stem borehole (no cores or SP & $4 / 4 / 1972$ & 15.0 & 345330 & 6787596 & & $\square$ & $\square$ & $\square$ & $\square$ \\
\hline 1631 & HB012 & Solid stem borehole (no cores or SP & $4 / 3 / 1972$ & 16.0 & 345373 & 6787614 & & $\square$ & $\square$ & $\square$ & $\square$ \\
\hline 1631 & HB013 & Solid stem borehole (no cores or SP & $4 / 5 / 1972$ & 10.0 & 345405 & 6787646 & & $\square$ & $\square$ & $\square$ & $\square$ \\
\hline 1631 & HB014 & Solid stem borehole (no cores or SP & 8/17/1971 & 8.0 & 345406 & 6787631 & 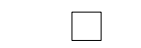 & $\square$ & $\square$ & $\square$ & $\square$ \\
\hline 1631 & HB015 & Solid stem borehole (no cores or SP & 4/5/1972 & 10.0 & 345392 & 6787584 & & $\square$ & $\square$ & $\square$ & $\square$ \\
\hline 1631 & HB016 & Solid stem borehole (no cores or SP & 8/17/1971 & 8.0 & 345423 & 6787578 & & $\square$ & $\square$ & $\square$ & $\square$ \\
\hline 1631 & HB017 & Solid stem borehole (no cores or SP & 8/17/1971 & 8.0 & 345442 & 6787582 & & $\square$ & $\square$ & $\square$ & $\square$ \\
\hline 1631 & HB018 & Solid stem borehole (no cores or SP & 8/17/1971 & 8.0 & 345481 & 6787606 & & $\square$ & $\square$ & $\square$ & $\square$ \\
\hline 1631 & HB019 & Solid stem borehole (no cores or SP & 8/18/1971 & 13.0 & 345549 & 6787577 & & $\square$ & $\square$ & $\square$ & $\square$ \\
\hline 1631 & HB020 & Solid stem borehole (no cores or SP & 8/18/1971 & 13.0 & 345646 & 6787574 & & $\square$ & $\square$ & $\square$ & $\square$ \\
\hline 1631 & HB021 & Solid stem borehole (no cores or SP & 8/18/1971 & 13.0 & 345729 & 6787554 & $\square$ & $\square$ & $\square$ & $\square$ & $\square$ \\
\hline 1631 & HB022 & Solid stem borehole (no cores or SP & 8/18/1971 & 13.0 & 345827 & 6787570 & $\square$ & $\square$ & $\square$ & $\square$ & $\square$ \\
\hline 1631 & HB023 & Solid stem borehole (no cores or SP & 8/18/1971 & 13.0 & 345870 & 6787568 & $\square$ & $\square$ & $\square$ & $\square$ & $\square$ \\
\hline 1631 & $\mathrm{HB} 024$ & Solid stem borehole (no cores or SP & 8/18/1971 & 13.0 & 345925 & 6787568 & $\square$ & $\square$ & $\square$ & $\square$ & $\square$ \\
\hline 1631 & HB035 & Solid stem borehole (no cores or SP & $7 / 9 / 1958$ & 7.5 & 345451 & 6787779 & $\square$ & $\square$ & $\square$ & $\square$ & $\square$ \\
\hline 1631 & HB040 & Solid stem borehole (no cores or SP & $7 / 10 / 1966$ & 5.0 & 345677 & 6787775 & $\square$ & $\square$ & $\square$ & $\square$ & $\square$ \\
\hline
\end{tabular}




\begin{tabular}{|c|c|c|c|c|c|c|c|c|c|c|c|}
\hline \multirow[b]{2}{*}{$\begin{array}{c}\text { MOA } \\
\text { grid }\end{array}$} & \multirow[b]{2}{*}{$\begin{array}{c}\text { DGGS } \\
\text { number }\end{array}$} & \multirow[b]{2}{*}{ Hole Type } & \multirow[b]{2}{*}{$\begin{array}{c}\text { Date } \\
\text { Completed }\end{array}$} & \multirow[b]{2}{*}{$\begin{array}{c}\text { Total } \\
\text { depth }(\mathrm{ft})\end{array}$} & \multicolumn{2}{|c|}{ UTM coordinates (m) } & \multicolumn{5}{|c|}{ Data entered } \\
\hline & & & & & East & North & Lithology & SPT & $\begin{array}{c}\text { Sample } \\
\text { test }\end{array}$ & $\begin{array}{c}\text { Water } \\
\text { level }\end{array}$ & Velocity \\
\hline 1631 & HB042 & Solid stem borehole (no cores or SP & $7 / 10 / 1958$ & 6.0 & 345730 & 6787773 & $\square$ & $\square$ & $\square$ & $\square$ & $\square$ \\
\hline 1631 & HB052 & Hollow stem borehole w/SPT & $1 / 1 / 1975$ & 21.5 & 345898 & 6787102 & $\square$ & $\square$ & $\square$ & $\square$ & $\square$ \\
\hline 1631 & HB053 & Hollow stem borehole w/SPT & $1 / 1 / 1975$ & 21.5 & 345889 & 6787078 & & $\square$ & $\square$ & $\square$ & $\square$ \\
\hline 1631 & HB054 & Hollow stem borehole w/SPT & $1 / 1 / 1975$ & 31.5 & 345906 & 6787080 & $\square$ & $\square$ & $\square$ & $\square$ & $\square$ \\
\hline 1631 & HB055 & Hollow stem borehole w/SPT & $1 / 1 / 1975$ & 21.5 & 345925 & 6787084 & & $\square$ & $\square$ & $\square$ & $\square$ \\
\hline 1631 & HB056 & Hollow stem borehole w/SPT & $1 / 1 / 1975$ & 21.5 & 345936 & 6787072 & $\square$ & $\square$ & $\square$ & $\square$ & $\square$ \\
\hline 1631 & HB057 & Hollow stem borehole w/SPT & $1 / 1 / 1975$ & 21.5 & 345894 & 6787064 & $\square$ & $\square$ & $\square$ & $\square$ & $\square$ \\
\hline 1631 & HB058 & Hollow stem borehole w/SPT & $1 / 1 / 1975$ & 21.5 & 345916 & 6787062 & $\square$ & $\square$ & $\square$ & $\square$ & $\square$ \\
\hline 1631 & HB059 & Hollow stem borehole w/SPT & $4 / 7 / 1977$ & 95.0 & 345073 & 6787750 & $\nabla$ & $\checkmark$ & $\square$ & $\checkmark$ & $\square$ \\
\hline 1631 & HB063 & Hollow stem borehole w/SPT & $7 / 30 / 1982$ & 40.5 & 345708 & 6787174 & & $\square$ & $\square$ & $\square$ & $\square$ \\
\hline 1632 & 1310 & Hollow stem borehole w/SPT & $6 / 1 / 1975$ & 31.5 & 346425 & 6787138 & $\square$ & $\square$ & $\square$ & $\square$ & $\square$ \\
\hline 1632 & HA001 & Solid stem borehole (no cores or SP & 8/19/1971 & 13.0 & 346155 & 6787546 & & $\square$ & $\square$ & $\square$ & $\square$ \\
\hline 1632 & HA002 & Solid stem borehole (no cores or SP & 8/19/1971 & 18.0 & 346264 & 6787542 & $\square$ & $\square$ & $\square$ & $\square$ & $\square$ \\
\hline 1632 & HA003 & Solid stem borehole (no cores or SP & $8 / 19 / 1971$ & 13.0 & 346308 & 6787580 & & $\square$ & $\square$ & $\square$ & $\square$ \\
\hline 1632 & HA004 & Solid stem borehole (no cores or SP & 8/19/1971 & 13.0 & 346340 & 6787597 & $\square$ & $\square$ & $\square$ & $\square$ & $\square$ \\
\hline 1632 & HA005 & Solid stem borehole (no cores or SP & 8/19/1971 & 13.0 & 346417 & 6787666 & $\square$ & $\square$ & $\square$ & $\square$ & $\square$ \\
\hline 1632 & HA012 & Hollow stem borehole w/SPT & $1 / 30 / 1982$ & 56.5 & 346226 & 6787414 & $\boldsymbol{V}$ & $\nabla$ & $\boldsymbol{V}$ & 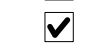 & $\square$ \\
\hline 1632 & HA013 & Hollow stem borehole w/SPT & $1 / 29 / 1982$ & 56.5 & 346230 & 6787480 & $\nabla$ & $\checkmark$ & $\nabla$ & $\nabla$ & $\square$ \\
\hline 1632 & HA014 & Hollow stem borehole w/SPT & $2 / 5 / 1982$ & 114.0 & 346198 & 6787447 & $\checkmark$ & $\checkmark$ & $\square$ & $\checkmark$ & $\square$ \\
\hline 1632 & HA015 & Hollow stem borehole w/SPT & $2 / 1 / 1982$ & 66.0 & 346158 & 6787423 & $\checkmark$ & $\checkmark$ & $\checkmark$ & $\checkmark$ & $\square$ \\
\hline 1632 & HA016 & Hollow stem borehole w/SPT & $2 / 2 / 1982$ & 66.5 & 346180 & 6787479 & $\checkmark$ & $\checkmark$ & $\square$ & $\checkmark$ & $\square$ \\
\hline 1632 & HA017 & Hollow stem borehole w/SPT & $2 / 6 / 1982$ & 70.7 & 346106 & 6787358 & $\checkmark$ & $\nabla$ & $\nabla$ & $\nabla$ & $\square$ \\
\hline 1632 & HA018 & Hollow stem borehole w/SPT & $2 / 9 / 1982$ & 80.5 & 346118 & 6787448 & 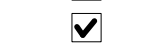 & $\checkmark$ & $\nabla$ & 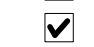 & $\square$ \\
\hline 1632 & НА019 & Hollow stem borehole w/SPT & $2 / 10 / 1982$ & 16.5 & 346313 & 6787451 & & $\square$ & $\square$ & $\square$ & $\square$ \\
\hline 1632 & HA020 & Hollow stem borehole w/SPT & $2 / 10 / 1982$ & 14.0 & 346301 & 6787273 & & $\square$ & $\square$ & $\square$ & $\square$ \\
\hline 1632 & HA021 & Hollow stem borehole w/SPT & $2 / 11 / 1982$ & 13.9 & 346305 & 6787162 & $\square$ & $\square$ & $\square$ & $\square$ & $\square$ \\
\hline 1632 & HA022 & Hollow stem borehole w/SPT & $2 / 11 / 1982$ & 14.0 & 346230 & 6787068 & $\square$ & $\square$ & $\square$ & $\square$ & $\square$ \\
\hline 1632 & HA023 & Hollow stem borehole w/SPT & 2/9/1982 & 16.5 & 346040 & 6787234 & $\square$ & $\square$ & $\square$ & $\square$ & $\square$ \\
\hline 1632 & HA024 & Hollow stem borehole w/SPT & 2/9/1982 & 30.0 & 346045 & 6787430 & $\square$ & $\square$ & $\square$ & $\square$ & $\square$ \\
\hline 1632 & HA025 & Hollow stem borehole w/SPT & $2 / 10 / 1982$ & 13.9 & 346194 & 6787150 & $\square$ & $\square$ & $\square$ & $\square$ & $\square$ \\
\hline 1632 & HA026 & Hollow stem borehole w/SPT & $2 / 10 / 1982$ & 14.0 & 346190 & 6787248 & $\square$ & $\square$ & $\square$ & $\square$ & $\square$ \\
\hline 1632 & HA027 & Hollow stem borehole w/SPT & $6 / 1 / 1976$ & 90.0 & 346273 & 6787020 & 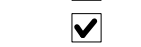 & $\checkmark$ & $\square$ & $\sqrt{v}$ & $\square$ \\
\hline 1632 & HB025 & Solid stem borehole (no cores or SP & 8/19/1971 & 13.0 & 345993 & 6787552 & $\square$ & $\square$ & $\square$ & $\square$ & $\square$ \\
\hline 1633 & GZ007 & Solid stem borehole (no cores or SP & 2/3/1976 & 10.5 & 347553 & 6787015 & $\square$ & $\square$ & $\square$ & $\square$ & $\square$ \\
\hline 1633 & GZ008 & Solid stem borehole (no cores or SP & 2/4/1976 & 10.5 & 347549 & 6787138 & $\square$ & $\square$ & $\square$ & $\square$ & $\square$ \\
\hline 1634 & 1311 & Hollow stem borehole w/SPT & 8/26/1991 & 51.0 & 348178 & 6787025 & $\boldsymbol{V}$ & $\boldsymbol{V}$ & $\square$ & $\checkmark$ & $\square$ \\
\hline
\end{tabular}




\begin{tabular}{|c|c|c|c|c|c|c|c|c|c|c|c|}
\hline \multirow[b]{2}{*}{$\begin{array}{l}\text { MOA } \\
\text { grid }\end{array}$} & \multirow[b]{2}{*}{$\begin{array}{c}\text { DGGS } \\
\text { number }\end{array}$} & \multirow[b]{2}{*}{ Hole Type } & \multirow[b]{2}{*}{$\begin{array}{c}\text { Date } \\
\text { Completed }\end{array}$} & \multirow[b]{2}{*}{$\begin{array}{c}\text { Total } \\
\text { depth (ft) }\end{array}$} & \multicolumn{2}{|c|}{ UTM coordinates (m) } & \multicolumn{5}{|c|}{ Data entered } \\
\hline & & & & & East & North & Lithology & SPT & $\begin{array}{l}\text { Sample } \\
\text { test }\end{array}$ & $\begin{array}{l}\text { Water } \\
\text { level }\end{array}$ & Velocity \\
\hline 1634 & 1312 & Hollow stem borehole w/SPT & 8/27/1981 & 50.5 & 348205 & 6787033 & $\boldsymbol{V}$ & $\boldsymbol{V}$ & $\nabla$ & $\checkmark$ & $\square$ \\
\hline 1634 & 1313 & Hollow stem borehole w/SPT & 9/4/1981 & 50.5 & 348186 & 6787048 & $\boldsymbol{V}$ & $\checkmark$ & $\nabla$ & $\checkmark$ & $\square$ \\
\hline 1634 & 1314 & Hollow stem borehole w/SPT & 9/1/1981 & 51.0 & 348166 & 6787066 & 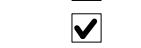 & $\checkmark$ & $\checkmark$ & $\checkmark$ & $\square$ \\
\hline 1634 & 1315 & Hollow stem borehole w/SPT & $8 / 31 / 1981$ & 50.5 & 348195 & 6787069 & $\nabla$ & $\nabla$ & $\square$ & $\nabla$ & $\square$ \\
\hline 1634 & 1316 & Hollow stem borehole w/SPT & $1 / 17 / 1975$ & 28.5 & 348172 & 6787113 & $\square$ & $\square$ & $\square$ & $\square$ & $\square$ \\
\hline 1634 & 1317 & Hollow stem borehole w/SPT & $1 / 16 / 1975$ & 37.5 & 348072 & 6787072 & $\boldsymbol{V}$ & $\checkmark$ & $\square$ & $\checkmark$ & $\square$ \\
\hline 1634 & 1318 & Hollow stem borehole w/SPT & $1 / 3 / 1975$ & 34.5 & 348027 & 6787113 & $\square$ & $\square$ & $\square$ & $\square$ & $\square$ \\
\hline 1634 & 1319 & Hollow stem borehole w/SPT & $1 / 7 / 1975$ & 49.5 & 348245 & 6787123 & 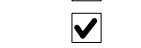 & $\checkmark$ & $\square$ & $\square$ & $\square$ \\
\hline 1634 & 2360 & Hollow stem borehole w/SPT & $8 / 21 / 2000$ & 96.5 & 347667 & 6787522 & $\checkmark$ & $\checkmark$ & $\checkmark$ & $\checkmark$ & $\square$ \\
\hline 1634 & 4409 & Water well & $5 / 3 / 1973$ & 211.0 & 347682 & 6786987 & $\boldsymbol{V}$ & $\square$ & $\square$ & $\checkmark$ & $\square$ \\
\hline 1634 & GK005 & Hollow stem borehole w/SPT & 9/18/1981 & 60.0 & 347984 & 6786960 & $\nabla$ & $\nabla$ & $\nabla$ & $\checkmark$ & $\square$ \\
\hline 1634 & GK012 & Solid stem borehole (no cores or SP & 2/3/1976 & 10.5 & 347550 & 6786932 & $\square$ & $\square$ & $\square$ & $\square$ & $\square$ \\
\hline 1634 & GZ009 & Solid stem borehole (no cores or SP & 2/4/1976 & 10.5 & 347571 & 6787361 & $\square$ & $\square$ & $\square$ & $\square$ & $\square$ \\
\hline 1634 & GZ010 & Solid stem borehole (no cores or SP & 2/4/1976 & 10.5 & 347582 & 6787682 & $\square$ & $\square$ & $\square$ & $\square$ & $\square$ \\
\hline 1634 & GZ011 & Solid stem borehole (no cores or SP & 2/4/1976 & 10.6 & 347873 & 6787234 & $\square$ & $\square$ & $\square$ & $\square$ & $\square$ \\
\hline 1634 & GZ012 & Cone Penetration Test (CPT) & $3 / 28 / 1986$ & 6.0 & 347626 & 6787451 & $\square$ & $\square$ & $\square$ & $\square$ & $\square$ \\
\hline 1635 & 1320 & Hollow stem borehole w/SPT & 10/6/1992 & 75.5 & 348568 & 6786904 & $\checkmark$ & $\checkmark$ & $\nabla$ & $\sqrt{V}$ & $\square$ \\
\hline 1635 & 1321 & Hollow stem borehole w/SPT & $9 / 21 / 1992$ & 76.0 & 348607 & 6786904 & $\checkmark$ & $\checkmark$ & $\checkmark$ & $\checkmark$ & $\square$ \\
\hline 1635 & 2025 & Water well & 8/1/1994 & 553.5 & 348667 & 6786962 & $\nabla$ & $\square$ & $\nabla$ & $\nabla$ & $\square$ \\
\hline 1635 & GL004 & Hollow stem borehole w/SPT & $8 / 26 / 1983$ & 35.5 & 348611 & 6786874 & $\square$ & $\square$ & $\square$ & $\square$ & $\square$ \\
\hline 1635 & GL005 & Hollow stem borehole w/SPT & $7 / 11 / 1984$ & 31.0 & 348545 & 6786880 & $\square$ & $\square$ & $\square$ & $\square$ & $\square$ \\
\hline 1635 & GL006 & Hollow stem borehole w/SPT & $7 / 11 / 1984$ & 30.7 & 348512 & 6786856 & $\square$ & $\square$ & $\square$ & $\square$ & $\square$ \\
\hline 1635 & GY002 & Hollow stem borehole w/SPT & $4 / 26 / 1983$ & 50.9 & 348924 & 6787402 & $\checkmark$ & $\checkmark$ & $\square$ & $\checkmark$ & $\square$ \\
\hline 1635 & GY003 & Hollow stem borehole w/SPT & $4 / 20 / 1983$ & 50.9 & 348963 & 6787312 & $\boldsymbol{V}$ & $\checkmark$ & $\square$ & $\checkmark$ & $\square$ \\
\hline 1635 & GY004 & Hollow stem borehole w/SPT & $4 / 22 / 1983$ & 50.7 & 348975 & 6787431 & $\nabla$ & $\checkmark$ & $\square$ & $\nabla$ & $\square$ \\
\hline 1636 & 4455 & Water well & $3 / 1 / 1978$ & 300.0 & 349399 & 6786913 & 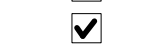 & $\square$ & $\square$ & $\square$ & $\square$ \\
\hline 1636 & GM001 & Hollow stem borehole w/SPT & $3 / 17 / 1978$ & 300.0 & 349411 & 6786846 & $\boldsymbol{V}$ & $\square$ & $\square$ & $\square$ & $\square$ \\
\hline 1636 & GM002 & Hollow stem borehole w/SPT & 9/6/1983 & 51.0 & 349397 & 6786928 & $\nabla$ & $\nabla$ & $\nabla$ & $\nabla$ & $\square$ \\
\hline 1637 & 1247 & Hollow stem borehole w/SPT & $2 / 28 / 1985$ & 36.5 & 350709 & 6786783 & $\square$ & $\square$ & $\square$ & $\square$ & $\square$ \\
\hline 1637 & 1322 & Hollow stem borehole w/SPT & $3 / 26 / 1974$ & 30.0 & 350310 & 6786790 & 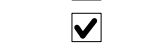 & $\square$ & $\square$ & $\checkmark$ & $\square$ \\
\hline 1637 & 1323 & Hollow stem borehole w/SPT & $3 / 27 / 1974$ & 30.0 & 350231 & 6786808 & $\square$ & $\square$ & $\square$ & $\square$ & $\square$ \\
\hline 1638 & 1248 & Hollow stem borehole w/SPT & $2 / 22 / 1985$ & 31.5 & 350785 & 6786899 & $\square$ & $\square$ & $\square$ & $\square$ & $\square$ \\
\hline 1638 & 1324 & Hollow stem borehole w/SPT & 9/12/1977 & 39.5 & 350837 & 6787133 & $\square$ & $\square$ & $\square$ & $\square$ & $\square$ \\
\hline 1638 & 1325 & Hollow stem borehole w/SPT & 9/13/1977 & 35.0 & 350861 & 6787103 & $\square$ & $\square$ & $\square$ & $\square$ & $\square$ \\
\hline 1638 & 4410 & Water well & $7 / 16 / 1970$ & 242.0 & 351019 & 6787029 & $\checkmark$ & $\square$ & $\square$ & $\checkmark$ & $\square$ \\
\hline 1639 & 1326 & Hollow stem borehole w/SPT & $5 / 13 / 1994$ & 41.5 & 352164 & 6786825 & $\checkmark$ & $\nabla$ & $\nabla$ & $\nabla$ & $\square$ \\
\hline
\end{tabular}




\begin{tabular}{|c|c|c|c|c|c|c|c|c|c|c|c|}
\hline \multirow[b]{2}{*}{$\begin{array}{l}\text { MOA } \\
\text { grid }\end{array}$} & \multirow[b]{2}{*}{$\begin{array}{l}\text { DGGS } \\
\text { number }\end{array}$} & \multirow[b]{2}{*}{ Hole Type } & \multirow[b]{2}{*}{$\begin{array}{c}\text { Date } \\
\text { Completed }\end{array}$} & \multirow[b]{2}{*}{$\begin{array}{c}\text { Total } \\
\text { depth }(\mathrm{ft})\end{array}$} & \multicolumn{2}{|c|}{ UTM coordinates (m) } & \multicolumn{5}{|c|}{ Data entered } \\
\hline & & & & & East & North & Lithology & SPT & $\begin{array}{c}\text { Sample } \\
\text { test }\end{array}$ & $\begin{array}{l}\text { Water } \\
\text { level }\end{array}$ & Velocity \\
\hline 1639 & 1327 & Hollow stem borehole w/SPT & $5 / 13 / 1994$ & 41.5 & 352148 & 6786779 & $\square$ & & $\square$ & $\square$ & \\
\hline 1639 & 1328 & Hollow stem borehole w/SPT & $1 / 29 / 1995$ & 36.5 & 352302 & 6786928 & $\square$ & $\square$ & $\square$ & $\square$ & $\square$ \\
\hline 1639 & 1329 & Hollow stem borehole w/SPT & $1 / 29 / 1995$ & 36.5 & 352337 & 6786915 & $\square$ & & $\square$ & $\square$ & \\
\hline 1639 & 1330 & Hollow stem borehole w/SPT & $1 / 29 / 1995$ & 36.5 & 352369 & 6786920 & $\square$ & $\square$ & $\square$ & $\square$ & $\square$ \\
\hline 1639 & 1331 & Hollow stem borehole w/SPT & $7 / 27 / 1983$ & 31.5 & 351739 & 6787491 & $\checkmark$ & $\checkmark$ & $\square$ & $\nabla$ & $\square$ \\
\hline 1639 & 1384 & Hollow stem borehole w/SPT & $12 / 13 / 1975$ & 30.0 & 352145 & 6786715 & $\nabla$ & $\checkmark$ & $\checkmark$ & $\checkmark$ & $\square$ \\
\hline 1639 & 1385 & Hollow stem borehole w/SPT & $11 / 20 / 1975$ & 30.0 & 352173 & 6786748 & $\square$ & $\square$ & $\square$ & $\square$ & $\square$ \\
\hline 1639 & 1386 & Hollow stem borehole w/SPT & $11 / 25 / 1975$ & 41.5 & 352296 & 6786718 & $\checkmark$ & $\checkmark$ & $\checkmark$ & $\nabla$ & $\square$ \\
\hline 1639 & 1387 & Hollow stem borehole w/SPT & $11 / 28 / 1975$ & 41.0 & 352328 & 6786754 & $\square$ & ᄂ & $\square$ & $\square$ & $\square$ \\
\hline 1640 & 1332 & Hollow stem borehole w/SPT & $4 / 26 / 1984$ & 31.5 & 353109 & 6787136 & $\square$ & & $\square$ & $\square$ & $\square$ \\
\hline 1641 & 1294 & Hollow stem borehole w/SPT & $8 / 16 / 1994$ & 31.4 & 353203 & 6787067 & $\square$ & $\sqcup$ & $\square$ & $\square$ & $\square$ \\
\hline 1641 & 1333 & Hollow stem borehole w/SPT & 8/1/1977 & 41.5 & 353798 & 6786912 & $\square$ & & $\square$ & $\square$ & $\square$ \\
\hline 1641 & 1334 & Hollow stem borehole w/SPT & 8/3/1977 & 41.5 & 353775 & 6786792 & $\square$ & & $\square$ & $\square$ & $\square$ \\
\hline 1641 & 1335 & Hollow stem borehole w/SPT & 8/5/1977 & 38.0 & 353770 & 6786701 & $\square$ & $\square$ & $\square$ & $\square$ & $\square$ \\
\hline 1641 & 1391 & Hollow stem borehole, no SPT & $2 / 5 / 1975$ & 20.0 & 353211 & 6786789 & $\square$ & & $\square$ & $\square$ & $\square$ \\
\hline 1641 & 1392 & Hollow stem borehole, no SPT & $6 / 22 / 1971$ & 15.0 & 353216 & 6787260 & $\square$ & & $\square$ & $\square$ & $\square$ \\
\hline 1641 & 1610 & Hollow stem borehole w/SPT & $5 / 29 / 1996$ & 36.0 & 353521 & 6787344 & $\square$ & & $\square$ & $\square$ & $\square$ \\
\hline 1722 & GB001 & Solid stem borehole (no cores or SP & $7 / 10 / 1968$ & 23.0 & 338691 & 6786982 & $\square$ & & $\square$ & $\square$ & $\square$ \\
\hline 1722 & GB004 & Solid stem borehole (no cores or SP & 2/7/1973 & 40.0 & 338375 & 6786650 & $\square$ & & $\square$ & $\square$ & $\square$ \\
\hline 1722 & GB006 & Solid stem borehole (no cores or SP & 2/7/1973 & 30.0 & 338348 & 6786774 & $\square$ & $\checkmark$ & $\square$ & $\square$ & $\square$ \\
\hline 1722 & GB007 & Solid stem borehole (no cores or SP & 2/7/1973 & 30.0 & 338312 & 6786918 & $\square$ & & $\square$ & $\square$ & \\
\hline 1722 & GB010 & Solid stem borehole (no cores or SP & $2 / 23 / 1973$ & 30.0 & 338668 & 6786637 & $\square$ & & $\square$ & $\square$ & $\square$ \\
\hline 1722 & GB011 & Solid stem borehole (no cores or SP & $2 / 23 / 1973$ & 25.0 & 338593 & 6786890 & $\square$ & & $\square$ & $\square$ & $\square$ \\
\hline 1722 & GB039 & Solid stem borehole (no cores or SP & $11 / 8 / 1977$ & 10.0 & 338154 & 6786598 & $\square$ & $\square$ & $\square$ & $\square$ & $\square$ \\
\hline 1722 & GB040 & Solid stem borehole (no cores or SP & $11 / 8 / 1977$ & 15.0 & 338161 & 6786724 & $\square$ & & $\square$ & $\square$ & $\square$ \\
\hline 1722 & GB041 & Solid stem borehole (no cores or SP & $11 / 8 / 1977$ & 10.0 & 338147 & 6786946 & $\square$ & & $\square$ & $\square$ & \\
\hline 1722 & HIO03 & Solid stem borehole (no cores or SP & $7 / 12 / 1968$ & 28.0 & 338578 & 6787276 & $\square$ & $\square$ & $\square$ & $\square$ & $\square$ \\
\hline 1722 & HIO04 & Solid stem borehole (no cores or SP & $7 / 12 / 1968$ & 23.5 & 338632 & 6787121 & $\square$ & $\square$ & $\square$ & $\square$ & $\square$ \\
\hline 1722 & HI022 & Hollow stem borehole w/SPT & $3 / 9 / 1973$ & 61.5 & 338360 & 6787298 & $\checkmark$ & $\checkmark$ & $\checkmark$ & $\checkmark$ & $\square$ \\
\hline 1722 & $\mathrm{HI} 023$ & Solid stem borehole (no cores or SP & $1 / 31 / 1973$ & 40.0 & 338471 & 6787050 & $\square$ & & $\square$ & $\square$ & $\square$ \\
\hline 1722 & HI024 & Solid stem borehole (no cores or SP & 2/7/1973 & 25.0 & 338267 & 6787080 & $\square$ & $\square$ & $\square$ & $\square$ & $\square$ \\
\hline 1722 & HIO25 & Solid stem borehole (no cores or SP & 2/7/1973 & 30.0 & 338239 & 6787236 & $\square$ & $\square$ & $\square$ & $\square$ & $\square$ \\
\hline 1722 & HI035 & Solid stem borehole (no cores or SP & $11 / 8 / 1977$ & 10.0 & 338131 & 6787039 & $\square$ & $\square$ & $\square$ & $\square$ & $\square$ \\
\hline 1722 & HIO36 & Solid stem borehole (no cores or SP & $11 / 9 / 1977$ & 15.0 & 338130 & 6787097 & $\square$ & $\square$ & $\square$ & $\square$ & $\square$ \\
\hline 1722 & HI037 & Solid stem borehole (no cores or SP & $11 / 9 / 1977$ & 15.0 & 338120 & 6787190 & $\square$ & $\square$ & $\square$ & $\square$ & $\square$ \\
\hline 1722 & HI038 & Solid stem borehole (no cores or SP & $11 / 9 / 1977$ & 20.0 & 338105 & 6787257 & $\square$ & $\square$ & $\square$ & $\square$ & $\square$ \\
\hline
\end{tabular}




\begin{tabular}{|c|c|c|c|c|c|c|c|c|c|c|c|}
\hline \multirow[b]{2}{*}{$\begin{array}{l}\text { MOA } \\
\text { grid }\end{array}$} & \multirow[b]{2}{*}{$\begin{array}{l}\text { DGGS } \\
\text { number }\end{array}$} & \multirow[b]{2}{*}{ Hole Type } & \multirow[b]{2}{*}{$\begin{array}{c}\text { Date } \\
\text { Completed }\end{array}$} & \multirow[b]{2}{*}{$\begin{array}{c}\text { Total } \\
\text { depth }(\mathrm{ft})\end{array}$} & \multicolumn{2}{|c|}{ UTM coordinates (m) } & \multicolumn{5}{|c|}{ Data entered } \\
\hline & & & & & East & North & Lithology & SPT & $\begin{array}{c}\text { Sample } \\
\text { test }\end{array}$ & $\begin{array}{l}\text { Water } \\
\text { level }\end{array}$ & Velocity \\
\hline 1722 & HI039 & Solid stem borehole (no cores or SP & $11 / 9 / 1977$ & 15.0 & 338099 & 6787318 & $\square$ & & $\square$ & $\square$ & $\square$ \\
\hline 1723 & 2057 & Solid stem borehole (no cores or SP & $4 / 2 / 1984$ & 20.0 & 339403 & 6787131 & $\checkmark$ & L & $\square$ & $\checkmark$ & $\square$ \\
\hline 1723 & 2062 & Hollow stem borehole, no SPT & $8 / 11 / 1982$ & 20.0 & 339431 & 6787025 & $\checkmark$ & & $\nabla$ & $\bar{\nabla}$ & \\
\hline 1723 & 2063 & Hollow stem borehole, no SPT & $8 / 12 / 1982$ & 20.0 & 339414 & 6787145 & $\checkmark$ & 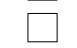 & $\nabla$ & $\boldsymbol{V}$ & $\square$ \\
\hline 1723 & GB002 & Solid stem borehole (no cores or SP & $7 / 10 / 1968$ & 13.0 & 338770 & 6786844 & $\square$ & $\square$ & $\square$ & $\square$ & $\square$ \\
\hline 1723 & GB003 & Solid stem borehole (no cores or SP & $7 / 2 / 1968$ & 23.0 & 338873 & 6786977 & $\square$ & $\square$ & $\square$ & $\square$ & $\square$ \\
\hline 1723 & GB008 & Solid stem borehole (no cores or SP & $2 / 20 / 1973$ & 20.0 & 338754 & 6786749 & $\square$ & $\square$ & $\square$ & $\square$ & $\square$ \\
\hline 1723 & GB025 & Hollow stem borehole w/SPT & $12 / 21 / 1966$ & 21.5 & 338910 & 6786823 & $\square$ & & $\square$ & $\square$ & $\square$ \\
\hline 1723 & GB026 & Hollow stem borehole w/SPT & $8 / 14 / 1967$ & 14.0 & 338943 & 6786731 & $\square$ & 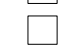 & $\square$ & $\square$ & $\square$ \\
\hline 1723 & GB027 & Hollow stem borehole w/SPT & $8 / 29 / 1967$ & 14.0 & 338915 & 6786570 & $\square$ & & $\square$ & $\square$ & \\
\hline 1723 & GC017 & Solid stem borehole (no cores or SP & $7 / 10 / 1968$ & 9.0 & 338999 & 6786966 & $\square$ & $\square$ & $\square$ & $\square$ & $\square$ \\
\hline 1723 & GC018 & Hollow stem borehole w/SPT & $8 / 24 / 1967$ & 45.0 & 339019 & 6786780 & $\checkmark$ & $\square$ & $\square$ & $\bar{\nabla}$ & $\square$ \\
\hline 1723 & GC019 & Hollow stem borehole w/SPT & $8 / 22 / 1967$ & 45.0 & 339083 & 6786624 & $\checkmark$ & $\checkmark$ & $\square$ & $\checkmark$ & $\square$ \\
\hline 1723 & HH003 & Solid stem borehole (no cores or SP & $9 / 4 / 1968$ & 20.0 & 339465 & 6787214 & $\square$ & $\square$ & $\square$ & $\square$ & $\square$ \\
\hline 1723 & HH022 & Solid stem borehole (no cores or SP & $4 / 14 / 1972$ & 20.0 & 339187 & 6787249 & $\square$ & 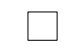 & $\square$ & $\square$ & $\square$ \\
\hline 1723 & HI005 & Solid stem borehole (no cores or SP & $7 / 2 / 1968$ & 18.0 & 338789 & 6787108 & $\square$ & & $\square$ & $\square$ & $\square$ \\
\hline 1723 & HI006 & Solid stem borehole (no cores or SP & $7 / 2 / 1968$ & 13.0 & 338749 & 6787232 & $\square$ & 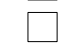 & $\square$ & $\square$ & $\square$ \\
\hline 1723 & HI012 & Solid stem borehole (no cores or SP & $7 / 10 / 1968$ & 8.0 & 338884 & 6787204 & $\square$ & - & $\square$ & $\square$ & $\square$ \\
\hline 1723 & HI013 & Solid stem borehole (no cores or SP & $7 / 10 / 1968$ & 9.0 & 338932 & 6787084 & $\square$ & $\square$ & $\square$ & $\square$ & $\square$ \\
\hline 1724 & 2127 & Hollow stem borehole w/SPT & 6/6/1994 & 31.5 & 339554 & 6786897 & $\checkmark$ & $\nabla$ & $\checkmark$ & $\checkmark$ & $\square$ \\
\hline 1724 & 2142 & Hollow stem borehole w/SPT & 3/7/1995 & 41.5 & 339584 & 6786967 & $\checkmark$ & $\checkmark$ & $\bar{\nabla}$ & $\bar{\nabla}$ & \\
\hline 1724 & 2143 & Hollow stem borehole w/SPT & 3/7/1995 & 41.5 & 339620 & 6786968 & $\checkmark$ & $\checkmark$ & $\checkmark$ & $\checkmark$ & $\square$ \\
\hline 1724 & 2144 & Hollow stem borehole w/SPT & 3/8/1995 & 41.5 & 339626 & 6786897 & $\checkmark$ & $\checkmark$ & $\nabla$ & $\nabla$ & $\square$ \\
\hline 1724 & GC010 & Solid stem borehole (no cores or SP & 9/4/1968 & 18.0 & 339730 & 6786443 & $\square$ & 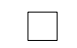 & $\square$ & $\square$ & $\square$ \\
\hline 1724 & GC011 & Solid stem borehole (no cores or SP & 9/6/1968 & 20.0 & 339630 & 6786469 & $\checkmark$ & & $\square$ & 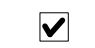 & $\square$ \\
\hline 1724 & GC012 & Solid stem borehole (no cores or SP & 9/4/1968 & 18.0 & 339722 & 6786590 & $\square$ & & $\square$ & $\square$ & $\square$ \\
\hline 1724 & GC013 & Solid stem borehole (no cores or SP & 9/6/1968 & 20.0 & 339567 & 6786662 & $\square$ & $\square$ & $\square$ & $\square$ & $\square$ \\
\hline 1724 & GC014 & Solid stem borehole (no cores or SP & 9/4/1968 & 15.0 & 339614 & 6786706 & $\square$ & & $\square$ & $\square$ & $\square$ \\
\hline 1724 & GC015 & Solid stem borehole (no cores or SP & 9/4/1968 & 23.0 & 339601 & 6786852 & $\checkmark$ & & $\square$ & $\checkmark$ & $\square$ \\
\hline 1724 & GC016 & Solid stem borehole (no cores or SP & 9/4/1968 & 18.0 & 339584 & 6786981 & $\square$ & & $\square$ & $\square$ & $\square$ \\
\hline 1724 & HH001 & Solid stem borehole (no cores or SP & 9/6/1968 & 20.0 & 339622 & 6787060 & $\square$ & $\square$ & $\square$ & $\square$ & $\square$ \\
\hline 1724 & HH002 & Solid stem borehole (no cores or SP & $9 / 4 / 1968$ & 18.0 & 339521 & 6787094 & $\square$ & $\square$ & $\square$ & $\square$ & $\square$ \\
\hline 1725 & HG002 & Solid stem borehole (no cores or SP & $9 / 23 / 1968$ & 23.0 & 340357 & 6787211 & $\checkmark$ & $\square$ & $\square$ & $\bar{\square}$ & $\square$ \\
\hline 1726 & 2314 & Hollow stem borehole w/SPT & $2 / 5 / 1969$ & 51.5 & 341902 & 6786746 & $\checkmark$ & $\checkmark$ & $\checkmark$ & $\bar{\nabla}$ & $\square$ \\
\hline 1726 & 2330 & Hollow stem borehole w/SPT & $2 / 21 / 1969$ & 31.5 & 341920 & 6787119 & $\square$ & $\square$ & $\square$ & $\square$ & $\square$ \\
\hline 1726 & 2331 & Hollow stem borehole w/SPT & $2 / 22 / 1969$ & 31.5 & 341916 & 6787012 & $\square$ & $\square$ & $\square$ & $\square$ & $\square$ \\
\hline
\end{tabular}




\begin{tabular}{|c|c|c|c|c|c|c|c|c|c|c|c|}
\hline \multirow[b]{2}{*}{$\begin{array}{c}\text { MOA } \\
\text { grid }\end{array}$} & \multirow[b]{2}{*}{$\begin{array}{c}\text { DGGS } \\
\text { number }\end{array}$} & \multirow[b]{2}{*}{ Hole Type } & \multirow[b]{2}{*}{$\begin{array}{c}\text { Date } \\
\text { Completed }\end{array}$} & \multirow[b]{2}{*}{$\begin{array}{c}\text { Total } \\
\text { depth }(\mathrm{ft})\end{array}$} & \multicolumn{2}{|c|}{ UTM coordinates (m) } & \multicolumn{5}{|c|}{ Data entered } \\
\hline & & & & & East & North & Lithology & SPT & $\begin{array}{c}\text { Sample } \\
\text { test }\end{array}$ & $\begin{array}{l}\text { Water } \\
\text { level }\end{array}$ & Velocity \\
\hline 1726 & 2332 & Hollow stem borehole w/SPT & $2 / 22 / 1969$ & 31.5 & 341909 & 6786891 & $\square$ & $\square$ & $\square$ & $\square$ & $\square$ \\
\hline 1726 & 2333 & Hollow stem borehole w/SPT & $2 / 22 / 1969$ & 31.5 & 341897 & 6786632 & $\square$ & $\square$ & $\square$ & $\square$ & $\square$ \\
\hline 1726 & 2334 & Hollow stem borehole w/SPT & $2 / 24 / 1969$ & 31.5 & 341892 & 6786525 & $\square$ & $\square$ & $\square$ & $\square$ & $\square$ \\
\hline 1726 & 2335 & Hollow stem borehole w/SPT & $2 / 24 / 1969$ & 31.5 & 341887 & 6786403 & $\square$ & $\square$ & $\square$ & $\square$ & $\square$ \\
\hline 1727 & 1807 & Solid stem borehole (no cores or SP & $10 / 23 / 1981$ & 15.0 & 342304 & 6786416 & $\square$ & $\square$ & $\square$ & $\square$ & $\square$ \\
\hline 1727 & 1808 & Solid stem borehole (no cores or SP & $10 / 5 / 1981$ & 10.0 & 342598 & 6786499 & $\square$ & $\square$ & $\square$ & $\square$ & $\square$ \\
\hline 1727 & 4407 & Water well & $2 / 28 / 1966$ & 107.0 & 342365 & 6786787 & $\checkmark$ & $\square$ & $\square$ & $\checkmark$ & $\square$ \\
\hline 1728 & 1809 & Solid stem borehole (no cores or SP & $10 / 5 / 1981$ & 10.0 & 342848 & 6786696 & $\square$ & $\square$ & $\square$ & $\square$ & $\square$ \\
\hline 1728 & 1810 & Solid stem borehole (no cores or SP & $12 / 11 / 1978$ & 10.0 & 342949 & 6786776 & $\square$ & $\square$ & $\square$ & $\square$ & $\square$ \\
\hline 1729 & 3024 & Water well & $3 / 27 / 1974$ & 139.0 & 343876 & 6786782 & $\checkmark$ & $\square$ & $\square$ & $\checkmark$ & $\square$ \\
\hline 1729 & 4405 & Water well & $2 / 2 / 1965$ & 125.0 & 344110 & 6786678 & $\checkmark$ & $\square$ & $\square$ & $\checkmark$ & $\square$ \\
\hline 1729 & GH001 & Hollow stem borehole w/SPT & $3 / 26 / 1971$ & 24.5 & 344031 & 6786262 & $\square$ & $\square$ & $\square$ & $\square$ & $\square$ \\
\hline 1730 & 1336 & Hollow stem borehole w/SPT & $5 / 13 / 1992$ & 41.5 & 344823 & 6786407 & $\square$ & $\square$ & $\square$ & $\square$ & $\square$ \\
\hline 1730 & 1337 & Hollow stem borehole w/SPT & $1 / 21 / 1983$ & 36.5 & 344763 & 6786288 & $\square$ & $\square$ & $\square$ & $\square$ & $\square$ \\
\hline 1730 & 1784 & Solid stem borehole (no cores or SP & 8/5/1982 & 20.0 & 344989 & 6786712 & $\square$ & $\square$ & $\square$ & $\square$ & $\square$ \\
\hline 1730 & 2176 & Hollow stem borehole w/SPT & 9/8/1999 & 46.5 & 344307 & 6786240 & $\checkmark$ & $\checkmark$ & $\square$ & $\checkmark$ & $\square$ \\
\hline 1730 & 4456 & Water well & $5 / 1 / 1977$ & 305.0 & 344940 & 6786518 & $\checkmark$ & $\square$ & $\square$ & $\checkmark$ & $\square$ \\
\hline 1730 & 4457 & Water well & $5 / 1 / 1979$ & 312.0 & 344939 & 6786487 & $\nabla$ & $\square$ & $\square$ & $\nabla$ & $\square$ \\
\hline 1730 & GH003 & Hollow stem borehole, no SPT & & 37.0 & 344975 & 6786984 & $\square$ & $\square$ & $\square$ & $\square$ & $\square$ \\
\hline 1730 & GH004 & Hollow stem borehole, no SPT & & 50.0 & 344970 & 6786920 & $\checkmark$ & $\square$ & $\checkmark$ & $\square$ & $\square$ \\
\hline 1730 & GH005 & Hollow stem borehole, no SPT & & 51.5 & 344969 & 6786870 & $\checkmark$ & $\square$ & $\square$ & $\square$ & $\square$ \\
\hline 1730 & GH013 & Hollow stem borehole w/SPT & $1 / 29 / 1980$ & 49.5 & 344870 & 6786488 & $\square$ & $\square$ & $\square$ & $\square$ & $\square$ \\
\hline 1730 & GH014 & Hollow stem borehole w/SPT & $1 / 28 / 1980$ & 26.5 & 344872 & 6786424 & $\square$ & $\square$ & $\square$ & $\square$ & $\square$ \\
\hline 1730 & GH015 & Hollow stem borehole w/SPT & $1 / 28 / 1980$ & 26.5 & 344902 & 6786482 & $\square$ & $\square$ & $\square$ & $\square$ & $\square$ \\
\hline 1730 & GH016 & Hollow stem borehole w/SPT & $1 / 24 / 1980$ & 26.5 & 344785 & 6786570 & $\square$ & $\square$ & $\square$ & $\square$ & $\square$ \\
\hline 1730 & GH017 & Hollow stem borehole w/SPT & $1 / 25 / 1980$ & 51.5 & 344781 & 6786483 & $\boldsymbol{V}$ & 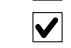 & $\nabla$ & $\checkmark$ & $\square$ \\
\hline 1730 & GH018 & Hollow stem borehole w/SPT & $1 / 28 / 1980$ & 26.5 & 344770 & 6786400 & $\square$ & $\square$ & $\square$ & $\square$ & $\square$ \\
\hline 1730 & GH020 & Hollow stem borehole w/SPT & $3 / 29 / 1982$ & 51.5 & 344800 & 6786552 & $\checkmark$ & $\checkmark$ & $\checkmark$ & $\checkmark$ & $\square$ \\
\hline 1730 & GI001 & Hollow stem borehole, no SPT & & 51.0 & 345045 & 6786976 & $\boldsymbol{V}$ & $\square$ & $\square$ & $\square$ & $\square$ \\
\hline 1730 & GI002 & Hollow stem borehole, no SPT & & 70.0 & 345098 & 6786984 & $\checkmark$ & $\square$ & $\checkmark$ & $\square$ & $\square$ \\
\hline 1730 & GI003 & Hollow stem borehole, no SPT & & 49.0 & 345035 & 6786916 & $\square$ & $\square$ & $\square$ & $\square$ & $\square$ \\
\hline 1730 & GI004 & Hollow stem borehole, no SPT & & 51.0 & 345091 & 6786922 & 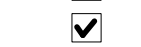 & $\square$ & $\square$ & $\square$ & $\square$ \\
\hline 1730 & GI005 & Hollow stem borehole, no SPT & & 51.5 & 345036 & 6786862 & $\checkmark$ & $\square$ & $\square$ & $\square$ & $\square$ \\
\hline 1730 & GI006 & Hollow stem borehole, no SPT & & 51.5 & 345092 & 6786870 & $\checkmark$ & $\square$ & $\square$ & $\square$ & $\square$ \\
\hline 1730 & GI007 & Hollow stem borehole, no SPT & & 130.0 & 345089 & 6786950 & $\boldsymbol{V}$ & $\square$ & $\square$ & $\square$ & $\square$ \\
\hline 1730 & GI033 & Hollow stem borehole w/SPT & $1 / 6 / 1982$ & 80.5 & 344980 & 6786280 & $\checkmark$ & $\nabla$ & $\nabla$ & $\nabla$ & $\square$ \\
\hline
\end{tabular}




\begin{tabular}{|c|c|c|c|c|c|c|c|c|c|c|c|}
\hline \multirow[b]{2}{*}{$\begin{array}{c}\text { MOA } \\
\text { grid }\end{array}$} & \multirow[b]{2}{*}{$\begin{array}{c}\text { DGGS } \\
\text { number }\end{array}$} & \multirow[b]{2}{*}{ Hole Type } & \multirow[b]{2}{*}{$\begin{array}{c}\text { Date } \\
\text { Completed }\end{array}$} & \multirow[b]{2}{*}{$\begin{array}{c}\text { Total } \\
\text { depth }(\mathrm{ft})\end{array}$} & \multicolumn{2}{|c|}{ UTM coordinates (m) } & \multicolumn{5}{|c|}{ Data entered } \\
\hline & & & & & East & North & Lithology & SPT & $\begin{array}{l}\text { Sample } \\
\text { test }\end{array}$ & $\begin{array}{l}\text { Water } \\
\text { level }\end{array}$ & Velocity \\
\hline 1731 & 1344 & Hollow stem borehole w/SPT & $4 / 25 / 1983$ & 50.8 & 345360 & 6786893 & $\checkmark$ & $\checkmark$ & $\checkmark$ & $\checkmark$ & $\square$ \\
\hline 1731 & 1345 & Hollow stem borehole w/SPT & $4 / 20 / 1983$ & 50.0 & 345380 & 6786928 & $\nabla$ & $\checkmark$ & $\square$ & $\checkmark$ & $\square$ \\
\hline 1731 & 1346 & Hollow stem borehole w/SPT & $2 / 23 / 1994$ & 61.0 & 345725 & 6786323 & $\checkmark$ & $\checkmark$ & $\square$ & $\checkmark$ & $\square$ \\
\hline 1731 & 1347 & Hollow stem borehole w/SPT & $2 / 22 / 1994$ & 50.5 & 345755 & 6786314 & $\nabla$ & $\checkmark$ & $\square$ & $\nabla$ & $\square$ \\
\hline 1731 & 1348 & Hollow stem borehole w/SPT & $2 / 22 / 1994$ & 51.0 & 345809 & 6786313 & $\checkmark$ & $\checkmark$ & $\square$ & $\nabla$ & $\square$ \\
\hline 1731 & 1621 & Hollow stem borehole w/SPT & $11 / 15 / 1996$ & 26.5 & 345651 & 6786555 & $\square$ & $\square$ & $\square$ & $\square$ & $\square$ \\
\hline 1731 & 1622 & Hollow stem borehole w/SPT & $11 / 19 / 1996$ & 25.9 & 345656 & 6786463 & $\square$ & $\square$ & $\square$ & $\square$ & $\square$ \\
\hline 1731 & 1786 & Solid stem borehole (no cores or SP & $10 / 22 / 1982$ & 20.0 & 345136 & 6786979 & $\square$ & $\square$ & $\square$ & $\square$ & $\square$ \\
\hline 1731 & 3025 & Water well & 9/1/1974 & 300.0 & 345467 & 6786588 & $\nabla$ & $\square$ & $\square$ & $\checkmark$ & $\square$ \\
\hline 1731 & 4403 & Water well & $10 / 1 / 1977$ & 316.0 & 345840 & 6786231 & $\checkmark$ & $\square$ & $\square$ & $\checkmark$ & $\square$ \\
\hline 1731 & GI011 & Hollow stem borehole w/SPT & $6 / 26 / 1984$ & 31.0 & 345803 & 6786206 & $\square$ & $\square$ & $\square$ & $\square$ & $\square$ \\
\hline 1731 & GI012 & Hollow stem borehole w/SPT & $6 / 26 / 1984$ & 26.0 & 345808 & 6786260 & $\square$ & $\square$ & $\square$ & $\square$ & $\square$ \\
\hline 1731 & GI013 & Hollow stem borehole w/SPT & $6 / 26 / 1984$ & 21.0 & 345809 & 6786324 & $\square$ & $\square$ & $\square$ & $\square$ & $\square$ \\
\hline 1731 & GI014 & Hollow stem borehole w/SPT & $6 / 26 / 1984$ & 26.0 & 345865 & 6786310 & $\square$ & $\square$ & $\square$ & $\square$ & $\square$ \\
\hline 1731 & GI015 & Hollow stem borehole w/SPT & $6 / 26 / 1984$ & 31.0 & 345863 & 6786268 & $\square$ & $\square$ & $\square$ & $\square$ & $\square$ \\
\hline 1731 & GI016 & Hollow stem borehole w/SPT & $6 / 27 / 1984$ & 26.0 & 345866 & 6786186 & $\square$ & $\square$ & $\square$ & $\square$ & $\square$ \\
\hline 1731 & GI018 & Hollow stem borehole w/SPT & $6 / 2 / 1982$ & 45.8 & 345430 & 6786880 & $\square$ & $\square$ & $\square$ & $\square$ & $\square$ \\
\hline 1731 & GI019 & Hollow stem borehole w/SPT & $6 / 3 / 1982$ & 30.8 & 345339 & 6786882 & $\square$ & $\square$ & $\square$ & $\square$ & $\square$ \\
\hline 1731 & GI020 & Hollow stem borehole w/SPT & 6/4/1982 & 31.0 & 345428 & 6786836 & $\square$ & $\square$ & $\square$ & $\square$ & $\square$ \\
\hline 1731 & GI021 & Hollow stem borehole w/SPT & $6 / 3 / 1982$ & 36.0 & 345383 & 6786838 & $\square$ & $\square$ & $\square$ & $\square$ & $\square$ \\
\hline 1731 & GI022 & Hollow stem borehole w/SPT & $6 / 2 / 1982$ & 40.0 & 345397 & 6786916 & $\square$ & $\square$ & $\square$ & $\square$ & $\square$ \\
\hline 1731 & GI023 & Hollow stem borehole w/SPT & $4 / 24 / 1983$ & 80.0 & 345367 & 6786938 & $\nabla$ & $\nabla$ & $\checkmark$ & $\nabla$ & $\square$ \\
\hline 1731 & GI026 & Hollow stem borehole w/SPT & $6 / 18 / 1984$ & 76.5 & 345821 & 6786482 & 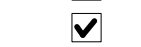 & $\checkmark$ & $\nabla$ & $\square$ & $\square$ \\
\hline 1731 & GI027 & Hollow stem borehole w/SPT & $6 / 20 / 1984$ & 51.5 & 345821 & 6786452 & $\checkmark$ & $\checkmark$ & $\square$ & $\checkmark$ & $\square$ \\
\hline 1731 & GI028 & Hollow stem borehole w/SPT & $6 / 19 / 1984$ & 51.5 & 345864 & 6786436 & $\checkmark$ & $\checkmark$ & $\square$ & $\checkmark$ & $\square$ \\
\hline 1731 & GI029 & Hollow stem borehole w/SPT & $6 / 22 / 1984$ & 51.5 & 345843 & 6786462 & $\checkmark$ & $\checkmark$ & $\checkmark$ & $\checkmark$ & $\square$ \\
\hline 1731 & GI030 & Hollow stem borehole w/SPT & $6 / 26 / 1984$ & 56.5 & 345795 & 6786462 & $\nabla$ & $\checkmark$ & $\square$ & $\nabla$ & $\square$ \\
\hline 1731 & GI031 & Hollow stem borehole w/SPT & $6 / 25 / 1984$ & 51.5 & 345810 & 6786506 & $\checkmark$ & $\checkmark$ & $\square$ & $\checkmark$ & $\square$ \\
\hline 1731 & GI032 & Hollow stem borehole w/SPT & $6 / 26 / 1984$ & 56.5 & 345844 & 6786500 & $\boldsymbol{V}$ & $\nabla$ & $\square$ & $\nabla$ & $\square$ \\
\hline 1731 & GI034 & Hollow stem borehole w/SPT & 2/16/1977 & 46.5 & 345879 & 6786372 & $\square$ & $\square$ & $\square$ & $\square$ & $\square$ \\
\hline 1731 & GI035 & Hollow stem borehole w/SPT & 2/16/1977 & 46.5 & 345790 & 6786395 & $\square$ & $\square$ & $\square$ & $\square$ & $\square$ \\
\hline 1732 & 1688 & Hollow stem borehole w/SPT & 9/18/1976 & 41.5 & 346213 & 6786874 & $\square$ & $\square$ & $\square$ & $\square$ & $\square$ \\
\hline 1732 & 3028 & Water well & $9 / 11 / 1964$ & 217.0 & 345979 & 6786317 & $\checkmark$ & $\square$ & $\square$ & $\checkmark$ & $\square$ \\
\hline 1732 & 4402 & Water well & $12 / 6 / 1965$ & 123.0 & 346182 & 6786185 & $\checkmark$ & $\square$ & $\square$ & $\checkmark$ & $\square$ \\
\hline 1732 & GJ003 & Hollow stem borehole w/SPT & $3 / 26 / 1971$ & 13.5 & 346102 & 6786810 & $\square$ & $\square$ & $\square$ & $\square$ & $\square$ \\
\hline 1732 & GJ004 & Hollow stem borehole w/SPT & $4 / 12 / 1971$ & 61.5 & 346062 & 6786810 & $\nabla$ & $\checkmark$ & $\checkmark$ & $\checkmark$ & $\square$ \\
\hline
\end{tabular}




\begin{tabular}{|c|c|c|c|c|c|c|c|c|c|c|c|}
\hline \multirow[b]{2}{*}{$\begin{array}{l}\text { MOA } \\
\text { grid }\end{array}$} & \multirow[b]{2}{*}{$\begin{array}{l}\text { DGGS } \\
\text { number }\end{array}$} & \multirow[b]{2}{*}{ Hole Type } & \multirow[b]{2}{*}{$\begin{array}{c}\text { Date } \\
\text { Completed }\end{array}$} & \multirow[b]{2}{*}{$\begin{array}{c}\text { Total } \\
\text { depth }(\mathrm{ft})\end{array}$} & \multicolumn{2}{|c|}{ UTM coordinates (m) } & \multicolumn{5}{|c|}{ Data entered } \\
\hline & & & & & East & North & Lithology & SPT & $\begin{array}{c}\text { Sample } \\
\text { test }\end{array}$ & $\begin{array}{l}\text { Water } \\
\text { level }\end{array}$ & Velocity \\
\hline 1732 & GJ005 & Hollow stem borehole w/SPT & $4 / 8 / 1971$ & 56.0 & 346103 & 6786700 & $\checkmark$ & $\checkmark$ & $\checkmark$ & $\checkmark$ & \\
\hline 1732 & GJ006 & Hollow stem borehole w/SPT & $3 / 26 / 1971$ & 14.0 & 346055 & 6786698 & $\square$ & $\square$ & $\square$ & $\square$ & $\square$ \\
\hline 1732 & GJ007 & Hollow stem borehole w/SPT & $4 / 1 / 1971$ & 26.5 & 346094 & 6786646 & $\square$ & $\square$ & $\square$ & $\square$ & \\
\hline 1732 & GJ008 & Hollow stem borehole w/SPT & $4 / 1 / 1971$ & 67.0 & 346047 & 6786642 & $\nabla$ & 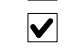 & $\nabla$ & $\sqrt{\nabla}$ & $\square$ \\
\hline 1732 & GJ009 & Hollow stem borehole w/SPT & $4 / 1 / 1971$ & 23.8 & 346110 & 6786590 & $\square$ & $\square$ & $\square$ & $\square$ & $\square$ \\
\hline 1732 & GJ010 & Hollow stem borehole w/SPT & 4/6/1971 & 24.0 & 346044 & 6786598 & $\square$ & $\square$ & $\square$ & $\square$ & $\square$ \\
\hline 1732 & GJ011 & Hollow stem borehole w/SPT & $11 / 4 / 1975$ & 50.4 & 346228 & 6786787 & $\square$ & L & $\square$ & $\square$ & $\square$ \\
\hline 1732 & GJ012 & Hollow stem borehole w/SPT & $9 / 17 / 1976$ & 41.5 & 346208 & 6786874 & $\square$ & & $\square$ & $\square$ & $\checkmark$ \\
\hline 1732 & GJ013 & Hollow stem borehole w/SPT & $9 / 18 / 1976$ & 36.5 & 346180 & 6786912 & $\square$ & $\checkmark$ & $\square$ & $\square$ & $\square$ \\
\hline 1732 & GJ014 & Hollow stem borehole w/SPT & 6/7/1973 & 20.0 & 346518 & 6786180 & $\square$ & $\square$ & $\square$ & $\square$ & $\square$ \\
\hline 1732 & GJ015 & Hollow stem borehole w/SPT & $6 / 12 / 1973$ & 41.5 & 346507 & 6786208 & $\square$ & $\square$ & $\square$ & $\square$ & $\square$ \\
\hline 1732 & GJ016 & Hollow stem borehole w/SPT & $6 / 12 / 1973$ & 20.0 & 346487 & 6786176 & $\square$ & $\square$ & $\square$ & $\square$ & \\
\hline 1732 & GJ017 & Hollow stem borehole w/SPT & $10 / 3 / 1975$ & 42.0 & 346212 & 6786304 & $\square$ & $\square$ & $\square$ & $\square$ & $\square$ \\
\hline 1732 & GJ018 & Hollow stem borehole w/SPT & $10 / 3 / 1975$ & 41.5 & 346211 & 6786228 & $\square$ & $\square$ & $\square$ & $\square$ & $\square$ \\
\hline 1732 & GJ019 & Hollow stem borehole w/SPT & $10 / 3 / 1975$ & 42.0 & 346185 & 6786228 & $\square$ & $\square$ & $\square$ & $\square$ & $\square$ \\
\hline 1732 & GJ020 & Hollow stem borehole w/SPT & $10 / 3 / 1975$ & 40.0 & 346194 & 6786307 & $\square$ & $\square$ & $\square$ & $\square$ & $\square$ \\
\hline 1732 & GJ021 & Hollow stem borehole w/SPT & $6 / 7 / 1977$ & 36.0 & 346223 & 6786327 & $\square$ & $\square$ & $\square$ & $\square$ & $\checkmark$ \\
\hline 1732 & GJ022 & Hollow stem borehole w/SPT & 6/7/1977 & 36.0 & 346180 & 6786330 & $\square$ & $\square$ & $\square$ & $\square$ & $\square$ \\
\hline 1732 & GJ024 & Hollow stem borehole w/SPT & 9/1/1975 & 41.0 & 346619 & 6786596 & $\square$ & $\square$ & $\square$ & $\square$ & $\square$ \\
\hline 1732 & GJ025 & Hollow stem borehole w/SPT & 9/1/1975 & 42.0 & 346567 & 6786760 & $\square$ & $\square$ & $\square$ & $\square$ & $\square$ \\
\hline 1732 & GJ026 & Hollow stem borehole w/SPT & 9/1/1975 & 32.0 & 346368 & 6786560 & $\square$ & $\square$ & $\square$ & $\square$ & \\
\hline 1732 & GJ027 & Hollow stem borehole w/SPT & $12 / 6 / 1971$ & 46.5 & 346590 & 6786214 & $\nabla$ & $\checkmark$ & $\square$ & $\square$ & $\square$ \\
\hline 1733 & 1351 & Hollow stem borehole w/SPT & $5 / 29 / 1987$ & 40.0 & 347365 & 6786166 & $\checkmark$ & $\nabla$ & $\nabla$ & $\nabla$ & $\square$ \\
\hline 1733 & 1352 & Hollow stem borehole w/SPT & $7 / 8 / 1977$ & 83.0 & 347422 & 6786358 & $\nabla$ & $\bar{\square}$ & $\bar{\nabla}$ & $\checkmark$ & $\square$ \\
\hline 1733 & 1353 & Hollow stem borehole w/SPT & $7 / 12 / 1977$ & 59.5 & 347404 & 6786308 & $\checkmark$ & $\checkmark$ & $\square$ & $\checkmark$ & $\square$ \\
\hline 1733 & 1354 & Hollow stem borehole w/SPT & $7 / 15 / 1977$ & 60.0 & 347434 & 6786308 & $\checkmark$ & $\checkmark$ & $\square$ & $\checkmark$ & $\square$ \\
\hline 1733 & 1355 & Hollow stem borehole w/SPT & $7 / 16 / 1977$ & 60.0 & 347434 & 6786393 & $\checkmark$ & 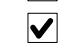 & $\square$ & $\checkmark$ & $\square$ \\
\hline 1733 & 1356 & Hollow stem borehole w/SPT & $7 / 18 / 1977$ & 60.0 & 347403 & 6786395 & $\checkmark$ & $\bar{\nabla}$ & $\square$ & $\checkmark$ & \\
\hline 1733 & GK003 & Solid stem borehole (no cores or SP & 2/17/1976 & 15.0 & 347518 & 6786345 & $\square$ & Г & $\square$ & $\square$ & $\square$ \\
\hline 1733 & GK004 & Solid stem borehole (no cores or SP & 2/6/1976 & 20.5 & 347536 & 6786654 & $\checkmark$ & 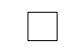 & $\checkmark$ & $\square$ & $\square$ \\
\hline 1733 & GK009 & Solid stem borehole (no cores or SP & 2/6/1976 & 10.5 & 347513 & 6786160 & $\square$ & $\square$ & $\square$ & $\square$ & $\square$ \\
\hline 1733 & GK010 & Solid stem borehole (no cores or SP & $2 / 5 / 1976$ & 15.5 & 347527 & 6786489 & $\square$ & $\square$ & $\square$ & $\square$ & $\square$ \\
\hline 1733 & GK011 & Solid stem borehole (no cores or SP & 2/5/1976 & 15.5 & 347529 & 6786830 & $\square$ & $\square$ & $\square$ & $\square$ & $\square$ \\
\hline 1733 & GK014 & Natural exposure & $7 / 15 / 1971$ & 17.0 & 347447 & 6786100 & $\square$ & $\square$ & $\square$ & $\square$ & $\square$ \\
\hline 1734 & 1350 & Hollow stem borehole w/SPT & $5 / 22 / 1974$ & 42.0 & 347530 & 6786110 & $\square$ & $\square$ & $\square$ & $\square$ & $\square$ \\
\hline 1734 & GK002 & Solid stem borehole (no cores or SP & $7 / 16 / 1971$ & 8.0 & 347853 & 6786082 & $\square$ & $\square$ & $\square$ & $\square$ & $\square$ \\
\hline
\end{tabular}




\begin{tabular}{|c|c|c|c|c|c|c|c|c|c|c|c|}
\hline \multirow[b]{2}{*}{$\begin{array}{c}\text { MOA } \\
\text { grid }\end{array}$} & \multirow[b]{2}{*}{$\begin{array}{l}\text { DGGS } \\
\text { number }\end{array}$} & \multirow[b]{2}{*}{ Hole Type } & \multirow[b]{2}{*}{$\begin{array}{c}\text { Date } \\
\text { Completed }\end{array}$} & \multirow[b]{2}{*}{$\begin{array}{c}\text { Total } \\
\text { depth (ft) }\end{array}$} & \multicolumn{2}{|c|}{ UTM coordinates (m) } & \multicolumn{5}{|c|}{ Data entered } \\
\hline & & & & & East & North & Lithology & SPT & $\begin{array}{c}\text { Sample } \\
\text { test }\end{array}$ & $\begin{array}{l}\text { Water } \\
\text { level }\end{array}$ & Velocity \\
\hline 1734 & GK006 & Hollow stem borehole w/SPT & $3 / 11 / 1983$ & 40.5 & 347831 & 6786426 & $\boldsymbol{V}$ & $\nabla$ & $\nabla$ & $\checkmark$ & $\square$ \\
\hline 1734 & GK007 & Hollow stem borehole w/SPT & $7 / 18 / 1979$ & 21.5 & 347568 & 6786376 & $\square$ & $\square$ & $\square$ & $\square$ & $\square$ \\
\hline 1734 & GK008 & Hollow stem borehole w/SPT & $7 / 19 / 1979$ & 21.5 & 347628 & 6786403 & $\square$ & $\square$ & $\square$ & $\square$ & $\square$ \\
\hline 1734 & GK015 & Solid stem borehole (no cores or SP & $7 / 16 / 1971$ & 13.0 & 347554 & 6786098 & $\square$ & $\square$ & $\square$ & $\square$ & $\square$ \\
\hline 1735 & 1245 & Hollow stem borehole w/SPT & $12 / 18 / 1992$ & 20.0 & 348958 & 6786338 & $\square$ & $\square$ & $\square$ & $\square$ & $\square$ \\
\hline 1735 & 1357 & Hollow stem borehole w/SPT & $3 / 5 / 1984$ & 30.0 & 348944 & 6786552 & $\square$ & $\square$ & $\square$ & $\square$ & $\square$ \\
\hline 1735 & 1358 & Hollow stem borehole w/SPT & $3 / 6 / 1984$ & 29.5 & 348949 & 6786505 & $\square$ & $\square$ & $\square$ & $\square$ & $\square$ \\
\hline 1735 & 1359 & Hollow stem borehole w/SPT & $3 / 8 / 1984$ & 20.0 & 348858 & 6786450 & $\boldsymbol{V}$ & $\nabla$ & $\nabla$ & $\nabla$ & $\square$ \\
\hline 1735 & 1626 & Hollow stem borehole w/SPT & 6/27/1996 & 31.5 & 348873 & 6786385 & $\boldsymbol{V}$ & $\checkmark$ & $\square$ & $\checkmark$ & $\square$ \\
\hline 1735 & 1627 & Hollow stem borehole w/SPT & 6/27/1996 & 31.5 & 349008 & 6786316 & $\square$ & $\square$ & $\square$ & $\square$ & $\square$ \\
\hline 1735 & GL001 & Solid stem borehole (no cores or SP & $12 / 10 / 1973$ & 48.0 & 348436 & 6786056 & $\boldsymbol{V}$ & $\square$ & $\nabla$ & $\square$ & $\square$ \\
\hline 1735 & GL003 & Hollow stem borehole w/SPT & $8 / 24 / 1983$ & 75.8 & 348610 & 6786836 & $\boldsymbol{V}$ & $\checkmark$ & $\square$ & $\checkmark$ & $\square$ \\
\hline 1735 & GL007 & Hollow stem borehole w/SPT & $7 / 10 / 1984$ & 50.5 & 348540 & 6786853 & $\boldsymbol{V}$ & $\checkmark$ & $\nabla$ & 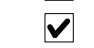 & $\square$ \\
\hline 1736 & 1360 & Hollow stem borehole w/SPT & $11 / 30 / 1990$ & 49.5 & 349575 & 6786291 & $\square$ & $\square$ & $\square$ & $\square$ & $\square$ \\
\hline 1736 & 1361 & Hollow stem borehole w/SPT & $12 / 3 / 1990$ & 50.0 & 349615 & 6786272 & $\boldsymbol{V}$ & $\square$ & $\nabla$ & $\nabla$ & $\square$ \\
\hline 1736 & 1362 & Hollow stem borehole w/SPT & $12 / 4 / 1990$ & 50.5 & 349512 & 6786272 & $\checkmark$ & $\checkmark$ & $\checkmark$ & $\checkmark$ & $\square$ \\
\hline 1736 & 1363 & Hollow stem borehole w/SPT & 2/2/1991 & 213.5 & 349574 & 6786269 & $\boldsymbol{V}$ & $\checkmark$ & $\boldsymbol{V}$ & $\nabla$ & $\boldsymbol{V}$ \\
\hline 1736 & 1364 & Hollow stem borehole w/SPT & $12 / 11 / 1990$ & 49.5 & 349508 & 6786245 & $\square$ & $\square$ & $\square$ & $\square$ & $\square$ \\
\hline 1736 & 1365 & Hollow stem borehole w/SPT & $12 / 12 / 1990$ & 50.5 & 349615 & 6786242 & $\boldsymbol{V}$ & $\checkmark$ & $\nabla$ & $\nabla$ & $\square$ \\
\hline 1737 & 1366 & Hollow stem borehole w/SPT & 8/11/1976 & 40.0 & 350327 & 6786611 & $\square$ & $\square$ & $\square$ & $\square$ & $\square$ \\
\hline 1737 & 1367 & Hollow stem borehole w/SPT & $10 / 10 / 1975$ & 31.0 & 350549 & 6786003 & $\boldsymbol{V}$ & $\checkmark$ & $\square$ & $\checkmark$ & $\square$ \\
\hline 1737 & 1368 & Hollow stem borehole w/SPT & $10 / 10 / 1975$ & 29.5 & 350558 & 6786072 & $\square$ & $\square$ & $\square$ & $\square$ & $\square$ \\
\hline 1737 & 1369 & Hollow stem borehole w/SPT & $10 / 10 / 1975$ & 31.5 & 350433 & 6786060 & 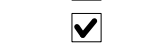 & $\checkmark$ & $\square$ & 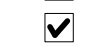 & $\square$ \\
\hline 1737 & 1370 & Hollow stem borehole w/SPT & $10 / 10 / 1975$ & 31.0 & 350470 & 6786085 & $\square$ & $\square$ & $\square$ & $\square$ & $\square$ \\
\hline 1738 & 1371 & Hollow stem borehole w/SPT & 4/29/1982 & 31.0 & 350860 & 6786347 & $\square$ & $\square$ & $\square$ & $\square$ & $\square$ \\
\hline 1738 & 1372 & Hollow stem borehole w/SPT & $4 / 30 / 1982$ & 31.0 & 350850 & 6786243 & $\boldsymbol{V}$ & $\checkmark$ & $\nabla$ & $\square$ & $\square$ \\
\hline 1738 & 1373 & Hollow stem borehole w/SPT & $5 / 3 / 1982$ & 31.0 & 350941 & 6786342 & $\square$ & $\square$ & $\square$ & $\square$ & $\square$ \\
\hline 1738 & 1374 & Hollow stem borehole w/SPT & $5 / 3 / 1982$ & 30.5 & 350950 & 6786251 & $\boldsymbol{V}$ & $\checkmark$ & $\square$ & $\nabla$ & $\square$ \\
\hline 1738 & 1375 & Hollow stem borehole w/SPT & $6 / 1 / 1976$ & 31.0 & 351020 & 6786117 & $\boldsymbol{V}$ & $\checkmark$ & $\square$ & $\square$ & $\square$ \\
\hline 1738 & 1376 & Hollow stem borehole w/SPT & 6/1/1976 & 31.5 & 351157 & 6786176 & 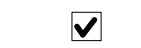 & $\nabla$ & $\square$ & $\nabla$ & $\square$ \\
\hline 1738 & 1377 & Hollow stem borehole w/SPT & $6 / 1 / 1976$ & 31.5 & 351210 & 6786312 & $\checkmark$ & $\checkmark$ & $\square$ & $\checkmark$ & $\square$ \\
\hline 1738 & 1378 & Hollow stem borehole w/SPT & $10 / 12 / 1977$ & 30.5 & 351413 & 6786311 & $\square$ & $\square$ & $\square$ & $\square$ & $\square$ \\
\hline 1738 & 1379 & Hollow stem borehole w/SPT & $10 / 12 / 1977$ & 31.0 & 351462 & 6786308 & $\square$ & $\square$ & $\square$ & $\square$ & $\square$ \\
\hline 1738 & 1380 & Hollow stem borehole w/SPT & $10 / 12 / 1977$ & 31.0 & 351403 & 6786200 & $\square$ & $\square$ & $\square$ & $\square$ & $\square$ \\
\hline 1738 & 1381 & Hollow stem borehole w/SPT & $10 / 12 / 1977$ & 31.0 & 351476 & 6786198 & $\boldsymbol{v}$ & $\checkmark$ & $\square$ & $\nabla$ & $\square$ \\
\hline 1738 & 4404 & Water well & $7 / 28 / 1977$ & 117.0 & 350812 & 6786387 & $\boldsymbol{V}$ & $\square$ & $\square$ & $\checkmark$ & $\square$ \\
\hline
\end{tabular}




\begin{tabular}{|c|c|c|c|c|c|c|c|c|c|c|c|}
\hline \multirow[b]{2}{*}{$\begin{array}{l}\text { MOA } \\
\text { grid }\end{array}$} & \multirow[b]{2}{*}{$\begin{array}{l}\text { DGGS } \\
\text { number }\end{array}$} & \multirow[b]{2}{*}{ Hole Type } & \multirow[b]{2}{*}{$\begin{array}{c}\text { Date } \\
\text { Completed }\end{array}$} & \multirow[b]{2}{*}{$\begin{array}{c}\text { Total } \\
\text { depth }(\mathrm{ft})\end{array}$} & \multicolumn{2}{|c|}{ UTM coordinates (m) } & \multicolumn{5}{|c|}{ Data entered } \\
\hline & & & & & East & North & Lithology & SPT & $\begin{array}{c}\text { Sample } \\
\text { test }\end{array}$ & $\begin{array}{l}\text { Water } \\
\text { level }\end{array}$ & Velocity \\
\hline 1739 & 1382 & Hollow stem borehole w/SPT & $5 / 20 / 1983$ & 30.1 & 352215 & 6786028 & $\nabla$ & $\checkmark$ & $\square$ & $\square$ & \\
\hline 1739 & 1383 & Hollow stem borehole w/SPT & $5 / 23 / 1983$ & 30.8 & 352174 & 6786029 & $\checkmark$ & 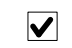 & $\square$ & $\square$ & $\square$ \\
\hline 1740 & 1292 & Hollow stem borehole w/SPT & $8 / 16 / 1994$ & 40.8 & 352415 & 6785882 & $\checkmark$ & $\nabla$ & $\square$ & $\bar{\nabla}$ & \\
\hline 1740 & 1293 & Hollow stem borehole w/SPT & $1 / 13 / 1994$ & 30.3 & 353021 & 6786129 & $\checkmark$ & 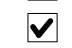 & $\nabla$ & $\boldsymbol{V}$ & $\square$ \\
\hline 1740 & 1390 & Hollow stem borehole, no SPT & $6 / 13 / 1974$ & 20.0 & 353164 & 6786358 & $\checkmark$ & $\square$ & $\square$ & $\boldsymbol{V}$ & $\square$ \\
\hline 1741 & 1395 & Hollow stem borehole w/SPT & $6 / 17 / 1977$ & 23.0 & 353235 & 6785983 & $\square$ & $\square$ & $\square$ & $\square$ & $\square$ \\
\hline 1741 & 3023 & Water well & $9 / 19 / 1973$ & 250.0 & 353305 & 6785911 & $\square$ & $\square$ & $\square$ & $\checkmark$ & $\square$ \\
\hline 1742 & 3022 & Water well & $11 / 5 / 1973$ & 183.0 & 354044 & 6786066 & $\checkmark$ & & $\square$ & $\checkmark$ & \\
\hline 1821 & 3033 & Water well & $3 / 27 / 1967$ & 197.0 & 337070 & 6785821 & $\checkmark$ & $\leftarrow$ & $\square$ & $\checkmark$ & $\square$ \\
\hline 1821 & FW005 & Solid stem borehole (no cores or SP & $6 / 13 / 1968$ & 13.0 & 337080 & 6785770 & $\square$ & $\square$ & $\square$ & $\square$ & $\square$ \\
\hline 1821 & FW007 & Solid stem borehole (no cores or SP & 6/7/1968 & 18.0 & 337184 & 6785760 & $\square$ & $\square$ & $\square$ & $\square$ & $\square$ \\
\hline 1821 & FW008 & Solid stem borehole (no cores or SP & 6/7/1968 & 18.0 & 337122 & 6785794 & $\square$ & & $\square$ & $\square$ & \\
\hline 1821 & FW009 & Solid stem borehole (no cores or SP & 6/7/1968 & 18.0 & 337175 & 6785807 & $\square$ & & $\square$ & $\square$ & \\
\hline 1821 & FW010 & Solid stem borehole (no cores or SP & $6 / 13 / 1968$ & 13.0 & 337069 & 6785830 & $\square$ & $\square$ & $\square$ & $\square$ & $\square$ \\
\hline 1821 & FW011 & Solid stem borehole (no cores or SP & $6 / 12 / 1968$ & 13.0 & 337148 & 6785853 & $\square$ & $\square$ & $\square$ & $\square$ & $\square$ \\
\hline 1821 & FW012 & Solid stem borehole (no cores or SP & $6 / 12 / 1968$ & 18.0 & 337217 & 6785848 & $\square$ & & $\square$ & $\square$ & $\square$ \\
\hline 1821 & FW013 & Solid stem borehole (no cores or SP & 6/7/1968 & 18.0 & 337226 & 6785807 & $\square$ & & $\square$ & $\square$ & \\
\hline 1821 & FW014 & Solid stem borehole (no cores or SP & $6 / 13 / 1968$ & 13.0 & 337091 & 6785884 & $\square$ & $\square$ & $\square$ & $\square$ & $\square$ \\
\hline 1821 & FW015 & Solid stem borehole (no cores or SP & $6 / 6 / 1968$ & 13.0 & 337171 & 6785884 & $\square$ & $\square$ & $\square$ & $\square$ & $\square$ \\
\hline 1821 & FW016 & Solid stem borehole (no cores or SP & $6 / 7 / 1968$ & 18.0 & 337227 & 6785887 & $\square$ & $\checkmark$ & $\square$ & $\square$ & $\square$ \\
\hline 1821 & FW017 & Solid stem borehole (no cores or SP & $6 / 13 / 1968$ & 13.0 & 337154 & 6785920 & $\square$ & & $\square$ & $\square$ & \\
\hline 1821 & FW018 & Solid stem borehole (no cores or SP & $6 / 13 / 1968$ & 18.0 & 337203 & 6785920 & $\square$ & $\checkmark$ & $\square$ & $\square$ & $\square$ \\
\hline 1821 & FW019 & Solid stem borehole (no cores or SP & $6 / 13 / 1968$ & 13.0 & 337247 & 6785912 & $\square$ & & $\square$ & $\square$ & $\square$ \\
\hline 1821 & FW020 & Solid stem borehole (no cores or SP & $6 / 14 / 1968$ & 13.0 & 337297 & 6785968 & $\square$ & $\square$ & $\square$ & $\square$ & $\square$ \\
\hline 1821 & FW021 & Solid stem borehole (no cores or SP & $6 / 14 / 1968$ & 13.0 & 337375 & 6785984 & $\square$ & & $\square$ & $\square$ & $\square$ \\
\hline 1821 & FW022 & Solid stem borehole (no cores or SP & $6 / 14 / 1968$ & 18.0 & 337462 & 6785982 & $\square$ & & $\square$ & $\square$ & $\square$ \\
\hline 1822 & 2128 & Hollow stem borehole w/SPT & $10 / 13 / 1993$ & 61.3 & 338085 & 6785780 & $\nabla$ & $\checkmark$ & $\square$ & $\checkmark$ & $\square$ \\
\hline 1822 & 4398 & Water well & $1 / 1 / 1971$ & 214.0 & 337899 & 6786278 & $\checkmark$ & & $\square$ & $\checkmark$ & \\
\hline 1822 & FV010 & Solid stem borehole (no cores or SP & 2/6/1973 & 20.0 & 338613 & 6785864 & $\square$ & & $\square$ & $\square$ & $\square$ \\
\hline 1822 & FV019 & Solid stem borehole (no cores or SP & $11 / 8 / 1977$ & 10.0 & 338236 & 6785811 & $\square$ & & $\square$ & $\square$ & $\square$ \\
\hline 1822 & FV020 & Solid stem borehole (no cores or SP & $11 / 8 / 1977$ & 10.0 & 338208 & 6785924 & $\square$ & $\square$ & $\square$ & $\square$ & $\square$ \\
\hline 1822 & GB005 & Solid stem borehole (no cores or SP & 2/7/1973 & 25.0 & 338431 & 6786448 & $\square$ & $\square$ & $\square$ & $\square$ & $\square$ \\
\hline 1822 & GB009 & Solid stem borehole (no cores or SP & $2 / 23 / 1973$ & 35.0 & 338671 & 6786476 & $\square$ & $\square$ & $\square$ & $\square$ & $\square$ \\
\hline 1822 & GB014 & Solid stem borehole (no cores or SP & $1 / 31 / 1973$ & 15.0 & 338657 & 6786198 & $\square$ & $\square$ & $\square$ & $\square$ & $\square$ \\
\hline 1822 & GB015 & Solid stem borehole (no cores or SP & $1 / 31 / 1973$ & 15.0 & 338622 & 6786272 & $\square$ & $\square$ & $\square$ & $\square$ & $\square$ \\
\hline 1822 & GB016 & Solid stem borehole (no cores or SP & 2/6/1973 & 20.0 & 338597 & 6786022 & $\square$ & $\square$ & $\square$ & $\square$ & $\square$ \\
\hline
\end{tabular}




\begin{tabular}{|c|c|c|c|c|c|c|c|c|c|c|c|}
\hline \multirow[b]{2}{*}{$\begin{array}{c}\text { MOA } \\
\text { grid }\end{array}$} & \multirow[b]{2}{*}{$\begin{array}{c}\text { DGGS } \\
\text { number }\end{array}$} & \multirow[b]{2}{*}{ Hole Type } & \multirow[b]{2}{*}{$\begin{array}{c}\text { Date } \\
\text { Completed }\end{array}$} & \multirow[b]{2}{*}{$\begin{array}{c}\text { Total } \\
\text { depth (ft) }\end{array}$} & \multicolumn{2}{|c|}{ UTM coordinates (m) } & \multicolumn{5}{|c|}{ Data entered } \\
\hline & & & & & East & North & Lithology & SPT & $\begin{array}{c}\text { Sample } \\
\text { test }\end{array}$ & $\begin{array}{c}\text { Water } \\
\text { level }\end{array}$ & Velocity \\
\hline 1822 & GB017 & Solid stem borehole (no cores or SP & $2 / 6 / 1973$ & 20.0 & 338567 & 6786087 & & $\square$ & $\square$ & $\square$ & $\square$ \\
\hline 1822 & GB018 & Solid stem borehole (no cores or SP & $2 / 6 / 1973$ & 20.0 & 338534 & 6786168 & & $\square$ & $\square$ & $\square$ & $\square$ \\
\hline 1822 & GB019 & Solid stem borehole (no cores or SP & $2 / 6 / 1973$ & 20.0 & 338512 & 6786236 & & $\square$ & $\square$ & $\square$ & $\square$ \\
\hline 1822 & GB020 & Solid stem borehole (no cores or SP & $2 / 7 / 1973$ & 20.0 & 338486 & 6786308 & $\square$ & $\square$ & $\square$ & $\square$ & $\square$ \\
\hline 1822 & GB022 & Solid stem borehole (no cores or SP & $1 / 31 / 1973$ & 25.0 & 338564 & 6786409 & & $\square$ & $\square$ & $\square$ & $\square$ \\
\hline 1822 & GB036 & Solid stem borehole (no cores or SP & $11 / 8 / 1977$ & 30.0 & 338180 & 6786002 & & $\square$ & $\square$ & $\square$ & $\square$ \\
\hline 1822 & GB037 & Solid stem borehole (no cores or SP & $11 / 8 / 1977$ & 25.0 & 338153 & 6786090 & & $\square$ & $\square$ & $\square$ & $\square$ \\
\hline 1822 & GB038 & Solid stem borehole (no cores or SP & $11 / 8 / 1977$ & 10.0 & 338108 & 6786330 & & $\square$ & $\square$ & $\square$ & $\square$ \\
\hline 1823 & FU017 & Solid stem borehole (no cores or SP & $2 / 20 / 1973$ & 25.0 & 339036 & 6785766 & $\square$ & $\square$ & $\square$ & $\square$ & $\square$ \\
\hline 1823 & FU018 & Solid stem borehole (no cores or SP & $2 / 20 / 1973$ & 25.0 & 339016 & 6785862 & & $\square$ & $\square$ & $\square$ & $\square$ \\
\hline 1823 & FU019 & Solid stem borehole (no cores or SP & $2 / 20 / 1973$ & 25.0 & 338996 & 6785950 & & $\square$ & $\square$ & $\square$ & $\square$ \\
\hline 1823 & FV007 & Solid stem borehole (no cores or SP & $1 / 31 / 1973$ & 15.0 & 338765 & 6785980 & & $\square$ & $\square$ & $\square$ & $\square$ \\
\hline 1823 & FV009 & Solid stem borehole (no cores or SP & $2 / 6 / 1973$ & 30.0 & 338654 & 6785718 & & $\square$ & $\square$ & $\square$ & $\square$ \\
\hline 1823 & FV012 & Solid stem borehole (no cores or SP & $12 / 16 / 1967$ & 21.5 & 338900 & 6785864 & & $\square$ & $\square$ & $\square$ & $\square$ \\
\hline 1823 & FV013 & Solid stem borehole (no cores or SP & $1 / 5 / 1973$ & 20.0 & 338870 & 6785959 & & $\square$ & $\square$ & $\square$ & $\square$ \\
\hline 1823 & FV014 & Solid stem borehole (no cores or SP & $1 / 31 / 1973$ & 15.0 & 338814 & 6785833 & & $\square$ & $\square$ & $\square$ & $\square$ \\
\hline 1823 & FV015 & Solid stem borehole (no cores or SP & $1 / 31 / 1973$ & 15.0 & 338870 & 6785686 & $\square$ & $\square$ & $\square$ & $\square$ & $\square$ \\
\hline 1823 & GB012 & Solid stem borehole (no cores or SP & $1 / 31 / 1973$ & 15.0 & 338741 & 6786054 & & $\square$ & $\square$ & $\square$ & $\square$ \\
\hline 1823 & GB013 & Solid stem borehole (no cores or SP & $1 / 31 / 1973$ & 15.0 & 338681 & 6786124 & & $\square$ & $\square$ & $\square$ & $\square$ \\
\hline 1823 & GB021 & Solid stem borehole (no cores or SP & 2/23/1973 & 20.0 & 338783 & 6786368 & & $\square$ & $\square$ & $\square$ & $\square$ \\
\hline 1823 & GB023 & Hollow stem borehole w/SPT & $3 / 8 / 1973$ & 56.5 & 338691 & 6786090 & $\nabla$ & $\checkmark$ & $\checkmark$ & $\checkmark$ & $\square$ \\
\hline 1823 & GB024 & Solid stem borehole (no cores or SP & $9 / 28 / 1948$ & 10.0 & 338889 & 6786438 & & $\square$ & $\square$ & $\square$ & $\square$ \\
\hline 1823 & GB028 & Solid stem borehole (no cores or SP & & 21.5 & 338861 & 6786285 & & $\square$ & $\square$ & $\square$ & $\square$ \\
\hline 1823 & GB029 & Solid stem borehole (no cores or SP & $8 / 29 / 1967$ & 14.0 & 338962 & 6786424 & & $\square$ & $\square$ & $\square$ & $\square$ \\
\hline 1823 & GB030 & Solid stem borehole (no cores or SP & $1 / 5 / 1973$ & 15.0 & 338860 & 6786138 & & $\square$ & $\square$ & $\square$ & $\square$ \\
\hline 1823 & GB031 & Solid stem borehole (no cores or SP & $1 / 5 / 1973$ & 15.0 & 338867 & 6786062 & & $\square$ & $\square$ & $\square$ & $\square$ \\
\hline 1823 & GB032 & Solid stem borehole (no cores or SP & $2 / 20 / 1973$ & 25.0 & 338974 & 6786020 & & $\square$ & $\square$ & $\square$ & $\square$ \\
\hline 1823 & GB033 & Solid stem borehole (no cores or SP & $2 / 21 / 1973$ & 25.0 & 338959 & 6786081 & & $\square$ & $\square$ & $\square$ & $\square$ \\
\hline 1823 & GB034 & Solid stem borehole (no cores or SP & $2 / 20 / 1973$ & 30.0 & 338942 & 6786190 & & $\square$ & $\square$ & $\square$ & $\square$ \\
\hline 1823 & GB035 & Solid stem borehole (no cores or SP & 2/20/1973 & 30.0 & 338894 & 6786319 & & $\square$ & $\square$ & $\square$ & $\square$ \\
\hline 1823 & GC020 & Solid stem borehole (no cores or SP & $12 / 21 / 1966$ & 22.0 & 339088 & 6786111 & $\square$ & $\square$ & $\square$ & $\square$ & $\square$ \\
\hline 1823 & GC021 & Solid stem borehole (no cores or SP & $9 / 28 / 1948$ & 10.0 & 339117 & 6786196 & & $\square$ & $\square$ & $\square$ & $\square$ \\
\hline 1823 & GC022 & Solid stem borehole (no cores or SP & $9 / 28 / 1948$ & 10.0 & 339013 & 6786226 & $\square$ & $\square$ & $\square$ & $\square$ & $\square$ \\
\hline 1823 & GC023 & Hollow stem borehole w/SPT & $8 / 27 / 1967$ & 14.0 & 339022 & 6786290 & $\square$ & $\square$ & $\square$ & $\square$ & $\square$ \\
\hline 1823 & GC024 & Solid stem borehole (no cores or SP & $8 / 29 / 1967$ & 14.0 & 339073 & 6786381 & $\square$ & $\square$ & $\square$ & $\square$ & $\square$ \\
\hline 1824 & 2027 & Water well & $8 / 1 / 1996$ & 720.0 & 340164 & 6786136 & 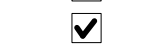 & $\square$ & $\square$ & $\checkmark$ & $\square$ \\
\hline
\end{tabular}




\begin{tabular}{|c|c|c|c|c|c|c|c|c|c|c|c|}
\hline \multirow[b]{2}{*}{$\begin{array}{l}\text { MOA } \\
\text { grid }\end{array}$} & \multirow[b]{2}{*}{$\begin{array}{l}\text { DGGS } \\
\text { number }\end{array}$} & \multirow[b]{2}{*}{ Hole Type } & \multirow[b]{2}{*}{$\begin{array}{c}\text { Date } \\
\text { Completed }\end{array}$} & \multirow[b]{2}{*}{$\begin{array}{c}\text { Total } \\
\text { depth }(\mathrm{ft})\end{array}$} & \multicolumn{2}{|c|}{ UTM coordinates (m) } & \multicolumn{5}{|c|}{ Data entered } \\
\hline & & & & & East & North & Lithology & SPT & $\begin{array}{c}\text { Sample } \\
\text { test }\end{array}$ & $\begin{array}{l}\text { Water } \\
\text { level }\end{array}$ & Velocity \\
\hline 1824 & 2056 & Hollow stem borehole w/SPT & 2/18/1999 & 101.5 & 339670 & 6785905 & $\checkmark$ & $\nabla$ & $\checkmark$ & $\checkmark$ & $\checkmark$ \\
\hline 1824 & 2117 & Hollow stem borehole w/SPT & 2/2/1999 & 51.5 & 339673 & 6785816 & $\checkmark$ & $\checkmark$ & $\checkmark$ & $\checkmark$ & $\square$ \\
\hline 1824 & 2119 & Hollow stem borehole w/SPT & 2/3/1999 & 51.5 & 339691 & 6785781 & $\checkmark$ & $\checkmark$ & $\nabla$ & $\checkmark$ & \\
\hline 1824 & 2120 & Hollow stem borehole w/SPT & 2/4/1999 & 51.5 & 339711 & 6785642 & $\checkmark$ & $\checkmark$ & $\nabla$ & $\square$ & $\square$ \\
\hline 1824 & 2121 & Hollow stem borehole w/SPT & 2/3/1999 & 51.5 & 339787 & 6785659 & $\checkmark$ & $\checkmark$ & $\checkmark$ & $\square$ & $\square$ \\
\hline 1824 & 2122 & Hollow stem borehole w/SPT & 2/4/1999 & 51.5 & 339762 & 6785697 & $\checkmark$ & $\checkmark$ & $\checkmark$ & $\square$ & $\square$ \\
\hline 1824 & 2123 & Hollow stem borehole w/SPT & $12 / 8 / 1999$ & 71.5 & 339764 & 6785661 & $\checkmark$ & $\checkmark$ & $\checkmark$ & $\checkmark$ & \\
\hline 1824 & 2124 & Hollow stem borehole w/SPT & $12 / 9 / 1999$ & 71.5 & 339772 & 6785639 & $\checkmark$ & $\checkmark$ & $\checkmark$ & $\checkmark$ & \\
\hline 1824 & 2192 & Hollow stem borehole w/SPT & $12 / 16 / 1998$ & 77.1 & 339761 & 6785711 & $\nabla$ & $\nabla$ & $\checkmark$ & $\checkmark$ & \\
\hline 1824 & 2193 & Hollow stem borehole w/SPT & $12 / 21 / 1998$ & 76.4 & 339774 & 6785732 & $\checkmark$ & $\nabla$ & $\checkmark$ & $\square$ & \\
\hline 1824 & 2197 & Hollow stem borehole w/SPT & $12 / 17 / 1998$ & 77.1 & 339754 & 6785738 & $\checkmark$ & $\nabla$ & $\bar{\nabla}$ & $\nabla$ & $\square$ \\
\hline 1824 & 2198 & Hollow stem borehole w/SPT & $12 / 15 / 1998$ & 102.0 & 339777 & 6785680 & $\checkmark$ & $\nabla$ & $\checkmark$ & $\checkmark$ & \\
\hline 1824 & 2199 & Hollow stem borehole w/SPT & $12 / 18 / 1998$ & 100.1 & 339728 & 6785801 & $\checkmark$ & $\nabla$ & $\checkmark$ & $\checkmark$ & $\square$ \\
\hline 1824 & 2208 & Hollow stem borehole w/SPT & $6 / 24 / 1985$ & 51.5 & 339863 & 6785777 & $\checkmark$ & $\square$ & $\checkmark$ & $\square$ & $\square$ \\
\hline 1824 & 2209 & Hollow stem borehole w/SPT & $9 / 27 / 1985$ & 75.0 & 339869 & 6785729 & $\nabla$ & $\checkmark$ & $\bar{\nabla}$ & $\checkmark$ & $\square$ \\
\hline 1824 & 2212 & Hollow stem borehole w/SPT & $8 / 13 / 1996$ & 60.0 & 339771 & 6785707 & $\checkmark$ & $\checkmark$ & $\square$ & $\square$ & $\square$ \\
\hline 1824 & 2213 & Hollow stem borehole w/SPT & 8/8/1996 & 77.0 & 339744 & 6785760 & $\checkmark$ & $\checkmark$ & $\square$ & $\checkmark$ & $\square$ \\
\hline 1824 & 2214 & Hollow stem borehole w/SPT & $8 / 11 / 1996$ & 76.5 & 339695 & 6785744 & $\checkmark$ & $\checkmark$ & $\square$ & $\checkmark$ & \\
\hline 1824 & 2217 & Hollow stem borehole w/SPT & $12 / 17 / 1998$ & 76.4 & 339735 & 6785776 & $\checkmark$ & $\nabla$ & $\checkmark$ & $\nabla$ & $\square$ \\
\hline 1824 & 2218 & Hollow stem borehole w/SPT & $12 / 18 / 1998$ & 76.4 & 339753 & 6785791 & $\checkmark$ & $\nabla$ & $\bar{\nabla}$ & $\square$ & $\square$ \\
\hline 1824 & 2219 & Hollow stem borehole w/SPT & $12 / 21 / 1998$ & 77.1 & 339748 & 6785829 & $\checkmark$ & $\checkmark$ & $\bar{\nabla}$ & $\bar{\nabla}$ & \\
\hline 1824 & 2220 & Hollow stem borehole w/SPT & $12 / 22 / 1998$ & 76.4 & 339726 & 6785830 & $\checkmark$ & $\nabla$ & $\checkmark$ & $\square$ & $\square$ \\
\hline 1824 & 2221 & Hollow stem borehole w/SPT & $9 / 29 / 1998$ & 50.9 & 339740 & 6785841 & $\checkmark$ & $\checkmark$ & $\nabla$ & $\square$ & $\square$ \\
\hline 1824 & FT027 & Solid stem borehole (no cores or SP & $9 / 19 / 1968$ & 23.0 & 340044 & 6785870 & $\square$ & $\square$ & $\square$ & $\square$ & $\square$ \\
\hline 1824 & FT028 & Solid stem borehole (no cores or SP & $9 / 19 / 1968$ & 23.0 & 340078 & 6785781 & $\square$ & $\square$ & $\square$ & $\square$ & $\square$ \\
\hline 1824 & FT029 & Solid stem borehole (no cores or SP & $9 / 19 / 1968$ & 23.0 & 340118 & 6785868 & $\square$ & $\square$ & $\square$ & $\square$ & $\square$ \\
\hline 1824 & FT030 & Solid stem borehole (no cores or SP & $9 / 20 / 1968$ & 23.0 & 340151 & 6785958 & $\square$ & $\square$ & $\square$ & $\square$ & $\square$ \\
\hline 1824 & FT031 & Solid stem borehole (no cores or SP & $9 / 20 / 1968$ & 23.0 & 340203 & 6785788 & $\square$ & $\square$ & $\square$ & $\square$ & \\
\hline 1824 & FT032 & Solid stem borehole (no cores or SP & $9 / 20 / 1968$ & 23.0 & 340228 & 6785864 & $\square$ & $\square$ & $\square$ & $\square$ & $\square$ \\
\hline 1824 & FT036 & Solid stem borehole (no cores or SP & $9 / 19 / 1968$ & 23.0 & 340004 & 6785950 & $\checkmark$ & $\square$ & $\square$ & $\square$ & $\square$ \\
\hline 1824 & FT045 & Hollow stem borehole w/SPT & $6 / 16 / 1977$ & 45.8 & 340234 & 6785624 & $\checkmark$ & $\nabla$ & $\checkmark$ & $\square$ & $\square$ \\
\hline 1824 & FT046 & Hollow stem borehole w/SPT & $6 / 17 / 1977$ & 51.0 & 340175 & 6785663 & $\checkmark$ & $\checkmark$ & $\checkmark$ & 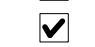 & $\square$ \\
\hline 1824 & FU005 & Solid stem borehole (no cores or SP & $9 / 19 / 1968$ & 23.0 & 339822 & 6785946 & $\square$ & $\square$ & $\square$ & $\square$ & $\square$ \\
\hline 1824 & FU006 & Solid stem borehole (no cores or SP & $9 / 19 / 1968$ & 23.0 & 339909 & 6785875 & $\square$ & $\square$ & $\square$ & $\square$ & $\square$ \\
\hline 1824 & GC001 & Solid stem borehole (no cores or SP & $9 / 18 / 1968$ & 23.0 & 339745 & 6786254 & $\checkmark$ & $\square$ & $\square$ & $\nabla$ & $\square$ \\
\hline 1824 & GC002 & Solid stem borehole (no cores or SP & $9 / 18 / 1968$ & 23.0 & 339874 & 6786328 & $\checkmark$ & $\square$ & $\square$ & $\square$ & $\square$ \\
\hline
\end{tabular}




\begin{tabular}{|c|c|c|c|c|c|c|c|c|c|c|c|}
\hline \multirow[b]{2}{*}{$\begin{array}{c}\text { MOA } \\
\text { grid }\end{array}$} & \multirow[b]{2}{*}{$\begin{array}{l}\text { DGGS } \\
\text { number }\end{array}$} & \multirow[b]{2}{*}{ Hole Type } & \multirow[b]{2}{*}{$\begin{array}{c}\text { Date } \\
\text { Completed }\end{array}$} & \multirow[b]{2}{*}{$\begin{array}{c}\text { Total } \\
\text { depth }(\mathrm{ft})\end{array}$} & \multicolumn{2}{|c|}{ UTM coordinates (m) } & \multicolumn{5}{|c|}{ Data entered } \\
\hline & & & & & East & North & Lithology & SPT & $\begin{array}{l}\text { Sample } \\
\text { test }\end{array}$ & $\begin{array}{l}\text { Water } \\
\text { level }\end{array}$ & Velocity \\
\hline 1824 & GC003 & Solid stem borehole (no cores or SP & $9 / 18 / 1968$ & 23.0 & 339790 & 6786084 & $\checkmark$ & & $\square$ & $\square$ & \\
\hline 1824 & GC004 & Solid stem borehole (no cores or SP & $9 / 18 / 1968$ & 23.0 & 339845 & 6786156 & $\square$ & & $\square$ & $\square$ & \\
\hline 1824 & GC005 & Solid stem borehole (no cores or SP & $9 / 18 / 1968$ & 23.0 & 339897 & 6786249 & $\square$ & & $\square$ & $\square$ & \\
\hline 1824 & GC006 & Solid stem borehole (no cores or SP & $9 / 18 / 1968$ & 23.0 & 339925 & 6786164 & $\checkmark$ & $\square$ & $\square$ & $\square$ & $\square$ \\
\hline 1824 & GC007 & Solid stem borehole (no cores or SP & $9 / 19 / 1968$ & 23.0 & 339886 & 6786012 & $\square$ & & $\square$ & $\square$ & $\square$ \\
\hline 1824 & GC008 & Solid stem borehole (no cores or SP & $9 / 18 / 1968$ & 23.0 & 339958 & 6786083 & $\square$ & & $\square$ & $\square$ & \\
\hline 1824 & GC009 & Solid stem borehole (no cores or SP & $9 / 19 / 1968$ & 23.0 & 339983 & 6786022 & $\square$ & $\square$ & $\square$ & $\square$ & $\square$ \\
\hline 1824 & GD003 & Solid stem borehole (no cores or SP & $9 / 18 / 1968$ & 23.0 & 340094 & 6786111 & $\square$ & & $\square$ & $\square$ & \\
\hline 1824 & GD004 & Solid stem borehole (no cores or SP & $9 / 19 / 1968$ & 23.0 & 340056 & 6786030 & $\square$ & $\square$ & $\square$ & $\square$ & $\square$ \\
\hline 1824 & GD005 & Solid stem borehole (no cores or SP & $9 / 18 / 1968$ & 23.0 & 340247 & 6786094 & $\square$ & & $\square$ & $\square$ & \\
\hline 1824 & GD006 & Solid stem borehole (no cores or SP & $9 / 20 / 1968$ & 23.0 & 340204 & 6786022 & $\square$ & $\square$ & $\square$ & $\square$ & $\square$ \\
\hline 1825 & 2177 & Hollow stem borehole w/SPT & $5 / 23 / 1995$ & 64.0 & 340422 & 6785675 & $\checkmark$ & $\bar{\nabla}$ & $\square$ & $\bar{\nabla}$ & $\square$ \\
\hline 1825 & 2178 & Hollow stem borehole w/SPT & $10 / 18 / 1995$ & 67.0 & 340417 & 6785739 & $\checkmark$ & $\checkmark$ & $\square$ & $\checkmark$ & \\
\hline 1825 & 2179 & Hollow stem borehole w/SPT & $10 / 19 / 1995$ & 67.0 & 340457 & 6785703 & $\checkmark$ & $\checkmark$ & $\square$ & $\nabla$ & $\square$ \\
\hline 1825 & FS016 & Solid stem borehole (no cores or SP & $9 / 17 / 1968$ & 22.0 & 341016 & 6785844 & $\square$ & & $\square$ & $\square$ & $\checkmark$ \\
\hline 1825 & FS018 & Solid stem borehole (no cores or SP & $9 / 16 / 1968$ & 23.0 & 341055 & 6785688 & $\checkmark$ & & $\square$ & 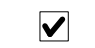 & $\square$ \\
\hline 1825 & FT016 & Solid stem borehole (no cores or SP & $9 / 12 / 1968$ & 23.0 & 340515 & 6785990 & $\square$ & & $\square$ & $\square$ & - \\
\hline 1825 & FT017 & Solid stem borehole (no cores or SP & $9 / 12 / 1968$ & 23.0 & 340472 & 6785931 & $\square$ & & $\square$ & $\square$ & \\
\hline 1825 & FT018 & Solid stem borehole (no cores or SP & $9 / 16 / 1968$ & 23.0 & 340602 & 6785932 & $\square$ & & $\square$ & $\square$ & \\
\hline 1825 & FT019 & Solid stem borehole (no cores or SP & $9 / 17 / 1968$ & 23.0 & 340408 & 6785850 & $\square$ & & $\square$ & $\square$ & $\checkmark$ \\
\hline 1825 & FT020 & Solid stem borehole (no cores or SP & $9 / 17 / 1968$ & 23.0 & 340537 & 6785863 & $\square$ & $\square$ & $\square$ & $\square$ & $\square$ \\
\hline 1825 & FT021 & Solid stem borehole (no cores or SP & $9 / 12 / 1968$ & 23.0 & 340511 & 6785778 & $\checkmark$ & $\square$ & $\square$ & $\square$ & $\square$ \\
\hline 1825 & FT022 & Solid stem borehole (no cores or SP & $9 / 12 / 1968$ & 23.0 & 340647 & 6785776 & $\square$ & & $\square$ & $\square$ & $\square$ \\
\hline 1825 & FT023 & Solid stem borehole (no cores or SP & $9 / 17 / 1968$ & 23.0 & 340702 & 6785864 & $\square$ & & $\square$ & $\square$ & $\square$ \\
\hline 1825 & FT024 & Solid stem borehole (no cores or SP & $9 / 16 / 1967$ & 23.0 & 340803 & 6785762 & $\checkmark$ & & $\square$ & $\square$ & $\square$ \\
\hline 1825 & FT025 & Solid stem borehole (no cores or SP & $9 / 17 / 1968$ & 23.0 & 340893 & 6785848 & $\square$ & & $\square$ & $\square$ & \\
\hline 1825 & FT026 & Solid stem borehole (no cores or SP & $9 / 16 / 1968$ & 23.0 & 340948 & 6785760 & $\square$ & & $\square$ & $\square$ & $\square$ \\
\hline 1825 & FT033 & Solid stem borehole (no cores or SP & $9 / 20 / 1968$ & 23.0 & 340295 & 6785969 & $\checkmark$ & & $\square$ & $\nabla$ & \\
\hline 1825 & FT034 & Solid stem borehole (no cores or SP & $9 / 16 / 1967$ & 23.0 & 340324 & 6785782 & $\square$ & $\square$ & $\square$ & $\square$ & $\square$ \\
\hline 1825 & FT035 & Solid stem borehole (no cores or SP & $9 / 23 / 1968$ & 33.0 & 340402 & 6785650 & $\checkmark$ & $\square$ & $\square$ & $\square$ & $\square$ \\
\hline 1825 & GD001 & Solid stem borehole (no cores or SP & $9 / 18 / 1968$ & 23.0 & 340392 & 6786103 & $\square$ & $\square$ & $\square$ & $\square$ & $\square$ \\
\hline 1825 & GD002 & Solid stem borehole (no cores or SP & $9 / 12 / 1968$ & 23.0 & 340556 & 6786048 & $\nabla$ & $\square$ & $\square$ & $\checkmark$ & $\square$ \\
\hline 1825 & GD007 & Solid stem borehole (no cores or SP & $9 / 23 / 1968$ & 23.0 & 340330 & 6786032 & $\square$ & $\square$ & $\square$ & $\square$ & $\square$ \\
\hline 1825 & GE005 & Solid stem borehole (no cores or SP & $4 / 27 / 1976$ & 10.0 & 341007 & 6786078 & $\square$ & $\square$ & $\square$ & $\square$ & $\square$ \\
\hline 1826 & 2315 & Hollow stem borehole w/SPT & 2/4/1969 & 51.3 & 341883 & 6786304 & $\checkmark$ & $\checkmark$ & $\square$ & $\nabla$ & $\square$ \\
\hline 1826 & 2316 & Hollow stem borehole w/SPT & 2/6/1969 & 51.5 & 341804 & 6785743 & $\checkmark$ & $\checkmark$ & $\checkmark$ & $\checkmark$ & $\square$ \\
\hline
\end{tabular}




\begin{tabular}{|c|c|c|c|c|c|c|c|c|c|c|c|}
\hline \multirow[b]{2}{*}{$\begin{array}{l}\text { MOA } \\
\text { grid }\end{array}$} & \multirow[b]{2}{*}{$\begin{array}{r}\text { DGGS } \\
\text { number }\end{array}$} & \multirow[b]{2}{*}{ Hole Type } & \multirow[b]{2}{*}{$\begin{array}{c}\text { Date } \\
\text { Completed }\end{array}$} & \multirow[b]{2}{*}{$\begin{array}{c}\text { Total } \\
\text { depth (ft) }\end{array}$} & \multicolumn{2}{|c|}{ UTM coordinates (m) } & \multicolumn{5}{|c|}{ Data entered } \\
\hline & & & & & East & North & Lithology & SPT & $\begin{array}{c}\text { Sample } \\
\text { test }\end{array}$ & $\begin{array}{l}\text { Water } \\
\text { level }\end{array}$ & Velocity \\
\hline 1826 & 2336 & Hollow stem borehole w/SPT & $3 / 6 / 1969$ & 31.5 & 341875 & 6786136 & & $\square$ & $\square$ & $\square$ & $\square$ \\
\hline 1826 & 2337 & Hollow stem borehole w/SPT & $3 / 6 / 1969$ & 31.5 & 341861 & 6786038 & $\square$ & $\square$ & $\square$ & $\square$ & $\square$ \\
\hline 1826 & 2338 & Hollow stem borehole w/SPT & $3 / 5 / 1969$ & 31.5 & 341822 & 6785955 & & $\square$ & $\square$ & $\square$ & $\square$ \\
\hline 1826 & 2339 & Hollow stem borehole w/SPT & $3 / 4 / 1969$ & 31.5 & 341790 & 6785859 & L & $\square$ & $\square$ & $\square$ & $\square$ \\
\hline 1826 & 2340 & Hollow stem borehole w/SPT & $3 / 4 / 1969$ & 36.5 & 341827 & 6785625 & & $\square$ & $\square$ & $\square$ & $\square$ \\
\hline 1826 & FS017 & Solid stem borehole (no cores or SP & 9/16/1968 & 23.0 & 341089 & 6785748 & $\square$ & $\square$ & $\square$ & $\square$ & $\square$ \\
\hline 1826 & FS024 & Hollow stem borehole w/SPT & $4 / 26 / 1976$ & 10.0 & 341583 & 6785807 & $\square$ & $\square$ & $\square$ & $\square$ & $\square$ \\
\hline 1826 & FS025 & Solid stem borehole (no cores or SP & $4 / 26 / 1976$ & 10.0 & 341528 & 6785768 & & $\square$ & $\square$ & $\square$ & $\square$ \\
\hline 1826 & FS026 & Hollow stem borehole w/SPT & $4 / 27 / 1976$ & 16.5 & 341500 & 6785734 & & $\square$ & $\square$ & $\square$ & $\square$ \\
\hline 1826 & FS027 & Hollow stem borehole w/SPT & 4/26/1976 & 10.0 & 341474 & 6785750 & $\square$ & $\square$ & $\square$ & $\square$ & $\square$ \\
\hline 1826 & FS028 & Hollow stem borehole w/SPT & $4 / 27 / 1976$ & 16.5 & 341472 & 6785722 & $\nabla$ & $\square$ & $\nabla$ & $\checkmark$ & $\square$ \\
\hline 1826 & FS029 & Hollow stem borehole w/SPT & 4/27/1976 & 11.5 & 341438 & 6785719 & - & $\square$ & $\square$ & $\square$ & $\square$ \\
\hline 1826 & FS030 & Solid stem borehole (no cores or SP & $4 / 26 / 1976$ & 10.0 & 341425 & 6785736 & & $\square$ & $\square$ & $\square$ & $\square$ \\
\hline 1826 & FS031 & Hollow stem borehole w/SPT & $4 / 26 / 1976$ & 11.5 & 341342 & 6785726 & & $\square$ & $\square$ & $\square$ & $\square$ \\
\hline 1826 & FS032 & Solid stem borehole (no cores or SP & $4 / 26 / 1976$ & 10.0 & 341299 & 6785772 & & $\square$ & $\square$ & $\square$ & $\square$ \\
\hline 1826 & FS033 & Solid stem borehole (no cores or SP & $4 / 26 / 1976$ & 10.0 & 341264 & 6785815 & & $\square$ & $\square$ & $\square$ & $\square$ \\
\hline 1826 & FS034 & Solid stem borehole (no cores or SP & $4 / 26 / 1976$ & 10.0 & 341239 & 6785868 & & $\square$ & $\square$ & $\square$ & $\square$ \\
\hline 1826 & FS035 & Hollow stem borehole w/SPT & $4 / 26 / 1976$ & 11.5 & 341206 & 6785927 & & $\square$ & $\square$ & $\square$ & $\square$ \\
\hline 1826 & FS036 & Hollow stem borehole w/SPT & $4 / 26 / 1976$ & 11.5 & 341191 & 6785946 & $\square$ & $\square$ & $\square$ & $\square$ & $\square$ \\
\hline 1826 & FS037 & Hollow stem borehole w/SPT & 4/27/1976 & 11.5 & 341167 & 6785988 & $\square$ & $\square$ & $\square$ & $\square$ & $\square$ \\
\hline 1826 & GE003 & Hollow stem borehole w/SPT & 4/27/1976 & 11.5 & 341117 & 6786022 & $\square$ & $\square$ & $\square$ & $\square$ & $\square$ \\
\hline 1826 & GE004 & Solid stem borehole (no cores or SP & $4 / 27 / 1976$ & 10.0 & 341084 & 6786056 & $\square$ & $\square$ & $\square$ & $\square$ & $\square$ \\
\hline 1827 & 1396 & Hollow stem borehole w/SPT & $8 / 26 / 1982$ & 31.5 & 342431 & 6786010 & $\nabla$ & $\checkmark$ & $\square$ & $\checkmark$ & $\square$ \\
\hline 1827 & 1397 & Hollow stem borehole w/SPT & $8 / 25 / 1982$ & 45.0 & 342510 & 6785987 & $\boldsymbol{V}$ & $\checkmark$ & $\square$ & $\checkmark$ & $\square$ \\
\hline 1827 & 1805 & Solid stem borehole (no cores or SP & $10 / 23 / 1981$ & 20.0 & 341958 & 6785936 & $\square$ & $\square$ & $\square$ & $\square$ & $\square$ \\
\hline 1827 & 1806 & Solid stem borehole (no cores or SP & $10 / 5 / 1981$ & 15.0 & 342103 & 6786184 & $\square$ & $\square$ & $\square$ & $\square$ & $\square$ \\
\hline 1827 & GF009 & Hollow stem borehole w/SPT & $5 / 13 / 1977$ & 27.0 & 342019 & 6786288 & $\square$ & $\square$ & $\square$ & $\square$ & $\square$ \\
\hline 1827 & GF010 & Hollow stem borehole w/SPT & $5 / 16 / 1977$ & 27.0 & 342017 & 6786246 & $\square$ & $\square$ & $\square$ & $\square$ & $\square$ \\
\hline 1827 & GF011 & Hollow stem borehole w/SPT & $5 / 16 / 1977$ & 52.0 & 342004 & 6786268 & $\boldsymbol{V}$ & $\checkmark$ & $\square$ & $\checkmark$ & $\square$ \\
\hline 1828 & 1412 & Hollow stem borehole w/SPT & 9/4/1978 & 21.0 & 343274 & 6785560 & $\square$ & $\square$ & $\square$ & $\square$ & $\square$ \\
\hline 1828 & 1859 & Hollow stem borehole w/SPT & $12 / 12 / 1990$ & 52.0 & 343498 & 6786209 & $\checkmark$ & $\checkmark$ & $\nabla$ & $\checkmark$ & $\square$ \\
\hline 1828 & 1860 & Hollow stem borehole w/SPT & $12 / 3 / 1991$ & 50.0 & 343432 & 6785833 & $\checkmark$ & $\checkmark$ & $\checkmark$ & $\checkmark$ & $\square$ \\
\hline 1828 & 1861 & Hollow stem borehole w/SPT & $11 / 20 / 1990$ & 42.0 & 343329 & 6785641 & $\nabla$ & $\nabla$ & $\nabla$ & $\nabla$ & $\square$ \\
\hline 1828 & 1862 & Hollow stem borehole w/SPT & $12 / 3 / 1990$ & 41.5 & 343370 & 6785628 & $\nabla$ & $\checkmark$ & $\square$ & $\checkmark$ & $\square$ \\
\hline 1828 & 1863 & Hollow stem borehole w/SPT & $12 / 4 / 1990$ & 41.0 & 343367 & 6785620 & $\square$ & $\square$ & $\square$ & $\square$ & $\square$ \\
\hline 1828 & 2186 & Hollow stem borehole w/SPT & $4 / 15 / 1988$ & 21.0 & 342699 & 6786151 & $\nabla$ & $\bar{v}$ & $\square$ & $\nabla$ & $\square$ \\
\hline
\end{tabular}




\begin{tabular}{|c|c|c|c|c|c|c|c|c|c|c|c|}
\hline \multirow[b]{2}{*}{$\begin{array}{c}\text { MOA } \\
\text { grid }\end{array}$} & \multirow[b]{2}{*}{$\begin{array}{c}\text { DGGS } \\
\text { number }\end{array}$} & \multirow[b]{2}{*}{ Hole Type } & \multirow[b]{2}{*}{$\begin{array}{c}\text { Date } \\
\text { Completed }\end{array}$} & \multirow[b]{2}{*}{$\begin{array}{c}\text { Total } \\
\text { depth (ft) }\end{array}$} & \multicolumn{2}{|c|}{ UTM coordinates (m) } & \multicolumn{5}{|c|}{ Data entered } \\
\hline & & & & & East & North & Lithology & SPT & $\begin{array}{c}\text { Sample } \\
\text { test }\end{array}$ & $\begin{array}{l}\text { Water } \\
\text { level }\end{array}$ & Velocity \\
\hline 1828 & 2187 & Hollow stem borehole w/SPT & $4 / 14 / 1988$ & 21.0 & 342818 & 6785947 & $\checkmark$ & $\nabla$ & $\square$ & $\nabla$ & $\square$ \\
\hline 1828 & 4401 & Water well & $3 / 5 / 1974$ & 260.0 & 342938 & 6786235 & $\boldsymbol{V}$ & $\square$ & $\square$ & $\nabla$ & $\square$ \\
\hline 1828 & 4458 & Water well & 8/14/1976 & 354.0 & 342974 & 6786017 & $\boldsymbol{V}$ & $\square$ & $\square$ & $\nabla$ & $\square$ \\
\hline 1828 & FQ009 & Solid stem borehole (no cores or SP & $10 / 1 / 1965$ & 24.0 & 343456 & 6785850 & $\square$ & $\square$ & $\square$ & $\square$ & $\square$ \\
\hline 1828 & FQ010 & Hollow stem borehole w/SPT & $1 / 19 / 1966$ & 95.0 & 343439 & 6785896 & $\checkmark$ & $\checkmark$ & $\square$ & $\nabla$ & $\square$ \\
\hline 1828 & FQ011 & Hollow stem borehole w/SPT & 2/7/1966 & 35.0 & 343451 & 6785880 & $\square$ & $\square$ & $\square$ & $\square$ & $\square$ \\
\hline 1828 & FQ012 & Hollow stem borehole w/SPT & $2 / 10 / 1966$ & 26.0 & 343435 & 6785846 & $\square$ & $\square$ & $\square$ & $\square$ & $\square$ \\
\hline 1828 & FQ013 & Hollow stem borehole w/SPT & 2/7/1966 & 50.0 & 343445 & 6785796 & $\boldsymbol{V}$ & $\nabla$ & $\nabla$ & $\nabla$ & $\square$ \\
\hline 1828 & FQ014 & Hollow stem borehole w/SPT & 2/8/1966 & 24.0 & 343461 & 6785804 & $\square$ & $\square$ & $\square$ & $\square$ & $\square$ \\
\hline 1828 & FQ015 & Hollow stem borehole w/SPT & $2 / 11 / 1966$ & 24.0 & 343462 & 6785766 & $\square$ & $\square$ & $\square$ & $\square$ & $\square$ \\
\hline 1828 & FQ016 & Hollow stem borehole w/SPT & $1 / 26 / 1966$ & 100.0 & 343455 & 6785756 & $\boldsymbol{V}$ & $\checkmark$ & $\square$ & $\nabla$ & $\square$ \\
\hline 1828 & FQ022 & Hollow stem borehole w/SPT & $11 / 2 / 1982$ & 31.5 & 343006 & 6785850 & $\boldsymbol{V}$ & $\checkmark$ & $\nabla$ & $\nabla$ & $\square$ \\
\hline 1828 & FQ023 & Hollow stem borehole w/SPT & $11 / 2 / 1982$ & 31.5 & 343119 & 6785850 & $\boldsymbol{V}$ & $\nabla$ & $\square$ & $\nabla$ & $\square$ \\
\hline 1828 & FQ024 & Hollow stem borehole w/SPT & $11 / 3 / 1982$ & 31.5 & 342997 & 6785735 & $\checkmark$ & $\checkmark$ & 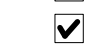 & $\checkmark$ & $\square$ \\
\hline 1828 & FQ025 & Hollow stem borehole w/SPT & $11 / 3 / 1982$ & 31.5 & 343104 & 6785732 & $\boldsymbol{V}$ & $\checkmark$ & $\nabla$ & $\nabla$ & $\square$ \\
\hline 1828 & FQ026 & Hollow stem borehole w/SPT & $11 / 3 / 1982$ & 31.0 & 343054 & 6785787 & $\checkmark$ & $\checkmark$ & $\checkmark$ & $\checkmark$ & $\square$ \\
\hline 1829 & 1416 & Hollow stem borehole w/SPT & $7 / 17 / 1978$ & 30.0 & 343948 & 6785451 & $\square$ & $\square$ & $\square$ & $\square$ & $\square$ \\
\hline 1829 & 1417 & Hollow stem borehole w/SPT & $7 / 17 / 1978$ & 30.0 & 344059 & 6785448 & $\square$ & $\square$ & $\square$ & $\square$ & $\square$ \\
\hline 1829 & 1418 & Hollow stem borehole w/SPT & 7/17/1978 & 30.0 & 344198 & 6785445 & $\square$ & $\square$ & $\square$ & $\square$ & $\square$ \\
\hline 1829 & 1858 & Hollow stem borehole w/SPT & $12 / 10 / 1990$ & 65.0 & 343500 & 6786157 & $\boldsymbol{V}$ & $\checkmark$ & $\nabla$ & $\nabla$ & $\square$ \\
\hline 1829 & 2175 & Hollow stem borehole w/SPT & $5 / 18 / 1999$ & 43.5 & 344301 & 6786232 & $\nabla$ & $\nabla$ & $\square$ & $\nabla$ & $\square$ \\
\hline 1829 & GH002 & Hollow stem borehole w/SPT & $3 / 27 / 1971$ & 24.5 & 344028 & 6786216 & $\boldsymbol{V}$ & $\nabla$ & $\square$ & $\square$ & $\square$ \\
\hline 1829 & GH021 & Cone Penetration Test (CPT) & $3 / 28 / 1986$ & 110.0 & 344252 & 6786029 & $\square$ & $\square$ & $\square$ & $\square$ & $\square$ \\
\hline 1830 & 1398 & Hollow stem borehole w/SPT & $8 / 25 / 1980$ & 51.5 & 344708 & 6785962 & $\nabla$ & $\checkmark$ & $\nabla$ & $\nabla$ & $\square$ \\
\hline 1830 & 1420 & Hollow stem borehole w/SPT & $12 / 9 / 1978$ & 15.0 & 345067 & 6785417 & & $\square$ & $\square$ & $\square$ & $\square$ \\
\hline 1830 & 1440 & Hollow stem borehole w/SPT & $2 / 10 / 1976$ & 14.0 & 344791 & 6785580 & ᄂ & $\square$ & $\square$ & $\square$ & $\square$ \\
\hline 1830 & 1441 & Hollow stem borehole w/SPT & $2 / 10 / 1976$ & 14.0 & 344855 & 6785580 & $\square$ & $\square$ & $\square$ & $\square$ & $\square$ \\
\hline 1830 & 1442 & Hollow stem borehole w/SPT & $2 / 10 / 1976$ & 20.0 & 344791 & 6785439 & & $\square$ & $\square$ & $\square$ & $\square$ \\
\hline 1830 & 1443 & Hollow stem borehole w/SPT & 2/10/1976 & 18.0 & 344855 & 6785438 & $\square$ & $\square$ & $\square$ & $\square$ & $\square$ \\
\hline 1830 & FO003 & Hollow stem borehole w/SPT & $7 / 30 / 1981$ & 26.5 & 345001 & 6785952 & $\square$ & $\square$ & $\square$ & $\square$ & $\square$ \\
\hline 1830 & FO004 & Hollow stem borehole w/SPT & $8 / 3 / 1981$ & 21.5 & 345052 & 6785892 & $\square$ & $\square$ & $\square$ & $\square$ & $\square$ \\
\hline 1830 & FO005 & Hollow stem borehole w/SPT & $8 / 4 / 1981$ & 51.0 & 345042 & 6785823 & 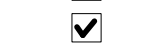 & $\checkmark$ & $\square$ & $\checkmark$ & $\square$ \\
\hline 1830 & FP001 & Hollow stem borehole w/SPT & $7 / 31 / 1981$ & 31.5 & 344990 & 6785880 & $\square$ & $\square$ & $\square$ & $\square$ & $\square$ \\
\hline 1830 & FP002 & Hollow stem borehole w/SPT & $8 / 3 / 1981$ & 21.5 & 344958 & 6785812 & $\square$ & $\square$ & $\square$ & $\square$ & $\square$ \\
\hline 1830 & FP004 & Hollow stem borehole w/SPT & $8 / 22 / 1980$ & 51.5 & 344701 & 6785983 & $\checkmark$ & $\checkmark$ & $\checkmark$ & $\nabla$ & $\square$ \\
\hline 1830 & GH006 & Solid stem borehole (no cores or SP & $12 / 10 / 1963$ & 12.0 & 344493 & 6786212 & $\square$ & $\square$ & $\square$ & $\square$ & $\square$ \\
\hline
\end{tabular}




\begin{tabular}{|c|c|c|c|c|c|c|c|c|c|c|c|}
\hline \multirow[b]{2}{*}{$\begin{array}{l}\text { MOA } \\
\text { grid }\end{array}$} & \multirow[b]{2}{*}{$\begin{array}{c}\text { DGGS } \\
\text { number }\end{array}$} & \multirow[b]{2}{*}{ Hole Type } & \multirow[b]{2}{*}{$\begin{array}{c}\text { Date } \\
\text { Completed }\end{array}$} & \multirow[b]{2}{*}{$\begin{array}{c}\text { Total } \\
\text { depth (ft) }\end{array}$} & \multicolumn{2}{|c|}{ UTM coordinates (m) } & \multicolumn{5}{|c|}{ Data entered } \\
\hline & & & & & East & North & Lithology & SPT & $\begin{array}{c}\text { Sample } \\
\text { test }\end{array}$ & $\begin{array}{l}\text { Water } \\
\text { level }\end{array}$ & Velocity \\
\hline 1830 & GH007 & Solid stem borehole (no cores or SP & $11 / 19 / 1963$ & 18.0 & 344652 & 6786212 & $\square$ & & $\square$ & $\square$ & \\
\hline 1830 & GH008 & Solid stem borehole (no cores or SP & $11 / 19 / 1963$ & 33.0 & 344779 & 6786200 & $\checkmark$ & $\square$ & $\square$ & $\square$ & $\square$ \\
\hline 1830 & GH009 & Solid stem borehole (no cores or SP & $11 / 19 / 1963$ & 13.0 & 344960 & 6786192 & $\square$ & 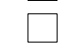 & $\square$ & $\square$ & \\
\hline 1830 & GH011 & Hollow stem borehole w/SPT & $7 / 29 / 1981$ & 31.5 & 344970 & 6786126 & $\square$ & $\square$ & $\square$ & $\square$ & $\checkmark$ \\
\hline 1830 & GH012 & Hollow stem borehole w/SPT & $7 / 31 / 1981$ & 50.0 & 344982 & 6786025 & $\checkmark$ & 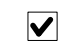 & $\square$ & $\checkmark$ & \\
\hline 1830 & GI008 & Solid stem borehole (no cores or SP & $11 / 19 / 1963$ & 18.0 & 345100 & 6786189 & $\square$ & $\square$ & $\square$ & $\square$ & $\square$ \\
\hline 1830 & GI024 & Hollow stem borehole w/SPT & 8/4/1981 & 21.5 & 345058 & 6786106 & $\square$ & $\square$ & $\square$ & $\square$ & $\square$ \\
\hline 1830 & GI025 & Hollow stem borehole w/SPT & $7 / 30 / 1981$ & 21.5 & 345061 & 6786022 & $\square$ & $\square$ & $\square$ & $\square$ & $\square$ \\
\hline 1831 & 1399 & Hollow stem borehole w/SPT & $11 / 9 / 1977$ & 30.5 & 345591 & 6786139 & $\checkmark$ & $\bar{\nabla}$ & $\square$ & $\square$ & $\square$ \\
\hline 1831 & 1822 & Solid stem borehole (no cores or SP & $10 / 27 / 1971$ & 18.0 & 345672 & 6785383 & $\square$ & & $\square$ & $\square$ & $\square$ \\
\hline 1831 & 1823 & Solid stem borehole (no cores or SP & $10 / 22 / 1971$ & 13.0 & 345859 & 6785369 & $\square$ & $\square$ & $\square$ & $\square$ & $\square$ \\
\hline 1831 & 4396 & Water well & $12 / 2 / 1968$ & 152.0 & 345315 & 6785510 & $\checkmark$ & $\square$ & $\square$ & 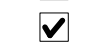 & $\square$ \\
\hline 1831 & FO002 & Hollow stem borehole w/SPT & $11 / 19 / 1984$ & 122.5 & 345081 & 6785558 & $\checkmark$ & $\checkmark$ & $\checkmark$ & $\checkmark$ & $\square$ \\
\hline 1831 & GI009 & Solid stem borehole (no cores or SP & $11 / 19 / 1963$ & 30.0 & 345441 & 6786174 & $\square$ & $\square$ & $\square$ & $\square$ & $\square$ \\
\hline 1831 & GI010 & Solid stem borehole (no cores or SP & 8/8/1963 & 13.0 & 345878 & 6786162 & $\square$ & $\square$ & $\square$ & $\square$ & $\square$ \\
\hline 1832 & 1349 & Hollow stem borehole, no SPT & $2 / 28 / 1967$ & 44.0 & 346330 & 6786025 & $\square$ & & $\square$ & $\square$ & 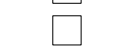 \\
\hline 1832 & 1400 & Hollow stem borehole w/SPT & $7 / 23 / 1982$ & 30.0 & 346602 & 6786058 & $\square$ & $\square$ & $\square$ & $\square$ & $\square$ \\
\hline 1832 & 1401 & Hollow stem borehole w/SPT & $1 / 27 / 1982$ & 60.0 & 346673 & 6786003 & $\checkmark$ & $\bar{\nabla}$ & $\checkmark$ & $\sqrt{V}$ & $\square$ \\
\hline 1832 & 1402 & Hollow stem borehole w/SPT & 2/3/1982 & 65.0 & 346652 & 6786009 & $\checkmark$ & $\bar{\nabla}$ & $\checkmark$ & 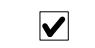 & \\
\hline 1832 & 1619 & Hollow stem borehole w/SPT & $2 / 22 / 1996$ & 41.5 & 346458 & 6786077 & $\square$ & $\square$ & $\square$ & $\square$ & $\square$ \\
\hline 1832 & 1620 & Hollow stem borehole w/SPT & $2 / 22 / 1996$ & 40.3 & 346454 & 6785999 & $\square$ & $\square$ & $\square$ & $\square$ & $\square$ \\
\hline 1832 & 1824 & Solid stem borehole (no cores or SP & 10/13/1971 & 33.0 & 346247 & 6785358 & $\square$ & $\square$ & $\square$ & $\square$ & $\square$ \\
\hline 1832 & 1825 & Solid stem borehole (no cores or SP & $10 / 28 / 1971$ & 32.0 & 346558 & 6785344 & $\square$ & $\square$ & $\square$ & $\square$ & \\
\hline 1832 & 4400 & Water well & $3 / 27 / 1965$ & 181.0 & 346383 & 6785990 & $\checkmark$ & $\square$ & $\square$ & $\bar{\nabla}$ & $\square$ \\
\hline 1832 & FN001 & Hollow stem borehole w/SPT & $12 / 27 / 1967$ & 50.0 & 346357 & 6785551 & $\checkmark$ & 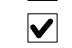 & $\nabla$ & $\sqrt{\square}$ & $\square$ \\
\hline 1832 & FN002 & Hollow stem borehole w/SPT & $1 / 2 / 1968$ & 46.5 & 346404 & 6785554 & $\square$ & $\square$ & $\square$ & $\square$ & $\square$ \\
\hline 1832 & FN003 & Hollow stem borehole w/SPT & $12 / 28 / 1967$ & 47.0 & 346365 & 6785521 & $\square$ & $\square$ & $\square$ & $\square$ & $\square$ \\
\hline 1832 & FN004 & Hollow stem borehole w/SPT & $12 / 29 / 1967$ & 52.0 & 346411 & 6785522 & $\checkmark$ & 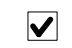 & $\nabla$ & $\bar{\square}$ & $\square$ \\
\hline 1832 & GJ001 & Hollow stem borehole w/SPT & $11 / 14 / 1973$ & 25.0 & 346328 & 6786090 & $\square$ & $\square$ & $\square$ & $\square$ & $\square$ \\
\hline 1832 & GJ001C & Cone Penetration Test (CPT) & $11 / 14 / 1973$ & 60.0 & 346328 & 6786090 & $\square$ & $\square$ & $\square$ & $\square$ & $\square$ \\
\hline 1832 & GJ023 & Solid stem borehole (no cores or SP & $7 / 13 / 1971$ & 8.0 & 346630 & 6786112 & $\square$ & $\square$ & $\square$ & $\square$ & $\square$ \\
\hline 1833 & 1446 & Hollow stem borehole w/SPT & $12 / 6 / 1977$ & 74.5 & 346843 & 6786085 & $\checkmark$ & $\checkmark$ & $\square$ & $\checkmark$ & $\square$ \\
\hline 1833 & 1447 & Hollow stem borehole w/SPT & $12 / 13 / 1977$ & 50.5 & 346833 & 6786054 & $\nabla$ & $\bar{\nabla}$ & $\square$ & $\bar{\nabla}$ & $\square$ \\
\hline 1833 & 1448 & Hollow stem borehole w/SPT & $12 / 9 / 1977$ & 70.5 & 346816 & 6786029 & $\checkmark$ & $\checkmark$ & $\square$ & $\checkmark$ & $\square$ \\
\hline 1833 & 1845 & Hollow stem borehole w/SPT & $6 / 2 / 1982$ & 21.0 & 347473 & 6785421 & $\square$ & $\square$ & $\square$ & $\square$ & $\square$ \\
\hline 1833 & FM001 & Hollow stem borehole w/SPT & $4 / 19 / 1963$ & 72.0 & 347496 & 6785756 & $\checkmark$ & $\checkmark$ & $\checkmark$ & $\checkmark$ & $\square$ \\
\hline
\end{tabular}




\begin{tabular}{|c|c|c|c|c|c|c|c|c|c|c|c|}
\hline \multirow[b]{2}{*}{$\begin{array}{c}\text { MOA } \\
\text { grid }\end{array}$} & \multirow[b]{2}{*}{$\begin{array}{l}\text { DGGS } \\
\text { number }\end{array}$} & \multirow[b]{2}{*}{ Hole Type } & \multirow[b]{2}{*}{$\begin{array}{c}\text { Date } \\
\text { Completed }\end{array}$} & \multirow[b]{2}{*}{$\begin{array}{c}\text { Total } \\
\text { depth }(\mathrm{ft})\end{array}$} & \multicolumn{2}{|c|}{ UTM coordinates (m) } & \multicolumn{5}{|c|}{ Data entered } \\
\hline & & & & & East & North & Lithology & SPT & $\begin{array}{c}\text { Sample } \\
\text { test }\end{array}$ & $\begin{array}{l}\text { Water } \\
\text { level }\end{array}$ & Velocity \\
\hline 1833 & FM003 & Cone Penetration Test (CPT) & $4 / 26 / 1963$ & 20.0 & 347486 & 6785731 & $\square$ & 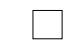 & $\square$ & $\square$ & $\square$ \\
\hline 1833 & GJ002 & Solid stem borehole (no cores or SP & $7 / 13 / 1971$ & 13.0 & 346779 & 6786122 & $\square$ & 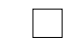 & $\square$ & $\square$ & $\square$ \\
\hline 1833 & GK001 & Solid stem borehole (no cores or SP & $7 / 14 / 1971$ & 20.0 & 347255 & 6786105 & $\square$ & 5 & $\square$ & $\square$ & \\
\hline 1833 & GK013 & Solid stem borehole (no cores or SP & $7 / 13 / 1971$ & 8.0 & 347084 & 6786104 & $\square$ & $\square$ & $\square$ & $\square$ & $\square$ \\
\hline 1834 & 1843 & Solid stem borehole (no cores or SP & 6/1/1982 & 35.0 & 347502 & 6785391 & $\square$ & $\square$ & $\square$ & $\square$ & $\square$ \\
\hline 1834 & 1844 & Hollow stem borehole w/SPT & $6 / 2 / 1982$ & 26.0 & 347500 & 6785414 & $\checkmark$ & $\checkmark$ & $\square$ & $\checkmark$ & $\square$ \\
\hline 1834 & 4394 & Water well & $7 / 24 / 1978$ & 122.0 & 347763 & 6785372 & $\checkmark$ & $\square$ & $\square$ & $\checkmark$ & $\square$ \\
\hline 1834 & 4395 & Water well & $11 / 1 / 1970$ & 206.0 & 347763 & 6785372 & $\checkmark$ & & $\square$ & $\nabla$ & \\
\hline 1834 & 4399 & Water well & $4 / 29 / 1969$ & 107.0 & 347679 & 6785871 & $\nabla$ & $\square$ & $\square$ & $\checkmark$ & $\square$ \\
\hline 1834 & FM002 & Cone Penetration Test (CPT) & $4 / 25 / 1963$ & 20.0 & 347517 & 6785760 & $\square$ & $\square$ & $\square$ & $\square$ & $\square$ \\
\hline 1834 & FM004 & Hollow stem borehole w/SPT & $4 / 23 / 1963$ & 51.0 & 347509 & 6785740 & $\checkmark$ & $\checkmark$ & $\checkmark$ & $\checkmark$ & $\square$ \\
\hline 1835 & 3031 & Water well & 3/1/1976 & 210.0 & 349099 & 6785500 & $\checkmark$ & $\square$ & $\square$ & $\bar{\nabla}$ & $\square$ \\
\hline 1835 & 4397 & Water well & $5 / 21 / 1980$ & 335.0 & 348778 & 6785700 & $\checkmark$ & 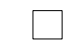 & $\square$ & $\square$ & $\square$ \\
\hline 1836 & 1403 & Hollow stem borehole, no SPT & $7 / 27 / 1983$ & 30.2 & 349217 & 6785863 & $\square$ & $\square$ & $\square$ & $\square$ & $\square$ \\
\hline 1836 & 1404 & Hollow stem borehole w/SPT & $7 / 18 / 1983$ & 31.5 & 349306 & 6785871 & $\checkmark$ & $\checkmark$ & $\square$ & $\square$ & $\square$ \\
\hline 1836 & 1405 & Hollow stem borehole, no SPT & $7 / 14 / 1983$ & 30.7 & 349360 & 6785793 & $\square$ & & $\square$ & $\square$ & $\square$ \\
\hline 1836 & 1406 & Hollow stem borehole, no SPT & $7 / 13 / 1983$ & 31.5 & 349221 & 6785694 & $\square$ & 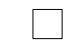 & $\square$ & $\square$ & $\square$ \\
\hline 1836 & 1407 & Hollow stem borehole w/SPT & $4 / 1 / 1984$ & 31.5 & 349216 & 6785735 & $\square$ & & $\square$ & $\square$ & $\square$ \\
\hline 1836 & 1408 & Hollow stem borehole w/SPT & $4 / 1 / 1984$ & 31.5 & 349252 & 6785709 & $\square$ & & $\square$ & $\square$ & \\
\hline 1836 & 1409 & Hollow stem borehole w/SPT & 4/1/1984 & 31.5 & 349223 & 6785676 & $\square$ & $\sqcup$ & $\square$ & $\square$ & $\square$ \\
\hline 1836 & 1410 & Hollow stem borehole w/SPT & $4 / 1 / 1984$ & 31.5 & 349195 & 6785688 & $\square$ & $\square$ & $\square$ & $\square$ & $\square$ \\
\hline 1837 & 3029 & Water well & $3 / 1 / 1976$ & 112.0 & 350704 & 6785587 & $\checkmark$ & $\square$ & $\square$ & $\nabla$ & $\square$ \\
\hline 1838 & 3027 & Water well & $6 / 15 / 1965$ & 225.0 & 351070 & 6785788 & $\checkmark$ & $\square$ & $\square$ & $\nabla$ & $\square$ \\
\hline 1839 & 1411 & Hollow stem borehole w/SPT & $4 / 16 / 1981$ & 44.0 & 352072 & 6785661 & $\checkmark$ & $\nabla$ & $\bar{\nabla}$ & $\checkmark$ & $\square$ \\
\hline 1840 & 1388 & Hollow stem borehole, no SPT & $6 / 12 / 1974$ & 15.0 & 352414 & 6785853 & $\square$ & & $\square$ & $\square$ & $\square$ \\
\hline 1840 & 1389 & Hollow stem borehole, no SPT & $6 / 12 / 1974$ & 20.0 & 352679 & 6785865 & $\square$ & & $\square$ & $\square$ & $\square$ \\
\hline 1841 & 1424 & Hollow stem borehole w/SPT & 6/9/1971 & 12.5 & 353739 & 6785392 & $\square$ & & $\square$ & $\square$ & $\square$ \\
\hline 1841 & 1425 & Hollow stem borehole w/SPT & $6 / 2 / 1971$ & 13.0 & 353807 & 6785172 & $\square$ & & $\square$ & $\square$ & \\
\hline 1841 & 3030 & Water well & $10 / 16 / 1968$ & 112.0 & 353888 & 6785205 & $\checkmark$ & $\square$ & $\square$ & $\checkmark$ & $\square$ \\
\hline 1842 & 1422 & Hollow stem borehole w/SPT & 7/7/1971 & 28.0 & 353963 & 6785201 & $\square$ & $\square$ & $\square$ & $\square$ & $\square$ \\
\hline 1842 & 1423 & Hollow stem borehole w/SPT & $6 / 11 / 1971$ & 13.0 & 353963 & 6785423 & $\checkmark$ & $\square$ & $\checkmark$ & $\square$ & $\square$ \\
\hline 1842 & 1426 & Hollow stem borehole w/SPT & $7 / 8 / 1971$ & 15.0 & 354019 & 6785143 & $\square$ & $\square$ & $\square$ & $\square$ & $\square$ \\
\hline 1842 & 1427 & Hollow stem borehole w/SPT & 7/8/1971 & 15.0 & 354067 & 6785088 & $\square$ & $\square$ & $\square$ & $\square$ & $\square$ \\
\hline 1842 & 1428 & Hollow stem borehole w/SPT & 7/9/1971 & 15.0 & 353963 & 6785028 & $\square$ & $\square$ & $\square$ & $\square$ & $\square$ \\
\hline 1842 & 1429 & Hollow stem borehole w/SPT & $6 / 16 / 1971$ & 12.5 & 354265 & 6785404 & $\square$ & $\square$ & $\square$ & $\square$ & $\square$ \\
\hline 1919 & 4462 & Oil \& gas exploratory well & $7 / 1 / 1968$ & 4744.0 & 335854 & 6785412 & $\checkmark$ & $\square$ & $\square$ & $\nabla$ & $\square$ \\
\hline
\end{tabular}




\begin{tabular}{|c|c|c|c|c|c|c|c|c|c|c|c|}
\hline \multirow[b]{2}{*}{$\begin{array}{l}\text { MOA } \\
\text { grid }\end{array}$} & \multirow[b]{2}{*}{$\begin{array}{l}\text { DGGS } \\
\text { number }\end{array}$} & \multirow[b]{2}{*}{ Hole Type } & \multirow[b]{2}{*}{$\begin{array}{c}\text { Date } \\
\text { Completed }\end{array}$} & \multirow[b]{2}{*}{$\begin{array}{c}\text { Total } \\
\text { depth }(\mathrm{ft})\end{array}$} & \multicolumn{2}{|c|}{ UTM coordinates (m) } & \multicolumn{5}{|c|}{ Data entered } \\
\hline & & & & & East & North & Lithology & SPT & $\begin{array}{c}\text { Sample } \\
\text { test }\end{array}$ & $\begin{array}{l}\text { Water } \\
\text { level }\end{array}$ & Velocity \\
\hline 1919 & FX041 & Solid stem borehole (no cores or SP & $6 / 5 / 1968$ & 9.0 & 336078 & 6785498 & $\square$ & & $\square$ & $\square$ & $\square$ \\
\hline 1919 & FX042 & Solid stem borehole (no cores or SP & $6 / 5 / 1968$ & 10.0 & 336195 & 6785495 & $\square$ & $\square$ & $\square$ & $\square$ & $\square$ \\
\hline 1919 & FX066 & Solid stem borehole (no cores or SP & 6/6/1968 & 18.0 & 336037 & 6785388 & $\square$ & & $\square$ & $\square$ & \\
\hline 1919 & FX067 & Solid stem borehole (no cores or SP & $6 / 6 / 1968$ & 13.0 & 336100 & 6785416 & $\square$ & 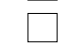 & $\square$ & $\square$ & $\square$ \\
\hline 1919 & FX068 & Solid stem borehole (no cores or SP & $6 / 6 / 1968$ & 13.0 & 336156 & 6785386 & $\square$ & $\square$ & $\square$ & $\square$ & $\square$ \\
\hline 1919 & FX070 & Solid stem borehole (no cores or SP & $5 / 28 / 1968$ & 23.0 & 336038 & 6785229 & $\square$ & $\square$ & $\square$ & $\square$ & $\square$ \\
\hline 1919 & FX071 & Pit or trench & $10 / 12 / 1967$ & 12.0 & 336104 & 6785257 & $\square$ & $\square$ & $\square$ & $\square$ & $\square$ \\
\hline 1919 & FX072 & Pit or trench & $10 / 11 / 1967$ & 11.0 & 336163 & 6785254 & $\square$ & & $\square$ & $\square$ & $\square$ \\
\hline 1919 & FX073 & Pit or trench & $10 / 12 / 1967$ & 11.0 & 336099 & 6785200 & $\square$ & 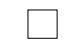 & $\square$ & $\square$ & $\square$ \\
\hline 1919 & FX074 & Solid stem borehole (no cores or SP & $5 / 28 / 1968$ & 13.0 & 336034 & 6785153 & $\square$ & $\square$ & $\square$ & $\square$ & $\square$ \\
\hline 1919 & FX075 & Pit or trench & $10 / 12 / 1967$ & 10.5 & 336098 & 6785159 & $\square$ & $\square$ & $\square$ & $\square$ & $\square$ \\
\hline 1919 & FX076 & Pit or trench & $10 / 12 / 1967$ & 11.0 & 336157 & 6785158 & $\square$ & & $\square$ & $\square$ & $\square$ \\
\hline 1919 & FX077 & Pit or trench & $10 / 11 / 1967$ & 9.5 & 336159 & 6785320 & $\square$ & 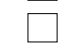 & $\square$ & $\square$ & $\square$ \\
\hline 1919 & FY002 & Solid stem borehole (no cores or SP & $5 / 28 / 1968$ & 13.0 & 335937 & 6785166 & $\square$ & $\square$ & $\square$ & $\square$ & $\square$ \\
\hline 1919 & FY003 & Solid stem borehole (no cores or SP & $12 / 4 / 1975$ & 40.0 & 335590 & 6785127 & $\square$ & 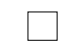 & $\square$ & $\square$ & $\square$ \\
\hline 1919 & FY004 & Solid stem borehole (no cores or SP & $12 / 5 / 1975$ & 40.0 & 335412 & 6785066 & $\square$ & & $\square$ & $\square$ & $\square$ \\
\hline 1919 & FY006 & Solid stem borehole (no cores or SP & $3 / 18 / 1977$ & 50.0 & 335746 & 6785052 & $\checkmark$ & & $\checkmark$ & $\nabla$ & $\square$ \\
\hline 1920 & FX001 & Pit or trench & $10 / 9 / 1967$ & 10.0 & 336290 & 6785357 & $\square$ & & $\square$ & $\square$ & $\square$ \\
\hline 1920 & FX002 & Pit or trench & $10 / 10 / 1967$ & 11.0 & 336345 & 6785360 & $\square$ & $\square$ & $\square$ & $\square$ & $\square$ \\
\hline 1920 & FX003 & Pit or trench & $10 / 10 / 1967$ & 11.5 & 336394 & 6785348 & $\square$ & $\square$ & $\square$ & $\square$ & $\square$ \\
\hline 1920 & FX004 & Pit or trench & $10 / 16 / 1967$ & 11.5 & 336450 & 6785345 & $\square$ & & $\square$ & $\square$ & $\square$ \\
\hline 1920 & FX005 & Pit or trench & $10 / 6 / 1967$ & 11.0 & 336496 & 6785344 & $\square$ & & $\square$ & $\square$ & $\square$ \\
\hline 1920 & FX006 & Pit or trench & $10 / 16 / 1967$ & 11.0 & 336543 & 6785348 & $\square$ & & $\square$ & $\square$ & $\square$ \\
\hline 1920 & FX007 & Pit or trench & $10 / 16 / 1967$ & 11.0 & 336602 & 6785346 & $\square$ & $\square$ & $\square$ & $\square$ & $\square$ \\
\hline 1920 & FX008 & Pit or trench & $10 / 16 / 1967$ & 11.0 & 336643 & 6785350 & $\square$ & & $\square$ & $\square$ & $\square$ \\
\hline 1920 & FX009 & Pit or trench & $10 / 9 / 1967$ & 11.5 & 336679 & 6785350 & $\square$ & & $\square$ & $\square$ & $\square$ \\
\hline 1920 & FX010 & Pit or trench & $10 / 16 / 1967$ & 11.5 & 336719 & 6785346 & $\square$ & $\square$ & $\square$ & $\square$ & $\square$ \\
\hline 1920 & FX011 & Pit or trench & $10 / 9 / 1967$ & 11.5 & 336775 & 6785342 & $\square$ & & $\square$ & $\square$ & $\square$ \\
\hline 1920 & FX012 & Solid stem borehole (no cores or SP & $6 / 13 / 1968$ & 18.0 & 336315 & 6785338 & $\square$ & & $\square$ & $\square$ & $\square$ \\
\hline 1920 & FX013 & Solid stem borehole (no cores or SP & $5 / 28 / 1968$ & 33.0 & 336415 & 6785326 & $\square$ & & $\square$ & $\square$ & $\square$ \\
\hline 1920 & FX014 & Pit or trench & $10 / 5 / 1967$ & 11.0 & 336292 & 6785309 & $\square$ & $\square$ & $\square$ & $\square$ & $\square$ \\
\hline 1920 & FX015 & Pit or trench & $10 / 16 / 1967$ & 11.5 & 336342 & 6785308 & $\square$ & $\square$ & $\square$ & $\square$ & $\square$ \\
\hline 1920 & FX016 & Pit or trench & $10 / 5 / 1967$ & 11.0 & 336397 & 6785305 & $\square$ & $\square$ & $\square$ & $\square$ & $\square$ \\
\hline 1920 & FX017 & Pit or trench & $10 / 16 / 1967$ & 11.0 & 336452 & 6785303 & $\square$ & $\square$ & $\square$ & $\square$ & $\square$ \\
\hline 1920 & FX018 & Pit or trench & $10 / 16 / 1967$ & 11.0 & 336498 & 6785302 & $\square$ & $\square$ & $\square$ & $\square$ & $\square$ \\
\hline 1920 & FX019 & Pit or trench & $10 / 5 / 1967$ & 11.0 & 336543 & 6785294 & $\square$ & $\square$ & $\square$ & $\square$ & $\square$ \\
\hline
\end{tabular}




\begin{tabular}{|c|c|c|c|c|c|c|c|c|c|c|c|}
\hline \multirow[b]{2}{*}{$\begin{array}{l}\text { MOA } \\
\text { grid }\end{array}$} & \multirow[b]{2}{*}{$\begin{array}{l}\text { DGGS } \\
\text { number }\end{array}$} & \multirow[b]{2}{*}{ Hole Type } & \multirow[b]{2}{*}{$\begin{array}{c}\text { Date } \\
\text { Completed }\end{array}$} & \multirow[b]{2}{*}{$\begin{array}{c}\text { Total } \\
\text { depth }(\mathrm{ft})\end{array}$} & \multicolumn{2}{|c|}{ UTM coordinates (m) } & \multicolumn{5}{|c|}{ Data entered } \\
\hline & & & & & East & North & Lithology & SPT & $\begin{array}{c}\text { Sample } \\
\text { test }\end{array}$ & $\begin{array}{l}\text { Water } \\
\text { level }\end{array}$ & Velocity \\
\hline 1920 & FX020 & Pit or trench & $10 / 10 / 1967$ & 11.0 & 336591 & 6785300 & $\square$ & & $\square$ & $\square$ & \\
\hline 1920 & FX021 & Pit or trench & $10 / 10 / 1967$ & 11.0 & 336639 & 6785298 & $\square$ & $\square$ & $\square$ & $\square$ & $\square$ \\
\hline 1920 & FX022 & Pit or trench & $10 / 10 / 1967$ & 10.5 & 336680 & 6785300 & $\square$ & & $\square$ & $\square$ & \\
\hline 1920 & FX023 & Pit or trench & $10 / 5 / 1967$ & 12.0 & 336719 & 6785296 & $\square$ & $\square$ & $\square$ & $\square$ & $\square$ \\
\hline 1920 & FX024 & Solid stem borehole (no cores or SP & $6 / 13 / 1968$ & 15.0 & 336392 & 6785274 & $\square$ & $\square$ & $\square$ & $\square$ & $\square$ \\
\hline 1920 & FX025 & Solid stem borehole (no cores or SP & $10 / 11 / 1967$ & 18.0 & 336290 & 6785240 & $\square$ & $\square$ & $\square$ & $\square$ & $\square$ \\
\hline 1920 & FX026 & Pit or trench & $10 / 9 / 1967$ & 11.0 & 336346 & 6785243 & $\square$ & $\square$ & $\square$ & $\square$ & $\square$ \\
\hline 1920 & FX027 & Solid stem borehole (no cores or SP & $10 / 11 / 1967$ & 22.0 & 336398 & 6785236 & $\square$ & & $\square$ & $\square$ & $\square$ \\
\hline 1920 & FX028 & Pit or trench & $10 / 9 / 1967$ & 11.5 & 336450 & 6785234 & $\square$ & $\checkmark$ & $\square$ & $\square$ & $\square$ \\
\hline 1920 & FX029 & Solid stem borehole (no cores or SP & $10 / 11 / 1967$ & 35.0 & 336502 & 6785230 & $\square$ & $\square$ & $\square$ & $\square$ & $\square$ \\
\hline 1920 & FX030 & Solid stem borehole (no cores or SP & $10 / 13 / 1967$ & 33.0 & 336551 & 6785242 & $\square$ & $\square$ & $\square$ & $\square$ & $\square$ \\
\hline 1920 & FX031 & Solid stem borehole (no cores or SP & $10 / 12 / 1967$ & 48.0 & 336594 & 6785240 & $\square$ & & $\square$ & $\square$ & $\square$ \\
\hline 1920 & FX032 & Solid stem borehole (no cores or SP & $10 / 12 / 1967$ & 48.0 & 336638 & 6785240 & $\square$ & & $\square$ & $\square$ & $\square$ \\
\hline 1920 & FX033 & Pit or trench & $10 / 9 / 1967$ & 11.0 & 336681 & 6785237 & $\square$ & $\square$ & $\square$ & $\square$ & $\square$ \\
\hline 1920 & FX034 & Solid stem borehole (no cores or SP & $10 / 12 / 1967$ & 18.5 & 336725 & 6785239 & $\square$ & & $\square$ & $\square$ & $\square$ \\
\hline 1920 & FX035 & Pit or trench & $10 / 9 / 1967$ & 11.0 & 336774 & 6785240 & $\square$ & & $\square$ & $\square$ & $\square$ \\
\hline 1920 & FX036 & Pit or trench & $10 / 6 / 1967$ & 1.0 & 336291 & 6785178 & $\square$ & & $\square$ & $\square$ & $\checkmark$ \\
\hline 1920 & FX037 & Pit or trench & $10 / 12 / 1967$ & 23.0 & 336344 & 6785174 & $\square$ & & $\square$ & $\square$ & $\square$ \\
\hline 1920 & FX038 & Pit or trench & $10 / 6 / 1967$ & 11.0 & 336395 & 6785174 & $\square$ & $\square$ & $\square$ & $\square$ & $\square$ \\
\hline 1920 & FX039 & Solid stem borehole (no cores or SP & $10 / 12 / 1967$ & 22.0 & 336447 & 6785174 & $\square$ & $\checkmark$ & $\square$ & $\square$ & $\square$ \\
\hline 1920 & FX040 & Pit or trench & $10 / 6 / 1967$ & 12.0 & 336497 & 6785172 & $\square$ & & $\square$ & $\square$ & $\square$ \\
\hline 1920 & FX043 & Solid stem borehole (no cores or SP & $6 / 5 / 1968$ & 8.0 & 336249 & 6785524 & $\square$ & & $\square$ & $\square$ & $\square$ \\
\hline 1920 & FX044 & Solid stem borehole (no cores or SP & $6 / 5 / 1968$ & 8.0 & 336248 & 6785465 & $\square$ & & $\square$ & $\square$ & $\square$ \\
\hline 1920 & FX045 & Solid stem borehole (no cores or SP & $6 / 5 / 1968$ & 13.0 & 336307 & 6785492 & $\square$ & $\checkmark$ & $\square$ & $\square$ & $\square$ \\
\hline 1920 & FX046 & Solid stem borehole (no cores or SP & $6 / 5 / 1968$ & 13.0 & 336360 & 6785513 & $\square$ & & $\square$ & $\square$ & $\square$ \\
\hline 1920 & FX047 & Solid stem borehole (no cores or SP & $6 / 3 / 1968$ & 23.0 & 336397 & 6785546 & $\square$ & & $\square$ & $\square$ & $\square$ \\
\hline 1920 & FX048 & Solid stem borehole (no cores or SP & $5 / 29 / 1968$ & 8.0 & 336439 & 6785570 & $\square$ & $\square$ & $\square$ & $\square$ & $\square$ \\
\hline 1920 & FX049 & Solid stem borehole (no cores or SP & $6 / 3 / 1968$ & 23.0 & 336489 & 6785532 & $\square$ & & $\square$ & $\square$ & $\square$ \\
\hline 1920 & FX050 & Solid stem borehole (no cores or SP & $5 / 29 / 1968$ & 18.0 & 336534 & 6785564 & $\square$ & & $\square$ & $\square$ & $\square$ \\
\hline 1920 & FX051 & Solid stem borehole (no cores or SP & $5 / 29 / 1968$ & 18.0 & 336580 & 6785548 & $\square$ & & $\square$ & $\square$ & $\square$ \\
\hline 1920 & FX052 & Solid stem borehole (no cores or SP & 6/6/1968 & 13.0 & 336232 & 6785394 & $\square$ & $\square$ & $\square$ & $\square$ & $\square$ \\
\hline 1920 & FX053 & Solid stem borehole (no cores or SP & $6 / 5 / 1968$ & 13.0 & 336359 & 6785452 & $\square$ & $\square$ & $\square$ & $\square$ & $\square$ \\
\hline 1920 & FX054 & Solid stem borehole (no cores or SP & $6 / 5 / 1968$ & 18.0 & 336412 & 6785480 & $\square$ & $\square$ & $\square$ & $\square$ & $\square$ \\
\hline 1920 & FX055 & Pit or trench & $10 / 10 / 1967$ & 10.5 & 336390 & 6785404 & $\square$ & $\square$ & $\square$ & $\square$ & $\square$ \\
\hline 1920 & FX056 & Pit or trench & $10 / 6 / 1967$ & 10.5 & 336496 & 6785403 & $\square$ & $\square$ & $\square$ & $\square$ & $\square$ \\
\hline 1920 & FX057 & Solid stem borehole (no cores or SP & $5 / 27 / 1968$ & 28.0 & 336545 & 6785398 & $\square$ & $\square$ & $\square$ & $\square$ & $\square$ \\
\hline
\end{tabular}




\begin{tabular}{|c|c|c|c|c|c|c|c|c|c|c|c|}
\hline \multirow[b]{2}{*}{$\begin{array}{c}\text { MOA } \\
\text { grid }\end{array}$} & \multirow[b]{2}{*}{$\begin{array}{l}\text { DGGS } \\
\text { number }\end{array}$} & \multirow[b]{2}{*}{ Hole Type } & \multirow[b]{2}{*}{$\begin{array}{c}\text { Date } \\
\text { Completed }\end{array}$} & \multirow[b]{2}{*}{$\begin{array}{c}\text { Total } \\
\text { depth (ft) }\end{array}$} & \multicolumn{2}{|c|}{ UTM coordinates (m) } & \multicolumn{5}{|c|}{ Data entered } \\
\hline & & & & & East & North & Lithology & SPT & $\begin{array}{c}\text { Sample } \\
\text { test }\end{array}$ & $\begin{array}{l}\text { Water } \\
\text { level }\end{array}$ & Velocity \\
\hline 1920 & FX058 & Solid stem borehole (no cores or SP & $5 / 27 / 1968$ & 38.0 & 336605 & 6785424 & & $\square$ & $\square$ & $\square$ & $\square$ \\
\hline 1920 & FX059 & Solid stem borehole (no cores or SP & $5 / 27 / 1968$ & 43.0 & 336650 & 6785440 & $\square$ & $\square$ & $\square$ & $\square$ & $\square$ \\
\hline 1920 & FX060 & Solid stem borehole (no cores or SP & $5 / 28 / 1968$ & 23.0 & 336729 & 6785472 & & $\square$ & $\square$ & $\square$ & $\square$ \\
\hline 1920 & FX061 & Solid stem borehole (no cores or SP & $5 / 29 / 1968$ & 18.0 & 336759 & 6785512 & L & $\square$ & $\square$ & $\square$ & $\square$ \\
\hline 1920 & FX062 & Solid stem borehole (no cores or SP & $5 / 29 / 1968$ & 23.0 & 336781 & 6785476 & & $\square$ & $\square$ & $\square$ & $\square$ \\
\hline 1920 & FX063 & Solid stem borehole (no cores or SP & $5 / 29 / 1968$ & 13.0 & 336823 & 6785504 & $\square$ & $\square$ & $\square$ & $\square$ & $\square$ \\
\hline 1920 & FX064 & Pit or trench & $10 / 16 / 1967$ & 10.5 & 336721 & 6785392 & & $\square$ & $\square$ & $\square$ & $\square$ \\
\hline 1920 & FX065 & Pit or trench & $10 / 16 / 1967$ & 10.5 & 336792 & 6785392 & & $\square$ & $\square$ & $\square$ & $\square$ \\
\hline 1920 & FX069 & Pit or trench & $10 / 10 / 1967$ & 10.0 & 336229 & 6785314 & & $\square$ & $\square$ & $\square$ & $\square$ \\
\hline 1921 & FW001 & Solid stem borehole (no cores or SP & 6/7/1968 & 18.0 & 337082 & 6785734 & & $\square$ & $\square$ & $\square$ & $\square$ \\
\hline 1921 & FW002 & Solid stem borehole (no cores or SP & $6 / 7 / 1968$ & 23.0 & 337171 & 6785736 & 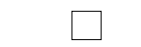 & $\square$ & $\square$ & $\square$ & $\square$ \\
\hline 1921 & FW003 & Solid stem borehole (no cores or SP & $6 / 7 / 1968$ & 18.0 & 337239 & 6785734 & & $\square$ & $\square$ & $\square$ & $\square$ \\
\hline 1921 & FW004 & Solid stem borehole (no cores or SP & $6 / 7 / 1968$ & 13.0 & 337268 & 6785732 & & $\square$ & $\square$ & $\square$ & $\square$ \\
\hline 1921 & FW006 & Solid stem borehole (no cores or SP & $6 / 12 / 1968$ & 18.0 & 337135 & 6785752 & $\square$ & $\square$ & $\square$ & $\square$ & $\square$ \\
\hline 1922 & FV002 & Solid stem borehole (no cores or SP & & 25.0 & 338262 & 6785268 & & $\square$ & $\square$ & $\square$ & $\square$ \\
\hline 1922 & FV003 & Solid stem borehole (no cores or SP & & 25.0 & 338415 & 6785263 & & $\square$ & $\square$ & $\square$ & $\square$ \\
\hline 1922 & FV004 & Solid stem borehole (no cores or SP & & 25.5 & 338585 & 6785260 & & $\square$ & $\square$ & $\square$ & $\square$ \\
\hline 1922 & FV017 & Solid stem borehole (no cores or SP & $11 / 8 / 1977$ & 15.0 & 338309 & 6785588 & & $\square$ & $\square$ & $\square$ & $\square$ \\
\hline 1922 & FV018 & Solid stem borehole (no cores or SP & $11 / 8 / 1977$ & 10.0 & 338278 & 6785670 & & $\square$ & $\square$ & $\square$ & $\square$ \\
\hline 1923 & FU007 & Solid stem borehole (no cores or SP & & 25.5 & 339056 & 6785238 & & $\square$ & $\square$ & $\square$ & $\square$ \\
\hline 1923 & FU012 & Solid stem borehole (no cores or SP & $1 / 27 / 1967$ & 31.5 & 339153 & 6785614 & & $\square$ & $\square$ & $\square$ & $\square$ \\
\hline 1923 & FU013 & Solid stem borehole (no cores or SP & $12 / 21 / 1966$ & 21.5 & 339260 & 6785594 & & $\square$ & $\square$ & $\square$ & $\square$ \\
\hline 1923 & FU014 & Solid stem borehole (no cores or SP & $9 / 28 / 1948$ & 7.0 & 339285 & 6785656 & & $\square$ & $\square$ & $\square$ & $\square$ \\
\hline 1923 & FU015 & Solid stem borehole (no cores or SP & $9 / 28 / 1948$ & 8.0 & 339342 & 6785628 & $\square$ & $\square$ & $\square$ & $\square$ & $\square$ \\
\hline 1923 & FU016 & Solid stem borehole (no cores or SP & $2 / 20 / 1973$ & 25.0 & 339028 & 6785529 & & $\square$ & $\square$ & $\square$ & $\square$ \\
\hline 1923 & FV005 & Solid stem borehole (no cores or SP & & 25.0 & 338768 & 6785259 & & $\square$ & $\square$ & $\square$ & $\square$ \\
\hline 1923 & FV006 & Solid stem borehole (no cores or SP & & 25.0 & 338918 & 6785242 & $\square$ & $\square$ & $\square$ & $\square$ & $\square$ \\
\hline 1923 & FV008 & Solid stem borehole (no cores or SP & $2 / 6 / 1973$ & 35.0 & 338729 & 6785586 & $\square$ & $\square$ & $\square$ & $\square$ & $\square$ \\
\hline 1923 & FV011 & Solid stem borehole (no cores or SP & $3 / 7 / 1973$ & 20.0 & 338756 & 6785508 & $\square$ & $\square$ & $\square$ & $\square$ & $\square$ \\
\hline 1923 & FV016 & Solid stem borehole (no cores or SP & $2 / 6 / 1973$ & 30.0 & 338926 & 6785508 & $\square$ & $\square$ & $\square$ & $\square$ & $\square$ \\
\hline 1924 & 2055 & Hollow stem borehole w/SPT & 2/23/1999 & 101.5 & 339744 & 6785620 & $\checkmark$ & $\checkmark$ & $\nabla$ & $\nabla$ & $\nabla$ \\
\hline 1924 & 2118 & Hollow stem borehole w/SPT & 2/3/1999 & 51.5 & 339729 & 6785607 & 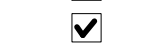 & $\checkmark$ & $\checkmark$ & $\square$ & $\square$ \\
\hline 1924 & 2215 & Hollow stem borehole w/SPT & $8 / 10 / 1996$ & 82.0 & 339754 & 6785623 & $\boldsymbol{V}$ & $\bar{v}$ & $\square$ & $\bar{\nabla}$ & $\square$ \\
\hline 1924 & FT037 & Solid stem borehole (no cores or SP & & 25.0 & 340030 & 6785200 & $\square$ & $\square$ & $\square$ & $\square$ & $\square$ \\
\hline 1924 & FT038 & Solid stem borehole (no cores or SP & & 25.0 & 340200 & 6785196 & $\square$ & $\square$ & $\square$ & $\square$ & $\square$ \\
\hline 1924 & FU008 & Solid stem borehole (no cores or SP & & 25.0 & 339582 & 6785210 & $\square$ & $\square$ & $\square$ & $\square$ & $\square$ \\
\hline
\end{tabular}




\begin{tabular}{|c|c|c|c|c|c|c|c|c|c|c|c|}
\hline \multirow[b]{2}{*}{$\begin{array}{l}\text { MOA } \\
\text { grid }\end{array}$} & \multirow[b]{2}{*}{$\begin{array}{l}\text { DGGS } \\
\text { number }\end{array}$} & \multirow[b]{2}{*}{ Hole Type } & \multirow[b]{2}{*}{$\begin{array}{c}\text { Date } \\
\text { Completed }\end{array}$} & \multirow[b]{2}{*}{$\begin{array}{c}\text { Total } \\
\text { depth }(\mathrm{ft})\end{array}$} & \multicolumn{2}{|c|}{ UTM coordinates (m) } & \multicolumn{5}{|c|}{ Data entered } \\
\hline & & & & & East & North & Lithology & SPT & $\begin{array}{c}\text { Sample } \\
\text { test }\end{array}$ & $\begin{array}{l}\text { Water } \\
\text { level }\end{array}$ & Velocity \\
\hline 1924 & FU009 & Solid stem borehole (no cores or SP & & 25.0 & 339722 & 6785208 & $\square$ & 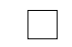 & $\square$ & $\square$ & 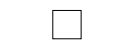 \\
\hline 1924 & FU010 & Solid stem borehole (no cores or SP & & 25.0 & 339876 & 6785202 & $\square$ & $\leftarrow$ & $\square$ & $\square$ & $\square$ \\
\hline 1925 & FT039 & Solid stem borehole (no cores or SP & & 25.0 & 340370 & 6785180 & $\square$ & $=$ & $\square$ & $\square$ & \\
\hline 1925 & FT040 & Solid stem borehole (no cores or SP & & 25.0 & 340557 & 6785177 & $\square$ & $\square$ & $\square$ & $\square$ & $\square$ \\
\hline 1925 & FT041 & Solid stem borehole (no cores or SP & & 24.5 & 340689 & 6785172 & $\square$ & $\square$ & $\square$ & $\square$ & $\square$ \\
\hline 1925 & FT042 & Solid stem borehole (no cores or SP & & 20.0 & 340817 & 6785166 & $\square$ & & $\square$ & $\square$ & $\square$ \\
\hline 1925 & FT043 & Solid stem borehole (no cores or SP & & 20.0 & 340936 & 6785160 & $\square$ & $\square$ & $\square$ & $\square$ & $\square$ \\
\hline 1926 & 2225 & Hollow stem borehole w/SPT & $7 / 31 / 1974$ & 30.5 & 341318 & 6784883 & $\checkmark$ & $\checkmark$ & $\checkmark$ & $\nabla$ & $\square$ \\
\hline 1926 & FS019 & Solid stem borehole (no cores or SP & & 15.3 & 341078 & 6785148 & $\square$ & $\square$ & $\square$ & $\square$ & $\square$ \\
\hline 1926 & FS020 & Solid stem borehole (no cores or SP & & 18.0 & 341209 & 6785141 & $\square$ & & $\square$ & $\square$ & \\
\hline 1926 & FS021 & Solid stem borehole (no cores or SP & & 15.0 & 341371 & 6785136 & $\square$ & $\square$ & $\square$ & $\square$ & $\square$ \\
\hline 1926 & FS022 & Solid stem borehole (no cores or SP & & 15.0 & 341535 & 6785127 & $\square$ & $\square$ & $\square$ & $\square$ & $\square$ \\
\hline 1927 & 2226 & Hollow stem borehole w/SPT & 2/4/1969 & 51.5 & 342448 & 6784833 & $\checkmark$ & $\checkmark$ & $\square$ & $\nabla$ & $\square$ \\
\hline 1927 & 2317 & Hollow stem borehole w/SPT & 2/1/1969 & 51.5 & 342087 & 6785237 & $\checkmark$ & $\checkmark$ & $\square$ & $\nabla$ & $\square$ \\
\hline 1927 & 2318 & Hollow stem borehole w/SPT & 2/4/1969 & 51.5 & 342593 & 6784919 & $\checkmark$ & $\bar{\square}$ & $\bar{\nabla}$ & $\checkmark$ & $\square$ \\
\hline 1927 & 2341 & Hollow stem borehole w/SPT & 3/3/1969 & 31.5 & 341876 & 6785488 & $\square$ & L & $\square$ & $\square$ & $\square$ \\
\hline 1927 & 2342 & Hollow stem borehole w/SPT & 3/3/1969 & 36.5 & 341951 & 6785390 & $\square$ & $\checkmark$ & $\square$ & $\square$ & $\square$ \\
\hline 1927 & 2343 & Hollow stem borehole w/SPT & 3/3/1969 & 36.5 & 342011 & 6785303 & $\square$ & & $\square$ & $\square$ & $\square$ \\
\hline 1927 & 2344 & Hollow stem borehole w/SPT & 2/2/1969 & 36.5 & 342194 & 6785181 & $\square$ & & $\square$ & $\square$ & \\
\hline 1927 & 2345 & Hollow stem borehole w/SPT & $2 / 25 / 1969$ & 36.5 & 342301 & 6785124 & $\square$ & $\square$ & $\square$ & $\square$ & $\square$ \\
\hline 1927 & 2346 & Hollow stem borehole w/SPT & $2 / 25 / 1969$ & 41.5 & 342407 & 6785067 & $\square$ & $\square$ & $\square$ & $\square$ & $\square$ \\
\hline 1927 & 2347 & Hollow stem borehole w/SPT & $2 / 26 / 1969$ & 41.5 & 342502 & 6784998 & $\square$ & $\square$ & $\square$ & $\square$ & $\square$ \\
\hline 1927 & FR001 & Hollow stem borehole, no SPT & & 15.0 & 342043 & 6785526 & $\square$ & $\square$ & $\square$ & $\square$ & $\square$ \\
\hline 1927 & FR002 & Hollow stem borehole, no SPT & & 20.0 & 342121 & 6785504 & $\square$ & $\square$ & $\square$ & $\square$ & $\square$ \\
\hline 1927 & FR003 & Hollow stem borehole, no SPT & & 20.0 & 342180 & 6785494 & $\square$ & $\square$ & $\square$ & $\square$ & $\square$ \\
\hline 1927 & FR004 & Hollow stem borehole, no SPT & & 15.0 & 342255 & 6785478 & $\square$ & $\square$ & $\square$ & $\square$ & $\square$ \\
\hline 1927 & FR005 & Hollow stem borehole, no SPT & & 15.0 & 342354 & 6785450 & $\square$ & $\square$ & $\square$ & $\square$ & $\square$ \\
\hline 1927 & FR006 & Hollow stem borehole, no SPT & & 15.0 & 342461 & 6785427 & $\square$ & $\square$ & $\square$ & $\square$ & \\
\hline 1927 & FR007 & Hollow stem borehole, no SPT & & 15.0 & 342575 & 6785410 & $\square$ & $\square$ & $\square$ & $\square$ & $\square$ \\
\hline 1928 & 1413 & Hollow stem borehole w/SPT & $9 / 13 / 1974$ & 20.0 & 343240 & 6785380 & $\square$ & $\square$ & $\square$ & $\square$ & $\square$ \\
\hline 1928 & 1414 & Hollow stem borehole w/SPT & $12 / 20 / 1966$ & 33.0 & 342909 & 6785373 & $\square$ & $\square$ & $\square$ & $\square$ & $\square$ \\
\hline 1928 & 1430 & Hollow stem borehole w/SPT & $2 / 25 / 1976$ & 70.0 & 343176 & 6785377 & $\nabla$ & $\checkmark$ & $\checkmark$ & $\checkmark$ & $\square$ \\
\hline 1928 & 1431 & Hollow stem borehole w/SPT & $2 / 23 / 1976$ & 70.0 & 343166 & 6785297 & $\checkmark$ & $\bar{\nabla}$ & $\bar{\nabla}$ & $\checkmark$ & $\square$ \\
\hline 1928 & 1432 & Hollow stem borehole w/SPT & $2 / 27 / 1976$ & 52.0 & 343234 & 6785373 & $\checkmark$ & $\checkmark$ & $\checkmark$ & $\boldsymbol{V}$ & $\square$ \\
\hline 1928 & 1433 & Hollow stem borehole w/SPT & $3 / 2 / 1976$ & 52.0 & 343204 & 6785264 & $\checkmark$ & $\checkmark$ & $\checkmark$ & $\nabla$ & $\square$ \\
\hline 1928 & 1434 & Hollow stem borehole w/SPT & 8/4/1986 & 60.0 & 342696 & 6785324 & $\checkmark$ & $\overline{\boldsymbol{V}}$ & $\bar{v}$ & $\bar{\nabla}$ & $\square$ \\
\hline
\end{tabular}




\begin{tabular}{|c|c|c|c|c|c|c|c|c|c|c|c|}
\hline \multirow[b]{2}{*}{$\begin{array}{l}\text { MOA } \\
\text { grid }\end{array}$} & \multirow[b]{2}{*}{$\begin{array}{l}\text { DGGS } \\
\text { number }\end{array}$} & \multirow[b]{2}{*}{ Hole Type } & \multirow[b]{2}{*}{$\begin{array}{c}\text { Date } \\
\text { Completed }\end{array}$} & \multirow[b]{2}{*}{$\begin{array}{c}\text { Total } \\
\text { depth }(\mathrm{ft})\end{array}$} & \multicolumn{2}{|c|}{ UTM coordinates (m) } & \multicolumn{5}{|c|}{ Data entered } \\
\hline & & & & & East & North & Lithology & SPT & $\begin{array}{c}\text { Sample } \\
\text { test }\end{array}$ & $\begin{array}{l}\text { Water } \\
\text { level }\end{array}$ & Velocity \\
\hline 1928 & 2068 & Hollow stem borehole w/SPT & $5 / 3 / 1998$ & 150.0 & 343234 & 6785334 & $\checkmark$ & $\checkmark$ & $\square$ & $\checkmark$ & \\
\hline 1928 & 2155 & Hollow stem borehole w/SPT & 3/19/1999 & 41.5 & 342674 & 6785083 & $\checkmark$ & $\checkmark$ & $\checkmark$ & $\square$ & $\square$ \\
\hline 1928 & 2348 & Hollow stem borehole w/SPT & $2 / 26 / 1969$ & 41.5 & 342684 & 6784839 & $\square$ & & $\square$ & $\square$ & \\
\hline 1928 & 2349 & Hollow stem borehole w/SPT & $2 / 27 / 1969$ & 41.5 & 342775 & 6784760 & $\square$ & $\square$ & $\square$ & $\square$ & $\square$ \\
\hline 1928 & 3036 & Water well & 4/1/1974 & 145.0 & 342961 & 6785057 & $\checkmark$ & $\square$ & $\square$ & $\nabla$ & $\square$ \\
\hline 1928 & 4392 & Water well & 6/29/1977 & 201.0 & 342662 & 6785070 & $\checkmark$ & $\square$ & $\square$ & $\checkmark$ & $\square$ \\
\hline 1928 & 4461 & Water well & $1 / 1 / 1966$ & 415.0 & 343029 & 6785240 & $\checkmark$ & $\square$ & $\square$ & $\square$ & $\square$ \\
\hline 1928 & FQ001 & Hollow stem borehole, no SPT & & 15.0 & 343072 & 6785354 & $\square$ & $\square$ & $\square$ & $\square$ & $\square$ \\
\hline 1928 & FQ002 & Hollow stem borehole, no SPT & & 15.0 & 343150 & 6785358 & $\square$ & $\square$ & $\square$ & $\square$ & $\square$ \\
\hline 1928 & FQ003 & Hollow stem borehole, no SPT & & 15.0 & 343191 & 6785368 & $\square$ & $\square$ & $\square$ & $\square$ & \\
\hline 1928 & FQ004 & Hollow stem borehole, no SPT & & 20.0 & 343247 & 6785368 & $\square$ & $\square$ & $\square$ & $\square$ & $\square$ \\
\hline 1928 & FQ005 & Hollow stem borehole, no SPT & & 25.0 & 343328 & 6785388 & $\square$ & $\square$ & $\square$ & $\square$ & $\square$ \\
\hline 1928 & FQ018 & Solid stem borehole (no cores or SP & 3/13/1978 & 15.0 & 343219 & 6785258 & $\square$ & $\square$ & $\square$ & $\square$ & $\square$ \\
\hline 1928 & FQ021 & Solid stem borehole (no cores or SP & $5 / 14 / 1974$ & 20.0 & 343219 & 6785194 & $\square$ & $\square$ & $\square$ & $\square$ & $\square$ \\
\hline 1928 & FR008 & Hollow stem borehole, no SPT & & 15.0 & 342692 & 6785390 & $\square$ & $\square$ & $\square$ & $\square$ & $\square$ \\
\hline 1928 & FR009 & Hollow stem borehole, no SPT & & 15.0 & 342816 & 6785363 & $\square$ & $\square$ & $\square$ & $\square$ & $\square$ \\
\hline 1928 & FR010 & Hollow stem borehole, no SPT & & 15.0 & 342965 & 6785326 & $\square$ & $\square$ & $\square$ & $\square$ & $\square$ \\
\hline 1929 & 1415 & Hollow stem borehole w/SPT & $7 / 17 / 1978$ & 30.0 & 343837 & 6785445 & $\square$ & $\square$ & $\square$ & $\square$ & $\square$ \\
\hline 1929 & 2066 & Hollow stem borehole w/SPT & $5 / 9 / 1991$ & 90.5 & 343605 & 6785393 & $\checkmark$ & 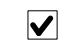 & $\square$ & $\nabla$ & $\square$ \\
\hline 1929 & 2067 & Hollow stem borehole w/SPT & $5 / 4 / 1991$ & 96.5 & 343581 & 6785386 & $\checkmark$ & $\bar{\nabla}$ & $\square$ & $\checkmark$ & $\square$ \\
\hline 1929 & FQ006 & Hollow stem borehole w/SPT & $3 / 23 / 1972$ & 62.0 & 343567 & 6785377 & $\checkmark$ & $\bar{\nabla}$ & $\bar{\nabla}$ & $\bar{\nabla}$ & \\
\hline 1929 & FQ007 & Hollow stem borehole w/SPT & $3 / 15 / 1972$ & 81.0 & 343612 & 6785358 & $\checkmark$ & $\checkmark$ & $\checkmark$ & $\nabla$ & $\square$ \\
\hline 1929 & FQ008 & Hollow stem borehole w/SPT & $3 / 17 / 1972$ & 61.5 & 343642 & 6785402 & $\checkmark$ & $\nabla$ & $\sqrt{V}$ & $\nabla$ & $\square$ \\
\hline 1930 & 1419 & Hollow stem borehole w/SPT & $7 / 24 / 1978$ & 20.0 & 344787 & 6785406 & $\square$ & $\square$ & $\square$ & $\square$ & $\square$ \\
\hline 1930 & 1435 & Hollow stem borehole w/SPT & $4 / 24 / 1987$ & 61.5 & 344782 & 6785232 & $\checkmark$ & $\checkmark$ & $\square$ & $\checkmark$ & $\square$ \\
\hline 1930 & 1436 & Hollow stem borehole w/SPT & $4 / 29 / 1987$ & 61.5 & 344830 & 6785235 & $\checkmark$ & $\checkmark$ & $\square$ & $\nabla$ & $\square$ \\
\hline 1930 & 1437 & Hollow stem borehole w/SPT & $4 / 30 / 1987$ & 76.5 & 344820 & 6785309 & $\checkmark$ & 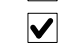 & $\checkmark$ & $\checkmark$ & $\square$ \\
\hline 1930 & 1438 & Hollow stem borehole w/SPT & $4 / 28 / 1987$ & 61.5 & 344768 & 6785354 & $\checkmark$ & $\bar{\nabla}$ & $\square$ & $\nabla$ & $\square$ \\
\hline 1930 & 1444 & Hollow stem borehole w/SPT & $3 / 2 / 1977$ & 19.5 & 345046 & 6785272 & $\square$ & $\square$ & $\square$ & $\square$ & $\square$ \\
\hline 1930 & 1445 & Hollow stem borehole w/SPT & $3 / 2 / 1977$ & 20.5 & 345042 & 6785252 & $\checkmark$ & $\bar{\nabla}$ & $\square$ & $\square$ & $\square$ \\
\hline 1930 & FP003 & Hollow stem borehole w/SPT & $1 / 10 / 1983$ & 41.5 & 344398 & 6785028 & $\square$ & $\square$ & $\square$ & $\square$ & $\square$ \\
\hline 1931 & 1421 & Hollow stem borehole w/SPT & $12 / 12 / 1977$ & 15.0 & 345491 & 6785360 & $\square$ & $\square$ & $\square$ & $\square$ & $\square$ \\
\hline 1931 & 4389 & Water well & $10 / 22 / 1974$ & 188.0 & 345290 & 6784613 & $\checkmark$ & $\square$ & $\square$ & $\bar{\square}$ & $\square$ \\
\hline 1931 & 4393 & Water well & $5 / 11 / 1976$ & 136.0 & 345218 & 6785019 & $\checkmark$ & $\square$ & $\square$ & $\nabla$ & $\square$ \\
\hline 1931 & EX001 & Hollow stem borehole w/SPT & $6 / 11 / 1981$ & 36.0 & 345616 & 6784843 & $\square$ & $\square$ & $\square$ & $\square$ & $\square$ \\
\hline 1931 & EX002 & Hollow stem borehole w/SPT & $10 / 1 / 1969$ & 17.5 & 345736 & 6784590 & $\square$ & $\square$ & $\square$ & $\square$ & $\square$ \\
\hline
\end{tabular}




\begin{tabular}{|c|c|c|c|c|c|c|c|c|c|c|c|}
\hline \multirow[b]{2}{*}{$\begin{array}{c}\text { MOA } \\
\text { grid }\end{array}$} & \multirow[b]{2}{*}{$\begin{array}{l}\text { DGGS } \\
\text { number }\end{array}$} & \multirow[b]{2}{*}{ Hole Type } & \multirow[b]{2}{*}{$\begin{array}{c}\text { Date } \\
\text { Completed }\end{array}$} & \multirow[b]{2}{*}{$\begin{array}{c}\text { Total } \\
\text { depth (ft) }\end{array}$} & \multicolumn{2}{|c|}{ UTM coordinates (m) } & \multicolumn{5}{|c|}{ Data entered } \\
\hline & & & & & East & North & Lithology & SPT & $\begin{array}{c}\text { Sample } \\
\text { test }\end{array}$ & $\begin{array}{l}\text { Water } \\
\text { level }\end{array}$ & Velocity \\
\hline 1931 & EX003 & Hollow stem borehole w/SPT & $10 / 1 / 1969$ & 15.5 & 345745 & 6784662 & $\square$ & $\square$ & $\square$ & $\square$ & $\square$ \\
\hline 1931 & EX004 & Hollow stem borehole w/SPT & $10 / 1 / 1969$ & 75.7 & 345760 & 6784636 & $\boldsymbol{V}$ & $\nabla$ & $\square$ & $\nabla$ & $\square$ \\
\hline 1931 & EX005 & Hollow stem borehole w/SPT & $10 / 1 / 1969$ & 15.5 & 345788 & 6784586 & & $\square$ & $\square$ & $\square$ & $\square$ \\
\hline 1931 & EX006 & Hollow stem borehole w/SPT & $10 / 1 / 1969$ & 14.5 & 345796 & 6784664 & 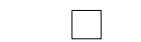 & $\square$ & $\square$ & $\square$ & $\square$ \\
\hline 1931 & FO001 & Hollow stem borehole, no SPT & & 16.5 & 345866 & 6785265 & $\square$ & $\square$ & $\square$ & $\square$ & $\square$ \\
\hline 1932 & 1449 & Hollow stem borehole w/SPT & $2 / 24 / 1983$ & 51.5 & 346601 & 6785156 & $\checkmark$ & $\checkmark$ & $\square$ & $\checkmark$ & $\square$ \\
\hline 1932 & 1826 & Solid stem borehole (no cores or SP & $10 / 20 / 1971$ & 13.0 & 346038 & 6785336 & $\square$ & $\square$ & $\square$ & $\square$ & $\square$ \\
\hline 1932 & 1850 & Hollow stem borehole w/SPT & $11 / 19 / 1973$ & 51.0 & 346489 & 6784539 & $\boldsymbol{V}$ & $\nabla$ & $\nabla$ & $\nabla$ & $\square$ \\
\hline 1933 & 4390 & Water well & $11 / 16 / 1981$ & 163.0 & 346998 & 6784662 & $\boldsymbol{v}$ & $\square$ & $\square$ & $\nabla$ & $\square$ \\
\hline 1933 & 4391 & Water well & $7 / 29 / 1969$ & 107.0 & 347022 & 6784847 & $\boldsymbol{V}$ & $\square$ & $\square$ & $\nabla$ & $\square$ \\
\hline 1933 & EY002 & Hollow stem borehole w/SPT & $2 / 17 / 1983$ & 29.5 & 346698 & 6784631 & $\square$ & $\square$ & $\square$ & $\square$ & $\square$ \\
\hline 1933 & EY003 & Hollow stem borehole w/SPT & $2 / 22 / 1983$ & 31.1 & 346701 & 6784716 & & $\square$ & $\square$ & $\square$ & $\square$ \\
\hline 1933 & EY004 & Hollow stem borehole w/SPT & $3 / 2 / 1983$ & 21.5 & 346709 & 6784948 & $\square$ & $\square$ & $\square$ & $\square$ & $\square$ \\
\hline 1933 & EY005 & Hollow stem borehole w/SPT & $3 / 2 / 1983$ & 16.5 & 346711 & 6784874 & $\square$ & $\square$ & $\square$ & $\square$ & $\square$ \\
\hline 1933 & EY006 & Hollow stem borehole w/SPT & $3 / 2 / 1983$ & 9.5 & 346703 & 6784796 & $\square$ & $\square$ & $\square$ & $\square$ & $\square$ \\
\hline 1934 & EZ001 & Hollow stem borehole w/SPT & $9 / 21 / 1983$ & 25.5 & 347500 & 6784680 & $\square$ & $\square$ & $\square$ & $\square$ & $\square$ \\
\hline 1934 & EZ002 & Hollow stem borehole w/SPT & $6 / 22 / 1984$ & 30.5 & 347586 & 6784705 & $\boldsymbol{V}$ & $\nabla$ & $\nabla$ & $\checkmark$ & $\square$ \\
\hline 1934 & EZ003 & Hollow stem borehole w/SPT & $6 / 22 / 1984$ & 30.0 & 347496 & 6784636 & $\square$ & $\square$ & $\square$ & $\square$ & $\square$ \\
\hline 1934 & EZ004 & Hollow stem borehole w/SPT & $6 / 21 / 1984$ & 30.5 & 347564 & 6784635 & $\boldsymbol{V}$ & $\checkmark$ & $\nabla$ & $\nabla$ & $\square$ \\
\hline 1934 & FM005 & Hollow stem borehole w/SPT & $11 / 16 / 1984$ & 49.0 & 347627 & 6785166 & $\boldsymbol{V}$ & $\nabla$ & $\nabla$ & $\nabla$ & $\square$ \\
\hline 1938 & 1450 & Hollow stem borehole w/SPT & $3 / 10 / 1995$ & 9.6 & 351457 & 6784646 & $\square$ & $\square$ & $\square$ & $\square$ & $\square$ \\
\hline 1938 & 1451 & Hollow stem borehole w/SPT & 3/9/1995 & 9.6 & 351452 & 6784609 & $\square$ & $\square$ & $\square$ & $\square$ & $\square$ \\
\hline 1938 & 1452 & Hollow stem borehole w/SPT & $3 / 17 / 1995$ & 9.6 & 351437 & 6784456 & $\square$ & $\square$ & $\square$ & $\square$ & $\square$ \\
\hline 1938 & 1453 & Hollow stem borehole w/SPT & $3 / 20 / 1995$ & 9.6 & 351424 & 6784459 & $\square$ & $\square$ & $\square$ & $\square$ & $\square$ \\
\hline 1939 & 4460 & Water well & $6 / 30 / 1969$ & 168.0 & 351503 & 6784345 & $\checkmark$ & $\square$ & $\square$ & $\checkmark$ & $\square$ \\
\hline 1941 & 3032 & Water well & $2 / 28 / 1971$ & 174.0 & 353595 & 6785000 & $\boldsymbol{V}$ & $\square$ & $\square$ & $\nabla$ & $\square$ \\
\hline 2017 & 1646 & Hollow stem borehole w/SPT & 9/7/1988 & 61.0 & 334833 & 6784258 & $\boldsymbol{V}$ & $\checkmark$ & 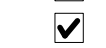 & 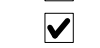 & $\square$ \\
\hline 2017 & 2112 & Hollow stem borehole, no SPT & $1 / 23 / 1975$ & 45.0 & 334939 & 6784368 & $\boldsymbol{V}$ & $\square$ & $\nabla$ & $\square$ & $\square$ \\
\hline 2018 & 2113 & Hollow stem borehole, no SPT & $7 / 30 / 1982$ & 50.0 & 335053 & 6784479 & $\boldsymbol{V}$ & $\square$ & $\nabla$ & $\nabla$ & $\square$ \\
\hline 2018 & EN001 & Solid stem borehole (no cores or SP & $12 / 17 / 1974$ & 40.0 & 335483 & 6784604 & $\square$ & $\square$ & $\square$ & $\square$ & $\square$ \\
\hline 2018 & ENO02 & Solid stem borehole (no cores or SP & $12 / 17 / 1974$ & 45.0 & 335481 & 6784321 & $\square$ & $\square$ & $\square$ & $\square$ & $\square$ \\
\hline 2018 & EN003 & Solid stem borehole (no cores or SP & $12 / 23 / 1974$ & 45.0 & 335269 & 6784320 & $\square$ & $\square$ & $\square$ & $\square$ & $\square$ \\
\hline 2018 & EN004 & Solid stem borehole (no cores or SP & $11 / 21 / 1975$ & 30.0 & 335310 & 6784648 & $\square$ & $\square$ & $\square$ & $\square$ & $\square$ \\
\hline 2018 & EN005 & Solid stem borehole (no cores or SP & $12 / 23 / 1974$ & 45.0 & 335267 & 6784495 & $\square$ & $\square$ & $\square$ & $\square$ & $\square$ \\
\hline 2018 & EN007 & Solid stem borehole (no cores or SP & $3 / 7 / 1977$ & 45.0 & 335604 & 6784545 & $\square$ & $\square$ & $\square$ & $\square$ & $\square$ \\
\hline 2018 & EN009 & Solid stem borehole (no cores or SP & $3 / 14 / 1977$ & 45.0 & 335207 & 6784318 & $\square$ & $\square$ & $\square$ & $\square$ & $\square$ \\
\hline
\end{tabular}




\begin{tabular}{|c|c|c|c|c|c|c|c|c|c|c|c|}
\hline \multirow[b]{2}{*}{$\begin{array}{c}\text { MOA } \\
\text { grid }\end{array}$} & \multirow[b]{2}{*}{$\begin{array}{c}\text { DGGS } \\
\text { number }\end{array}$} & \multirow[b]{2}{*}{ Hole Type } & \multirow[b]{2}{*}{$\begin{array}{c}\text { Date } \\
\text { Completed }\end{array}$} & \multirow[b]{2}{*}{$\begin{array}{c}\text { Total } \\
\text { depth (ft) }\end{array}$} & \multicolumn{2}{|c|}{ UTM coordinates (m) } & \multicolumn{5}{|c|}{ Data entered } \\
\hline & & & & & East & North & Lithology & SPT & $\begin{array}{c}\text { Sample } \\
\text { test }\end{array}$ & $\begin{array}{c}\text { Water } \\
\text { level }\end{array}$ & Velocity \\
\hline 2018 & EN011 & Solid stem borehole (no cores or SP & $1 / 24 / 1974$ & 40.0 & 335551 & 6784692 & $\square$ & $\square$ & $\square$ & $\square$ & $\square$ \\
\hline 2018 & EN012 & Solid stem borehole (no cores or SP & $3 / 16 / 1977$ & 50.0 & 335589 & 6784728 & $\boldsymbol{V}$ & $\square$ & $\nabla$ & $\nabla$ & $\square$ \\
\hline 2018 & EN015 & Solid stem borehole (no cores or SP & $1 / 28 / 1974$ & 40.0 & 335484 & 6784765 & $\square$ & $\square$ & $\square$ & $\square$ & $\square$ \\
\hline 2018 & EN016 & Solid stem borehole (no cores or SP & $11 / 24 / 1975$ & 45.0 & 335550 & 6784834 & $\square$ & $\square$ & $\square$ & $\square$ & $\square$ \\
\hline 2018 & EN017 & Solid stem borehole (no cores or SP & $11 / 24 / 1975$ & 30.0 & 335316 & 6784924 & $\square$ & $\square$ & $\square$ & $\square$ & $\square$ \\
\hline 2018 & FY005 & Solid stem borehole (no cores or SP & $12 / 2 / 1975$ & 30.0 & 335492 & 6785001 & $\square$ & $\square$ & $\square$ & $\square$ & $\square$ \\
\hline 2019 & EN010 & Solid stem borehole (no cores or SP & $3 / 14 / 1973$ & 45.0 & 335691 & 6784640 & $\square$ & $\square$ & $\square$ & $\square$ & $\square$ \\
\hline 2019 & EN013 & Solid stem borehole (no cores or SP & $1 / 24 / 1974$ & 35.0 & 335751 & 6784806 & $\square$ & $\square$ & $\square$ & $\square$ & $\square$ \\
\hline 2019 & EN014 & Solid stem borehole (no cores or SP & $11 / 10 / 1975$ & 60.0 & 335885 & 6784868 & $\boldsymbol{v}$ & $\square$ & $\nabla$ & $\nabla$ & $\square$ \\
\hline 2020 & EO003 & Pit or trench & $5 / 7 / 1956$ & 12.0 & 336856 & 6784162 & $\square$ & $\square$ & $\square$ & $\square$ & $\square$ \\
\hline 2020 & EO004 & Solid stem borehole (no cores or SP & $5 / 7 / 1956$ & 11.0 & 336813 & 6784176 & $\square$ & $\square$ & $\square$ & $\square$ & $\square$ \\
\hline 2021 & 2137 & Hollow stem borehole w/SPT & 6/1/1995 & 16.5 & 338064 & 6784106 & $\boldsymbol{V}$ & $\checkmark$ & $\nabla$ & $\square$ & $\square$ \\
\hline 2022 & 2138 & Hollow stem borehole w/SPT & $6 / 2 / 1995$ & 16.5 & 338536 & 6784085 & $\boldsymbol{V}$ & $\nabla$ & $\boldsymbol{V}$ & $\square$ & $\square$ \\
\hline 2022 & 4464 & Water well & $6 / 21 / 1965$ & 239.0 & 338473 & 6784114 & $\checkmark$ & $\square$ & $\square$ & $\checkmark$ & $\square$ \\
\hline 2023 & 2167 & Hollow stem borehole w/SPT & 4/3/1996 & 31.5 & 339434 & 6784598 & $\boldsymbol{V}$ & $\checkmark$ & $\square$ & $\square$ & $\square$ \\
\hline 2023 & 2206 & Hollow stem borehole w/SPT & $12 / 6 / 1983$ & 21.0 & 339434 & 6784565 & $\checkmark$ & $\checkmark$ & $\checkmark$ & $\square$ & $\square$ \\
\hline 2023 & 2207 & Hollow stem borehole w/SPT & $9 / 12 / 1983$ & 31.5 & 339511 & 6784480 & $\boldsymbol{V}$ & $\checkmark$ & $\square$ & $\square$ & $\square$ \\
\hline 2024 & 2162 & Hollow stem borehole w/SPT & $12 / 22 / 1997$ & 41.5 & 340004 & 6784709 & $\boldsymbol{V}$ & $\checkmark$ & $\nabla$ & $\square$ & $\square$ \\
\hline 2024 & 2163 & Hollow stem borehole w/SPT & $12 / 22 / 1997$ & 36.5 & 340066 & 6784684 & $\boldsymbol{V}$ & $\checkmark$ & $\square$ & $\square$ & $\square$ \\
\hline 2024 & ES003 & Water well & $8 / 13 / 1963$ & 287.0 & 340171 & 6784658 & $\boldsymbol{V}$ & $\square$ & $\nabla$ & $\nabla$ & $\square$ \\
\hline 2024 & ES020 & Water well & $5 / 25 / 1954$ & 270.0 & 340226 & 6784683 & $\boldsymbol{V}$ & $\square$ & $\square$ & $\nabla$ & $\square$ \\
\hline 2025 & 4367 & Water well & $10 / 4 / 1965$ & 205.0 & 340554 & 6784112 & 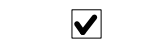 & $\square$ & $\square$ & $\square$ & $\square$ \\
\hline 2025 & 4372 & Water well & $10 / 18 / 1983$ & 218.0 & 340563 & 6784328 & 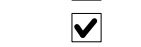 & $\square$ & $\square$ & $\nabla$ & $\square$ \\
\hline 2025 & 4377 & Water well & $11 / 24 / 1982$ & 224.0 & 340748 & 6784444 & $\nabla$ & $\square$ & $\square$ & $\square$ & $\square$ \\
\hline 2025 & 4380 & Water well & $9 / 25 / 1966$ & 200.0 & 340619 & 6784574 & $V$ & $\square$ & $\square$ & $\checkmark$ & $\square$ \\
\hline 2025 & 4381 & Water well & $8 / 12 / 1982$ & 130.0 & 340619 & 6784574 & $\boldsymbol{V}$ & $\square$ & $\square$ & $\nabla$ & $\square$ \\
\hline 2025 & ES004 & Solid stem borehole (no cores or SP & $4 / 2 / 1969$ & 23.0 & 340625 & 6784752 & $\square$ & $\square$ & $\square$ & $\square$ & $\square$ \\
\hline 2025 & ES005 & Solid stem borehole (no cores or SP & $4 / 3 / 1969$ & 23.0 & 340624 & 6784648 & & $\square$ & $\square$ & $\square$ & $\square$ \\
\hline 2025 & ES006 & Solid stem borehole (no cores or SP & $4 / 2 / 1969$ & 18.0 & 340622 & 6784562 & & $\square$ & $\square$ & $\square$ & $\square$ \\
\hline 2025 & ES007 & Solid stem borehole (no cores or SP & $4 / 3 / 1969$ & 8.0 & 340588 & 6784460 & $\square$ & $\square$ & $\square$ & $\square$ & $\square$ \\
\hline 2025 & ES008 & Solid stem borehole (no cores or SP & $4 / 2 / 1969$ & 23.0 & 340681 & 6784484 & $\square$ & $\square$ & $\square$ & $\square$ & $\square$ \\
\hline 2025 & ES009 & Solid stem borehole (no cores or SP & $4 / 2 / 1969$ & 33.0 & 340722 & 6784572 & $\square$ & $\square$ & $\square$ & $\square$ & $\square$ \\
\hline 2025 & ES010 & Solid stem borehole (no cores or SP & $4 / 2 / 1969$ & 23.0 & 340767 & 6784712 & $\square$ & $\square$ & $\square$ & $\square$ & $\square$ \\
\hline 2025 & ES011 & Solid stem borehole (no cores or SP & $4 / 3 / 1969$ & 13.0 & 340756 & 6784454 & $\square$ & $\square$ & $\square$ & $\square$ & $\square$ \\
\hline 2025 & ES012 & Solid stem borehole (no cores or SP & $4 / 2 / 1969$ & 23.0 & 340786 & 6784506 & $\square$ & $\square$ & $\square$ & $\square$ & $\square$ \\
\hline 2025 & ES013 & Solid stem borehole (no cores or SP & $4 / 3 / 1969$ & 23.0 & 340881 & 6784621 & $\square$ & $\square$ & $\square$ & $\square$ & $\square$ \\
\hline
\end{tabular}




\begin{tabular}{|c|c|c|c|c|c|c|c|c|c|c|c|}
\hline \multirow[b]{2}{*}{$\begin{array}{l}\text { MOA } \\
\text { grid }\end{array}$} & \multirow[b]{2}{*}{$\begin{array}{l}\text { DGGS } \\
\text { number }\end{array}$} & \multirow[b]{2}{*}{ Hole Type } & \multirow[b]{2}{*}{$\begin{array}{c}\text { Date } \\
\text { Completed }\end{array}$} & \multirow[b]{2}{*}{$\begin{array}{c}\text { Total } \\
\text { depth }(\mathrm{ft})\end{array}$} & \multicolumn{2}{|c|}{ UTM coordinates (m) } & \multicolumn{5}{|c|}{ Data entered } \\
\hline & & & & & East & North & Lithology & SPT & $\begin{array}{c}\text { Sample } \\
\text { test }\end{array}$ & $\begin{array}{l}\text { Water } \\
\text { level }\end{array}$ & Velocity \\
\hline 2025 & ES014 & Solid stem borehole (no cores or SP & $4 / 2 / 1969$ & 23.0 & 340962 & 6784634 & $\square$ & & $\square$ & $\square$ & \\
\hline 2025 & ES015 & Solid stem borehole (no cores or SP & $4 / 2 / 1969$ & 23.0 & 340896 & 6784778 & $\square$ & $\square$ & $\square$ & $\square$ & $\square$ \\
\hline 2025 & ES016 & Solid stem borehole (no cores or SP & $1 / 19 / 1970$ & 18.0 & 340736 & 6784283 & $\square$ & $\square$ & $\square$ & $\square$ & \\
\hline 2025 & ES017 & Solid stem borehole (no cores or SP & $1 / 19 / 1970$ & 13.0 & 340664 & 6784228 & $\square$ & $\square$ & $\square$ & $\square$ & $\square$ \\
\hline 2025 & ES018 & Solid stem borehole (no cores or SP & $1 / 19 / 1970$ & 23.0 & 340599 & 6784194 & $\checkmark$ & $\square$ & $\square$ & $\checkmark$ & $\square$ \\
\hline 2025 & ES019 & Solid stem borehole (no cores or SP & $1 / 19 / 1970$ & 18.0 & 340581 & 6784147 & $\square$ & $\square$ & $\square$ & $\square$ & $\square$ \\
\hline 2025 & ET001 & Solid stem borehole (no cores or SP & & 13.0 & 341281 & 6784355 & $\square$ & $\square$ & $\square$ & $\square$ & $\square$ \\
\hline 2025 & ET002 & Solid stem borehole (no cores or SP & & 13.0 & 341303 & 6784353 & $\square$ & $\square$ & $\square$ & $\square$ & $\checkmark$ \\
\hline 2025 & ET003 & Solid stem borehole (no cores or SP & & 18.0 & 341278 & 6784330 & $\square$ & $\square$ & $\square$ & $\square$ & $\square$ \\
\hline 2025 & ET004 & Solid stem borehole (no cores or SP & & 15.5 & 341299 & 6784329 & $\square$ & $\square$ & $\square$ & $\square$ & $\square$ \\
\hline 2025 & ET006 & Solid stem borehole (no cores or SP & & 23.0 & 341298 & 6784315 & $\square$ & $\square$ & $\square$ & $\square$ & $\square$ \\
\hline 2025 & ET007 & Solid stem borehole (no cores or SP & & 23.0 & 341296 & 6784299 & $\square$ & $\square$ & $\square$ & $\square$ & $\square$ \\
\hline 2026 & 4384 & Water well & $7 / 31 / 1965$ & 109.0 & 341911 & 6784670 & $\checkmark$ & $\square$ & $\square$ & $\checkmark$ & $\square$ \\
\hline 2026 & 4385 & Water well & $10 / 31 / 1978$ & 128.0 & 341672 & 6784681 & $\checkmark$ & $\square$ & $\square$ & $\checkmark$ & $\square$ \\
\hline 2026 & ET005 & Solid stem borehole (no cores or SP & & 23.0 & 341319 & 6784329 & $\square$ & $\square$ & $\square$ & $\square$ & $\square$ \\
\hline 2026 & ET014 & Hollow stem borehole w/SPT & $8 / 27 / 1973$ & 71.5 & 341554 & 6784460 & $\checkmark$ & $\checkmark$ & $\checkmark$ & $\square$ & $\square$ \\
\hline 2027 & 1484 & Hollow stem borehole w/SPT & $10 / 12 / 1972$ & 24.0 & 342504 & 6783932 & $\checkmark$ & $\checkmark$ & $\square$ & $\checkmark$ & $\square$ \\
\hline 2027 & 2350 & Hollow stem borehole w/SPT & $2 / 26 / 1969$ & 36.5 & 342867 & 6784680 & $\square$ & $\square$ & $\square$ & $\square$ & $\square$ \\
\hline 2028 & 1454 & Hollow stem borehole w/SPT & $6 / 19 / 1976$ & 50.0 & 343698 & 6784512 & $\checkmark$ & 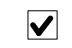 & $\square$ & $\nabla$ & $\square$ \\
\hline 2028 & 1488 & Hollow stem borehole w/SPT & $3 / 31 / 1989$ & 70.0 & 343418 & 6783874 & $\checkmark$ & $\checkmark$ & $\checkmark$ & $\nabla$ & $\square$ \\
\hline 2028 & 1490 & Hollow stem borehole w/SPT & $3 / 31 / 1989$ & 61.0 & 343377 & 6783878 & $\checkmark$ & $\checkmark$ & $\checkmark$ & $\checkmark$ & $\square$ \\
\hline 2028 & 2228 & Hollow stem borehole w/SPT & $2 / 28 / 1969$ & 36.5 & 342923 & 6784224 & $\nabla$ & $\checkmark$ & $\square$ & $\checkmark$ & $\square$ \\
\hline 2028 & 2229 & Hollow stem borehole w/SPT & $2 / 27 / 1969$ & 36.5 & 342998 & 6784117 & $\checkmark$ & $\nabla$ & $\square$ & $\checkmark$ & $\square$ \\
\hline 2028 & 2230 & Hollow stem borehole w/SPT & $2 / 27 / 1969$ & 36.5 & 343061 & 6783989 & $\checkmark$ & $\bar{\square}$ & $\square$ & $\checkmark$ & $\square$ \\
\hline 2028 & 2319 & Hollow stem borehole w/SPT & 2/6/1969 & 51.5 & 343050 & 6784520 & $\checkmark$ & $\checkmark$ & $\checkmark$ & $\checkmark$ & $\square$ \\
\hline 2028 & 2320 & Hollow stem borehole w/SPT & 2/6/1969 & 51.5 & 343426 & 6784191 & $\checkmark$ & $\checkmark$ & $\checkmark$ & $\nabla$ & $\square$ \\
\hline 2028 & 2351 & Hollow stem borehole w/SPT & $2 / 27 / 1969$ & 36.5 & 342956 & 6784601 & $\square$ & $\square$ & $\square$ & $\square$ & $\square$ \\
\hline 2028 & 2352 & Hollow stem borehole w/SPT & $2 / 28 / 1969$ & 36.5 & 343141 & 6784440 & $\square$ & & $\square$ & $\square$ & $\square$ \\
\hline 2028 & 2353 & Hollow stem borehole w/SPT & $2 / 27 / 1969$ & 36.5 & 343232 & 6784360 & $\square$ & & $\square$ & $\square$ & $\square$ \\
\hline 2028 & 2354 & Hollow stem borehole w/SPT & $2 / 27 / 1969$ & 36.5 & 343341 & 6784265 & $\square$ & $\square$ & $\square$ & $\square$ & $\square$ \\
\hline 2028 & 2355 & Hollow stem borehole w/SPT & $2 / 28 / 1969$ & 36.5 & 343398 & 6784073 & $\square$ & $\square$ & $\square$ & $\square$ & $\square$ \\
\hline 2028 & 2356 & Hollow stem borehole w/SPT & $2 / 28 / 1969$ & 37.0 & 343370 & 6783956 & $\square$ & $\square$ & $\square$ & $\square$ & $\square$ \\
\hline 2029 & 1455 & Hollow stem borehole w/SPT & $3 / 29 / 1976$ & 26.0 & 343988 & 6784593 & $\square$ & $\square$ & $\square$ & $\square$ & $\square$ \\
\hline 2029 & 1456 & Hollow stem borehole w/SPT & $3 / 29 / 1976$ & 25.5 & 344101 & 6784591 & $\square$ & $\square$ & $\square$ & $\square$ & $\square$ \\
\hline 2029 & 1457 & Hollow stem borehole w/SPT & $3 / 25 / 1976$ & 26.5 & 344040 & 6784560 & $\square$ & $\square$ & $\square$ & $\square$ & $\square$ \\
\hline 2029 & 1458 & Hollow stem borehole w/SPT & $3 / 29 / 1976$ & 26.0 & 343994 & 6784536 & $\square$ & $\square$ & $\square$ & $\square$ & $\square$ \\
\hline
\end{tabular}




\begin{tabular}{|c|c|c|c|c|c|c|c|c|c|c|c|}
\hline \multirow[b]{2}{*}{$\begin{array}{c}\text { MOA } \\
\text { grid }\end{array}$} & \multirow[b]{2}{*}{$\begin{array}{c}\text { DGGS } \\
\text { number }\end{array}$} & \multirow[b]{2}{*}{ Hole Type } & \multirow[b]{2}{*}{$\begin{array}{c}\text { Date } \\
\text { Completed }\end{array}$} & \multirow[b]{2}{*}{$\begin{array}{c}\text { Total } \\
\text { depth (ft) }\end{array}$} & \multicolumn{2}{|c|}{ UTM coordinates (m) } & \multicolumn{5}{|c|}{ Data entered } \\
\hline & & & & & East & North & Lithology & SPT & $\begin{array}{c}\text { Sample } \\
\text { test }\end{array}$ & $\begin{array}{c}\text { Water } \\
\text { level }\end{array}$ & Velocity \\
\hline 2029 & 1459 & Hollow stem borehole w/SPT & $3 / 25 / 1976$ & 26.0 & 344095 & 6784534 & 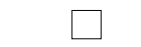 & $\square$ & $\square$ & $\square$ & $\square$ \\
\hline 2029 & 1498 & Hollow stem borehole w/SPT & $3 / 5 / 1985$ & 36.5 & 344057 & 6784405 & $\square$ & $\square$ & $\square$ & $\square$ & $\square$ \\
\hline 2029 & 1499 & Hollow stem borehole w/SPT & $3 / 1 / 1984$ & 30.0 & 344012 & 6784412 & $\square$ & $\square$ & $\square$ & $\square$ & $\square$ \\
\hline 2029 & 1500 & Hollow stem borehole w/SPT & $2 / 28 / 1984$ & 31.2 & 344036 & 6784360 & 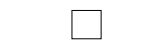 & $\square$ & $\square$ & $\square$ & $\square$ \\
\hline 2029 & 1501 & Hollow stem borehole w/SPT & $3 / 5 / 1984$ & 30.0 & 344004 & 6784358 & $\square$ & $\square$ & $\square$ & $\square$ & $\square$ \\
\hline 2029 & 1502 & Hollow stem borehole w/SPT & $3 / 1 / 1984$ & 31.0 & 344041 & 6784387 & $\square$ & $\square$ & $\square$ & $\square$ & $\square$ \\
\hline 2029 & 1503 & Hollow stem borehole w/SPT & $3 / 2 / 1984$ & 31.5 & 344041 & 6784421 & $\square$ & $\square$ & $\square$ & $\square$ & $\square$ \\
\hline 2029 & 2052 & Hollow stem borehole w/SPT & $2 / 14 / 1990$ & 100.0 & 343747 & 6784104 & $\boldsymbol{V}$ & $\nabla$ & $\nabla$ & $\nabla$ & $\square$ \\
\hline 2029 & 2053 & Hollow stem borehole w/SPT & $2 / 14 / 1990$ & 100.5 & 343804 & 6784060 & $\boldsymbol{v}$ & $\nabla$ & $\nabla$ & $\nabla$ & $\square$ \\
\hline 2029 & 2054 & Hollow stem borehole w/SPT & 4/4/1990 & 100.3 & 343864 & 6784059 & $\boldsymbol{V}$ & $\nabla$ & $\nabla$ & $\nabla$ & $\square$ \\
\hline 2029 & 4366 & Water well & $7 / 19 / 1974$ & 374.0 & 344165 & 6783888 & $\boldsymbol{V}$ & $\square$ & $\square$ & $\nabla$ & $\square$ \\
\hline 2030 & 1460 & Hollow stem borehole w/SPT & $4 / 8 / 1983$ & 32.0 & 345181 & 6784176 & $\boldsymbol{V}$ & $\checkmark$ & $\square$ & $\square$ & $\square$ \\
\hline 2030 & 1461 & Hollow stem borehole w/SPT & 6/9/1976 & 46.5 & 344594 & 6783870 & ـ & $\square$ & $\square$ & $\square$ & $\square$ \\
\hline 2030 & 1504 & Hollow stem borehole w/SPT & $1 / 20 / 1977$ & 55.5 & 344555 & 6783837 & $\checkmark$ & $\checkmark$ & 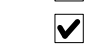 & $\checkmark$ & $\square$ \\
\hline 2030 & 1534 & Hollow stem borehole w/SPT & $5 / 11 / 1972$ & 44.5 & 344712 & 6783806 & $\square$ & $\square$ & $\square$ & $\square$ & $\square$ \\
\hline 2030 & 4378 & Water well & $5 / 15 / 1979$ & 119.0 & 345098 & 6784311 & $\checkmark$ & $\square$ & $\square$ & $\checkmark$ & $\square$ \\
\hline 2030 & EW002 & Hollow stem borehole, no SPT & $11 / 28 / 1975$ & 51.5 & 344819 & 6784149 & $\boldsymbol{V}$ & $\square$ & $\square$ & $\square$ & $\square$ \\
\hline 2031 & 1462 & Hollow stem borehole w/SPT & $12 / 20 / 1985$ & 31.0 & 345912 & 6784186 & & $\square$ & $\square$ & $\square$ & $\square$ \\
\hline 2031 & 1463 & Hollow stem borehole w/SPT & $12 / 20 / 1985$ & 31.5 & 345859 & 6784181 & $\square$ & $\square$ & $\square$ & $\square$ & $\square$ \\
\hline 2031 & 1464 & Hollow stem borehole w/SPT & $12 / 5 / 1977$ & 30.0 & 345963 & 6784126 & $\square$ & $\square$ & $\square$ & $\square$ & $\square$ \\
\hline 2031 & 1465 & Hollow stem borehole w/SPT & $12 / 5 / 1977$ & 31.5 & 345969 & 6784106 & & $\square$ & $\square$ & $\square$ & $\square$ \\
\hline 2031 & 1466 & Hollow stem borehole w/SPT & $5 / 19 / 1994$ & 31.0 & 345857 & 6783895 & $\square$ & $\square$ & $\square$ & $\square$ & $\square$ \\
\hline 2031 & 1467 & Hollow stem borehole w/SPT & $5 / 19 / 1994$ & 32.0 & 345797 & 6783883 & $\square$ & $\square$ & $\square$ & $\square$ & $\square$ \\
\hline 2031 & 1468 & Hollow stem borehole w/SPT & $5 / 19 / 1994$ & 32.0 & 345758 & 6783877 & $\square$ & $\square$ & $\square$ & $\square$ & $\square$ \\
\hline 2031 & 1469 & Hollow stem borehole w/SPT & $5 / 19 / 1994$ & 32.0 & 345667 & 6783885 & $\boldsymbol{V}$ & $\checkmark$ & $\boldsymbol{V}$ & $\nabla$ & $\square$ \\
\hline 2031 & 4388 & Water well & $1 / 8 / 1976$ & 141.0 & 345692 & 6784564 & $\boldsymbol{V}$ & $\square$ & $\square$ & $\square$ & $\square$ \\
\hline 2031 & EX009 & Water well & $6 / 1 / 1985$ & 147.0 & 345507 & 6784210 & $\boldsymbol{V}$ & $\square$ & $\square$ & $\nabla$ & $\square$ \\
\hline 2032 & 1470 & Hollow stem borehole w/SPT & $3 / 11 / 1983$ & 31.5 & 346261 & 6784022 & & $\square$ & $\square$ & $\square$ & $\square$ \\
\hline 2032 & 1471 & Hollow stem borehole w/SPT & $6 / 7 / 1982$ & 31.5 & 346208 & 6784234 & & $\square$ & $\square$ & $\square$ & $\square$ \\
\hline 2032 & 1472 & Hollow stem borehole w/SPT & $6 / 7 / 1982$ & 31.5 & 346208 & 6784252 & & $\square$ & $\square$ & $\square$ & $\square$ \\
\hline 2032 & 1473 & Hollow stem borehole, no SPT & $8 / 22 / 1975$ & 20.0 & 346646 & 6784487 & $\square$ & $\square$ & $\square$ & $\square$ & $\square$ \\
\hline 2032 & 1474 & Hollow stem borehole, no SPT & $8 / 22 / 1975$ & 20.0 & 346643 & 6784408 & $\square$ & $\square$ & $\square$ & $\square$ & $\square$ \\
\hline 2032 & 1475 & Hollow stem borehole w/SPT & $4 / 13 / 1984$ & 31.4 & 346532 & 6783850 & $\square$ & $\square$ & $\square$ & $\square$ & $\square$ \\
\hline 2032 & 1476 & Hollow stem borehole w/SPT & $4 / 13 / 1984$ & 31.4 & 346516 & 6783851 & $\square$ & $\square$ & $\square$ & $\square$ & $\square$ \\
\hline 2032 & 1673 & Hollow stem borehole w/SPT & $12 / 12 / 1985$ & 31.5 & 346748 & 6784040 & $\square$ & $\square$ & $\square$ & $\square$ & $\square$ \\
\hline 2032 & 1674 & Hollow stem borehole w/SPT & $12 / 13 / 1985$ & 31.5 & 346640 & 6784079 & $\square$ & $\square$ & $\square$ & $\square$ & $\square$ \\
\hline
\end{tabular}




\begin{tabular}{|c|c|c|c|c|c|c|c|c|c|c|c|}
\hline \multirow[b]{2}{*}{$\begin{array}{c}\text { MOA } \\
\text { grid }\end{array}$} & \multirow[b]{2}{*}{$\begin{array}{c}\text { DGGS } \\
\text { number }\end{array}$} & \multirow[b]{2}{*}{ Hole Type } & \multirow[b]{2}{*}{$\begin{array}{c}\text { Date } \\
\text { Completed }\end{array}$} & \multirow[b]{2}{*}{$\begin{array}{c}\text { Total } \\
\text { depth (ft) }\end{array}$} & \multicolumn{2}{|c|}{ UTM coordinates (m) } & \multicolumn{5}{|c|}{ Data entered } \\
\hline & & & & & East & North & Lithology & SPT & $\begin{array}{c}\text { Sample } \\
\text { test }\end{array}$ & $\begin{array}{l}\text { Water } \\
\text { level }\end{array}$ & Velocity \\
\hline 2032 & 4362 & Water well & 9/3/1982 & 143.0 & 346285 & 6783764 & $\checkmark$ & $\square$ & $\square$ & $\checkmark$ & $\square$ \\
\hline 2032 & 4363 & Water well & $4 / 5 / 1964$ & 161.0 & 346061 & 6783773 & $\nabla$ & $\square$ & $\square$ & $\checkmark$ & $\square$ \\
\hline 2032 & 4368 & Water well & 9/24/1982 & 105.0 & 346187 & 6783892 & $\checkmark$ & $\square$ & $\square$ & $\square$ & $\square$ \\
\hline 2032 & DZ001 & Water well & $5 / 11 / 1995$ & 140.0 & 346370 & 6783788 & $\nabla$ & $\square$ & $\square$ & $\nabla$ & $\square$ \\
\hline 2033 & 1477 & Hollow stem borehole w/SPT & 4/9/1982 & 30.0 & 347359 & 6784154 & $\square$ & $\square$ & $\square$ & $\square$ & $\square$ \\
\hline 2033 & 1478 & Hollow stem borehole w/SPT & $4 / 12 / 1982$ & 30.0 & 347383 & 6784137 & $\checkmark$ & $\checkmark$ & $\checkmark$ & $\checkmark$ & $\square$ \\
\hline 2033 & 1479 & Hollow stem borehole w/SPT & 8/7/1976 & 30.5 & 347182 & 6784450 & $\square$ & $\square$ & $\square$ & $\square$ & $\square$ \\
\hline 2033 & 1480 & Hollow stem borehole w/SPT & $8 / 7 / 1976$ & 28.5 & 347183 & 6784423 & $\square$ & $\square$ & $\square$ & $\square$ & $\square$ \\
\hline 2033 & 1481 & Hollow stem borehole w/SPT & 8/9/1976 & 29.5 & 347214 & 6784450 & $\square$ & $\square$ & $\square$ & $\square$ & $\square$ \\
\hline 2033 & 1482 & Hollow stem borehole w/SPT & $8 / 10 / 1976$ & 29.5 & 347214 & 6784418 & $\square$ & $\square$ & $\square$ & $\square$ & $\square$ \\
\hline 2033 & 1606 & Hollow stem borehole w/SPT & $4 / 12 / 1982$ & 30.0 & 347358 & 6784114 & $\square$ & $\square$ & $\square$ & $\square$ & $\square$ \\
\hline 2034 & 4364 & Water well & 4/21/1977 & 102.0 & 348005 & 6783720 & $\checkmark$ & $\square$ & $\square$ & $\checkmark$ & $\square$ \\
\hline 2034 & 4365 & Water well & 4/27/1977 & 102.0 & 347990 & 6783720 & $\boldsymbol{V}$ & $\square$ & $\square$ & 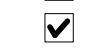 & $\square$ \\
\hline 2034 & 4370 & Water well & $2 / 20 / 1978$ & 106.0 & 348099 & 6783809 & $\checkmark$ & $\square$ & $\square$ & $\checkmark$ & $\square$ \\
\hline 2035 & 1483 & Hollow stem borehole, no SPT & $5 / 25 / 1977$ & 20.0 & 348897 & 6783942 & $\nabla$ & $\square$ & $\square$ & $\nabla$ & $\square$ \\
\hline 2035 & 3037 & Water well & $11 / 1 / 1982$ & 360.0 & 348485 & 6784102 & $\checkmark$ & $\square$ & $\square$ & $\checkmark$ & $\square$ \\
\hline 2035 & 4376 & Water well & $1 / 8 / 1982$ & 403.0 & 348499 & 6784070 & $\checkmark$ & $\square$ & $\square$ & $\checkmark$ & $\square$ \\
\hline 2037 & 1511 & Hollow stem borehole w/SPT & $9 / 20 / 1993$ & 41.5 & 350756 & 6784079 & $\square$ & $\square$ & $\square$ & $\square$ & $\square$ \\
\hline 2037 & 1512 & Hollow stem borehole w/SPT & $9 / 15 / 1993$ & 41.3 & 350697 & 6784017 & $\square$ & $\square$ & $\square$ & $\square$ & $\square$ \\
\hline 2037 & 1513 & Hollow stem borehole w/SPT & 9/20/1993 & 41.0 & 350711 & 6783981 & $\square$ & $\square$ & $\square$ & $\square$ & $\square$ \\
\hline 2037 & 1607 & Hollow stem borehole w/SPT & 9/17/1993 & 41.0 & 350686 & 6784082 & $\square$ & $\square$ & $\square$ & $\square$ & $\square$ \\
\hline 2042 & 4374 & Water well & $7 / 22 / 1978$ & 109.0 & 354727 & 6783776 & $\checkmark$ & $\square$ & $\square$ & $\nabla$ & $\square$ \\
\hline 2042 & 4379 & Water well & $6 / 1 / 1976$ & 194.0 & 354554 & 6783938 & 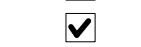 & $\square$ & $\square$ & 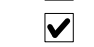 & $\square$ \\
\hline 2043 & 301 & Water well & $7 / 15 / 1984$ & 305.0 & 355040 & 6783580 & $V$ & $\square$ & $\square$ & $V$ & $\square$ \\
\hline 2043 & 3034 & Water well & $5 / 27 / 1980$ & 300.0 & 355519 & 6783775 & $V$ & $\square$ & $\square$ & $\square$ & $\square$ \\
\hline 2043 & 4369 & Water well & $12 / 1 / 1978$ & 355.0 & 355227 & 6783570 & 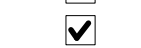 & $\square$ & $\square$ & $V$ & $\square$ \\
\hline 2043 & 4373 & Water well & 7/14/1973 & 150.0 & 355218 & 6783725 & $\checkmark$ & $\square$ & $\square$ & 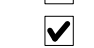 & $\square$ \\
\hline 2043 & 4375 & Water well & $11 / 15 / 1971$ & 145.0 & 355146 & 6783790 & $\checkmark$ & $\square$ & $\square$ & $V$ & $\square$ \\
\hline 2043 & 4383 & Water well & $6 / 22 / 1976$ & 310.0 & 355247 & 6784034 & $\square$ & $\square$ & $\square$ & $\nabla$ & $\square$ \\
\hline 2043 & 4386 & Water well & 9/2/1977 & 181.0 & 355369 & 6784122 & $\checkmark$ & $\square$ & $\square$ & $\checkmark$ & $\square$ \\
\hline 2043 & 4387 & Water well & 9/28/1976 & 103.0 & 355176 & 6784130 & $\checkmark$ & $\square$ & $\square$ & $\square$ & $\square$ \\
\hline 2117 & 2109 & Hollow stem borehole, no SPT & $7 / 21 / 1982$ & 50.0 & 334826 & 6784007 & 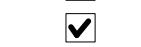 & $\square$ & $\square$ & $\sqrt{v}$ & $\square$ \\
\hline 2118 & 2110 & Hollow stem borehole, no SPT & $7 / 23 / 1982$ & 50.0 & 334990 & 6784058 & $\checkmark$ & $\square$ & $\checkmark$ & $\checkmark$ & $\square$ \\
\hline 2118 & 2111 & Hollow stem borehole, no SPT & $3 / 11 / 1977$ & 60.0 & 335206 & 6784212 & $\checkmark$ & $\square$ & $\checkmark$ & $\checkmark$ & $\square$ \\
\hline 2118 & EN006 & Solid stem borehole (no cores or SP & $1 / 22 / 1975$ & 49.0 & 335114 & 6784122 & $\square$ & $\square$ & $\square$ & $\square$ & $\square$ \\
\hline 2118 & EN008 & Solid stem borehole (no cores or SP & 3/11/1977 & 60.0 & 335194 & 6784124 & $\nabla$ & $\square$ & $\checkmark$ & $\checkmark$ & $\square$ \\
\hline
\end{tabular}




\begin{tabular}{|c|c|c|c|c|c|c|c|c|c|c|c|}
\hline \multirow[b]{2}{*}{$\begin{array}{c}\text { MOA } \\
\text { grid }\end{array}$} & \multirow[b]{2}{*}{$\begin{array}{c}\text { DGGS } \\
\text { number }\end{array}$} & \multirow[b]{2}{*}{ Hole Type } & \multirow[b]{2}{*}{$\begin{array}{c}\text { Date } \\
\text { Completed }\end{array}$} & \multirow[b]{2}{*}{$\begin{array}{c}\text { Total } \\
\text { depth (ft) }\end{array}$} & \multicolumn{2}{|c|}{ UTM coordinates (m) } & \multicolumn{5}{|c|}{ Data entered } \\
\hline & & & & & East & North & Lithology & SPT & $\begin{array}{c}\text { Sample } \\
\text { test }\end{array}$ & $\begin{array}{l}\text { Water } \\
\text { level }\end{array}$ & Velocity \\
\hline 2119 & 2132 & Hollow stem borehole w/SPT & $5 / 31 / 1995$ & 16.5 & 335830 & 6783653 & $\checkmark$ & $\nabla$ & $\nabla$ & $\square$ & $\square$ \\
\hline 2119 & 2133 & Hollow stem borehole w/SPT & $5 / 31 / 1995$ & 16.5 & 335979 & 6783649 & $\checkmark$ & $\checkmark$ & $\checkmark$ & $\square$ & $\square$ \\
\hline 2119 & EJ001 & Solid stem borehole (no cores or SP & & 20.0 & 336078 & 6783531 & $\boldsymbol{V}$ & $\square$ & $\square$ & $\square$ & $\square$ \\
\hline 2119 & EJ002 & Solid stem borehole (no cores or SP & & 10.0 & 336120 & 6783634 & $\square$ & $\square$ & $\square$ & $\square$ & $\square$ \\
\hline 2119 & EJ004 & Solid stem borehole (no cores or SP & & 20.0 & 336319 & 6783626 & $\checkmark$ & $\square$ & $\square$ & $\square$ & $\square$ \\
\hline 2119 & EJ005 & Solid stem borehole (no cores or SP & & 18.0 & 336245 & 6783718 & $\square$ & $\square$ & $\square$ & $\square$ & $\square$ \\
\hline 2119 & EK001 & Solid stem borehole (no cores or SP & $3 / 12 / 1959$ & 20.0 & 335828 & 6783701 & $\checkmark$ & $\square$ & $\square$ & $\square$ & $\square$ \\
\hline 2119 & EK002 & Solid stem borehole (no cores or SP & $3 / 12 / 1959$ & 20.0 & 335827 & 6783734 & & $\square$ & $\square$ & $\square$ & $\square$ \\
\hline 2119 & EK003 & Solid stem borehole (no cores or SP & & 19.0 & 335824 & 6783820 & $\square$ & $\square$ & $\square$ & $\square$ & $\square$ \\
\hline 2119 & EK004 & Solid stem borehole (no cores or SP & & 18.0 & 335837 & 6783908 & & $\square$ & $\square$ & $\square$ & $\square$ \\
\hline 2119 & EK005 & Solid stem borehole (no cores or SP & & 19.0 & 335929 & 6783490 & $\square$ & $\square$ & $\square$ & $\square$ & $\square$ \\
\hline 2119 & EK006 & Solid stem borehole (no cores or SP & & 19.0 & 335794 & 6783664 & $\square$ & $\square$ & $\square$ & $\square$ & $\square$ \\
\hline 2119 & EK007 & Solid stem borehole (no cores or SP & & 17.0 & 335940 & 6783897 & $\square$ & $\square$ & $\square$ & $\square$ & $\square$ \\
\hline 2119 & EK008 & Solid stem borehole (no cores or SP & & 20.0 & 335719 & 6783598 & $\checkmark$ & $\square$ & $\square$ & $\square$ & $\square$ \\
\hline 2119 & EK009 & Solid stem borehole (no cores or SP & & 20.0 & 335758 & 6783590 & $\square$ & $\square$ & $\square$ & $\square$ & $\square$ \\
\hline 2120 & 2134 & Hollow stem borehole w/SPT & $5 / 31 / 1995$ & 16.5 & 336673 & 6783727 & $\checkmark$ & $\checkmark$ & $\checkmark$ & $\square$ & $\square$ \\
\hline 2120 & 2135 & Hollow stem borehole w/SPT & $5 / 31 / 1995$ & 16.5 & 337062 & 6783554 & $\boldsymbol{V}$ & $\nabla$ & $\nabla$ & $\square$ & $\square$ \\
\hline 2120 & EI002 & Solid stem borehole (no cores or SP & & 9.0 & 337240 & 6783464 & $\square$ & $\square$ & $\square$ & $\square$ & $\square$ \\
\hline 2120 & EI003 & Solid stem borehole (no cores or SP & & 9.0 & 337038 & 6783576 & $\square$ & $\square$ & $\square$ & $\square$ & $\square$ \\
\hline 2120 & EJ003 & Solid stem borehole (no cores or SP & & 20.0 & 336480 & 6783634 & $\nabla$ & $\square$ & $\square$ & $\square$ & $\square$ \\
\hline 2120 & EJ006 & Solid stem borehole (no cores or SP & & 10.0 & 336792 & 6783698 & $\square$ & $\square$ & $\square$ & $\square$ & $\square$ \\
\hline 2120 & EO001 & Solid stem borehole (no cores or SP & $5 / 7 / 1956$ & 12.0 & 336930 & 6784068 & $\square$ & $\square$ & $\square$ & $\square$ & $\square$ \\
\hline 2120 & EO002 & Solid stem borehole (no cores or SP & $5 / 5 / 1956$ & 12.0 & 336856 & 6784114 & $\square$ & $\square$ & $\square$ & $\square$ & $\square$ \\
\hline 2120 & EO005 & Solid stem borehole (no cores or SP & $9 / 24 / 1965$ & 16.0 & 336824 & 6784154 & $\square$ & $\square$ & $\square$ & $\square$ & $\square$ \\
\hline 2120 & EO006 & Solid stem borehole (no cores or SP & $9 / 24 / 1965$ & 18.0 & 336816 & 6784110 & $\boldsymbol{V}$ & $\square$ & $\square$ & $\square$ & $\square$ \\
\hline 2120 & EO007 & Solid stem borehole (no cores or SP & & 19.5 & 336998 & 6784066 & $\square$ & $\square$ & $\square$ & $\square$ & $\square$ \\
\hline 2120 & EO008 & Solid stem borehole (no cores or SP & $5 / 7 / 1956$ & 13.5 & 336982 & 6784124 & $\square$ & $\square$ & $\square$ & $\square$ & $\square$ \\
\hline 2120 & EO009 & Solid stem borehole (no cores or SP & $5 / 7 / 1956$ & 19.5 & 336983 & 6784078 & $\boldsymbol{V}$ & $\square$ & $\square$ & $\square$ & $\square$ \\
\hline 2120 & EO010 & Solid stem borehole (no cores or SP & $5 / 5 / 1956$ & 14.0 & 336996 & 6784010 & $\square$ & $\square$ & $\square$ & $\square$ & $\square$ \\
\hline 2121 & 2136 & Hollow stem borehole w/SPT & $6 / 1 / 1995$ & 16.5 & 337755 & 6783694 & 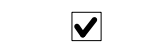 & $\nabla$ & $\square$ & $\square$ & $\square$ \\
\hline 2121 & EI001 & Solid stem borehole (no cores or SP & & 9.5 & 337375 & 6783504 & $\square$ & $\square$ & $\square$ & $\square$ & $\square$ \\
\hline 2121 & EI004 & Solid stem borehole (no cores or SP & & 9.5 & 337596 & 6783558 & $\square$ & $\square$ & $\square$ & $\square$ & $\square$ \\
\hline 2122 & 2012 & Natural exposure & $6 / 4 / 1997$ & 72.0 & 338467 & 6783613 & 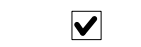 & $\square$ & $\square$ & $\square$ & $\square$ \\
\hline 2122 & 2295 & Water well & $5 / 11 / 1987$ & 543.0 & 338710 & 6783742 & $\boldsymbol{V}$ & $\square$ & $\square$ & $\nabla$ & $\square$ \\
\hline 2122 & 2296 & Water well & $12 / 7 / 1984$ & 280.0 & 338846 & 6783731 & $\boldsymbol{v}$ & $\square$ & $\square$ & $\nabla$ & $\square$ \\
\hline 2122 & EH008 & Pit or trench & $10 / 3 / 1967$ & 12.0 & 338636 & 6783963 & $\square$ & $\square$ & $\square$ & $\square$ & $\square$ \\
\hline
\end{tabular}




\begin{tabular}{|c|c|c|c|c|c|c|c|c|c|c|c|}
\hline \multirow[b]{2}{*}{$\begin{array}{l}\text { MOA } \\
\text { grid }\end{array}$} & \multirow[b]{2}{*}{$\begin{array}{l}\text { DGGS } \\
\text { number }\end{array}$} & \multirow[b]{2}{*}{ Hole Type } & \multirow[b]{2}{*}{$\begin{array}{c}\text { Date } \\
\text { Completed }\end{array}$} & \multirow[b]{2}{*}{$\begin{array}{c}\text { Total } \\
\text { depth }(\mathrm{ft})\end{array}$} & \multicolumn{2}{|c|}{ UTM coordinates (m) } & \multicolumn{5}{|c|}{ Data entered } \\
\hline & & & & & East & North & Lithology & SPT & $\begin{array}{c}\text { Sample } \\
\text { test }\end{array}$ & $\begin{array}{l}\text { Water } \\
\text { level }\end{array}$ & Velocity \\
\hline 2122 & EH009 & Solid stem borehole (no cores or SP & $10 / 4 / 1967$ & 37.0 & 338651 & 6783962 & $\square$ & & $\square$ & $\square$ & $\square$ \\
\hline 2122 & EH010 & Pit or trench & $10 / 3 / 1967$ & 11.5 & 338685 & 6783950 & $\square$ & $\square$ & $\square$ & $\square$ & $\square$ \\
\hline 2122 & EH011 & Pit or trench & $10 / 3 / 1967$ & 11.5 & 338769 & 6783955 & $\square$ & & $\square$ & $\square$ & \\
\hline 2122 & EH012 & Pit or trench & $10 / 8 / 1967$ & 11.0 & 338804 & 6783945 & $\square$ & 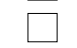 & $\square$ & $\square$ & $\square$ \\
\hline 2122 & EH013 & Pit or trench & $9 / 29 / 1967$ & 12.0 & 338484 & 6783882 & $\square$ & $\square$ & $\square$ & $\square$ & $\square$ \\
\hline 2122 & EH014 & Pit or trench & $10 / 3 / 1967$ & 11.5 & 338605 & 6783908 & $\square$ & $\square$ & $\square$ & $\square$ & $\square$ \\
\hline 2122 & EH015 & Pit or trench & $10 / 2 / 1967$ & 12.5 & 338747 & 6783900 & $\square$ & $\square$ & $\square$ & $\square$ & $\square$ \\
\hline 2122 & EH017 & Pit or trench & $10 / 3 / 1967$ & 12.0 & 338573 & 6783864 & $\square$ & & $\square$ & $\square$ & $\square$ \\
\hline 2122 & EH019 & Pit or trench & $9 / 29 / 1967$ & 13.5 & 338664 & 6783839 & $\square$ & 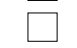 & $\square$ & $\square$ & $\square$ \\
\hline 2122 & EH020 & Pit or trench & $10 / 3 / 1967$ & 11.5 & 338738 & 6783843 & $\square$ & & $\square$ & $\square$ & $\square$ \\
\hline 2122 & EH021 & Pit or trench & $10 / 2 / 1967$ & 11.0 & 338789 & 6783842 & $\square$ & $\square$ & $\square$ & $\square$ & $\square$ \\
\hline 2122 & EH022 & Pit or trench & $10 / 3 / 1967$ & 12.0 & 338634 & 6783756 & $\square$ & & $\square$ & $\square$ & $\square$ \\
\hline 2122 & EH024 & Pit or trench & $10 / 8 / 1967$ & 11.3 & 338731 & 6783784 & $\square$ & 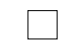 & $\square$ & $\square$ & $\square$ \\
\hline 2122 & EH025 & Pit or trench & $9 / 29 / 1967$ & 12.0 & 338783 & 6783780 & $\square$ & $\square$ & $\square$ & $\square$ & $\square$ \\
\hline 2122 & EH026 & Pit or trench & $9 / 29 / 1967$ & 12.0 & 338763 & 6783696 & $\square$ & 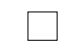 & $\square$ & $\square$ & $\square$ \\
\hline 2122 & EH027 & Pit or trench & $9 / 28 / 1967$ & 12.2 & 338288 & 6783642 & $\square$ & 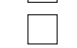 & $\square$ & $\square$ & $\square$ \\
\hline 2122 & EH028 & Pit or trench & $9 / 28 / 1967$ & 15.5 & 338420 & 6783632 & $\square$ & & $\square$ & $\square$ & $\square$ \\
\hline 2123 & 3048 & Water well & $6 / 25 / 1985$ & 160.0 & 339153 & 6783928 & $\checkmark$ & & $\square$ & $\vec{\nabla}$ & $\square$ \\
\hline 2123 & 4336 & Water well & 12/1/1977 & 112.0 & 339601 & 6783257 & $\checkmark$ & $\checkmark$ & $\square$ & $\nabla$ & $\square$ \\
\hline 2123 & 4348 & Water well & $6 / 30 / 1982$ & 173.0 & 339218 & 6783708 & $\checkmark$ & $\square$ & $\square$ & $\checkmark$ & $\square$ \\
\hline 2123 & 4352 & Water well & $6 / 20 / 1982$ & 157.0 & 339222 & 6783801 & $\checkmark$ & & $\square$ & $\bar{\nabla}$ & $\square$ \\
\hline 2123 & 4353 & Water well & $8 / 15 / 1965$ & 278.0 & 338938 & 6783814 & $\checkmark$ & & $\square$ & $\nabla$ & $\square$ \\
\hline 2123 & 4357 & Water well & $10 / 15 / 1965$ & 210.0 & 339245 & 6783986 & $\checkmark$ & & $\square$ & $\nabla$ & $\square$ \\
\hline 2123 & 4359 & Water well & $11 / 13 / 1981$ & 140.0 & 339245 & 6783986 & $\checkmark$ & $\square$ & $\square$ & $\square$ & $\square$ \\
\hline 2123 & EG003 & Solid stem borehole (no cores or SP & $10 / 6 / 1967$ & 36.0 & 339399 & 6783675 & $\square$ & & $\square$ & $\square$ & $\square$ \\
\hline 2123 & EG004 & Solid stem borehole (no cores or SP & $10 / 6 / 1967$ & 43.0 & 339474 & 6783663 & $\square$ & & $\square$ & $\square$ & $\square$ \\
\hline 2123 & EG005 & Solid stem borehole (no cores or SP & $10 / 5 / 1967$ & 48.0 & 339386 & 6783767 & $\square$ & $\square$ & $\square$ & $\square$ & $\square$ \\
\hline 2123 & EG006 & Solid stem borehole (no cores or SP & $10 / 5 / 1967$ & 48.0 & 339472 & 6783768 & $\square$ & & $\square$ & $\square$ & $\square$ \\
\hline 2123 & EG007 & Solid stem borehole (no cores or SP & $10 / 2 / 1967$ & 28.0 & 339528 & 6783774 & $\square$ & & $\square$ & $\square$ & $\square$ \\
\hline 2123 & EG008 & Solid stem borehole (no cores or SP & $10 / 10 / 1967$ & 23.0 & 339560 & 6783706 & $\square$ & & $\square$ & $\square$ & $\square$ \\
\hline 2123 & EG009 & Solid stem borehole (no cores or SP & $10 / 9 / 1967$ & 38.3 & 339629 & 6783750 & $\square$ & $\square$ & $\square$ & $\square$ & $\square$ \\
\hline 2123 & EG010 & Solid stem borehole (no cores or SP & $10 / 5 / 1967$ & 43.0 & 339404 & 6783875 & $\square$ & $\square$ & $\square$ & $\square$ & $\square$ \\
\hline 2123 & EG011 & Solid stem borehole (no cores or SP & $10 / 2 / 1967$ & 28.0 & 339467 & 6783864 & $\square$ & $\square$ & $\square$ & $\square$ & $\square$ \\
\hline 2123 & EG012 & Solid stem borehole (no cores or SP & $10 / 9 / 1967$ & 48.0 & 339535 & 6783850 & $\square$ & $\square$ & $\square$ & $\square$ & $\square$ \\
\hline 2123 & EG013 & Solid stem borehole (no cores or SP & $10 / 2 / 1967$ & 43.0 & 339634 & 6783826 & $\square$ & $\square$ & $\square$ & $\square$ & $\square$ \\
\hline 2123 & EG014 & Solid stem borehole (no cores or SP & $10 / 2 / 1967$ & 43.0 & 339515 & 6783916 & $\square$ & $\square$ & $\square$ & $\square$ & $\square$ \\
\hline
\end{tabular}




\begin{tabular}{|c|c|c|c|c|c|c|c|c|c|c|c|}
\hline \multirow[b]{2}{*}{$\begin{array}{l}\text { MOA } \\
\text { grid }\end{array}$} & \multirow[b]{2}{*}{$\begin{array}{l}\text { DGGS } \\
\text { number }\end{array}$} & \multirow[b]{2}{*}{ Hole Type } & \multirow[b]{2}{*}{$\begin{array}{c}\text { Date } \\
\text { Completed }\end{array}$} & \multirow[b]{2}{*}{$\begin{array}{c}\text { Total } \\
\text { depth }(\mathrm{ft})\end{array}$} & \multicolumn{2}{|c|}{ UTM coordinates (m) } & \multicolumn{5}{|c|}{ Data entered } \\
\hline & & & & & East & North & Lithology & SPT & $\begin{array}{c}\text { Sample } \\
\text { test }\end{array}$ & $\begin{array}{l}\text { Water } \\
\text { level }\end{array}$ & Velocity \\
\hline 2123 & EG015 & Solid stem borehole (no cores or SP & $10 / 10 / 1967$ & 38.0 & 339619 & 6783892 & $\square$ & 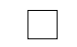 & $\square$ & $\square$ & $\square$ \\
\hline 2123 & EG016 & Solid stem borehole (no cores or SP & $10 / 9 / 1967$ & 48.0 & 339572 & 6783974 & $\square$ & $\square$ & $\square$ & $\square$ & $\square$ \\
\hline 2123 & ER001 & Solid stem borehole (no cores or SP & $10 / 5 / 1967$ & 18.0 & 339325 & 6784010 & $\square$ & & $\square$ & $\square$ & \\
\hline 2123 & ER002 & Solid stem borehole (no cores or SP & $10 / 5 / 1967$ & 33.0 & 339413 & 6784010 & $\square$ & $\square$ & $\square$ & $\square$ & $\square$ \\
\hline 2123 & ER003 & Solid stem borehole (no cores or SP & $10 / 2 / 1967$ & 38.0 & 339473 & 6784016 & $\square$ & $\square$ & $\square$ & $\square$ & $\square$ \\
\hline 2124 & 2227 & Water well & $12 / 5 / 1973$ & 99.0 & 340062 & 6783567 & $\checkmark$ & $\square$ & $\square$ & $\checkmark$ & $\square$ \\
\hline 2124 & 3051 & Water well & 6/4/1981 & 138.0 & 340043 & 6783422 & $\checkmark$ & $\square$ & $\square$ & $\square$ & $\square$ \\
\hline 2124 & 4334 & Water well & $7 / 21 / 1966$ & 178.0 & 340034 & 6783237 & $\checkmark$ & $\square$ & $\square$ & $\bar{\nabla}$ & $\square$ \\
\hline 2124 & 4340 & Water well & $10 / 8 / 1979$ & 200.0 & 339917 & 6783273 & $\checkmark$ & $\square$ & $\square$ & $\checkmark$ & $\square$ \\
\hline 2124 & 4341 & Water well & $10 / 23 / 1978$ & 125.0 & 339713 & 6783406 & $\checkmark$ & $\square$ & $\square$ & $\checkmark$ & $\square$ \\
\hline 2124 & 4345 & Water well & $1 / 1 / 1979$ & 125.0 & 339925 & 6783459 & $\checkmark$ & $\square$ & $\square$ & $\bar{V}$ & $\square$ \\
\hline 2125 & 2185 & Hollow stem borehole w/SPT & $1 / 24 / 1994$ & 18.0 & 341107 & 6783776 & $\checkmark$ & $\checkmark$ & $\square$ & $\square$ & $\square$ \\
\hline 2125 & 4351 & Water well & $7 / 14 / 1964$ & 186.0 & 340641 & 6783736 & $\checkmark$ & $\square$ & $\square$ & $\checkmark$ & $\square$ \\
\hline 2126 & 2180 & Hollow stem borehole w/SPT & $10 / 19 / 1994$ & 85.0 & 341353 & 6783942 & $\checkmark$ & $\checkmark$ & $\square$ & $\nabla$ & $\square$ \\
\hline 2126 & 2181 & Hollow stem borehole w/SPT & $10 / 18 / 1994$ & 94.0 & 341337 & 6783912 & $\checkmark$ & $\checkmark$ & $\square$ & $\checkmark$ & $\square$ \\
\hline 2126 & 2182 & Hollow stem borehole w/SPT & $2 / 11 / 1998$ & 94.5 & 341327 & 6783913 & $\checkmark$ & $\checkmark$ & $\square$ & $\checkmark$ & $\square$ \\
\hline 2126 & 4339 & Water well & $7 / 25 / 1965$ & 257.0 & 341620 & 6783196 & $\checkmark$ & $\square$ & $\square$ & 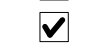 & $\square$ \\
\hline 2127 & 1485 & Hollow stem borehole w/SPT & $10 / 12 / 1972$ & 20.0 & 342496 & 6783793 & $\square$ & $\square$ & $\square$ & $\square$ & $\square$ \\
\hline 2127 & 1605 & Solid stem borehole (no cores or SP & $11 / 9 / 1978$ & 20.0 & 342652 & 6783896 & $\square$ & $\square$ & $\square$ & $\square$ & $\square$ \\
\hline 2127 & 2194 & Hollow stem borehole w/SPT & $12 / 17 / 1973$ & 16.0 & 342866 & 6783112 & $\checkmark$ & $\nabla$ & $\square$ & $\checkmark$ & $\square$ \\
\hline 2127 & 2195 & Hollow stem borehole w/SPT & $12 / 17 / 1973$ & 16.0 & 342488 & 6783624 & $\checkmark$ & $\square$ & $\square$ & $\square$ & $\square$ \\
\hline 2127 & 2196 & Hollow stem borehole w/SPT & $3 / 16 / 1972$ & 20.0 & 342641 & 6783612 & $\checkmark$ & $\square$ & $\square$ & $\checkmark$ & $\square$ \\
\hline 2127 & 4347 & Water well & $8 / 12 / 1973$ & 158.0 & 342743 & 6783548 & $\checkmark$ & $\square$ & $\square$ & $\nabla$ & $\square$ \\
\hline 2127 & 4360 & Water well & $9 / 22 / 1975$ & 202.0 & 342653 & 6783862 & $\checkmark$ & $\square$ & $\square$ & $\checkmark$ & $\square$ \\
\hline 2128 & 1486 & Hollow stem borehole w/SPT & $3 / 22 / 1989$ & 70.0 & 343418 & 6783844 & $\checkmark$ & $\nabla$ & $\checkmark$ & 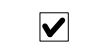 & $\square$ \\
\hline 2128 & 1487 & Hollow stem borehole w/SPT & $3 / 23 / 1989$ & 71.0 & 343377 & 6783862 & $\checkmark$ & $\nabla$ & $\nabla$ & 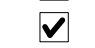 & $\square$ \\
\hline 2128 & 1489 & Hollow stem borehole w/SPT & $3 / 29 / 1989$ & 75.0 & 343376 & 6783844 & $\checkmark$ & $\checkmark$ & $\checkmark$ & $\checkmark$ & $\square$ \\
\hline 2128 & 2231 & Hollow stem borehole w/SPT & $2 / 28 / 1969$ & 36.5 & 343111 & 6783709 & $\checkmark$ & $\square$ & $\nabla$ & $\nabla$ & $\square$ \\
\hline 2128 & 2232 & Hollow stem borehole w/SPT & $5 / 20 / 1972$ & 25.8 & 343222 & 6783621 & $\nabla$ & $\nabla$ & $\square$ & $\checkmark$ & $\square$ \\
\hline 2128 & 2233 & Hollow stem borehole w/SPT & $5 / 22 / 1972$ & 26.5 & 343365 & 6783376 & $\checkmark$ & $\nabla$ & $\square$ & $\bar{\nabla}$ & $\square$ \\
\hline 2128 & 2312 & Hollow stem borehole w/SPT & 2/7/1969 & 51.5 & 343318 & 6783733 & $\checkmark$ & $\nabla$ & $\checkmark$ & $\nabla$ & $\square$ \\
\hline 2128 & 2357 & Hollow stem borehole w/SPT & $2 / 28 / 1969$ & 36.5 & 343343 & 6783837 & $\square$ & $\square$ & $\square$ & $\square$ & $\square$ \\
\hline 2129 & 1509 & Hollow stem borehole w/SPT & $1 / 20 / 1977$ & 54.5 & 344235 & 6783823 & $\checkmark$ & $\nabla$ & $\bar{\nabla}$ & $\bar{\square}$ & $\square$ \\
\hline 2129 & 1514 & Hollow stem borehole w/SPT & $4 / 23 / 1975$ & 41.0 & 343923 & 6783065 & $\square$ & $\square$ & $\square$ & $\square$ & $\square$ \\
\hline 2129 & 1515 & Hollow stem borehole w/SPT & $4 / 24 / 1975$ & 41.0 & 343743 & 6783421 & $\square$ & $\square$ & $\square$ & $\square$ & $\square$ \\
\hline 2129 & 1516 & Hollow stem borehole w/SPT & $4 / 24 / 1975$ & 41.0 & 343874 & 6783130 & $\square$ & $\square$ & $\square$ & $\square$ & $\square$ \\
\hline
\end{tabular}




\begin{tabular}{|c|c|c|c|c|c|c|c|c|c|c|c|}
\hline \multirow[b]{2}{*}{$\begin{array}{c}\text { MOA } \\
\text { grid }\end{array}$} & \multirow[b]{2}{*}{$\begin{array}{c}\text { DGGS } \\
\text { number }\end{array}$} & \multirow[b]{2}{*}{ Hole Type } & \multirow[b]{2}{*}{$\begin{array}{c}\text { Date } \\
\text { Completed }\end{array}$} & \multirow[b]{2}{*}{$\begin{array}{c}\text { Total } \\
\text { depth }(\mathrm{ft})\end{array}$} & \multicolumn{2}{|c|}{ UTM coordinates (m) } & \multicolumn{5}{|c|}{ Data entered } \\
\hline & & & & & East & North & Lithology & SPT & $\begin{array}{c}\text { Sample } \\
\text { test }\end{array}$ & $\begin{array}{l}\text { Water } \\
\text { level }\end{array}$ & Velocity \\
\hline 2129 & 1517 & Hollow stem borehole w/SPT & $4 / 25 / 1975$ & 41.0 & 343834 & 6783055 & $\square$ & $\square$ & $\square$ & $\square$ & $\square$ \\
\hline 2129 & 1518 & Hollow stem borehole w/SPT & $4 / 25 / 1975$ & 41.5 & 343784 & 6783114 & $\square$ & $\square$ & $\square$ & $\square$ & $\square$ \\
\hline 2129 & 1519 & Hollow stem borehole w/SPT & $4 / 26 / 1975$ & 41.0 & 343720 & 6783070 & $\square$ & $\square$ & $\square$ & $\square$ & $\square$ \\
\hline 2129 & 1520 & Hollow stem borehole w/SPT & $4 / 26 / 1975$ & 41.5 & 343701 & 6783180 & $\square$ & $\square$ & $\square$ & $\square$ & $\square$ \\
\hline 2129 & 1521 & Hollow stem borehole w/SPT & 4/27/1975 & 41.0 & 343750 & 6783212 & $\square$ & $\square$ & $\square$ & $\square$ & $\square$ \\
\hline 2129 & 1522 & Hollow stem borehole w/SPT & 4/27/1975 & 41.0 & 343795 & 6783256 & $\square$ & $\square$ & $\square$ & $\square$ & $\square$ \\
\hline 2129 & 1523 & Hollow stem borehole w/SPT & $4 / 28 / 1975$ & 41.0 & 343700 & 6783322 & $\square$ & $\square$ & $\square$ & $\square$ & $\square$ \\
\hline 2129 & 1524 & Hollow stem borehole w/SPT & $4 / 28 / 1975$ & 39.5 & 343826 & 6783374 & $\square$ & $\square$ & $\square$ & $\square$ & $\square$ \\
\hline 2129 & 1525 & Hollow stem borehole w/SPT & $4 / 29 / 1974$ & 40.0 & 343872 & 6783384 & $\square$ & $\square$ & $\square$ & $\square$ & $\square$ \\
\hline 2129 & 1526 & Hollow stem borehole w/SPT & $4 / 29 / 1975$ & 41.0 & 343862 & 6783274 & $\square$ & $\square$ & $\square$ & $\square$ & $\square$ \\
\hline 2129 & 1527 & Hollow stem borehole w/SPT & 4/30/1975 & 40.0 & 343876 & 6783168 & $\square$ & $\square$ & $\square$ & $\square$ & $\square$ \\
\hline 2129 & 1528 & Hollow stem borehole w/SPT & 4/30/1975 & 41.0 & 343938 & 6783225 & $\square$ & $\square$ & $\square$ & $\square$ & $\square$ \\
\hline 2129 & 1535 & Hollow stem borehole w/SPT & $5 / 10 / 1972$ & 43.5 & 344444 & 6783812 & $\square$ & $\square$ & $\square$ & $\square$ & $\square$ \\
\hline 2129 & 1590 & Hollow stem borehole w/SPT & $3 / 16 / 1983$ & 60.0 & 343733 & 6783845 & $\checkmark$ & $\nabla$ & $\square$ & $\square$ & $\square$ \\
\hline 2129 & 1591 & Hollow stem borehole w/SPT & $3 / 16 / 1983$ & 60.0 & 343853 & 6783840 & $\checkmark$ & $\checkmark$ & $\square$ & $\checkmark$ & $\square$ \\
\hline 2129 & 1592 & Hollow stem borehole w/SPT & $3 / 16 / 1983$ & 60.0 & 344157 & 6783826 & $\checkmark$ & $\checkmark$ & $\square$ & $\checkmark$ & $\square$ \\
\hline 2130 & 1082 & Hollow stem borehole w/SPT & $9 / 22 / 1981$ & 50.4 & 344873 & 6782991 & $\checkmark$ & $\checkmark$ & $\square$ & $\checkmark$ & $\square$ \\
\hline 2130 & 1505 & Hollow stem borehole w/SPT & $1 / 18 / 1976$ & 54.5 & 344775 & 6783784 & $\nabla$ & $\checkmark$ & $\checkmark$ & $\nabla$ & $\square$ \\
\hline 2130 & 1506 & Hollow stem borehole w/SPT & $1 / 17 / 1977$ & 53.0 & 344768 & 6783616 & $\checkmark$ & $\checkmark$ & $\checkmark$ & $\checkmark$ & $\square$ \\
\hline 2130 & 1507 & Hollow stem borehole w/SPT & $1 / 14 / 1977$ & 55.0 & 344758 & 6783415 & $\checkmark$ & $\checkmark$ & $\checkmark$ & $\checkmark$ & $\square$ \\
\hline 2130 & 1508 & Hollow stem borehole w/SPT & $1 / 18 / 1977$ & 44.0 & 345063 & 6783392 & $\checkmark$ & $\checkmark$ & $\checkmark$ & $\checkmark$ & $\square$ \\
\hline 2130 & 1532 & Hollow stem borehole w/SPT & $5 / 11 / 1972$ & 32.0 & 345040 & 6783602 & $\square$ & $\square$ & $\square$ & $\square$ & $\square$ \\
\hline 2130 & 1533 & Hollow stem borehole w/SPT & $5 / 11 / 1972$ & 41.5 & 344930 & 6783721 & $\square$ & $\square$ & $\square$ & $\square$ & $\square$ \\
\hline 2130 & 1593 & Hollow stem borehole w/SPT & $3 / 16 / 1983$ & 75.0 & 344598 & 6783807 & $\checkmark$ & $\checkmark$ & $\square$ & $\checkmark$ & $\square$ \\
\hline 2130 & 1594 & Hollow stem borehole w/SPT & $3 / 16 / 1983$ & 70.0 & 345096 & 6783780 & $\checkmark$ & $\checkmark$ & $\square$ & $\checkmark$ & $\square$ \\
\hline 2130 & 1595 & Hollow stem borehole w/SPT & $3 / 16 / 1983$ & 75.0 & 345089 & 6783632 & $\checkmark$ & $\checkmark$ & $\square$ & $\checkmark$ & $\square$ \\
\hline 2130 & 1600 & Hollow stem borehole w/SPT & $3 / 16 / 1983$ & 75.0 & 345197 & 6783516 & $\nabla$ & $\checkmark$ & $\square$ & $\nabla$ & $\square$ \\
\hline 2130 & 1645 & Hollow stem borehole w/SPT & $6 / 11 / 1982$ & 41.5 & 344825 & 6783793 & $\square$ & $\square$ & $\square$ & $\square$ & $\square$ \\
\hline 2130 & 1781 & Hollow stem borehole, no SPT & $11 / 19 / 1965$ & 48.0 & 344731 & 6782801 & $\square$ & $\square$ & $\square$ & $\square$ & $\square$ \\
\hline 2130 & 1782 & Hollow stem borehole, no SPT & $11 / 19 / 1965$ & 43.0 & 344732 & 6782851 & $\square$ & $\square$ & $\square$ & $\square$ & $\square$ \\
\hline 2130 & 1783 & Hollow stem borehole, no SPT & $11 / 19 / 1965$ & 36.0 & 344736 & 6782910 & $\square$ & $\square$ & $\square$ & $\square$ & $\square$ \\
\hline 2130 & 2084 & Hollow stem borehole w/SPT & $1 / 18 / 1977$ & 55.0 & 344910 & 6783392 & 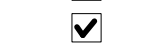 & $\checkmark$ & $\checkmark$ & $\sqrt{v}$ & $\square$ \\
\hline 2130 & 4346 & Water well & $11 / 30 / 1974$ & 118.0 & 344650 & 6783340 & $\checkmark$ & $\square$ & $\square$ & $\checkmark$ & $\square$ \\
\hline 2130 & EB001 & Water well & $12 / 1 / 1981$ & 450.0 & 344865 & 6783610 & $\checkmark$ & $\square$ & $\square$ & $\square$ & $\square$ \\
\hline 2131 & 1536 & Hollow stem borehole w/SPT & $2 / 16 / 1983$ & 73.5 & 345287 & 6783332 & $\boldsymbol{V}$ & $\nabla$ & $\square$ & $\checkmark$ & $\square$ \\
\hline 2131 & 1537 & Hollow stem borehole w/SPT & $2 / 18 / 1983$ & 85.0 & 345243 & 6783403 & $\nabla$ & $\nabla$ & $\square$ & $\nabla$ & $\square$ \\
\hline
\end{tabular}




\begin{tabular}{|c|c|c|c|c|c|c|c|c|c|c|c|}
\hline \multirow[b]{2}{*}{$\begin{array}{l}\text { MOA } \\
\text { grid }\end{array}$} & \multirow[b]{2}{*}{$\begin{array}{l}\text { DGGS } \\
\text { number }\end{array}$} & \multirow[b]{2}{*}{ Hole Type } & \multirow[b]{2}{*}{$\begin{array}{c}\text { Date } \\
\text { Completed }\end{array}$} & \multirow[b]{2}{*}{$\begin{array}{c}\text { Total } \\
\text { depth }(\mathrm{ft})\end{array}$} & \multicolumn{2}{|c|}{ UTM coordinates (m) } & \multicolumn{5}{|c|}{ Data entered } \\
\hline & & & & & East & North & Lithology & SPT & $\begin{array}{c}\text { Sample } \\
\text { test }\end{array}$ & $\begin{array}{l}\text { Water } \\
\text { level }\end{array}$ & Velocity \\
\hline 2131 & 1596 & Hollow stem borehole w/SPT & $3 / 16 / 1983$ & 70.0 & 345314 & 6783763 & $\checkmark$ & $\checkmark$ & $\square$ & $\nabla$ & $\square$ \\
\hline 2131 & 1597 & Hollow stem borehole w/SPT & $3 / 16 / 1983$ & 50.0 & 345363 & 6783644 & $\checkmark$ & $\checkmark$ & $\checkmark$ & $\sqrt{V}$ & $\square$ \\
\hline 2131 & 1598 & Hollow stem borehole w/SPT & $3 / 16 / 1983$ & 50.0 & 345380 & 6783522 & $\checkmark$ & $\checkmark$ & $\nabla$ & $\bar{\nabla}$ & \\
\hline 2131 & 1599 & Hollow stem borehole w/SPT & $3 / 16 / 1983$ & 73.0 & 345336 & 6783417 & $\checkmark$ & 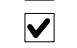 & $\square$ & $\sqrt{\nabla}$ & $\square$ \\
\hline 2131 & 4338 & Water well & $12 / 23 / 1976$ & 150.0 & 345490 & 6783024 & $\checkmark$ & $\square$ & $\square$ & $\square$ & $\square$ \\
\hline 2131 & 4344 & Water well & 9/6/1978 & 111.0 & 345693 & 6783201 & $\checkmark$ & $\square$ & $\square$ & $\checkmark$ & $\square$ \\
\hline 2132 & 1541 & Hollow stem borehole, no SPT & $1 / 23 / 1976$ & 24.0 & 346758 & 6783050 & $\square$ & $\square$ & $\square$ & $\square$ & $\square$ \\
\hline 2132 & 1542 & Hollow stem borehole w/SPT & $1 / 23 / 1976$ & 20.0 & 346779 & 6782954 & $\square$ & 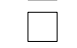 & $\square$ & $\square$ & $\square$ \\
\hline 2132 & 4333 & Water well & $9 / 11 / 1972$ & 114.0 & 346221 & 6782961 & $\checkmark$ & $\square$ & $\square$ & $\square$ & $\square$ \\
\hline 2132 & 4349 & Water well & $7 / 14 / 1981$ & 108.0 & 346075 & 6783401 & $\checkmark$ & & $\square$ & $\checkmark$ & \\
\hline 2132 & DZ002 & Water well & $6 / 5 / 1968$ & 171.0 & 346249 & 6783470 & $\checkmark$ & $\square$ & $\square$ & $\nabla$ & $\square$ \\
\hline 2133 & 1538 & Hollow stem borehole, no SPT & $1 / 23 / 1976$ & 21.0 & 347015 & 6782944 & $\square$ & $\square$ & $\square$ & $\square$ & $\square$ \\
\hline 2133 & 1539 & Hollow stem borehole, no SPT & $1 / 23 / 1976$ & 20.0 & 346961 & 6783034 & $\square$ & $\square$ & $\square$ & $\square$ & $\square$ \\
\hline 2133 & 1540 & Hollow stem borehole, no SPT & $1 / 23 / 1976$ & 20.0 & 346855 & 6783038 & $\square$ & $\square$ & $\square$ & $\square$ & $\square$ \\
\hline 2133 & 1543 & Hollow stem borehole, no SPT & $1 / 23 / 1976$ & 20.0 & 346860 & 6782947 & $\square$ & $\square$ & $\square$ & $\square$ & $\square$ \\
\hline 2133 & 1544 & Hollow stem borehole w/SPT & $12 / 8 / 1981$ & 20.0 & 347272 & 6783594 & $\square$ & $\square$ & $\square$ & $\square$ & $\square$ \\
\hline 2133 & 1545 & Hollow stem borehole w/SPT & $12 / 8 / 1981$ & 20.0 & 347266 & 6783468 & $\square$ & $\square$ & $\square$ & $\square$ & $\square$ \\
\hline 2133 & 1546 & Hollow stem borehole w/SPT & $12 / 9 / 1981$ & 20.0 & 347259 & 6783299 & $\square$ & $\square$ & $\square$ & $\square$ & $\square$ \\
\hline 2133 & 1547 & Hollow stem borehole w/SPT & $12 / 9 / 1981$ & 20.0 & 347386 & 6783281 & $\square$ & $\square$ & $\square$ & $\square$ & $\square$ \\
\hline 2133 & 3043 & Water well & $8 / 17 / 1970$ & 116.0 & 347248 & 6783505 & $\checkmark$ & $\square$ & $\square$ & $\checkmark$ & $\square$ \\
\hline 2133 & 3047 & Water well & $10 / 7 / 1983$ & 105.0 & 347322 & 6783161 & $\checkmark$ & & $\square$ & $\square$ & $\square$ \\
\hline 2133 & 3049 & Water well & $8 / 10 / 1976$ & 129.0 & 347356 & 6782912 & $\checkmark$ & $\square$ & $\square$ & $\nabla$ & $\square$ \\
\hline 2133 & 4343 & Water well & $7 / 23 / 1968$ & 118.0 & 347545 & 6783120 & $\checkmark$ & $\square$ & $\square$ & $\square$ & $\square$ \\
\hline 2133 & DY001 & Water well & $6 / 14 / 1985$ & 179.0 & 347253 & 6783442 & $\checkmark$ & $\square$ & $\square$ & $\checkmark$ & $\square$ \\
\hline 2134 & 4337 & Water well & $5 / 25 / 1965$ & 126.0 & 347851 & 6782921 & 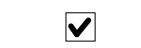 & 5 & $\square$ & $\bar{\nabla}$ & $\square$ \\
\hline 2134 & 4354 & Water well & $7 / 20 / 1969$ & 104.0 & 348029 & 6783595 & $\square$ & $\square$ & $\square$ & $\bar{V}$ & $\square$ \\
\hline 2134 & 4355 & Water well & $3 / 29 / 1982$ & 126.0 & 347969 & 6783598 & $\checkmark$ & $\square$ & $\square$ & $\square$ & $\square$ \\
\hline 2134 & 4356 & Water well & $11 / 30 / 1978$ & 104.0 & 347880 & 6783601 & $\checkmark$ & $\square$ & $\square$ & $\nabla$ & $\square$ \\
\hline 2134 & 4361 & Water well & $1 / 21 / 1977$ & 101.0 & 348002 & 6783658 & $\nabla$ & $\square$ & $\square$ & $\checkmark$ & $\square$ \\
\hline 2135 & 1113 & Hollow stem borehole w/SPT & $1 / 22 / 1986$ & 25.0 & 348426 & 6783224 & $\square$ & $\square$ & $\square$ & $\square$ & $\square$ \\
\hline 2136 & 1529 & Hollow stem borehole w/SPT & $2 / 20 / 1996$ & 21.5 & 349804 & 6783213 & $\checkmark$ & $\checkmark$ & $\square$ & $\square$ & $\square$ \\
\hline 2136 & 1530 & Hollow stem borehole w/SPT & $2 / 20 / 1996$ & 21.5 & 349746 & 6783306 & $\square$ & $\square$ & $\square$ & $\square$ & $\square$ \\
\hline 2136 & 1531 & Hollow stem borehole w/SPT & $2 / 20 / 1996$ & 21.5 & 349761 & 6783249 & $\checkmark$ & $\nabla$ & $\square$ & $\square$ & $\square$ \\
\hline 2136 & 3044 & Water well & $6 / 20 / 1966$ & 349.0 & 349869 & 6783206 & $\checkmark$ & $\square$ & $\square$ & $\bar{\nabla}$ & $\square$ \\
\hline 2136 & 4350 & Water well & 7/8/1966 & 169.0 & 349874 & 6783330 & $\checkmark$ & $\square$ & $\square$ & $\vec{\nabla}$ & $\square$ \\
\hline 2139 & 3046 & Water well & $12 / 26 / 1968$ & 260.0 & 352207 & 6782921 & $\checkmark$ & $\square$ & $\square$ & $\checkmark$ & $\square$ \\
\hline
\end{tabular}




\begin{tabular}{|c|c|c|c|c|c|c|c|c|c|c|c|}
\hline \multirow[b]{2}{*}{$\begin{array}{c}\text { MOA } \\
\text { grid }\end{array}$} & \multirow[b]{2}{*}{$\begin{array}{c}\text { DGGS } \\
\text { number }\end{array}$} & \multirow[b]{2}{*}{ Hole Type } & \multirow[b]{2}{*}{$\begin{array}{c}\text { Date } \\
\text { Completed }\end{array}$} & \multirow[b]{2}{*}{$\begin{array}{c}\text { Total } \\
\text { depth (ft) }\end{array}$} & \multicolumn{2}{|c|}{ UTM coordinates (m) } & \multicolumn{5}{|c|}{ Data entered } \\
\hline & & & & & East & North & Lithology & SPT & $\begin{array}{c}\text { Sample } \\
\text { test }\end{array}$ & $\begin{array}{l}\text { Water } \\
\text { level }\end{array}$ & Velocity \\
\hline 2142 & 3045 & Water well & $5 / 9 / 1984$ & 107.0 & 354219 & 6782713 & $\checkmark$ & $\square$ & $\square$ & $\nabla$ & $\square$ \\
\hline 2142 & 4342 & Water well & $6 / 6 / 1981$ & 111.0 & 354118 & 6782810 & $\checkmark$ & $\square$ & $\square$ & $\checkmark$ & $\square$ \\
\hline 2143 & 3039 & Water well & $10 / 22 / 1980$ & 300.0 & 355024 & 6783361 & $\boldsymbol{V}$ & $\square$ & $\square$ & $\nabla$ & $\square$ \\
\hline 2220 & DA001 & Solid stem borehole (no cores or SP & $8 / 12 / 1954$ & 20.0 & 336849 & 6782665 & $\square$ & $\square$ & $\square$ & $\square$ & $\square$ \\
\hline 2220 & DA002 & Solid stem borehole (no cores or SP & $8 / 12 / 1954$ & 10.0 & 336974 & 6782622 & $\square$ & $\square$ & $\square$ & $\square$ & $\square$ \\
\hline 2220 & DA003 & Solid stem borehole (no cores or SP & $8 / 12 / 1954$ & 11.0 & 336861 & 6782742 & $\square$ & $\square$ & $\square$ & $\square$ & $\square$ \\
\hline 2221 & DB014 & Solid stem borehole (no cores or SP & $8 / 12 / 1954$ & 15.0 & 337215 & 6782640 & $\square$ & $\square$ & $\square$ & $\square$ & $\square$ \\
\hline 2221 & DB015 & Solid stem borehole (no cores or SP & $8 / 12 / 1954$ & 10.0 & 337289 & 6782614 & $\square$ & $\square$ & $\square$ & $\square$ & $\square$ \\
\hline 2221 & DB016 & Solid stem borehole (no cores or SP & $8 / 12 / 1954$ & 10.0 & 337353 & 6782586 & $\square$ & $\square$ & $\square$ & $\square$ & $\square$ \\
\hline 2221 & DB017 & Solid stem borehole (no cores or SP & $8 / 12 / 1954$ & 10.0 & 337431 & 6782532 & $\square$ & $\square$ & $\square$ & $\square$ & $\square$ \\
\hline 2221 & DB018 & Solid stem borehole (no cores or SP & $8 / 12 / 1954$ & 10.0 & 337496 & 6782525 & $\square$ & $\square$ & $\square$ & $\square$ & $\square$ \\
\hline 2221 & DB021 & Solid stem borehole (no cores or SP & $8 / 12 / 1954$ & 11.0 & 337690 & 6782514 & $\square$ & $\square$ & $\square$ & $\square$ & $\square$ \\
\hline 2221 & DB022 & Solid stem borehole (no cores or SP & $8 / 12 / 1954$ & 10.0 & 337758 & 6782578 & $\square$ & $\square$ & $\square$ & $\square$ & $\square$ \\
\hline 2221 & DB023 & Solid stem borehole (no cores or SP & $8 / 12 / 1954$ & 12.0 & 337819 & 6782658 & $\square$ & $\square$ & $\square$ & $\square$ & $\square$ \\
\hline 2221 & DB024 & Solid stem borehole (no cores or SP & $8 / 12 / 1954$ & 10.0 & 337800 & 6782736 & $\square$ & $\square$ & $\square$ & $\square$ & $\square$ \\
\hline 2221 & DB025 & Solid stem borehole (no cores or SP & $8 / 12 / 1954$ & 10.0 & 337929 & 6782761 & $\square$ & $\square$ & $\square$ & $\square$ & $\square$ \\
\hline 2221 & DB026 & Solid stem borehole (no cores or SP & $8 / 12 / 1954$ & 9.0 & 337939 & 6782656 & $\square$ & $\square$ & $\square$ & $\square$ & $\square$ \\
\hline 2222 & 2200 & Solid stem borehole (no cores or SP & $2 / 22 / 1972$ & 58.0 & 338427 & 6782843 & $\boldsymbol{V}$ & $\square$ & $\square$ & $\square$ & $\square$ \\
\hline 2222 & 2201 & Solid stem borehole (no cores or SP & $2 / 22 / 1972$ & 57.0 & 338283 & 6782693 & $\boldsymbol{V}$ & $\square$ & $\square$ & $\square$ & $\square$ \\
\hline 2222 & 2202 & Solid stem borehole (no cores or SP & $3 / 28 / 1972$ & 72.0 & 338798 & 6782841 & $\boldsymbol{V}$ & $\square$ & $\square$ & $\square$ & $\square$ \\
\hline 2222 & 2203 & Solid stem borehole (no cores or SP & $3 / 29 / 1972$ & 120.0 & 338762 & 6782679 & $\boldsymbol{V}$ & $\square$ & $\square$ & $\square$ & $\square$ \\
\hline 2222 & 3054 & Water well & $4 / 1 / 1984$ & 287.0 & 338674 & 6783268 & $\boldsymbol{V}$ & $\square$ & $\square$ & $\square$ & $\square$ \\
\hline 2222 & 4273 & Water well & $8 / 1 / 1966$ & 294.0 & 338056 & 6782522 & 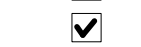 & $\square$ & $\square$ & $\nabla$ & $\square$ \\
\hline 2222 & 4311 & Water well & $6 / 16 / 1974$ & 280.0 & 338210 & 6782948 & $\nabla$ & $\square$ & $\square$ & $\nabla$ & $\square$ \\
\hline 2222 & 4315 & Water well & $5 / 22 / 1975$ & 226.0 & 338707 & 6783018 & $\checkmark$ & $\square$ & $\square$ & $\checkmark$ & $\square$ \\
\hline 2222 & 4322 & Water well & $8 / 5 / 1974$ & 195.0 & 338801 & 6783107 & $\boldsymbol{V}$ & $\square$ & $\square$ & $\nabla$ & $\square$ \\
\hline 2222 & 4323 & Water well & 8/13/1982 & 141.0 & 338801 & 6783107 & $\boldsymbol{V}$ & $\square$ & $\square$ & $\nabla$ & $\square$ \\
\hline 2222 & 4324 & Water well & $10 / 25 / 1978$ & 183.0 & 338682 & 6783113 & $\boldsymbol{V}$ & $\square$ & $\square$ & $\nabla$ & $\square$ \\
\hline 2222 & 4326 & Water well & 9/27/1978 & 192.0 & 338698 & 6783143 & $\boldsymbol{V}$ & $\square$ & $\square$ & $\square$ & $\square$ \\
\hline 2222 & 4330 & Water well & 8/19/1982 & 180.0 & 338805 & 6783200 & 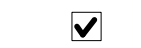 & $\square$ & $\square$ & $\nabla$ & $\square$ \\
\hline 2222 & 4331 & Water well & 8/23/1979 & 306.0 & 338491 & 6783214 & $\nabla$ & $\square$ & $\square$ & $\nabla$ & $\square$ \\
\hline 2223 & 2011 & Natural exposure & 6/3/1997 & 36.0 & 338978 & 6782990 & 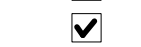 & $\square$ & $\square$ & $\square$ & $\square$ \\
\hline 2223 & 2204 & Hollow stem borehole w/SPT & 10/5/1999 & 21.0 & 339512 & 6783227 & 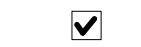 & $\bar{v}$ & $\nabla$ & $\square$ & $\square$ \\
\hline 2223 & 4328 & Water well & 9/26/1973 & 171.0 & 338910 & 6783195 & $\nabla$ & $\square$ & $\square$ & $\nabla$ & $\square$ \\
\hline 2224 & 2026 & Water well & 10/6/1997 & 554.0 & 339655 & 6782493 & $\boldsymbol{v}$ & $\square$ & $\square$ & $\nabla$ & $\square$ \\
\hline 2224 & 3056 & Water well & $9 / 14 / 1985$ & 190.7 & 339991 & 6782929 & $\boldsymbol{V}$ & $\square$ & $\square$ & $\checkmark$ & $\square$ \\
\hline
\end{tabular}




\begin{tabular}{|c|c|c|c|c|c|c|c|c|c|c|c|}
\hline \multirow[b]{2}{*}{$\begin{array}{l}\text { MOA } \\
\text { grid }\end{array}$} & \multirow[b]{2}{*}{$\begin{array}{l}\text { DGGS } \\
\text { number }\end{array}$} & \multirow[b]{2}{*}{ Hole Type } & \multirow[b]{2}{*}{$\begin{array}{c}\text { Date } \\
\text { Completed }\end{array}$} & \multirow[b]{2}{*}{$\begin{array}{c}\text { Total } \\
\text { depth (ft) }\end{array}$} & \multicolumn{2}{|c|}{ UTM coordinates (m) } & \multicolumn{5}{|c|}{ Data entered } \\
\hline & & & & & East & North & Lithology & SPT & $\begin{array}{c}\text { Sample } \\
\text { test }\end{array}$ & $\begin{array}{l}\text { Water } \\
\text { level }\end{array}$ & Velocity \\
\hline 2224 & 3063 & Water well & 4/6/1982 & 416.0 & 339791 & 6782814 & $\checkmark$ & $\square$ & $\square$ & $\square$ & $\square$ \\
\hline 2224 & 3071 & Water well & $4 / 2 / 1982$ & 414.0 & 339784 & 6782659 & $\checkmark$ & $\square$ & $\square$ & $\square$ & $\square$ \\
\hline 2224 & 3073 & Water well & $10 / 10 / 1978$ & 470.0 & 340006 & 6782618 & $\checkmark$ & $\square$ & $\square$ & $\square$ & $\square$ \\
\hline 2224 & 4270 & Water well & $6 / 1 / 1974$ & 120.0 & 340282 & 6782420 & $\nabla$ & $\square$ & $\square$ & $\nabla$ & $\square$ \\
\hline 2224 & 4271 & Water well & 8/6/1981 & 216.0 & 339879 & 6782439 & $\checkmark$ & $\square$ & $\square$ & $\bar{V}$ & $\square$ \\
\hline 2224 & 4272 & Water well & $8 / 13 / 1982$ & 300.0 & 339789 & 6782443 & $\checkmark$ & $\square$ & $\square$ & $\checkmark$ & $\square$ \\
\hline 2224 & 4281 & Water well & $10 / 22 / 1980$ & 263.0 & 339970 & 6782465 & $\checkmark$ & $\square$ & $\square$ & 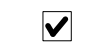 & $\square$ \\
\hline 2224 & 4283 & Water well & $1 / 20 / 1978$ & 294.0 & 339865 & 6782470 & $\checkmark$ & $\checkmark$ & $\square$ & $\nabla$ & $\square$ \\
\hline 2224 & 4285 & Water well & $6 / 15 / 1965$ & 361.0 & 340211 & 6782516 & $\nabla$ & $\square$ & $\square$ & $\nabla$ & $\square$ \\
\hline 2224 & 4286 & Water well & 7/10/1978 & 250.0 & 340211 & 6782516 & $\checkmark$ & $\square$ & $\square$ & $\square$ & $\square$ \\
\hline 2224 & 4288 & Water well & 1/13/1981 & 500.0 & 339883 & 6782531 & $\checkmark$ & $\square$ & $\square$ & $\checkmark$ & $\square$ \\
\hline 2224 & 4289 & Water well & 7/30/1980 & 332.0 & 339689 & 6782540 & $\checkmark$ & $\square$ & $\square$ & $\bar{\nabla}$ & $\square$ \\
\hline 2224 & 4291 & Water well & $5 / 6 / 1982$ & 224.0 & 339992 & 6782619 & $\checkmark$ & $\square$ & $\square$ & $\nabla$ & $\square$ \\
\hline 2224 & 4292 & Water well & $11 / 4 / 1981$ & 259.0 & 339782 & 6782628 & $\checkmark$ & $\square$ & $\square$ & $\nabla$ & $\square$ \\
\hline 2224 & 4294 & Water well & 10/1/1979 & 112.0 & 340202 & 6782640 & $\checkmark$ & $\checkmark$ & $\square$ & $\square$ & $\square$ \\
\hline 2224 & 4295 & Water well & $9 / 18 / 1982$ & 512.0 & 340082 & 6782646 & $\checkmark$ & $\square$ & $\square$ & $\square$ & $\square$ \\
\hline 2224 & 4297 & Water well & $12 / 22 / 1981$ & 510.0 & 339889 & 6782655 & $\checkmark$ & $\checkmark$ & $\square$ & $\boldsymbol{V}$ & $\square$ \\
\hline 2224 & 4301 & Water well & $10 / 11 / 1979$ & 340.0 & 340192 & 6782734 & $\checkmark$ & $\square$ & $\square$ & $\nabla$ & $\square$ \\
\hline 2224 & 4302 & Water well & 10/9/1981 & 410.0 & 339893 & 6782748 & $\checkmark$ & $\square$ & $\square$ & $\nabla$ & $\square$ \\
\hline 2224 & 4304 & Water well & $7 / 20 / 1981$ & 275.0 & 339788 & 6782752 & $\checkmark$ & $\square$ & $\square$ & $\boldsymbol{V}$ & $\square$ \\
\hline 2224 & 4305 & Water well & $11 / 29 / 1983$ & 253.0 & 339684 & 6782757 & $\checkmark$ & $\square$ & $\square$ & $\bar{\nabla}$ & $\square$ \\
\hline 2224 & 4307 & Water well & $6 / 27 / 1981$ & 156.0 & 340091 & 6782831 & $\checkmark$ & $\square$ & $\square$ & $\nabla$ & $\square$ \\
\hline 2224 & 4308 & Water well & $9 / 28 / 1973$ & 244.0 & 340016 & 6782835 & $\checkmark$ & $\square$ & $\square$ & $\nabla$ & $\square$ \\
\hline 2224 & 4309 & Water well & $8 / 17 / 1974$ & 390.0 & 340016 & 6782835 & $\checkmark$ & $\square$ & $\square$ & $\checkmark$ & $\square$ \\
\hline 2224 & 4466 & Water well & $10 / 18 / 1970$ & 250.0 & 340104 & 6783110 & $\checkmark$ & $\square$ & $\square$ & 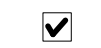 & $\square$ \\
\hline 2225 & 3077 & Water well & $6 / 9 / 1983$ & 280.0 & 339795 & 6782574 & $\checkmark$ & $\square$ & $\square$ & $\nabla$ & $\square$ \\
\hline 2226 & 3052 & Water well & $6 / 17 / 1970$ & 250.0 & 341361 & 6783115 & $\checkmark$ & $\square$ & $\square$ & $\checkmark$ & $\square$ \\
\hline 2226 & 3053 & Water well & $4 / 6 / 1983$ & 319.0 & 341330 & 6783085 & $\checkmark$ & $\square$ & $\square$ & $\nabla$ & $\square$ \\
\hline 2226 & 4332 & Water well & $7 / 7 / 1975$ & 146.0 & 341421 & 6783112 & $\checkmark$ & $\square$ & $\square$ & $\checkmark$ & $\square$ \\
\hline 2226 & 4467 & Water well & $11 / 11 / 1975$ & 276.0 & 341619 & 6782855 & $\checkmark$ & $\square$ & $\square$ & $\checkmark$ & $\square$ \\
\hline 2228 & 1548 & Hollow stem borehole w/SPT & 4/8/1986 & 51.5 & 342858 & 6782516 & $\checkmark$ & $\checkmark$ & $\checkmark$ & $\nabla$ & $\square$ \\
\hline 2228 & 1549 & Hollow stem borehole, no SPT & $10 / 24 / 1975$ & 15.0 & 342848 & 6782508 & $\square$ & $\square$ & $\square$ & $\square$ & $\square$ \\
\hline 2228 & 1550 & Hollow stem borehole w/SPT & $4 / 9 / 1986$ & 51.5 & 342871 & 6782498 & $\checkmark$ & $\bar{\nabla}$ & $\square$ & $\bar{\square}$ & $\square$ \\
\hline 2228 & 1551 & Hollow stem borehole w/SPT & $4 / 7 / 1986$ & 51.5 & 342866 & 6782490 & $\checkmark$ & $\checkmark$ & $\checkmark$ & $\boldsymbol{V}$ & $\square$ \\
\hline 2228 & 1604 & Solid stem borehole (no cores or SP & $10 / 11 / 1978$ & 30.0 & 343586 & 6782354 & $\square$ & $\square$ & $\square$ & $\square$ & $\square$ \\
\hline 2228 & 2089 & Hollow stem borehole w/SPT & $1 / 11 / 1984$ & 41.5 & 342932 & 6782993 & $\checkmark$ & $\checkmark$ & $\checkmark$ & $\checkmark$ & $\square$ \\
\hline
\end{tabular}




\begin{tabular}{|c|c|c|c|c|c|c|c|c|c|c|c|}
\hline \multirow[b]{2}{*}{$\begin{array}{l}\text { MOA } \\
\text { grid }\end{array}$} & \multirow[b]{2}{*}{$\begin{array}{c}\text { DGGS } \\
\text { number }\end{array}$} & \multirow[b]{2}{*}{ Hole Type } & \multirow[b]{2}{*}{$\begin{array}{c}\text { Date } \\
\text { Completed }\end{array}$} & \multirow[b]{2}{*}{$\begin{array}{c}\text { Total } \\
\text { depth (ft) }\end{array}$} & \multicolumn{2}{|c|}{ UTM coordinates (m) } & \multicolumn{5}{|c|}{ Data entered } \\
\hline & & & & & East & North & Lithology & SPT & $\begin{array}{c}\text { Sample } \\
\text { test }\end{array}$ & $\begin{array}{c}\text { Water } \\
\text { level }\end{array}$ & Velocity \\
\hline 2228 & 2090 & Hollow stem borehole w/SPT & $1 / 9 / 1984$ & 46.5 & 342973 & 6783039 & $\boldsymbol{V}$ & $\nabla$ & $\nabla$ & $\checkmark$ & $\square$ \\
\hline 2228 & 2091 & Hollow stem borehole w/SPT & $3 / 15 / 1984$ & 51.5 & 342855 & 6782587 & $\checkmark$ & $\checkmark$ & $\checkmark$ & $\sqrt{v}$ & $\square$ \\
\hline 2228 & 2092 & Hollow stem borehole w/SPT & $3 / 14 / 1984$ & 51.5 & 342849 & 6782491 & $\nabla$ & $\checkmark$ & $\checkmark$ & $\square$ & $\square$ \\
\hline 2228 & 2234 & Hollow stem borehole w/SPT & $12 / 7 / 1982$ & 32.0 & 343409 & 6782850 & $\checkmark$ & $\checkmark$ & $\square$ & $\checkmark$ & $\square$ \\
\hline 2229 & 1559 & Hollow stem borehole w/SPT & $4 / 28 / 1978$ & 50.0 & 344441 & 6782354 & $\checkmark$ & $\checkmark$ & $\square$ & $\checkmark$ & $\square$ \\
\hline 2229 & 1560 & Hollow stem borehole w/SPT & $8 / 26 / 1974$ & 41.5 & 343739 & 6782365 & $\square$ & $\square$ & $\square$ & $\square$ & $\square$ \\
\hline 2229 & 1563 & Hollow stem borehole w/SPT & $8 / 28 / 1974$ & 40.0 & 343794 & 6782559 & $\square$ & $\square$ & $\square$ & $\square$ & $\square$ \\
\hline 2229 & 1564 & Hollow stem borehole w/SPT & $8 / 28 / 1974$ & 40.0 & 343787 & 6782453 & $\square$ & $\square$ & $\square$ & $\square$ & $\square$ \\
\hline 2229 & 1565 & Hollow stem borehole w/SPT & $8 / 28 / 1974$ & 40.0 & 343938 & 6782541 & $\square$ & $\square$ & $\square$ & $\square$ & $\square$ \\
\hline 2229 & 1566 & Hollow stem borehole w/SPT & $8 / 29 / 1974$ & 40.0 & 344028 & 6782525 & $\square$ & $\square$ & $\square$ & $\square$ & $\square$ \\
\hline 2229 & 1567 & Hollow stem borehole w/SPT & $8 / 29 / 1974$ & 40.0 & 344018 & 6782612 & $\square$ & $\square$ & $\square$ & $\square$ & $\square$ \\
\hline 2229 & 1568 & Hollow stem borehole w/SPT & $8 / 28 / 1974$ & 40.0 & 343836 & 6782618 & $\square$ & $\square$ & $\square$ & $\square$ & $\square$ \\
\hline 2229 & 1569 & Hollow stem borehole w/SPT & $8 / 29 / 1974$ & 40.0 & 343685 & 6782624 & $\square$ & $\square$ & $\square$ & $\square$ & $\square$ \\
\hline 2229 & 1570 & Hollow stem borehole w/SPT & $8 / 30 / 1974$ & 40.0 & 343670 & 6782405 & $\square$ & $\square$ & $\square$ & $\square$ & $\square$ \\
\hline 2229 & 1571 & Hollow stem borehole w/SPT & $8 / 30 / 1974$ & 40.0 & 343780 & 6782260 & $\square$ & $\square$ & $\square$ & $\square$ & $\square$ \\
\hline 2229 & 1572 & Hollow stem borehole w/SPT & 7/7/1982 & 31.5 & 344369 & 6782528 & $\square$ & $\square$ & $\square$ & $\square$ & $\square$ \\
\hline 2229 & 4314 & Water well & $6 / 20 / 1969$ & 111.0 & 344444 & 6782729 & 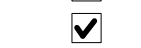 & $\square$ & $\square$ & $\checkmark$ & $\square$ \\
\hline 2230 & 1558 & Hollow stem borehole w/SPT & $4 / 26 / 1978$ & 50.0 & 344451 & 6782378 & $\nabla$ & $\nabla$ & $\square$ & $\nabla$ & $\square$ \\
\hline 2230 & 1573 & Hollow stem borehole w/SPT & $3 / 18 / 1982$ & 35.5 & 344863 & 6782211 & $\checkmark$ & $\checkmark$ & $\square$ & $\checkmark$ & $\square$ \\
\hline 2230 & 1574 & Hollow stem borehole w/SPT & 6/8/1966 & 50.0 & 344723 & 6782536 & $\boldsymbol{V}$ & $\nabla$ & $\nabla$ & $\nabla$ & $\square$ \\
\hline 2230 & 1575 & Hollow stem borehole w/SPT & 6/16/1966 & 45.0 & 344718 & 6782593 & $\square$ & $\square$ & $\square$ & $\square$ & $\square$ \\
\hline 2230 & 1576 & Hollow stem borehole w/SPT & $6 / 24 / 1966$ & 50.0 & 344721 & 6782575 & $\checkmark$ & $\checkmark$ & $\nabla$ & $\square$ & $\square$ \\
\hline 2230 & 1780 & Hollow stem borehole, no SPT & $11 / 20 / 1965$ & 45.0 & 344722 & 6782766 & $\square$ & $\square$ & $\square$ & $\square$ & $\square$ \\
\hline 2231 & 1097 & Hollow stem borehole w/SPT & $3 / 30 / 1983$ & 72.0 & 345442 & 6782207 & $\boldsymbol{V}$ & $\checkmark$ & $\square$ & $\checkmark$ & $\square$ \\
\hline 2231 & 1103 & Hollow stem borehole w/SPT & 4/27/1979 & 30.0 & 345438 & 6782171 & $\square$ & $\square$ & $\square$ & $\square$ & $\square$ \\
\hline 2231 & 1553 & Hollow stem borehole w/SPT & $3 / 22 / 1983$ & 35.0 & 345929 & 6782730 & $\square$ & $\square$ & $\square$ & $\square$ & $\square$ \\
\hline 2231 & 1672 & Hollow stem borehole w/SPT & 9/9/1975 & 52.0 & 345878 & 6782253 & $\boldsymbol{V}$ & $\nabla$ & $\square$ & $\nabla$ & $\square$ \\
\hline 2231 & 4313 & Water well & $9 / 24 / 1973$ & 111.0 & 345744 & 6782672 & $\checkmark$ & $\square$ & $\square$ & $\sqrt{v}$ & $\square$ \\
\hline 2231 & 4316 & Water well & $6 / 6 / 1965$ & 172.0 & 345854 & 6782791 & $\boldsymbol{V}$ & $\square$ & $\square$ & $\checkmark$ & $\square$ \\
\hline 2231 & 4321 & Water well & 8/26/1977 & 168.0 & 345764 & 6782795 & 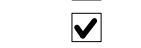 & $\square$ & $\square$ & $\checkmark$ & $\square$ \\
\hline 2231 & DJ001 & Water well & $11 / 4 / 1982$ & 180.0 & 345822 & 6782618 & $\checkmark$ & $\square$ & $\square$ & $\checkmark$ & $\square$ \\
\hline 2232 & 1554 & Hollow stem borehole w/SPT & $7 / 15 / 1980$ & 30.5 & 346137 & 6782325 & $\checkmark$ & $\checkmark$ & $\checkmark$ & $\square$ & $\square$ \\
\hline 2232 & 1555 & Hollow stem borehole w/SPT & $12 / 20 / 1976$ & 27.5 & 346113 & 6782861 & $\square$ & $\square$ & $\square$ & $\square$ & $\square$ \\
\hline 2232 & 1556 & Hollow stem borehole w/SPT & $12 / 20 / 1976$ & 31.2 & 346150 & 6782860 & $\square$ & $\square$ & $\square$ & $\square$ & $\square$ \\
\hline 2232 & 1557 & Hollow stem borehole w/SPT & $12 / 16 / 1976$ & 32.0 & 346113 & 6782921 & $\square$ & $\square$ & $\square$ & $\square$ & $\square$ \\
\hline 2232 & 1577 & Hollow stem borehole w/SPT & $10 / 22 / 1984$ & 42.0 & 346329 & 6782171 & $\checkmark$ & $\checkmark$ & $\nabla$ & $\nabla$ & $\square$ \\
\hline
\end{tabular}




\begin{tabular}{|c|c|c|c|c|c|c|c|c|c|c|c|}
\hline \multirow[b]{2}{*}{$\begin{array}{c}\text { MOA } \\
\text { grid }\end{array}$} & \multirow[b]{2}{*}{$\begin{array}{c}\text { DGGS } \\
\text { number }\end{array}$} & \multirow[b]{2}{*}{ Hole Type } & \multirow[b]{2}{*}{$\begin{array}{c}\text { Date } \\
\text { Completed }\end{array}$} & \multirow[b]{2}{*}{$\begin{array}{c}\text { Total } \\
\text { depth (ft) }\end{array}$} & \multicolumn{2}{|c|}{ UTM coordinates (m) } & \multicolumn{5}{|c|}{ Data entered } \\
\hline & & & & & East & North & Lithology & SPT & $\begin{array}{c}\text { Sample } \\
\text { test }\end{array}$ & $\begin{array}{l}\text { Water } \\
\text { level }\end{array}$ & Velocity \\
\hline 2232 & 1580 & Hollow stem borehole w/SPT & $10 / 31 / 1984$ & 42.0 & 346392 & 6782169 & $\checkmark$ & $\nabla$ & $\nabla$ & $\square$ & $\square$ \\
\hline 2232 & 4293 & Water well & $9 / 1 / 1982$ & 112.0 & 346120 & 6782377 & $\checkmark$ & $\square$ & $\square$ & $\nabla$ & $\square$ \\
\hline 2233 & 1552 & Hollow stem borehole w/SPT & 4/8/1977 & 30.5 & 346827 & 6782281 & $\square$ & $\square$ & $\square$ & $\square$ & $\square$ \\
\hline 2233 & 3050 & Water well & $11 / 5 / 1980$ & 120.0 & 347396 & 6782786 & $\boldsymbol{V}$ & $\square$ & $\square$ & $\square$ & $\square$ \\
\hline 2233 & 4284 & Water well & $10 / 8 / 1974$ & 107.0 & 346950 & 6782185 & $\checkmark$ & $\square$ & $\square$ & $\nabla$ & $\square$ \\
\hline 2233 & 4317 & Water well & 4/28/1966 & 105.0 & 347363 & 6782725 & $\boldsymbol{V}$ & $\square$ & $\square$ & $\nabla$ & $\square$ \\
\hline 2233 & 4318 & Water well & $10 / 23 / 1974$ & 123.0 & 347333 & 6782727 & $\checkmark$ & $\square$ & $\square$ & $\checkmark$ & $\square$ \\
\hline 2233 & 4320 & Water well & $6 / 21 / 1967$ & 112.0 & 347258 & 6782730 & $\boldsymbol{V}$ & $\square$ & $\square$ & $\square$ & $\square$ \\
\hline 2233 & 4325 & Water well & $4 / 6 / 1965$ & 115.0 & 347499 & 6782750 & $\boldsymbol{V}$ & $\square$ & $\square$ & $\square$ & $\square$ \\
\hline 2233 & 4465 & Water well & $5 / 1 / 1977$ & 324.0 & 346945 & 6782743 & $\checkmark$ & $\square$ & $\square$ & $\sqrt{v}$ & $\square$ \\
\hline 2233 & 4468 & Water well & $5 / 29 / 1971$ & 164.0 & 347252 & 6782235 & 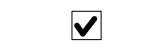 & $\square$ & $\square$ & $\nabla$ & $\square$ \\
\hline 2233 & DK001 & Water well & $3 / 28 / 1970$ & 198.0 & 346972 & 6782130 & $\nabla$ & $\square$ & $\square$ & $\nabla$ & $\square$ \\
\hline 2234 & 4274 & Water well & 4/28/1980 & 108.0 & 347921 & 6782112 & $\nabla$ & $\square$ & $\square$ & $\nabla$ & $\square$ \\
\hline 2234 & 4275 & Water well & $6 / 18 / 1970$ & 198.0 & 347681 & 6782123 & 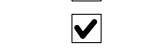 & $\square$ & $\square$ & $\checkmark$ & $\square$ \\
\hline 2234 & 4276 & Water well & $10 / 19 / 1973$ & 165.0 & 347577 & 6782127 & $\nabla$ & $\square$ & $\square$ & $\nabla$ & $\square$ \\
\hline 2234 & 4299 & Water well & 4/1/1964 & 123.0 & 347977 & 6782389 & $\checkmark$ & $\square$ & $\square$ & $\checkmark$ & $\square$ \\
\hline 2234 & 4300 & Water well & $5 / 9 / 1964$ & 165.0 & 347618 & 6782405 & $\boldsymbol{v}$ & $\square$ & $\square$ & $\square$ & $\square$ \\
\hline 2235 & 4298 & Water well & 9/20/1974 & 305.0 & 348948 & 6782347 & $\nabla$ & $\square$ & $\square$ & $\square$ & $\square$ \\
\hline 2235 & 4312 & Water well & $5 / 21 / 1981$ & 102.0 & 348449 & 6782555 & $\boldsymbol{V}$ & $\square$ & $\square$ & $\square$ & $\square$ \\
\hline 2236 & 4247 & Water well & $8 / 4 / 1975$ & 145.0 & 349595 & 6781718 & $\boldsymbol{V}$ & $\square$ & $\square$ & $\nabla$ & $\square$ \\
\hline 2241 & 2007 & Natural exposure & 6/1/1997 & 10.0 & 353453 & 6782531 & $\boldsymbol{V}$ & $\square$ & $\square$ & $\square$ & $\square$ \\
\hline 2321 & DB001 & Solid stem borehole (no cores or SP & $8 / 12 / 1954$ & 15.0 & 337331 & 6782492 & $\square$ & $\square$ & $\square$ & $\square$ & $\square$ \\
\hline 2321 & DB002 & Solid stem borehole (no cores or SP & $8 / 12 / 1954$ & 10.0 & 337216 & 6782496 & $\square$ & $\square$ & $\square$ & $\square$ & $\square$ \\
\hline 2321 & DB003 & Solid stem borehole (no cores or SP & $8 / 12 / 1954$ & 18.0 & 337286 & 6782460 & $\square$ & $\square$ & $\square$ & $\square$ & $\square$ \\
\hline 2321 & DB004 & Solid stem borehole (no cores or SP & $8 / 12 / 1954$ & 20.0 & 337341 & 6782430 & $\square$ & $\square$ & $\square$ & $\square$ & $\square$ \\
\hline 2321 & DB005 & Solid stem borehole (no cores or SP & $8 / 12 / 1954$ & 11.0 & 337527 & 6782270 & & $\square$ & $\square$ & $\square$ & $\square$ \\
\hline 2321 & DB006 & Solid stem borehole (no cores or SP & $8 / 12 / 1954$ & 10.0 & 337533 & 6782306 & $\square$ & $\square$ & $\square$ & $\square$ & $\square$ \\
\hline 2321 & DB007 & Solid stem borehole (no cores or SP & $8 / 12 / 1954$ & 16.0 & 337463 & 6782358 & & $\square$ & $\square$ & $\square$ & $\square$ \\
\hline 2321 & DB008 & Solid stem borehole (no cores or SP & $8 / 12 / 1954$ & 10.0 & 337490 & 6782348 & $\square$ & $\square$ & $\square$ & $\square$ & $\square$ \\
\hline 2321 & DB009 & Solid stem borehole (no cores or SP & $8 / 12 / 1954$ & 10.0 & 337477 & 6782415 & $\square$ & $\square$ & $\square$ & $\square$ & $\square$ \\
\hline 2321 & DB010 & Solid stem borehole (no cores or SP & $8 / 12 / 1954$ & 11.0 & 337510 & 6782410 & $\square$ & $\square$ & $\square$ & $\square$ & $\square$ \\
\hline 2321 & DB011 & Solid stem borehole (no cores or SP & $8 / 12 / 1954$ & 10.0 & 337527 & 6782398 & $\square$ & $\square$ & $\square$ & $\square$ & $\square$ \\
\hline 2321 & DB012 & Solid stem borehole (no cores or SP & $8 / 12 / 1954$ & 12.0 & 337555 & 6782368 & $\square$ & $\square$ & $\square$ & $\square$ & $\square$ \\
\hline 2321 & DB019 & Solid stem borehole (no cores or SP & $8 / 12 / 1954$ & 10.0 & 337584 & 6782466 & $\square$ & $\square$ & $\square$ & $\square$ & $\square$ \\
\hline 2321 & DB020 & Solid stem borehole (no cores or SP & $8 / 12 / 1954$ & 10.0 & 337632 & 6782436 & $\square$ & $\square$ & $\square$ & $\square$ & $\square$ \\
\hline 2321 & DB028 & Solid stem borehole (no cores or SP & $8 / 12 / 1954$ & 15.0 & 337810 & 6782481 & $\square$ & $\square$ & $\square$ & $\square$ & $\square$ \\
\hline
\end{tabular}




\begin{tabular}{|c|c|c|c|c|c|c|c|c|c|c|c|}
\hline \multirow[b]{2}{*}{$\begin{array}{l}\text { MOA } \\
\text { grid }\end{array}$} & \multirow[b]{2}{*}{$\begin{array}{l}\text { DGGS } \\
\text { number }\end{array}$} & \multirow[b]{2}{*}{ Hole Type } & \multirow[b]{2}{*}{$\begin{array}{c}\text { Date } \\
\text { Completed }\end{array}$} & \multirow[b]{2}{*}{$\begin{array}{c}\text { Total } \\
\text { depth }(\mathrm{ft})\end{array}$} & \multicolumn{2}{|c|}{ UTM coordinates (m) } & \multicolumn{5}{|c|}{ Data entered } \\
\hline & & & & & East & North & Lithology & SPT & $\begin{array}{c}\text { Sample } \\
\text { test }\end{array}$ & $\begin{array}{l}\text { Water } \\
\text { level }\end{array}$ & Velocity \\
\hline 2321 & DB029 & Solid stem borehole (no cores or SP & $8 / 12 / 1954$ & 10.0 & 337653 & 6782390 & $\square$ & & $\square$ & $\square$ & $\square$ \\
\hline 2321 & DB030 & Solid stem borehole (no cores or SP & $8 / 12 / 1954$ & 11.0 & 337691 & 6782350 & $\square$ & $\square$ & $\square$ & $\square$ & $\square$ \\
\hline 2321 & DB031 & Solid stem borehole (no cores or SP & $8 / 12 / 1954$ & 10.0 & 337750 & 6782330 & $\square$ & & $\square$ & $\square$ & \\
\hline 2321 & DB032 & Solid stem borehole (no cores or SP & $8 / 12 / 1954$ & 12.0 & 337818 & 6782316 & $\square$ & 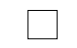 & $\square$ & $\square$ & $\square$ \\
\hline 2321 & DB033 & Solid stem borehole (no cores or SP & $8 / 12 / 1954$ & 11.0 & 337880 & 6782315 & $\square$ & $\square$ & $\square$ & $\square$ & $\square$ \\
\hline 2321 & DB034 & Solid stem borehole (no cores or SP & $8 / 12 / 1954$ & 8.0 & 337942 & 6782357 & $\square$ & $\square$ & $\square$ & $\square$ & $\square$ \\
\hline 2322 & 3127 & Water well & $7 / 12 / 1976$ & 158.0 & 338666 & 6782122 & $\checkmark$ & $\square$ & $\square$ & $\checkmark$ & $\square$ \\
\hline 2322 & 4253 & Water well & $9 / 14 / 1965$ & 253.0 & 338670 & 6782215 & $\checkmark$ & & $\square$ & $\square$ & $\square$ \\
\hline 2322 & 4254 & Water well & $5 / 27 / 1982$ & 351.0 & 338058 & 6782243 & $\checkmark$ & 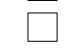 & $\square$ & $\sqrt{\square}$ & $\square$ \\
\hline 2322 & 4256 & Water well & $9 / 17 / 1973$ & 225.0 & 338582 & 6782250 & $\checkmark$ & & $\square$ & $\checkmark$ & $\square$ \\
\hline 2322 & 4260 & Water well & $5 / 12 / 1977$ & 325.0 & 338062 & 6782336 & $\checkmark$ & $\square$ & $\square$ & $\nabla$ & $\square$ \\
\hline 2322 & 4267 & Water well & $12 / 27 / 1981$ & 315.0 & 338156 & 6782424 & $\checkmark$ & & $\square$ & $\bar{\nabla}$ & $\square$ \\
\hline 2322 & 4269 & Water well & $10 / 30 / 1975$ & 328.0 & 338067 & 6782459 & $\checkmark$ & 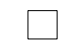 & $\square$ & $\square$ & $\square$ \\
\hline 2323 & 2010 & Natural exposure & $6 / 3 / 1997$ & 61.0 & 338884 & 6782336 & $\checkmark$ & $\square$ & $\square$ & $\square$ & $\square$ \\
\hline 2323 & 2205 & Solid stem borehole (no cores or SP & $5 / 16 / 1974$ & 30.0 & 339184 & 6781911 & $\checkmark$ & 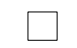 & $\square$ & $\square$ & $\square$ \\
\hline 2323 & 303 & Water well & $4 / 10 / 1976$ & 474.0 & 339340 & 6781570 & $\checkmark$ & & $\square$ & $\checkmark$ & $\square$ \\
\hline 2323 & 4206 & Water well & $6 / 16 / 1969$ & 304.0 & 339126 & 6781698 & $\checkmark$ & & $\square$ & 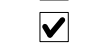 & $\square$ \\
\hline 2323 & 4220 & Water well & $6 / 6 / 1980$ & 260.0 & 338940 & 6781892 & $\checkmark$ & & $\square$ & $\square$ & $\square$ \\
\hline 2323 & 4225 & Water well & $9 / 13 / 1970$ & 243.0 & 339391 & 6781934 & $\checkmark$ & $\square$ & $\square$ & $\nabla$ & $\square$ \\
\hline 2323 & 4517 & Oil \& gas exploratory well & & 11566.0 & 339407 & 6781813 & $\square$ & $\square$ & $\square$ & $\square$ & $\square$ \\
\hline 2324 & 2129 & Hollow stem borehole w/SPT & $5 / 27 / 1998$ & 61.5 & 340078 & 6781707 & $\checkmark$ & $\checkmark$ & $\bar{\nabla}$ & $\bar{\nabla}$ & $\square$ \\
\hline 2324 & 3094 & Water well & $9 / 12 / 1977$ & 273.0 & 340294 & 6782358 & $\checkmark$ & & $\square$ & $\nabla$ & $\square$ \\
\hline 2324 & 4233 & Water well & $8 / 15 / 1973$ & 244.0 & 340349 & 6781921 & $\checkmark$ & & $\square$ & $\nabla$ & $\square$ \\
\hline 2324 & 4234 & Water well & $10 / 20 / 1977$ & 243.0 & 340350 & 6781952 & $\checkmark$ & $\square$ & $\square$ & $\checkmark$ & $\square$ \\
\hline 2324 & 4252 & Water well & $7 / 21 / 1982$ & 280.0 & 340060 & 6782151 & $\checkmark$ & & $\square$ & $\bar{\nabla}$ & $\square$ \\
\hline 2324 & 4261 & Water well & 4/1/1968 & 262.0 & 340169 & 6782239 & $\checkmark$ & & $\square$ & $\bar{V}$ & $\square$ \\
\hline 2324 & 4262 & Water well & $12 / 13 / 1965$ & 271.0 & 340351 & 6782293 & $\checkmark$ & $\square$ & $\square$ & $\checkmark$ & $\square$ \\
\hline 2324 & 4266 & Water well & $5 / 7 / 1981$ & 265.0 & 340293 & 6782327 & $\checkmark$ & & $\square$ & $\nabla$ & $\square$ \\
\hline 2324 & CX017 & Solid stem borehole (no cores or SP & $3 / 7 / 1962$ & 6.0 & 340008 & 6781636 & $\square$ & & $\square$ & $\square$ & $\square$ \\
\hline 2324 & CX018 & Solid stem borehole (no cores or SP & $3 / 7 / 1962$ & 25.0 & 340102 & 6781630 & $\square$ & & $\square$ & $\square$ & $\square$ \\
\hline 2324 & CX019 & Solid stem borehole (no cores or SP & $3 / 7 / 1962$ & 28.0 & 340039 & 6781662 & $\square$ & $\square$ & $\square$ & $\square$ & $\square$ \\
\hline 2324 & CX020 & Solid stem borehole (no cores or SP & $3 / 7 / 1962$ & 10.0 & 340002 & 6781694 & $\square$ & $\square$ & $\square$ & $\square$ & $\square$ \\
\hline 2324 & CX021 & Solid stem borehole (no cores or SP & 3/7/1962 & 3.5 & 340140 & 6781702 & $\square$ & $\square$ & $\square$ & $\square$ & $\square$ \\
\hline 2324 & CX022 & Solid stem borehole (no cores or SP & $3 / 7 / 1962$ & 26.0 & 340066 & 6781723 & $\square$ & $\square$ & $\square$ & $\square$ & $\square$ \\
\hline 2324 & CX023 & Solid stem borehole (no cores or SP & $3 / 7 / 1962$ & 3.5 & 340020 & 6781750 & $\square$ & $\square$ & $\square$ & $\square$ & $\square$ \\
\hline 2324 & CX024 & Solid stem borehole (no cores or SP & $3 / 7 / 1962$ & 10.0 & 340128 & 6781763 & $\square$ & $\square$ & $\square$ & $\square$ & $\square$ \\
\hline
\end{tabular}




\begin{tabular}{|c|c|c|c|c|c|c|c|c|c|c|c|}
\hline \multirow[b]{2}{*}{$\begin{array}{c}\text { MOA } \\
\text { grid }\end{array}$} & \multirow[b]{2}{*}{$\begin{array}{l}\text { DGGS } \\
\text { number }\end{array}$} & \multirow[b]{2}{*}{ Hole Type } & \multirow[b]{2}{*}{$\begin{array}{c}\text { Date } \\
\text { Completed }\end{array}$} & \multirow[b]{2}{*}{$\begin{array}{c}\text { Total } \\
\text { depth (ft) }\end{array}$} & \multicolumn{2}{|c|}{ UTM coordinates (m) } & \multicolumn{5}{|c|}{ Data entered } \\
\hline & & & & & East & North & Lithology & SPT & $\begin{array}{c}\text { Sample } \\
\text { test }\end{array}$ & $\begin{array}{l}\text { Water } \\
\text { level }\end{array}$ & Velocity \\
\hline 2324 & CX025 & Solid stem borehole (no cores or SP & $3 / 7 / 1962$ & 12.5 & 340007 & 6781810 & $\square$ & $\square$ & $\square$ & $\square$ & $\square$ \\
\hline 2324 & CX026 & Solid stem borehole (no cores or SP & $3 / 7 / 1962$ & 30.0 & 340117 & 6781818 & $\square$ & $\square$ & $\square$ & $\square$ & $\square$ \\
\hline 2324 & $\mathrm{CX} 027$ & Solid stem borehole (no cores or SP & $3 / 7 / 1962$ & 15.0 & 340054 & 6781858 & $\square$ & $\square$ & $\square$ & $\square$ & $\square$ \\
\hline 2324 & CX028 & Solid stem borehole (no cores or SP & $3 / 7 / 1962$ & 4.0 & 340150 & 6781867 & $\square$ & $\square$ & $\square$ & $\square$ & $\square$ \\
\hline 2325 & 2242 & Hollow stem borehole w/SPT & $6 / 3 / 1971$ & 26.5 & 341143 & 6781837 & $\checkmark$ & $\checkmark$ & $\square$ & $\checkmark$ & $\square$ \\
\hline 2325 & 2243 & Hollow stem borehole w/SPT & & 31.5 & 340802 & 6782066 & $\boldsymbol{V}$ & $\checkmark$ & $\square$ & $\nabla$ & $\square$ \\
\hline 2325 & 2244 & Hollow stem borehole w/SPT & & 31.5 & 340806 & 6782167 & $\nabla$ & $\nabla$ & $\square$ & $\nabla$ & $\square$ \\
\hline 2325 & 2245 & Hollow stem borehole w/SPT & $12 / 16 / 1981$ & 35.0 & 340853 & 6782207 & $\boldsymbol{V}$ & $\nabla$ & $\square$ & $\nabla$ & $\square$ \\
\hline 2325 & 2246 & Hollow stem borehole w/SPT & $3 / 17 / 1983$ & 31.5 & 340813 & 6782257 & $\boldsymbol{v}$ & $\nabla$ & $\square$ & $\nabla$ & $\square$ \\
\hline 2325 & 2247 & Hollow stem borehole w/SPT & & 31.5 & 340816 & 6782359 & $\boldsymbol{V}$ & $\nabla$ & $\square$ & $\checkmark$ & $\square$ \\
\hline 2325 & 3118 & Water well & $6 / 27 / 1979$ & 247.0 & 340473 & 6782009 & $\boldsymbol{V}$ & $\square$ & $\square$ & $\checkmark$ & $\square$ \\
\hline 2325 & 3119 & Water well & $1 / 25 / 1978$ & 265.0 & 340444 & 6782010 & $\nabla$ & $\square$ & $\square$ & $\checkmark$ & $\square$ \\
\hline 2325 & 3129 & Water well & $10 / 27 / 1976$ & 403.0 & 340454 & 6781916 & $\boldsymbol{V}$ & $\square$ & $\square$ & $V$ & $\square$ \\
\hline 2325 & 3130 & Water well & $9 / 5 / 1981$ & 330.0 & 340439 & 6781917 & 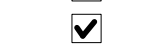 & $\square$ & $\square$ & $V$ & $\square$ \\
\hline 2325 & 4210 & Water well & $6 / 21 / 1973$ & 235.0 & 341102 & 6781670 & $\boldsymbol{V}$ & $\square$ & $\square$ & $\nabla$ & $\square$ \\
\hline 2325 & 4211 & Water well & 7/11/1978 & 459.0 & 340833 & 6781683 & 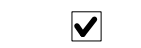 & $\square$ & $\square$ & $v$ & $\square$ \\
\hline 2325 & 4219 & Water well & $10 / 25 / 1976$ & 216.0 & 340749 & 6781810 & $\boldsymbol{V}$ & $\square$ & $\square$ & 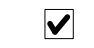 & $\square$ \\
\hline 2325 & 4224 & Water well & 11/3/1971 & 230.0 & 340752 & 6781872 & $\nabla$ & $\square$ & $\square$ & $\nabla$ & $\square$ \\
\hline 2325 & 4242 & Water well & $7 / 3 / 1979$ & 236.0 & 340446 & 6782072 & $\boldsymbol{V}$ & $\square$ & $\square$ & $V$ & $\square$ \\
\hline 2326 & 2210 & Hollow stem borehole w/SPT & $6 / 25 / 1998$ & 31.5 & 341990 & 6781940 & $\boldsymbol{V}$ & $\checkmark$ & $\checkmark$ & $\checkmark$ & $\square$ \\
\hline 2326 & 2239 & Hollow stem borehole w/SPT & $10 / 16 / 1974$ & 46.5 & 341665 & 6781556 & $\boldsymbol{V}$ & $\checkmark$ & $\square$ & $\checkmark$ & $\square$ \\
\hline 2326 & 2240 & Hollow stem borehole w/SPT & $4 / 6 / 1983$ & 31.5 & 341265 & 6781823 & $\boldsymbol{V}$ & $\nabla$ & $\square$ & $\square$ & $\square$ \\
\hline 2326 & 2241 & Hollow stem borehole w/SPT & $4 / 5 / 1983$ & 31.5 & 341313 & 6781913 & 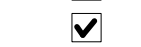 & $\checkmark$ & $\square$ & 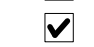 & $\square$ \\
\hline 2327 & 1583 & Hollow stem borehole w/SPT & $3 / 12 / 1982$ & 82.0 & 342619 & 6781497 & $\nabla$ & $\checkmark$ & $\square$ & $\nabla$ & $\square$ \\
\hline 2327 & 2211 & Hollow stem borehole w/SPT & $6 / 26 / 1998$ & 31.5 & 342375 & 6782122 & $\boldsymbol{V}$ & $\checkmark$ & $\nabla$ & $\nabla$ & $\square$ \\
\hline 2327 & 2216 & Hollow stem borehole w/SPT & 8/19/1999 & 100.0 & 342089 & 6782104 & $\boldsymbol{V}$ & $\checkmark$ & $\nabla$ & $\square$ & $\square$ \\
\hline 2327 & 3096 & Water well & $5 / 26 / 1965$ & 597.0 & 342097 & 6782152 & $\nabla$ & $\square$ & $\square$ & $\nabla$ & $\square$ \\
\hline 2328 & 1087 & Hollow stem borehole w/SPT & $4 / 22 / 1983$ & 75.0 & 343554 & 6781792 & $\boldsymbol{V}$ & $\checkmark$ & $\square$ & $\nabla$ & $\square$ \\
\hline 2328 & 1088 & Hollow stem borehole w/SPT & $4 / 26 / 1983$ & 70.0 & 343586 & 6781706 & $\boldsymbol{V}$ & $\checkmark$ & $\square$ & $\nabla$ & $\square$ \\
\hline 2328 & 1089 & Hollow stem borehole w/SPT & 6/9/1993 & 85.0 & 343510 & 6781679 & 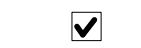 & $\nabla$ & $\square$ & $\nabla$ & $\square$ \\
\hline 2328 & 1099 & Hollow stem borehole w/SPT & $5 / 1 / 1979$ & 30.0 & 343577 & 6781774 & $\square$ & $\square$ & $\square$ & $\square$ & $\square$ \\
\hline 2328 & 1581 & Hollow stem borehole w/SPT & $5 / 18 / 1983$ & 31.5 & 343077 & 6781584 & $\square$ & $\square$ & $\square$ & $\square$ & $\square$ \\
\hline 2328 & 1584 & Hollow stem borehole w/SPT & 8/30/1979 & 66.3 & 343570 & 6781893 & 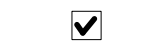 & $\nabla$ & $\nabla$ & $\nabla$ & $\square$ \\
\hline 2328 & 1585 & Hollow stem borehole w/SPT & 8/22/1979 & 60.0 & 343588 & 6781918 & $\nabla$ & $\checkmark$ & $\nabla$ & $\nabla$ & $\square$ \\
\hline 2328 & 1586 & Hollow stem borehole w/SPT & $8 / 24 / 1979$ & 76.3 & 343610 & 6781889 & $\boldsymbol{v}$ & $\checkmark$ & $\nabla$ & $\nabla$ & $\square$ \\
\hline 2328 & 1587 & Hollow stem borehole w/SPT & 8/17/1979 & 71.3 & 343606 & 6781918 & $\boldsymbol{V}$ & $\boldsymbol{V}$ & $\nabla$ & $\checkmark$ & $\square$ \\
\hline
\end{tabular}




\begin{tabular}{|c|c|c|c|c|c|c|c|c|c|c|c|}
\hline \multirow[b]{2}{*}{$\begin{array}{c}\text { MOA } \\
\text { grid }\end{array}$} & \multirow[b]{2}{*}{$\begin{array}{c}\text { DGGS } \\
\text { number }\end{array}$} & \multirow[b]{2}{*}{ Hole Type } & \multirow[b]{2}{*}{$\begin{array}{c}\text { Date } \\
\text { Completed }\end{array}$} & \multirow[b]{2}{*}{$\begin{array}{c}\text { Total } \\
\text { depth (ft) }\end{array}$} & \multicolumn{2}{|c|}{ UTM coordinates (m) } & \multicolumn{5}{|c|}{ Data entered } \\
\hline & & & & & East & North & Lithology & SPT & $\begin{array}{c}\text { Sample } \\
\text { test }\end{array}$ & $\begin{array}{l}\text { Water } \\
\text { level }\end{array}$ & Velocity \\
\hline 2328 & 1588 & Hollow stem borehole w/SPT & 8/29/1979 & 65.5 & 343549 & 6781894 & $\checkmark$ & $\nabla$ & $\nabla$ & $\nabla$ & $\square$ \\
\hline 2328 & 1589 & Hollow stem borehole w/SPT & $8 / 21 / 1979$ & 71.3 & 343558 & 6781922 & $\checkmark$ & $\checkmark$ & $\sqrt{v}$ & $\checkmark$ & $\square$ \\
\hline 2328 & 1601 & Solid stem borehole (no cores or SP & $10 / 16 / 1978$ & 25.0 & 343535 & 6781664 & & $\square$ & $\square$ & $\square$ & $\square$ \\
\hline 2328 & 1602 & Solid stem borehole (no cores or SP & $10 / 11 / 1978$ & 30.0 & 343580 & 6782113 & $\square$ & $\square$ & $\square$ & $\square$ & $\square$ \\
\hline 2328 & 1603 & Solid stem borehole (no cores or SP & $10 / 11 / 1978$ & 30.0 & 343580 & 6782140 & $\square$ & $\square$ & $\square$ & $\square$ & $\square$ \\
\hline 2328 & 1851 & Hollow stem borehole w/SPT & $7 / 11 / 1983$ & 36.0 & 343551 & 6782112 & $\square$ & $\square$ & $\square$ & $\square$ & $\square$ \\
\hline 2328 & 1852 & Hollow stem borehole w/SPT & $7 / 14 / 1983$ & 36.0 & 343570 & 6781925 & $\square$ & $\square$ & $\square$ & $\square$ & $\square$ \\
\hline 2328 & 1853 & Hollow stem borehole w/SPT & $7 / 12 / 1983$ & 51.0 & 343508 & 6781666 & $\boldsymbol{V}$ & $\nabla$ & $\square$ & $\nabla$ & $\square$ \\
\hline 2328 & 2250 & Hollow stem borehole w/SPT & $3 / 17 / 1979$ & 32.0 & 343282 & 6781529 & $\boldsymbol{V}$ & $\checkmark$ & $\square$ & $\square$ & $\square$ \\
\hline 2328 & 4218 & Water well & $1 / 20 / 1967$ & 254.0 & 343424 & 6781690 & $\boldsymbol{V}$ & $\square$ & $\square$ & $\nabla$ & $\square$ \\
\hline 2328 & CV032 & Water well & & 461.0 & 342830 & 6781600 & $\boldsymbol{V}$ & $\square$ & $\square$ & $\square$ & $\square$ \\
\hline 2329 & 1100 & Hollow stem borehole w/SPT & $8 / 1 / 1983$ & 32.0 & 344041 & 6782013 & $\square$ & $\square$ & $\square$ & $\square$ & $\square$ \\
\hline 2329 & 1101 & Hollow stem borehole w/SPT & $8 / 3 / 1983$ & 37.0 & 343838 & 6781900 & $\square$ & $\square$ & $\square$ & $\square$ & $\square$ \\
\hline 2329 & 1102 & Hollow stem borehole w/SPT & $8 / 3 / 1983$ & 32.0 & 344090 & 6782036 & $\square$ & $\square$ & $\square$ & $\square$ & $\square$ \\
\hline 2329 & 1561 & Hollow stem borehole w/SPT & 8/30/1974 & 40.0 & 343729 & 6782145 & $\square$ & $\square$ & $\square$ & $\square$ & $\square$ \\
\hline 2329 & 1562 & Hollow stem borehole w/SPT & 8/30/1974 & 40.0 & 343676 & 6782102 & $\square$ & $\square$ & $\square$ & $\square$ & $\square$ \\
\hline 2329 & 1685 & Hollow stem borehole w/SPT & 9/9/1983 & 31.0 & 344119 & 6781852 & $\square$ & $\square$ & $\square$ & $\square$ & $\square$ \\
\hline 2329 & 3085 & Water well & $8 / 15 / 1972$ & 220.0 & 344353 & 6782021 & $\boldsymbol{V}$ & $\square$ & $\square$ & $\nabla$ & $\square$ \\
\hline 2329 & 3102 & Water well & $4 / 10 / 1984$ & 160.0 & 344091 & 6781877 & $\boldsymbol{V}$ & $\square$ & $\square$ & $\nabla$ & $\square$ \\
\hline 2329 & 4235 & Water well & $7 / 13 / 1970$ & 215.0 & 343880 & 6781825 & $\boldsymbol{V}$ & $\square$ & $\square$ & $\nabla$ & $\square$ \\
\hline 2330 & 1090 & Hollow stem borehole w/SPT & 10/6/1981 & 15.0 & 344571 & 6781781 & $\square$ & $\square$ & $\square$ & $\square$ & $\square$ \\
\hline 2330 & 1091 & Hollow stem borehole w/SPT & $10 / 6 / 1981$ & 15.0 & 344567 & 6781695 & $\square$ & $\square$ & $\square$ & $\square$ & $\square$ \\
\hline 2330 & 1092 & Hollow stem borehole w/SPT & 10/6/1981 & 15.0 & 344718 & 6781720 & $\square$ & $\square$ & $\square$ & $\square$ & $\square$ \\
\hline 2330 & 1093 & Hollow stem borehole w/SPT & $10 / 6 / 1981$ & 15.0 & 344453 & 6781731 & $\square$ & $\square$ & $\square$ & $\square$ & $\square$ \\
\hline 2330 & 1094 & Hollow stem borehole w/SPT & 10/6/1981 & 15.0 & 344458 & 6781840 & $\square$ & $\square$ & $\square$ & $\square$ & $\square$ \\
\hline 2330 & 1095 & Hollow stem borehole w/SPT & 10/6/1981 & 15.0 & 344723 & 6781830 & $\square$ & $\square$ & $\square$ & $\square$ & $\square$ \\
\hline 2330 & 2251 & Hollow stem borehole w/SPT & $1 / 14 / 1983$ & 31.5 & 344668 & 6782028 & $\boldsymbol{V}$ & $\checkmark$ & $\square$ & $\nabla$ & $\square$ \\
\hline 2330 & 4205 & Water well & 8/13/1982 & 219.0 & 344880 & 6781409 & $\boldsymbol{V}$ & $\square$ & $\square$ & $\nabla$ & $\square$ \\
\hline 2330 & 4245 & Water well & $9 / 2 / 1976$ & 170.0 & 344573 & 6781918 & $\nabla$ & $\square$ & $\square$ & $\nabla$ & $\square$ \\
\hline 2330 & 4246 & Water well & $6 / 17 / 1976$ & 145.0 & 344498 & 6781921 & 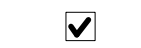 & $\square$ & $\square$ & $\nabla$ & $\square$ \\
\hline 2330 & 4251 & Water well & $8 / 22 / 1969$ & 208.0 & 344484 & 6781953 & $\nabla$ & $\square$ & $\square$ & $\square$ & $\square$ \\
\hline 2330 & СТ001 & Water well & 8/13/1982 & 214.0 & 344500 & 6781418 & 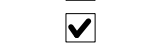 & $\square$ & $\square$ & $\checkmark$ & $\square$ \\
\hline 2331 & 1096 & Hollow stem borehole w/SPT & $3 / 29 / 1983$ & 70.0 & 345485 & 6782129 & 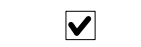 & $\nabla$ & $\square$ & $\bar{\nabla}$ & $\square$ \\
\hline 2331 & 1098 & Hollow stem borehole w/SPT & $6 / 8 / 1983$ & 66.0 & 345442 & 6782097 & $\checkmark$ & $\boldsymbol{V}$ & $\square$ & $\nabla$ & $\square$ \\
\hline 2331 & 1109 & Hollow stem borehole w/SPT & 8/19/1992 & 41.0 & 345567 & 6782052 & $\square$ & $\square$ & $\square$ & $\square$ & $\square$ \\
\hline 2331 & 1110 & Hollow stem borehole w/SPT & 8/19/1992 & 40.0 & 345721 & 6782051 & $\square$ & $\square$ & $\square$ & $\square$ & $\square$ \\
\hline
\end{tabular}




\begin{tabular}{|c|c|c|c|c|c|c|c|c|c|c|c|}
\hline \multirow[b]{2}{*}{$\begin{array}{c}\text { MOA } \\
\text { grid }\end{array}$} & \multirow[b]{2}{*}{$\begin{array}{c}\text { DGGS } \\
\text { number }\end{array}$} & \multirow[b]{2}{*}{ Hole Type } & \multirow[b]{2}{*}{$\begin{array}{c}\text { Date } \\
\text { Completed }\end{array}$} & \multirow[b]{2}{*}{$\begin{array}{c}\text { Total } \\
\text { depth (ft) }\end{array}$} & \multicolumn{2}{|c|}{ UTM coordinates (m) } & \multicolumn{5}{|c|}{ Data entered } \\
\hline & & & & & East & North & Lithology & SPT & $\begin{array}{c}\text { Sample } \\
\text { test }\end{array}$ & $\begin{array}{l}\text { Water } \\
\text { level }\end{array}$ & Velocity \\
\hline 2331 & 1111 & Hollow stem borehole w/SPT & 8/13/1992 & 40.5 & 345567 & 6781927 & $\square$ & $\square$ & $\square$ & $\square$ & $\square$ \\
\hline 2331 & 1112 & Hollow stem borehole w/SPT & 8/13/1992 & 41.0 & 345727 & 6781935 & $\square$ & $\square$ & $\square$ & $\square$ & $\square$ \\
\hline 2331 & 1510 & Hollow stem borehole w/SPT & $4 / 30 / 1997$ & 21.5 & 345888 & 6781663 & $\square$ & $\square$ & $\square$ & $\square$ & $\square$ \\
\hline 2331 & 1675 & Hollow stem borehole w/SPT & $1 / 25 / 1977$ & 25.5 & 345487 & 6782081 & $\square$ & $\square$ & $\square$ & $\square$ & $\square$ \\
\hline 2331 & 1676 & Hollow stem borehole w/SPT & $1 / 25 / 1977$ & 25.5 & 345490 & 6782007 & $\square$ & $\square$ & $\square$ & $\square$ & $\square$ \\
\hline 2331 & 1677 & Hollow stem borehole w/SPT & $1 / 25 / 1977$ & 25.5 & 345497 & 6781892 & $\square$ & $\square$ & $\square$ & $\square$ & $\square$ \\
\hline 2331 & 1678 & Hollow stem borehole w/SPT & $1 / 25 / 1977$ & 25.5 & 345499 & 6781755 & $\checkmark$ & $\checkmark$ & $\checkmark$ & $\checkmark$ & $\square$ \\
\hline 2331 & 1679 & Hollow stem borehole w/SPT & $1 / 25 / 1977$ & 20.5 & 345653 & 6781746 & $\boldsymbol{V}$ & $\nabla$ & 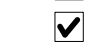 & $\nabla$ & $\square$ \\
\hline 2331 & 1680 & Hollow stem borehole w/SPT & $1 / 26 / 1977$ & 20.5 & 345849 & 6781737 & $\square$ & $\square$ & $\square$ & $\square$ & $\square$ \\
\hline 2331 & 4217 & Water well & $4 / 5 / 1978$ & 103.0 & 345188 & 6781612 & $\boldsymbol{V}$ & $\square$ & $\square$ & $\nabla$ & $\square$ \\
\hline 2332 & 1104 & Hollow stem borehole w/SPT & $7 / 11 / 1979$ & 50.0 & 346059 & 6782132 & $\boldsymbol{V}$ & $\square$ & $\nabla$ & $\nabla$ & $\square$ \\
\hline 2332 & 1105 & Hollow stem borehole w/SPT & $7 / 3 / 1979$ & 36.5 & 346356 & 6782095 & $\square$ & $\square$ & $\square$ & $\square$ & $\square$ \\
\hline 2332 & 1106 & Hollow stem borehole w/SPT & $7 / 23 / 1992$ & 46.0 & 346159 & 6781926 & $\square$ & $\square$ & $\square$ & $\square$ & $\square$ \\
\hline 2332 & 1107 & Hollow stem borehole w/SPT & $7 / 23 / 1992$ & 46.0 & 346140 & 6781865 & $\square$ & $\square$ & $\square$ & $\square$ & $\square$ \\
\hline 2332 & 1108 & Hollow stem borehole w/SPT & 7/23/1992 & 46.0 & 346179 & 6781792 & $\square$ & $\square$ & $\square$ & $\square$ & $\square$ \\
\hline 2332 & 1578 & Hollow stem borehole w/SPT & $10 / 26 / 1984$ & 42.0 & 346327 & 6782068 & $\square$ & $\square$ & $\square$ & $\square$ & $\square$ \\
\hline 2332 & 1579 & Hollow stem borehole w/SPT & $10 / 30 / 1984$ & 42.0 & 346391 & 6782068 & $\square$ & $\square$ & $\square$ & $\square$ & $\square$ \\
\hline 2332 & 1681 & Hollow stem borehole w/SPT & $1 / 28 / 1977$ & 20.5 & 346050 & 6781730 & $\square$ & $\square$ & $\square$ & $\square$ & $\square$ \\
\hline 2332 & 1682 & Hollow stem borehole w/SPT & $1 / 28 / 1977$ & 20.5 & 346180 & 6781725 & $\square$ & $\square$ & $\square$ & $\square$ & $\square$ \\
\hline 2332 & CR002 & Water well & $10 / 19 / 1972$ & 159.0 & 346549 & 6781455 & $\boldsymbol{V}$ & $\square$ & $\square$ & $\nabla$ & $\square$ \\
\hline 2333 & 1117 & Hollow stem borehole w/SPT & $1 / 22 / 1986$ & 25.0 & 347497 & 6781681 & $\boldsymbol{V}$ & $\checkmark$ & $\square$ & $\nabla$ & $\square$ \\
\hline 2333 & 1614 & Hollow stem borehole w/SPT & $6 / 4 / 1986$ & 40.5 & 347536 & 6781983 & $\square$ & $\square$ & $\square$ & $\square$ & $\square$ \\
\hline 2333 & 3074 & Water well & $3 / 13 / 1974$ & 153.0 & 347325 & 6781828 & 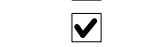 & $\square$ & $\square$ & $\nabla$ & $\square$ \\
\hline 2333 & 4222 & Water well & $4 / 3 / 1964$ & 103.0 & 346820 & 6781603 & $\nabla$ & $\square$ & $\square$ & $\nabla$ & $\square$ \\
\hline 2333 & 4223 & Water well & 10/1/1970 & 156.0 & 346820 & 6781603 & 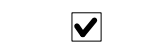 & $\square$ & $\square$ & $\nabla$ & $\square$ \\
\hline 2333 & 4239 & Water well & $9 / 1 / 1967$ & 162.0 & 347215 & 6781740 & $\boldsymbol{V}$ & $\square$ & $\square$ & $\nabla$ & $\square$ \\
\hline 2333 & 4241 & Water well & 1/1/1969 & 198.0 & 346993 & 6781781 & $\boldsymbol{V}$ & $\square$ & $\square$ & $\nabla$ & $\square$ \\
\hline 2333 & 4255 & Water well & $5 / 24 / 1965$ & 153.0 & 347310 & 6781860 & $\boldsymbol{V}$ & $\square$ & $\square$ & $\nabla$ & $\square$ \\
\hline 2333 & 4268 & Water well & 8/23/1974 & 117.0 & 346840 & 6782066 & $\nabla$ & $\square$ & $\square$ & $\nabla$ & $\square$ \\
\hline 2334 & 1115 & Hollow stem borehole w/SPT & $1 / 20 / 1986$ & 25.5 & 348164 & 6781642 & $\square$ & $\square$ & $\square$ & $\square$ & $\square$ \\
\hline 2334 & 1116 & Hollow stem borehole w/SPT & $1 / 22 / 1986$ & 25.5 & 347645 & 6781672 & $\checkmark$ & $\checkmark$ & $\square$ & $\square$ & $\square$ \\
\hline 2334 & 1613 & Hollow stem borehole w/SPT & $5 / 19 / 1986$ & 41.0 & 347567 & 6781982 & 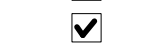 & $\checkmark$ & $\square$ & $\checkmark$ & $\square$ \\
\hline 2334 & 3058 & Water well & $4 / 21 / 1981$ & 111.0 & 348199 & 6781977 & 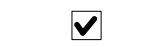 & $\square$ & $\square$ & $\bar{\nabla}$ & $\square$ \\
\hline 2334 & 3064 & Water well & $4 / 29 / 1981$ & 108.0 & 348226 & 6781913 & $\nabla$ & $\square$ & $\square$ & $\nabla$ & $\square$ \\
\hline 2334 & 4207 & Water well & $11 / 1 / 1977$ & 182.0 & 347993 & 6781366 & $\boldsymbol{v}$ & $\square$ & $\square$ & $\nabla$ & $\square$ \\
\hline 2334 & 4231 & Water well & $5 / 18 / 1974$ & 178.0 & 348092 & 6781578 & $\boldsymbol{V}$ & $\square$ & $\square$ & $\nabla$ & $\square$ \\
\hline
\end{tabular}




\begin{tabular}{|c|c|c|c|c|c|c|c|c|c|c|c|}
\hline \multirow[b]{2}{*}{$\begin{array}{l}\text { MOA } \\
\text { grid }\end{array}$} & \multirow[b]{2}{*}{$\begin{array}{l}\text { DGGS } \\
\text { number }\end{array}$} & \multirow[b]{2}{*}{ Hole Type } & \multirow[b]{2}{*}{$\begin{array}{c}\text { Date } \\
\text { Completed }\end{array}$} & \multirow[b]{2}{*}{$\begin{array}{c}\text { Total } \\
\text { depth }(\mathrm{ft})\end{array}$} & \multicolumn{2}{|c|}{ UTM coordinates (m) } & \multicolumn{5}{|c|}{ Data entered } \\
\hline & & & & & East & North & Lithology & SPT & $\begin{array}{c}\text { Sample } \\
\text { test }\end{array}$ & $\begin{array}{l}\text { Water } \\
\text { level }\end{array}$ & Velocity \\
\hline 2334 & 4232 & Water well & $5 / 26 / 1977$ & 111.0 & 347793 & 6781591 & $\checkmark$ & 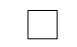 & $\square$ & $\checkmark$ & $\square$ \\
\hline 2334 & 4244 & Water well & $8 / 9 / 1969$ & 148.0 & 347577 & 6781786 & $\checkmark$ & $\square$ & $\square$ & $\checkmark$ & $\square$ \\
\hline 2334 & 4263 & Water well & $4 / 21 / 1981$ & 111.0 & 348214 & 6781976 & $\checkmark$ & & $\square$ & 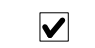 & \\
\hline 2334 & 4265 & Water well & $7 / 16 / 1970$ & 125.0 & 348080 & 6781982 & $\checkmark$ & $\square$ & $\square$ & $\sqrt{\nabla}$ & $\square$ \\
\hline 2335 & 1114 & Hollow stem borehole w/SPT & $1 / 21 / 1986$ & 20.5 & 348360 & 6781938 & $\square$ & $\square$ & $\square$ & $\square$ & $\square$ \\
\hline 2335 & 1118 & Hollow stem borehole w/SPT & 2/11/1977 & 40.5 & 349039 & 6781968 & $\square$ & $\square$ & $\square$ & $\square$ & $\square$ \\
\hline 2335 & 3081 & Water well & $8 / 15 / 1973$ & 175.0 & 349094 & 6781535 & $\checkmark$ & $\square$ & $\square$ & $\checkmark$ & $\square$ \\
\hline 2335 & 4230 & Water well & $11 / 23 / 1974$ & 143.0 & 348511 & 6781560 & $\checkmark$ & & $\square$ & $\bar{\nabla}$ & $\square$ \\
\hline 2335 & 4237 & Water well & 7/6/1972 & 184.0 & 348604 & 6781649 & $\checkmark$ & $\square$ & $\square$ & $\sqrt{\square}$ & $\square$ \\
\hline 2335 & 4250 & Water well & $8 / 31 / 1976$ & 155.0 & 348520 & 6781777 & $\checkmark$ & & $\square$ & $\checkmark$ & $\square$ \\
\hline 2336 & 3055 & Water well & $5 / 1 / 1985$ & 140.0 & 349398 & 6781987 & $\checkmark$ & $\square$ & $\square$ & $\square$ & $\square$ \\
\hline 2336 & 3057 & Water well & $7 / 3 / 1973$ & 110.0 & 349304 & 6781898 & $\checkmark$ & & $\square$ & $\bar{\nabla}$ & $\square$ \\
\hline 2336 & 3059 & Water well & $12 / 13 / 1972$ & 160.0 & 349466 & 6781829 & $\checkmark$ & $\square$ & $\square$ & $\nabla$ & $\square$ \\
\hline 2336 & 3060 & Water well & $9 / 22 / 1973$ & 122.0 & 349391 & 6781833 & $\checkmark$ & $\square$ & $\square$ & $\nabla$ & $\square$ \\
\hline 2336 & 3061 & Water well & $6 / 25 / 1976$ & 155.0 & 349404 & 6781801 & $\checkmark$ & $\square$ & $\square$ & $\square$ & $\square$ \\
\hline 2336 & 3065 & Water well & $9 / 16 / 1985$ & 141.0 & 349493 & 6781766 & $\checkmark$ & & $\square$ & $\checkmark$ & $\square$ \\
\hline 2336 & 3066 & Water well & $5 / 28 / 1975$ & 163.0 & 349207 & 6781747 & $\checkmark$ & - & $\square$ & $\bar{V}$ & $\square$ \\
\hline 2336 & 3067 & Water well & 9/6/1973 & 141.0 & 349610 & 6781699 & $\checkmark$ & ᄂ & $\square$ & $\vec{\nabla}$ & $\square$ \\
\hline 2336 & 3068 & Water well & $1 / 1 / 1986$ & 165.0 & 349520 & 6781703 & $\checkmark$ & $\square$ & $\square$ & $\square$ & $\square$ \\
\hline 2336 & 3069 & Water well & $10 / 24 / 1973$ & 160.0 & 349355 & 6781710 & $\checkmark$ & $\square$ & $\square$ & $\checkmark$ & $\square$ \\
\hline 2336 & 3072 & Water well & $10 / 2 / 1981$ & 177.0 & 349651 & 6781604 & $\checkmark$ & & $\square$ & $\bar{\nabla}$ & $\square$ \\
\hline 2336 & 3075 & Water well & 7/17/1978 & 170.0 & 349618 & 6781544 & $\checkmark$ & 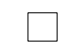 & $\square$ & $\nabla$ & $\square$ \\
\hline 2336 & 3076 & Water well & $8 / 25 / 1973$ & 163.0 & 349588 & 6781545 & $\checkmark$ & $\square$ & $\square$ & $\nabla$ & $\square$ \\
\hline 2336 & 3078 & Water well & $5 / 18 / 1983$ & 171.0 & 349543 & 6781547 & $\checkmark$ & $\square$ & $\square$ & $\checkmark$ & $\square$ \\
\hline 2336 & 3079 & Water well & $10 / 26 / 1973$ & 180.0 & 349379 & 6781554 & $\checkmark$ & & $\square$ & $\checkmark$ & $\square$ \\
\hline 2336 & 3080 & Water well & 9/16/1972 & 161.0 & 349690 & 6781479 & $\checkmark$ & & $\square$ & $\bar{V}$ & $\square$ \\
\hline 2336 & 3082 & Water well & 9/6/1973 & 178.0 & 349555 & 6781485 & $\checkmark$ & $\square$ & $\square$ & $\checkmark$ & $\square$ \\
\hline 2336 & 3086 & Water well & 10/4/1972 & 164.0 & 349674 & 6781449 & $\checkmark$ & & $\square$ & $\nabla$ & $\square$ \\
\hline 2336 & 3087 & Water well & 8/1/1971 & 196.0 & 349585 & 6781452 & $\checkmark$ & $\square$ & $\square$ & $\checkmark$ & $\square$ \\
\hline 2336 & 3089 & Water well & 8/20/1977 & 113.0 & 349465 & 6781458 & $\checkmark$ & $\square$ & $\square$ & $\square$ & $\square$ \\
\hline 2336 & 3090 & Water well & $5 / 8 / 1978$ & 180.0 & 349538 & 6781423 & $\checkmark$ & $\square$ & $\square$ & $\checkmark$ & $\square$ \\
\hline 2336 & 3091 & Water well & 8/10/1978 & 134.0 & 349493 & 6781425 & $\checkmark$ & $\square$ & $\square$ & $\square$ & $\square$ \\
\hline 2336 & 3092 & Water well & $5 / 25 / 1973$ & 173.0 & 349403 & 6781429 & $\checkmark$ & $\square$ & $\square$ & $\bar{\nabla}$ & $\square$ \\
\hline 2336 & 3093 & Water well & $11 / 2 / 1972$ & 140.0 & 349432 & 6781397 & $\checkmark$ & $\square$ & $\square$ & $\bar{\nabla}$ & $\square$ \\
\hline 2336 & 3095 & Water well & $2 / 27 / 1968$ & 118.0 & 349223 & 6781406 & $\checkmark$ & $\square$ & $\square$ & $\boldsymbol{V}$ & $\square$ \\
\hline 2336 & 3097 & Water well & $5 / 31 / 1979$ & 160.0 & 349475 & 6781364 & $\checkmark$ & $\square$ & $\square$ & $\nabla$ & $\square$ \\
\hline
\end{tabular}




\begin{tabular}{|c|c|c|c|c|c|c|c|c|c|c|c|}
\hline \multirow[b]{2}{*}{$\begin{array}{c}\text { MOA } \\
\text { grid }\end{array}$} & \multirow[b]{2}{*}{$\begin{array}{c}\text { DGGS } \\
\text { number }\end{array}$} & \multirow[b]{2}{*}{ Hole Type } & \multirow[b]{2}{*}{$\begin{array}{c}\text { Date } \\
\text { Completed }\end{array}$} & \multirow[b]{2}{*}{$\begin{array}{c}\text { Total } \\
\text { depth (ft) }\end{array}$} & \multicolumn{2}{|c|}{ UTM coordinates (m) } & \multicolumn{5}{|c|}{ Data entered } \\
\hline & & & & & East & North & Lithology & SPT & $\begin{array}{c}\text { Sample } \\
\text { test }\end{array}$ & $\begin{array}{l}\text { Water } \\
\text { level }\end{array}$ & Velocity \\
\hline 2336 & 3099 & Water well & $7 / 31 / 1972$ & 146.0 & 349459 & 6781334 & $\checkmark$ & $\square$ & $\square$ & $\nabla$ & $\square$ \\
\hline 2336 & 3100 & Water well & $11 / 20 / 1979$ & 164.0 & 349370 & 6781338 & $\checkmark$ & $\square$ & $\square$ & $\sqrt{ }$ & $\square$ \\
\hline 2336 & 4213 & Water well & $5 / 17 / 1973$ & 176.0 & 349689 & 6781448 & $\boldsymbol{V}$ & $\square$ & $\square$ & $\nabla$ & $\square$ \\
\hline 2336 & 4214 & Water well & 8/1/1971 & 202.0 & 349613 & 6781420 & $\boldsymbol{V}$ & $\square$ & $\square$ & $\nabla$ & $\square$ \\
\hline 2336 & 4216 & Water well & $11 / 9 / 1981$ & 150.0 & 349508 & 6781425 & $\checkmark$ & $\square$ & $\square$ & $\square$ & $\square$ \\
\hline 2336 & 4221 & Water well & 9/13/1973 & 125.0 & 349481 & 6781488 & $\boldsymbol{V}$ & $\square$ & $\square$ & $\nabla$ & $\square$ \\
\hline 2336 & 4227 & Water well & 4/7/1979 & 169.0 & 349497 & 6781518 & $\checkmark$ & $\square$ & $\square$ & $\checkmark$ & $\square$ \\
\hline 2336 & 4228 & Water well & $5 / 16 / 1982$ & 106.0 & 349422 & 6781521 & $\boldsymbol{V}$ & $\square$ & $\square$ & $\nabla$ & $\square$ \\
\hline 2336 & 4229 & Water well & $6 / 16 / 1975$ & 110.0 & 349273 & 6781528 & $\boldsymbol{V}$ & $\square$ & $\square$ & $\sqrt{ }$ & $\square$ \\
\hline 2336 & 4236 & Water well & $11 / 10 / 1981$ & 175.0 & 349710 & 6781602 & $\boldsymbol{V}$ & $\square$ & $\square$ & $\square$ & $\square$ \\
\hline 2336 & 4238 & Water well & $10 / 25 / 1982$ & 102.0 & 349323 & 6781650 & 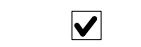 & $\square$ & $\square$ & $\nabla$ & $\square$ \\
\hline 2336 & 4240 & Water well & $10 / 28 / 1975$ & 119.0 & 349324 & 6781681 & $\nabla$ & $\square$ & $\square$ & $\nabla$ & $\square$ \\
\hline 2336 & 4243 & Water well & 4/7/1978 & 126.0 & 349626 & 6781729 & $\nabla$ & $\square$ & $\square$ & $\nabla$ & $\square$ \\
\hline 2336 & 4249 & Water well & $7 / 3 / 1974$ & 152.0 & 349402 & 6781739 & 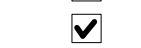 & $\square$ & $\square$ & $\checkmark$ & $\square$ \\
\hline 2336 & 4257 & Water well & $5 / 12 / 1974$ & 124.0 & 349346 & 6781835 & $\boldsymbol{V}$ & $\square$ & $\square$ & $\nabla$ & $\square$ \\
\hline 2336 & 4471 & Water well & $10 / 12 / 1978$ & 137.0 & 349522 & 6781734 & $\checkmark$ & $\square$ & $\square$ & $\checkmark$ & $\square$ \\
\hline 2336 & 4472 & Water well & 8/15/1974 & 135.0 & 349518 & 6781641 & $\boldsymbol{v}$ & $\square$ & $\square$ & $\nabla$ & $\square$ \\
\hline 2336 & 4474 & Water well & $5 / 18 / 1974$ & 164.0 & 349397 & 6781615 & 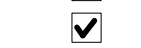 & $\square$ & $\square$ & $\nabla$ & $\square$ \\
\hline 2336 & 4475 & Water well & $9 / 16 / 1974$ & 176.0 & 349572 & 6781515 & $\boldsymbol{V}$ & $\square$ & $\square$ & $\nabla$ & $\square$ \\
\hline 2336 & 4476 & Water well & 9/20/1975 & 128.0 & 349314 & 6781433 & $\boldsymbol{V}$ & $\square$ & $\square$ & $\nabla$ & $\square$ \\
\hline 2336 & 4477 & Water well & 8/1/1971 & 155.0 & 349657 & 6781387 & $\boldsymbol{V}$ & $\square$ & $\square$ & $\nabla$ & $\square$ \\
\hline 2337 & 1119 & Hollow stem borehole w/SPT & $8 / 4 / 1982$ & 30.0 & 350533 & 6781341 & $\square$ & $\square$ & $\square$ & $\square$ & $\square$ \\
\hline 2337 & 3084 & Water well & $2 / 1 / 1969$ & 347.0 & 350330 & 6781390 & 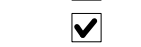 & $\square$ & $\square$ & $\nabla$ & $\square$ \\
\hline 2338 & 1706 & Hollow stem borehole w/SPT & $3 / 25 / 1985$ & 20.5 & 350750 & 6781197 & $\nabla$ & $\bar{V}$ & $\nabla$ & $\nabla$ & $\square$ \\
\hline 2339 & 1689 & Pit or trench & $7 / 25 / 1962$ & 13.0 & 351803 & 6781166 & $\square$ & $\square$ & $\square$ & $\square$ & $\square$ \\
\hline 2339 & 1690 & Pit or trench & $7 / 25 / 1962$ & 14.0 & 351904 & 6781219 & $\square$ & $\square$ & $\square$ & $\square$ & $\square$ \\
\hline 2339 & 1691 & Pit or trench & $7 / 25 / 1962$ & 14.0 & 352119 & 6781309 & $\square$ & $\square$ & $\square$ & $\square$ & $\square$ \\
\hline 2339 & 1692 & Pit or trench & $7 / 25 / 1962$ & 11.0 & 352056 & 6781377 & $\square$ & $\square$ & $\square$ & $\square$ & $\square$ \\
\hline 2339 & 1693 & Pit or trench & $7 / 25 / 1962$ & 14.0 & 352048 & 6781305 & $\square$ & $\square$ & $\square$ & $\square$ & $\square$ \\
\hline 2339 & 1694 & Pit or trench & 7/25/1962 & 14.0 & 351833 & 6781369 & $\square$ & $\square$ & $\square$ & $\square$ & $\square$ \\
\hline 2339 & 1695 & Pit or trench & 7/25/1962 & 13.0 & 352074 & 6781137 & $\square$ & $\square$ & $\square$ & $\square$ & $\square$ \\
\hline 2339 & 1696 & Pit or trench & 7/25/1962 & 13.0 & 352114 & 6781105 & $\square$ & $\square$ & $\square$ & $\square$ & $\square$ \\
\hline 2424 & 2235 & Natural exposure & 4/28/1980 & 100.0 & 339923 & 6781275 & 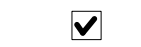 & $\square$ & $\square$ & $\square$ & $\square$ \\
\hline 2424 & 304 & Water well & 2/11/1995 & 528.0 & 339990 & 6781520 & $\nabla$ & $\square$ & $\square$ & $\nabla$ & $\square$ \\
\hline 2424 & CY009 & Hollow stem borehole w/SPT & $2 / 3 / 1975$ & 26.5 & 339627 & 6781551 & $\nabla$ & $\nabla$ & $\square$ & $\square$ & $\square$ \\
\hline 2424 & CY010 & Hollow stem borehole w/SPT & $2 / 3 / 1975$ & 26.5 & 339673 & 6781546 & $\square$ & $\square$ & $\square$ & $\square$ & $\square$ \\
\hline
\end{tabular}




\begin{tabular}{|c|c|c|c|c|c|c|c|c|c|c|c|}
\hline \multirow[b]{2}{*}{$\begin{array}{c}\text { MOA } \\
\text { grid }\end{array}$} & \multirow[b]{2}{*}{$\begin{array}{l}\text { DGGS } \\
\text { number }\end{array}$} & \multirow[b]{2}{*}{ Hole Type } & \multirow[b]{2}{*}{$\begin{array}{c}\text { Date } \\
\text { Completed }\end{array}$} & \multirow[b]{2}{*}{$\begin{array}{c}\text { Total } \\
\text { depth (ft) }\end{array}$} & \multicolumn{2}{|c|}{ UTM coordinates (m) } & \multicolumn{5}{|c|}{ Data entered } \\
\hline & & & & & East & North & Lithology & SPT & $\begin{array}{c}\text { Sample } \\
\text { test }\end{array}$ & $\begin{array}{l}\text { Water } \\
\text { level }\end{array}$ & Velocity \\
\hline 2424 & CY011 & Hollow stem borehole w/SPT & $2 / 3 / 1975$ & 26.5 & 339601 & 6781504 & $\square$ & $\square$ & $\square$ & $\square$ & $\square$ \\
\hline 2424 & CY012 & Hollow stem borehole w/SPT & $2 / 3 / 1975$ & 26.5 & 339664 & 6781494 & $\square$ & $\square$ & $\square$ & $\square$ & $\square$ \\
\hline 2424 & CY013 & Hollow stem borehole w/SPT & $2 / 3 / 1975$ & 26.5 & 339589 & 6781451 & $\square$ & $\square$ & $\square$ & $\square$ & $\square$ \\
\hline 2424 & CY014 & Hollow stem borehole w/SPT & $2 / 3 / 1975$ & 26.5 & 339662 & 6781414 & $\boldsymbol{V}$ & $\checkmark$ & $\square$ & $\square$ & $\square$ \\
\hline 2425 & 2125 & Hollow stem borehole w/SPT & $1 / 5 / 1979$ & 42.0 & 340971 & 6781477 & $\checkmark$ & $\square$ & $\nabla$ & $\nabla$ & $\square$ \\
\hline 2425 & 2126 & Hollow stem borehole w/SPT & & 26.5 & 341131 & 6781383 & $\boldsymbol{V}$ & $\checkmark$ & $\square$ & $\nabla$ & $\square$ \\
\hline 2426 & 2183 & Hollow stem borehole w/SPT & $1 / 23 / 1985$ & 31.5 & 341250 & 6781493 & $\checkmark$ & $\checkmark$ & $\checkmark$ & $\checkmark$ & $\square$ \\
\hline 2426 & 2236 & Hollow stem borehole w/SPT & $2 / 5 / 1982$ & 31.5 & 341517 & 6781491 & $\boldsymbol{V}$ & $\nabla$ & $\square$ & $\nabla$ & $\square$ \\
\hline 2426 & BX022 & Hollow stem borehole w/SPT & $3 / 16 / 1983$ & 130.7 & 341604 & 6780910 & $\boldsymbol{V}$ & $\nabla$ & $\square$ & $\nabla$ & $\square$ \\
\hline 2426 & BX023 & Hollow stem borehole w/SPT & $4 / 5 / 1983$ & 61.5 & 341473 & 6780796 & $\boldsymbol{V}$ & $\nabla$ & $\nabla$ & $\nabla$ & $\square$ \\
\hline 2426 & BX024 & Hollow stem borehole w/SPT & $4 / 11 / 1983$ & 61.5 & 341650 & 6780924 & $\nabla$ & $\nabla$ & $\nabla$ & $\nabla$ & $\square$ \\
\hline 2426 & BX026 & Hollow stem borehole w/SPT & $6 / 3 / 1983$ & 76.5 & 341457 & 6780722 & $\boldsymbol{V}$ & $\nabla$ & $\nabla$ & $\nabla$ & $\square$ \\
\hline 2427 & 1120 & Hollow stem borehole w/SPT & 9/9/1974 & 31.5 & 342444 & 6781419 & $\nabla$ & $\nabla$ & $\nabla$ & $\nabla$ & $\square$ \\
\hline 2427 & 1121 & Hollow stem borehole w/SPT & 9/10/1974 & 31.5 & 342404 & 6781310 & $\square$ & $\square$ & $\square$ & $\square$ & $\square$ \\
\hline 2427 & 1582 & Hollow stem borehole w/SPT & 3/8/1982 & 85.0 & 342655 & 6781453 & $\boldsymbol{V}$ & $\checkmark$ & $\square$ & $\nabla$ & $\square$ \\
\hline 2427 & 2248 & Hollow stem borehole w/SPT & $12 / 23 / 1977$ & 36.0 & 342337 & 6781436 & $\checkmark$ & $\checkmark$ & $\square$ & $\square$ & $\square$ \\
\hline 2427 & BY035 & Hollow stem borehole w/SPT & 6/8/1983 & 76.5 & 342088 & 6780721 & $\boldsymbol{V}$ & $\nabla$ & $\nabla$ & $\nabla$ & $\square$ \\
\hline 2427 & CV024 & Hollow stem borehole w/SPT & $11 / 18 / 1975$ & 39.5 & 342191 & 6781095 & $\boldsymbol{V}$ & $\checkmark$ & $\nabla$ & $\nabla$ & $\square$ \\
\hline 2427 & CV025 & Hollow stem borehole w/SPT & $11 / 18 / 1975$ & 41.0 & 342214 & 6781177 & $\boldsymbol{V}$ & $\checkmark$ & $\nabla$ & $\nabla$ & $\square$ \\
\hline 2427 & CV026 & Hollow stem borehole w/SPT & $11 / 18 / 1975$ & 41.0 & 342299 & 6781175 & $\nabla$ & $\nabla$ & $\nabla$ & $\nabla$ & $\square$ \\
\hline 2427 & CV027 & Hollow stem borehole w/SPT & $11 / 18 / 1975$ & 41.0 & 342349 & 6781114 & $\boldsymbol{V}$ & $\checkmark$ & $\nabla$ & $\square$ & $\square$ \\
\hline 2428 & 1713 & Hollow stem borehole w/SPT & $4 / 15 / 1983$ & 52.0 & 343461 & 6780641 & $\nabla$ & $\checkmark$ & $\nabla$ & $\nabla$ & $\square$ \\
\hline 2428 & 1854 & Hollow stem borehole w/SPT & $7 / 19 / 1983$ & 52.0 & 343546 & 6781397 & 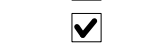 & $\checkmark$ & $\square$ & $\nabla$ & $\square$ \\
\hline 2428 & 1855 & Hollow stem borehole w/SPT & $7 / 20 / 1983$ & 37.0 & 343546 & 6781344 & $\nabla$ & $\checkmark$ & $\square$ & $\nabla$ & $\square$ \\
\hline 2428 & 2249 & Hollow stem borehole w/SPT & & 32.0 & 342868 & 6781215 & $\boldsymbol{V}$ & $\checkmark$ & $\square$ & $\nabla$ & $\square$ \\
\hline 2428 & 308 & Water well & $10 / 26 / 1980$ & 253.0 & 352820 & 6780820 & $\boldsymbol{V}$ & $\square$ & $\square$ & $\nabla$ & $\square$ \\
\hline 2429 & 1712 & Hollow stem borehole w/SPT & $3 / 22 / 1982$ & 71.0 & 343584 & 6780635 & $\boldsymbol{V}$ & $\checkmark$ & $\nabla$ & $\nabla$ & $\square$ \\
\hline 2430 & 2188 & Hollow stem borehole w/SPT & 2/8/1996 & 41.5 & 344645 & 6780901 & $\boldsymbol{V}$ & $\checkmark$ & $\square$ & $\nabla$ & $\square$ \\
\hline 2430 & 2190 & Hollow stem borehole w/SPT & $1 / 31 / 1996$ & 25.0 & 344585 & 6780853 & $\nabla$ & $\nabla$ & $\nabla$ & $\square$ & $\square$ \\
\hline 2430 & 2191 & Hollow stem borehole w/SPT & 2/2/1996 & 20.5 & 344676 & 6780614 & 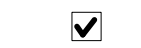 & $\nabla$ & $\nabla$ & $\nabla$ & $\square$ \\
\hline 2431 & 1122 & Hollow stem borehole w/SPT & 4/29/1993 & 31.0 & 345818 & 6781159 & $\nabla$ & $\checkmark$ & $\square$ & $\nabla$ & $\square$ \\
\hline 2431 & 1123 & Hollow stem borehole w/SPT & 4/28/1993 & 31.0 & 345866 & 6781159 & $\square$ & $\square$ & $\square$ & $\square$ & $\square$ \\
\hline 2431 & 1124 & Hollow stem borehole w/SPT & $4 / 28 / 1993$ & 30.8 & 345817 & 6781139 & $\square$ & $\square$ & $\square$ & $\square$ & $\square$ \\
\hline 2431 & 1125 & Hollow stem borehole w/SPT & 4/27/1993 & 31.0 & 345866 & 6781139 & $\square$ & $\square$ & $\square$ & $\square$ & $\square$ \\
\hline 2431 & 1720 & Hollow stem borehole w/SPT & $5 / 14 / 1979$ & 16.0 & 345534 & 6781088 & $\square$ & $\square$ & $\square$ & $\square$ & $\square$ \\
\hline 2431 & 1721 & Hollow stem borehole w/SPT & $5 / 14 / 1979$ & 16.0 & 345544 & 6780874 & $\square$ & $\square$ & $\square$ & $\square$ & $\square$ \\
\hline
\end{tabular}




\begin{tabular}{|c|c|c|c|c|c|c|c|c|c|c|c|}
\hline \multirow[b]{2}{*}{$\begin{array}{c}\text { MOA } \\
\text { grid }\end{array}$} & \multirow[b]{2}{*}{$\begin{array}{c}\text { DGGS } \\
\text { number }\end{array}$} & \multirow[b]{2}{*}{ Hole Type } & \multirow[b]{2}{*}{$\begin{array}{c}\text { Date } \\
\text { Completed }\end{array}$} & \multirow[b]{2}{*}{$\begin{array}{c}\text { Total } \\
\text { depth (ft) }\end{array}$} & \multicolumn{2}{|c|}{ UTM coordinates (m) } & \multicolumn{5}{|c|}{ Data entered } \\
\hline & & & & & East & North & Lithology & SPT & $\begin{array}{c}\text { Sample } \\
\text { test }\end{array}$ & $\begin{array}{l}\text { Water } \\
\text { level }\end{array}$ & Velocity \\
\hline 2431 & 1722 & Hollow stem borehole w/SPT & $5 / 14 / 1979$ & 15.5 & 345553 & 6780588 & $\square$ & $\square$ & $\square$ & $\square$ & $\square$ \\
\hline 2431 & 1773 & Hollow stem borehole w/SPT & 9/27/1993 & 15.0 & 345901 & 6781126 & $\square$ & $\square$ & $\square$ & $\square$ & $\square$ \\
\hline 2431 & 1774 & Hollow stem borehole w/SPT & $12 / 13 / 1993$ & 15.0 & 345899 & 6781001 & $\square$ & $\square$ & $\square$ & $\square$ & $\square$ \\
\hline 2431 & 1775 & Hollow stem borehole w/SPT & $12 / 9 / 1993$ & 15.0 & 345882 & 6780594 & $\square$ & $\square$ & $\square$ & $\square$ & $\square$ \\
\hline 2431 & CB001 & Water well & $8 / 30 / 1975$ & 305.0 & 345127 & 6780960 & $\nabla$ & $\square$ & $\square$ & $\nabla$ & $\square$ \\
\hline 2432 & 302 & Water well & $1 / 1 / 1965$ & 172.0 & 346210 & 6781220 & $\boldsymbol{V}$ & $\square$ & $\square$ & $\nabla$ & $\square$ \\
\hline 2432 & 4181 & Water well & $5 / 29 / 1974$ & 125.0 & 346157 & 6781167 & $\checkmark$ & $\square$ & $\square$ & $\checkmark$ & $\square$ \\
\hline 2432 & 4182 & Water well & $5 / 20 / 1974$ & 112.0 & 346082 & 6781170 & $\boldsymbol{V}$ & $\square$ & $\square$ & $\nabla$ & $\square$ \\
\hline 2432 & 4200 & Water well & $5 / 16 / 1974$ & 112.0 & 346191 & 6781258 & $\boldsymbol{V}$ & $\square$ & $\square$ & $\sqrt{ }$ & $\square$ \\
\hline 2432 & 4201 & Water well & $5 / 4 / 1971$ & 132.0 & 346176 & 6781259 & $\square$ & $\square$ & $\square$ & $\nabla$ & $\square$ \\
\hline 2432 & 4202 & Water well & 9/10/1974 & 115.0 & 345982 & 6781268 & 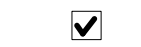 & $\square$ & $\square$ & $\nabla$ & $\square$ \\
\hline 2432 & CC001 & Water well & 4/22/1977 & 444.0 & 346403 & 6780544 & $\nabla$ & $\square$ & $\square$ & $\nabla$ & $\square$ \\
\hline 2432 & CR001 & Water well & 8/18/1974 & 150.0 & 346090 & 6781150 & $\nabla$ & $\square$ & $\square$ & $\nabla$ & $\square$ \\
\hline 2432 & CR003 & Water well & 7/19/1975 & 220.0 & 346515 & 6781153 & 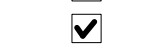 & $\square$ & $\square$ & $v$ & $\square$ \\
\hline 2433 & 1126 & Hollow stem borehole w/SPT & 9/27/1983 & 21.0 & 347208 & 6780902 & $\square$ & $\square$ & $\square$ & $\square$ & $\square$ \\
\hline 2433 & 1127 & Hollow stem borehole w/SPT & $9 / 27 / 1983$ & 30.2 & 347240 & 6780945 & $\checkmark$ & $\checkmark$ & $\checkmark$ & $\checkmark$ & $\square$ \\
\hline 2433 & 1128 & Hollow stem borehole w/SPT & 9/27/1983 & 30.3 & 347225 & 6781014 & $\boldsymbol{V}$ & $\nabla$ & $\nabla$ & $\nabla$ & $\square$ \\
\hline 2433 & 1129 & Hollow stem borehole w/SPT & 9/27/1983 & 20.2 & 347250 & 6780975 & $\square$ & $\square$ & $\square$ & $\square$ & $\square$ \\
\hline 2433 & 1130 & Hollow stem borehole w/SPT & $8 / 23 / 1991$ & 26.5 & 347433 & 6780803 & $\boldsymbol{V}$ & $\checkmark$ & $\nabla$ & $\nabla$ & $\square$ \\
\hline 2433 & 1131 & Hollow stem borehole w/SPT & $8 / 26 / 1991$ & 16.5 & 347387 & 6780792 & $\square$ & $\square$ & $\square$ & $\square$ & $\square$ \\
\hline 2433 & 4125 & Water well & $11 / 4 / 1982$ & 119.0 & 346849 & 6780548 & $\boldsymbol{V}$ & $\square$ & $\square$ & $\nabla$ & $\square$ \\
\hline 2433 & CQ001 & Water well & & 220.0 & 347348 & 6781236 & $\nabla$ & $\square$ & $\square$ & $\nabla$ & $\square$ \\
\hline 2434 & 1132 & Hollow stem borehole w/SPT & $7 / 5 / 1984$ & 31.5 & 347816 & 6780778 & $\square$ & $\square$ & $\square$ & $\square$ & $\square$ \\
\hline 2434 & 1133 & Hollow stem borehole w/SPT & $7 / 2 / 1984$ & 31.5 & 347682 & 6780725 & $\square$ & $\square$ & $\square$ & $\square$ & $\square$ \\
\hline 2434 & 1134 & Hollow stem borehole w/SPT & $7 / 6 / 1984$ & 31.5 & 347770 & 6780747 & $\square$ & $\square$ & $\square$ & $\square$ & $\square$ \\
\hline 2434 & 1735 & Hollow stem borehole w/SPT & $6 / 18 / 1982$ & 46.5 & 347697 & 6780460 & $\boldsymbol{V}$ & 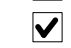 & $\square$ & $\nabla$ & $\square$ \\
\hline 2434 & 1736 & Hollow stem borehole w/SPT & $6 / 21 / 1982$ & 46.5 & 347633 & 6780490 & $\square$ & $\square$ & $\square$ & $\square$ & $\square$ \\
\hline 2434 & 4478 & Water well & $1 / 3 / 1983$ & 261.0 & 347859 & 6780690 & $\boldsymbol{V}$ & $\square$ & $\square$ & $\nabla$ & $\square$ \\
\hline 2435 & 3139 & Water well & $9 / 24 / 1984$ & 217.5 & 348992 & 6780920 & $\boldsymbol{V}$ & $\square$ & $\square$ & $\square$ & $\square$ \\
\hline 2435 & 4199 & Water well & $11 / 19 / 1971$ & 176.0 & 349047 & 6781135 & 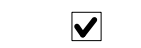 & $\square$ & $\square$ & $\nabla$ & $\square$ \\
\hline 2436 & 3108 & Water well & $7 / 27 / 1984$ & 235.0 & 349885 & 6781130 & $\checkmark$ & $\square$ & $\square$ & $\nabla$ & $\square$ \\
\hline 2436 & 3109 & Water well & $7 / 31 / 1984$ & 239.5 & 349810 & 6781133 & 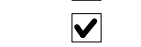 & $\square$ & $\square$ & $\checkmark$ & $\square$ \\
\hline 2436 & 3110 & Water well & 9/21/1977 & 173.0 & 349198 & 6781159 & 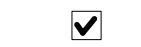 & $\square$ & $\square$ & $\bar{v}$ & $\square$ \\
\hline 2436 & 3115 & Water well & $8 / 2 / 1984$ & 252.0 & 349881 & 6781037 & $\nabla$ & $\square$ & $\square$ & $\nabla$ & $\square$ \\
\hline 2436 & 3116 & Water well & $12 / 16 / 1983$ & 254.0 & 349821 & 6781039 & $\boldsymbol{v}$ & $\square$ & $\square$ & $\nabla$ & $\square$ \\
\hline 2436 & 3117 & Water well & $8 / 14 / 1965$ & 280.0 & 349672 & 6781046 & $\boldsymbol{V}$ & $\square$ & $\square$ & $\nabla$ & $\square$ \\
\hline
\end{tabular}




\begin{tabular}{|c|c|c|c|c|c|c|c|c|c|c|c|}
\hline \multirow[b]{2}{*}{$\begin{array}{l}\text { MOA } \\
\text { grid }\end{array}$} & \multirow[b]{2}{*}{$\begin{array}{c}\text { DGGS } \\
\text { number }\end{array}$} & \multirow[b]{2}{*}{ Hole Type } & \multirow[b]{2}{*}{$\begin{array}{c}\text { Date } \\
\text { Completed }\end{array}$} & \multirow[b]{2}{*}{$\begin{array}{c}\text { Total } \\
\text { depth }(\mathrm{ft})\end{array}$} & \multicolumn{2}{|c|}{ UTM coordinates (m) } & \multicolumn{5}{|c|}{ Data entered } \\
\hline & & & & & East & North & Lithology & SPT & $\begin{array}{c}\text { Sample } \\
\text { test }\end{array}$ & $\begin{array}{l}\text { Water } \\
\text { level }\end{array}$ & Velocity \\
\hline 2436 & 3120 & Water well & $12 / 22 / 1983$ & 243.0 & 349775 & 6781010 & $\checkmark$ & $\square$ & $\square$ & $\checkmark$ & $\square$ \\
\hline 2436 & 3123 & Water well & $6 / 2 / 1965$ & 194.0 & 349251 & 6781033 & $\checkmark$ & $\square$ & $\square$ & $\checkmark$ & $\square$ \\
\hline 2436 & 3124 & Water well & $4 / 2 / 1984$ & 251.0 & 349729 & 6780982 & $\checkmark$ & & $\square$ & $\checkmark$ & \\
\hline 2436 & 3125 & Water well & $1 / 26 / 1984$ & 240.0 & 349877 & 6780944 & $\checkmark$ & $\square$ & $\square$ & $\square$ & $\square$ \\
\hline 2436 & 3131 & Water well & $12 / 13 / 1983$ & 253.0 & 349771 & 6780918 & $\checkmark$ & $\square$ & $\square$ & $\checkmark$ & $\square$ \\
\hline 2436 & 3136 & Water well & $9 / 28 / 1984$ & 205.0 & 349844 & 6780853 & $\checkmark$ & $\square$ & $\square$ & $\checkmark$ & $\square$ \\
\hline 2436 & 3137 & Water well & $7 / 25 / 1984$ & 239.5 & 349709 & 6780858 & $\checkmark$ & $\square$ & $\square$ & $\checkmark$ & $\square$ \\
\hline 2436 & 3140 & Water well & $8 / 27 / 1984$ & 200.0 & 349857 & 6780821 & $\checkmark$ & 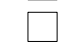 & $\square$ & $\bar{V}$ & $\square$ \\
\hline 2436 & 3141 & Water well & $8 / 24 / 1965$ & 227.0 & 349320 & 6780875 & $\checkmark$ & $\square$ & $\square$ & $\checkmark$ & $\square$ \\
\hline 2436 & 3143 & Water well & $9 / 20 / 1985$ & 191.0 & 349736 & 6780796 & $\checkmark$ & & $\square$ & $\checkmark$ & $\square$ \\
\hline 2436 & 3144 & Water well & $10 / 29 / 1984$ & 179.0 & 349854 & 6780759 & $\checkmark$ & $\square$ & $\square$ & $\bar{V}$ & $\square$ \\
\hline 2436 & 3145 & Water well & 9/10/1984 & 180.0 & 349749 & 6780764 & $\checkmark$ & & $\square$ & $\bar{\nabla}$ & $\square$ \\
\hline 2436 & 3149 & Water well & $9 / 14 / 1985$ & 179.0 & 349747 & 6780702 & $\checkmark$ & $\square$ & $\square$ & $\vec{V}$ & $\square$ \\
\hline 2436 & 3150 & Water well & $10 / 4 / 1975$ & 240.0 & 349403 & 6780717 & $\checkmark$ & $\square$ & $\square$ & $\nabla$ & $\square$ \\
\hline 2436 & 3151 & Water well & $3 / 20 / 1986$ & 199.0 & 349850 & 6780666 & $\checkmark$ & $\square$ & $\square$ & $\nabla$ & $\square$ \\
\hline 2436 & 3153 & Water well & $8 / 22 / 1986$ & 179.0 & 349729 & 6780641 & $\checkmark$ & 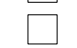 & $\square$ & $\checkmark$ & $\square$ \\
\hline 2436 & 3156 & Water well & $11 / 26 / 1984$ & 200.0 & 349668 & 6780613 & $\checkmark$ & $\square$ & $\square$ & $\checkmark$ & $\square$ \\
\hline 2436 & 3157 & Water well & 9/5/1984 & 220.0 & 349624 & 6780614 & $\checkmark$ & ᄂ & $\square$ & $\nabla$ & $\square$ \\
\hline 2436 & 3158 & Water well & $8 / 31 / 1984$ & 200.0 & 349564 & 6780617 & $\checkmark$ & $\square$ & $\square$ & $\checkmark$ & $\square$ \\
\hline 2436 & 3159 & Water well & $2 / 5 / 1985$ & 220.0 & 349518 & 6780619 & $\checkmark$ & $\square$ & $\square$ & $\nabla$ & $\square$ \\
\hline 2436 & 3162 & Water well & $10 / 3 / 1983$ & 233.0 & 349143 & 6780604 & $\checkmark$ & & $\square$ & $\checkmark$ & $\square$ \\
\hline 2436 & 3165 & Water well & 7/19/1984 & 219.0 & 349859 & 6780511 & $\checkmark$ & $\checkmark$ & $\square$ & $\bar{V}$ & $\square$ \\
\hline 2436 & 3167 & Water well & $10 / 30 / 1984$ & 159.0 & 349649 & 6780520 & $\checkmark$ & & $\square$ & $\nabla$ & $\square$ \\
\hline 2436 & 3168 & Water well & $8 / 28 / 1984$ & 200.0 & 349514 & 6780526 & $\nabla$ & $\square$ & $\square$ & $\nabla$ & $\square$ \\
\hline 2436 & 3169 & Water well & 2/3/1984 & 219.0 & 349812 & 6780482 & $\checkmark$ & & $\square$ & $\checkmark$ & $\square$ \\
\hline 2436 & 3170 & Water well & $5 / 28 / 1983$ & 239.0 & 349171 & 6780541 & $\checkmark$ & & $\square$ & $\checkmark$ & $\square$ \\
\hline 2436 & 3171 & Water well & $10 / 1 / 1984$ & 159.0 & 349618 & 6780490 & $\checkmark$ & $\square$ & $\square$ & $\checkmark$ & $\square$ \\
\hline 2436 & 3172 & Water well & $9 / 13 / 1984$ & 200.0 & 349573 & 6780492 & $\checkmark$ & & $\square$ & $\nabla$ & $\square$ \\
\hline 2436 & 3174 & Water well & $12 / 7 / 1969$ & 241.0 & 349378 & 6780501 & $\checkmark$ & $\square$ & $\square$ & $\checkmark$ & $\square$ \\
\hline 2436 & 3175 & Water well & $9 / 17 / 1985$ & 199.0 & 349736 & 6780455 & $\checkmark$ & & $\square$ & $\bar{\nabla}$ & $\square$ \\
\hline 2436 & 3179 & Water well & $1 / 31 / 1985$ & 181.0 & 349511 & 6780433 & $\checkmark$ & $\square$ & $\square$ & $\bar{\nabla}$ & $\square$ \\
\hline 2436 & 3180 & Water well & $5 / 18 / 1984$ & 240.0 & 349853 & 6780388 & $\checkmark$ & $\square$ & $\square$ & $\checkmark$ & $\square$ \\
\hline 2436 & 3181 & Water well & $2 / 1 / 1984$ & 224.0 & 349808 & 6780390 & $\checkmark$ & $\square$ & $\square$ & $\bar{\nabla}$ & $\square$ \\
\hline 2436 & 3182 & Water well & $10 / 5 / 1984$ & 219.0 & 349764 & 6780391 & $\checkmark$ & $\square$ & $\square$ & $\checkmark$ & $\square$ \\
\hline 2436 & 3183 & Water well & $9 / 12 / 1984$ & 180.0 & 349704 & 6780394 & $\checkmark$ & $\square$ & $\square$ & $\checkmark$ & $\square$ \\
\hline 2436 & 3184 & Water well & $8 / 27 / 1985$ & 179.0 & 349644 & 6780397 & $\checkmark$ & $\square$ & $\square$ & $\nabla$ & $\square$ \\
\hline
\end{tabular}




\begin{tabular}{|c|c|c|c|c|c|c|c|c|c|c|c|}
\hline \multirow[b]{2}{*}{$\begin{array}{l}\text { MOA } \\
\text { grid }\end{array}$} & \multirow[b]{2}{*}{$\begin{array}{l}\text { DGGS } \\
\text { number }\end{array}$} & \multirow[b]{2}{*}{ Hole Type } & \multirow[b]{2}{*}{$\begin{array}{c}\text { Date } \\
\text { Completed }\end{array}$} & \multirow[b]{2}{*}{$\begin{array}{c}\text { Total } \\
\text { depth }(\mathrm{ft})\end{array}$} & \multicolumn{2}{|c|}{ UTM coordinates (m) } & \multicolumn{5}{|c|}{ Data entered } \\
\hline & & & & & East & North & Lithology & SPT & $\begin{array}{c}\text { Sample } \\
\text { test }\end{array}$ & $\begin{array}{l}\text { Water } \\
\text { level }\end{array}$ & Velocity \\
\hline 2436 & 3186 & Water well & $8 / 21 / 1984$ & 180.0 & 349569 & 6780400 & $\checkmark$ & & $\square$ & $\nabla$ & $\square$ \\
\hline 2437 & 3112 & Water well & $1 / 1 / 1973$ & 235.0 & 350047 & 6781061 & $\checkmark$ & $\square$ & $\square$ & $\checkmark$ & $\square$ \\
\hline 2437 & 3154 & Water well & $5 / 3 / 1986$ & 240.0 & 349937 & 6780601 & $\checkmark$ & $\square$ & $\square$ & $\bar{V}$ & \\
\hline 2437 & 3155 & Water well & $10 / 16 / 1982$ & 103.0 & 350325 & 6780553 & $\nabla$ & $\square$ & $\square$ & $\checkmark$ & $\square$ \\
\hline 2437 & 3160 & Water well & $8 / 14 / 1975$ & 290.0 & 350307 & 6780492 & $\checkmark$ & $\square$ & $\square$ & 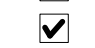 & $\square$ \\
\hline 2437 & 3164 & Water well & $6 / 1 / 1981$ & 124.0 & 349963 & 6780507 & $\checkmark$ & $\square$ & $\square$ & $\checkmark$ & $\square$ \\
\hline 2437 & 3166 & Water well & $1 / 12 / 1984$ & 146.0 & 350171 & 6780467 & $\checkmark$ & $\square$ & $\square$ & $\checkmark$ & $\square$ \\
\hline 2437 & 3173 & Water well & $7 / 18 / 1978$ & 306.0 & 350363 & 6780397 & $\checkmark$ & $\square$ & $\square$ & $\bar{\nabla}$ & $\square$ \\
\hline 2437 & 3176 & Water well & $8 / 20 / 1986$ & 312.0 & 350362 & 6780366 & $\checkmark$ & $\square$ & $\square$ & $\sqrt{\square}$ & $\square$ \\
\hline 2437 & 3178 & Water well & 7/16/1982 & 140.0 & 350242 & 6780371 & $\checkmark$ & & $\square$ & $\checkmark$ & $\square$ \\
\hline 2437 & 4128 & Water well & $5 / 3 / 1968$ & 107.0 & 350471 & 6780485 & $\checkmark$ & $\square$ & $\square$ & $\bar{V}$ & $\square$ \\
\hline 2437 & 4134 & Water well & $9 / 4 / 1975$ & 118.0 & 350008 & 6780505 & $\checkmark$ & & $\square$ & $\checkmark$ & $\square$ \\
\hline 2437 & 4141 & Water well & 10/1/1981 & 107.0 & 350461 & 6780579 & $\checkmark$ & $\square$ & $\square$ & $\boldsymbol{V}$ & $\square$ \\
\hline 2437 & 4479 & Water well & $1 / 1 / 1970$ & 110.0 & 350125 & 6780438 & $\square$ & $\square$ & $\square$ & $\checkmark$ & $\square$ \\
\hline 2438 & 1705 & Hollow stem borehole w/SPT & $3 / 26 / 1985$ & 21.0 & 350753 & 6781059 & $\checkmark$ & $\checkmark$ & $\square$ & $\checkmark$ & $\square$ \\
\hline 2438 & 3134 & Water well & $5 / 21 / 1983$ & 101.0 & 351171 & 6780735 & $\checkmark$ & & $\square$ & $\square$ & $\square$ \\
\hline 2438 & 4147 & Water well & 9/8/1976 & 154.0 & 350883 & 6780654 & $\checkmark$ & $\square$ & $\square$ & $\bar{\nabla}$ & $\square$ \\
\hline 2438 & 4164 & Water well & $12 / 1 / 1976$ & 107.0 & 351008 & 6780772 & $\checkmark$ & L & $\square$ & $\nabla$ & $\square$ \\
\hline 2438 & 4171 & Water well & 8/1/1982 & 285.0 & 351489 & 6780814 & $\checkmark$ & $\square$ & $\square$ & $\nabla$ & $\square$ \\
\hline 2439 & 1707 & Hollow stem borehole w/SPT & $7 / 21 / 1981$ & 26.0 & 352318 & 6780601 & $\square$ & $\square$ & $\square$ & $\square$ & $\square$ \\
\hline 2439 & 3113 & Water well & $8 / 18 / 1978$ & 120.0 & 352193 & 6780815 & $\checkmark$ & & $\square$ & $\bar{\nabla}$ & $\square$ \\
\hline 2439 & 3121 & Water well & $5 / 24 / 1978$ & 118.5 & 352206 & 6780753 & $\checkmark$ & $\checkmark$ & $\square$ & $\bar{V}$ & $\square$ \\
\hline 2439 & 3126 & Water well & $10 / 26 / 1979$ & 173.0 & 352293 & 6780687 & $\checkmark$ & & $\square$ & $\bar{V}$ & $\square$ \\
\hline 2439 & 3146 & Water well & 8/5/1981 & 111.0 & 352180 & 6780506 & $\checkmark$ & $\square$ & $\square$ & $\checkmark$ & $\square$ \\
\hline 2439 & 3148 & Water well & $7 / 10 / 1981$ & 116.0 & 352237 & 6780442 & $\checkmark$ & & $\square$ & $\checkmark$ & $\square$ \\
\hline 2439 & 3152 & Water well & $3 / 13 / 1984$ & 131.0 & 352205 & 6780381 & $\checkmark$ & & $\square$ & $\checkmark$ & $\square$ \\
\hline 2439 & 4121 & Water well & $12 / 1 / 1978$ & 234.0 & 351663 & 6780311 & $\checkmark$ & $\square$ & $\square$ & $\checkmark$ & $\square$ \\
\hline 2439 & 4122 & Water well & $7 / 1 / 1979$ & 134.0 & 351663 & 6780311 & $\checkmark$ & & $\square$ & $\nabla$ & $\square$ \\
\hline 2439 & 4131 & Water well & $7 / 12 / 1982$ & 130.0 & 352266 & 6780409 & $\checkmark$ & & $\square$ & $\checkmark$ & $\square$ \\
\hline 2439 & 4132 & Water well & $7 / 12 / 1981$ & 125.0 & 352161 & 6780414 & $\checkmark$ & $\square$ & $\square$ & 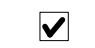 & $\square$ \\
\hline 2439 & 4135 & Water well & $9 / 27 / 1978$ & 298.0 & 352147 & 6780445 & $\checkmark$ & $\square$ & $\square$ & $\nabla$ & $\square$ \\
\hline 2439 & 4136 & Water well & 8/7/1978 & 204.0 & 351581 & 6780500 & $\checkmark$ & $\square$ & $\square$ & $\checkmark$ & $\square$ \\
\hline 2439 & 4139 & Water well & 9/9/1981 & 116.0 & 352270 & 6780502 & $\checkmark$ & $\square$ & $\square$ & $\bar{\nabla}$ & $\square$ \\
\hline 2439 & 4146 & Water well & $10 / 31 / 1981$ & 140.0 & 352274 & 6780595 & $\checkmark$ & $\square$ & $\square$ & $\nabla$ & $\square$ \\
\hline 2439 & 4150 & Water well & $12 / 2 / 1977$ & 163.0 & 351887 & 6780642 & $\checkmark$ & $\square$ & $\square$ & $\vec{V}$ & $\square$ \\
\hline 2439 & 4153 & Water well & $8 / 25 / 1978$ & 170.0 & 351768 & 6780678 & $\checkmark$ & $\square$ & $\square$ & $\nabla$ & $\square$ \\
\hline
\end{tabular}




\begin{tabular}{|c|c|c|c|c|c|c|c|c|c|c|c|}
\hline \multirow[b]{2}{*}{$\begin{array}{c}\text { MOA } \\
\text { grid }\end{array}$} & \multirow[b]{2}{*}{$\begin{array}{c}\text { DGGS } \\
\text { number }\end{array}$} & \multirow[b]{2}{*}{ Hole Type } & \multirow[b]{2}{*}{$\begin{array}{c}\text { Date } \\
\text { Completed }\end{array}$} & \multirow[b]{2}{*}{$\begin{array}{c}\text { Total } \\
\text { depth (ft) }\end{array}$} & \multicolumn{2}{|c|}{ UTM coordinates (m) } & \multicolumn{5}{|c|}{ Data entered } \\
\hline & & & & & East & North & Lithology & SPT & $\begin{array}{c}\text { Sample } \\
\text { test }\end{array}$ & $\begin{array}{l}\text { Water } \\
\text { level }\end{array}$ & Velocity \\
\hline 2439 & 4159 & Water well & $7 / 18 / 1977$ & 170.0 & 351755 & 6780710 & $\checkmark$ & $\square$ & $\square$ & $\nabla$ & $\square$ \\
\hline 2439 & 4160 & Water well & $7 / 25 / 1977$ & 173.0 & 351665 & 6780714 & $\checkmark$ & $\square$ & $\square$ & $\sqrt{ }$ & $\square$ \\
\hline 2439 & 4162 & Water well & $6 / 4 / 1980$ & 112.0 & 352279 & 6780719 & $\boldsymbol{V}$ & $\square$ & $\square$ & $\nabla$ & $\square$ \\
\hline 2439 & 4163 & Water well & $5 / 23 / 1980$ & 104.0 & 352190 & 6780722 & $\nabla$ & $\square$ & $\square$ & $\nabla$ & $\square$ \\
\hline 2439 & 4173 & Water well & $11 / 21 / 1977$ & 141.0 & 351849 & 6780830 & $\checkmark$ & $\square$ & $\square$ & $\nabla$ & $\square$ \\
\hline 2439 & 4179 & Water well & 8/11/1978 & 178.0 & 351988 & 6780917 & $\boldsymbol{V}$ & $\square$ & $\square$ & $\nabla$ & $\square$ \\
\hline 2439 & 4180 & Water well & 9/28/1977 & 174.0 & 351808 & 6780924 & $\checkmark$ & $\square$ & $\square$ & $\checkmark$ & $\square$ \\
\hline 2439 & 4184 & Water well & $8 / 31 / 1978$ & 298.0 & 352108 & 6780943 & $\boldsymbol{V}$ & $\square$ & $\square$ & $\square$ & $\square$ \\
\hline 2439 & 4185 & Water well & 9/15/1978 & 129.0 & 351900 & 6780951 & $\boldsymbol{v}$ & $\square$ & $\square$ & $\sqrt{ }$ & $\square$ \\
\hline 2439 & 4187 & Water well & 8/25/1978 & 101.0 & 352110 & 6780974 & $\boldsymbol{V}$ & $\square$ & $\square$ & $\nabla$ & $\square$ \\
\hline 2439 & 4195 & Water well & 12/8/1977 & 119.0 & 351992 & 6781010 & 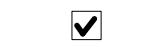 & $\square$ & $\square$ & $\nabla$ & $\square$ \\
\hline 2439 & 4197 & Water well & $10 / 12 / 1977$ & 107.0 & 351782 & 6781018 & $\bar{V}$ & $\square$ & $\square$ & $\nabla$ & $\square$ \\
\hline 2439 & 4203 & Water well & 8/17/1978 & 138.0 & 352112 & 6781036 & $\nabla$ & $\square$ & $\square$ & $\nabla$ & $\square$ \\
\hline 2439 & 4204 & Water well & 9/25/1981 & 120.0 & 352096 & 6781005 & 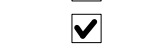 & $\square$ & $\square$ & $\checkmark$ & $\square$ \\
\hline 2440 & 1708 & Hollow stem borehole, no SPT & 4/7/1976 & 18.0 & 352889 & 6780988 & $\square$ & $\square$ & $\square$ & $\square$ & $\square$ \\
\hline 2440 & 3098 & Water well & $10 / 15 / 1980$ & 195.0 & 353128 & 6780962 & $\checkmark$ & $\square$ & $\square$ & $\checkmark$ & $\square$ \\
\hline 2440 & 3103 & Water well & $9 / 11 / 1980$ & 223.0 & 352634 & 6780952 & $\boldsymbol{V}$ & $\square$ & $\square$ & $\square$ & $\square$ \\
\hline 2440 & 3104 & Water well & $3 / 31 / 1978$ & 460.0 & 352871 & 6780911 & $\boldsymbol{V}$ & $\square$ & $\square$ & $\square$ & $\square$ \\
\hline 2440 & 3106 & Water well & $11 / 13 / 1980$ & 291.0 & 352898 & 6780848 & $\boldsymbol{V}$ & $\square$ & $\square$ & $\square$ & $\square$ \\
\hline 2440 & 3111 & Water well & 9/11/1976 & 425.0 & 352582 & 6780799 & $\boldsymbol{V}$ & $\square$ & $\square$ & $\nabla$ & $\square$ \\
\hline 2440 & 3114 & Water well & $2 / 1 / 1983$ & 370.0 & 353013 & 6780719 & $\boldsymbol{V}$ & $\square$ & $\square$ & $\nabla$ & $\square$ \\
\hline 2440 & 3128 & Water well & 9/22/1977 & 268.0 & 352903 & 6780600 & 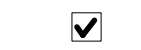 & $\square$ & $\square$ & $\nabla$ & $\square$ \\
\hline 2440 & 3132 & Water well & $9 / 4 / 1983$ & 172.0 & 352379 & 6780622 & $\nabla$ & $\square$ & $\square$ & $\nabla$ & $\square$ \\
\hline 2440 & 3135 & Water well & 7/14/1984 & 200.0 & 352498 & 6780586 & $\nabla$ & $\square$ & $\square$ & $\square$ & $\square$ \\
\hline 2440 & 3142 & Water well & $6 / 25 / 1981$ & 170.0 & 352793 & 6780480 & 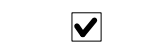 & $\square$ & $\square$ & $\nabla$ & $\square$ \\
\hline 2440 & 4124 & Water well & 8/2/1978 & 251.0 & 352771 & 6780296 & $\boldsymbol{V}$ & $\square$ & $\square$ & $\nabla$ & $\square$ \\
\hline 2440 & 4127 & Water well & 7/29/1981 & 223.0 & 352968 & 6780349 & $\nabla$ & $\square$ & $\square$ & $\checkmark$ & $\square$ \\
\hline 2440 & 4130 & Water well & 7/1/1974 & 300.0 & 352744 & 6780389 & $\boldsymbol{V}$ & $\square$ & $\square$ & $\nabla$ & $\square$ \\
\hline 2440 & 4137 & Water well & $6 / 12 / 1973$ & 211.0 & 352449 & 6780495 & $\nabla$ & $\square$ & $\square$ & $\nabla$ & $\square$ \\
\hline 2440 & 4138 & Water well & 9/3/1970 & 270.0 & 352449 & 6780495 & 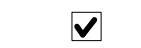 & $\square$ & $\square$ & $\nabla$ & $\square$ \\
\hline 2440 & 4142 & Water well & 7/20/1979 & 198.0 & 352361 & 6780530 & 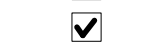 & $\square$ & $\square$ & $\nabla$ & $\square$ \\
\hline 2440 & 4144 & Water well & $3 / 15 / 1975$ & 301.0 & 352767 & 6780574 & 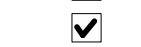 & $\square$ & $\square$ & $\checkmark$ & $\square$ \\
\hline 2440 & 4145 & Water well & $12 / 15 / 1981$ & 450.0 & 352693 & 6780577 & 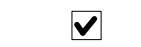 & $\square$ & $\square$ & $\square$ & $\square$ \\
\hline 2440 & 4149 & Water well & 11/1/1969 & 215.0 & 352933 & 6780598 & $\boldsymbol{V}$ & $\square$ & $\square$ & $\nabla$ & $\square$ \\
\hline 2440 & 4152 & Water well & $2 / 26 / 1973$ & 231.0 & 352563 & 6780707 & $\boldsymbol{v}$ & $\square$ & $\square$ & $\nabla$ & $\square$ \\
\hline 2440 & 4157 & Water well & $5 / 1 / 1979$ & 320.0 & 352771 & 6780667 & $\checkmark$ & $\square$ & $\square$ & $\nabla$ & $\square$ \\
\hline
\end{tabular}




\begin{tabular}{|c|c|c|c|c|c|c|c|c|c|c|c|}
\hline \multirow[b]{2}{*}{$\begin{array}{l}\text { MOA } \\
\text { grid }\end{array}$} & \multirow[b]{2}{*}{$\begin{array}{l}\text { DGGS } \\
\text { number }\end{array}$} & \multirow[b]{2}{*}{ Hole Type } & \multirow[b]{2}{*}{$\begin{array}{c}\text { Date } \\
\text { Completed }\end{array}$} & \multirow[b]{2}{*}{$\begin{array}{c}\text { Total } \\
\text { depth }(\mathrm{ft})\end{array}$} & \multicolumn{2}{|c|}{ UTM coordinates (m) } & \multicolumn{5}{|c|}{ Data entered } \\
\hline & & & & & East & North & Lithology & SPT & $\begin{array}{c}\text { Sample } \\
\text { test }\end{array}$ & $\begin{array}{l}\text { Water } \\
\text { level }\end{array}$ & Velocity \\
\hline 2440 & 4158 & Water well & $5 / 11 / 1978$ & 350.0 & 352701 & 6780763 & $\checkmark$ & $\square$ & $\square$ & $\square$ & $\square$ \\
\hline 2440 & 4161 & Water well & $9 / 25 / 1978$ & 115.0 & 353072 & 6780685 & $\checkmark$ & $\square$ & $\square$ & $\square$ & $\square$ \\
\hline 2440 & 4167 & Water well & $12 / 26 / 1979$ & 498.0 & 352985 & 6780751 & $\bar{\nabla}$ & & $\square$ & $\square$ & \\
\hline 2440 & 4168 & Water well & $2 / 23 / 1983$ & 340.0 & 352894 & 6780755 & $\nabla$ & $\square$ & $\square$ & $\square$ & $\square$ \\
\hline 2440 & 4169 & Water well & $11 / 18 / 1977$ & 398.0 & 352775 & 6780760 & $\checkmark$ & $\square$ & $\square$ & $\checkmark$ & $\square$ \\
\hline 2440 & 4170 & Water well & $11 / 5 / 1965$ & 143.0 & 352416 & 6780775 & $\checkmark$ & $\square$ & $\square$ & $\checkmark$ & $\square$ \\
\hline 2440 & 4172 & Water well & $5 / 5 / 1979$ & 156.0 & 353061 & 6780779 & $\checkmark$ & $\square$ & $\square$ & $\checkmark$ & $\square$ \\
\hline 2440 & 4175 & Water well & 6/12/1976 & 287.0 & 352779 & 6780853 & $\checkmark$ & $\square$ & $\square$ & $\bar{\nabla}$ & $\square$ \\
\hline 2440 & 4177 & Water well & $4 / 19 / 1978$ & 190.0 & 352974 & 6780875 & $\checkmark$ & $\square$ & $\square$ & $\checkmark$ & $\square$ \\
\hline 2440 & 4178 & Water well & $7 / 16 / 1976$ & 197.0 & 352481 & 6780896 & $\checkmark$ & $\square$ & $\square$ & $\vec{V}$ & $\square$ \\
\hline 2440 & 4192 & Water well & $12 / 11 / 1978$ & 183.0 & 352859 & 6780973 & $\checkmark$ & $\square$ & $\square$ & $\bar{V}$ & $\square$ \\
\hline 2440 & 4193 & Water well & $3 / 12 / 1974$ & 426.0 & 352843 & 6780974 & $\checkmark$ & & $\square$ & $\bar{\nabla}$ & $\square$ \\
\hline 2440 & 4194 & Water well & $9 / 24 / 1975$ & 354.0 & 352679 & 6780981 & $\checkmark$ & $\square$ & $\square$ & $\vec{V}$ & $\square$ \\
\hline 2440 & 4327 & Water well & $6 / 13 / 1979$ & 350.0 & 352859 & 6780973 & $\square$ & $\square$ & $\square$ & $\nabla$ & $\square$ \\
\hline 2441 & 1759 & Hollow stem borehole w/SPT & $12 / 12 / 1990$ & 15.2 & 353718 & 6780302 & $\nabla$ & $\nabla$ & $\square$ & $\checkmark$ & $\square$ \\
\hline 2441 & 1760 & Hollow stem borehole w/SPT & $12 / 12 / 1990$ & 20.5 & 353773 & 6780374 & $\checkmark$ & $\checkmark$ & $\square$ & $\checkmark$ & $\square$ \\
\hline 2441 & 3101 & Water well & $9 / 17 / 1979$ & 146.1 & 353619 & 6780880 & $\checkmark$ & & $\square$ & 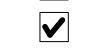 & $\square$ \\
\hline 2441 & 3122 & Water well & 8/5/1979 & 254.0 & 353278 & 6780615 & $\checkmark$ & ᄂ & $\square$ & $\vec{\nabla}$ & $\square$ \\
\hline 2441 & 3133 & Water well & $7 / 23 / 1979$ & 280.0 & 353707 & 6780473 & $\checkmark$ & $\square$ & $\square$ & $\nabla$ & $\square$ \\
\hline 2441 & 3138 & Water well & 8/3/1979 & 399.0 & 353406 & 6780455 & $\square$ & $\square$ & $\square$ & $\checkmark$ & $\square$ \\
\hline 2441 & 3147 & Water well & 7/6/1977 & 200.0 & 353610 & 6780322 & $\checkmark$ & & $\square$ & $\bar{\nabla}$ & $\square$ \\
\hline 2441 & 4120 & Water well & $5 / 30 / 1982$ & 250.0 & 353562 & 6780231 & $\checkmark$ & $\checkmark$ & $\square$ & $\bar{V}$ & $\square$ \\
\hline 2441 & 4123 & Water well & 4/1/1971 & 170.0 & 353458 & 6780267 & $\checkmark$ & & $\square$ & $\nabla$ & $\square$ \\
\hline 2441 & 4126 & Water well & $7 / 13 / 1973$ & 340.0 & 353371 & 6780332 & $\checkmark$ & $\square$ & $\square$ & $\checkmark$ & $\square$ \\
\hline 2441 & 4129 & Water well & $7 / 12 / 1969$ & 200.0 & 353283 & 6780367 & $\checkmark$ & & $\square$ & $\checkmark$ & $\square$ \\
\hline 2441 & 4143 & Water well & $9 / 27 / 1973$ & 175.0 & 353485 & 6780544 & $\checkmark$ & & $\square$ & $\bar{V}$ & $\square$ \\
\hline 2441 & 4151 & Water well & $7 / 16 / 1973$ & 425.0 & 353428 & 6780609 & $\checkmark$ & $\square$ & $\square$ & $\checkmark$ & $\square$ \\
\hline 2441 & 4154 & Water well & 8/8/1973 & 225.0 & 353683 & 6780629 & $\checkmark$ & & $\square$ & $\nabla$ & $\square$ \\
\hline 2441 & 4156 & Water well & 8/5/1978 & 608.0 & 353399 & 6780641 & $\square$ & $\square$ & $\square$ & $\checkmark$ & $\square$ \\
\hline 2441 & 4165 & Water well & $6 / 26 / 1977$ & 343.0 & 353388 & 6780734 & $\checkmark$ & & $\square$ & $\bar{\nabla}$ & $\square$ \\
\hline 2441 & 4166 & Water well & $5 / 23 / 1978$ & 333.0 & 353193 & 6780742 & $\checkmark$ & $\square$ & $\square$ & $\bar{\nabla}$ & $\square$ \\
\hline 2441 & 4174 & Water well & $5 / 10 / 1981$ & 320.0 & 353691 & 6780815 & $\checkmark$ & $\square$ & $\square$ & $\checkmark$ & $\square$ \\
\hline 2441 & 4176 & Water well & $5 / 12 / 1975$ & 225.0 & 353289 & 6780862 & $\checkmark$ & $\square$ & $\square$ & $\bar{\nabla}$ & $\square$ \\
\hline 2441 & 4188 & Water well & $5 / 25 / 1978$ & 405.0 & 353711 & 6780938 & $\checkmark$ & $\square$ & $\square$ & $\bar{\nabla}$ & $\square$ \\
\hline 2441 & 4189 & Water well & $8 / 22 / 1981$ & 595.0 & 353501 & 6780947 & $\checkmark$ & $\square$ & $\square$ & $\vec{V}$ & $\square$ \\
\hline 2441 & 4190 & Water well & $5 / 15 / 1970$ & 105.0 & 353367 & 6780952 & $\square$ & $\square$ & $\square$ & $\nabla$ & $\square$ \\
\hline
\end{tabular}




\begin{tabular}{|c|c|c|c|c|c|c|c|c|c|c|c|}
\hline \multirow[b]{2}{*}{$\begin{array}{c}\text { MOA } \\
\text { grid }\end{array}$} & \multirow[b]{2}{*}{$\begin{array}{c}\text { DGGS } \\
\text { number }\end{array}$} & \multirow[b]{2}{*}{ Hole Type } & \multirow[b]{2}{*}{$\begin{array}{c}\text { Date } \\
\text { Completed }\end{array}$} & \multirow[b]{2}{*}{$\begin{array}{c}\text { Total } \\
\text { depth }(\mathrm{ft})\end{array}$} & \multicolumn{2}{|c|}{ UTM coordinates (m) } & \multicolumn{5}{|c|}{ Data entered } \\
\hline & & & & & East & North & Lithology & SPT & $\begin{array}{l}\text { Sample } \\
\text { test }\end{array}$ & $\begin{array}{l}\text { Water } \\
\text { level }\end{array}$ & Velocity \\
\hline 2441 & 4191 & Water well & $7 / 17 / 1973$ & 140.0 & 353307 & 6780955 & $\checkmark$ & $\square$ & $\square$ & $\checkmark$ & $\square$ \\
\hline 2525 & 2237 & Hollow stem borehole w/SPT & $8 / 2 / 1983$ & 26.5 & 340936 & 6780546 & $\nabla$ & $\checkmark$ & $\square$ & $\square$ & $\square$ \\
\hline 2525 & 2238 & Hollow stem borehole w/SPT & $12 / 22 / 1981$ & 26.5 & 340919 & 6780705 & $\checkmark$ & $\checkmark$ & $\checkmark$ & $\nabla$ & $\square$ \\
\hline 2525 & 4115 & Water well & $7 / 25 / 1972$ & 107.0 & 340728 & 6780696 & $\nabla$ & $\square$ & $\square$ & $\nabla$ & $\square$ \\
\hline 2526 & 2098 & Hollow stem borehole w/SPT & & 60.0 & 341716 & 6780159 & $\checkmark$ & $\checkmark$ & $\square$ & $\checkmark$ & $\square$ \\
\hline 2526 & 2099 & Hollow stem borehole, no SPT & $11 / 1 / 1973$ & 136.0 & 341721 & 6780173 & $\checkmark$ & $\square$ & $\square$ & $\checkmark$ & $\square$ \\
\hline 2526 & 2100 & Hollow stem borehole w/SPT & $10 / 21 / 1973$ & 55.0 & 341744 & 6780183 & $\checkmark$ & $\checkmark$ & $\square$ & $\checkmark$ & $\square$ \\
\hline 2526 & 2222 & Hollow stem borehole w/SPT & & 61.5 & 341204 & 6779969 & $\checkmark$ & $\checkmark$ & $\square$ & $\checkmark$ & $\square$ \\
\hline 2526 & 2253 & Hollow stem borehole w/SPT & $8 / 28 / 1977$ & 32.0 & 341321 & 6780019 & $\nabla$ & $\checkmark$ & $\square$ & $\checkmark$ & $\square$ \\
\hline 2526 & ВТ013 & Hollow stem borehole w/SPT & & 31.5 & 341756 & 6779955 & $\checkmark$ & $\checkmark$ & $\square$ & $\checkmark$ & $\square$ \\
\hline 2526 & ВТ014 & Hollow stem borehole w/SPT & & 31.5 & 341836 & 6779947 & $\square$ & $\square$ & $\square$ & $\square$ & $\square$ \\
\hline 2526 & BT015 & Hollow stem borehole w/SPT & & 31.5 & 341879 & 6779899 & $\checkmark$ & $\square$ & $\square$ & $\square$ & $\square$ \\
\hline 2526 & BX001 & Hollow stem borehole, no SPT & & 15.0 & 341814 & 6780584 & $\square$ & $\square$ & $\square$ & $\square$ & $\square$ \\
\hline 2526 & BX002 & Hollow stem borehole w/SPT & & 30.0 & 341895 & 6780513 & $\checkmark$ & $\checkmark$ & $\square$ & $\square$ & $\square$ \\
\hline 2526 & BX003 & Hollow stem borehole, no SPT & & 15.0 & 341803 & 6780444 & $\square$ & $\square$ & $\square$ & $\square$ & $\square$ \\
\hline 2526 & BX004 & Hollow stem borehole, no SPT & & 12.0 & 341859 & 6780369 & $\square$ & $\square$ & $\square$ & $\square$ & $\square$ \\
\hline 2526 & BX005 & Hollow stem borehole w/SPT & & 26.5 & 341885 & 6780388 & $\square$ & $\square$ & $\square$ & $\square$ & $\square$ \\
\hline 2526 & BX006 & Hollow stem borehole w/SPT & & 16.5 & 341918 & 6780346 & $\square$ & $\square$ & $\square$ & $\square$ & $\square$ \\
\hline 2526 & BX007 & Hollow stem borehole, no SPT & & 12.0 & 341854 & 6780314 & $\square$ & $\square$ & $\square$ & $\square$ & $\square$ \\
\hline 2526 & BX008 & Hollow stem borehole w/SPT & & 16.5 & 341807 & 6780306 & $\square$ & $\square$ & $\square$ & $\square$ & $\square$ \\
\hline 2526 & BX009 & Hollow stem borehole w/SPT & & 26.5 & 341830 & 6780260 & $\square$ & $\square$ & $\square$ & $\square$ & $\square$ \\
\hline 2526 & BX010 & Hollow stem borehole w/SPT & & 26.5 & 341861 & 6780206 & $\square$ & $\square$ & $\square$ & $\square$ & $\square$ \\
\hline 2526 & BX011 & Hollow stem borehole w/SPT & & 26.5 & 341870 & 6780140 & $\square$ & $\square$ & $\square$ & $\square$ & $\square$ \\
\hline 2526 & BX012 & Hollow stem borehole w/SPT & & 26.5 & 341909 & 6780300 & $\square$ & $\square$ & $\square$ & $\square$ & $\square$ \\
\hline 2526 & BX013 & Hollow stem borehole w/SPT & & 26.5 & 341904 & 6780222 & $\square$ & $\square$ & $\square$ & $\square$ & $\square$ \\
\hline 2526 & BX014 & Hollow stem borehole w/SPT & & 16.5 & 341925 & 6780204 & $\square$ & $\square$ & $\square$ & $\square$ & $\square$ \\
\hline 2526 & ВX020 & Hollow stem borehole, no SPT & & 22.0 & 341920 & 6780128 & $\square$ & $\square$ & $\square$ & $\square$ & $\square$ \\
\hline 2527 & 1709 & Hollow stem borehole w/SPT & $6 / 21 / 1983$ & 52.0 & 342244 & 6780293 & $\checkmark$ & $\checkmark$ & $\checkmark$ & $\checkmark$ & $\square$ \\
\hline 2527 & 1710 & Hollow stem borehole w/SPT & $8 / 15 / 1983$ & 51.5 & 342218 & 6780484 & $\boldsymbol{V}$ & $\nabla$ & $\nabla$ & $\nabla$ & $\square$ \\
\hline 2527 & 2157 & Hollow stem borehole w/SPT & 7/10/1996 & 90.9 & 342188 & 6780061 & $\checkmark$ & $\checkmark$ & $\square$ & $\square$ & $\square$ \\
\hline 2527 & 2255 & Hollow stem borehole w/SPT & $7 / 18 / 1985$ & 31.5 & 342325 & 6780040 & $\checkmark$ & $\nabla$ & $\square$ & $\nabla$ & $\square$ \\
\hline 2527 & 2256 & Hollow stem borehole w/SPT & $3 / 8 / 1983$ & 31.0 & 342351 & 6780492 & $\checkmark$ & $\checkmark$ & $\square$ & $\sqrt{v}$ & $\square$ \\
\hline 2527 & 2257 & Hollow stem borehole w/SPT & $7 / 24 / 1985$ & 31.5 & 342492 & 6780318 & $\checkmark$ & $\checkmark$ & $\square$ & $\checkmark$ & $\square$ \\
\hline 2527 & 2258 & Hollow stem borehole w/SPT & $7 / 22 / 1985$ & 31.5 & 342450 & 6780181 & $\checkmark$ & $\checkmark$ & $\square$ & $\checkmark$ & $\square$ \\
\hline 2527 & 2259 & Hollow stem borehole w/SPT & $7 / 10 / 1985$ & 31.5 & 342566 & 6780076 & $\checkmark$ & $\checkmark$ & $\square$ & $\checkmark$ & $\square$ \\
\hline 2527 & 2260 & Hollow stem borehole w/SPT & 7/12/1985 & 31.5 & 342563 & 6780008 & $\nabla$ & $\nabla$ & $\square$ & $v$ & $\square$ \\
\hline
\end{tabular}




\begin{tabular}{|c|c|c|c|c|c|c|c|c|c|c|c|}
\hline \multirow[b]{2}{*}{$\begin{array}{c}\text { MOA } \\
\text { grid }\end{array}$} & \multirow[b]{2}{*}{$\begin{array}{l}\text { DGGS } \\
\text { number }\end{array}$} & \multirow[b]{2}{*}{ Hole Type } & \multirow[b]{2}{*}{$\begin{array}{c}\text { Date } \\
\text { Completed }\end{array}$} & \multirow[b]{2}{*}{$\begin{array}{c}\text { Total } \\
\text { depth }(\mathrm{ft})\end{array}$} & \multicolumn{2}{|c|}{ UTM coordinates (m) } & \multicolumn{5}{|c|}{ Data entered } \\
\hline & & & & & East & North & Lithology & SPT & $\begin{array}{c}\text { Sample } \\
\text { test }\end{array}$ & $\begin{array}{l}\text { Water } \\
\text { level }\end{array}$ & Velocity \\
\hline 2527 & BS001 & Hollow stem borehole w/SPT & & 41.5 & 342091 & 6779899 & $\checkmark$ & $\square$ & $\square$ & $\square$ & $\square$ \\
\hline 2527 & BS002 & Hollow stem borehole w/SPT & & 36.5 & 342160 & 6779894 & $\square$ & $\square$ & $\square$ & $\square$ & $\square$ \\
\hline 2527 & BS003 & Hollow stem borehole w/SPT & & 36.5 & 342244 & 6779892 & & $\square$ & $\square$ & $\square$ & $\square$ \\
\hline 2527 & BS016 & Hollow stem borehole w/SPT & & 16.5 & 342093 & 6779994 & $\square$ & $\square$ & $\square$ & $\square$ & $\square$ \\
\hline 2527 & BS017 & Hollow stem borehole w/SPT & & 21.5 & 342158 & 6779990 & $\square$ & $\square$ & $\square$ & $\square$ & $\square$ \\
\hline 2527 & BS018 & Hollow stem borehole w/SPT & & 16.5 & 342243 & 6779983 & $\square$ & $\square$ & $\square$ & $\square$ & $\square$ \\
\hline 2527 & BS019 & Hollow stem borehole w/SPT & & 16.5 & 342510 & 6779987 & $\square$ & $\square$ & $\square$ & $\square$ & $\square$ \\
\hline 2527 & BS020 & Hollow stem borehole w/SPT & & 26.5 & 342669 & 6779962 & & $\square$ & $\square$ & $\square$ & $\square$ \\
\hline 2527 & BS021 & Hollow stem borehole w/SPT & & 21.5 & 342341 & 6779887 & $\square$ & $\square$ & $\square$ & $\square$ & $\square$ \\
\hline 2527 & BS022 & Hollow stem borehole w/SPT & & 16.5 & 342505 & 6779882 & & $\square$ & $\square$ & $\square$ & $\square$ \\
\hline 2527 & BS023 & Hollow stem borehole w/SPT & & 16.5 & 342589 & 6779874 & $\square$ & $\square$ & $\square$ & $\square$ & $\square$ \\
\hline 2527 & BS024 & Hollow stem borehole w/SPT & & 5.0 & 342678 & 6779873 & & $\square$ & $\square$ & $\square$ & $\square$ \\
\hline 2527 & ВT023 & Hollow stem borehole w/SPT & & 21.5 & 341933 & 6779996 & $\square$ & $\square$ & $\square$ & $\square$ & $\square$ \\
\hline 2527 & ВT024 & Hollow stem borehole w/SPT & & 26.5 & 341926 & 6779906 & $\square$ & $\square$ & $\square$ & $\square$ & $\square$ \\
\hline 2527 & BX015 & Pit or trench & & 15.0 & 341963 & 6780218 & $\square$ & $\square$ & $\square$ & $\square$ & $\square$ \\
\hline 2527 & BX016 & Hollow stem borehole w/SPT & & 26.5 & 341970 & 6780252 & $\checkmark$ & $\checkmark$ & $\square$ & $\square$ & $\square$ \\
\hline 2527 & BX017 & Hollow stem borehole w/SPT & & 26.5 & 341963 & 6780348 & & $\square$ & $\square$ & $\square$ & $\square$ \\
\hline 2527 & BX018 & Hollow stem borehole, no SPT & & 12.0 & 341973 & 6780326 & & $\square$ & $\square$ & $\square$ & $\square$ \\
\hline 2527 & BX019 & Hollow stem borehole w/SPT & & 22.0 & 341974 & 6780160 & 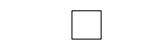 & $\square$ & $\square$ & $\square$ & $\square$ \\
\hline 2527 & BX021 & Hollow stem borehole w/SPT & & 16.5 & 341937 & 6780090 & $\square$ & $\square$ & $\square$ & $\square$ & $\square$ \\
\hline 2527 & BY001 & Hollow stem borehole w/SPT & & 26.5 & 341990 & 6780652 & & $\square$ & $\square$ & $\square$ & $\square$ \\
\hline 2527 & BY002 & Hollow stem borehole w/SPT & & 26.5 & 342029 & 6780549 & 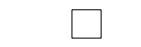 & $\square$ & $\square$ & $\square$ & $\square$ \\
\hline 2527 & BY003 & Hollow stem borehole, no SPT & & 12.0 & 342003 & 6780396 & & $\square$ & $\square$ & $\square$ & $\square$ \\
\hline 2527 & BY004 & Hollow stem borehole w/SPT & & 26.5 & 342147 & 6780568 & $\square$ & $\square$ & $\square$ & $\square$ & $\square$ \\
\hline 2527 & BY005 & Hollow stem borehole w/SPT & & 26.5 & 342132 & 6780493 & & $\square$ & $\square$ & $\square$ & $\square$ \\
\hline 2527 & BY006 & Hollow stem borehole w/SPT & & 26.5 & 342082 & 6780424 & $\square$ & $\square$ & $\square$ & $\square$ & $\square$ \\
\hline 2527 & BY007 & Hollow stem borehole, no SPT & & 21.0 & 342073 & 6780310 & $\square$ & $\square$ & $\square$ & $\square$ & $\square$ \\
\hline 2527 & BY008 & Hollow stem borehole w/SPT & & 26.5 & 342149 & 6780360 & $\boldsymbol{V}$ & $\checkmark$ & $\square$ & $\square$ & $\square$ \\
\hline 2527 & BY009 & Hollow stem borehole w/SPT & & 26.5 & 342192 & 6780438 & & $\square$ & $\square$ & $\square$ & $\square$ \\
\hline 2527 & BY010 & Hollow stem borehole w/SPT & & 26.5 & 342250 & 6780514 & & $\square$ & $\square$ & $\square$ & $\square$ \\
\hline 2527 & BY011 & Hollow stem borehole w/SPT & & 26.5 & 342252 & 6780573 & $\square$ & $\square$ & $\square$ & $\square$ & $\square$ \\
\hline 2527 & BY012 & Hollow stem borehole, no SPT & & 15.0 & 342302 & 6780664 & $\square$ & $\square$ & $\square$ & $\square$ & $\square$ \\
\hline 2527 & BY013 & Hollow stem borehole w/SPT & & 26.5 & 342307 & 6780629 & $\square$ & $\square$ & $\square$ & $\square$ & $\square$ \\
\hline 2527 & BY014 & Hollow stem borehole, no SPT & & 13.0 & 342351 & 6780573 & $\square$ & $\square$ & $\square$ & $\square$ & $\square$ \\
\hline 2527 & BY015 & Hollow stem borehole w/SPT & & 26.5 & 342330 & 6780530 & $\square$ & $\square$ & $\square$ & $\square$ & $\square$ \\
\hline 2527 & BY016 & Hollow stem borehole, no SPT & & 15.0 & 342303 & 6780514 & $\square$ & $\square$ & $\square$ & $\square$ & $\square$ \\
\hline
\end{tabular}




\begin{tabular}{|c|c|c|c|c|c|c|c|c|c|c|c|}
\hline \multirow[b]{2}{*}{$\begin{array}{l}\text { MOA } \\
\text { grid }\end{array}$} & \multirow[b]{2}{*}{$\begin{array}{l}\text { DGGS } \\
\text { number }\end{array}$} & \multirow[b]{2}{*}{ Hole Type } & \multirow[b]{2}{*}{$\begin{array}{c}\text { Date } \\
\text { Completed }\end{array}$} & \multirow[b]{2}{*}{$\begin{array}{c}\text { Total } \\
\text { depth }(\mathrm{ft})\end{array}$} & \multicolumn{2}{|c|}{ UTM coordinates (m) } & \multicolumn{5}{|c|}{ Data entered } \\
\hline & & & & & East & North & Lithology & SPT & $\begin{array}{c}\text { Sample } \\
\text { test }\end{array}$ & $\begin{array}{l}\text { Water } \\
\text { level }\end{array}$ & Velocity \\
\hline 2527 & BY017 & Hollow stem borehole w/SPT & & 26.5 & 342289 & 6780416 & $\square$ & & $\square$ & $\square$ & \\
\hline 2527 & BY018 & Hollow stem borehole w/SPT & & 26.5 & 342256 & 6780360 & $\square$ & $\square$ & $\square$ & $\square$ & $\square$ \\
\hline 2527 & BY019 & Hollow stem borehole, no SPT & & 13.0 & 342359 & 6780344 & $\square$ & & $\square$ & $\square$ & \\
\hline 2527 & BY020 & Pit or trench & & 15.0 & 342249 & 6780240 & $\square$ & $\square$ & $\square$ & $\square$ & $\square$ \\
\hline 2527 & BY021 & Hollow stem borehole w/SPT & & 26.5 & 342094 & 6780663 & $\square$ & $\square$ & $\square$ & $\square$ & $\square$ \\
\hline 2527 & BY022 & Hollow stem borehole w/SPT & & 26.5 & 341989 & 6780470 & $\square$ & $\square$ & $\square$ & $\square$ & $\square$ \\
\hline 2527 & BY023 & Hollow stem borehole w/SPT & & 21.5 & 342161 & 6780200 & $\square$ & $\square$ & $\square$ & $\square$ & $\square$ \\
\hline 2527 & BY024 & Hollow stem borehole w/SPT & & 21.5 & 342243 & 6780104 & $\square$ & $\checkmark$ & $\square$ & $\square$ & $\square$ \\
\hline 2527 & BY025 & Hollow stem borehole w/SPT & & 16.5 & 342440 & 6780094 & $\square$ & $\square$ & $\square$ & $\square$ & $\square$ \\
\hline 2527 & BY026 & Hollow stem borehole w/SPT & & 21.5 & 342590 & 6780078 & $\square$ & & $\square$ & $\square$ & $\square$ \\
\hline 2527 & BY027 & Hollow stem borehole w/SPT & & 16.5 & 342518 & 6780184 & $\square$ & $\square$ & $\square$ & $\square$ & $\square$ \\
\hline 2527 & BY028 & Hollow stem borehole w/SPT & & 26.5 & 342658 & 6780163 & $\square$ & $\square$ & $\square$ & $\square$ & $\square$ \\
\hline 2527 & BY029 & Hollow stem borehole w/SPT & & 21.5 & 342444 & 6780272 & $\square$ & $\square$ & $\square$ & $\square$ & $\square$ \\
\hline 2527 & BY030 & Hollow stem borehole w/SPT & & 16.5 & 342596 & 6780259 & $\square$ & $\square$ & $\square$ & $\square$ & $\square$ \\
\hline 2527 & BY031 & Hollow stem borehole w/SPT & & 26.5 & 342668 & 6780341 & $\square$ & $\square$ & $\square$ & $\square$ & $\square$ \\
\hline 2527 & BY032 & Hollow stem borehole w/SPT & & 26.5 & 342676 & 6780531 & $\square$ & $\square$ & $\square$ & $\square$ & $\square$ \\
\hline 2527 & BY033 & Hollow stem borehole w/SPT & & 26.5 & 342670 & 6780624 & $\square$ & $\square$ & $\square$ & $\square$ & $\square$ \\
\hline 2528 & 1711 & Hollow stem borehole w/SPT & $3 / 18 / 1982$ & 75.0 & 343462 & 6780610 & $\checkmark$ & 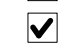 & $\nabla$ & $\vec{\nabla}$ & $\square$ \\
\hline 2528 & 2015 & Water well & 2/18/1994 & 200.0 & 343100 & 6780100 & $\checkmark$ & $\square$ & $\square$ & $\nabla$ & $\square$ \\
\hline 2528 & 2261 & Hollow stem borehole w/SPT & $1 / 16 / 1984$ & 31.5 & 343375 & 6780095 & $\checkmark$ & $\bar{\nabla}$ & $\square$ & $\checkmark$ & $\square$ \\
\hline 2530 & 1640 & Hollow stem borehole w/SPT & $5 / 9 / 1997$ & 17.0 & 344761 & 6780518 & $\checkmark$ & $\bar{\nabla}$ & $\square$ & $\bar{\nabla}$ & $\square$ \\
\hline 2530 & 1641 & Hollow stem borehole w/SPT & $5 / 9 / 1997$ & 16.5 & 345022 & 6780499 & $\square$ & $\square$ & $\square$ & $\square$ & $\square$ \\
\hline 2530 & 1642 & Hollow stem borehole w/SPT & $5 / 8 / 1997$ & 16.5 & 344735 & 6780254 & $\checkmark$ & $\nabla$ & $\square$ & $\nabla$ & $\square$ \\
\hline 2530 & 1643 & Hollow stem borehole w/SPT & $5 / 8 / 1997$ & 16.5 & 345022 & 6780251 & $\checkmark$ & $\bar{\square}$ & $\square$ & $\square$ & $\square$ \\
\hline 2531 & 1715 & Hollow stem borehole w/SPT & $12 / 9 / 1981$ & 100.0 & 345507 & 6779786 & $\checkmark$ & 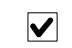 & $\checkmark$ & 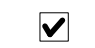 & $\square$ \\
\hline 2531 & 1716 & Hollow stem borehole w/SPT & $12 / 16 / 1981$ & 100.0 & 345517 & 6779906 & $\checkmark$ & $\checkmark$ & $\checkmark$ & $\checkmark$ & $\square$ \\
\hline 2531 & 1718 & Hollow stem borehole w/SPT & $1 / 12 / 1982$ & 81.0 & 345550 & 6779848 & $\checkmark$ & $\checkmark$ & $\checkmark$ & $\checkmark$ & $\square$ \\
\hline 2531 & 1719 & Hollow stem borehole w/SPT & $1 / 15 / 1982$ & 100.0 & 345558 & 6779954 & $\checkmark$ & $\bar{\nabla}$ & $\nabla$ & $\nabla$ & $\square$ \\
\hline 2531 & 1723 & Hollow stem borehole w/SPT & $5 / 15 / 1979$ & 26.0 & 345540 & 6780374 & $\square$ & 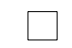 & $\square$ & $\square$ & $\square$ \\
\hline 2531 & 1724 & Hollow stem borehole w/SPT & $5 / 16 / 1979$ & 19.0 & 345552 & 6780238 & $\square$ & $\square$ & $\square$ & $\square$ & $\square$ \\
\hline 2531 & 1725 & Hollow stem borehole w/SPT & $5 / 16 / 1979$ & 19.5 & 345571 & 6780111 & $\square$ & $\square$ & $\square$ & $\square$ & $\square$ \\
\hline 2531 & 1726 & Hollow stem borehole w/SPT & $5 / 16 / 1979$ & 26.0 & 345577 & 6779983 & $\square$ & $\square$ & $\square$ & $\square$ & $\square$ \\
\hline 2531 & 1776 & Hollow stem borehole w/SPT & $12 / 9 / 1993$ & 16.5 & 345869 & 6780251 & $\square$ & $\square$ & $\square$ & $\square$ & $\square$ \\
\hline 2531 & 1777 & Hollow stem borehole w/SPT & $1 / 19 / 1994$ & 21.5 & 345863 & 6780175 & $\square$ & $\square$ & $\square$ & $\square$ & $\square$ \\
\hline 2531 & 1778 & Hollow stem borehole w/SPT & $12 / 13 / 1993$ & 19.0 & 345854 & 6780078 & $\square$ & $\square$ & $\square$ & $\square$ & $\square$ \\
\hline 2531 & 1779 & Hollow stem borehole w/SPT & $12 / 10 / 1993$ & 15.0 & 345850 & 6779979 & $\square$ & $\square$ & $\square$ & $\square$ & $\square$ \\
\hline
\end{tabular}




\begin{tabular}{|c|c|c|c|c|c|c|c|c|c|c|c|}
\hline \multirow[b]{2}{*}{$\begin{array}{c}\text { MOA } \\
\text { grid }\end{array}$} & \multirow[b]{2}{*}{$\begin{array}{c}\text { DGGS } \\
\text { number }\end{array}$} & \multirow[b]{2}{*}{ Hole Type } & \multirow[b]{2}{*}{$\begin{array}{c}\text { Date } \\
\text { Completed }\end{array}$} & \multirow[b]{2}{*}{$\begin{array}{c}\text { Total } \\
\text { depth (ft) }\end{array}$} & \multicolumn{2}{|c|}{ UTM coordinates (m) } & \multicolumn{5}{|c|}{ Data entered } \\
\hline & & & & & East & North & Lithology & SPT & $\begin{array}{c}\text { Sample } \\
\text { test }\end{array}$ & $\begin{array}{l}\text { Water } \\
\text { level }\end{array}$ & Velocity \\
\hline 2531 & 1799 & Hollow stem borehole w/SPT & $7 / 11 / 1995$ & 31.5 & 345228 & 6780077 & $\checkmark$ & $\nabla$ & $\square$ & $\nabla$ & $\square$ \\
\hline 2531 & 1800 & Hollow stem borehole w/SPT & $7 / 11 / 1995$ & 31.5 & 345390 & 6780077 & $\checkmark$ & $\checkmark$ & $\square$ & $\sqrt{V}$ & $\square$ \\
\hline 2531 & 1801 & Hollow stem borehole w/SPT & $7 / 11 / 1995$ & 31.5 & 345305 & 6780038 & $\square$ & $\square$ & $\square$ & $\square$ & $\square$ \\
\hline 2531 & 1802 & Hollow stem borehole w/SPT & $7 / 11 / 1995$ & 31.0 & 345227 & 6779972 & $\boldsymbol{V}$ & $\checkmark$ & $\square$ & $\nabla$ & $\square$ \\
\hline 2531 & 1803 & Hollow stem borehole w/SPT & $7 / 11 / 1995$ & 31.3 & 345302 & 6779973 & $\square$ & $\square$ & $\square$ & $\square$ & $\square$ \\
\hline 2531 & 1804 & Hollow stem borehole w/SPT & $7 / 11 / 1995$ & 31.0 & 345387 & 6779969 & $\square$ & $\square$ & $\square$ & $\square$ & $\square$ \\
\hline 2531 & 4101 & Water well & $12 / 22 / 1982$ & 299.0 & 345815 & 6780190 & $\checkmark$ & $\square$ & $\square$ & $\checkmark$ & $\square$ \\
\hline 2531 & CB002 & Water well & & 491.0 & 345735 & 6780366 & $\boldsymbol{V}$ & $\square$ & $\square$ & $\nabla$ & $\square$ \\
\hline 2532 & 1846 & Hollow stem borehole w/SPT & $12 / 15 / 1977$ & 55.5 & 346239 & 6779724 & $\boldsymbol{V}$ & $\checkmark$ & $\nabla$ & $\checkmark$ & $\square$ \\
\hline 2532 & 1847 & Hollow stem borehole w/SPT & $12 / 14 / 1977$ & 60.0 & 346282 & 6779723 & $\boldsymbol{V}$ & $\nabla$ & $\nabla$ & $\nabla$ & $\square$ \\
\hline 2532 & CB003 & Water well & $8 / 26 / 1976$ & 502.0 & 345952 & 6780098 & $\boldsymbol{V}$ & $\square$ & $\square$ & $\nabla$ & $\square$ \\
\hline 2533 & 1727 & Hollow stem borehole w/SPT & $3 / 31 / 1993$ & 21.5 & 347448 & 6779965 & $\boldsymbol{V}$ & $\checkmark$ & $\square$ & $\nabla$ & $\square$ \\
\hline 2533 & 1728 & Hollow stem borehole w/SPT & $6 / 28 / 1993$ & 21.5 & 347302 & 6779941 & $\square$ & $\square$ & $\square$ & $\square$ & $\square$ \\
\hline 2533 & 1729 & Hollow stem borehole w/SPT & $6 / 25 / 1993$ & 31.5 & 347346 & 6780079 & $\square$ & $\square$ & $\square$ & $\square$ & $\square$ \\
\hline 2533 & 1730 & Hollow stem borehole w/SPT & $6 / 25 / 1993$ & 31.5 & 347423 & 6780094 & $\boldsymbol{V}$ & $\checkmark$ & $\square$ & $\nabla$ & $\square$ \\
\hline 2533 & 1731 & Hollow stem borehole w/SPT & $3 / 24 / 1993$ & 16.5 & 347120 & 6780118 & $\square$ & $\square$ & $\square$ & $\square$ & $\square$ \\
\hline 2534 & 1733 & Hollow stem borehole w/SPT & $6 / 11 / 1982$ & 45.5 & 347795 & 6780405 & $\square$ & $\square$ & $\square$ & $\square$ & $\square$ \\
\hline 2534 & 1734 & Hollow stem borehole w/SPT & $6 / 11 / 1982$ & 46.0 & 347721 & 6780410 & $\square$ & $\square$ & $\square$ & $\square$ & $\square$ \\
\hline 2535 & 3208 & Water well & $6 / 20 / 1985$ & 240.0 & 348631 & 6780192 & $\boldsymbol{V}$ & $\square$ & $\square$ & $\nabla$ & $\square$ \\
\hline 2535 & 4089 & Water well & $7 / 24 / 1985$ & 239.0 & 348530 & 6779918 & $\boldsymbol{V}$ & $\square$ & $\square$ & $\nabla$ & $\square$ \\
\hline 2536 & 1732 & Hollow stem borehole w/SPT & $2 / 20 / 1976$ & 21.5 & 349740 & 6779900 & $\square$ & $\square$ & $\square$ & $\square$ & $\square$ \\
\hline 2536 & 3190 & Water well & $7 / 30 / 1982$ & 159.0 & 349536 & 6780308 & $\nabla$ & $\square$ & $\square$ & $\nabla$ & $\square$ \\
\hline 2536 & 3193 & Water well & $9 / 26 / 1967$ & 210.0 & 349501 & 6780217 & 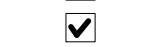 & $\square$ & $\square$ & $\nabla$ & $\square$ \\
\hline 2536 & 3200 & Water well & $5 / 19 / 1981$ & 272.0 & 349423 & 6780127 & $\nabla$ & $\square$ & $\square$ & $\nabla$ & $\square$ \\
\hline 2536 & 3202 & Water well & 9/13/1978 & 292.0 & 349840 & 6780079 & 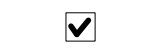 & $\square$ & $\square$ & $\nabla$ & $\square$ \\
\hline 2536 & 3203 & Water well & $11 / 1 / 1985$ & 257.0 & 349348 & 6780131 & $\boldsymbol{V}$ & $\square$ & $\square$ & $\nabla$ & $\square$ \\
\hline 2536 & 3204 & Water well & $5 / 16 / 1984$ & 260.0 & 349795 & 6780080 & $\boldsymbol{V}$ & $\square$ & $\square$ & $\nabla$ & $\square$ \\
\hline 2536 & 3206 & Water well & $9 / 3 / 1984$ & 250.0 & 349751 & 6780082 & $\boldsymbol{V}$ & $\square$ & $\square$ & $\nabla$ & $\square$ \\
\hline 2536 & 3209 & Water well & $10 / 14 / 1972$ & 201.0 & 349601 & 6780089 & $\nabla$ & $\square$ & $\square$ & $\nabla$ & $\square$ \\
\hline 2536 & 3216 & Water well & $6 / 3 / 1981$ & 237.0 & 349227 & 6780105 & 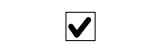 & $\square$ & $\square$ & $\nabla$ & $\square$ \\
\hline 2536 & 3218 & Water well & 7/29/1977 & 268.0 & 349867 & 6780015 & $\nabla$ & $\square$ & $\square$ & $\checkmark$ & $\square$ \\
\hline 2536 & 3219 & Water well & $5 / 23 / 1985$ & 255.0 & 349613 & 6780026 & 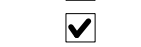 & $\square$ & $\square$ & $\checkmark$ & $\square$ \\
\hline 2536 & 3221 & Water well & $6 / 24 / 1980$ & 207.0 & 349240 & 6780042 & 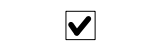 & $\square$ & $\square$ & $\square$ & $\square$ \\
\hline 2536 & 3224 & Water well & 8/17/1981 & 161.0 & 349193 & 6780013 & $\nabla$ & $\square$ & $\square$ & $\square$ & $\square$ \\
\hline 2536 & 3226 & Water well & 9/17/1976 & 238.0 & 349744 & 6779928 & $\boldsymbol{v}$ & $\square$ & $\square$ & $\nabla$ & $\square$ \\
\hline 2536 & 3227 & Water well & $9 / 22 / 1987$ & 295.0 & 349252 & 6779980 & $\boldsymbol{V}$ & $\square$ & $\square$ & $\nabla$ & $\square$ \\
\hline
\end{tabular}




\begin{tabular}{|c|c|c|c|c|c|c|c|c|c|c|c|}
\hline \multirow[b]{2}{*}{$\begin{array}{c}\text { MOA } \\
\text { grid }\end{array}$} & \multirow[b]{2}{*}{$\begin{array}{c}\text { DGGS } \\
\text { number }\end{array}$} & \multirow[b]{2}{*}{ Hole Type } & \multirow[b]{2}{*}{$\begin{array}{c}\text { Date } \\
\text { Completed }\end{array}$} & \multirow[b]{2}{*}{$\begin{array}{c}\text { Total } \\
\text { depth (ft) }\end{array}$} & \multicolumn{2}{|c|}{ UTM coordinates (m) } & \multicolumn{5}{|c|}{ Data entered } \\
\hline & & & & & East & North & Lithology & SPT & $\begin{array}{c}\text { Sample } \\
\text { test }\end{array}$ & $\begin{array}{l}\text { Water } \\
\text { level }\end{array}$ & Velocity \\
\hline 2536 & 3231 & Water well & $6 / 19 / 1979$ & 340.0 & 349652 & 6779870 & $\checkmark$ & $\square$ & $\square$ & $\checkmark$ & $\square$ \\
\hline 2536 & 3234 & Water well & $6 / 28 / 1984$ & 180.0 & 349202 & 6779889 & $\checkmark$ & $\square$ & $\square$ & $\checkmark$ & $\square$ \\
\hline 2536 & 3235 & Water well & 6/18/1976 & 269.0 & 349858 & 6779799 & $\boldsymbol{V}$ & $\square$ & $\square$ & $\checkmark$ & $\square$ \\
\hline 2536 & 3237 & Water well & $6 / 30 / 1984$ & 184.0 & 349230 & 6779825 & $\boldsymbol{V}$ & $\square$ & $\square$ & 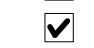 & $\square$ \\
\hline 2536 & 3241 & Water well & $3 / 28 / 1984$ & 220.0 & 349178 & 6779673 & $\checkmark$ & $\square$ & $\square$ & $\checkmark$ & $\square$ \\
\hline 2536 & 3242 & Water well & $10 / 20 / 1981$ & 180.0 & 349102 & 6779645 & $\boldsymbol{V}$ & $\square$ & $\square$ & $\nabla$ & $\square$ \\
\hline 2536 & 3243 & Water well & $6 / 15 / 1983$ & 240.0 & 349400 & 6779602 & $\checkmark$ & $\square$ & $\square$ & $\square$ & $\square$ \\
\hline 2536 & 3244 & Water well & $9 / 3 / 1967$ & 221.0 & 349236 & 6779609 & $\boldsymbol{V}$ & $\square$ & $\square$ & $\nabla$ & $\square$ \\
\hline 2536 & 4084 & Water well & 8/3/1982 & 190.0 & 349137 & 6779737 & $\boldsymbol{V}$ & $\square$ & $\square$ & $\checkmark$ & $\square$ \\
\hline 2536 & 4086 & Water well & $7 / 16 / 1976$ & 236.0 & 349828 & 6779800 & $\boldsymbol{V}$ & $\square$ & $\square$ & $\checkmark$ & $\square$ \\
\hline 2536 & 4095 & Water well & 9/17/1976 & 175.0 & 349174 & 6779921 & $\boldsymbol{V}$ & $\square$ & $\square$ & $\checkmark$ & $\square$ \\
\hline 2537 & 1753 & Hollow stem borehole, no SPT & $1 / 31 / 1977$ & 20.0 & 349899 & 6779590 & $\square$ & $\square$ & $\square$ & $\square$ & $\square$ \\
\hline 2537 & 3185 & Water well & $6 / 28 / 1985$ & 140.0 & 350106 & 6780346 & $\boldsymbol{V}$ & $\square$ & $\square$ & $\square$ & $\square$ \\
\hline 2537 & 3188 & Water well & $10 / 4 / 1981$ & 180.0 & 350148 & 6780282 & 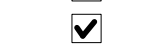 & $\square$ & $\square$ & $V$ & $\square$ \\
\hline 2537 & 3195 & Water well & $8 / 16 / 1983$ & 275.0 & 350052 & 6780131 & $\boldsymbol{V}$ & $\square$ & $\square$ & $\nabla$ & $\square$ \\
\hline 2537 & 3201 & Water well & 9/24/1977 & 300.0 & 349900 & 6780076 & $\checkmark$ & $\square$ & $\square$ & $V$ & $\square$ \\
\hline 2537 & 3210 & Water well & $6 / 10 / 1984$ & 285.0 & 350078 & 6780037 & $\boldsymbol{v}$ & $\square$ & $\square$ & $V$ & $\square$ \\
\hline 2537 & 3211 & Water well & 8/1/1984 & 112.0 & 350556 & 6779986 & 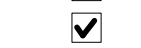 & $\square$ & $\square$ & $\square$ & $\square$ \\
\hline 2537 & 3213 & Water well & $10 / 17 / 1974$ & 275.0 & 350018 & 6780040 & $\boldsymbol{V}$ & $\square$ & $\square$ & $\boldsymbol{V}$ & $\square$ \\
\hline 2537 & 3217 & Water well & 6/19/1974 & 275.0 & 350091 & 6780006 & $\boldsymbol{V}$ & $\square$ & $\square$ & $\nabla$ & $\square$ \\
\hline 2537 & 3220 & Water well & 4/1/1983 & 101.0 & 350478 & 6779928 & $\boldsymbol{V}$ & $\square$ & $\square$ & $\checkmark$ & $\square$ \\
\hline 2537 & 3238 & Water well & $8 / 27 / 1981$ & 124.0 & 350617 & 6779674 & 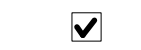 & $\square$ & $\square$ & $\square$ & $\square$ \\
\hline 2537 & 3240 & Water well & 4/21/1981 & 128.0 & 350612 & 6779550 & 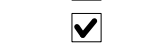 & $\square$ & $\square$ & $\bar{v}$ & $\square$ \\
\hline 2537 & 4083 & Water well & $6 / 28 / 1982$ & 134.0 & 350632 & 6779673 & $\nabla$ & $\square$ & $\square$ & $\nabla$ & $\square$ \\
\hline 2537 & 4102 & Water well & $3 / 27 / 1975$ & 275.0 & 350081 & 6780099 & 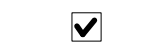 & $\square$ & $\square$ & $V$ & $\square$ \\
\hline 2537 & 4103 & Water well & 8/27/1982 & 111.0 & 350486 & 6780113 & $\boldsymbol{V}$ & $\square$ & $\square$ & $\checkmark$ & $\square$ \\
\hline 2537 & 4109 & Water well & $11 / 20 / 1978$ & 285.0 & 350144 & 6780189 & $\boldsymbol{V}$ & $\square$ & $\square$ & $\nabla$ & $\square$ \\
\hline 2538 & 1754 & Hollow stem borehole, no SPT & & 20.0 & 351197 & 6779720 & $\square$ & $\square$ & $\square$ & $\square$ & $\square$ \\
\hline 2538 & 1755 & Hollow stem borehole, no SPT & & 20.0 & 351173 & 6779842 & $\square$ & $\square$ & $\square$ & $\square$ & $\square$ \\
\hline 2539 & 1756 & Hollow stem borehole w/SPT & $10 / 16 / 1972$ & 21.5 & 352132 & 6779786 & $\square$ & $\square$ & $\square$ & $\square$ & $\square$ \\
\hline 2539 & 1757 & Hollow stem borehole w/SPT & $10 / 17 / 1972$ & 21.5 & 352137 & 6779982 & $\square$ & $\square$ & $\square$ & $\square$ & $\square$ \\
\hline 2539 & 3177 & Water well & 8/24/1977 & 233.0 & 352285 & 6780161 & 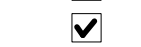 & $\square$ & $\square$ & $V$ & $\square$ \\
\hline 2539 & 3189 & Water well & 10/7/1981 & 152.0 & 351997 & 6780080 & 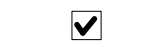 & $\square$ & $\square$ & 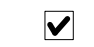 & $\square$ \\
\hline 2539 & 3225 & Water well & 4/12/1972 & 179.0 & 351505 & 6779760 & $\nabla$ & $\square$ & $\square$ & 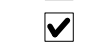 & $\square$ \\
\hline 2539 & 3230 & Water well & 7/30/1986 & 237.0 & 352262 & 6779604 & $\boldsymbol{v}$ & $\square$ & $\square$ & 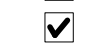 & $\square$ \\
\hline 2539 & 4078 & Water well & $9 / 1 / 1969$ & 240.0 & 352232 & 6779606 & $\checkmark$ & $\square$ & $\square$ & $v$ & $\square$ \\
\hline
\end{tabular}




\begin{tabular}{|c|c|c|c|c|c|c|c|c|c|c|c|}
\hline \multirow[b]{2}{*}{$\begin{array}{c}\text { MOA } \\
\text { grid }\end{array}$} & \multirow[b]{2}{*}{$\begin{array}{c}\text { DGGS } \\
\text { number }\end{array}$} & \multirow[b]{2}{*}{ Hole Type } & \multirow[b]{2}{*}{$\begin{array}{c}\text { Date } \\
\text { Completed }\end{array}$} & \multirow[b]{2}{*}{$\begin{array}{c}\text { Total } \\
\text { depth (ft) }\end{array}$} & \multicolumn{2}{|c|}{ UTM coordinates (m) } & \multicolumn{5}{|c|}{ Data entered } \\
\hline & & & & & East & North & Lithology & SPT & $\begin{array}{c}\text { Sample } \\
\text { test }\end{array}$ & $\begin{array}{l}\text { Water } \\
\text { level }\end{array}$ & Velocity \\
\hline 2539 & 4080 & Water well & 7/17/1979 & 281.0 & 352038 & 6779614 & $\checkmark$ & $\square$ & $\square$ & $\square$ & $\square$ \\
\hline 2539 & 4087 & Water well & $6 / 22 / 1977$ & 256.0 & 352252 & 6779728 & $\checkmark$ & $\square$ & $\square$ & $\sqrt{V}$ & $\square$ \\
\hline 2539 & 4094 & Water well & $8 / 1 / 1969$ & 225.0 & 352240 & 6779791 & $\nabla$ & $\square$ & $\square$ & $\nabla$ & $\square$ \\
\hline 2539 & 4098 & Water well & $7 / 1 / 1969$ & 200.0 & 352261 & 6779945 & $\nabla$ & $\square$ & $\square$ & $\nabla$ & $\square$ \\
\hline 2539 & 4104 & Water well & $10 / 14 / 1964$ & 150.0 & 352102 & 6780076 & $\checkmark$ & $\square$ & $\square$ & $\nabla$ & $\square$ \\
\hline 2539 & 4108 & Water well & $1 / 1 / 1978$ & 239.0 & 352058 & 6780108 & $\nabla$ & $\square$ & $\square$ & $\nabla$ & $\square$ \\
\hline 2539 & 4114 & Water well & 2/1/1978 & 217.0 & 352062 & 6780201 & $V$ & $\square$ & $\square$ & $\checkmark$ & $\square$ \\
\hline 2539 & 4119 & Water well & 4/1/1978 & 168.0 & 352153 & 6780228 & $\boldsymbol{V}$ & $\square$ & $\square$ & $\nabla$ & $\square$ \\
\hline 2540 & 3187 & Water well & 8/18/1983 & 187.0 & 352611 & 6780054 & $\boldsymbol{V}$ & $\square$ & $\square$ & $\sqrt{ }$ & $\square$ \\
\hline 2540 & 3191 & Water well & $10 / 14 / 1980$ & 208.0 & 352560 & 6779932 & $\checkmark$ & $\square$ & $\square$ & $\sqrt{v}$ & $\square$ \\
\hline 2540 & 3194 & Water well & $6 / 23 / 1983$ & 156.0 & 352543 & 6779871 & $\bar{v}$ & $\square$ & $\square$ & $\nabla$ & $\square$ \\
\hline 2540 & 3196 & Water well & 10/1/1981 & 209.1 & 352379 & 6779878 & $\nabla$ & $\square$ & $\square$ & $\nabla$ & $\square$ \\
\hline 2540 & 3197 & Water well & 8/6/1980 & 118.0 & 352646 & 6779836 & $\nabla$ & $\square$ & $\square$ & $\nabla$ & $\square$ \\
\hline 2540 & 3199 & Water well & 7/15/1983 & 192.0 & 352376 & 6779816 & 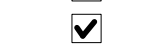 & $\square$ & $\square$ & $\checkmark$ & $\square$ \\
\hline 2540 & 3215 & Water well & $10 / 4 / 1985$ & 158.0 & 352822 & 6779736 & 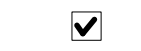 & $\square$ & $\square$ & $\nabla$ & $\square$ \\
\hline 2540 & 3222 & Water well & 7/19/1981 & 260.0 & 352997 & 6779636 & $\checkmark$ & $\square$ & $\square$ & $\checkmark$ & $\square$ \\
\hline 2540 & 3236 & Water well & 9/25/1979 & 450.0 & 352930 & 6779452 & $\boldsymbol{v}$ & $\square$ & $\square$ & $\nabla$ & $\square$ \\
\hline 2540 & 4077 & Water well & $1 / 1 / 1965$ & 150.0 & 353052 & 6779509 & $\square$ & $\square$ & $\square$ & $\nabla$ & $\square$ \\
\hline 2540 & 4079 & Water well & $3 / 1 / 1965$ & 198.0 & 352335 & 6779570 & $\checkmark$ & $\square$ & $\square$ & $\checkmark$ & $\square$ \\
\hline 2540 & 4082 & Water well & $11 / 10 / 1978$ & 129.0 & 352442 & 6779597 & $\bar{V}$ & $\square$ & $\square$ & $\nabla$ & $\square$ \\
\hline 2540 & 4097 & Water well & $5 / 17 / 1980$ & 400.0 & 352451 & 6779813 & $\boldsymbol{V}$ & $\square$ & $\square$ & $\nabla$ & $\square$ \\
\hline 2540 & 4100 & Water well & $11 / 11 / 1980$ & 186.0 & 352470 & 6779905 & 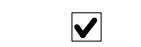 & $\square$ & $\square$ & $\sqrt{V}$ & $\square$ \\
\hline 2540 & 4107 & Water well & 9/23/1981 & 145.0 & 352672 & 6780083 & $\nabla$ & $\square$ & $\square$ & $\nabla$ & $\square$ \\
\hline 2540 & 4111 & Water well & 8/14/1982 & 139.0 & 352571 & 6780180 & $\nabla$ & $\square$ & $\square$ & $\nabla$ & $\square$ \\
\hline 2540 & 4112 & Water well & $10 / 30 / 1981$ & 233.0 & 352466 & 6780184 & 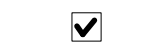 & $\square$ & $\square$ & $\nabla$ & $\square$ \\
\hline 2540 & 4118 & Water well & $9 / 25 / 1975$ & 300.0 & 352662 & 6780207 & $\boldsymbol{V}$ & $\square$ & $\square$ & $\nabla$ & $\square$ \\
\hline 2541 & 1758 & Hollow stem borehole, no SPT & $8 / 25 / 1977$ & 14.5 & 353166 & 6779462 & $\boldsymbol{V}$ & $\square$ & $\square$ & $\nabla$ & $\square$ \\
\hline 2541 & 3161 & Water well & $6 / 3 / 1984$ & 175.0 & 353848 & 6780120 & $\checkmark$ & $\square$ & $\square$ & $\nabla$ & $\square$ \\
\hline 2541 & 3163 & Water well & $7 / 27 / 1985$ & 202.0 & 353642 & 6780137 & $\boldsymbol{V}$ & $\square$ & $\square$ & $\nabla$ & $\square$ \\
\hline 2541 & 3192 & Water well & 8/8/1979 & 280.0 & 353216 & 6779843 & $\nabla$ & $\square$ & $\square$ & $\nabla$ & $\square$ \\
\hline 2541 & 3198 & Water well & $8 / 1 / 1980$ & 340.0 & 353407 & 6779742 & $\nabla$ & $\square$ & $\square$ & $\nabla$ & $\square$ \\
\hline 2541 & 3205 & Water well & $11 / 10 / 1978$ & 532.0 & 353151 & 6779722 & $\nabla$ & $\square$ & $\square$ & $\checkmark$ & $\square$ \\
\hline 2541 & 3214 & Water well & $5 / 19 / 1976$ & 200.0 & 353897 & 6779629 & $\bar{v}$ & $\square$ & $\square$ & $\bar{v}$ & $\square$ \\
\hline 2541 & 3223 & Water well & $10 / 19 / 1983$ & 306.0 & 353788 & 6779541 & $\nabla$ & $\square$ & $\square$ & $\nabla$ & $\square$ \\
\hline 2541 & 3228 & Water well & 9/1/1979 & 325.0 & 353173 & 6779535 & $\boldsymbol{v}$ & $\square$ & $\square$ & $\square$ & $\square$ \\
\hline 2541 & 3229 & Water well & $2 / 3 / 1976$ & 405.0 & 353591 & 6779487 & $\checkmark$ & $\square$ & $\square$ & $\square$ & $\square$ \\
\hline
\end{tabular}




\begin{tabular}{|c|c|c|c|c|c|c|c|c|c|c|c|}
\hline \multirow[b]{2}{*}{$\begin{array}{l}\text { MOA } \\
\text { grid }\end{array}$} & \multirow[b]{2}{*}{$\begin{array}{c}\text { DGGS } \\
\text { number }\end{array}$} & \multirow[b]{2}{*}{ Hole Type } & \multirow[b]{2}{*}{$\begin{array}{c}\text { Date } \\
\text { Completed }\end{array}$} & \multirow[b]{2}{*}{$\begin{array}{c}\text { Total } \\
\text { depth (ft) }\end{array}$} & \multicolumn{2}{|c|}{ UTM coordinates (m) } & \multicolumn{5}{|c|}{ Data entered } \\
\hline & & & & & East & North & Lithology & SPT & $\begin{array}{c}\text { Sample } \\
\text { test }\end{array}$ & $\begin{array}{l}\text { Water } \\
\text { level }\end{array}$ & Velocity \\
\hline 2541 & 3232 & Water well & $8 / 20 / 1985$ & 431.0 & 353470 & 6779461 & $\checkmark$ & $\checkmark$ & $\square$ & $\checkmark$ & $\square$ \\
\hline 2541 & 4076 & Water well & $7 / 17 / 1975$ & 150.0 & 353545 & 6779458 & $\checkmark$ & $\square$ & $\square$ & $\nabla$ & $\square$ \\
\hline 2541 & 4081 & Water well & $9 / 18 / 1978$ & 138.0 & 353728 & 6779543 & $\checkmark$ & & $\square$ & $\nabla$ & \\
\hline 2541 & 4085 & Water well & 7/7/1980 & 390.0 & 353552 & 6779643 & $\checkmark$ & $\square$ & $\square$ & $\nabla$ & $\square$ \\
\hline 2541 & 4088 & Water well & 8/9/1978 & 198.0 & 353675 & 6779700 & $\square$ & $\square$ & $\square$ & $\checkmark$ & $\square$ \\
\hline 2541 & 4091 & Water well & $5 / 8 / 1972$ & 125.0 & 353840 & 6779724 & $\checkmark$ & $\square$ & $\square$ & $\checkmark$ & $\square$ \\
\hline 2541 & 4092 & Water well & $4 / 11 / 1979$ & 125.0 & 353661 & 6779732 & $\square$ & $\square$ & $\square$ & $\checkmark$ & $\square$ \\
\hline 2541 & 4093 & Water well & $11 / 30 / 1978$ & 140.0 & 353317 & 6779746 & $\square$ & $\square$ & $\square$ & $\checkmark$ & $\square$ \\
\hline 2541 & 4096 & Water well & $4 / 19 / 1972$ & 158.0 & 353153 & 6779784 & $\checkmark$ & $\square$ & $\square$ & $\checkmark$ & $\square$ \\
\hline 2541 & 4099 & Water well & 8/1/1979 & 280.0 & 353156 & 6779846 & $\checkmark$ & & $\square$ & $\checkmark$ & $\square$ \\
\hline 2541 & 4105 & Water well & 4/6/1979 & 156.0 & 353480 & 6780049 & $\square$ & $\square$ & $\square$ & 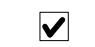 & $\square$ \\
\hline 2541 & 4106 & Water well & $6 / 26 / 1974$ & 178.0 & 353449 & 6780050 & $\checkmark$ & & $\square$ & $\checkmark$ & $\square$ \\
\hline 2541 & 4116 & Water well & $3 / 23 / 1976$ & 605.0 & 353350 & 6780178 & $\checkmark$ & $\square$ & $\square$ & $\checkmark$ & $\square$ \\
\hline 2542 & 3233 & Water well & $10 / 5 / 1971$ & 400.0 & 353861 & 6779570 & $\checkmark$ & $\square$ & $\square$ & $\nabla$ & $\square$ \\
\hline 2626 & 2223 & Hollow stem borehole w/SPT & & 51.5 & 341223 & 6779866 & $\checkmark$ & $\checkmark$ & $\square$ & $\nabla$ & $\square$ \\
\hline 2626 & 2254 & Hollow stem borehole w/SPT & $10 / 24 / 1975$ & 32.0 & 341439 & 6779776 & $\checkmark$ & $\checkmark$ & $\square$ & $\checkmark$ & $\square$ \\
\hline 2626 & 4066 & Water well & $3 / 4 / 1975$ & 550.0 & 341795 & 6779780 & $\checkmark$ & & $\square$ & $\checkmark$ & $\square$ \\
\hline 2626 & ВТ001 & Hollow stem borehole w/SPT & & 41.5 & 341840 & 6779808 & $\square$ & $\square$ & $\square$ & $\square$ & $\square$ \\
\hline 2626 & ВТ002 & Hollow stem borehole w/SPT & & 31.5 & 341914 & 6779790 & $\square$ & $\square$ & $\square$ & $\square$ & $\square$ \\
\hline 2626 & ВТ003 & Hollow stem borehole w/SPT & & 41.5 & 341793 & 6779678 & $\square$ & $\square$ & $\square$ & $\square$ & $\square$ \\
\hline 2626 & ВТ004 & Hollow stem borehole w/SPT & & 40.0 & 341686 & 6779571 & $\square$ & $\square$ & $\square$ & $\square$ & $\square$ \\
\hline 2626 & ВT005 & Hollow stem borehole w/SPT & & 41.5 & 341523 & 6779608 & $\checkmark$ & $\checkmark$ & $\square$ & $\square$ & $\square$ \\
\hline 2626 & ВT006 & Hollow stem borehole w/SPT & & 41.5 & 341644 & 6779458 & $\square$ & & $\square$ & $\square$ & $\square$ \\
\hline 2626 & ВТ007 & Hollow stem borehole w/SPT & & 41.5 & 341727 & 6779453 & $\square$ & $\square$ & $\square$ & $\square$ & $\square$ \\
\hline 2626 & ВТ008 & Hollow stem borehole w/SPT & & 30.0 & 341719 & 6779422 & $\square$ & $\square$ & $\square$ & $\square$ & $\square$ \\
\hline 2626 & ВТ009 & Hollow stem borehole w/SPT & & 35.0 & 341850 & 6779486 & $\square$ & $\square$ & $\square$ & $\square$ & $\square$ \\
\hline 2626 & ВT011 & Hollow stem borehole w/SPT & & 41.5 & 341707 & 6779360 & $\checkmark$ & $\checkmark$ & $\square$ & $\square$ & $\square$ \\
\hline 2626 & ВТ012 & Hollow stem borehole w/SPT & & 40.0 & 341706 & 6779256 & $\square$ & & $\square$ & $\square$ & $\square$ \\
\hline 2626 & ВT016 & Hollow stem borehole w/SPT & & 31.5 & 341789 & 6779897 & $\square$ & $\square$ & $\square$ & $\square$ & $\square$ \\
\hline 2626 & ВТ017 & Hollow stem borehole w/SPT & & 31.5 & 341744 & 6779850 & $\square$ & $\square$ & $\square$ & $\square$ & $\square$ \\
\hline 2626 & ВT018 & Hollow stem borehole w/SPT & & 31.5 & 341827 & 6779854 & $\square$ & $\square$ & $\square$ & $\square$ & $\square$ \\
\hline 2626 & ВТ019 & Hollow stem borehole w/SPT & & 30.0 & 341739 & 6779807 & $\square$ & $\square$ & $\square$ & $\square$ & $\square$ \\
\hline 2626 & ВТ020 & Hollow stem borehole w/SPT & & 31.5 & 341785 & 6779759 & $\checkmark$ & $\bar{\nabla}$ & $\square$ & $\bar{\nabla}$ & $\square$ \\
\hline 2626 & ВТ021 & Hollow stem borehole w/SPT & & 21.5 & 341639 & 6779728 & $\square$ & $\square$ & $\square$ & $\square$ & $\square$ \\
\hline 2626 & ВT022 & Hollow stem borehole w/SPT & & 21.5 & 341630 & 6779625 & $\square$ & $\square$ & $\square$ & $\square$ & $\square$ \\
\hline 2627 & 1737 & Hollow stem borehole w/SPT & $1 / 4 / 1985$ & 31.5 & 342296 & 6779680 & $\checkmark$ & $\checkmark$ & $\square$ & $\checkmark$ & $\square$ \\
\hline
\end{tabular}




\begin{tabular}{|c|c|c|c|c|c|c|c|c|c|c|c|}
\hline \multirow[b]{2}{*}{$\begin{array}{c}\text { MOA } \\
\text { grid }\end{array}$} & \multirow[b]{2}{*}{$\begin{array}{c}\text { DGGS } \\
\text { number }\end{array}$} & \multirow[b]{2}{*}{ Hole Type } & \multirow[b]{2}{*}{$\begin{array}{c}\text { Date } \\
\text { Completed }\end{array}$} & \multirow[b]{2}{*}{$\begin{array}{c}\text { Total } \\
\text { depth (ft) }\end{array}$} & \multicolumn{2}{|c|}{ UTM coordinates (m) } & \multicolumn{5}{|c|}{ Data entered } \\
\hline & & & & & East & North & Lithology & SPT & $\begin{array}{c}\text { Sample } \\
\text { test }\end{array}$ & $\begin{array}{c}\text { Water } \\
\text { level }\end{array}$ & Velocity \\
\hline 2627 & 1738 & Hollow stem borehole w/SPT & $1 / 17 / 1985$ & 53.0 & 341999 & 6779461 & $\checkmark$ & $\nabla$ & $\nabla$ & $\nabla$ & $\square$ \\
\hline 2627 & 1739 & Hollow stem borehole w/SPT & $1 / 8 / 1985$ & 52.0 & 341991 & 6779453 & $\checkmark$ & $\checkmark$ & $\sqrt{v}$ & $\sqrt{V}$ & $\square$ \\
\hline 2627 & 1740 & Hollow stem borehole w/SPT & $1 / 9 / 1985$ & 31.5 & 341997 & 6779314 & $\boldsymbol{V}$ & $\checkmark$ & $\square$ & $\nabla$ & $\square$ \\
\hline 2627 & 2130 & Hollow stem borehole w/SPT & $8 / 30 / 1998$ & 52.0 & 341944 & 6779195 & $\boldsymbol{V}$ & $\checkmark$ & $\square$ & $\square$ & $\square$ \\
\hline 2627 & 2131 & Hollow stem borehole w/SPT & 9/1/1998 & 41.5 & 341924 & 6779247 & $\checkmark$ & $\checkmark$ & $\square$ & $\square$ & $\square$ \\
\hline 2627 & BS004 & Hollow stem borehole w/SPT & & 30.0 & 342294 & 6779862 & $\square$ & $\square$ & $\square$ & $\square$ & $\square$ \\
\hline 2627 & BSO05 & Hollow stem borehole w/SPT & & 31.5 & 342239 & 6779808 & $\square$ & $\square$ & $\square$ & $\square$ & $\square$ \\
\hline 2627 & BS006 & Hollow stem borehole w/SPT & & 41.5 & 341993 & 6779717 & $\boldsymbol{V}$ & $\nabla$ & $\square$ & $\square$ & $\square$ \\
\hline 2627 & BS007 & Hollow stem borehole w/SPT & & 30.0 & 342145 & 6779564 & $\square$ & $\square$ & $\square$ & $\square$ & $\square$ \\
\hline 2627 & BS008 & Hollow stem borehole w/SPT & & 30.0 & 342148 & 6779501 & $\square$ & $\square$ & $\square$ & $\square$ & $\square$ \\
\hline 2627 & BS009 & Hollow stem borehole w/SPT & & 41.5 & 342033 & 6779323 & $\square$ & $\square$ & $\square$ & $\square$ & $\square$ \\
\hline 2627 & BS010 & Hollow stem borehole w/SPT & & 41.5 & 342187 & 6779366 & $\square$ & $\square$ & $\square$ & $\square$ & $\square$ \\
\hline 2627 & BS011 & Hollow stem borehole w/SPT & & 41.5 & 342287 & 6779336 & $\square$ & $\square$ & $\square$ & $\square$ & $\square$ \\
\hline 2627 & BS012 & Hollow stem borehole w/SPT & & 41.5 & 342285 & 6779130 & $\checkmark$ & $\checkmark$ & $\square$ & $\checkmark$ & $\square$ \\
\hline 2627 & BS013 & Hollow stem borehole w/SPT & & 41.5 & 342450 & 6779629 & $\square$ & $\square$ & $\square$ & $\square$ & $\square$ \\
\hline 2627 & BS014 & Hollow stem borehole w/SPT & & 41.5 & 342478 & 6779501 & $\checkmark$ & $\checkmark$ & $\square$ & $\checkmark$ & $\square$ \\
\hline 2627 & BS015 & Hollow stem borehole w/SPT & & 30.0 & 342617 & 6779677 & $\square$ & $\square$ & $\square$ & $\square$ & $\square$ \\
\hline 2627 & BS025 & Hollow stem borehole w/SPT & & 26.5 & 342094 & 6779680 & $\square$ & $\square$ & $\square$ & $\square$ & $\square$ \\
\hline 2627 & BS026 & Hollow stem borehole w/SPT & & 26.5 & 342207 & 6779686 & $\square$ & $\square$ & $\square$ & $\square$ & $\square$ \\
\hline 2627 & BS027 & Hollow stem borehole w/SPT & & 16.5 & 342356 & 6779732 & $\square$ & $\square$ & $\square$ & $\square$ & $\square$ \\
\hline 2627 & BSO28 & Hollow stem borehole w/SPT & & 26.5 & 342032 & 6779486 & $\square$ & $\square$ & $\square$ & $\square$ & $\square$ \\
\hline 2627 & BS029 & Hollow stem borehole w/SPT & & 25.0 & 342207 & 6779502 & $\square$ & $\square$ & $\square$ & $\square$ & $\square$ \\
\hline 2627 & $\mathrm{BS} 030$ & Hollow stem borehole w/SPT & & 21.0 & 342296 & 6779498 & $\square$ & $\square$ & $\square$ & $\square$ & $\square$ \\
\hline 2627 & BS031 & Hollow stem borehole w/SPT & & 21.0 & 342363 & 6779494 & $\square$ & $\square$ & $\square$ & $\square$ & $\square$ \\
\hline 2627 & ВT010 & Hollow stem borehole w/SPT & & 41.0 & 341924 & 6779486 & $\square$ & $\square$ & $\square$ & $\square$ & $\square$ \\
\hline 2627 & ВT025 & Hollow stem borehole w/SPT & & 25.0 & 341909 & 6779242 & $\square$ & $\square$ & $\square$ & $\square$ & $\square$ \\
\hline 2628 & 1743 & Hollow stem borehole w/SPT & $10 / 12 / 1984$ & 31.5 & 342818 & 6779812 & $\checkmark$ & $\nabla$ & $\checkmark$ & $\checkmark$ & $\square$ \\
\hline 2628 & 1744 & Hollow stem borehole w/SPT & $10 / 10 / 1984$ & 30.0 & 342942 & 6779789 & $\boldsymbol{V}$ & $\checkmark$ & $\nabla$ & $\nabla$ & $\square$ \\
\hline 2628 & 1745 & Hollow stem borehole w/SPT & $10 / 12 / 1984$ & 31.5 & 342772 & 6779749 & $\nabla$ & $\checkmark$ & $\checkmark$ & $\checkmark$ & $\square$ \\
\hline 2628 & 1746 & Hollow stem borehole w/SPT & $10 / 15 / 1984$ & 31.5 & 342977 & 6779703 & 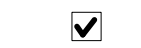 & $\nabla$ & $\nabla$ & $\nabla$ & $\square$ \\
\hline 2628 & 1747 & Hollow stem borehole w/SPT & $10 / 11 / 1984$ & 31.2 & 343113 & 6779691 & $\checkmark$ & $\checkmark$ & $\nabla$ & $\nabla$ & $\square$ \\
\hline 2628 & 2140 & Hollow stem borehole w/SPT & $3 / 29 / 1995$ & 31.5 & 343337 & 6779047 & 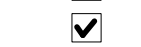 & $\checkmark$ & $\square$ & $\square$ & $\square$ \\
\hline 2629 & 2036 & Hollow stem borehole w/SPT & 2/8/1999 & 51.5 & 343996 & 6779471 & 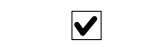 & $\nabla$ & $\square$ & $\bar{\nabla}$ & $\square$ \\
\hline 2629 & 2037 & Hollow stem borehole w/SPT & 2/4/1999 & 51.0 & 344118 & 6779442 & $\boldsymbol{V}$ & $\nabla$ & $\square$ & $\nabla$ & $\square$ \\
\hline 2629 & 2038 & Hollow stem borehole w/SPT & 2/9/1999 & 80.0 & 344047 & 6779419 & $\boldsymbol{V}$ & $\checkmark$ & $\nabla$ & $\nabla$ & $\square$ \\
\hline 2629 & 2039 & Hollow stem borehole w/SPT & 2/3/1999 & 51.5 & 344028 & 6779349 & $\boldsymbol{V}$ & $\boldsymbol{V}$ & $\square$ & $\nabla$ & $\square$ \\
\hline
\end{tabular}




\begin{tabular}{|c|c|c|c|c|c|c|c|c|c|c|c|}
\hline \multirow[b]{2}{*}{$\begin{array}{l}\text { MOA } \\
\text { grid }\end{array}$} & \multirow[b]{2}{*}{$\begin{array}{l}\text { DGGS } \\
\text { number }\end{array}$} & \multirow[b]{2}{*}{ Hole Type } & \multirow[b]{2}{*}{$\begin{array}{c}\text { Date } \\
\text { Completed }\end{array}$} & \multirow[b]{2}{*}{$\begin{array}{c}\text { Total } \\
\text { depth }(\mathrm{ft})\end{array}$} & \multicolumn{2}{|c|}{ UTM coordinates (m) } & \multicolumn{5}{|c|}{ Data entered } \\
\hline & & & & & East & North & Lithology & SPT & $\begin{array}{c}\text { Sample } \\
\text { test }\end{array}$ & $\begin{array}{l}\text { Water } \\
\text { level }\end{array}$ & Velocity \\
\hline 2629 & 2139 & Hollow stem borehole w/SPT & $3 / 27 / 1995$ & 26.5 & 343756 & 6779021 & $\checkmark$ & $\nabla$ & $\square$ & $\square$ & $\square$ \\
\hline 2629 & 2141 & Hollow stem borehole w/SPT & $11 / 7 / 1994$ & 41.5 & 344137 & 6779024 & $\checkmark$ & $\checkmark$ & $\checkmark$ & $\checkmark$ & $\square$ \\
\hline 2629 & 2168 & Hollow stem borehole w/SPT & $11 / 4 / 1994$ & 41.5 & 344185 & 6778997 & $\checkmark$ & $\checkmark$ & $\nabla$ & $\bar{\nabla}$ & \\
\hline 2629 & 2252 & Hollow stem borehole w/SPT & $10 / 24 / 1980$ & 51.5 & 343932 & 6779378 & $\nabla$ & $\nabla$ & $\checkmark$ & $\checkmark$ & $\square$ \\
\hline 2629 & 2262 & Hollow stem borehole w/SPT & $10 / 24 / 1980$ & 46.5 & 344029 & 6779252 & $\checkmark$ & $\checkmark$ & $\checkmark$ & $\nabla$ & $\square$ \\
\hline 2630 & 2189 & Hollow stem borehole w/SPT & $1 / 22 / 1998$ & 46.5 & 344309 & 6779019 & $\checkmark$ & $\checkmark$ & $\checkmark$ & $\checkmark$ & $\square$ \\
\hline 2631 & 1714 & Hollow stem borehole w/SPT & $12 / 3 / 1981$ & 100.0 & 345503 & 6779703 & $\checkmark$ & $\checkmark$ & $\checkmark$ & $\checkmark$ & $\square$ \\
\hline 2631 & 1717 & Hollow stem borehole w/SPT & $12 / 18 / 1981$ & 100.0 & 345578 & 6779702 & $\checkmark$ & $\nabla$ & $\nabla$ & $\bar{\nabla}$ & $\square$ \\
\hline 2631 & 1748 & Hollow stem borehole w/SPT & $11 / 13 / 1984$ & 41.2 & 345379 & 6779350 & $\checkmark$ & $\checkmark$ & $\checkmark$ & $\checkmark$ & $\square$ \\
\hline 2631 & 2266 & Hollow stem borehole w/SPT & $6 / 28 / 1990$ & 51.5 & 345637 & 6778967 & $\checkmark$ & $\checkmark$ & $\square$ & $\sqrt{\square}$ & $\square$ \\
\hline 2631 & 2294 & Water well & $12 / 9 / 1985$ & 718.0 & 345293 & 6779194 & $\checkmark$ & $\square$ & $\square$ & $\nabla$ & $\square$ \\
\hline 2631 & 3287 & Water well & $12 / 9 / 1985$ & 718.0 & 345384 & 6779249 & $\checkmark$ & $\square$ & $\square$ & $\bar{\nabla}$ & \\
\hline 2632 & 1628 & Hollow stem borehole w/SPT & 9/3/1996 & 21.5 & 346322 & 6779497 & $\square$ & $\square$ & $\square$ & $\square$ & $\square$ \\
\hline 2632 & 1742 & Hollow stem borehole, no SPT & $7 / 21 / 1977$ & 40.0 & 346283 & 6779692 & $\square$ & $\square$ & $\square$ & $\square$ & $\square$ \\
\hline 2632 & 1751 & Hollow stem borehole, no SPT & $11 / 30 / 1976$ & 31.5 & 346349 & 6779657 & $\square$ & $\square$ & $\square$ & $\square$ & $\square$ \\
\hline 2632 & 1752 & Hollow stem borehole, no SPT & $12 / 2 / 1976$ & 31.5 & 346418 & 6779649 & $\square$ & $\square$ & $\square$ & $\square$ & $\square$ \\
\hline 2632 & 1848 & Hollow stem borehole w/SPT & $12 / 20 / 1977$ & 44.0 & 346240 & 6779677 & $\square$ & $\square$ & $\square$ & $\square$ & $\square$ \\
\hline 2632 & 1849 & Hollow stem borehole w/SPT & $12 / 13 / 1977$ & 56.5 & 346280 & 6779676 & $\checkmark$ & $\checkmark$ & $\nabla$ & $\vec{\nabla}$ & $\square$ \\
\hline 2632 & 3301 & Water well & $4 / 16 / 1982$ & 305.0 & 346166 & 6778967 & $\checkmark$ & $\square$ & $\square$ & $\nabla$ & $\square$ \\
\hline 2632 & 3302 & Water well & $12 / 12 / 1980$ & 350.0 & 346136 & 6778968 & $\checkmark$ & $\square$ & $\square$ & $\square$ & $\square$ \\
\hline 2632 & 3986 & Water well & $11 / 26 / 1978$ & 122.0 & 345976 & 6779068 & $\checkmark$ & & $\square$ & $\bar{\nabla}$ & $\square$ \\
\hline 2632 & 3990 & Water well & $9 / 27 / 1965$ & 122.0 & 345885 & 6779041 & $\checkmark$ & $\square$ & $\square$ & $\sqrt{\nabla}$ & $\square$ \\
\hline 2633 & 3254 & Water well & $11 / 1 / 1969$ & 127.0 & 347241 & 6779570 & $\checkmark$ & $\square$ & $\square$ & $\nabla$ & $\square$ \\
\hline 2633 & 3274 & Water well & 8/18/1972 & 130.0 & 347080 & 6779299 & $\nabla$ & $\square$ & $\square$ & $\checkmark$ & $\square$ \\
\hline 2633 & 3285 & Water well & $10 / 19 / 1980$ & 203.0 & 346834 & 6779155 & $\checkmark$ & $\square$ & $\square$ & $\square$ & $\square$ \\
\hline 2633 & 3286 & Water well & 9/6/1974 & 236.0 & 346982 & 6779117 & $\checkmark$ & $\square$ & $\square$ & $\bar{V}$ & $\square$ \\
\hline 2633 & 3290 & Water well & $8 / 3 / 1973$ & 198.0 & 346934 & 6779058 & $\checkmark$ & $\square$ & $\square$ & $\checkmark$ & $\square$ \\
\hline 2633 & 4005 & Water well & 1/1/1979 & 178.0 & 347208 & 6779138 & $\checkmark$ & $\square$ & $\square$ & $\nabla$ & $\square$ \\
\hline 2633 & 4018 & Water well & $3 / 28 / 1978$ & 156.0 & 346986 & 6779210 & $\checkmark$ & $\square$ & $\square$ & $\checkmark$ & $\square$ \\
\hline 2633 & 4034 & Water well & $6 / 24 / 1974$ & 132.0 & 347395 & 6779316 & $\checkmark$ & $\square$ & $\square$ & $\bar{\nabla}$ & $\square$ \\
\hline 2633 & 4048 & Water well & 8/10/1979 & 149.0 & 347278 & 6779383 & $\checkmark$ & $\square$ & $\square$ & $\bar{\nabla}$ & $\square$ \\
\hline 2633 & 4062 & Water well & $7 / 12 / 1974$ & 158.0 & 347313 & 6779506 & $\checkmark$ & $\square$ & $\square$ & $\checkmark$ & $\square$ \\
\hline 2633 & 4063 & Water well & $10 / 5 / 1983$ & 160.0 & 347299 & 6779506 & $\checkmark$ & $\square$ & $\square$ & $\bar{\nabla}$ & $\square$ \\
\hline 2633 & 4075 & Water well & $9 / 1 / 1965$ & 159.0 & 347410 & 6779656 & $\checkmark$ & $\square$ & $\square$ & $\nabla$ & $\square$ \\
\hline 2634 & 3251 & Water well & $5 / 1 / 1986$ & 195.0 & 348021 & 6779599 & $\checkmark$ & $\square$ & $\square$ & $\square$ & $\square$ \\
\hline 2634 & 3255 & Water well & $6 / 18 / 1981$ & 200.0 & 347910 & 6779449 & $\checkmark$ & $\square$ & $\square$ & $\square$ & $\square$ \\
\hline
\end{tabular}




\begin{tabular}{|c|c|c|c|c|c|c|c|c|c|c|c|}
\hline \multirow[b]{2}{*}{$\begin{array}{c}\text { MOA } \\
\text { grid }\end{array}$} & \multirow[b]{2}{*}{$\begin{array}{c}\text { DGGS } \\
\text { number }\end{array}$} & \multirow[b]{2}{*}{ Hole Type } & \multirow[b]{2}{*}{$\begin{array}{c}\text { Date } \\
\text { Completed }\end{array}$} & \multirow[b]{2}{*}{$\begin{array}{c}\text { Total } \\
\text { depth (ft) }\end{array}$} & \multicolumn{2}{|c|}{ UTM coordinates (m) } & \multicolumn{5}{|c|}{ Data entered } \\
\hline & & & & & East & North & Lithology & SPT & $\begin{array}{c}\text { Sample } \\
\text { test }\end{array}$ & $\begin{array}{l}\text { Water } \\
\text { level }\end{array}$ & Velocity \\
\hline 2634 & 3267 & Water well & $9 / 6 / 1985$ & 236.0 & 348217 & 6779281 & $\checkmark$ & $\square$ & $\square$ & $\nabla$ & $\square$ \\
\hline 2634 & 3269 & Water well & $5 / 26 / 1978$ & 230.0 & 347889 & 6779295 & $\checkmark$ & $\square$ & $\square$ & $\square$ & $\square$ \\
\hline 2634 & 3273 & Water well & $5 / 31 / 1984$ & 240.0 & 347887 & 6779264 & $\nabla$ & $\square$ & $\square$ & $\nabla$ & $\square$ \\
\hline 2634 & 4024 & Water well & $7 / 15 / 1982$ & 260.0 & 348201 & 6779250 & $\nabla$ & $\square$ & $\square$ & $\nabla$ & $\square$ \\
\hline 2634 & 4025 & Water well & $10 / 27 / 1973$ & 278.0 & 348007 & 6779259 & $\checkmark$ & $\square$ & $\square$ & $\nabla$ & $\square$ \\
\hline 2634 & 4026 & Water well & 9/1/1971 & 240.0 & 347917 & 6779263 & 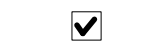 & $\square$ & $\square$ & $\nabla$ & $\square$ \\
\hline 2634 & 4033 & Water well & $7 / 27 / 1968$ & 103.0 & 347694 & 6779303 & $\checkmark$ & $\square$ & $\square$ & $\square$ & $\square$ \\
\hline 2634 & 4039 & Water well & 9/28/1972 & 167.0 & 347479 & 6779189 & $\boldsymbol{V}$ & $\square$ & $\square$ & $\nabla$ & $\square$ \\
\hline 2634 & 4045 & Water well & 9/19/1981 & 203.0 & 347921 & 6779356 & $\boldsymbol{v}$ & $\square$ & $\square$ & $\sqrt{ }$ & $\square$ \\
\hline 2634 & 4046 & Water well & $10 / 13 / 1978$ & 216.0 & 347697 & 6779365 & 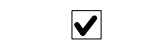 & $\square$ & $\square$ & $\nabla$ & $\square$ \\
\hline 2634 & 4053 & Water well & 7/22/1982 & 180.0 & 348207 & 6779374 & $\bar{v}$ & $\square$ & $\square$ & $\nabla$ & $\square$ \\
\hline 2634 & 4073 & Water well & 7/23/1973 & 195.0 & 348110 & 6779564 & $\bar{V}$ & $\square$ & $\square$ & $\nabla$ & $\square$ \\
\hline 2635 & 1764 & Hollow stem borehole w/SPT & $10 / 20 / 1989$ & 60.3 & 348376 & 6779154 & $\nabla$ & $\nabla$ & $\square$ & $\nabla$ & $\square$ \\
\hline 2635 & 3258 & Water well & $6 / 21 / 1984$ & 186.0 & 348610 & 6779326 & $\checkmark$ & $\square$ & $\square$ & $\checkmark$ & $\square$ \\
\hline 2635 & 3260 & Water well & 7/9/1975 & 265.5 & 348311 & 6779339 & $\bar{v}$ & $\square$ & $\square$ & $\nabla$ & $\square$ \\
\hline 2635 & 3263 & Water well & $7 / 21 / 1973$ & 200.0 & 349025 & 6779246 & $\checkmark$ & $\square$ & $\square$ & $\checkmark$ & $\square$ \\
\hline 2635 & 3265 & Water well & $9 / 25 / 1984$ & 206.5 & 348876 & 6779252 & $\boldsymbol{V}$ & $\square$ & $\square$ & $\nabla$ & $\square$ \\
\hline 2635 & 3266 & Water well & 10/1/1978 & 187.0 & 348591 & 6779265 & $\nabla$ & $\square$ & $\square$ & $\nabla$ & $\square$ \\
\hline 2635 & 3268 & Water well & 7/10/1985 & 187.0 & 348635 & 6779232 & $\boldsymbol{V}$ & $\square$ & $\square$ & $\nabla$ & $\square$ \\
\hline 2635 & 3271 & Water well & $5 / 24 / 1980$ & 175.0 & 348589 & 6779203 & $\bar{V}$ & $\square$ & $\square$ & $\nabla$ & $\square$ \\
\hline 2635 & 3280 & Water well & $6 / 15 / 1984$ & 238.0 & 348985 & 6779000 & $\bar{V}$ & $\square$ & $\square$ & $\nabla$ & $\square$ \\
\hline 2635 & 3281 & Water well & $1 / 6 / 1984$ & 171.0 & 348895 & 6779003 & 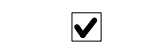 & $\square$ & $\square$ & $\sqrt{V}$ & $\square$ \\
\hline 2635 & 3289 & Water well & 7/13/1984 & 266.0 & 348993 & 6778844 & $\nabla$ & $\square$ & $\square$ & $\nabla$ & $\square$ \\
\hline 2635 & 3291 & Water well & $6 / 25 / 1983$ & 117.0 & 348679 & 6778858 & $\bar{v}$ & $\square$ & $\square$ & $\nabla$ & $\square$ \\
\hline 2635 & 3292 & Water well & 7/1/1986 & 180.0 & 348590 & 6778862 & 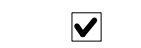 & $\square$ & $\square$ & $\nabla$ & $\square$ \\
\hline 2635 & 3984 & Water well & 7/13/1965 & 110.0 & 348424 & 6778869 & $\boldsymbol{V}$ & $\square$ & $\square$ & $\nabla$ & $\square$ \\
\hline 2635 & 4017 & Water well & $3 / 5 / 1975$ & 103.0 & 348511 & 6779144 & $\boldsymbol{V}$ & $\square$ & $\square$ & $\nabla$ & $\square$ \\
\hline 2635 & 4044 & Water well & 10/1/1981 & 209.0 & 348998 & 6779309 & $\boldsymbol{V}$ & $\square$ & $\square$ & $\nabla$ & $\square$ \\
\hline 2635 & 4061 & Water well & 8/7/1976 & 231.0 & 349018 & 6779432 & $\nabla$ & $\square$ & $\square$ & $\nabla$ & $\square$ \\
\hline 2635 & 4065 & Water well & 11/8/1978 & 214.0 & 348511 & 6779485 & $\nabla$ & $\square$ & $\square$ & $\nabla$ & $\square$ \\
\hline 2635 & 4486 & Water well & $8 / 30 / 1971$ & 168.0 & 349038 & 6779183 & $\boldsymbol{V}$ & $\square$ & $\square$ & $\nabla$ & $\square$ \\
\hline 2635 & 4488 & Water well & $6 / 23 / 1978$ & 176.0 & 348886 & 6779128 & $\nabla$ & $\square$ & $\square$ & $\checkmark$ & $\square$ \\
\hline 2636 & 1765 & Hollow stem borehole, no SPT & $4 / 16 / 1975$ & 20.0 & 349313 & 6779255 & $\square$ & $\square$ & $\square$ & $\square$ & $\square$ \\
\hline 2636 & 3246 & Water well & $4 / 22 / 1986$ & 297.0 & 349711 & 6779495 & $\nabla$ & $\square$ & $\square$ & $\nabla$ & $\square$ \\
\hline 2636 & 3247 & Water well & $6 / 2 / 1964$ & 242.0 & 349620 & 6779499 & $\boldsymbol{V}$ & $\square$ & $\square$ & $\nabla$ & $\square$ \\
\hline 2636 & 3250 & Water well & $6 / 19 / 1972$ & 255.0 & 349440 & 6779476 & $\checkmark$ & $\square$ & $\square$ & $\nabla$ & $\square$ \\
\hline
\end{tabular}




\begin{tabular}{|c|c|c|c|c|c|c|c|c|c|c|c|}
\hline \multirow[b]{2}{*}{$\begin{array}{l}\text { MOA } \\
\text { grid }\end{array}$} & \multirow[b]{2}{*}{$\begin{array}{c}\text { DGGS } \\
\text { number }\end{array}$} & \multirow[b]{2}{*}{ Hole Type } & \multirow[b]{2}{*}{$\begin{array}{c}\text { Date } \\
\text { Completed }\end{array}$} & \multirow[b]{2}{*}{$\begin{array}{c}\text { Total } \\
\text { depth }(\mathrm{ft})\end{array}$} & \multicolumn{2}{|c|}{ UTM coordinates (m) } & \multicolumn{5}{|c|}{ Data entered } \\
\hline & & & & & East & North & Lithology & SPT & $\begin{array}{c}\text { Sample } \\
\text { test }\end{array}$ & $\begin{array}{l}\text { Water } \\
\text { level }\end{array}$ & Velocity \\
\hline 2636 & 3252 & Water well & $5 / 25 / 1978$ & 295.0 & 349811 & 6779398 & $\checkmark$ & 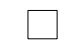 & $\square$ & $\checkmark$ & $\square$ \\
\hline 2636 & 3253 & Water well & $6 / 14 / 1980$ & 288.0 & 349707 & 6779403 & $\checkmark$ & $\square$ & $\square$ & $\checkmark$ & $\square$ \\
\hline 2636 & 3264 & Water well & $4 / 1 / 1975$ & 168.0 & 349518 & 6779194 & $\checkmark$ & & $\square$ & $\bar{V}$ & \\
\hline 2636 & 3276 & Water well & $6 / 3 / 1972$ & 258.0 & 349256 & 6779019 & $\checkmark$ & $\square$ & $\square$ & $\bar{\nabla}$ & $\square$ \\
\hline 2636 & 3277 & Water well & $8 / 10 / 1981$ & 221.0 & 349091 & 6779026 & $\checkmark$ & $\square$ & $\square$ & $\square$ & $\square$ \\
\hline 2636 & 3283 & Water well & $3 / 24 / 1982$ & 280.0 & 349311 & 6778924 & $\nabla$ & $\square$ & $\square$ & $\square$ & $\square$ \\
\hline 2636 & 3284 & Water well & $6 / 1 / 1980$ & 249.0 & 349177 & 6778930 & $\checkmark$ & $\square$ & $\square$ & $\checkmark$ & $\square$ \\
\hline 2636 & 3288 & Water well & $11 / 3 / 1979$ & 264.0 & 349157 & 6778837 & $\checkmark$ & & $\square$ & $\square$ & $\square$ \\
\hline 2636 & 3983 & Water well & $7 / 29 / 1976$ & 156.0 & 349771 & 6778811 & $\checkmark$ & $\square$ & $\square$ & $\sqrt{\square}$ & $\square$ \\
\hline 2636 & 3985 & Water well & $6 / 21 / 1973$ & 125.0 & 349742 & 6778843 & $\checkmark$ & & $\square$ & $\checkmark$ & $\square$ \\
\hline 2636 & 3997 & Water well & 10/1/1979 & 253.0 & 349207 & 6778928 & $\checkmark$ & $\square$ & $\square$ & $\square$ & $\square$ \\
\hline 2636 & 4016 & Water well & $1 / 1 / 1967$ & 280.0 & 349708 & 6779093 & $\square$ & $\square$ & $\square$ & $\bar{\nabla}$ & $\square$ \\
\hline 2636 & 4020 & Water well & $6 / 14 / 1973$ & 125.0 & 349470 & 6779134 & $\checkmark$ & $\square$ & $\square$ & $\checkmark$ & $\square$ \\
\hline 2636 & 4043 & Water well & 8/7/1976 & 163.0 & 349611 & 6779283 & $\checkmark$ & $\square$ & $\square$ & $\nabla$ & $\square$ \\
\hline 2636 & 4052 & Water well & $7 / 28 / 1964$ & 243.0 & 349299 & 6779327 & $\checkmark$ & $\square$ & $\square$ & $\checkmark$ & $\square$ \\
\hline 2636 & 4060 & Water well & $12 / 6 / 1969$ & 289.0 & 349796 & 6779399 & $\checkmark$ & 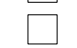 & $\square$ & $\checkmark$ & $\square$ \\
\hline 2636 & 4483 & Water well & $7 / 26 / 1971$ & 224.0 & 349243 & 6779423 & $\checkmark$ & 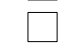 & $\square$ & $\bar{V}$ & $\square$ \\
\hline 2636 & 4484 & Water well & 11/1/1979 & 249.0 & 349428 & 6779198 & $\checkmark$ & 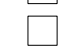 & $\square$ & $\checkmark$ & $\square$ \\
\hline 2636 & 4487 & Water well & 8/8/1974 & 278.0 & 349485 & 6779133 & $\checkmark$ & $\square$ & $\square$ & $\nabla$ & $\square$ \\
\hline 2637 & 3245 & Water well & $10 / 4 / 1985$ & 300.0 & 350324 & 6779469 & $\checkmark$ & $\square$ & $\square$ & $\checkmark$ & $\square$ \\
\hline 2637 & 3272 & Water well & $5 / 24 / 1978$ & 358.0 & 349871 & 6779055 & $\checkmark$ & & $\square$ & $\bar{\nabla}$ & $\square$ \\
\hline 2637 & 3278 & Water well & $9 / 19 / 1981$ & 172.3 & 350538 & 6778872 & $\checkmark$ & 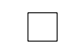 & $\square$ & $\nabla$ & $\square$ \\
\hline 2637 & 3982 & Water well & $9 / 16 / 1972$ & 110.0 & 350010 & 6778801 & $\checkmark$ & $\square$ & $\square$ & $\nabla$ & $\square$ \\
\hline 2637 & 3996 & Water well & $6 / 19 / 1973$ & 200.0 & 350314 & 6778881 & $\checkmark$ & $\square$ & $\square$ & $\checkmark$ & $\square$ \\
\hline 2637 & 4015 & Water well & 8/1/1976 & 104.0 & 350127 & 6779075 & $\checkmark$ & & $\square$ & $\bar{\nabla}$ & $\square$ \\
\hline 2637 & 4059 & Water well & $7 / 22 / 1964$ & 127.0 & 349916 & 6779394 & $\checkmark$ & & $\square$ & $\square$ & $\square$ \\
\hline 2637 & 4482 & Water well & $11 / 15 / 1971$ & 284.0 & 350027 & 6779544 & $\checkmark$ & $\square$ & $\square$ & $\checkmark$ & $\square$ \\
\hline 2637 & 4485 & Water well & $9 / 29 / 1973$ & 155.0 & 350202 & 6779072 & $\checkmark$ & & $\square$ & $\nabla$ & $\square$ \\
\hline 2637 & 4489 & Water well & 9/1/1976 & 146.0 & 350598 & 6778869 & $\checkmark$ & $\square$ & $\square$ & $\checkmark$ & $\square$ \\
\hline 2637 & 4490 & Water well & $10 / 11 / 1974$ & 203.0 & 350507 & 6778842 & $\checkmark$ & $\square$ & $\square$ & $\bar{\nabla}$ & $\square$ \\
\hline 2638 & 1644 & Hollow stem borehole w/SPT & $12 / 2 / 1982$ & 41.0 & 350957 & 6779207 & $\square$ & $\square$ & $\square$ & $\square$ & $\square$ \\
\hline 2638 & 3256 & Water well & $3 / 23 / 1972$ & 175.0 & 351117 & 6779095 & $\checkmark$ & $\square$ & $\square$ & $\checkmark$ & $\square$ \\
\hline 2638 & 3259 & Water well & $5 / 4 / 1977$ & 189.0 & 351399 & 6779021 & $\checkmark$ & $\square$ & $\square$ & $\bar{\square}$ & $\square$ \\
\hline 2638 & 3261 & Water well & $3 / 20 / 1981$ & 176.0 & 351144 & 6779032 & $\checkmark$ & $\square$ & $\square$ & $\nabla$ & $\square$ \\
\hline 2638 & 3275 & Water well & $2 / 10 / 1982$ & 201.0 & 351240 & 6778811 & $\checkmark$ & $\square$ & $\square$ & $\vec{\nabla}$ & $\square$ \\
\hline 2638 & 3279 & Water well & $9 / 1 / 1979$ & 216.0 & 350926 & 6778824 & $\checkmark$ & $\square$ & $\square$ & $\nabla$ & $\square$ \\
\hline
\end{tabular}




\begin{tabular}{|c|c|c|c|c|c|c|c|c|c|c|c|}
\hline \multirow[b]{2}{*}{$\begin{array}{l}\text { MOA } \\
\text { grid }\end{array}$} & \multirow[b]{2}{*}{$\begin{array}{l}\text { DGGS } \\
\text { number }\end{array}$} & \multirow[b]{2}{*}{ Hole Type } & \multirow[b]{2}{*}{$\begin{array}{c}\text { Date } \\
\text { Completed }\end{array}$} & \multirow[b]{2}{*}{$\begin{array}{c}\text { Total } \\
\text { depth }(\mathrm{ft})\end{array}$} & \multicolumn{2}{|c|}{ UTM coordinates (m) } & \multicolumn{5}{|c|}{ Data entered } \\
\hline & & & & & East & North & Lithology & SPT & $\begin{array}{c}\text { Sample } \\
\text { test }\end{array}$ & $\begin{array}{l}\text { Water } \\
\text { level }\end{array}$ & Velocity \\
\hline 2638 & 3282 & Water well & $5 / 30 / 1983$ & 199.0 & 350998 & 6778759 & $\checkmark$ & & $\square$ & $\checkmark$ & $\square$ \\
\hline 2638 & 3979 & Water well & $1 / 4 / 1979$ & 200.0 & 351072 & 6778725 & $\checkmark$ & $\square$ & $\square$ & $\square$ & $\square$ \\
\hline 2638 & 3988 & Water well & 2/13/1979 & 211.0 & 351180 & 6778813 & $\checkmark$ & $\square$ & $\square$ & 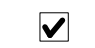 & $\square$ \\
\hline 2638 & 3989 & Water well & $9 / 12 / 1981$ & 177.0 & 351105 & 6778817 & $\checkmark$ & $\square$ & $\square$ & $\boldsymbol{V}$ & $\square$ \\
\hline 2638 & 3995 & Water well & $10 / 3 / 1978$ & 108.0 & 351391 & 6778836 & $\checkmark$ & $\square$ & $\square$ & $\square$ & $\square$ \\
\hline 2638 & 4003 & Water well & $11 / 19 / 1983$ & 185.0 & 351200 & 6778936 & $\checkmark$ & $\square$ & $\square$ & $\checkmark$ & $\square$ \\
\hline 2638 & 4023 & Water well & $8 / 25 / 1975$ & 170.0 & 351193 & 6779123 & $\checkmark$ & $\square$ & $\square$ & $\checkmark$ & $\square$ \\
\hline 2638 & 4032 & Water well & 9/1/1966 & 142.0 & 351015 & 6779161 & $\checkmark$ & 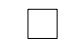 & $\square$ & $\nabla$ & $\square$ \\
\hline 2638 & 4051 & Water well & $7 / 16 / 1974$ & 169.0 & 351393 & 6779238 & $\checkmark$ & $\square$ & $\square$ & $\sqrt{\square}$ & $\square$ \\
\hline 2638 & 4055 & Water well & 8/9/1973 & 162.0 & 351394 & 6779269 & $\checkmark$ & & $\square$ & $\checkmark$ & $\square$ \\
\hline 2638 & 4064 & Water well & $5 / 18 / 1973$ & 199.0 & 351338 & 6779364 & $\checkmark$ & $\square$ & $\square$ & $\bar{V}$ & $\square$ \\
\hline 2638 & 4067 & Water well & 9/9/1966 & 126.0 & 351441 & 6779329 & $\checkmark$ & & $\square$ & $\checkmark$ & $\square$ \\
\hline 2638 & 4071 & Water well & $7 / 24 / 1978$ & 162.0 & 351416 & 6779423 & $\checkmark$ & $\square$ & $\square$ & $\nabla$ & $\square$ \\
\hline 2638 & 4072 & Water well & $9 / 2 / 1982$ & 126.0 & 351027 & 6779439 & $\checkmark$ & $\square$ & $\square$ & $\checkmark$ & $\square$ \\
\hline 2638 & 4491 & Water well & $1 / 11 / 1979$ & 207.0 & 351161 & 6778721 & $\checkmark$ & $\square$ & $\square$ & $\checkmark$ & $\square$ \\
\hline 2639 & 3987 & Water well & $9 / 24 / 1981$ & 148.0 & 351913 & 6778783 & $\checkmark$ & - & $\square$ & $\checkmark$ & $\square$ \\
\hline 2639 & 4000 & Water well & 7/30/1977 & 192.0 & 351722 & 6778883 & $\checkmark$ & & $\square$ & $\boldsymbol{V}$ & $\square$ \\
\hline 2639 & 4001 & Water well & 7/10/1981 & 155.0 & 351918 & 6778906 & $\checkmark$ & $\square$ & $\square$ & $\square$ & $\square$ \\
\hline 2639 & 4002 & Water well & $4 / 25 / 1974$ & 153.0 & 351604 & 6778919 & $\checkmark$ & $\checkmark$ & $\square$ & $\nabla$ & $\square$ \\
\hline 2639 & 4014 & Water well & 4/29/1978 & 145.0 & 351698 & 6779009 & $\checkmark$ & $\square$ & $\square$ & $\checkmark$ & $\square$ \\
\hline 2639 & 4029 & Water well & $3 / 30 / 1970$ & 214.0 & 351838 & 6779126 & $\checkmark$ & & $\square$ & $\bar{\nabla}$ & $\square$ \\
\hline 2639 & 4031 & Water well & $10 / 22 / 1982$ & 202.0 & 351703 & 6779132 & $\checkmark$ & 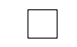 & $\square$ & $\nabla$ & $\square$ \\
\hline 2639 & 4036 & Water well & 8/1/1970 & 197.0 & 351722 & 6779224 & $\checkmark$ & & $\square$ & $\nabla$ & $\square$ \\
\hline 2639 & 4037 & Water well & $9 / 14 / 1983$ & 170.0 & 351525 & 6779171 & $\checkmark$ & $\square$ & $\square$ & $\checkmark$ & $\square$ \\
\hline 2639 & 4038 & Water well & 8/8/1964 & 200.0 & 351540 & 6779170 & $\checkmark$ & & $\square$ & $\checkmark$ & $\square$ \\
\hline 2639 & 4069 & Water well & $10 / 20 / 1972$ & 235.0 & 352103 & 6779394 & $\checkmark$ & & $\square$ & $\nabla$ & $\square$ \\
\hline 2639 & 4070 & Water well & $6 / 14 / 1979$ & 205.0 & 351729 & 6779410 & $\checkmark$ & $\square$ & $\square$ & $\vec{V}$ & $\square$ \\
\hline 2640 & 3262 & Water well & $6 / 1 / 1981$ & 200.0 & 352949 & 6778832 & $\checkmark$ & & $\square$ & $\nabla$ & $\square$ \\
\hline 2640 & 3994 & Water well & $8 / 11 / 1978$ & 198.0 & 352692 & 6778781 & $\square$ & & $\square$ & $\checkmark$ & $\square$ \\
\hline 2640 & 3999 & Water well & $7 / 17 / 1965$ & 117.0 & 352828 & 6778806 & $\checkmark$ & $\square$ & $\square$ & $\bar{\nabla}$ & $\square$ \\
\hline 2640 & 4004 & Water well & $6 / 1 / 1966$ & 113.0 & 352667 & 6778906 & $\checkmark$ & $\square$ & $\square$ & $\nabla$ & $\square$ \\
\hline 2640 & 4006 & Water well & $7 / 26 / 1978$ & 101.0 & 352639 & 6778938 & $\checkmark$ & $\square$ & $\square$ & $\square$ & $\square$ \\
\hline 2640 & 4012 & Water well & $11 / 2 / 1981$ & 325.0 & 352939 & 6778957 & $\checkmark$ & $\square$ & $\square$ & $\bar{\nabla}$ & $\square$ \\
\hline 2640 & 4013 & Water well & $4 / 24 / 1981$ & 240.0 & 352819 & 6778962 & $\checkmark$ & $\square$ & $\square$ & $\nabla$ & $\square$ \\
\hline 2640 & 4022 & Water well & $8 / 13 / 1975$ & 160.0 & 352794 & 6779055 & $\checkmark$ & $\square$ & $\square$ & $\boldsymbol{V}$ & $\square$ \\
\hline 2640 & 4027 & Water well & $4 / 22 / 1977$ & 200.0 & 352795 & 6779086 & $\checkmark$ & $\square$ & $\square$ & $\nabla$ & $\square$ \\
\hline
\end{tabular}




\begin{tabular}{|c|c|c|c|c|c|c|c|c|c|c|c|}
\hline \multirow[b]{2}{*}{$\begin{array}{l}\text { MOA } \\
\text { grid }\end{array}$} & \multirow[b]{2}{*}{$\begin{array}{c}\text { DGGS } \\
\text { number }\end{array}$} & \multirow[b]{2}{*}{ Hole Type } & \multirow[b]{2}{*}{$\begin{array}{c}\text { Date } \\
\text { Completed }\end{array}$} & \multirow[b]{2}{*}{$\begin{array}{c}\text { Total } \\
\text { depth (ft) }\end{array}$} & \multicolumn{2}{|c|}{ UTM coordinates (m) } & \multicolumn{5}{|c|}{ Data entered } \\
\hline & & & & & East & North & Lithology & SPT & $\begin{array}{c}\text { Sample } \\
\text { test }\end{array}$ & $\begin{array}{l}\text { Water } \\
\text { level }\end{array}$ & Velocity \\
\hline 2640 & 4035 & Water well & $7 / 31 / 1975$ & 190.0 & 352916 & 6779112 & $\boldsymbol{V}$ & $\square$ & $\square$ & $\checkmark$ & $\square$ \\
\hline 2640 & 4049 & Water well & $10 / 29 / 1972$ & 275.0 & 352619 & 6779186 & $\checkmark$ & $\square$ & $\square$ & $\checkmark$ & $\square$ \\
\hline 2640 & 4050 & Water well & $10 / 30 / 1981$ & 232.0 & 352529 & 6779190 & $\checkmark$ & $\square$ & $\square$ & $\checkmark$ & $\square$ \\
\hline 2640 & 4054 & Water well & $7 / 29 / 1965$ & 106.0 & 352631 & 6779093 & $\checkmark$ & $\square$ & $\square$ & $V$ & $\square$ \\
\hline 2640 & 4068 & Water well & $10 / 28 / 1969$ & 186.0 & 352836 & 6779363 & $\checkmark$ & $\square$ & $\square$ & $\checkmark$ & $\square$ \\
\hline 2641 & 1766 & Hollow stem borehole, no SPT & $12 / 28 / 1978$ & 20.0 & 353629 & 6778958 & $\square$ & $\square$ & $\square$ & $\square$ & $\square$ \\
\hline 2641 & 1767 & Hollow stem borehole, no SPT & $12 / 29 / 1978$ & 20.0 & 353755 & 6778917 & $\square$ & $\square$ & $\square$ & $\square$ & $\square$ \\
\hline 2641 & 1768 & Hollow stem borehole, no SPT & $12 / 29 / 1978$ & 20.0 & 353768 & 6778824 & 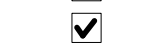 & $\square$ & $\square$ & $\checkmark$ & $\square$ \\
\hline 2641 & 1769 & Hollow stem borehole, no SPT & $12 / 29 / 1978$ & 20.0 & 353766 & 6778783 & $\square$ & $\square$ & $\square$ & $\square$ & $\square$ \\
\hline 2641 & 1770 & Hollow stem borehole, no SPT & $12 / 28 / 1978$ & 20.0 & 353628 & 6778711 & $\square$ & $\square$ & $\square$ & $\square$ & $\square$ \\
\hline 2641 & 3239 & Water well & 7/8/1975 & 246.0 & 353809 & 6779323 & $\checkmark$ & $\square$ & $\square$ & $\checkmark$ & $\square$ \\
\hline 2641 & 3248 & Water well & 9/12/1978 & 298.0 & 353782 & 6779045 & $\square$ & $\square$ & $\square$ & $\checkmark$ & $\square$ \\
\hline 2641 & 3249 & Water well & 7/15/1980 & 550.0 & 353260 & 6779098 & $V$ & $\square$ & $\square$ & $V$ & $\square$ \\
\hline 2641 & 3257 & Water well & 4/30/1983 & 175.0 & 353519 & 6778839 & $\checkmark$ & $\square$ & $\square$ & $V$ & $\square$ \\
\hline 2641 & 3270 & Water well & 4/10/1984 & 195.0 & 353677 & 6778678 & $\boldsymbol{v}$ & $\square$ & $\square$ & $V$ & $\square$ \\
\hline 2641 & 3980 & Water well & 8/13/1982 & 183.0 & 353810 & 6778641 & $\checkmark$ & $\square$ & $\square$ & $V$ & $\square$ \\
\hline 2641 & 3981 & Water well & 4/5/1982 & 153.0 & 353526 & 6778653 & 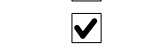 & $\square$ & $\square$ & $V$ & $\square$ \\
\hline 2641 & 3991 & Water well & $11 / 13 / 1981$ & 240.0 & 353814 & 6778734 & $\checkmark$ & $\square$ & $\square$ & $V$ & $\square$ \\
\hline 2641 & 3992 & Water well & $6 / 19 / 1982$ & 140.0 & 353814 & 6778825 & $\checkmark$ & $\square$ & $\square$ & $V$ & $\square$ \\
\hline 2641 & 3993 & Water well & $5 / 9 / 1975$ & 112.0 & 353201 & 6778760 & 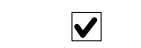 & $\square$ & $\square$ & $\nabla$ & $\square$ \\
\hline 2641 & 3998 & Water well & 4/29/1973 & 202.0 & 353309 & 6778848 & $\checkmark$ & $\square$ & $\square$ & $\checkmark$ & $\square$ \\
\hline 2641 & 4007 & Water well & 8/21/1979 & 120.0 & 353807 & 6778920 & $\checkmark$ & $\square$ & $\square$ & $\sqrt{v}$ & $\square$ \\
\hline 2641 & 4008 & Water well & 9/8/1983 & 212.0 & 353718 & 6778924 & $V$ & $\square$ & $\square$ & $V$ & $\square$ \\
\hline 2641 & 4009 & Water well & 4/14/1979 & 114.0 & 353193 & 6778946 & $\square$ & $\square$ & $\square$ & $\nabla$ & $\square$ \\
\hline 2641 & 4010 & Water well & 6/11/1982 & 134.0 & 353134 & 6778948 & $\nabla$ & $\square$ & $\square$ & $v$ & $\square$ \\
\hline 2641 & 4011 & Water well & 12/9/1977 & 263.0 & 353104 & 6778950 & 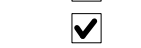 & $\square$ & $\square$ & $V$ & $\square$ \\
\hline 2641 & 4019 & Water well & 8/17/1982 & 119.0 & 353419 & 6778967 & $\checkmark$ & $\square$ & $\square$ & $V$ & $\square$ \\
\hline 2641 & 4021 & Water well & $10 / 5 / 1981$ & 225.0 & 353721 & 6779017 & $\checkmark$ & $\square$ & $\square$ & $\checkmark$ & $\square$ \\
\hline 2641 & 4041 & Water well & $5 / 15 / 1982$ & 250.0 & 353830 & 6779105 & $\boldsymbol{V}$ & $\square$ & $\square$ & $V$ & $\square$ \\
\hline 2641 & 4042 & Water well & $11 / 15 / 1981$ & 225.0 & 353740 & 6779109 & $\sqrt{v}$ & $\square$ & $\square$ & $\checkmark$ & $\square$ \\
\hline 2641 & 4056 & Water well & 7/12/1975 & 255.0 & 353820 & 6779230 & $\nabla$ & $\square$ & $\square$ & $\nabla$ & $\square$ \\
\hline 2641 & 4057 & Water well & 9/15/1978 & 350.0 & 353625 & 6779238 & $\square$ & $\square$ & $\square$ & $V$ & $\square$ \\
\hline 2641 & 4058 & Water well & 2/18/1978 & 178.0 & 353117 & 6779259 & $\nabla$ & $\square$ & $\square$ & 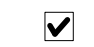 & $\square$ \\
\hline 2641 & 4074 & Water well & $9 / 22 / 1978$ & 250.0 & 353137 & 6779382 & $\checkmark$ & $\square$ & $\square$ & $\checkmark$ & $\square$ \\
\hline 2727 & 2009 & Natural exposure & 6/2/1997 & 10.0 & 341983 & 6779030 & $\checkmark$ & $\square$ & $\square$ & $\square$ & $\square$ \\
\hline 2727 & 2087 & Hollow stem borehole w/SPT & $5 / 12 / 1986$ & 71.5 & 341948 & 6778930 & $\checkmark$ & $\checkmark$ & $\checkmark$ & $\checkmark$ & $\square$ \\
\hline
\end{tabular}




\begin{tabular}{|c|c|c|c|c|c|c|c|c|c|c|c|}
\hline \multirow[b]{2}{*}{$\begin{array}{l}\text { MOA } \\
\text { grid }\end{array}$} & \multirow[b]{2}{*}{$\begin{array}{l}\text { DGGS } \\
\text { number }\end{array}$} & \multirow[b]{2}{*}{ Hole Type } & \multirow[b]{2}{*}{$\begin{array}{c}\text { Date } \\
\text { Completed }\end{array}$} & \multirow[b]{2}{*}{$\begin{array}{c}\text { Total } \\
\text { depth }(\mathrm{ft})\end{array}$} & \multicolumn{2}{|c|}{ UTM coordinates (m) } & \multicolumn{5}{|c|}{ Data entered } \\
\hline & & & & & East & North & Lithology & SPT & $\begin{array}{l}\text { Sample } \\
\text { test }\end{array}$ & $\begin{array}{l}\text { Water } \\
\text { level }\end{array}$ & Velocity \\
\hline 2727 & 2088 & Hollow stem borehole w/SPT & $5 / 9 / 1986$ & 71.0 & 342198 & 6778837 & $\checkmark$ & $\nabla$ & $\checkmark$ & $\checkmark$ & 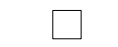 \\
\hline 2727 & 2103 & Hollow stem borehole w/SPT & 2/3/1983 & 21.5 & 342667 & 6778893 & $\checkmark$ & $\checkmark$ & $\square$ & $\nabla$ & $\square$ \\
\hline 2727 & 2104 & Hollow stem borehole w/SPT & $2 / 9 / 1983$ & 56.0 & 342594 & 6778608 & $\checkmark$ & $\checkmark$ & $\nabla$ & $\bar{\nabla}$ & \\
\hline 2727 & 2105 & Hollow stem borehole w/SPT & 2/10/1983 & 61.5 & 342540 & 6778635 & $\nabla$ & $\nabla$ & $\checkmark$ & $\nabla$ & $\square$ \\
\hline 2727 & 2106 & Hollow stem borehole w/SPT & 2/3/1983 & 27.0 & 342570 & 6778836 & $\checkmark$ & $\checkmark$ & $\square$ & $\nabla$ & $\square$ \\
\hline 2727 & 2107 & Hollow stem borehole w/SPT & 2/7/1983 & 21.5 & 342343 & 6778817 & $\checkmark$ & $\checkmark$ & $\square$ & $\checkmark$ & $\square$ \\
\hline 2727 & 2224 & Hollow stem borehole w/SPT & $1 / 8 / 1982$ & 72.0 & 341952 & 6779028 & $\checkmark$ & $\nabla$ & $\checkmark$ & $\square$ & $\square$ \\
\hline 2728 & 1635 & Hollow stem borehole w/SPT & $2 / 22 / 1984$ & 68.0 & 343133 & 6778331 & $\checkmark$ & $\checkmark$ & $\checkmark$ & $\nabla$ & $\square$ \\
\hline 2728 & 1636 & Hollow stem borehole w/SPT & 2/27/1984 & 40.0 & 343125 & 6778313 & $\square$ & $\square$ & $\square$ & $\square$ & $\square$ \\
\hline 2728 & 1637 & Hollow stem borehole w/SPT & 2/23/1984 & 68.0 & 343043 & 6778367 & $\checkmark$ & $\nabla$ & $\checkmark$ & $\checkmark$ & $\square$ \\
\hline 2728 & 1638 & Hollow stem borehole w/SPT & 2/28/1984 & 40.5 & 343034 & 6778348 & $\square$ & $\square$ & $\square$ & $\square$ & $\square$ \\
\hline 2728 & 1639 & Hollow stem borehole w/SPT & 2/29/1984 & 40.5 & 342962 & 6778388 & $\square$ & $\square$ & $\square$ & $\square$ & $\square$ \\
\hline 2728 & 2075 & Hollow stem borehole w/SPT & $5 / 21 / 1982$ & 51.0 & 343300 & 6778695 & $\checkmark$ & $\nabla$ & $\checkmark$ & $\nabla$ & $\square$ \\
\hline 2728 & 2076 & Hollow stem borehole w/SPT & $5 / 27 / 1982$ & 51.0 & 343383 & 6778665 & $\checkmark$ & $\nabla$ & $\checkmark$ & $\nabla$ & $\square$ \\
\hline 2728 & 2101 & Hollow stem borehole w/SPT & 2/4/1983 & 26.5 & 342833 & 6778814 & $\checkmark$ & $\checkmark$ & $\square$ & $\checkmark$ & $\square$ \\
\hline 2728 & 2102 & Hollow stem borehole w/SPT & $2 / 1 / 1983$ & 21.5 & 342729 & 6778995 & $\checkmark$ & $\checkmark$ & $\square$ & $\nabla$ & $\square$ \\
\hline 2729 & 2263 & Hollow stem borehole w/SPT & 2/28/1978 & 30.0 & 344162 & 6778616 & $\checkmark$ & $\checkmark$ & $\square$ & $\nabla$ & $\square$ \\
\hline 2729 & 3920 & Water well & $5 / 1 / 1969$ & 120.0 & 343689 & 6778208 & $\checkmark$ & $\square$ & $\square$ & $\nabla$ & $\square$ \\
\hline 2729 & 3932 & Water well & 7/16/1974 & 210.0 & 343712 & 6778393 & $\checkmark$ & $\square$ & $\square$ & $\nabla$ & \\
\hline 2730 & 3956 & Water well & $11 / 17 / 1972$ & 105.0 & 344728 & 6778658 & $\checkmark$ & $\square$ & $\square$ & $\nabla$ & $\square$ \\
\hline 2730 & 3965 & Water well & $4 / 10 / 1974$ & 113.0 & 344746 & 6778719 & $\checkmark$ & $\square$ & $\square$ & $\bar{\nabla}$ & $\square$ \\
\hline 2730 & 3968 & Water well & $11 / 24 / 1972$ & 117.0 & 344643 & 6778755 & $\checkmark$ & $\square$ & $\square$ & $\nabla$ & $\square$ \\
\hline 2731 & 2008 & Natural exposure & 6/2/1997 & 100.0 & 345417 & 6778641 & $\checkmark$ & $\square$ & $\square$ & $\square$ & $\square$ \\
\hline 2731 & 2264 & Hollow stem borehole w/SPT & & 34.5 & 345084 & 6778933 & $\checkmark$ & $\nabla$ & $\square$ & $\checkmark$ & $\square$ \\
\hline 2731 & 2265 & Hollow stem borehole w/SPT & & 36.5 & 345175 & 6778927 & $\checkmark$ & $\checkmark$ & $\square$ & $\checkmark$ & $\square$ \\
\hline 2731 & 2267 & Water well & $6 / 20 / 1977$ & 80.0 & 345712 & 6778797 & $\checkmark$ & $\square$ & $\square$ & $\square$ & $\square$ \\
\hline 2731 & 3337 & Water well & 4/11/1977 & 110.0 & 345511 & 6778376 & $\checkmark$ & $\square$ & $\square$ & $\checkmark$ & $\square$ \\
\hline 2731 & 3919 & Water well & $4 / 16 / 1982$ & 114.0 & 345428 & 6778194 & $\checkmark$ & $\square$ & $\square$ & $\nabla$ & $\square$ \\
\hline 2731 & 3923 & Water well & $10 / 16 / 1979$ & 438.0 & 345476 & 6778254 & $\checkmark$ & $\square$ & $\square$ & $\square$ & $\square$ \\
\hline 2731 & 3936 & Water well & 6/9/1981 & 115.0 & 345750 & 6778365 & $\checkmark$ & $\square$ & $\square$ & $\bar{\nabla}$ & $\square$ \\
\hline 2731 & 3939 & Water well & $3 / 16 / 1967$ & 104.0 & 345468 & 6778440 & $\checkmark$ & $\square$ & $\square$ & $\nabla$ & $\square$ \\
\hline 2731 & 3940 & Water well & $6 / 30 / 1975$ & 103.0 & 345439 & 6778441 & $\checkmark$ & $\square$ & $\square$ & $\checkmark$ & $\square$ \\
\hline 2731 & 4505 & Water well & $12 / 10 / 1970$ & 630.0 & 345133 & 6778640 & $\square$ & $\square$ & $\square$ & $\bar{\square}$ & $\square$ \\
\hline 2732 & 1741 & Hollow stem borehole, no SPT & 7/18/1977 & 20.0 & 346329 & 6778473 & $\square$ & $\square$ & $\square$ & $\square$ & $\square$ \\
\hline 2732 & 1842 & Hollow stem borehole w/SPT & $1 / 17 / 1980$ & 55.6 & 346518 & 6778107 & $\checkmark$ & $\checkmark$ & $\checkmark$ & $\nabla$ & $\square$ \\
\hline 2732 & 2268 & Hollow stem borehole w/SPT & $3 / 4 / 1986$ & 31.5 & 346206 & 6778594 & $\checkmark$ & $\checkmark$ & $\square$ & $\checkmark$ & $\square$ \\
\hline
\end{tabular}




\begin{tabular}{|c|c|c|c|c|c|c|c|c|c|c|c|}
\hline \multirow[b]{2}{*}{$\begin{array}{c}\text { MOA } \\
\text { grid }\end{array}$} & \multirow[b]{2}{*}{$\begin{array}{c}\text { DGGS } \\
\text { number }\end{array}$} & \multirow[b]{2}{*}{ Hole Type } & \multirow[b]{2}{*}{$\begin{array}{c}\text { Date } \\
\text { Completed }\end{array}$} & \multirow[b]{2}{*}{$\begin{array}{c}\text { Total } \\
\text { depth (ft) }\end{array}$} & \multicolumn{2}{|c|}{ UTM coordinates (m) } & \multicolumn{5}{|c|}{ Data entered } \\
\hline & & & & & East & North & Lithology & SPT & $\begin{array}{c}\text { Sample } \\
\text { test }\end{array}$ & $\begin{array}{l}\text { Water } \\
\text { level }\end{array}$ & Velocity \\
\hline 2732 & 2269 & Hollow stem borehole w/SPT & $7 / 5 / 1984$ & 31.5 & 346323 & 6778342 & $\checkmark$ & $\nabla$ & $\square$ & $\nabla$ & $\square$ \\
\hline 2732 & 3341 & Water well & $5 / 28 / 1974$ & 550.0 & 346090 & 6778257 & $\checkmark$ & $\square$ & $\square$ & $\sqrt{V}$ & $\square$ \\
\hline 2732 & 3930 & Water well & $4 / 1 / 1974$ & 286.0 & 346225 & 6778252 & $\nabla$ & $\square$ & $\square$ & $\nabla$ & $\square$ \\
\hline 2733 & 1761 & Hollow stem borehole w/SPT & $4 / 3 / 1984$ & 31.5 & 346811 & 6778770 & $\nabla$ & $\checkmark$ & $\nabla$ & $\nabla$ & $\square$ \\
\hline 2733 & 1762 & Hollow stem borehole w/SPT & $4 / 2 / 1984$ & 31.0 & 346775 & 6778763 & $\checkmark$ & $\checkmark$ & $\checkmark$ & $\nabla$ & $\square$ \\
\hline 2733 & 1763 & Hollow stem borehole w/SPT & $4 / 3 / 1984$ & 56.5 & 346724 & 6778764 & $\nabla$ & $\nabla$ & $\checkmark$ & $\nabla$ & $\square$ \\
\hline 2733 & 1811 & Solid stem borehole (no cores or SP & $11 / 21 / 1984$ & 15.0 & 347223 & 6778057 & $\square$ & $\square$ & $\square$ & $\square$ & $\square$ \\
\hline 2733 & 3321 & Water well & $5 / 18 / 1984$ & 114.0 & 347178 & 6778458 & $\nabla$ & $\square$ & $\square$ & $\nabla$ & $\square$ \\
\hline 2733 & 3964 & Water well & $1 / 14 / 1979$ & 107.0 & 347364 & 6778605 & $\boldsymbol{V}$ & $\square$ & $\square$ & $\nabla$ & $\square$ \\
\hline 2733 & 3967 & Water well & 4/6/1982 & 123.0 & 347276 & 6778640 & $\checkmark$ & $\square$ & $\square$ & $\sqrt{V}$ & $\square$ \\
\hline 2734 & 1771 & Hollow stem borehole w/SPT & $5 / 4 / 1995$ & 40.0 & 348205 & 6778639 & $\square$ & $\square$ & $\square$ & $\square$ & $\square$ \\
\hline 2734 & 3300 & Water well & $9 / 27 / 1983$ & 220.0 & 348195 & 6778755 & $\boldsymbol{V}$ & $\square$ & $\square$ & $\nabla$ & $\square$ \\
\hline 2734 & 3306 & Water well & $4 / 17 / 1985$ & 140.0 & 348179 & 6778725 & $\boldsymbol{V}$ & $\square$ & $\square$ & $\nabla$ & $\square$ \\
\hline 2734 & 3311 & Water well & $4 / 12 / 1979$ & 182.0 & 348173 & 6778601 & $\checkmark$ & $\square$ & $\square$ & $\checkmark$ & $\square$ \\
\hline 2734 & 3974 & Water well & $8 / 16 / 1973$ & 169.0 & 348073 & 6778698 & $\bar{v}$ & $\square$ & $\square$ & $\nabla$ & $\square$ \\
\hline 2735 & 3293 & Water well & $4 / 19 / 1985$ & 278.0 & 348990 & 6778783 & $\checkmark$ & $\square$ & $\square$ & $\checkmark$ & $\square$ \\
\hline 2735 & 3297 & Water well & $5 / 9 / 1985$ & 220.0 & 348346 & 6778779 & $\boldsymbol{v}$ & $\square$ & $\square$ & $\nabla$ & $\square$ \\
\hline 2735 & 3316 & Water well & 6/6/1977 & 179.0 & 348276 & 6778534 & 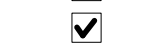 & $\square$ & $\square$ & $\nabla$ & $\square$ \\
\hline 2735 & 3319 & Water well & $9 / 1 / 1983$ & 107.0 & 348583 & 6778366 & $\checkmark$ & $\square$ & $\square$ & $\checkmark$ & $\square$ \\
\hline 2735 & 3331 & Water well & $10 / 1 / 1976$ & 258.0 & 348351 & 6778190 & $\bar{V}$ & $\square$ & $\square$ & $\nabla$ & $\square$ \\
\hline 2735 & 3942 & Water well & $8 / 5 / 1976$ & 108.0 & 348582 & 6778335 & $\boldsymbol{V}$ & $\square$ & $\square$ & $\nabla$ & $\square$ \\
\hline 2736 & 3299 & Water well & $10 / 10 / 1977$ & 280.0 & 349061 & 6778687 & 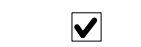 & $\square$ & $\square$ & $\sqrt{V}$ & $\square$ \\
\hline 2736 & 3305 & Water well & $6 / 22 / 1975$ & 117.0 & 349328 & 6778614 & $\nabla$ & $\square$ & $\square$ & $\nabla$ & $\square$ \\
\hline 2736 & 3308 & Water well & 6/8/1977 & 150.0 & 349460 & 6778546 & $\nabla$ & $\square$ & $\square$ & $\nabla$ & $\square$ \\
\hline 2736 & 3312 & Water well & 7/26/1975 & 136.0 & 349441 & 6778453 & 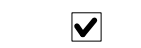 & $\square$ & $\square$ & $\nabla$ & $\square$ \\
\hline 2736 & 3315 & Water well & 8/26/1974 & 124.0 & 349365 & 6778426 & $\boldsymbol{V}$ & $\square$ & $\square$ & $\nabla$ & $\square$ \\
\hline 2736 & 3322 & Water well & $5 / 22 / 1984$ & 181.0 & 349640 & 6778197 & $\boldsymbol{V}$ & $\square$ & $\square$ & $\nabla$ & $\square$ \\
\hline 2736 & 3929 & Water well & $5 / 12 / 1979$ & 141.0 & 349367 & 6778116 & $\boldsymbol{V}$ & $\square$ & $\square$ & $\nabla$ & $\square$ \\
\hline 2736 & 3937 & Water well & 8/31/1977 & 109.0 & 349357 & 6778240 & $\nabla$ & $\square$ & $\square$ & $\nabla$ & $\square$ \\
\hline 2736 & 3941 & Water well & 7/14/1976 & 134.0 & 349375 & 6778301 & 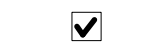 & $\square$ & $\square$ & $\nabla$ & $\square$ \\
\hline 2736 & 3945 & Water well & $5 / 29 / 1973$ & 104.0 & 349421 & 6778330 & 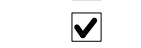 & $\square$ & $\square$ & $\nabla$ & $\square$ \\
\hline 2736 & 3946 & Water well & $5 / 24 / 1974$ & 115.0 & 349376 & 6778332 & $\nabla$ & $\square$ & $\square$ & $\checkmark$ & $\square$ \\
\hline 2736 & 3947 & Water well & $11 / 29 / 1974$ & 145.0 & 349648 & 6778383 & $\bar{v}$ & $\square$ & $\square$ & $\bar{v}$ & $\square$ \\
\hline 2736 & 3948 & Water well & $11 / 1 / 1973$ & 138.0 & 349498 & 6778389 & $\nabla$ & $\square$ & $\square$ & $\nabla$ & $\square$ \\
\hline 2736 & 3953 & Water well & 8/21/1976 & 135.0 & 349665 & 6778413 & $\boldsymbol{v}$ & $\square$ & $\square$ & $\nabla$ & $\square$ \\
\hline 2736 & 3954 & Water well & 8/15/1977 & 176.0 & 349065 & 6778439 & $\nabla$ & $\square$ & $\square$ & $\nabla$ & $\square$ \\
\hline
\end{tabular}




\begin{tabular}{|c|c|c|c|c|c|c|c|c|c|c|c|}
\hline \multirow[b]{2}{*}{$\begin{array}{l}\text { MOA } \\
\text { grid }\end{array}$} & \multirow[b]{2}{*}{$\begin{array}{l}\text { DGGS } \\
\text { number }\end{array}$} & \multirow[b]{2}{*}{ Hole Type } & \multirow[b]{2}{*}{$\begin{array}{c}\text { Date } \\
\text { Completed }\end{array}$} & \multirow[b]{2}{*}{$\begin{array}{c}\text { Total } \\
\text { depth }(\mathrm{ft})\end{array}$} & \multicolumn{2}{|c|}{ UTM coordinates (m) } & \multicolumn{5}{|c|}{ Data entered } \\
\hline & & & & & East & North & Lithology & SPT & $\begin{array}{c}\text { Sample } \\
\text { test }\end{array}$ & $\begin{array}{l}\text { Water } \\
\text { level }\end{array}$ & Velocity \\
\hline 2736 & 3961 & Water well & $8 / 27 / 1974$ & 130.0 & 349459 & 6778515 & $\checkmark$ & 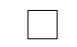 & $\square$ & $\nabla$ & $\square$ \\
\hline 2736 & 3962 & Water well & $8 / 26 / 1976$ & 149.0 & 349443 & 6778515 & $\checkmark$ & $\square$ & $\square$ & $\checkmark$ & $\square$ \\
\hline 2736 & 3963 & Water well & 6/8/1976 & 135.0 & 349369 & 6778519 & $\checkmark$ & & $\square$ & $\bar{\nabla}$ & \\
\hline 2736 & 3971 & Water well & $5 / 29 / 1979$ & 118.0 & 349193 & 6778619 & $\checkmark$ & $\square$ & $\square$ & $\bar{\nabla}$ & $\square$ \\
\hline 2736 & 3973 & Water well & 6/6/1977 & 146.0 & 349374 & 6778642 & $\checkmark$ & $\square$ & $\square$ & 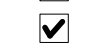 & $\square$ \\
\hline 2736 & 3978 & Water well & $2 / 2 / 1979$ & 140.0 & 349691 & 6778691 & $\checkmark$ & $\square$ & $\square$ & $\checkmark$ & $\square$ \\
\hline 2736 & 4502 & Water well & $5 / 25 / 1974$ & 115.0 & 349366 & 6778457 & $\checkmark$ & $\square$ & $\square$ & $\checkmark$ & $\square$ \\
\hline 2737 & 3303 & Water well & $4 / 27 / 1981$ & 206.0 & 350522 & 6778501 & $\checkmark$ & 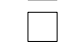 & $\square$ & $\bar{V}$ & $\square$ \\
\hline 2737 & 3307 & Water well & $6 / 21 / 1981$ & 215.0 & 350565 & 6778437 & $\checkmark$ & $\square$ & $\square$ & $\sqrt{\square}$ & $\square$ \\
\hline 2737 & 3309 & Water well & $5 / 8 / 1982$ & 173.0 & 350280 & 6778449 & $\checkmark$ & & $\square$ & $\vec{V}$ & $\square$ \\
\hline 2737 & 3310 & Water well & $5 / 28 / 1981$ & 223.0 & 350487 & 6778378 & $\checkmark$ & $\square$ & $\square$ & $\bar{V}$ & $\square$ \\
\hline 2737 & 3313 & Water well & 6/10/1977 & 264.0 & 350545 & 6778314 & $\checkmark$ & & $\square$ & $\bar{\nabla}$ & \\
\hline 2737 & 3325 & Water well & 6/6/1974 & 224.0 & 350191 & 6778112 & $\checkmark$ & $\square$ & $\square$ & $\vec{V}$ & $\square$ \\
\hline 2737 & 3330 & Water well & $5 / 15 / 1973$ & 258.0 & 350335 & 6777982 & $\checkmark$ & $\square$ & $\square$ & $\nabla$ & $\square$ \\
\hline 2737 & 3332 & Water well & 8/19/1977 & 251.0 & 350261 & 6777985 & $\nabla$ & $\square$ & $\square$ & $\nabla$ & $\square$ \\
\hline 2737 & 3333 & Water well & $6 / 16 / 1971$ & 214.0 & 350186 & 6777988 & $\checkmark$ & 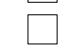 & $\square$ & $\checkmark$ & $\square$ \\
\hline 2737 & 3334 & Water well & $4 / 24 / 1973$ & 250.0 & 350334 & 6777951 & $\checkmark$ & & $\square$ & $\bar{V}$ & $\square$ \\
\hline 2737 & 3927 & Water well & $7 / 30 / 1965$ & 232.0 & 350130 & 6778083 & $\checkmark$ & L & $\square$ & $\checkmark$ & $\square$ \\
\hline 2737 & 3933 & Water well & $4 / 22 / 1973$ & 238.0 & 350161 & 6778113 & $\checkmark$ & $\square$ & $\square$ & $\checkmark$ & $\square$ \\
\hline 2737 & 3950 & Water well & $10 / 15 / 1981$ & 230.0 & 350577 & 6778374 & $\checkmark$ & $\square$ & $\square$ & $\nabla$ & $\square$ \\
\hline 2737 & 3951 & Water well & $12 / 5 / 1981$ & 225.0 & 350472 & 6778379 & $\checkmark$ & & $\square$ & $\checkmark$ & $\square$ \\
\hline 2737 & 3952 & Water well & $6 / 26 / 1982$ & 225.0 & 350367 & 6778383 & $\checkmark$ & $\checkmark$ & $\square$ & $\bar{V}$ & $\square$ \\
\hline 2737 & 3960 & Water well & $11 / 19 / 1981$ & 201.0 & 350386 & 6778475 & $\checkmark$ & $\square$ & $\square$ & $\checkmark$ & $\square$ \\
\hline 2737 & 4493 & Water well & 6/6/1979 & 175.0 & 350394 & 6778661 & $\checkmark$ & $\square$ & $\square$ & $\nabla$ & $\square$ \\
\hline 2737 & 4499 & Water well & $5 / 31 / 1974$ & 198.0 & 350355 & 6778446 & $\checkmark$ & & $\square$ & $\checkmark$ & $\square$ \\
\hline 2737 & 4500 & Water well & 6/6/1974 & 194.0 & 350278 & 6778387 & $\checkmark$ & & $\square$ & $\checkmark$ & $\square$ \\
\hline 2737 & 4501 & Water well & $10 / 5 / 1973$ & 216.0 & 350546 & 6778345 & $\checkmark$ & $\square$ & $\square$ & $\nabla$ & $\square$ \\
\hline 2737 & 4507 & Water well & $10 / 26 / 1972$ & 214.0 & 350245 & 6777955 & $\checkmark$ & & $\square$ & $\nabla$ & $\square$ \\
\hline 2738 & 3294 & Water well & $4 / 9 / 1969$ & 186.0 & 350988 & 6778512 & $\checkmark$ & $\square$ & $\square$ & $\checkmark$ & $\square$ \\
\hline 2738 & 3298 & Water well & $6 / 27 / 1972$ & 177.0 & 351226 & 6778471 & $\checkmark$ & & $\square$ & 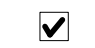 & $\square$ \\
\hline 2738 & 3972 & Water well & 8/1/1983 & 198.0 & 351080 & 6778570 & $\checkmark$ & $\square$ & $\square$ & $\bar{\nabla}$ & $\square$ \\
\hline 2738 & 3976 & Water well & $12 / 8 / 1977$ & 136.0 & 351367 & 6778620 & $\checkmark$ & $\square$ & $\square$ & $\checkmark$ & $\square$ \\
\hline 2738 & 3977 & Water well & $5 / 29 / 1974$ & 200.0 & 351112 & 6778630 & $\checkmark$ & $\square$ & $\square$ & $\bar{\nabla}$ & $\square$ \\
\hline 2738 & 4492 & Water well & $12 / 27 / 1976$ & 158.0 & 351186 & 6778596 & $\checkmark$ & $\square$ & $\square$ & $\bar{\nabla}$ & $\square$ \\
\hline 2738 & 4495 & Water well & 4/1/1983 & 158.0 & 351302 & 6778499 & $\checkmark$ & $\square$ & $\square$ & $\checkmark$ & $\square$ \\
\hline 2738 & 4496 & Water well & $10 / 25 / 1976$ & 149.0 & 351254 & 6778439 & $\checkmark$ & $\square$ & $\square$ & $\nabla$ & $\square$ \\
\hline
\end{tabular}




\begin{tabular}{|c|c|c|c|c|c|c|c|c|c|c|c|}
\hline \multirow[b]{2}{*}{$\begin{array}{c}\text { MOA } \\
\text { grid }\end{array}$} & \multirow[b]{2}{*}{$\begin{array}{c}\text { DGGS } \\
\text { number }\end{array}$} & \multirow[b]{2}{*}{ Hole Type } & \multirow[b]{2}{*}{$\begin{array}{c}\text { Date } \\
\text { Completed }\end{array}$} & \multirow[b]{2}{*}{$\begin{array}{c}\text { Total } \\
\text { depth (ft) }\end{array}$} & \multicolumn{2}{|c|}{ UTM coordinates (m) } & \multicolumn{5}{|c|}{ Data entered } \\
\hline & & & & & East & North & Lithology & SPT & $\begin{array}{c}\text { Sample } \\
\text { test }\end{array}$ & $\begin{array}{l}\text { Water } \\
\text { level }\end{array}$ & Velocity \\
\hline 2738 & 4497 & Water well & $3 / 11 / 1983$ & 105.0 & 351386 & 6778371 & $\checkmark$ & $\square$ & $\square$ & $\nabla$ & $\square$ \\
\hline 2738 & 4498 & Water well & 9/20/1978 & 285.0 & 351191 & 6778379 & $\checkmark$ & $\square$ & $\square$ & $\sqrt{ }$ & $\square$ \\
\hline 2738 & 4503 & Water well & $9 / 1 / 1970$ & 320.0 & 351094 & 6778198 & $\nabla$ & $\square$ & $\square$ & $\nabla$ & $\square$ \\
\hline 2738 & 4504 & Water well & $5 / 15 / 1976$ & 193.0 & 350959 & 6778203 & $\nabla$ & $\square$ & $\square$ & $\nabla$ & $\square$ \\
\hline 2738 & 4506 & Water well & $7 / 8 / 1965$ & 126.0 & 350978 & 6777924 & $\checkmark$ & $\square$ & $\square$ & $\nabla$ & $\square$ \\
\hline 2739 & 3296 & Water well & $10 / 12 / 1983$ & 238.0 & 351808 & 6778415 & 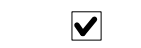 & $\square$ & $\square$ & $\nabla$ & $\square$ \\
\hline 2739 & 3327 & Water well & 7/17/1984 & 129.0 & 352071 & 6777909 & $\checkmark$ & $\square$ & $\square$ & $\checkmark$ & $\square$ \\
\hline 2739 & 3329 & Water well & 8/6/1972 & 128.0 & 352145 & 6777874 & $\boldsymbol{V}$ & $\square$ & $\square$ & $\nabla$ & $\square$ \\
\hline 2739 & 3915 & Water well & 6/6/1981 & 160.0 & 351965 & 6777882 & $\boldsymbol{V}$ & $\square$ & $\square$ & $\sqrt{ }$ & $\square$ \\
\hline 2739 & 3916 & Water well & $9 / 2 / 1978$ & 211.0 & 351755 & 6777891 & $\square$ & $\square$ & $\square$ & $\nabla$ & $\square$ \\
\hline 2739 & 3931 & Water well & $10 / 24 / 1983$ & 103.0 & 351553 & 6778054 & $\nabla$ & $\square$ & $\square$ & $\nabla$ & $\square$ \\
\hline 2739 & 3935 & Water well & $10 / 26 / 1982$ & 200.0 & 351465 & 6778120 & $\bar{V}$ & $\square$ & $\square$ & $\nabla$ & $\square$ \\
\hline 2739 & 3955 & Water well & $3 / 30 / 1983$ & 117.0 & 351550 & 6778364 & $\nabla$ & $\square$ & $\square$ & $\nabla$ & $\square$ \\
\hline 2739 & 3959 & Water well & $2 / 18 / 1983$ & 144.0 & 351538 & 6778427 & 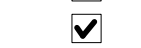 & $\square$ & $\square$ & $\checkmark$ & $\square$ \\
\hline 2739 & 3969 & Water well & $12 / 17 / 1982$ & 106.0 & 351556 & 6778488 & $\bar{v}$ & $\square$ & $\square$ & $\nabla$ & $\square$ \\
\hline 2739 & 3970 & Water well & $2 / 1 / 1983$ & 113.0 & 351587 & 6778518 & $\checkmark$ & $\square$ & $\square$ & $\checkmark$ & $\square$ \\
\hline 2739 & 3975 & Water well & $1 / 15 / 1967$ & 225.0 & 351814 & 6778570 & $\nabla$ & $\square$ & $\square$ & $\nabla$ & $\square$ \\
\hline 2740 & 3295 & Water well & $8 / 2 / 1981$ & 310.0 & 352837 & 6778310 & 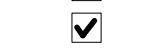 & $\square$ & $\square$ & $\nabla$ & $\square$ \\
\hline 2740 & 3304 & Water well & $5 / 28 / 1985$ & 430.0 & 352790 & 6778250 & $\checkmark$ & $\square$ & $\square$ & $\checkmark$ & $\square$ \\
\hline 2740 & 3317 & Water well & 7/1/1983 & 198.0 & 352378 & 6778082 & $\boldsymbol{V}$ & $\square$ & $\square$ & $\nabla$ & $\square$ \\
\hline 2740 & 3324 & Water well & 10/8/1980 & 269.0 & 352520 & 6777890 & $\boldsymbol{V}$ & $\square$ & $\square$ & $\nabla$ & $\square$ \\
\hline 2740 & 3864 & Water well & 8/1/1971 & 175.0 & 352683 & 6778193 & $\sqrt{v}$ & $\square$ & $\square$ & $\sqrt{V}$ & $\square$ \\
\hline 2740 & 3934 & Water well & $6 / 21 / 1982$ & 200.0 & 352797 & 6778064 & $\nabla$ & $\square$ & $\square$ & $\nabla$ & $\square$ \\
\hline 2741 & 1772 & Hollow stem borehole w/SPT & $5 / 8 / 1995$ & 31.5 & 353071 & 6778337 & $\square$ & $\square$ & $\square$ & $\square$ & $\square$ \\
\hline 2741 & 3314 & Water well & $11 / 5 / 1985$ & 261.0 & 353424 & 6778007 & $\boldsymbol{V}$ & $\square$ & $\square$ & $\nabla$ & $\square$ \\
\hline 2741 & 3318 & Water well & $8 / 4 / 1984$ & 370.0 & 353570 & 6777908 & $\boldsymbol{V}$ & $\square$ & $\square$ & $\nabla$ & $\square$ \\
\hline 2741 & 3320 & Water well & $5 / 15 / 1977$ & 230.0 & 353805 & 6777805 & $\boldsymbol{V}$ & $\square$ & $\square$ & $\nabla$ & $\square$ \\
\hline 2741 & 3914 & Water well & 6/17/1977 & 180.0 & 353597 & 6777814 & $\boldsymbol{V}$ & $\square$ & $\square$ & $\nabla$ & $\square$ \\
\hline 2741 & 3917 & Water well & 9/1/1981 & 300.0 & 353673 & 6777842 & $\nabla$ & $\square$ & $\square$ & $\nabla$ & $\square$ \\
\hline 2741 & 3921 & Water well & $5 / 19 / 1976$ & 258.0 & 353284 & 6777889 & 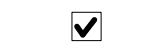 & $\square$ & $\square$ & $\nabla$ & $\square$ \\
\hline 2741 & 3922 & Water well & $5 / 24 / 1976$ & 200.0 & 353585 & 6777907 & $\nabla$ & $\square$ & $\square$ & $\nabla$ & $\square$ \\
\hline 2741 & 3925 & Water well & $5 / 18 / 1976$ & 548.0 & 353362 & 6777947 & $\nabla$ & $\square$ & $\square$ & $\checkmark$ & $\square$ \\
\hline 2741 & 3926 & Water well & 8/5/1977 & 355.0 & 353376 & 6777947 & $\bar{v}$ & $\square$ & $\square$ & $\square$ & $\square$ \\
\hline 2741 & 3958 & Water well & $10 / 13 / 1983$ & 135.0 & 353798 & 6778332 & $\nabla$ & $\square$ & $\square$ & $\nabla$ & $\square$ \\
\hline 2829 & 2270 & Water well & 8/19/1975 & 75.0 & 343615 & 6778162 & $\boldsymbol{v}$ & $\square$ & $\square$ & $\nabla$ & $\square$ \\
\hline 2829 & 2271 & Water well & $5 / 21 / 1973$ & 59.0 & 343739 & 6778109 & $\checkmark$ & $\square$ & $\square$ & $\nabla$ & $\square$ \\
\hline
\end{tabular}




\begin{tabular}{|c|c|c|c|c|c|c|c|c|c|c|c|}
\hline \multirow[b]{2}{*}{$\begin{array}{c}\text { MOA } \\
\text { grid }\end{array}$} & \multirow[b]{2}{*}{$\begin{array}{c}\text { DGGS } \\
\text { number }\end{array}$} & \multirow[b]{2}{*}{ Hole Type } & \multirow[b]{2}{*}{$\begin{array}{c}\text { Date } \\
\text { Completed }\end{array}$} & \multirow[b]{2}{*}{$\begin{array}{c}\text { Total } \\
\text { depth (ft) }\end{array}$} & \multicolumn{2}{|c|}{ UTM coordinates (m) } & \multicolumn{5}{|c|}{ Data entered } \\
\hline & & & & & East & North & Lithology & SPT & $\begin{array}{c}\text { Sample } \\
\text { test }\end{array}$ & $\begin{array}{l}\text { Water } \\
\text { level }\end{array}$ & Velocity \\
\hline 2829 & 2272 & Water well & $8 / 29 / 1975$ & 57.0 & 343831 & 6778073 & $\checkmark$ & $\square$ & $\square$ & $\nabla$ & $\square$ \\
\hline 2831 & 2273 & Hollow stem borehole w/SPT & $12 / 2 / 1976$ & 30.0 & 345023 & 6777946 & $\checkmark$ & $\checkmark$ & $\square$ & $\sqrt{V}$ & $\square$ \\
\hline 2831 & 2274 & Hollow stem borehole w/SPT & $12 / 9 / 1976$ & 28.5 & 345237 & 6777951 & $\boldsymbol{V}$ & $\checkmark$ & $\square$ & $\nabla$ & $\square$ \\
\hline 2831 & 2275 & Hollow stem borehole w/SPT & $12 / 2 / 1976$ & 31.0 & 345343 & 6777988 & $\boldsymbol{V}$ & $\checkmark$ & $\square$ & $\nabla$ & $\square$ \\
\hline 2831 & 2276 & Hollow stem borehole w/SPT & $7 / 24 / 1981$ & 26.5 & 345631 & 6778068 & $\checkmark$ & $\checkmark$ & $\square$ & $\nabla$ & $\square$ \\
\hline 2831 & 2277 & Hollow stem borehole w/SPT & $12 / 10 / 1976$ & 25.0 & 345687 & 6777762 & $\boldsymbol{V}$ & 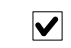 & $\square$ & $\nabla$ & $\square$ \\
\hline 2832 & 1654 & Hollow stem borehole, no SPT & $2 / 20 / 1980$ & 30.0 & 346477 & 6778043 & $\square$ & $\square$ & $\square$ & $\square$ & $\square$ \\
\hline 2832 & 1841 & Hollow stem borehole w/SPT & $1 / 21 / 1980$ & 50.0 & 346537 & 6778065 & $\nabla$ & $\nabla$ & $\nabla$ & $\nabla$ & $\square$ \\
\hline 2832 & 2278 & Hollow stem borehole w/SPT & $12 / 17 / 1984$ & 30.0 & 345926 & 6778030 & $\boldsymbol{V}$ & $\checkmark$ & $\square$ & $\nabla$ & $\square$ \\
\hline 2832 & 2279 & Hollow stem borehole w/SPT & 2/28/1990 & 26.5 & 345991 & 6777608 & $\boldsymbol{V}$ & $\nabla$ & $\square$ & $\nabla$ & $\square$ \\
\hline 2832 & 3378 & Water well & 7/7/1973 & 224.0 & 346012 & 6777858 & $\boldsymbol{V}$ & $\square$ & $\square$ & $\nabla$ & $\square$ \\
\hline 2832 & 3883 & Water well & $11 / 13 / 1982$ & 585.0 & 346042 & 6777857 & $\bar{V}$ & $\square$ & $\square$ & $\square$ & $\square$ \\
\hline 2833 & 1653 & Hollow stem borehole, no SPT & $2 / 20 / 1980$ & 25.0 & 346610 & 6777803 & $\square$ & $\square$ & $\square$ & $\square$ & $\square$ \\
\hline 2833 & 3413 & Water well & $3 / 20 / 1980$ & 550.0 & 347356 & 6777366 & $\checkmark$ & $\square$ & $\square$ & $\nabla$ & $\square$ \\
\hline 2833 & 3419 & Water well & 9/1/1964 & 228.0 & 346577 & 6777400 & $\boldsymbol{V}$ & $\square$ & $\square$ & $\square$ & $\square$ \\
\hline 2833 & 3899 & Water well & $11 / 13 / 1974$ & 103.0 & 347139 & 6777902 & $\checkmark$ & $\square$ & $\square$ & $\checkmark$ & $\square$ \\
\hline 2834 & 1812 & Solid stem borehole (no cores or SP & $11 / 6 / 1984$ & 14.5 & 347403 & 6778042 & $\square$ & $\square$ & $\square$ & $\square$ & $\square$ \\
\hline 2834 & 1813 & Solid stem borehole (no cores or SP & $11 / 26 / 1984$ & 15.0 & 347792 & 6778026 & $\square$ & $\square$ & $\square$ & $\square$ & $\square$ \\
\hline 2835 & 1633 & Hollow stem borehole w/SPT & $12 / 19 / 1995$ & 26.5 & 348272 & 6777888 & $\boldsymbol{V}$ & $\nabla$ & $\square$ & $\square$ & $\square$ \\
\hline 2835 & 1814 & Solid stem borehole (no cores or SP & $11 / 27 / 1984$ & 10.0 & 348414 & 6778003 & $\square$ & $\square$ & $\square$ & $\square$ & $\square$ \\
\hline 2835 & 1815 & Solid stem borehole (no cores or SP & $11 / 28 / 1984$ & 15.0 & 348871 & 6777976 & $\square$ & $\square$ & $\square$ & $\square$ & $\square$ \\
\hline 2835 & 3342 & Water well & $5 / 31 / 1978$ & 233.0 & 348893 & 6777920 & 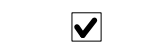 & $\square$ & $\square$ & $\sqrt{V}$ & $\square$ \\
\hline 2835 & 3346 & Water well & 6/23/1977 & 193.0 & 348772 & 6777863 & 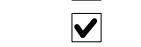 & $\square$ & $\square$ & $\nabla$ & $\square$ \\
\hline 2835 & 3350 & Water well & 6/7/1979 & 112.0 & 348650 & 6777837 & $\nabla$ & $\square$ & $\square$ & $\nabla$ & $\square$ \\
\hline 2835 & 3362 & Water well & 8/18/1978 & 138.0 & 348840 & 6777705 & 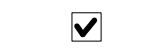 & $\square$ & $\square$ & $\nabla$ & $\square$ \\
\hline 2835 & 3374 & Water well & 7/26/1975 & 184.0 & 348820 & 6777582 & $\boldsymbol{V}$ & $\square$ & $\square$ & $\nabla$ & $\square$ \\
\hline 2835 & 3379 & Water well & $6 / 14 / 1975$ & 221.0 & 348833 & 6777550 & $\nabla$ & $\square$ & $\square$ & $\checkmark$ & $\square$ \\
\hline 2835 & 3383 & Water well & 6/14/1978 & 201.0 & 348696 & 6777494 & $\boldsymbol{V}$ & $\square$ & $\square$ & $\nabla$ & $\square$ \\
\hline 2835 & 3385 & Water well & 4/16/1977 & 167.0 & 348619 & 6777466 & $\nabla$ & $\square$ & $\square$ & $\nabla$ & $\square$ \\
\hline 2835 & 3386 & Water well & 8/18/1977 & 160.0 & 348530 & 6777470 & 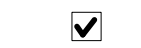 & $\square$ & $\square$ & $\nabla$ & $\square$ \\
\hline 2835 & 3391 & Water well & $10 / 30 / 1976$ & 100.5 & 348931 & 6777391 & $\nabla$ & $\square$ & $\square$ & $\nabla$ & $\square$ \\
\hline 2835 & 3410 & Water well & 9/15/1977 & 152.0 & 348595 & 6777251 & 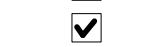 & $\square$ & $\square$ & $\checkmark$ & $\square$ \\
\hline 2835 & 3412 & Water well & $7 / 2 / 1978$ & 181.0 & 348833 & 6777210 & $\bar{v}$ & $\square$ & $\square$ & $\bar{v}$ & $\square$ \\
\hline 2835 & 3795 & Water well & 4/12/1978 & 175.0 & 348924 & 6777237 & $\nabla$ & $\square$ & $\square$ & $\nabla$ & $\square$ \\
\hline 2835 & 3796 & Water well & 4/21/1978 & 201.0 & 348909 & 6777237 & $\boldsymbol{v}$ & $\square$ & $\square$ & $\nabla$ & $\square$ \\
\hline 2835 & 3798 & Water well & 9/23/1977 & 150.0 & 348505 & 6777255 & $\checkmark$ & $\square$ & $\square$ & $\nabla$ & $\square$ \\
\hline
\end{tabular}




\begin{tabular}{|c|c|c|c|c|c|c|c|c|c|c|c|}
\hline \multirow[b]{2}{*}{$\begin{array}{c}\text { MOA } \\
\text { grid }\end{array}$} & \multirow[b]{2}{*}{$\begin{array}{c}\text { DGGS } \\
\text { number }\end{array}$} & \multirow[b]{2}{*}{ Hole Type } & \multirow[b]{2}{*}{$\begin{array}{c}\text { Date } \\
\text { Completed }\end{array}$} & \multirow[b]{2}{*}{$\begin{array}{c}\text { Total } \\
\text { depth (ft) }\end{array}$} & \multicolumn{2}{|c|}{ UTM coordinates (m) } & \multicolumn{5}{|c|}{ Data entered } \\
\hline & & & & & East & North & Lithology & SPT & $\begin{array}{c}\text { Sample } \\
\text { test }\end{array}$ & $\begin{array}{l}\text { Water } \\
\text { level }\end{array}$ & Velocity \\
\hline 2835 & 3802 & Water well & $10 / 5 / 1977$ & 155.0 & 348598 & 6777312 & $\checkmark$ & $\square$ & $\square$ & $\nabla$ & $\square$ \\
\hline 2835 & 3809 & Water well & $12 / 16 / 1977$ & 175.0 & 348749 & 6777337 & $\checkmark$ & $\square$ & $\square$ & $\sqrt{ }$ & $\square$ \\
\hline 2835 & 3810 & Water well & $12 / 18 / 1977$ & 186.0 & 348733 & 6777338 & $\nabla$ & $\square$ & $\square$ & $\nabla$ & $\square$ \\
\hline 2835 & 3811 & Water well & $11 / 11 / 1977$ & 177.0 & 348614 & 6777343 & $\nabla$ & $\square$ & $\square$ & $\nabla$ & $\square$ \\
\hline 2835 & 3816 & Water well & $11 / 8 / 1977$ & 175.0 & 348825 & 6777365 & $\checkmark$ & $\square$ & $\square$ & $\nabla$ & $\square$ \\
\hline 2835 & 3830 & Water well & $1 / 4 / 1978$ & 160.0 & 348618 & 6777436 & 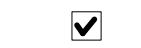 & $\square$ & $\square$ & $\nabla$ & $\square$ \\
\hline 2835 & 3832 & Water well & $10 / 16 / 1975$ & 183.0 & 348829 & 6777457 & $\checkmark$ & $\square$ & $\square$ & $\checkmark$ & $\square$ \\
\hline 2835 & 3841 & Water well & $6 / 30 / 1975$ & 179.0 & 348712 & 6777524 & $\boldsymbol{V}$ & $\square$ & $\square$ & $\nabla$ & $\square$ \\
\hline 2835 & 3842 & Water well & $11 / 29 / 1976$ & 101.0 & 348442 & 6777536 & $\boldsymbol{V}$ & $\square$ & $\square$ & $\square$ & $\square$ \\
\hline 2835 & 3852 & Water well & 8/20/1974 & 179.0 & 348743 & 6777554 & $\checkmark$ & $\square$ & $\square$ & $V$ & $\square$ \\
\hline 2835 & 3853 & Water well & 10/7/1974 & 183.0 & 348728 & 6777555 & 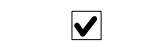 & $\square$ & $\square$ & $\nabla$ & $\square$ \\
\hline 2835 & 3855 & Water well & $5 / 9 / 1980$ & 210.0 & 348653 & 6777558 & $\bar{V}$ & $\square$ & $\square$ & $\nabla$ & $\square$ \\
\hline 2835 & 3858 & Water well & $6 / 14 / 1975$ & 187.0 & 348834 & 6777581 & $\nabla$ & $\square$ & $\square$ & $\nabla$ & $\square$ \\
\hline 2835 & 3866 & Water well & 8/29/1974 & 185.0 & 348837 & 6777643 & 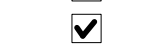 & $\square$ & $\square$ & $\checkmark$ & $\square$ \\
\hline 2835 & 3867 & Water well & $9 / 24 / 1974$ & 175.0 & 348522 & 6777656 & $\bar{v}$ & $\square$ & $\square$ & $\nabla$ & $\square$ \\
\hline 2835 & 3872 & Water well & $3 / 28 / 1974$ & 175.0 & 348510 & 6777719 & $\checkmark$ & $\square$ & $\square$ & $\checkmark$ & $\square$ \\
\hline 2835 & 3880 & Water well & 6/23/1976 & 180.0 & 348661 & 6777744 & $\boldsymbol{v}$ & $\square$ & $\square$ & $\nabla$ & $\square$ \\
\hline 2835 & 3881 & Water well & $10 / 14 / 1974$ & 171.0 & 348542 & 6777749 & 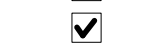 & $\square$ & $\square$ & $\nabla$ & $\square$ \\
\hline 2835 & 3882 & Water well & 7/6/1976 & 172.0 & 348437 & 6777753 & $\checkmark$ & $\square$ & $\square$ & $\checkmark$ & $\square$ \\
\hline 2835 & 3891 & Water well & $7 / 20 / 1974$ & 178.0 & 348737 & 6777771 & $\bar{V}$ & $\square$ & $\square$ & $\nabla$ & $\square$ \\
\hline 2835 & 3898 & Water well & 8/6/1980 & 203.0 & 348770 & 6777832 & $\boldsymbol{V}$ & $\square$ & $\square$ & $\nabla$ & $\square$ \\
\hline 2835 & 3902 & Water well & $5 / 18 / 1968$ & 202.0 & 348936 & 6777856 & 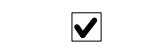 & $\square$ & $\square$ & $\sqrt{V}$ & $\square$ \\
\hline 2835 & 3909 & Water well & 9/13/1982 & 182.0 & 348265 & 6777947 & $\nabla$ & $\square$ & $\square$ & $\nabla$ & $\square$ \\
\hline 2835 & 3912 & Water well & $6 / 5 / 1976$ & 256.0 & 348969 & 6777947 & $\nabla$ & $\square$ & $\square$ & $\nabla$ & $\square$ \\
\hline 2835 & 4509 & Water well & 8/3/1977 & 115.0 & 348931 & 6777732 & 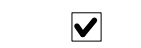 & $\square$ & $\square$ & $\nabla$ & $\square$ \\
\hline 2835 & 4513 & Water well & $10 / 1 / 1980$ & 101.0 & 348638 & 6777559 & $\boldsymbol{V}$ & $\square$ & $\square$ & $\nabla$ & $\square$ \\
\hline 2835 & 4515 & Water well & 7/10/1975 & 177.0 & 348724 & 6777462 & $\boldsymbol{V}$ & $\square$ & $\square$ & $\nabla$ & $\square$ \\
\hline 2836 & 1816 & Solid stem borehole (no cores or SP & $11 / 8 / 1984$ & 9.5 & 349072 & 6777972 & $\square$ & $\square$ & $\square$ & $\square$ & $\square$ \\
\hline 2836 & 3345 & Water well & $7 / 12 / 1971$ & 178.0 & 349548 & 6777798 & $\nabla$ & $\square$ & $\square$ & $\nabla$ & $\square$ \\
\hline 2836 & 3351 & Water well & 7/7/1975 & 173.0 & 349545 & 6777737 & $\nabla$ & $\square$ & $\square$ & $\nabla$ & $\square$ \\
\hline 2836 & 3356 & Water well & $10 / 11 / 1977$ & 135.0 & 349035 & 6777728 & $\nabla$ & $\square$ & $\square$ & $\checkmark$ & $\square$ \\
\hline 2836 & 3357 & Water well & $5 / 5 / 1978$ & 207.0 & 349751 & 6777635 & $\nabla$ & $\square$ & $\square$ & $\checkmark$ & $\square$ \\
\hline 2836 & 3363 & Water well & 8/24/1979 & 180.0 & 349645 & 6777608 & 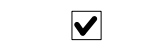 & $\square$ & $\square$ & $\square$ & $\square$ \\
\hline 2836 & 3364 & Water well & 10/5/1976 & 145.0 & 349570 & 6777612 & $\nabla$ & $\square$ & $\square$ & $\nabla$ & $\square$ \\
\hline 2836 & 3366 & Water well & 6/22/1979 & 125.0 & 349346 & 6777621 & $\boldsymbol{v}$ & $\square$ & $\square$ & $\nabla$ & $\square$ \\
\hline 2836 & 3367 & Water well & $10 / 20 / 1975$ & 231.0 & 349733 & 6777574 & $\nabla$ & $\square$ & $\square$ & $\square$ & $\square$ \\
\hline
\end{tabular}




\begin{tabular}{|c|c|c|c|c|c|c|c|c|c|c|c|}
\hline \multirow[b]{2}{*}{$\begin{array}{l}\text { MOA } \\
\text { grid }\end{array}$} & \multirow[b]{2}{*}{$\begin{array}{l}\text { DGGS } \\
\text { number }\end{array}$} & \multirow[b]{2}{*}{ Hole Type } & \multirow[b]{2}{*}{$\begin{array}{c}\text { Date } \\
\text { Completed }\end{array}$} & \multirow[b]{2}{*}{$\begin{array}{c}\text { Total } \\
\text { depth }(\mathrm{ft})\end{array}$} & \multicolumn{2}{|c|}{ UTM coordinates (m) } & \multicolumn{5}{|c|}{ Data entered } \\
\hline & & & & & East & North & Lithology & SPT & $\begin{array}{c}\text { Sample } \\
\text { test }\end{array}$ & $\begin{array}{l}\text { Water } \\
\text { level }\end{array}$ & Velocity \\
\hline 2836 & 3396 & Water well & $7 / 15 / 1981$ & 332.0 & 349525 & 6777273 & $\checkmark$ & 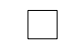 & $\square$ & $\nabla$ & $\square$ \\
\hline 2836 & 3398 & Water well & $6 / 1 / 1975$ & 187.0 & 349301 & 6777282 & $\checkmark$ & $\square$ & $\square$ & $\sqrt{ }$ & $\square$ \\
\hline 2836 & 3399 & Water well & 2/8/1985 & 117.0 & 349122 & 6777290 & $\checkmark$ & & $\square$ & $\bar{V}$ & \\
\hline 2836 & 3401 & Water well & $11 / 10 / 1978$ & 410.0 & 349628 & 6777207 & $\nabla$ & $\square$ & $\square$ & $\checkmark$ & $\square$ \\
\hline 2836 & 3404 & Water well & $9 / 28 / 1979$ & 240.0 & 349418 & 6777216 & $\checkmark$ & $\square$ & $\square$ & 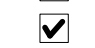 & $\square$ \\
\hline 2836 & 3405 & Water well & 8/3/1977 & 205.0 & 349029 & 6777232 & $\checkmark$ & $\square$ & $\square$ & $\checkmark$ & $\square$ \\
\hline 2836 & 3407 & Water well & $6 / 18 / 1984$ & 507.0 & 349417 & 6777185 & $\checkmark$ & $\square$ & $\square$ & $\checkmark$ & $\square$ \\
\hline 2836 & 3408 & Water well & $10 / 13 / 1978$ & 201.0 & 349327 & 6777188 & $\checkmark$ & & $\square$ & $\bar{\nabla}$ & $\square$ \\
\hline 2836 & 3409 & Water well & 12/1/1981 & 205.0 & 349730 & 6777140 & $\checkmark$ & $\square$ & $\square$ & $\sqrt{\square}$ & $\square$ \\
\hline 2836 & 3808 & Water well & $10 / 14 / 1978$ & 248.0 & 349632 & 6777299 & $\checkmark$ & & $\square$ & $\checkmark$ & $\square$ \\
\hline 2836 & 3814 & Water well & $6 / 23 / 1978$ & 286.0 & 349618 & 6777331 & $\checkmark$ & $\square$ & $\square$ & $\bar{V}$ & $\square$ \\
\hline 2836 & 3815 & Water well & $6 / 23 / 1970$ & 266.0 & 349469 & 6777337 & $\square$ & & $\square$ & $\checkmark$ & $\square$ \\
\hline 2836 & 3829 & Water well & $10 / 14 / 1977$ & 248.0 & 349636 & 6777392 & $\checkmark$ & $\square$ & $\square$ & $\checkmark$ & $\square$ \\
\hline 2836 & 3840 & Water well & 8/26/1974 & 225.0 & 349131 & 6777507 & $\checkmark$ & $\square$ & $\square$ & $\checkmark$ & $\square$ \\
\hline 2836 & 3865 & Water well & $10 / 1 / 1980$ & 155.0 & 349136 & 6777630 & $\checkmark$ & $\square$ & $\square$ & $\checkmark$ & $\square$ \\
\hline 2836 & 3871 & Water well & $10 / 23 / 1975$ & 103.0 & 349349 & 6777683 & $\checkmark$ & 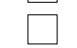 & $\square$ & $\checkmark$ & $\square$ \\
\hline 2836 & 3893 & Water well & $9 / 23 / 1975$ & 163.0 & 349666 & 6777762 & $\checkmark$ & & $\square$ & $\bar{V}$ & $\square$ \\
\hline 2836 & 3897 & Water well & $8 / 16 / 1982$ & 179.0 & 349743 & 6777790 & $\checkmark$ & $\square$ & $\square$ & $\vec{\nabla}$ & $\square$ \\
\hline 2836 & 3907 & Water well & $7 / 3 / 1981$ & 156.0 & 349657 & 6777887 & $\checkmark$ & $\square$ & $\square$ & $\checkmark$ & $\square$ \\
\hline 2836 & 3908 & Water well & 11/30/1977 & 115.0 & 349163 & 6777908 & $\checkmark$ & $\square$ & $\square$ & $\square$ & $\square$ \\
\hline 2836 & 4510 & Water well & $7 / 9 / 1974$ & 150.0 & 349660 & 6777608 & $\checkmark$ & & $\square$ & $\bar{\nabla}$ & $\square$ \\
\hline 2836 & 4512 & Water well & 7/14/1982 & 140.0 & 349325 & 6777498 & $\checkmark$ & $\checkmark$ & $\square$ & $\bar{V}$ & $\square$ \\
\hline 2836 & 4514 & Water well & $4 / 29 / 1982$ & 110.0 & 349026 & 6777511 & $\checkmark$ & $\square$ & $\square$ & $\nabla$ & $\square$ \\
\hline 2837 & 3335 & Water well & $8 / 24 / 1972$ & 245.0 & 350063 & 6777931 & $\checkmark$ & $\square$ & $\square$ & $\square$ & $\square$ \\
\hline 2837 & 3344 & Water well & $5 / 29 / 1984$ & 232.0 & 350130 & 6777743 & $\checkmark$ & & $\square$ & $\bar{\nabla}$ & $\square$ \\
\hline 2837 & 3353 & Water well & $7 / 12 / 1973$ & 400.0 & 350022 & 6777654 & $\square$ & $\square$ & $\square$ & $\bar{V}$ & $\square$ \\
\hline 2837 & 3365 & Water well & $5 / 13 / 1972$ & 209.0 & 349868 & 6777568 & $\checkmark$ & $\square$ & $\square$ & $\nabla$ & $\square$ \\
\hline 2837 & 3370 & Water well & 4/3/1985 & 258.0 & 350223 & 6777460 & $\checkmark$ & & $\square$ & $\nabla$ & $\square$ \\
\hline 2837 & 3371 & Water well & 6/7/1973 & 355.0 & 350118 & 6777464 & $\checkmark$ & $\square$ & $\square$ & $\square$ & $\square$ \\
\hline 2837 & 3380 & Water well & $10 / 14 / 1983$ & 390.0 & 350234 & 6777367 & $\checkmark$ & $\square$ & $\square$ & $\bar{\nabla}$ & $\square$ \\
\hline 2837 & 3388 & Water well & $7 / 27 / 1981$ & 342.0 & 349827 & 6777322 & $\checkmark$ & $\square$ & $\square$ & $\nabla$ & $\square$ \\
\hline 2837 & 3395 & Water well & $5 / 20 / 1980$ & 200.0 & 349825 & 6777260 & $\checkmark$ & $\square$ & $\square$ & $\checkmark$ & $\square$ \\
\hline 2837 & 3397 & Water well & $5 / 3 / 1979$ & 122.0 & 350316 & 6777177 & $\checkmark$ & $\square$ & $\square$ & $\bar{\nabla}$ & $\square$ \\
\hline 2837 & 3667 & Water well & $10 / 1 / 1971$ & 222.0 & 350012 & 6777779 & $\checkmark$ & $\square$ & $\square$ & $\bar{\nabla}$ & $\square$ \\
\hline 2837 & 3794 & Water well & $10 / 26 / 1976$ & 400.0 & 350421 & 6777173 & $\checkmark$ & $\square$ & $\square$ & $\vec{V}$ & $\square$ \\
\hline 2837 & 3861 & Water well & 10/11/1977 & 348.0 & 350062 & 6777560 & $\boldsymbol{V}$ & $\square$ & $\square$ & $\nabla$ & $\square$ \\
\hline
\end{tabular}




\begin{tabular}{|c|c|c|c|c|c|c|c|c|c|c|c|}
\hline \multirow[b]{2}{*}{$\begin{array}{l}\text { MOA } \\
\text { grid }\end{array}$} & \multirow[b]{2}{*}{$\begin{array}{l}\text { DGGS } \\
\text { number }\end{array}$} & \multirow[b]{2}{*}{ Hole Type } & \multirow[b]{2}{*}{$\begin{array}{c}\text { Date } \\
\text { Completed }\end{array}$} & \multirow[b]{2}{*}{$\begin{array}{c}\text { Total } \\
\text { depth }(\mathrm{ft})\end{array}$} & \multicolumn{2}{|c|}{ UTM coordinates (m) } & \multicolumn{5}{|c|}{ Data entered } \\
\hline & & & & & East & North & Lithology & SPT & $\begin{array}{c}\text { Sample } \\
\text { test }\end{array}$ & $\begin{array}{l}\text { Water } \\
\text { level }\end{array}$ & Velocity \\
\hline 2837 & 3862 & Water well & $11 / 12 / 1977$ & 340.0 & 350047 & 6777561 & $\checkmark$ & - & $\square$ & $\checkmark$ & $\square$ \\
\hline 2837 & 3868 & Water well & $6 / 20 / 1973$ & 275.0 & 350499 & 6777603 & $\checkmark$ & $\square$ & $\square$ & $\checkmark$ & $\square$ \\
\hline 2837 & 3870 & Water well & $3 / 28 / 1976$ & 405.0 & 349947 & 6777658 & $\checkmark$ & $\square$ & $\square$ & $\bar{V}$ & \\
\hline 2837 & 3890 & Water well & $5 / 15 / 1965$ & 187.0 & 350084 & 6777714 & $\nabla$ & $\square$ & $\square$ & $\checkmark$ & $\square$ \\
\hline 2837 & 3896 & Water well & $7 / 8 / 1982$ & 220.0 & 349847 & 6777786 & $\checkmark$ & $\square$ & $\square$ & 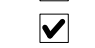 & $\square$ \\
\hline 2838 & 3339 & Water well & $10 / 15 / 1976$ & 213.0 & 350747 & 6777778 & $\checkmark$ & $\square$ & $\square$ & $\checkmark$ & $\square$ \\
\hline 2838 & 3340 & Water well & $11 / 2 / 1978$ & 220.0 & 351089 & 6777733 & $\checkmark$ & $\square$ & $\square$ & $\checkmark$ & $\square$ \\
\hline 2838 & 3347 & Water well & $8 / 30 / 1983$ & 143.0 & 350830 & 6777620 & $\checkmark$ & & $\square$ & $\checkmark$ & $\square$ \\
\hline 2838 & 3354 & Water well & $5 / 26 / 1976$ & 460.0 & 350932 & 6777554 & $\checkmark$ & $\square$ & $\square$ & $\sqrt{\square}$ & $\square$ \\
\hline 2838 & 3377 & Water well & 7/3/1981 & 245.0 & 351042 & 6777332 & $\checkmark$ & & $\square$ & $\checkmark$ & $\square$ \\
\hline 2838 & 3381 & Water well & $5 / 6 / 1985$ & 329.0 & 350682 & 6777317 & $\checkmark$ & $\square$ & $\square$ & $\bar{V}$ & $\square$ \\
\hline 2838 & 3384 & Water well & $10 / 26 / 1983$ & 200.0 & 351143 & 6777235 & $\checkmark$ & & $\square$ & $\bar{\nabla}$ & $\square$ \\
\hline 2838 & 3392 & Water well & $5 / 3 / 1984$ & 244.0 & 351349 & 6777134 & $\checkmark$ & $\square$ & $\square$ & $\nabla$ & $\square$ \\
\hline 2838 & 3393 & Water well & $6 / 19 / 1982$ & 200.0 & 350751 & 6777190 & $\checkmark$ & $\square$ & $\square$ & $\checkmark$ & $\square$ \\
\hline 2838 & 3394 & Water well & $4 / 14 / 1981$ & 238.0 & 351347 & 6777103 & $\checkmark$ & $\square$ & $\square$ & $\checkmark$ & $\square$ \\
\hline 2838 & 3783 & Water well & $3 / 1 / 1983$ & 242.0 & 351228 & 6777108 & $\checkmark$ & - & $\square$ & $\checkmark$ & $\square$ \\
\hline 2838 & 3784 & Water well & $5 / 26 / 1976$ & 167.0 & 350929 & 6777120 & $\checkmark$ & & $\square$ & $\boldsymbol{V}$ & $\square$ \\
\hline 2838 & 3787 & Water well & $8 / 25 / 1981$ & 200.0 & 350838 & 6777124 & $\checkmark$ & ᄂ & $\square$ & $\checkmark$ & $\square$ \\
\hline 2838 & 3788 & Water well & $3 / 5 / 1976$ & 326.0 & 350719 & 6777129 & $\checkmark$ & $\checkmark$ & $\square$ & $\square$ & $\square$ \\
\hline 2838 & 3789 & Water well & $12 / 10 / 1972$ & 300.0 & 350629 & 6777133 & $\checkmark$ & $\square$ & $\square$ & $\checkmark$ & $\square$ \\
\hline 2838 & 3800 & Water well & $5 / 25 / 1982$ & 180.0 & 350842 & 6777217 & $\checkmark$ & & $\square$ & $\bar{\nabla}$ & $\square$ \\
\hline 2838 & 3801 & Water well & 10/30/1975 & 284.0 & 350738 & 6777221 & $\checkmark$ & 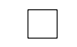 & $\square$ & $\square$ & $\square$ \\
\hline 2838 & 3806 & Water well & $5 / 18 / 1974$ & 290.0 & 350650 & 6777256 & $\checkmark$ & & $\square$ & $\nabla$ & $\square$ \\
\hline 2838 & 3807 & Water well & $6 / 7 / 1982$ & 137.0 & 350634 & 6777257 & $\checkmark$ & $\square$ & $\square$ & $\checkmark$ & $\square$ \\
\hline 2838 & 3826 & Water well & $10 / 16 / 1981$ & 538.0 & 351267 & 6777323 & $\checkmark$ & & $\square$ & $\square$ & $\square$ \\
\hline 2838 & 3827 & Water well & $10 / 17 / 1975$ & 175.0 & 350893 & 6777339 & $\checkmark$ & & $\square$ & $\nabla$ & $\square$ \\
\hline 2838 & 3828 & Water well & $8 / 15 / 1977$ & 181.0 & 350833 & 6777341 & $\checkmark$ & $\square$ & $\square$ & $\square$ & $\square$ \\
\hline 2838 & 3831 & Water well & $8 / 15 / 1976$ & 305.0 & 350714 & 6777377 & $\checkmark$ & & $\square$ & $\nabla$ & $\square$ \\
\hline 2838 & 3839 & Water well & $8 / 2 / 1974$ & 247.0 & 350972 & 6777428 & $\checkmark$ & & $\square$ & $\checkmark$ & $\square$ \\
\hline 2838 & 3847 & Water well & $1 / 1 / 1975$ & 200.0 & 350838 & 6777465 & $\checkmark$ & 5 & $\square$ & $\bar{\nabla}$ & $\square$ \\
\hline 2838 & 3848 & Water well & 2/12/1976 & 271.0 & 350734 & 6777469 & $\checkmark$ & $\square$ & $\square$ & $\nabla$ & $\square$ \\
\hline 2838 & 3850 & Water well & $10 / 12 / 1981$ & 260.0 & 350659 & 6777472 & $\checkmark$ & $\square$ & $\square$ & $\checkmark$ & $\square$ \\
\hline 2838 & 3878 & Water well & $6 / 14 / 1982$ & 164.0 & 350861 & 6777650 & $\checkmark$ & $\square$ & $\square$ & $\nabla$ & $\square$ \\
\hline 2838 & 3879 & Water well & 7/11/1979 & 216.0 & 350771 & 6777654 & $\checkmark$ & $\square$ & $\square$ & $\square$ & $\square$ \\
\hline 2838 & 3892 & Water well & $10 / 24 / 1973$ & 302.0 & 350983 & 6777706 & $\square$ & $\square$ & $\square$ & $\boldsymbol{V}$ & $\square$ \\
\hline 2838 & 3895 & Water well & 11/10/1981 & 179.0 & 350775 & 6777746 & $\checkmark$ & $\square$ & $\square$ & $\nabla$ & $\square$ \\
\hline
\end{tabular}




\begin{tabular}{|c|c|c|c|c|c|c|c|c|c|c|c|}
\hline \multirow[b]{2}{*}{$\begin{array}{l}\text { MOA } \\
\text { grid }\end{array}$} & \multirow[b]{2}{*}{$\begin{array}{l}\text { DGGS } \\
\text { number }\end{array}$} & \multirow[b]{2}{*}{ Hole Type } & \multirow[b]{2}{*}{$\begin{array}{c}\text { Date } \\
\text { Completed }\end{array}$} & \multirow[b]{2}{*}{$\begin{array}{c}\text { Total } \\
\text { depth }(\mathrm{ft})\end{array}$} & \multicolumn{2}{|c|}{ UTM coordinates (m) } & \multicolumn{5}{|c|}{ Data entered } \\
\hline & & & & & East & North & Lithology & SPT & $\begin{array}{c}\text { Sample } \\
\text { test }\end{array}$ & $\begin{array}{l}\text { Water } \\
\text { level }\end{array}$ & Velocity \\
\hline 2838 & 3900 & Water well & $10 / 22 / 1976$ & 248.0 & 350851 & 6777774 & $\square$ & $\square$ & $\square$ & $\nabla$ & $\square$ \\
\hline 2838 & 3901 & Water well & $10 / 19 / 1976$ & 323.0 & 350822 & 6777775 & $\square$ & $\square$ & $\square$ & $\checkmark$ & $\square$ \\
\hline 2838 & 3906 & Water well & $7 / 9 / 1973$ & 180.0 & 351093 & 6777826 & $\checkmark$ & 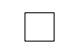 & $\square$ & $\bar{V}$ & \\
\hline 2839 & 3336 & Water well & $1 / 1 / 1984$ & 146.0 & 351690 & 6777739 & $\checkmark$ & $\square$ & $\square$ & $\boldsymbol{V}$ & $\square$ \\
\hline 2839 & 3338 & Water well & $5 / 27 / 1981$ & 180.0 & 352061 & 6777661 & $\checkmark$ & $\square$ & $\square$ & 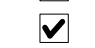 & $\square$ \\
\hline 2839 & 3343 & Water well & 7/7/1978 & 140.0 & 351967 & 6777572 & $\checkmark$ & $\square$ & $\square$ & $\checkmark$ & $\square$ \\
\hline 2839 & 3348 & Water well & $1 / 1 / 1986$ & 150.0 & 352098 & 6777474 & $\checkmark$ & $\square$ & $\square$ & $\square$ & $\square$ \\
\hline 2839 & 3349 & Water well & $7 / 9 / 1981$ & 400.0 & 352083 & 6777475 & $\checkmark$ & & $\square$ & $\bar{\nabla}$ & $\square$ \\
\hline 2839 & 3352 & Water well & $6 / 3 / 1981$ & 160.0 & 351904 & 6777482 & $\checkmark$ & $\square$ & $\square$ & $\sqrt{\square}$ & $\square$ \\
\hline 2839 & 3355 & Water well & $10 / 17 / 1972$ & 250.0 & 352125 & 6777411 & $\checkmark$ & & $\square$ & $\checkmark$ & $\square$ \\
\hline 2839 & 3358 & Water well & 7/30/1980 & 405.0 & 352169 & 6777378 & $\checkmark$ & $\square$ & $\square$ & $\bar{V}$ & $\square$ \\
\hline 2839 & 3359 & Water well & 8/4/1975 & 325.0 & 352094 & 6777381 & $\checkmark$ & & $\square$ & $\bar{\nabla}$ & $\square$ \\
\hline 2839 & 3361 & Water well & $8 / 27 / 1981$ & 310.0 & 352064 & 6777382 & $\checkmark$ & $\square$ & $\square$ & $\square$ & $\square$ \\
\hline 2839 & 3368 & Water well & 8/7/1978 & 305.0 & 352090 & 6777288 & $\checkmark$ & $\square$ & $\square$ & $\nabla$ & $\square$ \\
\hline 2839 & 3369 & Water well & 7/17/1978 & 298.0 & 352075 & 6777289 & $\checkmark$ & $\square$ & $\square$ & $\checkmark$ & $\square$ \\
\hline 2839 & 3372 & Water well & $11 / 29 / 1978$ & 545.0 & 352044 & 6777259 & $\checkmark$ & - & $\square$ & $\checkmark$ & $\square$ \\
\hline 2839 & 3375 & Water well & 10/1/1976 & 238.0 & 352072 & 6777227 & $\checkmark$ & & $\square$ & $\bar{V}$ & $\square$ \\
\hline 2839 & 3781 & Water well & 7/19/1978 & 200.0 & 352036 & 6777074 & $\checkmark$ & ᄂ & $\square$ & $\vec{\nabla}$ & $\square$ \\
\hline 2839 & 3782 & Water well & $1 / 1 / 1980$ & 210.0 & 351842 & 6777082 & $\checkmark$ & $\square$ & $\square$ & $\square$ & $\square$ \\
\hline 2839 & 3793 & Water well & $10 / 1 / 1976$ & 251.0 & 352022 & 6777105 & $\checkmark$ & $\square$ & $\square$ & $\checkmark$ & $\square$ \\
\hline 2839 & 3799 & Water well & 6/19/1974 & 280.0 & 352023 & 6777136 & $\checkmark$ & & $\square$ & $\bar{\nabla}$ & $\square$ \\
\hline 2839 & 3804 & Water well & 8/13/1982 & 280.0 & 352071 & 6777196 & $\checkmark$ & 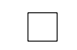 & $\square$ & $\nabla$ & $\square$ \\
\hline 2839 & 3805 & Water well & 9/1/1979 & 260.0 & 351936 & 6777202 & $\checkmark$ & & $\square$ & $\nabla$ & $\square$ \\
\hline 2839 & 3817 & Water well & 7/13/1978 & 338.0 & 352074 & 6777258 & $\checkmark$ & $\square$ & $\square$ & $\checkmark$ & $\square$ \\
\hline 2839 & 3824 & Water well & 7/19/1982 & 201.0 & 351836 & 6777299 & $\checkmark$ & & $\square$ & $\checkmark$ & $\square$ \\
\hline 2839 & 3835 & Water well & $8 / 4 / 1975$ & 225.0 & 352064 & 6777382 & $\square$ & $\square$ & $\square$ & $\bar{V}$ & $\square$ \\
\hline 2839 & 3836 & Water well & 2/27/1979 & 338.0 & 351975 & 6777386 & $\checkmark$ & $\square$ & $\square$ & $\checkmark$ & $\square$ \\
\hline 2839 & 3837 & Water well & $12 / 20 / 1978$ & 400.0 & 351869 & 6777390 & $\square$ & & $\square$ & $\nabla$ & $\square$ \\
\hline 2839 & 3838 & Water well & 2/20/1979 & 218.0 & 351854 & 6777391 & $\checkmark$ & $\square$ & $\square$ & $\checkmark$ & $\square$ \\
\hline 2839 & 3857 & Water well & 6/1/1972 & 275.0 & 351857 & 6777453 & $\checkmark$ & $\square$ & $\square$ & $\square$ & $\square$ \\
\hline 2839 & 3874 & Water well & $10 / 23 / 1977$ & 418.0 & 352058 & 6777599 & $\square$ & $\square$ & $\square$ & $\checkmark$ & $\square$ \\
\hline 2839 & 3875 & Water well & $10 / 30 / 1977$ & 176.0 & 351938 & 6777604 & $\square$ & $\square$ & $\square$ & $\checkmark$ & $\square$ \\
\hline 2839 & 3877 & Water well & $5 / 7 / 1982$ & 104.0 & 351864 & 6777608 & $\checkmark$ & $\square$ & $\square$ & $\bar{\nabla}$ & $\square$ \\
\hline 2839 & 3886 & Water well & $6 / 27 / 1973$ & 140.0 & 352164 & 6777626 & $\checkmark$ & $\square$ & $\square$ & $\bar{\nabla}$ & $\square$ \\
\hline 2839 & 3887 & Water well & 10/17/1977 & 379.0 & 352060 & 6777630 & $\square$ & $\square$ & $\square$ & $\boldsymbol{V}$ & $\square$ \\
\hline 2839 & 3888 & Water well & 8/8/1979 & 358.0 & 351955 & 6777635 & $\checkmark$ & $\square$ & $\square$ & $\nabla$ & $\square$ \\
\hline
\end{tabular}




\begin{tabular}{|c|c|c|c|c|c|c|c|c|c|c|c|}
\hline \multirow[b]{2}{*}{$\begin{array}{l}\text { MOA } \\
\text { grid }\end{array}$} & \multirow[b]{2}{*}{$\begin{array}{l}\text { DGGS } \\
\text { number }\end{array}$} & \multirow[b]{2}{*}{ Hole Type } & \multirow[b]{2}{*}{$\begin{array}{c}\text { Date } \\
\text { Completed }\end{array}$} & \multirow[b]{2}{*}{$\begin{array}{c}\text { Total } \\
\text { depth }(\mathrm{ft})\end{array}$} & \multicolumn{2}{|c|}{ UTM coordinates (m) } & \multicolumn{5}{|c|}{ Data entered } \\
\hline & & & & & East & North & Lithology & SPT & $\begin{array}{c}\text { Sample } \\
\text { test }\end{array}$ & $\begin{array}{l}\text { Water } \\
\text { level }\end{array}$ & Velocity \\
\hline 2839 & 3905 & Water well & 2/9/1982 & 450.0 & 352051 & 6777786 & $\checkmark$ & $\square$ & $\square$ & $\nabla$ & $\square$ \\
\hline 2840 & 3387 & Water well & $9 / 20 / 1973$ & 173.0 & 352321 & 6777062 & $\checkmark$ & $\square$ & $\square$ & $\nabla$ & $\square$ \\
\hline 2840 & 3390 & Water well & $8 / 26 / 1983$ & 200.0 & 352454 & 6777025 & $\checkmark$ & 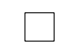 & $\square$ & $\bar{V}$ & \\
\hline 2840 & 3779 & Water well & 2/1/1982 & 195.0 & 352246 & 6777065 & $\nabla$ & $\square$ & $\square$ & $\checkmark$ & $\square$ \\
\hline 2840 & 3780 & Water well & $5 / 21 / 1975$ & 220.0 & 352230 & 6777066 & $\checkmark$ & $\square$ & $\square$ & 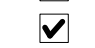 & $\square$ \\
\hline 2840 & 3791 & Water well & 8/1/1974 & 237.0 & 352861 & 6777070 & $\checkmark$ & $\square$ & $\square$ & $\checkmark$ & $\square$ \\
\hline 2840 & 3792 & Water well & $10 / 30 / 1981$ & 150.0 & 352456 & 6777087 & $\checkmark$ & $\square$ & $\square$ & $\checkmark$ & $\square$ \\
\hline 2840 & 3812 & Water well & $4 / 30 / 1973$ & 200.0 & 352866 & 6777194 & $\checkmark$ & 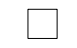 & $\square$ & $\nabla$ & $\square$ \\
\hline 2840 & 3813 & Water well & $5 / 19 / 1979$ & 348.0 & 352536 & 6777208 & $\square$ & $\square$ & $\square$ & $\sqrt{\square}$ & $\square$ \\
\hline 2840 & 3823 & Water well & $5 / 8 / 1979$ & 347.0 & 352584 & 6777268 & $\checkmark$ & $\square$ & $\square$ & $\checkmark$ & $\square$ \\
\hline 2840 & 3845 & Water well & $8 / 19 / 1976$ & 400.0 & 352768 & 6777384 & $\checkmark$ & $\square$ & $\square$ & $\bar{V}$ & $\square$ \\
\hline 2840 & 3846 & Water well & $3 / 1 / 1979$ & 105.0 & 352439 & 6777397 & $\checkmark$ & & $\square$ & $\bar{\nabla}$ & $\square$ \\
\hline 2840 & 3859 & Water well & $6 / 1 / 1970$ & 185.0 & 352677 & 6777356 & $\checkmark$ & $\square$ & $\square$ & $\nabla$ & $\square$ \\
\hline 2840 & 3860 & Water well & $8 / 28 / 1978$ & 125.0 & 352457 & 6777459 & $\square$ & $\square$ & $\square$ & $\checkmark$ & $\square$ \\
\hline 2840 & 3869 & Water well & $3 / 4 / 1979$ & 230.0 & 352476 & 6777551 & $\checkmark$ & $\square$ & $\square$ & $\checkmark$ & $\square$ \\
\hline 2840 & 3873 & Water well & $8 / 24 / 1982$ & 227.0 & 352986 & 6777561 & $\checkmark$ & - & $\square$ & $\checkmark$ & $\square$ \\
\hline 2840 & 3903 & Water well & $7 / 21 / 1978$ & 318.0 & 352964 & 6777747 & $\square$ & 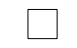 & $\square$ & $\boldsymbol{V}$ & $\square$ \\
\hline 2840 & 3904 & Water well & 8/2/1978 & 598.0 & 352320 & 6777774 & $\square$ & L & $\square$ & $\vec{\nabla}$ & $\square$ \\
\hline 2840 & 3911 & Water well & $10 / 15 / 1977$ & 175.0 & 352546 & 6777796 & $\checkmark$ & $\square$ & $\square$ & $\nabla$ & $\square$ \\
\hline 2841 & 3323 & Water well & $7 / 31 / 1984$ & 342.0 & 353490 & 6777787 & $\checkmark$ & $\square$ & $\square$ & $\checkmark$ & $\square$ \\
\hline 2841 & 3360 & Water well & $10 / 28 / 1980$ & 120.0 & 353064 & 6777278 & $\checkmark$ & & $\square$ & $\bar{\nabla}$ & $\square$ \\
\hline 2841 & 3376 & Water well & $12 / 27 / 1975$ & 101.0 & 353491 & 6777075 & $\checkmark$ & 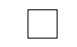 & $\square$ & $\nabla$ & $\square$ \\
\hline 2841 & 3790 & Water well & $7 / 19 / 1978$ & 238.0 & 353639 & 6777038 & $\square$ & $\square$ & $\square$ & $\nabla$ & $\square$ \\
\hline 2841 & 3818 & Water well & 8/5/1981 & 350.0 & 353647 & 6777223 & $\checkmark$ & $\square$ & $\square$ & $\checkmark$ & $\square$ \\
\hline 2841 & 3819 & Water well & $5 / 30 / 1982$ & 250.0 & 353452 & 6777231 & $\checkmark$ & & $\square$ & $\bar{\nabla}$ & $\square$ \\
\hline 2841 & 3820 & Water well & 7/18/1983 & 243.0 & 353153 & 6777244 & $\checkmark$ & & $\square$ & $\nabla$ & $\square$ \\
\hline 2841 & 3822 & Water well & $7 / 19 / 1983$ & 268.0 & 353063 & 6777248 & $\checkmark$ & $\square$ & $\square$ & $\checkmark$ & $\square$ \\
\hline 2841 & 3834 & Water well & $7 / 2 / 1981$ & 125.0 & 353157 & 6777336 & $\checkmark$ & & $\square$ & $\nabla$ & $\square$ \\
\hline 2841 & 3843 & Water well & $6 / 18 / 1975$ & 150.0 & 353053 & 6777372 & $\checkmark$ & $\square$ & $\square$ & $\checkmark$ & $\square$ \\
\hline 2841 & 3844 & Water well & $10 / 1 / 1978$ & 110.0 & 353067 & 6777340 & $\checkmark$ & - & $\square$ & $\bar{\nabla}$ & $\square$ \\
\hline 2841 & 3863 & Water well & $6 / 4 / 1975$ & 225.0 & 353281 & 6777455 & $\square$ & $\square$ & $\square$ & $\nabla$ & $\square$ \\
\hline 2841 & 3884 & Water well & 7/30/1974 & 432.0 & 353182 & 6777583 & $\checkmark$ & $\square$ & $\square$ & $\checkmark$ & $\square$ \\
\hline 2841 & 3885 & Water well & 10/9/1974 & 200.0 & 353151 & 6777554 & $\square$ & $\square$ & $\square$ & $\bar{\nabla}$ & $\square$ \\
\hline 2841 & 4508 & Water well & $3 / 1 / 1967$ & 125.0 & 353357 & 6777483 & $\checkmark$ & $\square$ & $\square$ & $\bar{\nabla}$ & $\square$ \\
\hline 2842 & 3910 & Water well & $6 / 15 / 1973$ & 125.0 & 353968 & 6777737 & $\checkmark$ & $\square$ & $\square$ & $\boldsymbol{V}$ & $\square$ \\
\hline 2843 & 3373 & Water well & $4 / 21 / 1983$ & 315.0 & 355253 & 6776909 & $\checkmark$ & $\square$ & $\square$ & $\nabla$ & $\square$ \\
\hline
\end{tabular}




\begin{tabular}{|c|c|c|c|c|c|c|c|c|c|c|c|}
\hline \multirow[b]{2}{*}{$\begin{array}{l}\text { MOA } \\
\text { grid }\end{array}$} & \multirow[b]{2}{*}{$\begin{array}{l}\text { DGGS } \\
\text { number }\end{array}$} & \multirow[b]{2}{*}{ Hole Type } & \multirow[b]{2}{*}{$\begin{array}{c}\text { Date } \\
\text { Completed }\end{array}$} & \multirow[b]{2}{*}{$\begin{array}{c}\text { Total } \\
\text { depth }(\mathrm{ft})\end{array}$} & \multicolumn{2}{|c|}{ UTM coordinates (m) } & \multicolumn{5}{|c|}{ Data entered } \\
\hline & & & & & East & North & Lithology & SPT & $\begin{array}{c}\text { Sample } \\
\text { test }\end{array}$ & $\begin{array}{l}\text { Water } \\
\text { level }\end{array}$ & Velocity \\
\hline 2933 & 1856 & Hollow stem borehole w/SPT & $5 / 24 / 1971$ & 25.5 & 347056 & 6776771 & $\square$ & $\checkmark$ & $\square$ & $\square$ & $\square$ \\
\hline 2933 & 1857 & Hollow stem borehole w/SPT & $4 / 25 / 1971$ & 25.1 & 347127 & 6776793 & $\square$ & $\square$ & $\square$ & $\square$ & $\square$ \\
\hline 2933 & 2096 & Hollow stem borehole w/SPT & $11 / 11 / 1982$ & 31.2 & 347210 & 6776716 & $\checkmark$ & $\checkmark$ & $\nabla$ & 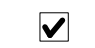 & \\
\hline 2933 & 2097 & Hollow stem borehole w/SPT & $11 / 12 / 1982$ & 40.7 & 347209 & 6776693 & $\checkmark$ & $\checkmark$ & $\checkmark$ & $\sqrt{\nabla}$ & $\square$ \\
\hline 2933 & 2280 & Hollow stem borehole w/SPT & $2 / 14 / 1977$ & 31.0 & 346648 & 6776789 & $\checkmark$ & $\checkmark$ & $\square$ & $\square$ & $\square$ \\
\hline 2933 & 2281 & Hollow stem borehole w/SPT & $2 / 12 / 1977$ & 28.5 & 346923 & 6776804 & $\checkmark$ & $\checkmark$ & $\square$ & $\square$ & $\square$ \\
\hline 2933 & 3714 & Water well & $9 / 2 / 1976$ & 159.0 & 347273 & 6776502 & $\checkmark$ & $\square$ & $\square$ & $\checkmark$ & $\square$ \\
\hline 2933 & 3715 & Water well & $9 / 15 / 1975$ & 163.0 & 347243 & 6776503 & $\checkmark$ & $\square$ & $\square$ & $\bar{\nabla}$ & $\square$ \\
\hline 2933 & 3720 & Water well & $11 / 13 / 1973$ & 162.0 & 347262 & 6776596 & $\checkmark$ & $\square$ & $\square$ & $\sqrt{\square}$ & $\square$ \\
\hline 2933 & 3721 & Water well & $9 / 10 / 1972$ & 155.0 & 347218 & 6776629 & $\checkmark$ & $\square$ & $\square$ & $\vec{V}$ & $\square$ \\
\hline 2933 & 3722 & Water well & 8/1/1970 & 150.0 & 347084 & 6776635 & $\checkmark$ & $\square$ & $\square$ & $\bar{V}$ & $\square$ \\
\hline 2933 & 3724 & Water well & $9 / 24 / 1971$ & 280.0 & 346685 & 6776776 & $\checkmark$ & 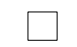 & $\square$ & $\bar{\nabla}$ & $\square$ \\
\hline 2934 & 3730 & Water well & $12 / 10 / 1975$ & 301.0 & 347799 & 6776882 & $\checkmark$ & $\square$ & $\square$ & $\vec{V}$ & $\square$ \\
\hline 2935 & 2169 & Hollow stem borehole w/SPT & $1 / 15 / 1996$ & 31.5 & 348881 & 6776857 & $\checkmark$ & $\checkmark$ & $\checkmark$ & $\nabla$ & $\square$ \\
\hline 2935 & 2170 & Hollow stem borehole w/SPT & $1 / 16 / 1996$ & 31.5 & 348893 & 6776804 & $\nabla$ & $\nabla$ & $\checkmark$ & $\square$ & $\square$ \\
\hline 2935 & 2358 & Hollow stem borehole w/SPT & $5 / 27 / 2000$ & 101.0 & 348810 & 6777030 & $\checkmark$ & $\checkmark$ & $\checkmark$ & 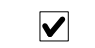 & $\checkmark$ \\
\hline 2935 & 2359 & Hollow stem borehole w/SPT & $5 / 31 / 2000$ & 49.3 & 348875 & 6777057 & $\checkmark$ & $\nabla$ & $\checkmark$ & 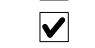 & $\square$ \\
\hline 2935 & 3716 & Water well & $8 / 20 / 1964$ & 125.0 & 348352 & 6776487 & $\checkmark$ & $\square$ & $\square$ & $\vec{\nabla}$ & $\square$ \\
\hline 2936 & 2001 & Natural exposure & $5 / 31 / 1997$ & 12.0 & 349734 & 6776725 & $\checkmark$ & $\square$ & $\square$ & $\square$ & $\square$ \\
\hline 2936 & 3415 & Water well & $5 / 17 / 1985$ & 550.0 & 350530 & 6777092 & $\checkmark$ & $\square$ & $\square$ & $\checkmark$ & $\square$ \\
\hline 2936 & 3416 & Water well & $3 / 30 / 1981$ & 228.0 & 349609 & 6777114 & $\checkmark$ & & $\square$ & $\bar{\nabla}$ & $\square$ \\
\hline 2936 & 3420 & Water well & $11 / 17 / 1980$ & 204.0 & 349323 & 6777095 & $\checkmark$ & $\square$ & $\square$ & $\bar{V}$ & $\square$ \\
\hline 2936 & 3424 & Water well & $8 / 20 / 1984$ & 276.0 & 349724 & 6777017 & $\checkmark$ & $\square$ & $\square$ & $\nabla$ & $\square$ \\
\hline 2936 & 3426 & Water well & $8 / 3 / 1980$ & 203.0 & 349336 & 6777033 & $\checkmark$ & $\square$ & $\square$ & $\checkmark$ & $\square$ \\
\hline 2936 & 3427 & Water well & $11 / 28 / 1978$ & 350.0 & 349619 & 6776990 & $\checkmark$ & & $\square$ & $\checkmark$ & $\square$ \\
\hline 2936 & 3428 & Water well & $12 / 13 / 1982$ & 345.0 & 349588 & 6776991 & $\checkmark$ & $\square$ & $\square$ & $\square$ & $\square$ \\
\hline 2936 & 3432 & Water well & $5 / 22 / 1981$ & 191.0 & 349615 & 6776897 & $\checkmark$ & $\square$ & $\square$ & $\square$ & $\square$ \\
\hline 2936 & 3433 & Water well & $1 / 1 / 1979$ & 225.0 & 349315 & 6776910 & $\checkmark$ & & $\square$ & $\nabla$ & $\square$ \\
\hline 2936 & 3435 & Water well & 9/7/1979 & 176.0 & 349225 & 6776914 & $\checkmark$ & $\square$ & $\square$ & $\checkmark$ & $\square$ \\
\hline 2936 & 3436 & Water well & $10 / 20 / 1980$ & 207.0 & 349046 & 6776922 & $\checkmark$ & $\square$ & $\square$ & $\bar{\nabla}$ & $\square$ \\
\hline 2936 & 3438 & Water well & $4 / 28 / 1979$ & 165.0 & 349537 & 6776839 & $\checkmark$ & $\square$ & $\square$ & $\nabla$ & $\square$ \\
\hline 2936 & 3445 & Water well & $8 / 11 / 1982$ & 200.0 & 348976 & 6776708 & $\checkmark$ & $\square$ & $\square$ & $\square$ & $\square$ \\
\hline 2936 & 3446 & Water well & $6 / 21 / 1975$ & 189.0 & 349659 & 6776524 & $\checkmark$ & $\square$ & $\square$ & $\bar{\nabla}$ & $\square$ \\
\hline 2936 & 3447 & Water well & $11 / 20 / 1985$ & 241.0 & 349596 & 6776464 & $\checkmark$ & $\square$ & $\square$ & $\nabla$ & $\square$ \\
\hline 2936 & 3450 & Water well & $5 / 15 / 1984$ & 111.0 & 349397 & 6776380 & $\checkmark$ & $\square$ & $\square$ & $\vec{V}$ & $\square$ \\
\hline 2936 & 3711 & Water well & $9 / 1 / 1979$ & 143.0 & 349294 & 6776415 & $\checkmark$ & $\square$ & $\square$ & $\checkmark$ & $\square$ \\
\hline
\end{tabular}




\begin{tabular}{|c|c|c|c|c|c|c|c|c|c|c|c|}
\hline \multirow[b]{2}{*}{$\begin{array}{l}\text { MOA } \\
\text { grid }\end{array}$} & \multirow[b]{2}{*}{$\begin{array}{l}\text { DGGS } \\
\text { number }\end{array}$} & \multirow[b]{2}{*}{ Hole Type } & \multirow[b]{2}{*}{$\begin{array}{c}\text { Date } \\
\text { Completed }\end{array}$} & \multirow[b]{2}{*}{$\begin{array}{c}\text { Total } \\
\text { depth }(\mathrm{ft})\end{array}$} & \multicolumn{2}{|c|}{ UTM coordinates (m) } & \multicolumn{5}{|c|}{ Data entered } \\
\hline & & & & & East & North & Lithology & SPT & $\begin{array}{c}\text { Sample } \\
\text { test }\end{array}$ & $\begin{array}{l}\text { Water } \\
\text { level }\end{array}$ & Velocity \\
\hline 2936 & 3712 & Water well & 7/17/1981 & 118.0 & 349085 & 6776424 & $\checkmark$ & 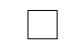 & $\square$ & $\nabla$ & $\square$ \\
\hline 2936 & 3713 & Water well & 8/20/1977 & 160.0 & 348995 & 6776428 & $\checkmark$ & $\square$ & $\square$ & $\sqrt{ }$ & $\square$ \\
\hline 2936 & 3717 & Water well & $7 / 22 / 1965$ & 144.0 & 349177 & 6776482 & $\checkmark$ & & $\square$ & $\bar{V}$ & \\
\hline 2936 & 3719 & Water well & 8/20/1974 & 145.0 & 349403 & 6776504 & $\nabla$ & $\square$ & $\square$ & $\checkmark$ & $\square$ \\
\hline 2936 & 3726 & Water well & $9 / 15 / 1972$ & 175.0 & 349624 & 6776773 & $\checkmark$ & $\square$ & $\square$ & 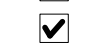 & $\square$ \\
\hline 2936 & 3729 & Water well & 9/11/1979 & 166.0 & 349312 & 6776817 & $\checkmark$ & $\square$ & $\square$ & $\checkmark$ & $\square$ \\
\hline 2936 & 3741 & Water well & $6 / 26 / 1976$ & 145.0 & 349420 & 6776906 & $\checkmark$ & $\square$ & $\square$ & $\checkmark$ & $\square$ \\
\hline 2936 & 3760 & Water well & 6/7/1979 & 400.0 & 349756 & 6777046 & $\square$ & 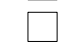 & $\square$ & $\bar{\nabla}$ & $\square$ \\
\hline 2936 & 3761 & Water well & 11/8/1977 & 168.0 & 349516 & 6777057 & $\checkmark$ & $\square$ & $\square$ & $\checkmark$ & $\square$ \\
\hline 2936 & 3770 & Water well & $6 / 26 / 1981$ & 350.0 & 349727 & 6777078 & $\checkmark$ & & $\square$ & $\square$ & $\square$ \\
\hline 2936 & 3771 & Water well & 9/8/1977 & 250.0 & 349533 & 6777086 & $\checkmark$ & $\square$ & $\square$ & $\nabla$ & $\square$ \\
\hline 2936 & 3772 & Water well & $10 / 1 / 1975$ & 212.0 & 349428 & 6777091 & $\checkmark$ & & $\square$ & $\checkmark$ & \\
\hline 2936 & 3775 & Water well & $5 / 23 / 1978$ & 278.0 & 349639 & 6777113 & $\checkmark$ & $\square$ & $\square$ & $\vec{V}$ & $\square$ \\
\hline 2936 & 3776 & Water well & 10/5/1982 & 201.0 & 349219 & 6777131 & $\checkmark$ & $\square$ & $\square$ & $\nabla$ & $\square$ \\
\hline 2937 & 3403 & Water well & 6/15/1981 & 320.0 & 350433 & 6777110 & $\checkmark$ & $\square$ & $\square$ & $\checkmark$ & $\square$ \\
\hline 2937 & 3406 & Water well & $8 / 15 / 1985$ & 180.0 & 350014 & 6777128 & $\checkmark$ & & $\square$ & $\checkmark$ & $\square$ \\
\hline 2937 & 3411 & Water well & $6 / 22 / 1981$ & 339.0 & 350431 & 6777048 & $\checkmark$ & & $\square$ & $\bar{V}$ & $\square$ \\
\hline 2937 & 3417 & Water well & 8/16/1979 & 273.0 & 350534 & 6777013 & $\checkmark$ & - & $\square$ & $\vec{\nabla}$ & $\square$ \\
\hline 2937 & 3418 & Water well & 8/19/1983 & 205.0 & 350340 & 6777021 & $\checkmark$ & 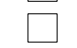 & $\square$ & $\checkmark$ & $\square$ \\
\hline 2937 & 3421 & Water well & $11 / 2 / 1980$ & 310.0 & 350502 & 6776952 & $\checkmark$ & $\square$ & $\square$ & $\checkmark$ & $\square$ \\
\hline 2937 & 3422 & Water well & $10 / 6 / 1980$ & 269.0 & 350412 & 6776956 & $\checkmark$ & & $\square$ & $\bar{\nabla}$ & $\square$ \\
\hline 2937 & 3423 & Water well & 9/28/1981 & 239.0 & 350217 & 6776965 & $\checkmark$ & $\checkmark$ & $\square$ & $\bar{V}$ & $\square$ \\
\hline 2937 & 3429 & Water well & $12 / 3 / 1983$ & 270.0 & 350125 & 6776906 & $\checkmark$ & & $\square$ & $\nabla$ & $\square$ \\
\hline 2937 & 3430 & Water well & $12 / 21 / 1984$ & 240.0 & 349930 & 6776915 & $\checkmark$ & $\square$ & $\square$ & $\checkmark$ & $\square$ \\
\hline 2937 & 3437 & Water well & $6 / 12 / 1985$ & 240.0 & 349926 & 6776822 & $\checkmark$ & & $\square$ & $\checkmark$ & $\square$ \\
\hline 2937 & 3441 & Water well & $5 / 13 / 1973$ & 160.5 & 349906 & 6776699 & $\checkmark$ & & $\square$ & $\checkmark$ & $\square$ \\
\hline 2937 & 3443 & Water well & $10 / 1 / 1974$ & 400.0 & 350219 & 6776655 & $\checkmark$ & $\square$ & $\square$ & $\nabla$ & $\square$ \\
\hline 2937 & 3750 & Water well & $9 / 22 / 1981$ & 319.0 & 350307 & 6776961 & $\checkmark$ & & $\square$ & $\nabla$ & $\square$ \\
\hline 2937 & 3751 & Water well & $11 / 12 / 1981$ & 358.0 & 350113 & 6776969 & $\checkmark$ & $\square$ & $\square$ & $\checkmark$ & $\square$ \\
\hline 2937 & 3758 & Water well & 9/25/1981 & 326.0 & 350009 & 6777004 & $\checkmark$ & 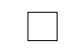 & $\square$ & $\bar{\nabla}$ & $\square$ \\
\hline 2937 & 3759 & Water well & $10 / 1 / 1978$ & 373.0 & 350549 & 6777013 & $\checkmark$ & $\square$ & $\square$ & $\nabla$ & $\square$ \\
\hline 2938 & 3425 & Water well & $3 / 23 / 1981$ & 291.0 & 350858 & 6776875 & $\checkmark$ & $\square$ & $\square$ & $\checkmark$ & $\square$ \\
\hline 2938 & 3728 & Water well & $12 / 22 / 1975$ & 288.0 & 350704 & 6776758 & $\checkmark$ & $\square$ & $\square$ & $\bar{\nabla}$ & $\square$ \\
\hline 2938 & 3731 & Water well & 9/26/1974 & 260.0 & 350915 & 6776780 & $\checkmark$ & $\square$ & $\square$ & $\bar{\nabla}$ & $\square$ \\
\hline 2938 & 3732 & Water well & 8/18/1977 & 223.0 & 350915 & 6776780 & $\checkmark$ & $\square$ & $\square$ & $\checkmark$ & $\square$ \\
\hline 2938 & 3733 & Water well & $3 / 1 / 1967$ & 290.0 & 350869 & 6776782 & $\checkmark$ & $\square$ & $\square$ & $\nabla$ & $\square$ \\
\hline
\end{tabular}




\begin{tabular}{|c|c|c|c|c|c|c|c|c|c|c|c|}
\hline \multirow[b]{2}{*}{$\begin{array}{l}\text { MOA } \\
\text { grid }\end{array}$} & \multirow[b]{2}{*}{$\begin{array}{l}\text { DGGS } \\
\text { number }\end{array}$} & \multirow[b]{2}{*}{ Hole Type } & \multirow[b]{2}{*}{$\begin{array}{c}\text { Date } \\
\text { Completed }\end{array}$} & \multirow[b]{2}{*}{$\begin{array}{c}\text { Total } \\
\text { depth }(\mathrm{ft})\end{array}$} & \multicolumn{2}{|c|}{ UTM coordinates (m) } & \multicolumn{5}{|c|}{ Data entered } \\
\hline & & & & & East & North & Lithology & SPT & $\begin{array}{c}\text { Sample } \\
\text { test }\end{array}$ & $\begin{array}{l}\text { Water } \\
\text { level }\end{array}$ & Velocity \\
\hline 2938 & 3740 & Water well & $10 / 25 / 1972$ & 300.0 & 350902 & 6776843 & $\checkmark$ & & $\square$ & $\checkmark$ & $\square$ \\
\hline 2938 & 3744 & Water well & $10 / 4 / 1973$ & 287.0 & 350784 & 6776878 & $\checkmark$ & $\square$ & $\square$ & $\nabla$ & $\square$ \\
\hline 2938 & 3749 & Water well & $6 / 12 / 1979$ & 198.0 & 350726 & 6776943 & $\checkmark$ & $\square$ & $\square$ & $\bar{V}$ & $\square$ \\
\hline 2938 & 3766 & Water well & $3 / 1 / 1976$ & 380.0 & 351239 & 6777014 & $\checkmark$ & $\square$ & $\square$ & $\boldsymbol{V}$ & $\square$ \\
\hline 2938 & 3767 & Water well & $6 / 15 / 1977$ & 221.0 & 350940 & 6777027 & $\checkmark$ & $\square$ & $\square$ & $\checkmark$ & $\square$ \\
\hline 2938 & 3768 & Water well & $5 / 28 / 1977$ & 305.0 & 350790 & 6777033 & $\checkmark$ & $\square$ & $\square$ & $\checkmark$ & $\square$ \\
\hline 2938 & 3769 & Water well & $7 / 2 / 1979$ & 260.0 & 350730 & 6777036 & $\checkmark$ & $\square$ & $\square$ & $\checkmark$ & $\square$ \\
\hline 2939 & 3431 & Water well & 8/18/1986 & 220.0 & 351407 & 6776728 & $\checkmark$ & & $\square$ & $\checkmark$ & $\square$ \\
\hline 2939 & 3718 & Water well & $4 / 15 / 1975$ & 180.0 & 352008 & 6776393 & $\checkmark$ & $\square$ & $\square$ & $\checkmark$ & $\square$ \\
\hline 2939 & 3727 & Water well & $7 / 19 / 1979$ & 150.0 & 352110 & 6776699 & $\checkmark$ & & $\square$ & $\nabla$ & $\square$ \\
\hline 2939 & 3735 & Water well & $9 / 12 / 1977$ & 340.0 & 351829 & 6776773 & $\checkmark$ & $\square$ & $\square$ & $\square$ & $\square$ \\
\hline 2939 & 3743 & Water well & 10/20/1982 & 179.0 & 352116 & 6776822 & $\checkmark$ & & $\square$ & $\bar{\nabla}$ & $\square$ \\
\hline 2939 & 3746 & Water well & $5 / 15 / 1976$ & 358.0 & 351982 & 6776859 & $\checkmark$ & $\square$ & $\square$ & $\square$ & $\square$ \\
\hline 2939 & 3757 & Water well & $12 / 1 / 1980$ & 124.0 & 351835 & 6776927 & $\checkmark$ & $\square$ & $\square$ & $\nabla$ & $\square$ \\
\hline 2939 & 3763 & Water well & $7 / 9 / 1979$ & 260.0 & 351838 & 6776989 & $\checkmark$ & $\square$ & $\square$ & $\checkmark$ & $\square$ \\
\hline 2939 & 3774 & Water well & $10 / 18 / 1975$ & 240.0 & 351914 & 6777017 & $\checkmark$ & - & $\square$ & $\checkmark$ & $\square$ \\
\hline 2939 & 3777 & Water well & $5 / 20 / 1978$ & 141.0 & 351840 & 6777051 & $\checkmark$ & & $\square$ & $\nabla$ & $\square$ \\
\hline 2940 & 2002 & Natural exposure & $5 / 31 / 1997$ & 43.0 & 352533 & 6776721 & $\checkmark$ & ᄂ & $\square$ & $\square$ & $\square$ \\
\hline 2940 & 3402 & Water well & 6/28/1974 & 130.0 & 352448 & 6776902 & $\checkmark$ & $\square$ & $\square$ & $\nabla$ & $\square$ \\
\hline 2940 & 3414 & Water well & $1 / 5 / 1986$ & 120.0 & 352714 & 6776797 & $\checkmark$ & $\square$ & $\square$ & $\checkmark$ & $\square$ \\
\hline 2940 & 3440 & Water well & 6/10/1981 & 260.0 & 352221 & 6776477 & $\checkmark$ & & $\square$ & $\bar{\nabla}$ & $\square$ \\
\hline 2940 & 3442 & Water well & $7 / 30 / 1985$ & 247.0 & 352817 & 6776391 & $\checkmark$ & 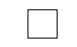 & $\square$ & $\nabla$ & $\square$ \\
\hline 2940 & 3444 & Water well & $11 / 29 / 1985$ & 260.0 & 352771 & 6776361 & $\checkmark$ & & $\square$ & $\nabla$ & $\square$ \\
\hline 2940 & 3710 & Water well & $8 / 23 / 1981$ & 340.0 & 352227 & 6776260 & $\checkmark$ & $\square$ & $\square$ & $\checkmark$ & $\square$ \\
\hline 2940 & 3723 & Water well & 3/27/1971 & 113.0 & 352296 & 6776474 & $\checkmark$ & & $\square$ & $\checkmark$ & $\square$ \\
\hline 2940 & 3739 & Water well & 1/6/1977 & 124.0 & 352713 & 6776767 & $\checkmark$ & & $\square$ & $\nabla$ & $\square$ \\
\hline 2940 & 3745 & Water well & $7 / 29 / 1970$ & 109.0 & 352835 & 6776823 & $\checkmark$ & $\square$ & $\square$ & $\checkmark$ & $\square$ \\
\hline 2940 & 3752 & Water well & $7 / 1 / 1970$ & 196.0 & 352943 & 6776881 & $\checkmark$ & & $\square$ & $\nabla$ & $\square$ \\
\hline 2940 & 3753 & Water well & $6 / 26 / 1970$ & 224.0 & 352928 & 6776882 & $\checkmark$ & $\square$ & $\square$ & $\checkmark$ & $\square$ \\
\hline 2940 & 3754 & Water well & 1/6/1977 & 111.0 & 352748 & 6776889 & $\checkmark$ & 5 & $\square$ & $\bar{\nabla}$ & $\square$ \\
\hline 2940 & 3755 & Water well & 4/6/1973 & 150.0 & 352628 & 6776894 & $\checkmark$ & $\square$ & $\square$ & $\nabla$ & $\square$ \\
\hline 2942 & 3737 & Water well & $11 / 27 / 1971$ & 175.0 & 353970 & 6776714 & $\checkmark$ & $\square$ & $\square$ & $\checkmark$ & $\square$ \\
\hline 2942 & 3738 & Water well & 11/7/1974 & 250.0 & 353851 & 6776719 & $\checkmark$ & $\square$ & $\square$ & $\bar{\nabla}$ & $\square$ \\
\hline 2942 & 3748 & Water well & $8 / 23 / 1974$ & 375.0 & 353839 & 6776813 & $\checkmark$ & $\square$ & $\square$ & $\bar{\nabla}$ & $\square$ \\
\hline 2943 & 3382 & Water well & $8 / 27 / 1985$ & 198.0 & 354846 & 6776864 & $\checkmark$ & $\square$ & $\square$ & $\boldsymbol{V}$ & $\square$ \\
\hline 2943 & 3747 & Water well & $5 / 5 / 1975$ & 150.0 & 355067 & 6776762 & $\checkmark$ & $\square$ & $\square$ & $\nabla$ & $\square$ \\
\hline
\end{tabular}




\begin{tabular}{|c|c|c|c|c|c|c|c|c|c|c|c|}
\hline \multirow[b]{2}{*}{$\begin{array}{c}\text { MOA } \\
\text { grid }\end{array}$} & \multirow[b]{2}{*}{$\begin{array}{c}\text { DGGS } \\
\text { number }\end{array}$} & \multirow[b]{2}{*}{ Hole Type } & \multirow[b]{2}{*}{$\begin{array}{c}\text { Date } \\
\text { Completed }\end{array}$} & \multirow[b]{2}{*}{$\begin{array}{c}\text { Total } \\
\text { depth (ft) }\end{array}$} & \multicolumn{2}{|c|}{ UTM coordinates (m) } & \multicolumn{5}{|c|}{ Data entered } \\
\hline & & & & & East & North & Lithology & SPT & $\begin{array}{c}\text { Sample } \\
\text { test }\end{array}$ & $\begin{array}{l}\text { Water } \\
\text { level }\end{array}$ & Velocity \\
\hline 2943 & 3762 & Water well & $5 / 1 / 1971$ & 125.0 & 355190 & 6776850 & $\checkmark$ & $\square$ & $\square$ & $\nabla$ & $\square$ \\
\hline 2943 & 3773 & Water well & $6 / 5 / 1974$ & 202.0 & 354878 & 6776893 & $\bar{v}$ & $\square$ & $\square$ & $\checkmark$ & $\square$ \\
\hline 3032 & 3707 & Water well & $11 / 15 / 1981$ & 231.0 & 346474 & 6776413 & $\boldsymbol{V}$ & $\square$ & $\square$ & $\nabla$ & $\square$ \\
\hline 3033 & 1650 & Hollow stem borehole w/SPT & $3 / 18 / 1985$ & 51.5 & 347277 & 6775703 & $\boldsymbol{V}$ & $\nabla$ & $\nabla$ & $\nabla$ & $\square$ \\
\hline 3033 & 1651 & Hollow stem borehole w/SPT & $3 / 20 / 1985$ & 87.0 & 347226 & 6775716 & $\checkmark$ & $\checkmark$ & $\checkmark$ & $\nabla$ & $\square$ \\
\hline 3033 & 1700 & Hollow stem borehole w/SPT & $11 / 5 / 1987$ & 51.0 & 347294 & 6775782 & $\boldsymbol{V}$ & $\checkmark$ & $\nabla$ & $\nabla$ & $\square$ \\
\hline 3033 & 1701 & Hollow stem borehole w/SPT & $11 / 6 / 1987$ & 51.5 & 347284 & 6775808 & $\checkmark$ & $\checkmark$ & $\checkmark$ & $\checkmark$ & $\square$ \\
\hline 3033 & 1702 & Hollow stem borehole w/SPT & $11 / 10 / 1987$ & 41.8 & 347277 & 6775873 & $\boldsymbol{V}$ & $\nabla$ & $\nabla$ & $\nabla$ & $\square$ \\
\hline 3033 & 1703 & Hollow stem borehole w/SPT & $11 / 12 / 1987$ & 52.0 & 347273 & 6775946 & $\boldsymbol{V}$ & $\checkmark$ & $\nabla$ & $\nabla$ & $\square$ \\
\hline 3033 & 1704 & Hollow stem borehole w/SPT & $11 / 16 / 1987$ & 62.0 & 347281 & 6776031 & $\boldsymbol{V}$ & $\nabla$ & $\nabla$ & $\square$ & $\square$ \\
\hline 3033 & 1749 & Hollow stem borehole w/SPT & $4 / 5 / 1985$ & 52.0 & 347155 & 6776432 & $\nabla$ & $\checkmark$ & $\nabla$ & $\nabla$ & $\square$ \\
\hline 3033 & 1750 & Hollow stem borehole w/SPT & $4 / 11 / 1985$ & 76.0 & 347240 & 6776407 & $\boldsymbol{V}$ & $\checkmark$ & $\nabla$ & $\nabla$ & $\square$ \\
\hline 3033 & 1818 & Hollow stem borehole w/SPT & $2 / 28 / 1986$ & 52.0 & 347246 & 6775897 & $\boldsymbol{V}$ & $\nabla$ & $\boldsymbol{V}$ & $\nabla$ & $\square$ \\
\hline 3033 & 1819 & Hollow stem borehole w/SPT & $3 / 3 / 1986$ & 67.0 & 347239 & 6776155 & $\checkmark$ & $\checkmark$ & $\checkmark$ & $\checkmark$ & $\square$ \\
\hline 3033 & 2005 & Natural exposure & $5 / 31 / 1997$ & 47.0 & 346710 & 6776141 & $\boldsymbol{V}$ & $\square$ & $\square$ & $\square$ & $\square$ \\
\hline 3033 & 2017 & Hollow stem borehole w/SPT & $7 / 1 / 1964$ & 117.0 & 347023 & 6775708 & $\checkmark$ & $\checkmark$ & $\square$ & $\checkmark$ & $\square$ \\
\hline 3033 & 2018 & Hollow stem borehole w/SPT & $7 / 3 / 1964$ & 51.0 & 346863 & 6775933 & $\boldsymbol{V}$ & $\nabla$ & $\square$ & $\nabla$ & $\square$ \\
\hline 3033 & 2019 & Hollow stem borehole w/SPT & 6/6/1964 & 51.0 & 346844 & 6775912 & $\boldsymbol{V}$ & $\checkmark$ & $\square$ & $\nabla$ & $\square$ \\
\hline 3033 & 2020 & Hollow stem borehole w/SPT & $7 / 6 / 1964$ & 73.0 & 346770 & 6776005 & $\boldsymbol{V}$ & $\checkmark$ & $\square$ & $\nabla$ & $\square$ \\
\hline 3033 & 2021 & Hollow stem borehole w/SPT & $7 / 4 / 1964$ & 101.0 & 346525 & 6776271 & $\boldsymbol{V}$ & $\nabla$ & $\square$ & $\nabla$ & $\square$ \\
\hline 3033 & 2282 & Water well & $8 / 5 / 1976$ & 156.0 & 346800 & 6776346 & $\boldsymbol{V}$ & $\square$ & $\square$ & $\nabla$ & $\square$ \\
\hline 3033 & 3456 & Water well & $1 / 10 / 1984$ & 141.0 & 346759 & 6776401 & $\nabla$ & $\square$ & $\square$ & $\nabla$ & $\square$ \\
\hline 3033 & 3457 & Water well & 9/8/1978 & 147.0 & 347174 & 6776290 & 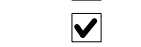 & $\square$ & $\square$ & $\nabla$ & $\square$ \\
\hline 3033 & 3460 & Water well & $8 / 13 / 1972$ & 171.0 & 346589 & 6776284 & $\nabla$ & $\square$ & $\square$ & $\nabla$ & $\square$ \\
\hline 3033 & 3461 & Water well & $6 / 25 / 1979$ & 179.0 & 347065 & 6776201 & 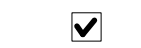 & $\square$ & $\square$ & $\nabla$ & $\square$ \\
\hline 3033 & 3462 & Water well & 9/15/1979 & 151.0 & 346661 & 6776219 & $\boldsymbol{V}$ & $\square$ & $\square$ & $\nabla$ & $\square$ \\
\hline 3033 & 3469 & Water well & $5 / 31 / 1979$ & 165.0 & 347055 & 6775985 & $\boldsymbol{V}$ & $\square$ & $\square$ & $\nabla$ & $\square$ \\
\hline 3033 & 3473 & Water well & $1 / 6 / 1974$ & 152.0 & 347124 & 6775827 & $\boldsymbol{V}$ & $\square$ & $\square$ & $\nabla$ & $\square$ \\
\hline 3033 & 3673 & Water well & 1/1/1977 & 167.0 & 347161 & 6775981 & $\nabla$ & $\square$ & $\square$ & $\nabla$ & $\square$ \\
\hline 3033 & 3675 & Water well & 8/14/1978 & 116.0 & 347043 & 6776047 & 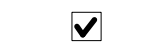 & $\square$ & $\square$ & $\nabla$ & $\square$ \\
\hline 3033 & 3681 & Water well & 10/1/1976 & 129.0 & 346910 & 6776084 & 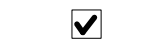 & $\square$ & $\square$ & $\nabla$ & $\square$ \\
\hline 3033 & 3682 & Water well & 10/1/1976 & 111.0 & 346880 & 6776086 & 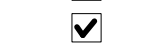 & $\square$ & $\square$ & $\checkmark$ & $\square$ \\
\hline 3033 & 3683 & Water well & 4/20/1983 & 111.0 & 346851 & 6776087 & 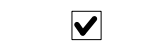 & $\square$ & $\square$ & $\bar{v}$ & $\square$ \\
\hline 3033 & 3691 & Water well & 6/30/1978 & 155.0 & 347098 & 6776262 & $\nabla$ & $\square$ & $\square$ & $\nabla$ & $\square$ \\
\hline 3033 & 3692 & Water well & $8 / 26 / 1981$ & 187.0 & 347069 & 6776294 & $\boldsymbol{v}$ & $\square$ & $\square$ & $\nabla$ & $\square$ \\
\hline 3033 & 3693 & Water well & 1/15/1982 & 101.0 & 346769 & 6776307 & $\boldsymbol{V}$ & $\square$ & $\square$ & $\square$ & $\square$ \\
\hline
\end{tabular}




\begin{tabular}{|c|c|c|c|c|c|c|c|c|c|c|c|}
\hline \multirow[b]{2}{*}{$\begin{array}{l}\text { MOA } \\
\text { grid }\end{array}$} & \multirow[b]{2}{*}{$\begin{array}{l}\text { DGGS } \\
\text { number }\end{array}$} & \multirow[b]{2}{*}{ Hole Type } & \multirow[b]{2}{*}{$\begin{array}{c}\text { Date } \\
\text { Completed }\end{array}$} & \multirow[b]{2}{*}{$\begin{array}{c}\text { Total } \\
\text { depth }(\mathrm{ft})\end{array}$} & \multicolumn{2}{|c|}{ UTM coordinates (m) } & \multicolumn{5}{|c|}{ Data entered } \\
\hline & & & & & East & North & Lithology & SPT & $\begin{array}{c}\text { Sample } \\
\text { test }\end{array}$ & $\begin{array}{l}\text { Water } \\
\text { level }\end{array}$ & Velocity \\
\hline 3033 & 3694 & Water well & $8 / 26 / 1964$ & 102.0 & 346605 & 6776315 & $\checkmark$ & - & $\square$ & $\nabla$ & $\square$ \\
\hline 3033 & 3696 & Water well & $5 / 16 / 1973$ & 107.0 & 346712 & 6776341 & $\checkmark$ & $\square$ & $\square$ & $\nabla$ & $\square$ \\
\hline 3033 & 3697 & Water well & $9 / 13 / 1972$ & 181.0 & 346726 & 6776340 & $\checkmark$ & $\square$ & $\square$ & $\bar{\nabla}$ & $\square$ \\
\hline 3033 & 3703 & Water well & $5 / 7 / 1981$ & 147.0 & 346864 & 6776396 & $\nabla$ & $\square$ & $\square$ & $\checkmark$ & $\square$ \\
\hline 3033 & 3704 & Water well & $6 / 3 / 1983$ & 127.0 & 346848 & 6776397 & $\checkmark$ & $\square$ & $\square$ & 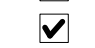 & $\square$ \\
\hline 3033 & 3706 & Water well & $6 / 24 / 1981$ & 180.0 & 346564 & 6776409 & $\checkmark$ & $\square$ & $\square$ & $\checkmark$ & $\square$ \\
\hline 3034 & 1817 & Hollow stem borehole w/SPT & $3 / 12 / 1986$ & 57.0 & 347323 & 6775650 & $\checkmark$ & $\checkmark$ & $\checkmark$ & $\checkmark$ & $\square$ \\
\hline 3034 & 3451 & Water well & $9 / 26 / 1983$ & 136.0 & 347495 & 6776431 & $\checkmark$ & & $\square$ & $\nabla$ & $\square$ \\
\hline 3034 & 3458 & Water well & $6 / 22 / 1985$ & 136.0 & 348068 & 6776158 & $\checkmark$ & $\square$ & $\square$ & $\checkmark$ & $\square$ \\
\hline 3034 & 3467 & Water well & $7 / 26 / 1979$ & 193.0 & 347370 & 6775972 & $\checkmark$ & $\square$ & $\square$ & $\checkmark$ & $\square$ \\
\hline 3034 & 3472 & Water well & $5 / 4 / 1983$ & 369.0 & 347573 & 6775808 & $\checkmark$ & $\square$ & $\square$ & $\nabla$ & $\square$ \\
\hline 3034 & 3666 & Water well & $1 / 1 / 1967$ & 176.0 & 347366 & 6775879 & $\checkmark$ & & $\square$ & $\nabla$ & $\square$ \\
\hline 3034 & 3679 & Water well & $9 / 16 / 1981$ & 176.0 & 347479 & 6776060 & $\checkmark$ & $\square$ & $\square$ & $\checkmark$ & $\square$ \\
\hline 3034 & 3680 & Water well & $5 / 22 / 1979$ & 159.0 & 347374 & 6776064 & $\checkmark$ & $\square$ & $\square$ & $\square$ & $\square$ \\
\hline 3034 & 3699 & Water well & $11 / 9 / 1964$ & 175.0 & 347536 & 6776336 & $\checkmark$ & $\square$ & $\square$ & $\checkmark$ & $\square$ \\
\hline 3034 & 3708 & Water well & $6 / 20 / 1972$ & 192.0 & 348077 & 6776375 & $\checkmark$ & - & $\square$ & $\checkmark$ & $\square$ \\
\hline 3034 & 3709 & Water well & $8 / 25 / 1982$ & 160.0 & 347658 & 6776393 & $\checkmark$ & 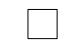 & $\square$ & $\nabla$ & $\square$ \\
\hline 3035 & 2003 & Natural exposure & $5 / 31 / 1997$ & 39.0 & 348887 & 6776008 & $\checkmark$ & 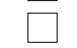 & $\square$ & $\square$ & $\square$ \\
\hline 3035 & 3686 & Water well & $3 / 1 / 1981$ & 117.0 & 348858 & 6776031 & $\checkmark$ & $\square$ & $\square$ & $\nabla$ & $\square$ \\
\hline 3035 & 3689 & Water well & $8 / 16 / 1965$ & 130.0 & 348728 & 6776161 & $\checkmark$ & $\square$ & $\square$ & $\nabla$ & $\square$ \\
\hline 3035 & 3690 & Water well & 9/2/1964 & 138.0 & 348295 & 6776210 & $\checkmark$ & & $\square$ & $\checkmark$ & $\square$ \\
\hline 3036 & 3459 & Water well & 7/15/1982 & 119.0 & 349172 & 6776018 & $\checkmark$ & 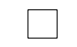 & $\square$ & $\nabla$ & $\square$ \\
\hline 3036 & 3466 & Water well & $7 / 25 / 1979$ & 110.0 & 349463 & 6775820 & $\checkmark$ & & $\square$ & $\nabla$ & $\square$ \\
\hline 3036 & 3470 & Water well & 8/13/1982 & 120.0 & 349638 & 6775688 & $\checkmark$ & $\square$ & $\square$ & $\checkmark$ & $\square$ \\
\hline 3036 & 3652 & Water well & 8/20/1982 & 117.0 & 349348 & 6775576 & $\checkmark$ & & $\square$ & $\checkmark$ & $\square$ \\
\hline 3036 & 3659 & Water well & $1 / 27 / 1976$ & 132.0 & 349426 & 6775635 & $\checkmark$ & & $\square$ & $\nabla$ & $\square$ \\
\hline 3036 & 3660 & Water well & $10 / 23 / 1978$ & 123.0 & 349157 & 6775678 & $\checkmark$ & $\square$ & $\square$ & $\checkmark$ & $\square$ \\
\hline 3036 & 3662 & Water well & 8/1/1973 & 138.0 & 349084 & 6775712 & $\checkmark$ & & $\square$ & $\nabla$ & $\square$ \\
\hline 3036 & 3663 & Water well & $12 / 9 / 1982$ & 124.0 & 349268 & 6775797 & $\checkmark$ & & $\square$ & $\checkmark$ & $\square$ \\
\hline 3036 & 3664 & Water well & 6/17/1976 & 128.0 & 349013 & 6775808 & $\checkmark$ & 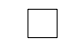 & $\square$ & $\checkmark$ & $\square$ \\
\hline 3036 & 3665 & Water well & 8/13/1982 & 134.0 & 348953 & 6775810 & $\checkmark$ & $\square$ & $\square$ & $\nabla$ & $\square$ \\
\hline 3036 & 3668 & Water well & 8/16/1977 & 122.0 & 349240 & 6775860 & $\checkmark$ & $\square$ & $\square$ & $\checkmark$ & $\square$ \\
\hline 3036 & 3669 & Water well & $4 / 22 / 1970$ & 105.0 & 349225 & 6775861 & $\checkmark$ & $\square$ & $\square$ & $\bar{\nabla}$ & $\square$ \\
\hline 3036 & 3670 & Water well & $6 / 28 / 1978$ & 161.0 & 349001 & 6775870 & $\checkmark$ & $\square$ & $\square$ & $\nabla$ & $\square$ \\
\hline 3036 & 3677 & Water well & 8/7/1973 & 110.0 & 349694 & 6775965 & $\checkmark$ & $\square$ & $\square$ & $\boldsymbol{V}$ & $\square$ \\
\hline 3036 & 3678 & Water well & $5 / 7 / 1967$ & 112.0 & 349335 & 6775980 & $\checkmark$ & $\square$ & $\square$ & $\nabla$ & $\square$ \\
\hline
\end{tabular}




\begin{tabular}{|c|c|c|c|c|c|c|c|c|c|c|c|}
\hline \multirow[b]{2}{*}{$\begin{array}{l}\text { MOA } \\
\text { grid }\end{array}$} & \multirow[b]{2}{*}{$\begin{array}{l}\text { DGGS } \\
\text { number }\end{array}$} & \multirow[b]{2}{*}{ Hole Type } & \multirow[b]{2}{*}{$\begin{array}{c}\text { Date } \\
\text { Completed }\end{array}$} & \multirow[b]{2}{*}{$\begin{array}{c}\text { Total } \\
\text { depth (ft) }\end{array}$} & \multicolumn{2}{|c|}{ UTM coordinates (m) } & \multicolumn{5}{|c|}{ Data entered } \\
\hline & & & & & East & North & Lithology & SPT & $\begin{array}{c}\text { Sample } \\
\text { test }\end{array}$ & $\begin{array}{l}\text { Water } \\
\text { level }\end{array}$ & Velocity \\
\hline 3036 & 3685 & Water well & $10 / 16 / 1981$ & 111.0 & 349562 & 6776001 & $\checkmark$ & $\square$ & $\square$ & $\checkmark$ & $\square$ \\
\hline 3036 & 3698 & Water well & $7 / 16 / 1968$ & 142.0 & 349228 & 6776263 & $\checkmark$ & $\leftarrow$ & $\square$ & $\checkmark$ & $\square$ \\
\hline 3036 & 3702 & Water well & 4/3/1979 & 201.0 & 349079 & 6776301 & $\checkmark$ & $\square$ & $\square$ & 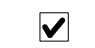 & \\
\hline 3037 & 3453 & Water well & 8/1/1982 & 146.0 & 350375 & 6776090 & $\nabla$ & $\checkmark$ & $\square$ & $\nabla$ & $\square$ \\
\hline 3037 & 3454 & Water well & $5 / 31 / 1984$ & 137.0 & 349851 & 6776113 & $\checkmark$ & $\square$ & $\square$ & 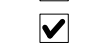 & $\square$ \\
\hline 3037 & 3463 & Water well & 2/9/1977 & 122.0 & 349975 & 6775860 & $\checkmark$ & & $\square$ & $\checkmark$ & $\square$ \\
\hline 3037 & 3651 & Water well & $11 / 2 / 1982$ & 140.0 & 350157 & 6775542 & $\checkmark$ & $\square$ & $\square$ & $\checkmark$ & $\square$ \\
\hline 3037 & 3658 & Water well & $6 / 25 / 1972$ & 138.0 & 349844 & 6775586 & $\checkmark$ & & $\square$ & $\nabla$ & $\square$ \\
\hline 3037 & 3661 & Water well & $11 / 8 / 1974$ & 111.0 & 349743 & 6775684 & $\nabla$ & $\square$ & $\square$ & $\nabla$ & $\square$ \\
\hline 3038 & 3655 & Water well & $1 / 26 / 1982$ & 268.0 & 351162 & 6775531 & $\checkmark$ & & $\square$ & $\square$ & $\square$ \\
\hline 3038 & 3674 & Water well & 3/6/1976 & 273.0 & 350545 & 6775866 & $\square$ & $\square$ & $\square$ & $\boldsymbol{V}$ & $\square$ \\
\hline 3038 & 3676 & Water well & $8 / 24 / 1974$ & 123.0 & 350593 & 6775926 & $\checkmark$ & $\square$ & $\square$ & $\bar{\nabla}$ & $\square$ \\
\hline 3038 & 3688 & Water well & $3 / 1 / 1970$ & 110.0 & 350808 & 6776041 & $\checkmark$ & $\checkmark$ & $\square$ & $\nabla$ & $\square$ \\
\hline 3038 & 3700 & Water well & $8 / 27 / 1982$ & 161.0 & 351115 & 6776214 & $\checkmark$ & $\square$ & $\square$ & $\nabla$ & $\square$ \\
\hline 3038 & 3701 & Water well & 9/2/1977 & 141.0 & 350921 & 6776222 & $\checkmark$ & $\checkmark$ & $\square$ & $\nabla$ & $\square$ \\
\hline 3038 & 4516 & Water well & $3 / 10 / 1968$ & 178.0 & 350544 & 6776176 & $\checkmark$ & $\square$ & $\square$ & $\bar{\nabla}$ & $\square$ \\
\hline 3039 & 3448 & Water well & 9/30/1972 & 265.0 & 351550 & 6776196 & $\checkmark$ & $\checkmark$ & $\square$ & $\boldsymbol{V}$ & $\square$ \\
\hline 3039 & 3452 & Water well & $5 / 28 / 1979$ & 310.0 & 351466 & 6775983 & $\checkmark$ & & $\square$ & $\nabla$ & $\square$ \\
\hline 3039 & 3455 & Water well & $4 / 16 / 1983$ & 305.0 & 351582 & 6775916 & $\checkmark$ & & $\square$ & $\square$ & $\pi$ \\
\hline 3039 & 3465 & Water well & $9 / 15 / 1975$ & 190.0 & 351465 & 6775611 & $\square$ & $\square$ & $\square$ & $\boldsymbol{V}$ & $\square$ \\
\hline 3039 & 3468 & Water well & $10 / 3 / 1981$ & 135.0 & 351565 & 6775513 & $\checkmark$ & $\square$ & $\square$ & $\bar{\nabla}$ & $\square$ \\
\hline 3039 & 3654 & Water well & 7/31/1981 & 128.0 & 351371 & 6775522 & $\checkmark$ & $\square$ & $\square$ & $\square$ & $\square$ \\
\hline 3039 & 3672 & Water well & $11 / 18 / 1982$ & 320.0 & 351458 & 6775797 & $\checkmark$ & $\square$ & $\square$ & $\square$ & $\square$ \\
\hline 3039 & 3684 & Water well & 9/29/1982 & 307.0 & 351478 & 6775920 & $\checkmark$ & $\square$ & $\square$ & $\checkmark$ & $\square$ \\
\hline 3039 & 3687 & Water well & $8 / 31 / 1974$ & 125.0 & 352078 & 6775926 & $\checkmark$ & $\square$ & $\square$ & $\boldsymbol{V}$ & $\square$ \\
\hline 3039 & 3695 & Water well & 9/1/1976 & 150.0 & 351876 & 6776120 & $\checkmark$ & $\square$ & $\square$ & $\nabla$ & $\square$ \\
\hline 3043 & 3653 & Water well & $8 / 12 / 1966$ & 150.0 & 354515 & 6775391 & $\checkmark$ & $\square$ & $\square$ & $\checkmark$ & $\square$ \\
\hline 3043 & 3671 & Water well & $7 / 31 / 1979$ & 103.0 & 354722 & 6775661 & $\checkmark$ & $\square$ & $\square$ & $\nabla$ & $\square$ \\
\hline 3133 & 2024 & Hollow stem borehole w/SPT & $7 / 9 / 1964$ & 80.0 & 347254 & 6775499 & $\checkmark$ & $\checkmark$ & $\square$ & $\checkmark$ & $\square$ \\
\hline 3134 & 1647 & Hollow stem borehole w/SPT & $3 / 12 / 1985$ & 52.0 & 347348 & 6775522 & $\checkmark$ & 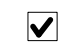 & $\checkmark$ & $\checkmark$ & $\square$ \\
\hline 3134 & 1648 & Hollow stem borehole w/SPT & $3 / 14 / 1985$ & 90.7 & 347300 & 6775615 & $\checkmark$ & $\checkmark$ & $\checkmark$ & $\checkmark$ & $\square$ \\
\hline 3134 & 1649 & Hollow stem borehole w/SPT & $3 / 15 / 1985$ & 52.0 & 347292 & 6775587 & $\checkmark$ & $\checkmark$ & $\checkmark$ & $\checkmark$ & $\square$ \\
\hline 3134 & 1652 & Hollow stem borehole w/SPT & $3 / 28 / 1985$ & 51.3 & 347346 & 6775559 & $\checkmark$ & $\checkmark$ & $\checkmark$ & $\nabla$ & $\square$ \\
\hline 3134 & 1820 & Solid stem borehole (no cores or SP & $4 / 29 / 1985$ & 61.5 & 347394 & 6775379 & $\checkmark$ & $\square$ & $\checkmark$ & $\square$ & $\square$ \\
\hline 3134 & 1821 & Solid stem borehole (no cores or SP & $5 / 13 / 1985$ & 61.5 & 347375 & 6775423 & $\checkmark$ & $\square$ & $\checkmark$ & $\square$ & $\square$ \\
\hline 3134 & 2022 & Hollow stem borehole w/SPT & $7 / 8 / 1964$ & 60.0 & 347341 & 6775241 & $\checkmark$ & $\checkmark$ & $\square$ & $\checkmark$ & $\square$ \\
\hline
\end{tabular}




\begin{tabular}{|c|c|c|c|c|c|c|c|c|c|c|c|}
\hline \multirow[b]{2}{*}{$\begin{array}{c}\text { MOA } \\
\text { grid }\end{array}$} & \multirow[b]{2}{*}{$\begin{array}{c}\text { DGGS } \\
\text { number }\end{array}$} & \multirow[b]{2}{*}{ Hole Type } & \multirow[b]{2}{*}{$\begin{array}{c}\text { Date } \\
\text { Completed }\end{array}$} & \multirow[b]{2}{*}{$\begin{array}{c}\text { Total } \\
\text { depth (ft) }\end{array}$} & \multicolumn{2}{|c|}{ UTM coordinates (m) } & \multicolumn{5}{|c|}{ Data entered } \\
\hline & & & & & East & North & Lithology & SPT & $\begin{array}{c}\text { Sample } \\
\text { test }\end{array}$ & $\begin{array}{c}\text { Water } \\
\text { level }\end{array}$ & Velocity \\
\hline 3134 & 2023 & Hollow stem borehole w/SPT & $7 / 9 / 1964$ & 68.0 & 347306 & 6775364 & $\checkmark$ & $\nabla$ & $\square$ & $\nabla$ & $\square$ \\
\hline 3134 & 3617 & Water well & $12 / 15 / 1964$ & 125.0 & 348021 & 6775076 & $\boldsymbol{V}$ & $\square$ & $\square$ & $\nabla$ & $\square$ \\
\hline 3134 & 3627 & Water well & $7 / 19 / 1973$ & 149.0 & 347505 & 6775284 & $\boldsymbol{V}$ & $\square$ & $\square$ & $\nabla$ & $\square$ \\
\hline 3134 & 3630 & Water well & $11 / 4 / 1972$ & 205.0 & 347433 & 6775349 & $\boldsymbol{V}$ & $\square$ & $\square$ & $\nabla$ & $\square$ \\
\hline 3134 & 3633 & Water well & $4 / 1 / 1969$ & 180.0 & 347929 & 6775359 & $\checkmark$ & $\square$ & $\square$ & $\nabla$ & $\square$ \\
\hline 3135 & 3475 & Water well & 7/1/1983 & 150.0 & 348507 & 6775551 & $\boldsymbol{V}$ & $\square$ & $\square$ & $\nabla$ & $\square$ \\
\hline 3135 & 3477 & Water well & 6/4/1976 & 425.0 & 348655 & 6775513 & $\checkmark$ & $\square$ & $\square$ & $\square$ & $\square$ \\
\hline 3135 & 3478 & Water well & $8 / 14 / 1979$ & 182.0 & 348236 & 6775531 & $\boldsymbol{V}$ & $\square$ & $\square$ & $\nabla$ & $\square$ \\
\hline 3135 & 3479 & Water well & 8/8/1981 & 223.4 & 348116 & 6775537 & $\boldsymbol{v}$ & $\square$ & $\square$ & $\sqrt{ }$ & $\square$ \\
\hline 3135 & 3495 & Water well & 8/1/1979 & 136.0 & 348179 & 6774914 & $\boldsymbol{V}$ & $\square$ & $\square$ & $\square$ & $\square$ \\
\hline 3135 & 3608 & Water well & $5 / 30 / 1975$ & 122.0 & 348432 & 6774841 & 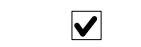 & $\square$ & $\square$ & $\nabla$ & $\square$ \\
\hline 3135 & 3619 & Water well & 4/19/1979 & 310.0 & 348552 & 6775208 & $\nabla$ & $\square$ & $\square$ & $\nabla$ & $\square$ \\
\hline 3135 & 3629 & Water well & $11 / 1 / 1964$ & 183.0 & 348481 & 6775304 & $\boldsymbol{V}$ & $\square$ & $\square$ & $\nabla$ & $\square$ \\
\hline 3135 & 3638 & Water well & $7 / 15 / 1965$ & 103.0 & 348442 & 6775430 & 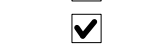 & $\square$ & $\square$ & $\checkmark$ & $\square$ \\
\hline 3135 & 3639 & Water well & 1/7/1966 & 179.0 & 348218 & 6775439 & $\boldsymbol{V}$ & $\square$ & $\square$ & $\nabla$ & $\square$ \\
\hline 3135 & 3646 & Water well & $10 / 5 / 1979$ & 191.0 & 348835 & 6775506 & $\checkmark$ & $\square$ & $\square$ & $\checkmark$ & $\square$ \\
\hline 3136 & 2172 & Hollow stem borehole w/SPT & $3 / 6 / 1996$ & 24.0 & 349654 & 6774787 & $\boldsymbol{V}$ & $\nabla$ & $\square$ & $\square$ & $\square$ \\
\hline 3136 & 3481 & Water well & $10 / 28 / 1981$ & 137.0 & 349445 & 6775387 & $\boldsymbol{V}$ & $\square$ & $\square$ & $\nabla$ & $\square$ \\
\hline 3136 & 3484 & Water well & 10/7/1982 & 120.0 & 349458 & 6775324 & $\boldsymbol{V}$ & $\square$ & $\square$ & $\nabla$ & $\square$ \\
\hline 3136 & 3485 & Water well & $11 / 10 / 1976$ & 106.0 & 349546 & 6775289 & $\nabla$ & $\square$ & $\square$ & $\nabla$ & $\square$ \\
\hline 3136 & 3487 & Water well & $4 / 17 / 1981$ & 108.0 & 349486 & 6775292 & $\boldsymbol{V}$ & $\square$ & $\square$ & $\nabla$ & $\square$ \\
\hline 3136 & 3488 & Water well & 6/14/1976 & 125.0 & 349440 & 6775263 & $\boldsymbol{V}$ & $\square$ & $\square$ & $\nabla$ & $\square$ \\
\hline 3136 & 3489 & Water well & 9/24/1980 & 300.0 & 349200 & 6775273 & 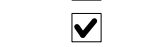 & $\square$ & $\square$ & $\square$ & $\square$ \\
\hline 3136 & 3490 & Water well & $8 / 28 / 1975$ & 102.0 & 349212 & 6775211 & $\nabla$ & $\square$ & $\square$ & $\square$ & $\square$ \\
\hline 3136 & 3491 & Water well & 7/10/1973 & 152.0 & 349256 & 6775178 & 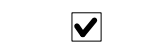 & $\square$ & $\square$ & $\nabla$ & $\square$ \\
\hline 3136 & 3493 & Water well & $8 / 1 / 1981$ & 200.0 & 349045 & 6775156 & $\boldsymbol{V}$ & $\square$ & $\square$ & $\nabla$ & $\square$ \\
\hline 3136 & 3494 & Water well & $5 / 1 / 1977$ & 167.0 & 348981 & 6775066 & $\boldsymbol{V}$ & $\square$ & $\square$ & $\nabla$ & $\square$ \\
\hline 3136 & 3605 & Water well & 7/1/1968 & 172.0 & 349314 & 6774773 & $\boldsymbol{V}$ & $\square$ & $\square$ & $\nabla$ & $\square$ \\
\hline 3136 & 3612 & Water well & 8/25/1978 & 270.0 & 349439 & 6774891 & $\boldsymbol{V}$ & $\square$ & $\square$ & $\checkmark$ & $\square$ \\
\hline 3136 & 3613 & Water well & 2/1/1967 & 154.0 & 349021 & 6774940 & 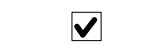 & $\square$ & $\square$ & $\nabla$ & $\square$ \\
\hline 3136 & 3615 & Water well & $12 / 5 / 1980$ & 176.0 & 348934 & 6775006 & 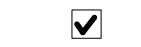 & $\square$ & $\square$ & $\nabla$ & $\square$ \\
\hline 3136 & 3616 & Water well & $3 / 17 / 1979$ & 138.0 & 349564 & 6775010 & 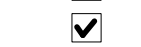 & $\square$ & $\square$ & $\square$ & $\square$ \\
\hline 3136 & 3623 & Water well & $6 / 24 / 1982$ & 101.0 & 349617 & 6775194 & 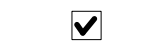 & $\square$ & $\square$ & $\bar{v}$ & $\square$ \\
\hline 3136 & 3624 & Water well & $12 / 14 / 1982$ & 120.0 & 349512 & 6775198 & $\nabla$ & $\square$ & $\square$ & $\nabla$ & $\square$ \\
\hline 3136 & 3626 & Water well & 9/11/1978 & 148.0 & 349123 & 6775215 & $\boldsymbol{v}$ & $\square$ & $\square$ & $\square$ & $\square$ \\
\hline 3136 & 3632 & Water well & $10 / 30 / 1982$ & 106.0 & 349636 & 6775286 & $\boldsymbol{V}$ & $\square$ & $\square$ & $\nabla$ & $\square$ \\
\hline
\end{tabular}




\begin{tabular}{|c|c|c|c|c|c|c|c|c|c|c|c|}
\hline \multirow[b]{2}{*}{$\begin{array}{c}\text { MOA } \\
\text { grid }\end{array}$} & \multirow[b]{2}{*}{$\begin{array}{c}\text { DGGS } \\
\text { number }\end{array}$} & \multirow[b]{2}{*}{ Hole Type } & \multirow[b]{2}{*}{$\begin{array}{c}\text { Date } \\
\text { Completed }\end{array}$} & \multirow[b]{2}{*}{$\begin{array}{c}\text { Total } \\
\text { depth (ft) }\end{array}$} & \multicolumn{2}{|c|}{ UTM coordinates (m) } & \multicolumn{5}{|c|}{ Data entered } \\
\hline & & & & & East & North & Lithology & SPT & $\begin{array}{c}\text { Sample } \\
\text { test }\end{array}$ & $\begin{array}{l}\text { Water } \\
\text { level }\end{array}$ & Velocity \\
\hline 3136 & 3634 & Water well & $7 / 11 / 1976$ & 133.0 & 349263 & 6775333 & $\checkmark$ & $\square$ & $\square$ & $\nabla$ & $\square$ \\
\hline 3136 & 3635 & Water well & $6 / 15 / 1977$ & 103.0 & 349549 & 6775351 & $\checkmark$ & $\square$ & $\square$ & $\sqrt{ }$ & $\square$ \\
\hline 3136 & 3636 & Water well & $5 / 15 / 1982$ & 140.0 & 349640 & 6775379 & $\boldsymbol{V}$ & $\square$ & $\square$ & $\nabla$ & $\square$ \\
\hline 3136 & 3637 & Water well & 9/3/1982 & 106.0 & 349235 & 6775396 & $\boldsymbol{V}$ & $\square$ & $\square$ & $\square$ & $\square$ \\
\hline 3136 & 3642 & Water well & 7/19/1977 & 123.0 & 349659 & 6775470 & $\checkmark$ & $\square$ & $\square$ & $\nabla$ & $\square$ \\
\hline 3136 & 3643 & Water well & $8 / 20 / 1974$ & 121.0 & 349584 & 6775474 & $\boldsymbol{V}$ & $\square$ & $\square$ & $\nabla$ & $\square$ \\
\hline 3136 & 3644 & Water well & 8/27/1977 & 107.0 & 349449 & 6775479 & $\checkmark$ & $\square$ & $\square$ & $\checkmark$ & $\square$ \\
\hline 3136 & 3645 & Water well & $12 / 12 / 1977$ & 109.0 & 349269 & 6775487 & $\boldsymbol{V}$ & $\square$ & $\square$ & $\nabla$ & $\square$ \\
\hline 3136 & 3649 & Water well & 6/6/1975 & 120.0 & 349481 & 6775509 & $\boldsymbol{V}$ & $\square$ & $\square$ & $\nabla$ & $\square$ \\
\hline 3136 & 3650 & Water well & $6 / 24 / 1974$ & 129.0 & 349197 & 6775552 & $\boldsymbol{V}$ & $\square$ & $\square$ & $\nabla$ & $\square$ \\
\hline 3137 & 2171 & Hollow stem borehole w/SPT & $1 / 31 / 1996$ & 25.0 & 349677 & 6774867 & $\boldsymbol{V}$ & $\nabla$ & $\square$ & $\square$ & $\square$ \\
\hline 3137 & 3480 & Water well & $12 / 14 / 1981$ & 126.9 & 350446 & 6775282 & $\boldsymbol{V}$ & $\square$ & $\square$ & $\nabla$ & $\square$ \\
\hline 3137 & 3492 & Water well & 9/29/1980 & 170.0 & 349823 & 6775092 & $\boldsymbol{V}$ & $\square$ & $\square$ & $\nabla$ & $\square$ \\
\hline 3137 & 3606 & Water well & $7 / 28 / 1977$ & 298.0 & 350334 & 6774760 & 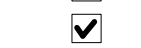 & $\square$ & $\square$ & $\checkmark$ & $\square$ \\
\hline 3137 & 3607 & Water well & $9 / 2 / 1981$ & 220.0 & 350199 & 6774766 & $\boldsymbol{V}$ & $\square$ & $\square$ & $\square$ & $\square$ \\
\hline 3137 & 3609 & Water well & $10 / 29 / 1970$ & 400.0 & 350230 & 6774796 & $\square$ & $\square$ & $\square$ & $\checkmark$ & $\square$ \\
\hline 3137 & 3614 & Water well & $6 / 1 / 1965$ & 122.0 & 350295 & 6774917 & $\square$ & $\square$ & $\square$ & $\nabla$ & $\square$ \\
\hline 3138 & 3471 & Water well & $5 / 29 / 1981$ & 128.0 & 350694 & 6775489 & 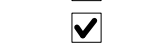 & $\square$ & $\square$ & $\nabla$ & $\square$ \\
\hline 3138 & 3474 & Water well & $3 / 26 / 1983$ & 196.0 & 350947 & 6775416 & $\boldsymbol{V}$ & $\square$ & $\square$ & $\square$ & $\square$ \\
\hline 3138 & 3476 & Water well & $10 / 17 / 1980$ & 174.0 & 350732 & 6775301 & $\boldsymbol{V}$ & $\square$ & $\square$ & $\nabla$ & $\square$ \\
\hline 3138 & 3483 & Water well & $3 / 17 / 1983$ & 182.0 & 350982 & 6775197 & $\boldsymbol{V}$ & $\square$ & $\square$ & $\nabla$ & $\square$ \\
\hline 3138 & 3610 & Water well & 8/7/1970 & 127.0 & 351026 & 6774824 & 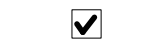 & $\square$ & $\square$ & $\nabla$ & $\square$ \\
\hline 3138 & 3611 & Water well & 8/14/1970 & 119.0 & 350817 & 6774833 & 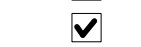 & $\square$ & $\square$ & $\nabla$ & $\square$ \\
\hline 3138 & 3618 & Water well & $6 / 30 / 1976$ & 117.0 & 351128 & 6775098 & $\nabla$ & $\square$ & $\square$ & $\nabla$ & $\square$ \\
\hline 3138 & 3621 & Water well & $5 / 22 / 1976$ & 123.0 & 351220 & 6775126 & 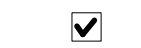 & $\square$ & $\square$ & $\nabla$ & $\square$ \\
\hline 3138 & 3631 & Water well & 9/14/1981 & 161.0 & 351058 & 6775225 & $\boldsymbol{V}$ & $\square$ & $\square$ & $\nabla$ & $\square$ \\
\hline 3138 & 3647 & Water well & $8 / 11 / 1980$ & 139.0 & 350888 & 6775449 & $\boldsymbol{V}$ & $\square$ & $\square$ & $\square$ & $\square$ \\
\hline 3138 & 3648 & Water well & $8 / 13 / 1982$ & 124.0 & 350649 & 6775459 & $\boldsymbol{V}$ & $\square$ & $\square$ & $\nabla$ & $\square$ \\
\hline 3139 & 2080 & Hollow stem borehole w/SPT & $2 / 28 / 1983$ & 50.5 & 351798 & 6775098 & $\nabla$ & $\checkmark$ & $\checkmark$ & $\checkmark$ & $\square$ \\
\hline 3139 & 2081 & Hollow stem borehole w/SPT & $3 / 1 / 1983$ & 50.5 & 351887 & 6775119 & 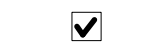 & $\nabla$ & $\nabla$ & $\nabla$ & $\square$ \\
\hline 3139 & 3482 & Water well & $1 / 1 / 1976$ & 138.0 & 351475 & 6775145 & $\checkmark$ & $\square$ & $\square$ & $\square$ & $\square$ \\
\hline 3139 & 3486 & Water well & $1 / 1 / 1975$ & 134.0 & 351443 & 6775085 & 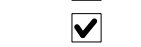 & $\square$ & $\square$ & $\checkmark$ & $\square$ \\
\hline 3139 & 3620 & Water well & $5 / 14 / 1976$ & 118.0 & 351339 & 6775121 & 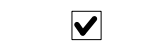 & $\square$ & $\square$ & $\bar{v}$ & $\square$ \\
\hline 3139 & 3640 & Water well & $3 / 6 / 1973$ & 175.0 & 351543 & 6775329 & $\nabla$ & $\square$ & $\square$ & $\nabla$ & $\square$ \\
\hline 3139 & 3641 & Water well & 7/5/1974 & 175.0 & 351575 & 6775389 & $\sqrt{ }$ & $\square$ & $\square$ & $\nabla$ & $\square$ \\
\hline 3235 & 3501 & Water well & 7/15/1977 & 125.0 & 348484 & 6774313 & $\square$ & $\square$ & $\square$ & $\nabla$ & $\square$ \\
\hline
\end{tabular}




\begin{tabular}{|c|c|c|c|c|c|c|c|c|c|c|c|}
\hline \multirow[b]{2}{*}{$\begin{array}{c}\text { MOA } \\
\text { grid }\end{array}$} & \multirow[b]{2}{*}{$\begin{array}{c}\text { DGGS } \\
\text { number }\end{array}$} & \multirow[b]{2}{*}{ Hole Type } & \multirow[b]{2}{*}{$\begin{array}{c}\text { Date } \\
\text { Completed }\end{array}$} & \multirow[b]{2}{*}{$\begin{array}{c}\text { Total } \\
\text { depth (ft) }\end{array}$} & \multicolumn{2}{|c|}{ UTM coordinates (m) } & \multicolumn{5}{|c|}{ Data entered } \\
\hline & & & & & East & North & Lithology & SPT & $\begin{array}{c}\text { Sample } \\
\text { test }\end{array}$ & $\begin{array}{l}\text { Water } \\
\text { level }\end{array}$ & Velocity \\
\hline 3235 & 3599 & Water well & 9/28/1977 & 390.0 & 348499 & 6774312 & $\checkmark$ & $\square$ & $\square$ & $\checkmark$ & $\square$ \\
\hline 3235 & 3601 & Water well & $7 / 2 / 1978$ & 330.0 & 348590 & 6774339 & $\square$ & $\square$ & $\square$ & $\nabla$ & $\square$ \\
\hline 3235 & 3602 & Water well & $4 / 12 / 1975$ & 350.0 & 348515 & 6774342 & $\nabla$ & $\square$ & $\square$ & $\checkmark$ & $\square$ \\
\hline 3236 & 2173 & Hollow stem borehole w/SPT & $3 / 15 / 1996$ & 30.2 & 349548 & 6774476 & $\boldsymbol{V}$ & $\checkmark$ & $\square$ & $\square$ & $\square$ \\
\hline 3236 & 2174 & Hollow stem borehole w/SPT & $3 / 19 / 1996$ & 35.5 & 349607 & 6774700 & $\checkmark$ & $\checkmark$ & $\nabla$ & $\square$ & $\square$ \\
\hline 3236 & 3585 & Water well & $5 / 31 / 1979$ & 533.0 & 348995 & 6773981 & $\square$ & $\square$ & $\square$ & $\nabla$ & $\square$ \\
\hline 3236 & 3594 & Water well & $5 / 18 / 1979$ & 373.0 & 349199 & 6774189 & $\square$ & $\square$ & $\square$ & $\checkmark$ & $\square$ \\
\hline 3236 & 3595 & Water well & $5 / 24 / 1979$ & 533.0 & 349004 & 6774197 & $\square$ & $\square$ & $\square$ & $\nabla$ & $\square$ \\
\hline 3236 & 3596 & Water well & $5 / 23 / 1979$ & 533.0 & 349005 & 6774228 & $\square$ & $\square$ & $\square$ & $V$ & $\square$ \\
\hline 3236 & 3598 & Water well & $5 / 14 / 1979$ & 373.0 & 348904 & 6774295 & $\square$ & $\square$ & $\square$ & $\nabla$ & $\square$ \\
\hline 3237 & 2077 & Water well & $12 / 16 / 1985$ & 301.0 & 350358 & 6774342 & 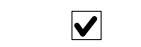 & $\square$ & $\square$ & $\checkmark$ & $\square$ \\
\hline 3237 & 2078 & Water well & $3 / 1 / 1985$ & 207.0 & 350306 & 6774354 & $\nabla$ & $\square$ & $\square$ & $\checkmark$ & $\square$ \\
\hline 3237 & 2079 & Water well & $2 / 15 / 1985$ & 207.0 & 350310 & 6774326 & $\boldsymbol{V}$ & $\square$ & $\square$ & $V$ & $\square$ \\
\hline 3237 & 3497 & Water well & 4/5/1982 & 405.0 & 350313 & 6774266 & 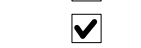 & $\square$ & $\square$ & $V$ & $\square$ \\
\hline 3238 & 3498 & Water well & $9 / 20 / 1975$ & 275.0 & 351209 & 6774166 & $\boldsymbol{V}$ & $\square$ & $\square$ & $\nabla$ & $\square$ \\
\hline 3238 & 3500 & Water well & $8 / 28 / 1976$ & 330.0 & 351071 & 6774110 & $\checkmark$ & $\square$ & $\square$ & $V$ & $\square$ \\
\hline 3238 & 3502 & Water well & $10 / 25 / 1984$ & 188.0 & 351126 & 6773983 & $\boldsymbol{v}$ & $\square$ & $\square$ & $V$ & $\square$ \\
\hline 3238 & 3586 & Water well & 7/27/1976 & 294.0 & 350899 & 6773931 & 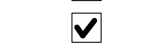 & $\square$ & $\square$ & $V$ & $\square$ \\
\hline 3238 & 3587 & Water well & 7/27/1979 & 105.0 & 350584 & 6773944 & $\boldsymbol{V}$ & $\square$ & $\square$ & $V$ & $\square$ \\
\hline 3238 & 3589 & Water well & 9/7/1981 & 175.0 & 351112 & 6774015 & $\boldsymbol{V}$ & $\square$ & $\square$ & $\nabla$ & $\square$ \\
\hline 3238 & 3590 & Water well & $5 / 14 / 1979$ & 318.0 & 350992 & 6774020 & $\boldsymbol{V}$ & $\square$ & $\square$ & $\checkmark$ & $\square$ \\
\hline 3238 & 3591 & Water well & $11 / 28 / 1975$ & 117.0 & 350693 & 6774032 & $\square$ & $\square$ & $\square$ & $v$ & $\square$ \\
\hline 3238 & 3593 & Water well & 9/29/1982 & 140.0 & 350996 & 6774113 & 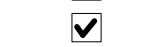 & $\square$ & $\square$ & 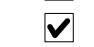 & $\square$ \\
\hline 3238 & 3597 & Water well & $10 / 2 / 1978$ & 238.0 & 350506 & 6774226 & $\nabla$ & $\square$ & $\square$ & $\square$ & $\square$ \\
\hline 3238 & 3603 & Water well & 11/1/1981 & 200.0 & 350819 & 6774523 & 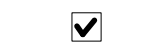 & $\square$ & $\square$ & $V$ & $\square$ \\
\hline 3238 & 3604 & Water well & $12 / 2 / 1972$ & 200.0 & 351016 & 6774577 & $\boldsymbol{V}$ & $\square$ & $\square$ & $V$ & $\square$ \\
\hline 3241 & 3496 & Water well & $11 / 13 / 1975$ & 155.0 & 353217 & 6774112 & $\boldsymbol{V}$ & $\square$ & $\square$ & 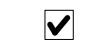 & $\square$ \\
\hline 3241 & 3588 & Water well & $3 / 31 / 1975$ & 125.0 & 353042 & 6773872 & $\boldsymbol{V}$ & $\square$ & $\square$ & $\nabla$ & $\square$ \\
\hline 3242 & 3499 & Water well & $3 / 10 / 1984$ & 162.0 & 353746 & 6773843 & $\boldsymbol{V}$ & $\square$ & $\square$ & $\nabla$ & $\square$ \\
\hline 3336 & 3505 & Water well & 8/9/1984 & 248.0 & 349560 & 6773864 & 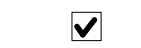 & $\square$ & $\square$ & $\nabla$ & $\square$ \\
\hline 3336 & 3506 & Water well & $6 / 2 / 1983$ & 255.0 & 349451 & 6773776 & 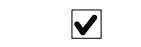 & $\square$ & $\square$ & $\nabla$ & $\square$ \\
\hline 3336 & 3508 & Water well & $11 / 28 / 1983$ & 120.0 & 349521 & 6773649 & 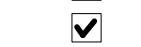 & $\square$ & $\square$ & $V$ & $\square$ \\
\hline 3336 & 3510 & Water well & $5 / 18 / 1979$ & 217.0 & 349067 & 6773575 & $\square$ & $\square$ & $\square$ & 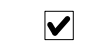 & $\square$ \\
\hline 3336 & 3512 & Water well & $5 / 17 / 1982$ & 250.0 & 348932 & 6773581 & $\checkmark$ & $\square$ & $\square$ & $\square$ & $\square$ \\
\hline 3336 & 3513 & Water well & 8/27/1978 & 440.0 & 349137 & 6773448 & $\square$ & $\square$ & $\square$ & 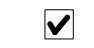 & $\square$ \\
\hline 3336 & 3514 & Water well & $5 / 23 / 1984$ & 180.0 & 348837 & 6773461 & $\boldsymbol{V}$ & $\square$ & $\square$ & 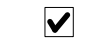 & $\square$ \\
\hline
\end{tabular}




\begin{tabular}{|c|c|c|c|c|c|c|c|c|c|c|c|}
\hline \multirow[b]{2}{*}{$\begin{array}{l}\text { MOA } \\
\text { grid }\end{array}$} & \multirow[b]{2}{*}{$\begin{array}{l}\text { DGGS } \\
\text { number }\end{array}$} & \multirow[b]{2}{*}{ Hole Type } & \multirow[b]{2}{*}{$\begin{array}{c}\text { Date } \\
\text { Completed }\end{array}$} & \multirow[b]{2}{*}{$\begin{array}{c}\text { Total } \\
\text { depth }(\mathrm{ft})\end{array}$} & \multicolumn{2}{|c|}{ UTM coordinates (m) } & \multicolumn{5}{|c|}{ Data entered } \\
\hline & & & & & East & North & Lithology & SPT & $\begin{array}{c}\text { Sample } \\
\text { test }\end{array}$ & $\begin{array}{l}\text { Water } \\
\text { level }\end{array}$ & Velocity \\
\hline 3336 & 3516 & Water well & $11 / 14 / 1977$ & 192.0 & 349136 & 6773417 & $\square$ & $\square$ & $\square$ & $\checkmark$ & $\square$ \\
\hline 3336 & 3517 & Water well & $8 / 1 / 1982$ & 200.0 & 349134 & 6773386 & $\checkmark$ & $\square$ & $\square$ & $\square$ & $\square$ \\
\hline 3336 & 3519 & Water well & $10 / 10 / 1979$ & 120.0 & 349144 & 6773262 & $\checkmark$ & 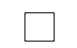 & $\square$ & 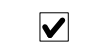 & \\
\hline 3336 & 3520 & Water well & $1 / 20 / 1978$ & 200.0 & 349054 & 6773266 & $\square$ & $\square$ & $\square$ & $\checkmark$ & $\square$ \\
\hline 3336 & 3570 & Water well & $5 / 30 / 1976$ & 266.0 & 349444 & 6773249 & $\checkmark$ & $\square$ & $\square$ & $\checkmark$ & $\square$ \\
\hline 3336 & 3573 & Water well & $6 / 11 / 1976$ & 198.0 & 349550 & 6773276 & $\checkmark$ & $\square$ & $\square$ & $\checkmark$ & $\square$ \\
\hline 3336 & 3574 & Water well & $7 / 22 / 1983$ & 175.0 & 349466 & 6773403 & $\checkmark$ & $\square$ & $\square$ & $\checkmark$ & $\square$ \\
\hline 3336 & 3575 & Water well & 9/17/1982 & 265.0 & 349063 & 6773482 & $\checkmark$ & 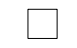 & $\square$ & $\nabla$ & $\square$ \\
\hline 3336 & 3576 & Water well & $10 / 8 / 1981$ & 340.0 & 349474 & 6773589 & $\checkmark$ & $\square$ & $\square$ & $\sqrt{\square}$ & $\square$ \\
\hline 3336 & 3578 & Water well & $5 / 20 / 1982$ & 305.0 & 348865 & 6773769 & $\checkmark$ & & $\square$ & $\checkmark$ & $\square$ \\
\hline 3337 & 3504 & Water well & $9 / 27 / 1982$ & 160.0 & 349681 & 6773890 & $\checkmark$ & $\square$ & $\square$ & $\square$ & $\square$ \\
\hline 3337 & 3509 & Water well & $8 / 13 / 1985$ & 285.0 & 349683 & 6773580 & $\checkmark$ & & $\square$ & $\checkmark$ & $\square$ \\
\hline 3337 & 3568 & Water well & $8 / 26 / 1981$ & 135.0 & 350355 & 6773148 & $\checkmark$ & $\square$ & $\square$ & $\nabla$ & $\square$ \\
\hline 3337 & 3579 & Water well & $6 / 22 / 1978$ & 132.0 & 350380 & 6773736 & $\square$ & $\square$ & $\square$ & $\nabla$ & $\square$ \\
\hline 3337 & 3580 & Water well & $6 / 22 / 1978$ & 180.0 & 350276 & 6773740 & $\square$ & $\square$ & $\square$ & $\checkmark$ & $\square$ \\
\hline 3337 & 3581 & Water well & 7/14/1978 & 310.0 & 350185 & 6773744 & $\square$ & $\square$ & $\square$ & $\checkmark$ & $\square$ \\
\hline 3337 & 3582 & Water well & $8 / 13 / 1982$ & 200.0 & 349661 & 6773767 & $\checkmark$ & & $\square$ & $\boldsymbol{V}$ & $\square$ \\
\hline 3338 & 3567 & Water well & 9/5/1980 & 226.0 & 350460 & 6773144 & $\checkmark$ & ᄂ & $\square$ & $\vec{\nabla}$ & $\square$ \\
\hline 3340 & 3503 & Water well & $11 / 1 / 1982$ & 102.0 & 352509 & 6773677 & $\checkmark$ & $\square$ & $\square$ & $\nabla$ & $\square$ \\
\hline 3340 & 3507 & Water well & $11 / 1 / 1983$ & 112.0 & 352768 & 6773419 & $\checkmark$ & $\square$ & $\square$ & $\checkmark$ & $\square$ \\
\hline 3340 & 3572 & Water well & $6 / 1 / 1979$ & 127.0 & 352742 & 6773141 & $\square$ & & $\square$ & $\bar{\nabla}$ & $\square$ \\
\hline 3340 & 3577 & Water well & $6 / 25 / 1976$ & 108.0 & 352671 & 6773609 & $\checkmark$ & $\square$ & $\square$ & $\square$ & $\square$ \\
\hline 3341 & 3515 & Water well & $7 / 18 / 1984$ & 185.0 & 353186 & 6772999 & $\checkmark$ & & $\square$ & $\square$ & $\square$ \\
\hline 3341 & 3571 & Water well & 9/25/1979 & 112.0 & 352967 & 6773132 & $\square$ & $\square$ & $\square$ & $\checkmark$ & $\square$ \\
\hline 3437 & 3559 & Water well & $10 / 15 / 1981$ & 140.0 & 350252 & 6772843 & $\checkmark$ & & $\square$ & $\bar{\nabla}$ & $\square$ \\
\hline 3437 & 3560 & Water well & $8 / 14 / 1983$ & 190.0 & 350242 & 6772937 & $\checkmark$ & & $\square$ & $\nabla$ & $\square$ \\
\hline 3437 & 3563 & Water well & 7/10/1976 & 180.0 & 350151 & 6772940 & $\square$ & $\square$ & $\square$ & $\checkmark$ & $\square$ \\
\hline 3437 & 3565 & Water well & 8/11/1979 & 140.0 & 350246 & 6773029 & $\square$ & & $\square$ & $\nabla$ & $\square$ \\
\hline 3438 & 3522 & Water well & $10 / 16 / 1983$ & 225.0 & 351136 & 6772434 & $\checkmark$ & $\square$ & $\square$ & $\checkmark$ & $\square$ \\
\hline 3438 & 3523 & Water well & $6 / 30 / 1983$ & 225.0 & 351114 & 6772280 & $\checkmark$ & $\square$ & $\square$ & 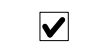 & $\square$ \\
\hline 3438 & 3561 & Water well & $6 / 13 / 1978$ & 205.0 & 350856 & 6772911 & $\square$ & $\square$ & $\square$ & $\nabla$ & $\square$ \\
\hline 3438 & 3564 & Water well & $7 / 27 / 1981$ & 162.0 & 350949 & 6772999 & $\checkmark$ & $\square$ & $\square$ & $\checkmark$ & $\square$ \\
\hline 3439 & 3521 & Water well & $6 / 16 / 1981$ & 180.0 & 351438 & 6772483 & $\checkmark$ & $\square$ & $\square$ & $\nabla$ & $\square$ \\
\hline 3439 & 3557 & Water well & 7/10/1981 & 280.0 & 351330 & 6772395 & $\checkmark$ & $\square$ & $\square$ & $\nabla$ & $\square$ \\
\hline 3439 & 3558 & Water well & $1 / 8 / 1982$ & 112.0 & 351438 & 6772483 & $\checkmark$ & $\square$ & $\square$ & $\square$ & $\square$ \\
\hline 3536 & 1634 & Pit or trench & 12/19/1996 & 15.0 & 349396 & 6771941 & $\square$ & $\square$ & $\square$ & $\square$ & $\square$ \\
\hline
\end{tabular}




\begin{tabular}{|c|c|c|c|c|c|c|c|c|c|c|c|}
\hline \multirow[b]{2}{*}{$\begin{array}{c}\text { MOA } \\
\text { grid }\end{array}$} & \multirow[b]{2}{*}{$\begin{array}{c}\text { DGGS } \\
\text { number }\end{array}$} & \multirow[b]{2}{*}{ Hole Type } & \multirow[b]{2}{*}{$\begin{array}{c}\text { Date } \\
\text { Completed }\end{array}$} & \multirow[b]{2}{*}{$\begin{array}{c}\text { Total } \\
\text { depth }(\mathrm{ft})\end{array}$} & \multicolumn{2}{|c|}{ UTM coordinates (m) } & \multicolumn{5}{|c|}{ Data entered } \\
\hline & & & & & East & North & Lithology & SPT & $\begin{array}{c}\text { Sample } \\
\text { test }\end{array}$ & $\begin{array}{c}\text { Water } \\
\text { level }\end{array}$ & Velocity \\
\hline 3536 & 2004 & Natural exposure & $5 / 31 / 1997$ & 49.0 & 349241 & 6772150 & $\checkmark$ & $\square$ & $\square$ & $\square$ & $\square$ \\
\hline 3536 & 3529 & Water well & $3 / 11 / 1986$ & 330.0 & 349482 & 6771668 & $\checkmark$ & $\square$ & $\square$ & $\checkmark$ & $\square$ \\
\hline 3537 & 3547 & Water well & $5 / 15 / 1984$ & 305.0 & 349887 & 6771650 & $\nabla$ & $\square$ & $\square$ & $\nabla$ & $\square$ \\
\hline 3538 & 3524 & Water well & $5 / 3 / 1983$ & 255.0 & 350884 & 6772166 & $\nabla$ & $\square$ & $\square$ & $V$ & $\square$ \\
\hline 3538 & 3525 & Water well & $5 / 12 / 1983$ & 185.0 & 350778 & 6772139 & $\checkmark$ & $\square$ & $\square$ & $\checkmark$ & $\square$ \\
\hline 3538 & 3526 & Water well & $11 / 16 / 1978$ & 150.0 & 351121 & 6772094 & $\square$ & $\square$ & $\square$ & $\checkmark$ & $\square$ \\
\hline 3538 & 3527 & Water well & $5 / 10 / 1985$ & 105.0 & 350523 & 6772150 & $\checkmark$ & $\square$ & $\square$ & $V$ & $\square$ \\
\hline 3538 & 3528 & Water well & $8 / 10 / 1985$ & 149.0 & 351114 & 6771909 & 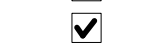 & $\square$ & $\square$ & $V$ & $\square$ \\
\hline 3538 & 3548 & Water well & $10 / 20 / 1979$ & 305.0 & 350608 & 6772023 & $\square$ & $\square$ & $\square$ & 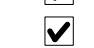 & $\square$ \\
\hline 3538 & 3549 & Water well & 9/19/1978 & 160.0 & 350518 & 6772026 & $\square$ & $\square$ & $\square$ & $V$ & $\square$ \\
\hline 3538 & 3550 & Water well & $11 / 19 / 1979$ & 198.0 & 350398 & 6772031 & $\nabla$ & $\square$ & $\square$ & $\checkmark$ & $\square$ \\
\hline 3538 & 3551 & Water well & $5 / 22 / 1973$ & 525.0 & 350984 & 6772038 & $\checkmark$ & $\square$ & $\square$ & $\checkmark$ & $\square$ \\
\hline 3538 & 3552 & Water well & $5 / 24 / 1973$ & 377.0 & 350521 & 6772088 & $\checkmark$ & $\square$ & $\square$ & $\square$ & $\square$ \\
\hline 3538 & 3553 & Water well & $1 / 20 / 1983$ & 276.0 & 350926 & 6772102 & $\checkmark$ & $\square$ & $\square$ & $V$ & $\square$ \\
\hline 3538 & 3554 & Water well & 9/10/1982 & 121.0 & 350836 & 6772106 & $\checkmark$ & $\square$ & $\square$ & $V$ & $\square$ \\
\hline 3538 & 3555 & Water well & 9/4/1976 & 230.0 & 350402 & 6772124 & $\square$ & $\square$ & $\square$ & $V$ & $\square$ \\
\hline 3539 & 3546 & Water well & 1/6/1977 & 253.0 & 351310 & 6771590 & 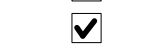 & $\square$ & $\square$ & $V$ & $\square$ \\
\hline 3637 & 3535 & Water well & $8 / 14 / 1981$ & 420.0 & 350148 & 6770741 & $\checkmark$ & $\square$ & $\square$ & $\square$ & $\square$ \\
\hline 3637 & 3537 & Water well & 7/7/1983 & 178.0 & 349976 & 6770934 & $\checkmark$ & $\square$ & $\square$ & $V$ & $\square$ \\
\hline 3638 & 3530 & Water well & 8/11/1981 & 520.0 & 351091 & 6771383 & 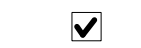 & $\square$ & $\square$ & $\square$ & $\square$ \\
\hline 3638 & 3544 & Water well & 4/12/1984 & 124.0 & 350398 & 6771319 & $\checkmark$ & $\square$ & $\square$ & $\square$ & $\square$ \\
\hline 3638 & 3545 & Water well & 8/6/1981 & 320.0 & 351002 & 6771387 & 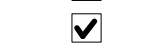 & $\square$ & $\square$ & $\square$ & $\square$ \\
\hline 3639 & 3531 & Water well & 7/17/1985 & 246.0 & 351593 & 6771176 & $V$ & $\square$ & $\square$ & $V$ & $\square$ \\
\hline 3639 & 3536 & Water well & $7 / 5 / 1983$ & 448.0 & 351865 & 6770855 & $\checkmark$ & $\square$ & $\square$ & $V$ & $\square$ \\
\hline 3639 & 3539 & Water well & $5 / 24 / 1970$ & 324.0 & 351582 & 6770897 & $\square$ & $\square$ & $\square$ & $v$ & $\square$ \\
\hline 3639 & 3541 & Water well & $7 / 21 / 1981$ & 280.0 & 351682 & 6771141 & 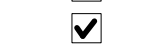 & $\square$ & $\square$ & $\square$ & $\square$ \\
\hline 3640 & 3538 & Water well & $7 / 21 / 1968$ & 125.0 & 352556 & 6770856 & $\boldsymbol{V}$ & $\square$ & $\square$ & $\square$ & $\square$ \\
\hline 3640 & 3540 & Water well & 4/23/1981 & 170.0 & 352309 & 6771053 & $\checkmark$ & $\square$ & $\square$ & $\square$ & $\square$ \\
\hline 3640 & 3542 & Water well & 7/24/1981 & 298.0 & 352208 & 6771150 & $\boldsymbol{V}$ & $\square$ & $\square$ & $V$ & $\square$ \\
\hline 3640 & 3543 & Water well & $5 / 4 / 1983$ & 361.0 & 352118 & 6771154 & 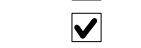 & $\square$ & $\square$ & $\square$ & $\square$ \\
\hline 3737 & 3532 & Water well & $10 / 20 / 1984$ & 209.0 & 350158 & 6770617 & $\checkmark$ & $\square$ & $\square$ & $\square$ & $\square$ \\
\hline 3740 & 3534 & Water well & 9/3/1977 & 306.0 & 351973 & 6770540 & $\square$ & $\square$ & $\square$ & $V$ & $\square$ \\
\hline
\end{tabular}

